NIST

PUBLICATIONS

\title{
PROCEEDINGS
}

\section{Workshop on Federal Programs Involving Supercritical Water Oxidation}

\author{
6-7 July 1992
}

Editor

Gregory J. Rosasco

U.S. DEPARTMENT OF COMMERCE Technology Administration

National Institute of Standards and Technology

Process Measurements Division

Gaithersburg, MD 20899

QC

. 456 



\section{PROCEEDINGS}

\section{Workshop on Federal Programs Involving Supercritical \\ Water Oxidation}

6-7 July 1992
Editor

Gregory J. Rosasco

U.S. DEPARTMENT OF COMMERCE Technology Administration National Institute of Standards and Technology Process Measurements Division Gaithersburg, MD 20899

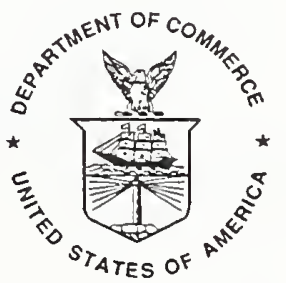

U.S. DEPARTMENT OF COMMERCE Barbara Hackman Franklin, Secretary TECHNOLOGY ADMINISTRATION Robert M. White, Under Secretary for Technology NATIONAL INSTITUTE OF STANDARDS

John W. Lyons, Director 


$$
\text { , }
$$




\title{
WORKSHOP ON FEDERAL PROGRAMS INVOLVING SUPERCRITICAL WATER OXIDATION
}

\author{
July $6-7,1992$ \\ National Institute of Standards and Technology \\ Gaithersburg, MD
}

Page

Executive Summary $\ldots \ldots \ldots \ldots \ldots \ldots \ldots \ldots$

scwo Track Chart $\ldots \ldots \ldots \ldots \ldots \ldots$

Chemical Reactions of Nitrogen Containing Compounds in Supercritical

Water, Dr. Steven J. Buelow, Los Alamos National Laboratory . . . . . 7

SCWO Experiments at Sandia National Laboratories,

Mr. Richard Steeper, Sandia National Laboratories . . . . . . . . . . . 46

DoE's Quest for Energy Recovery Using SCWO, Dr. Gideon Varga,

Department of Energy, Washington, DC . . . . . . . . . . . 58

Progress on the MODEC \& MODAR Supercritical Water Reactors,

Mr. Bob Chappell, DoE Idaho Operations . . . . . . . . . . . . 67

SCWO for Space Life Support Applications, Mr. T. Mark Hightower,

NASA Ames Research Center . . . . . . . . . . . . . . . 74

SCWO of Hazardous Waste

Mr. Richard E. Kirts, Naval Civil Engineering Laboratory . . . . . . . 111

SCWO System Development - Shipboard Applications,

Mr. Anthony T. Rodriguez, Naval Surface Warfare Center,

Carderock Division . . . . . . . . . . . . . . . . . . . . 138

Demilitarization R \& D Technology for Conventional Munitions

via SCWO of Colored Smokes, Dyes, and Pyrotechnics,

Mr. Crane Robinson, U.S. Army Armament Research, Development

and Engineering Center . . . . . . . . . . . . . . . . 160 
SCWO Decomposition of M31A1E1 Triple Base Propellant (US Army CERL), Dr. Roger Schneider, U.S. Army CERL

(Rho Sigma Associates) and Dr. Steven J. Buelow, Los Alamos

National Laboratory . . . . . . . . . . . . . . . . . . . . 176

Thermodynamics and Kinetics In SCWO Reactions,

Dr. Nina Bergan French, Sandia National Laboratory . . . . . . . . . 199

Interactive Computation of Thermophysical Properties for

Supercritical Aqueous Systems, Dr. J.M.H. Levelt Sengers and

Dr. Daniel Friend, National Institute of Standards and Technology . . 214

NIST Programs Relevant to SCWO, Dr. Gregory J. Rosasco,

National Institute of Standards and Technology . . . . . . . . . . . . . . 243

Army Research Office University Research Initiative in

SCWO--Unofficial Summary, Dr. Gregory J. Rosasco, National

Institute of Standards and Technology . . . . . . . . . . . . . 249

DARPA Supercritical Fluid Program, Dr. Richard Loda,

DARPA/DSO/MSD ....................... 255

Office of Environmental Restoration and Waste Management

Initiatives for Using Supercritical Water to Treat Mixed Wastes,

Dr. Hank Strauch, BDM International . . . . . . . . . . . . 287

List of Participants . . . . . . . . . . . . . . . . 300 


\section{EXECUTIVE SUMMARY}

\section{Background}

This Workshop on Federal Programs Involving Supercritical Water Oxidation was a follow-on to previous informal meetings held to discuss work in this area. The first was held at the Naval Civil Engineering Laboratory, Port Hueneme, CA in March 1990 and the second at Tyndall Air Force Base, FL in April 1991. As with past meetings, the focus of this Workshop was primarily programmatic not technical. Attendance at these meetings was by invitation, and by and large the list was determined by consultation with the Federal agency participants. To our knowledge all Agencies with a recent involvement in SCWO related technology were contacted. We received correspondence and information from a number of individuals or laboratories who were not able to attend either because of scheduling difficulties or requirements resulting from active contract negotiations. Input relevant to their interests was included in the presentations except in the case of the Environmental Protection Agency. In this regard, Mr. Ron Turner of the Risk Reduction Engineering Laboratory, Office of Research and Development, Cincinnati, OH informs us that an Engineering Bulletin on Supercritical Water Oxidation will be issued soon.

\section{Content of these proceedings}

The proceedings include the following:

- List of the attendees, their addresses and phone numbers

- Copies of the speakers' viewgraphs. Some speakers submitted additional material which also is included.

- an SCWO Track Chart which summarizes some of the organizations involved in SCWO technology development and their activities

The last item is derived from a viewgraph originally presented by Mr. Anthony Rodriguez of the Carderock Division of the Naval Surface Warfare Center. We circulated copies of this viewgraph for mark-up and return by the participants. However, the response was somewhat spotty. Therefore, while informative, this SCWO Track Chart is not all-inclusive either with regard to the participants or the nature of the activities in this field.

\section{Panel Discussion of Technical Challenges}

The final session in the Workshop was a Panel Discussion titled Technical Challenges in SCWO Implementation. The panel was composed of individuals who had personal familiarity with the design, development, operation, or evaluation of a SCWO reactor system (either direct hands-on experience or experience gained as a contract monitor). The panel did an excellent job in summarizing some of their experiences and concerns and in answering questions from the audience (which participated in the discussion at a very high level). 
There was no attempt to arrive at a formal consensus in terms of "technical challenges". However, many participants stressed the importance of a number of topics (in priority order as judged from the amount of discussion and a review of the taped proceedings):

1. materials performance (corrosion)

2. salt separation and reactor plugging

3. scale-up:

safety

economics-

energy costs

equipment costs

maintenance costs

lack of understanding, data, and validated models-

materials performance

phases (including kinetics of phase separation)

chemical kinetics

More detail concerning these topics will be presented below in an assessment of the research needs. However, first we note the absence of concern with respect to the destruction/removal efficiency (DRE) of the SCWO process for the waste materials of interest to the supporting agencies. This reflects the fact that the support for development of SCWO technology to this point has focussed principally on laboratory demonstrations of adequate DRE's for the agencyrelevant compounds. (In this context, it was observed by a number of participants that the successful demonstrations for agency-relevant wastes provide useful information which supports the use of this technology for treatment of a wide range of industrial, biological, and pharmacological waste streams.)

Further, we note that it is the view of most of those who have been involved in the development of SCWO that this is a technology "ripe for a successful demonstration project". This explains the fact that the current focus of the funding is on the construction of pilot facilities (two of these are in the $1000 \mathrm{gal} /$ day range). These facilities are seen as test beds critical for technology evaluation. Successful operation was seen as the enabler for an effort to select (or design) optimum technologies and for scale-up to even larger facilities $(50,000$ gal/day range facilities were discussed). The latter efforts, it was implied (hoped), would include work to enhance our understanding to a level commensurate with design goals, in terms of destruction efficiency, energy usage, reliability, throughput, safety, facility life-cycle, and 
maintenance. It was acknowledged that a firm basis for identifying and selecting technological alternatives and designing optimized SCWO reactors needs to be developed before this promising chemical process can be successfully deployed. While there are many uncertainties with respect to scale-up, there were consistent reports from many participants that the economics of SCWO-based remediation for a wide-spectrum of waste streams (including common industrial and municipal wastes) are very favorable.

Finally, we observe that a number of participants pointed out that the technical challenges enumerated above persist as major concerns in spite of claims by commercial proponents of the technology that these problems or issues have been "resolved". This observation led to the conclusion that there is a need for better communication among those involved in SCWO technology and that many problems are shared by all interested parties and thus there can be and should be common solutions to these problems.

\section{Research to Address Technical Issues}

In the following we summarize in more detail the discussion of the technical issues and the associated research needs which came up during the Workshop and in particular during the Panel session. Materials performance concerns focussed primarily on corrosion of the metals used for construction of these high pressure reactor vessels. There was also some discussion of designs based on corrosion resistant thin liners, e.g. gold, or coatings and designs based on having sacrificial sections in the reactor, which would be made of inexpensive materials replaced on a regular basis, and also of hybrid constructions using metals, elastomers, and ceramic liners. It is accurate to observe that little is known about materials performance in these types of environments. Further, it should be remembered that typical reactor systems provide a very large range of corrosion environments including regions with large chemical, thermal, and phase gradients along with conditions which could lead to stress corrosion cracking and fatigue. High density, high dielectric constant conditions with large ion concentrations are encountered as are conditions in the gas phase with strong oxidative chemistry in progress. Halides and sulfates are common and carbonates are ubiquitous in these systems. The presence of salt layers associated with phase separation and the possibility of surface catalyzed reactions also were considered important. In spite of these complexities, there were strong sentiments that the problems were not insurmountable. There appeared to be universal agreement that systematic studies were required and that microscopic examination and in situ characterization would be invaluable.

In designing large-scale reactors, information will be required about the location of the fluid-fluid and especially the fluid-solid phase boundaries. Crossing a phase boundary causes a phase separation which, in fact, is a vital aspect in the selection of SCWO technology for many of the agency-relevant wastes streams. However, fluid-solid phase boundaries, if encountered in the wrong region of the reactor also can lead to the observed reactor plugging by deposition of solids. Fluid-fluid phase boundaries will lead to the formation of brines which can greatly enhance corrosion as can the formation of salt layers. The kinetics of phase separations affects the carry-over of salts in the effluent stream. Phase behavior is thus 
intimately connected to the salt separation and reactor plugging issues. It is also important to the chemical behavior of the system. The formation of multiple phases in these inherently multicomponent streams can lead to demixing of reactants and reductions of efficiency. There was some speculation that demixing could lead to potentially dangerous reactant compositions (e.g. hot spots associated with hydrothermal flames and even the possibility of explosive conditions were discussed).

The NIST experts in this area stressed that modelling of phase separation in binary and multicomponent solutions is quite feasible; however, there currently is no modelling effort focussed on the conditions important to SCWO technology. The experts agree that validation by direct observation of phase separation in representative systems is a necessity. Thermophysical property data, enthalpy, density, viscosity, diffusivity, etc., are required for the design of all aspects of preparation of the reactants, the dimensions of reaction sections, and for design of heat recovery and cool down systems. There are few data available for the multicomponent systems of interest to SCWO; however, the prospects of developing a useful model appear good.

We are still lamentably lacking in understanding of the chemistry in a SCWO reactor. By and large, microscopic chemical kinetic models based on radical mechanisms have not been successful in predicting the observed destruction efficiencies. The large range of conditions encountered in typical flow reactor systems raises many important questions with respect to the chemistry. Catalytic effects associated with metal surfaces have been observed; however their contribution to the overall process efficiency is not understood. Conditions for both radical and ionic chemistry are encountered in typical process streams; the importance of each is not known. Stabilization against pyrolysis has been observed, but there is no basis for predicting behavior for the many systems of interest to this technology. It is difficult to specify the important process parameters, concentrations of reactants, temperatures, residence times, and perhaps even pressure, so as to warrant optimal performance in terms of DRE's, energy, and materials performance. In anticipation of this lack of predictive capability, practitioners are forced to design reactors that operate for longer residence times or at more extreme conditions of temperature with the potential for higher cost and higher risk.

All the above topics become increasingly more important as we talk about scale-up to either continuously operating or larger throughput systems. The risks also increase as we push in these directions. There is still a sense within the community that a major failure, either in terms of meeting DRE goals, or economic efficiency goals, and especially in terms of safety would do great harm to the future of this very promising technology. The need to understand the process and the processing system, in terms of materials behavior, phase behavior, or chemical behavior, came up over and over again each time the issues of failure avoidance and wide-scale deployment were raised. 
It was clear that SCWO processing holds great promise for waste treatment. As mentioned above, there is substantial confidence in the success of the pilot facilities currently under construction. It is hoped that these successes will provide the basis for the important scientific and engineering studies needed to translate this technology into a reality for safe, efficient hazardous waste treatment. 


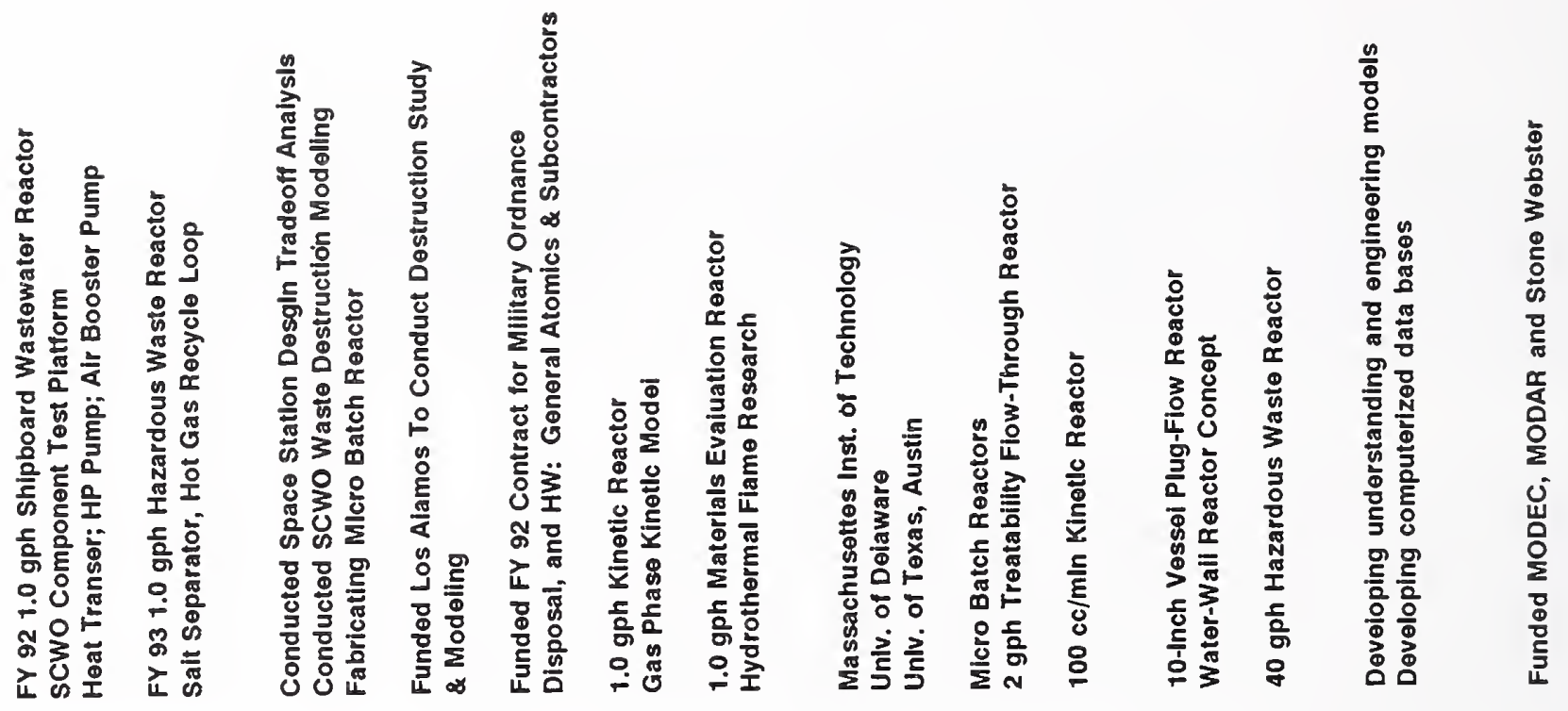

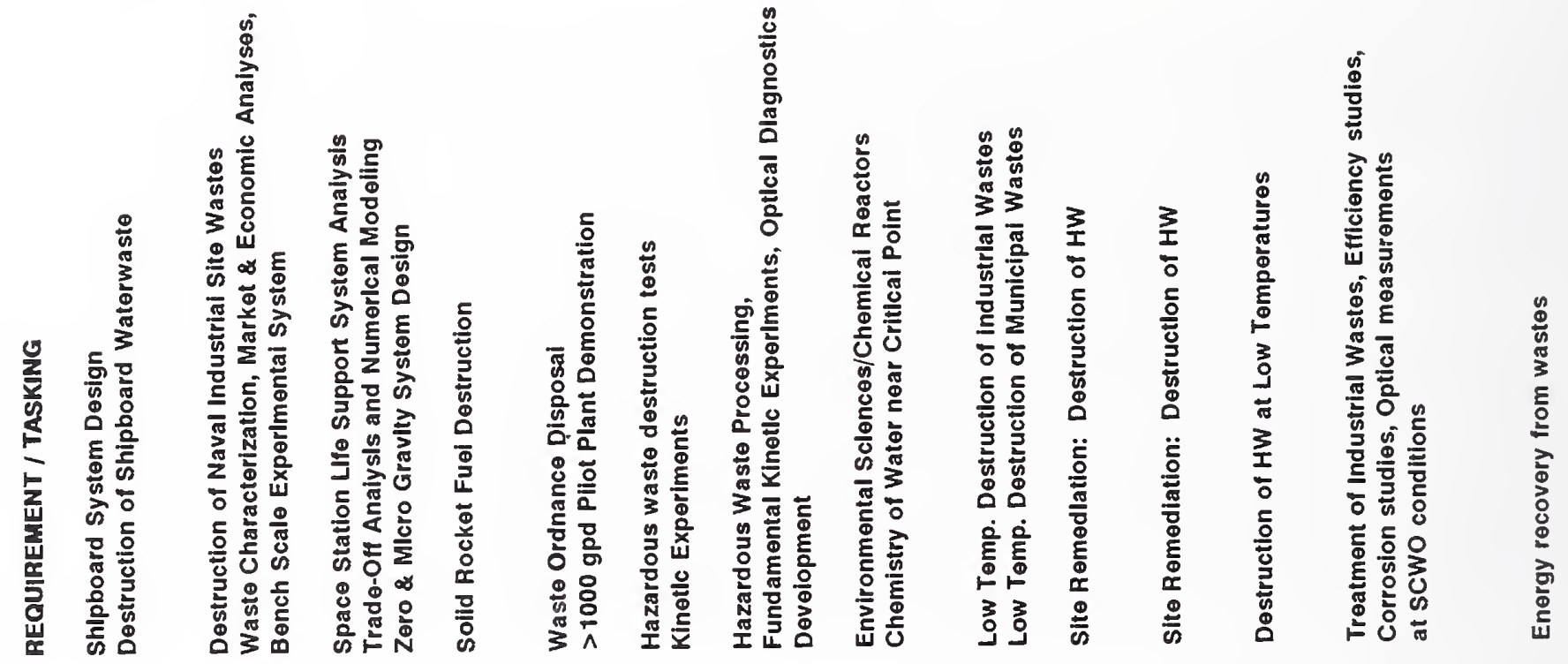

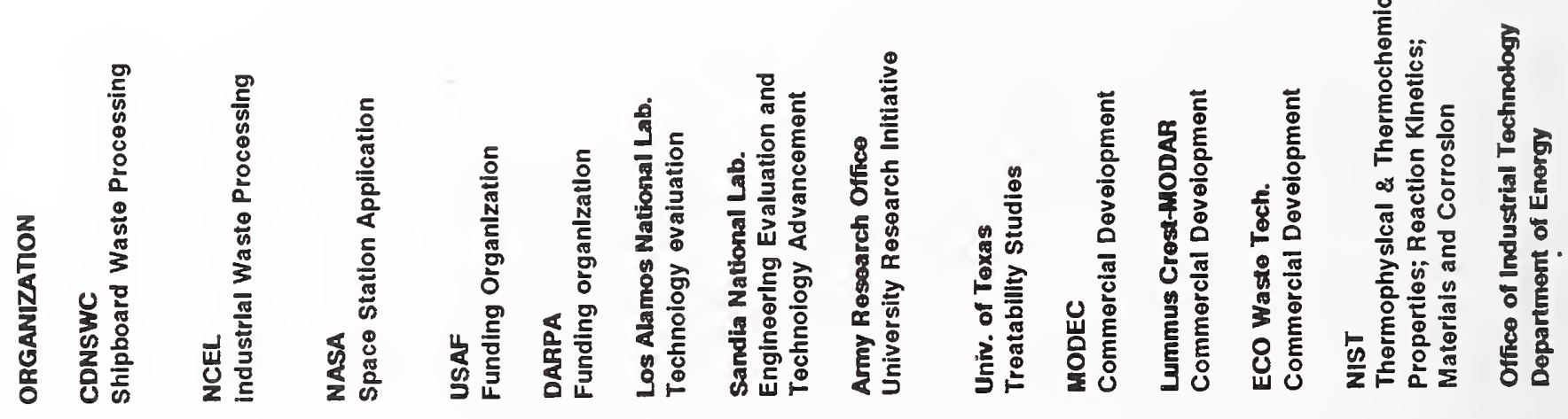




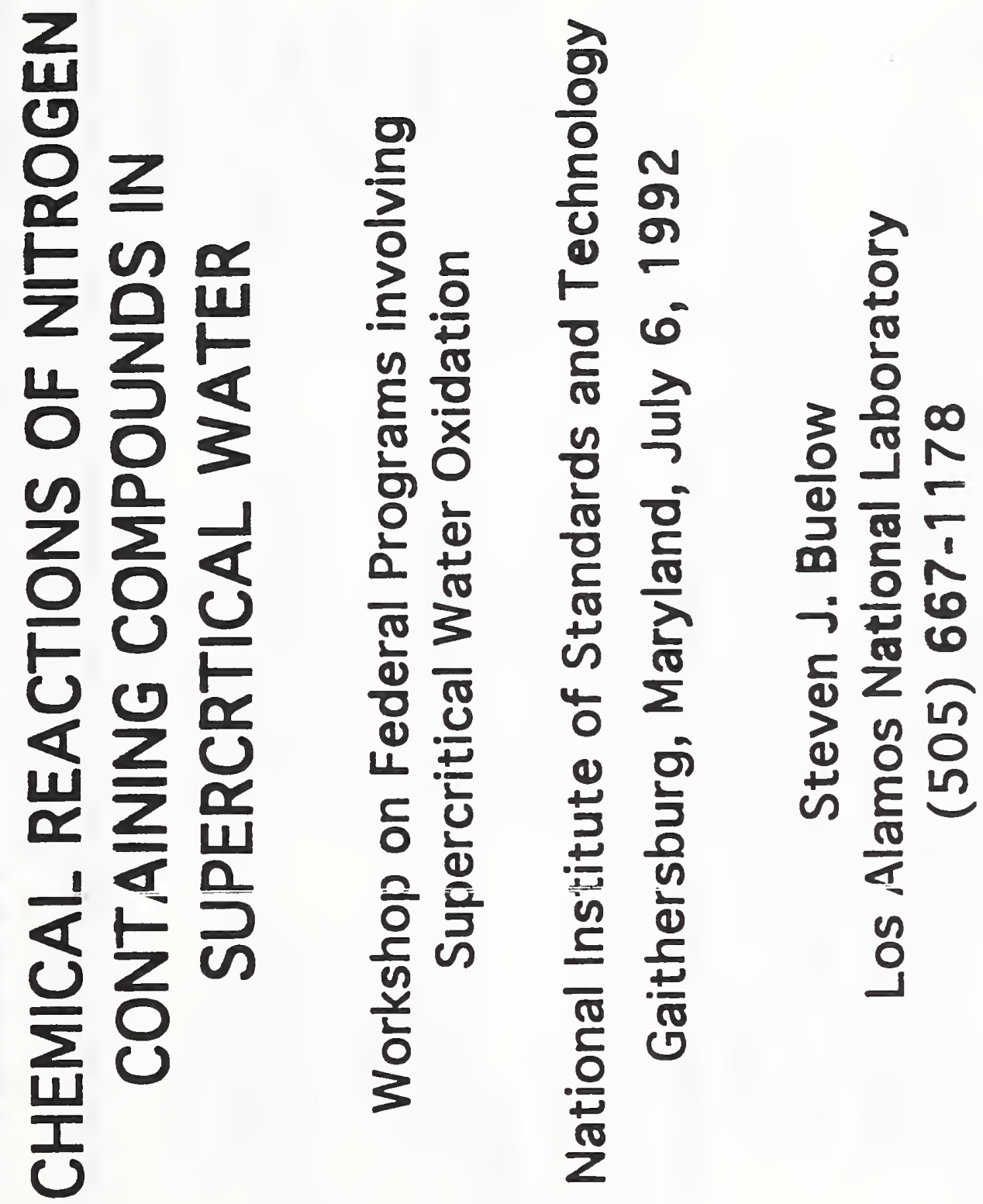

()

○

E

0

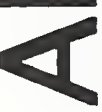

()

0

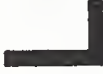




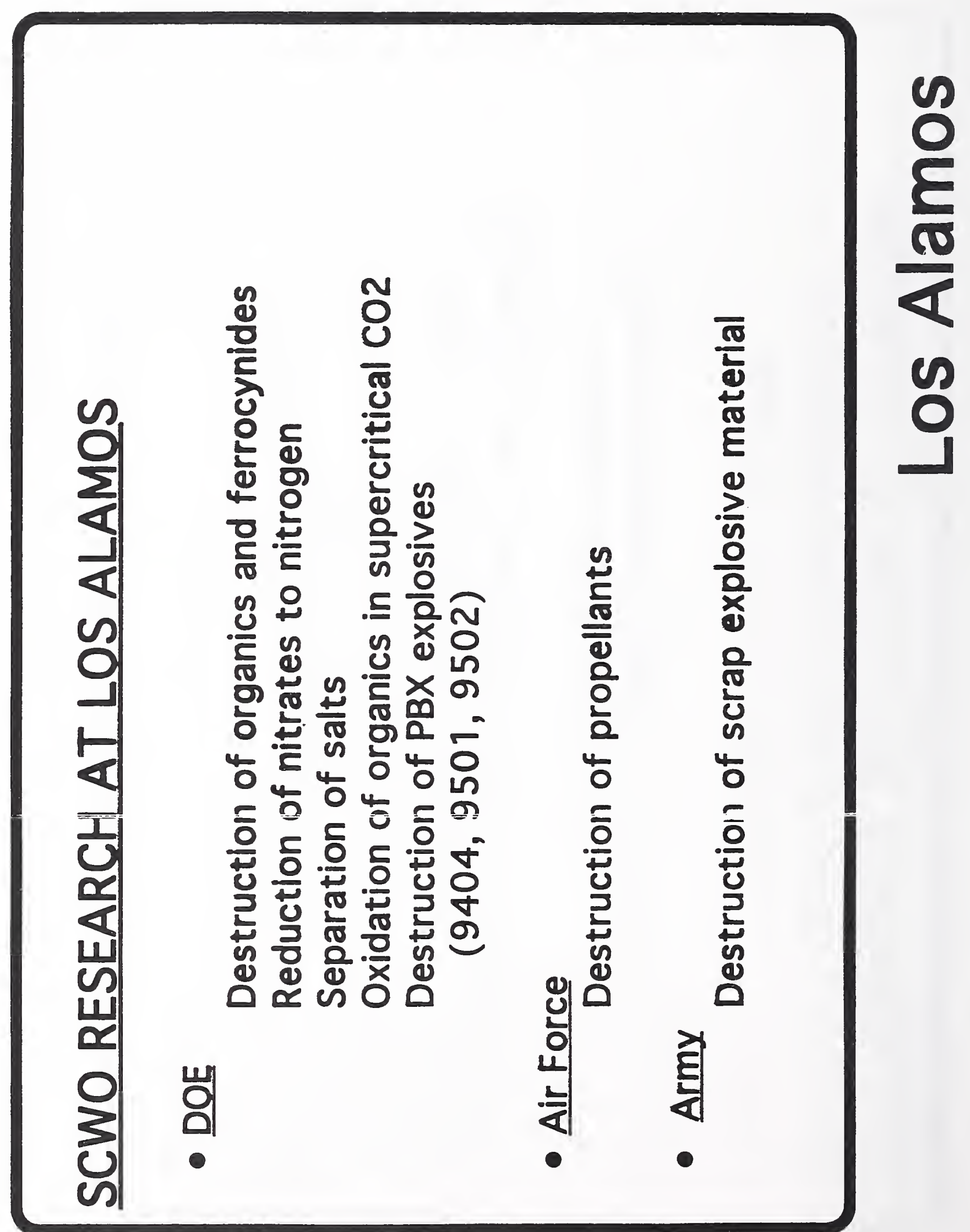




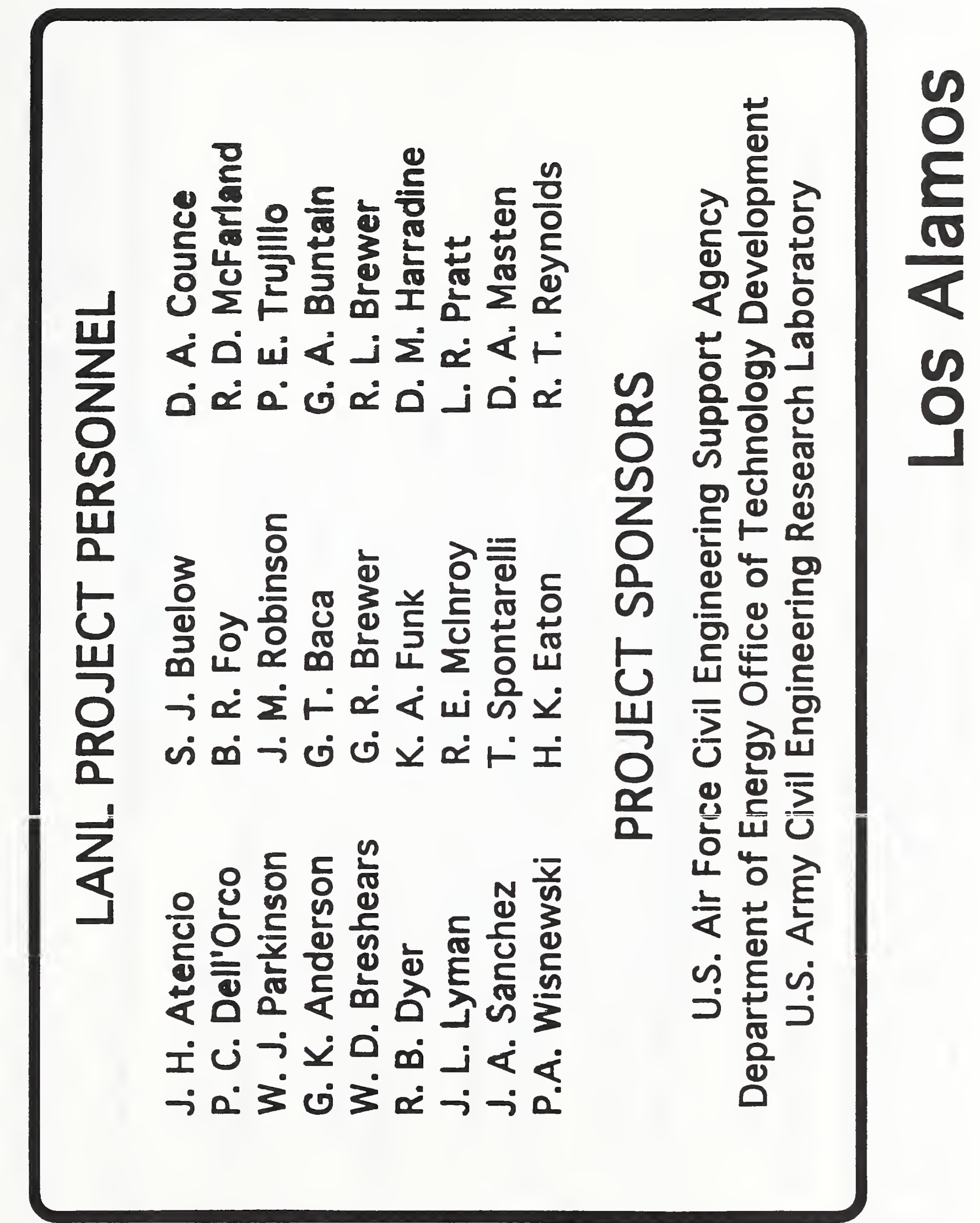




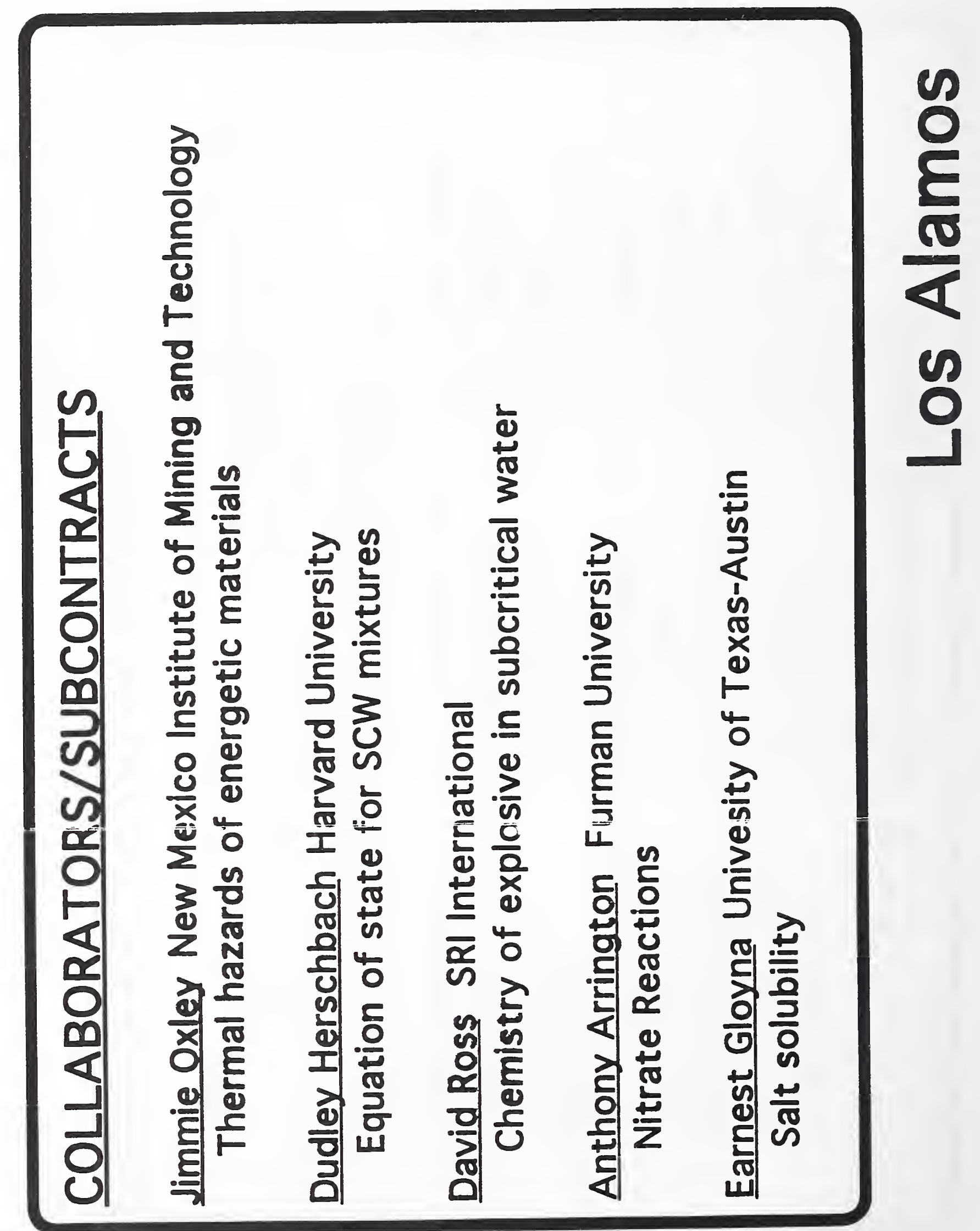




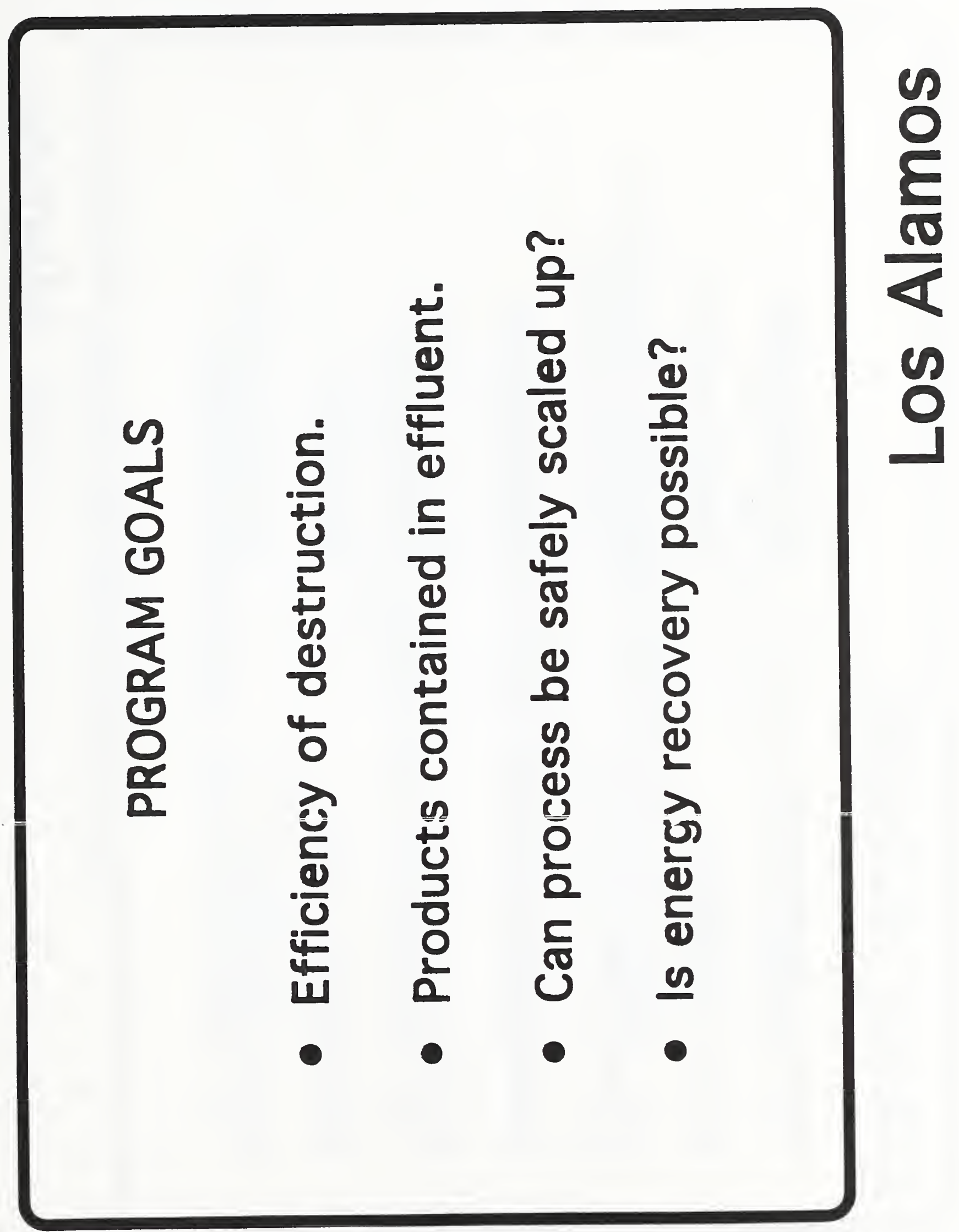



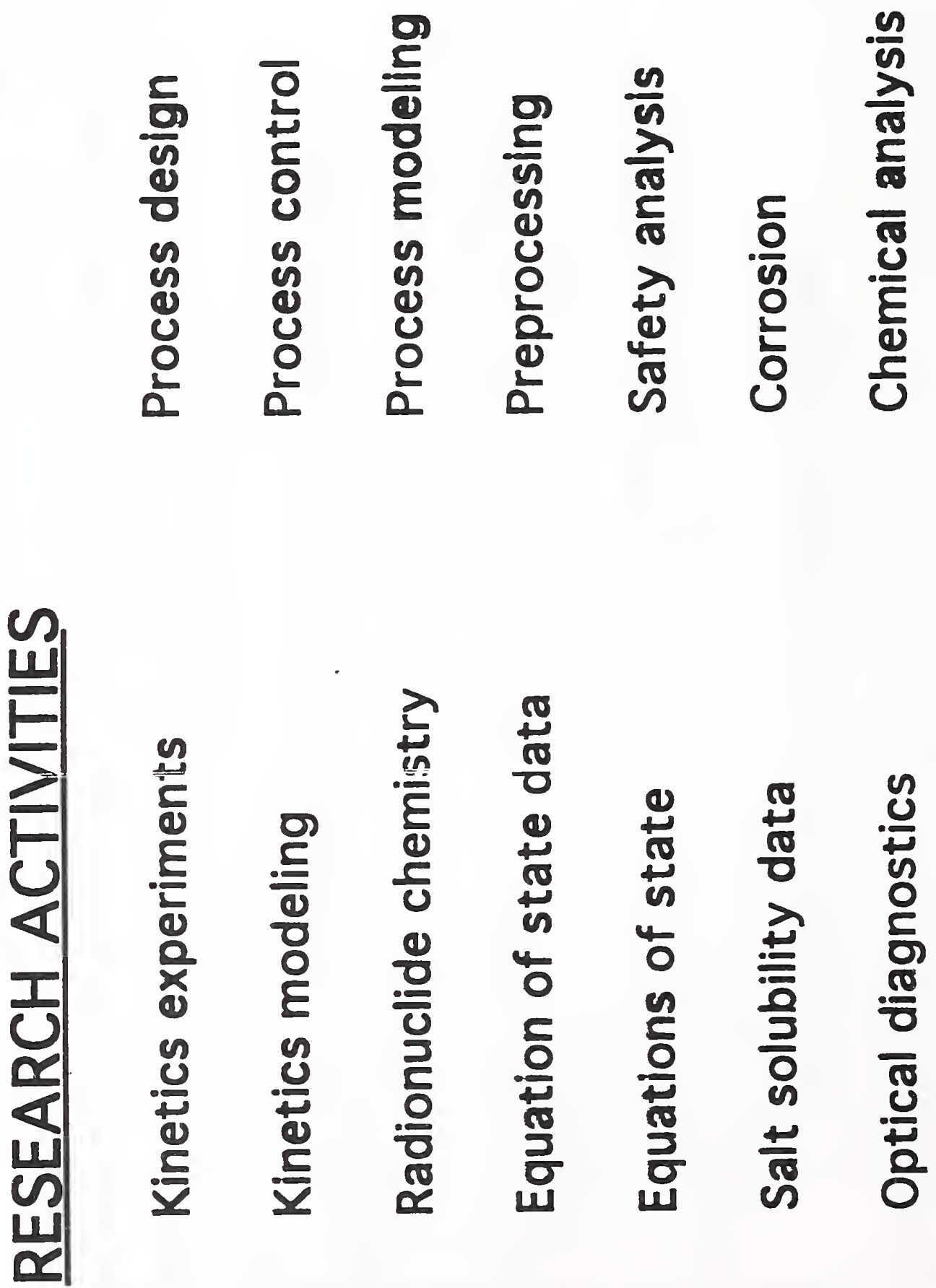


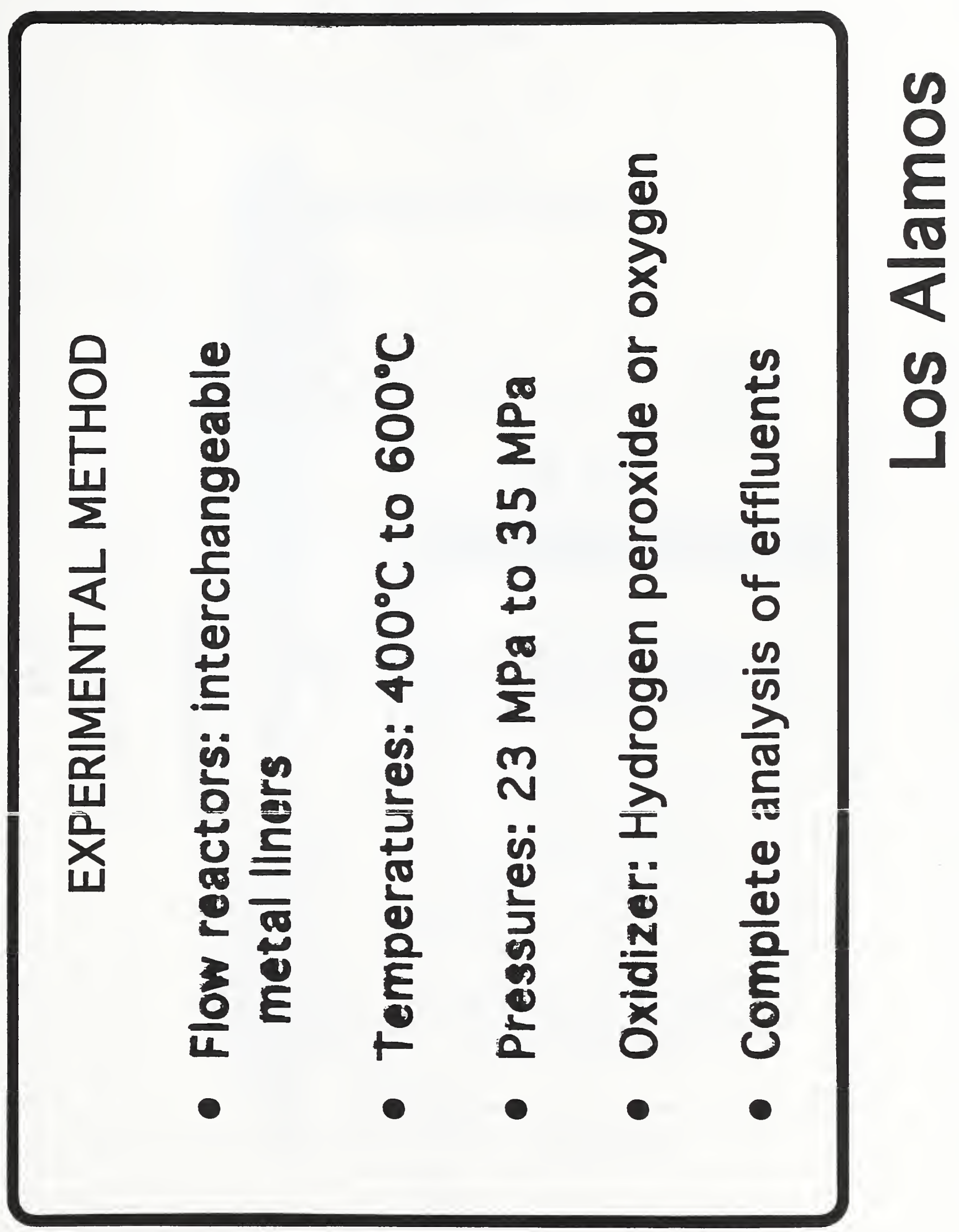




\section{Tubular Flow Reactor}

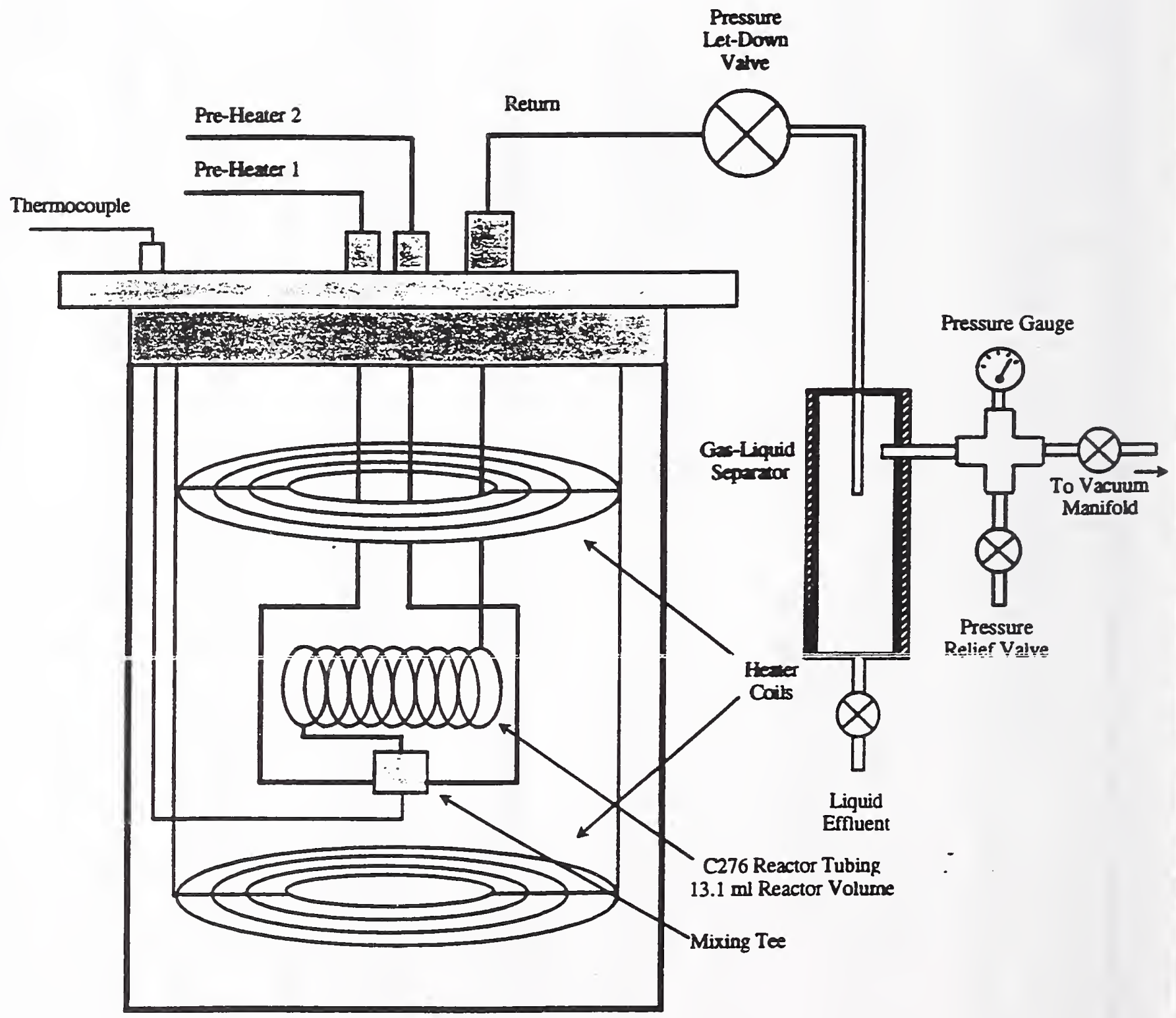

Fluidized Sand Bath 

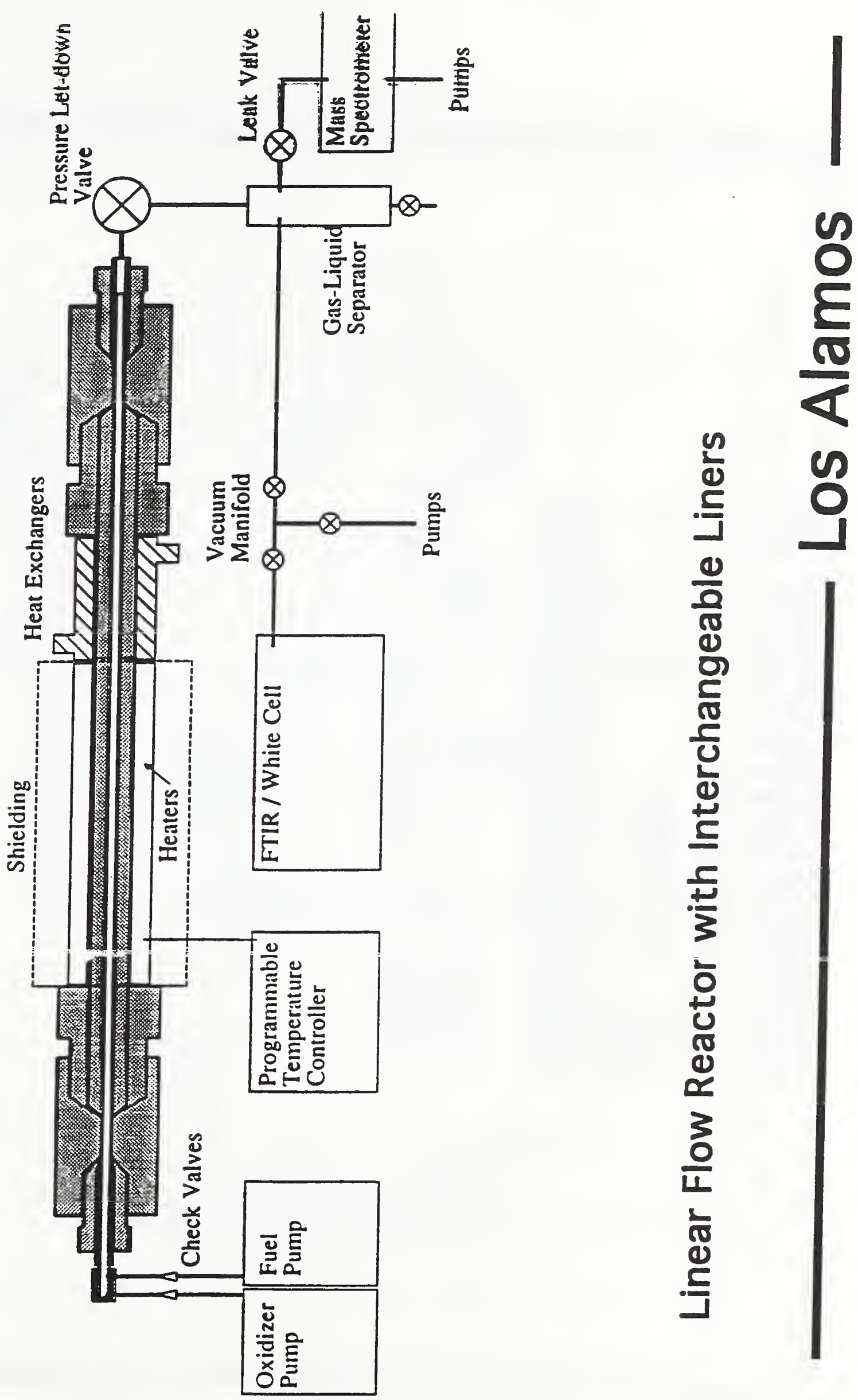

ऽั

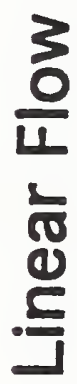




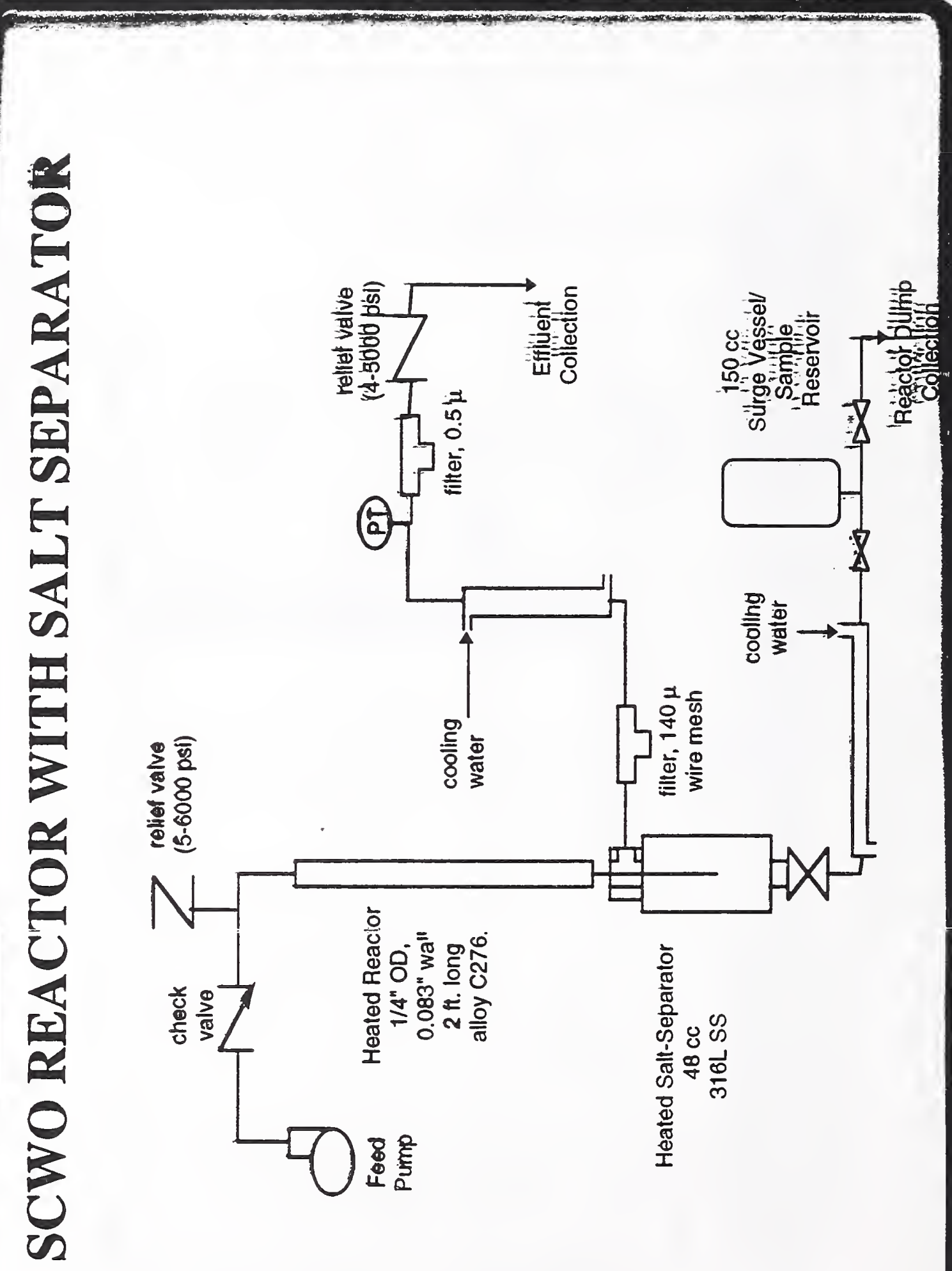




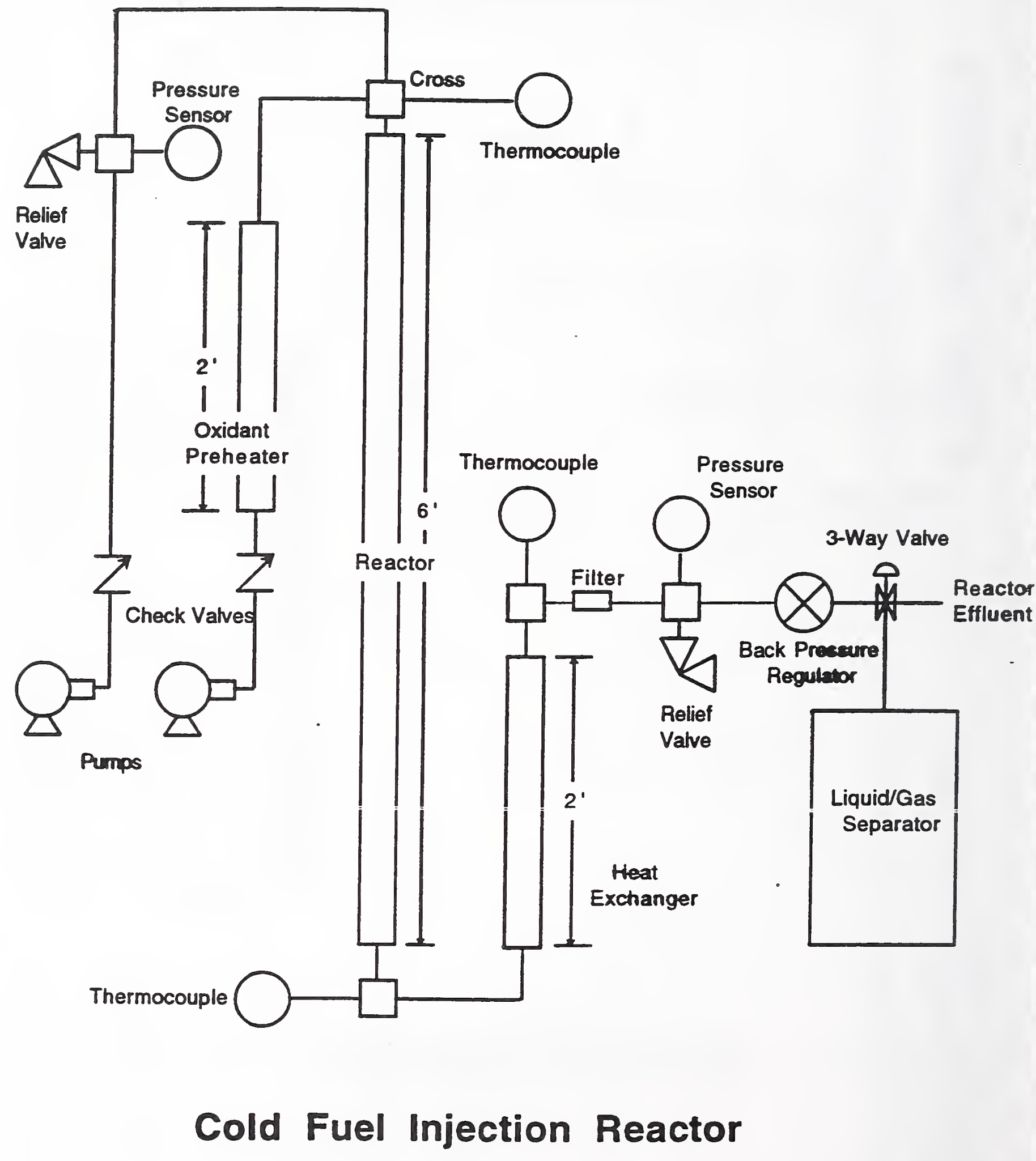



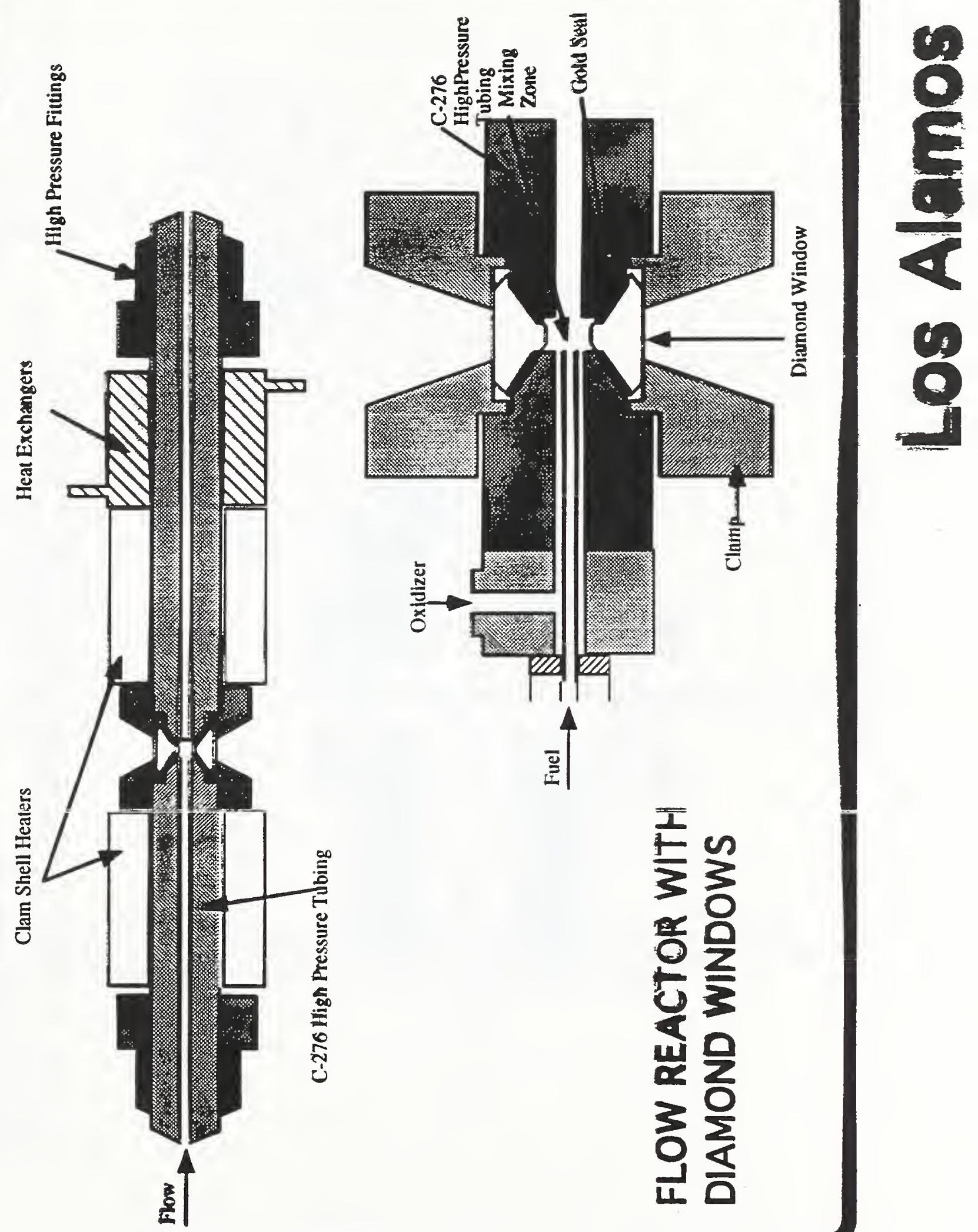


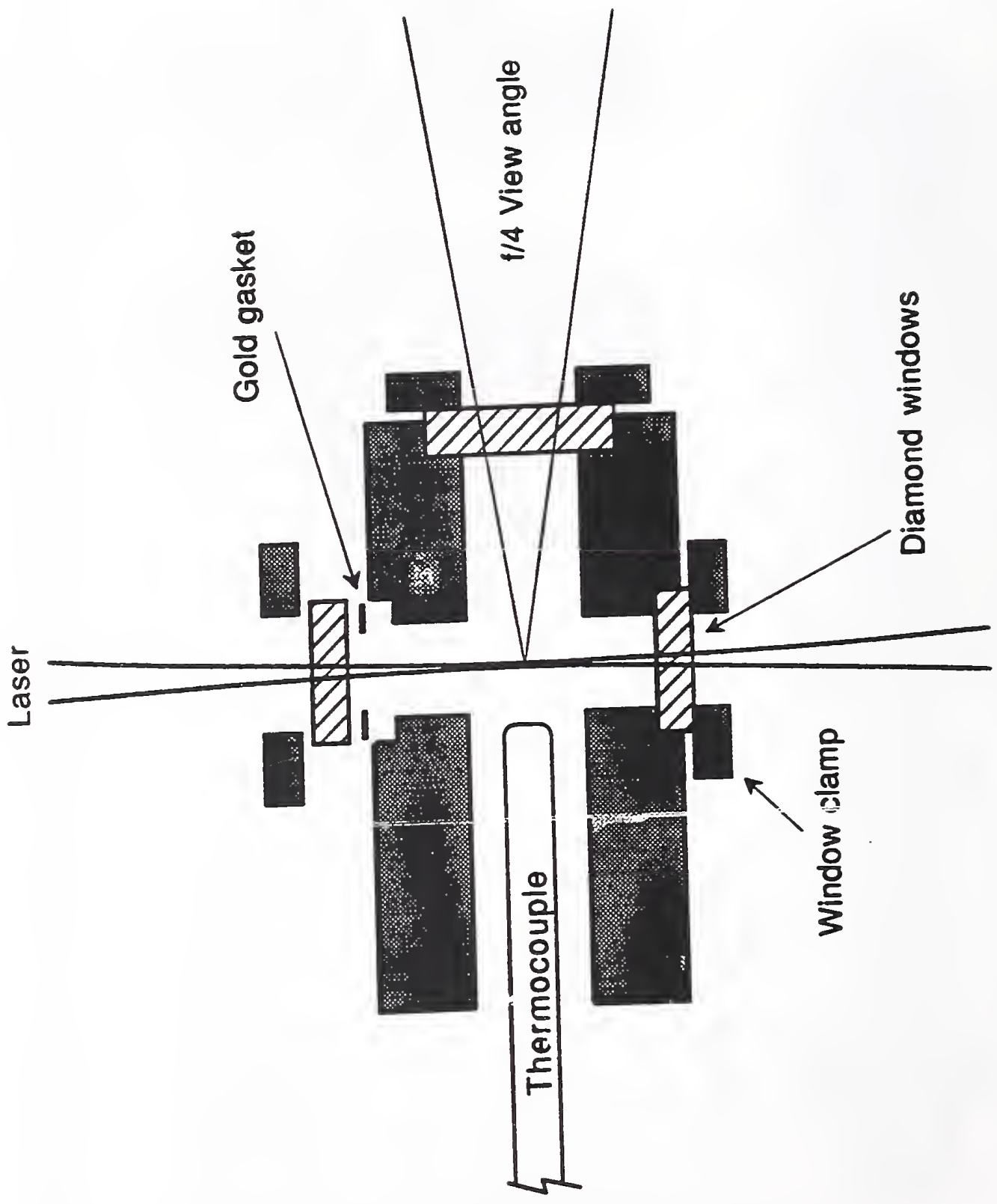

๙

동응 을 ह है 3 들 उ 市 范 ○ วิ응 을 혼 응 ثัن ह 응 造 - $E$ N 3 ह . (1) ธั क 을

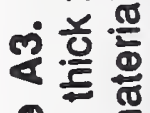
을

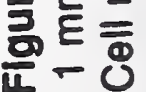




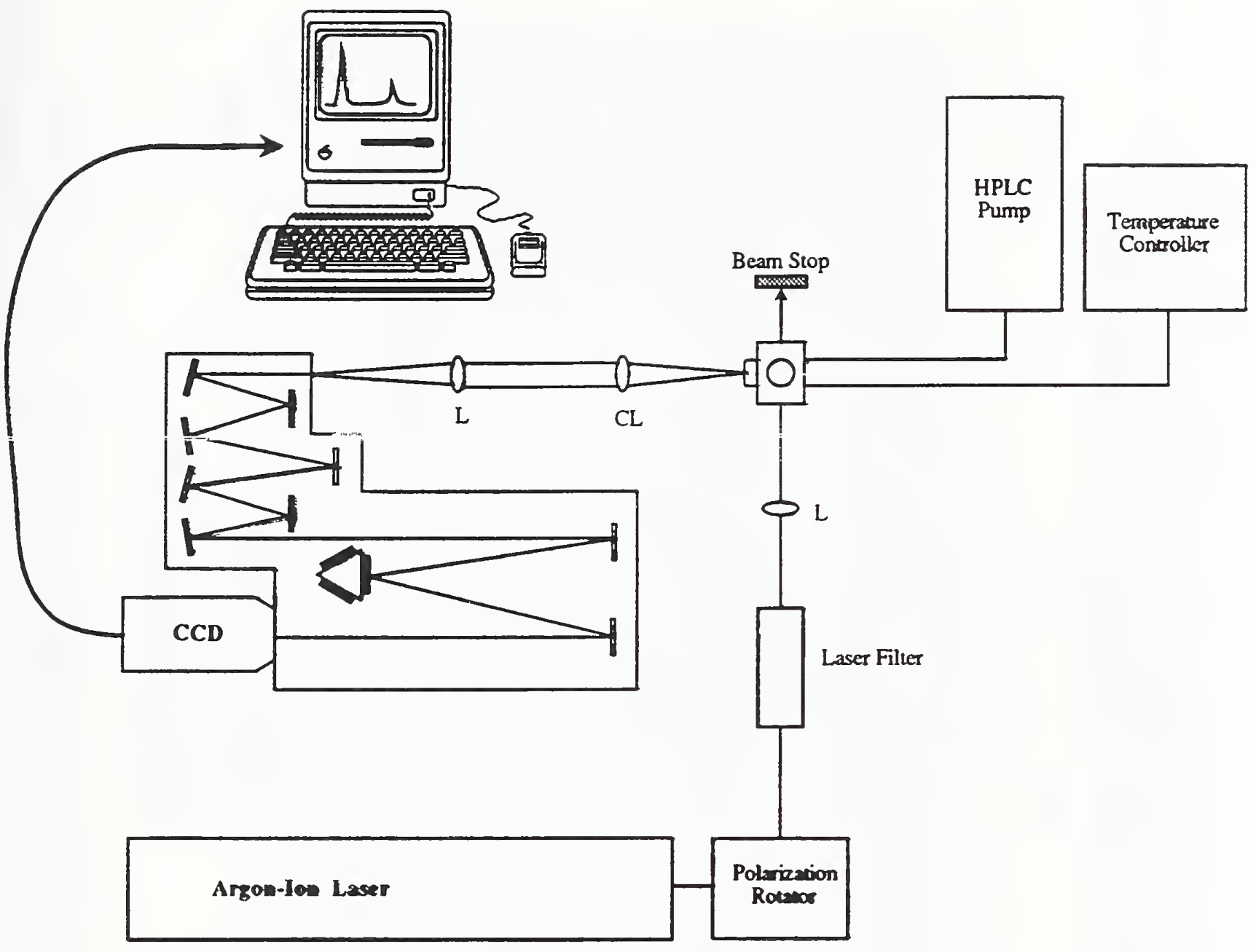

Figure 10. Raman apparatus. Polarization rotator: dorble romb half-wave retarder; Laser Filter. small monochromator, L: lens; $\mathrm{Cl}$ : collection lens; CCD: charge coupled device camera for detection. Data collection using macinosh II computer. 

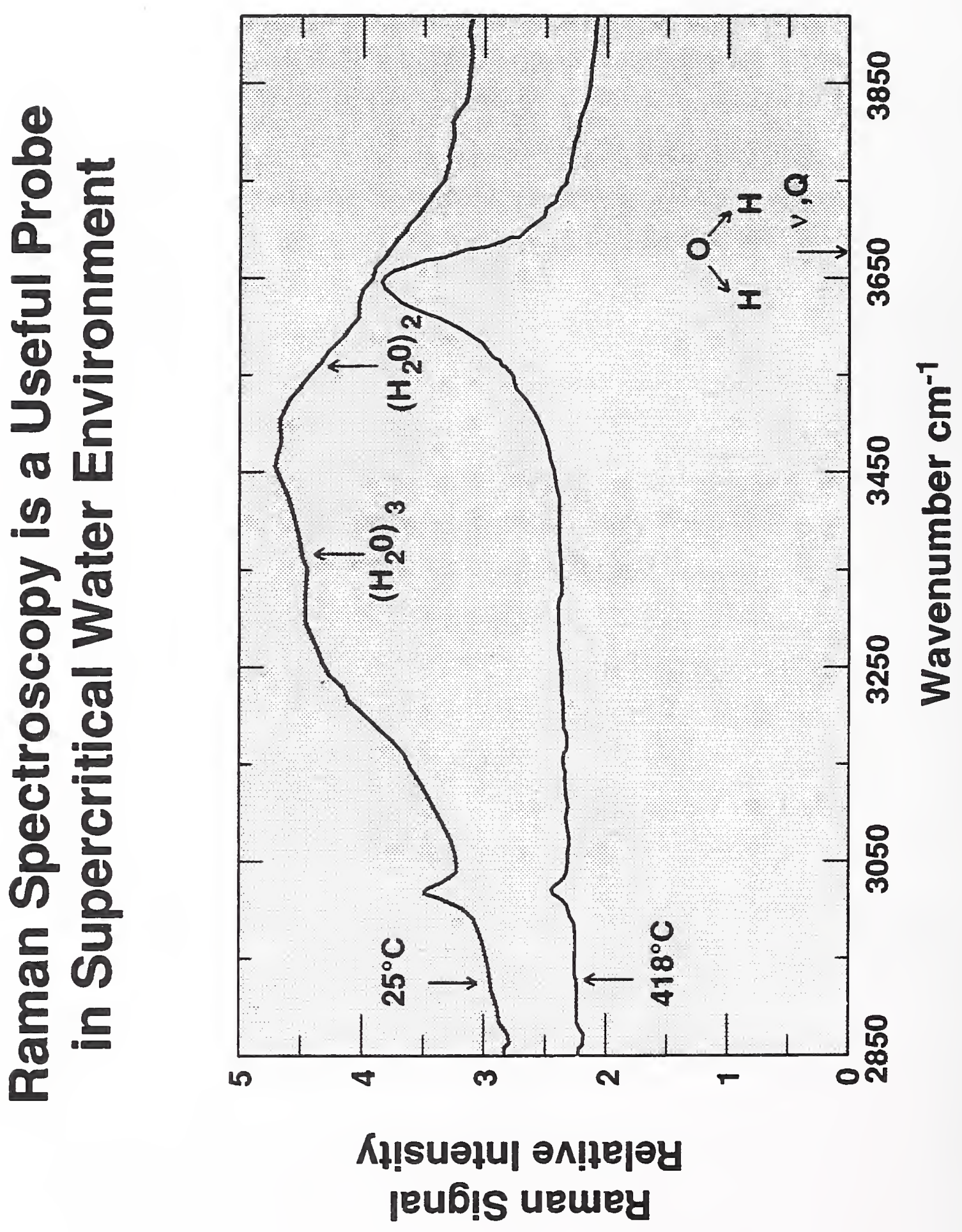

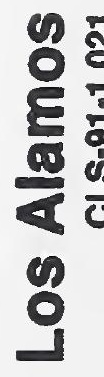




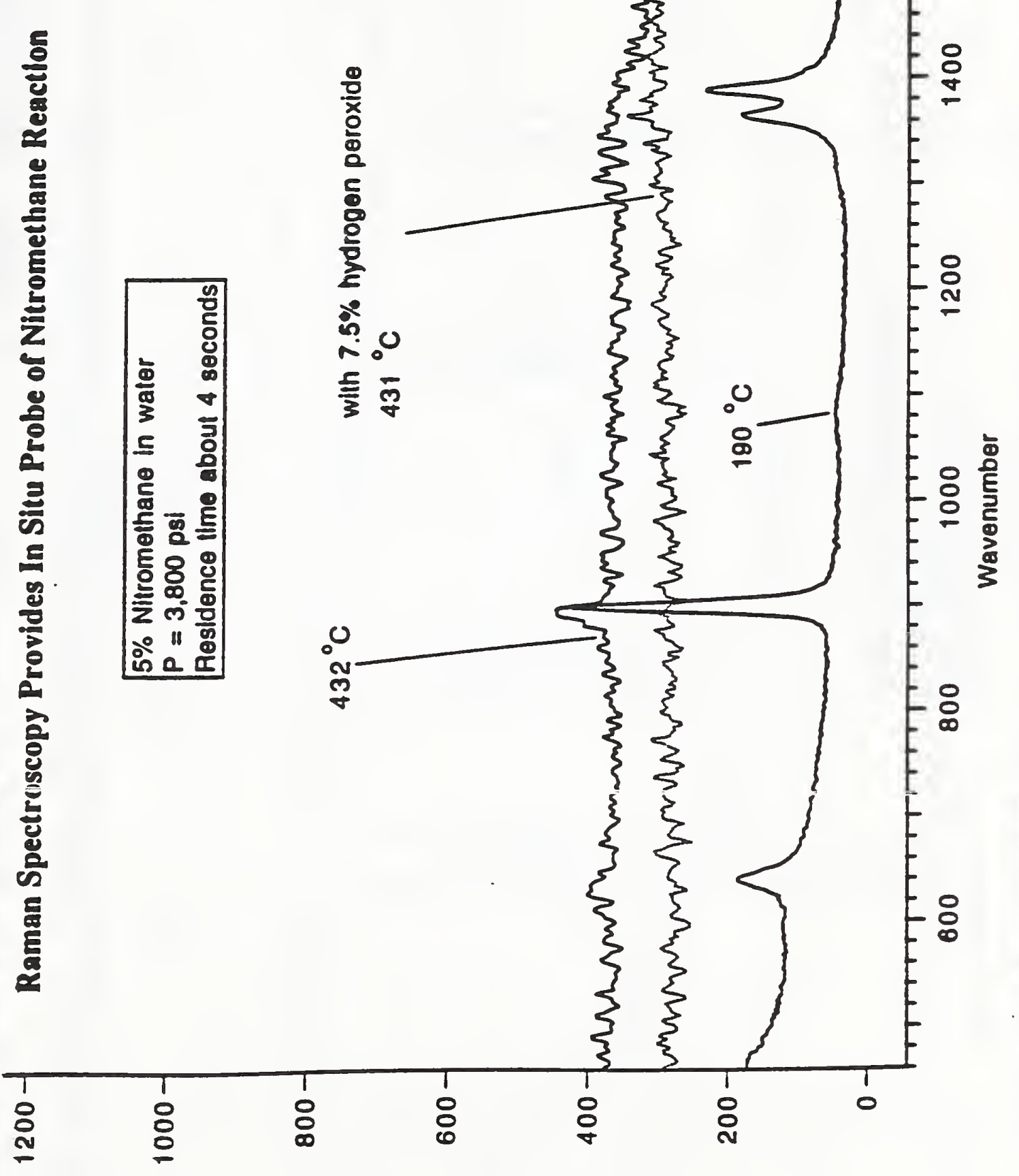




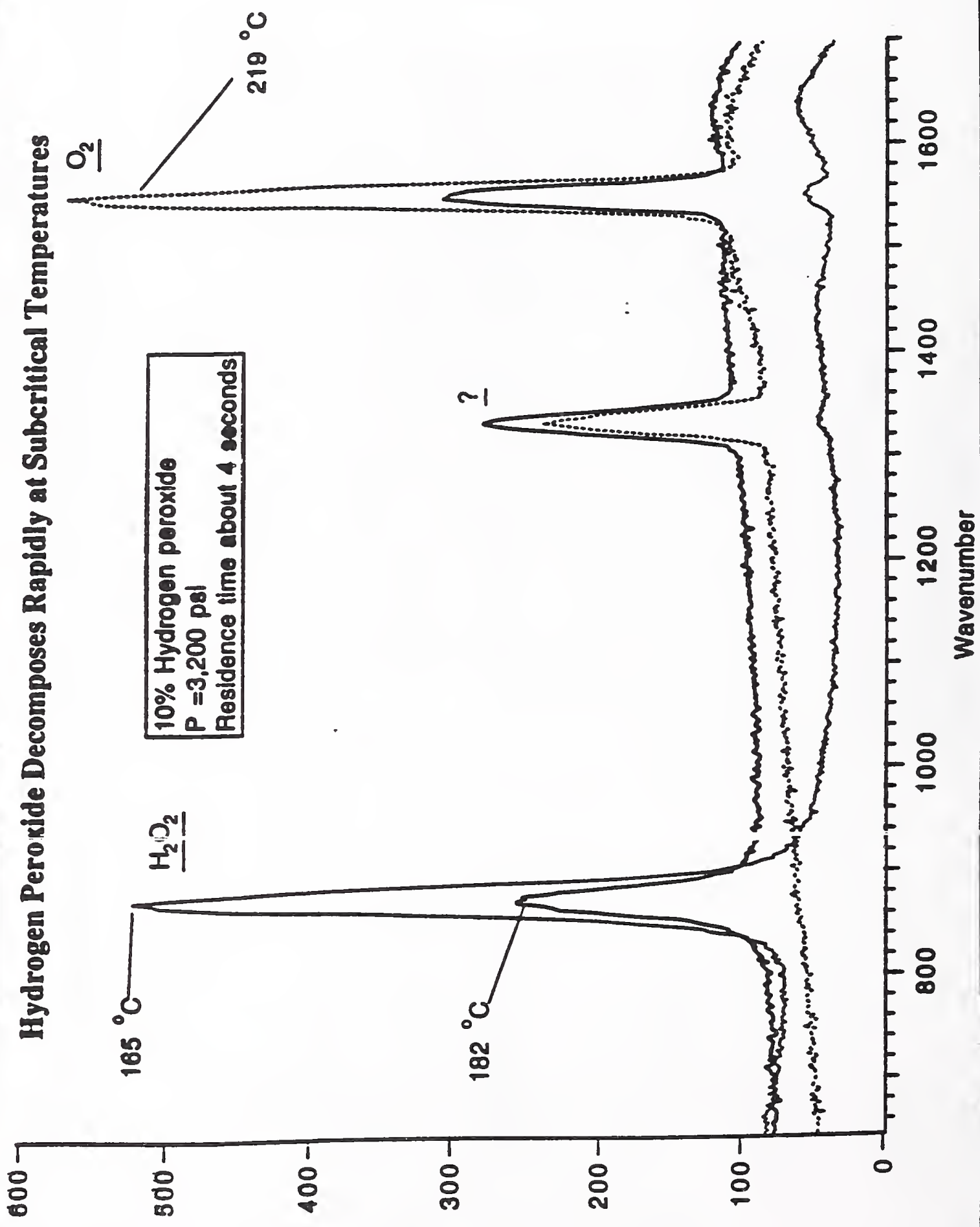



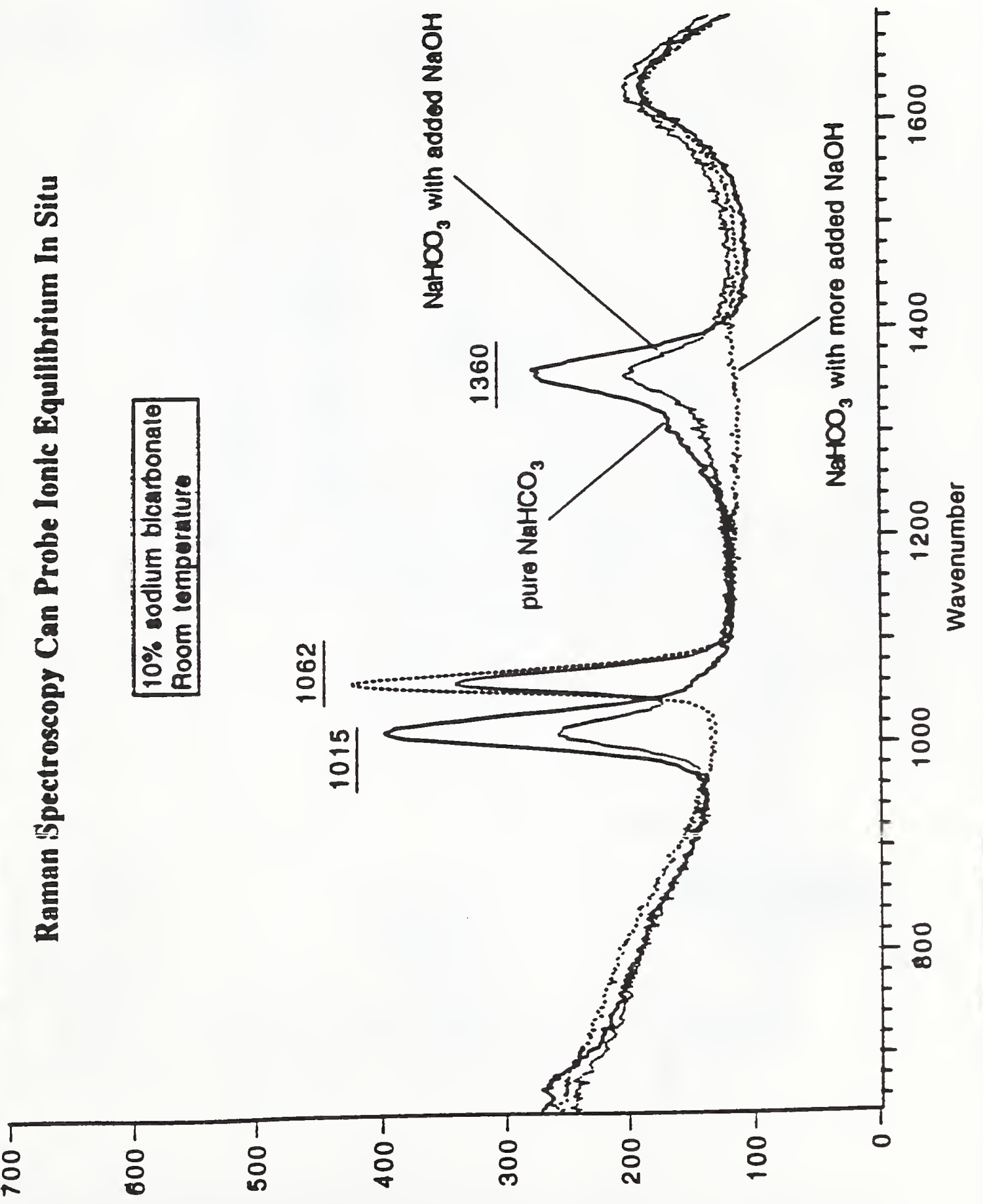


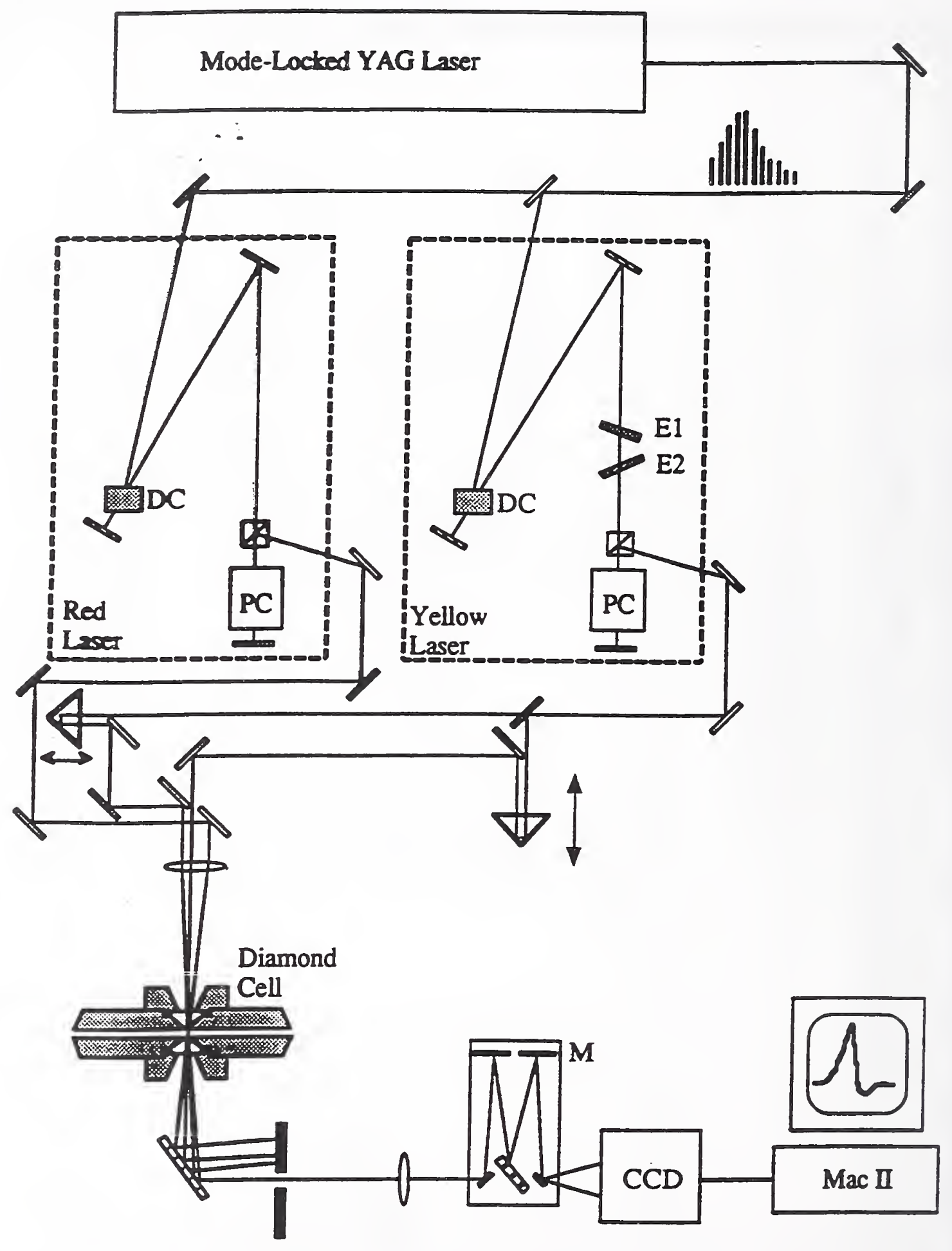

CARS apparatus. DC: dye cell, containing Sulforhodamine 640 for the red laser and Rbodamine 610 for the yellow laser. E1, E2: etalon tuning elements for the yellow laser, these are breat in the broad-band red laser. PC: Pockel's Cell used to fire the dye laser puise. M: monochromator used to disperse the broad-band CARS beam. CCD: charge-coupled device camera to detect CARS beam. Mac II: compurer for data acquisicion, tisplay, and analysis. 


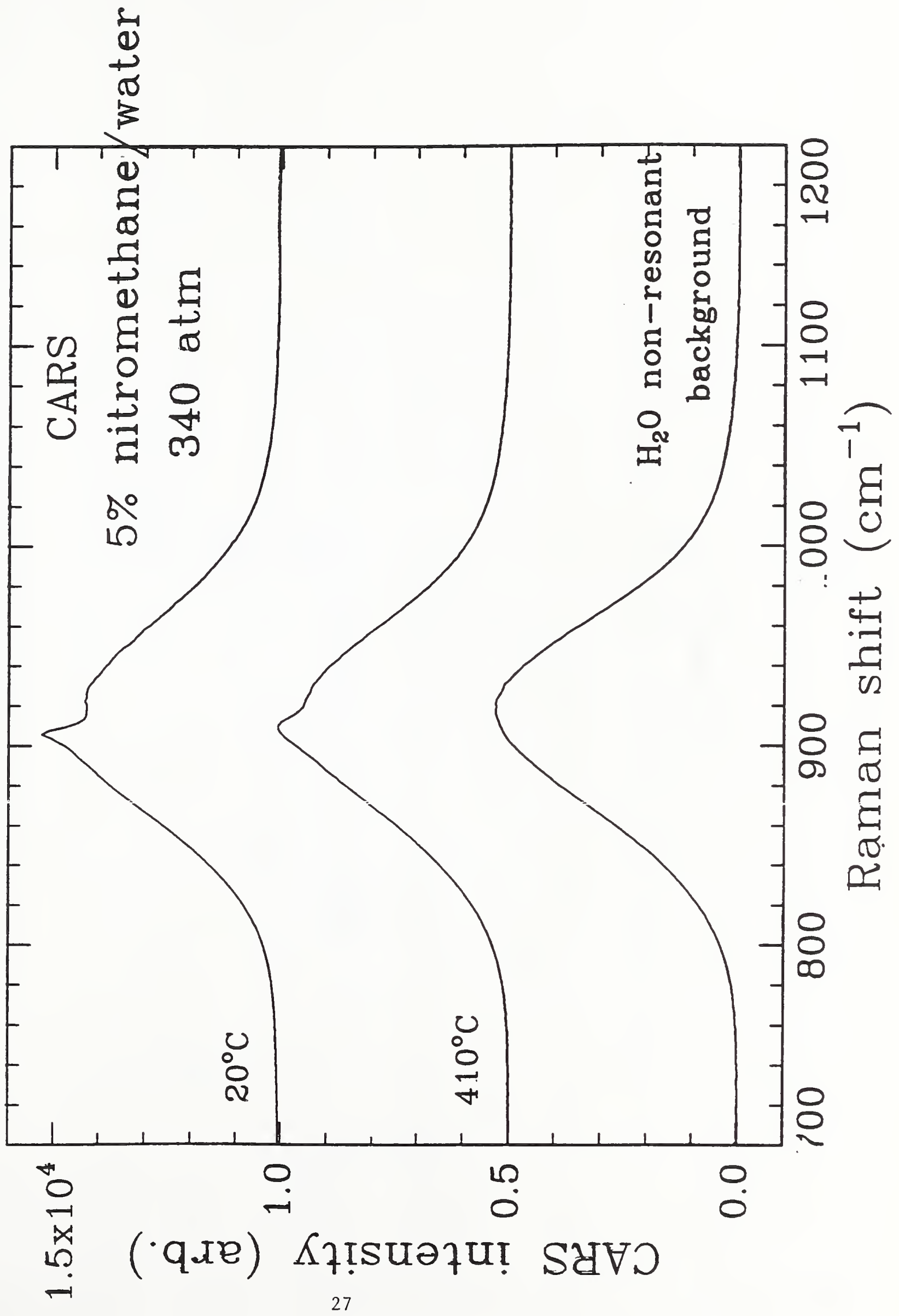




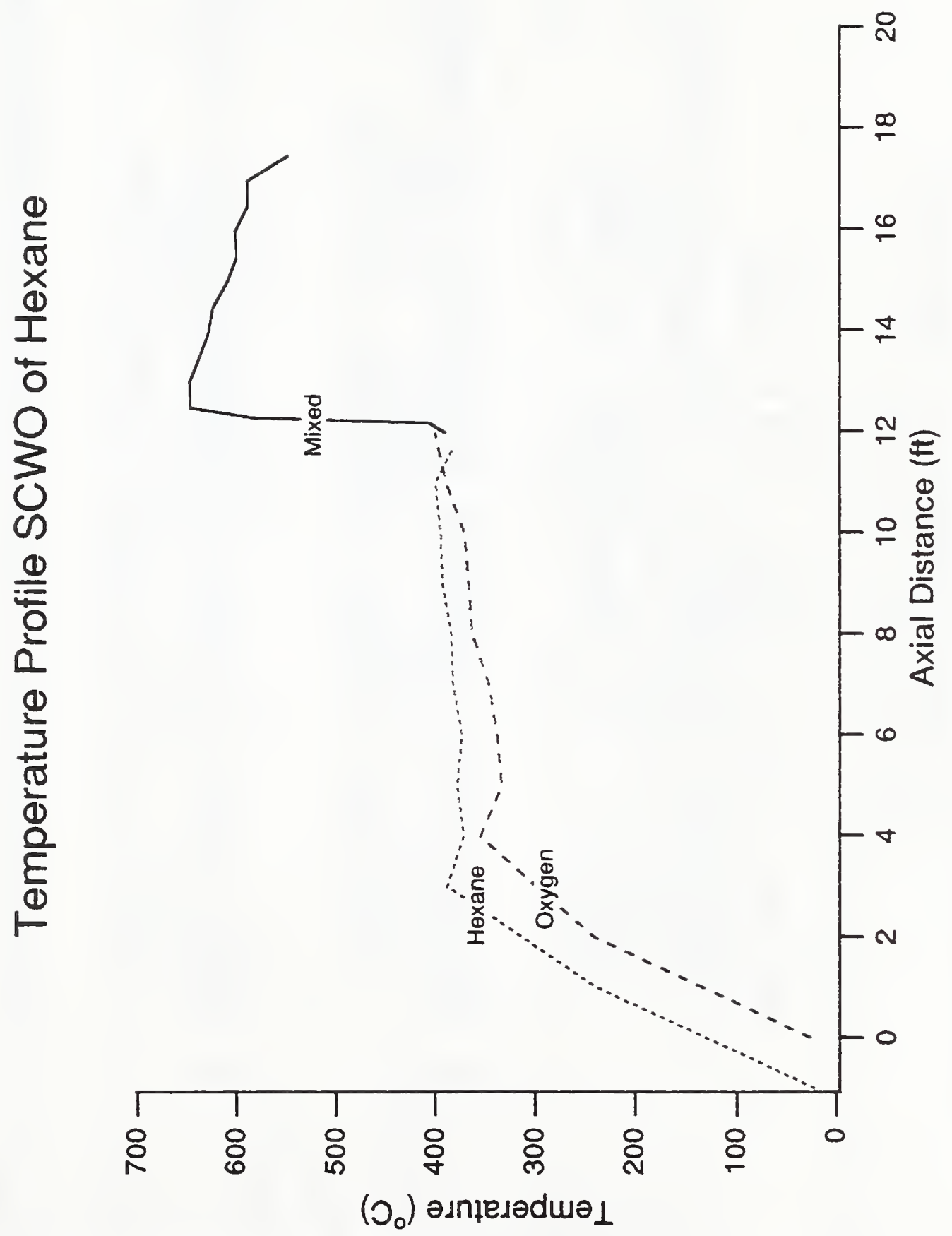


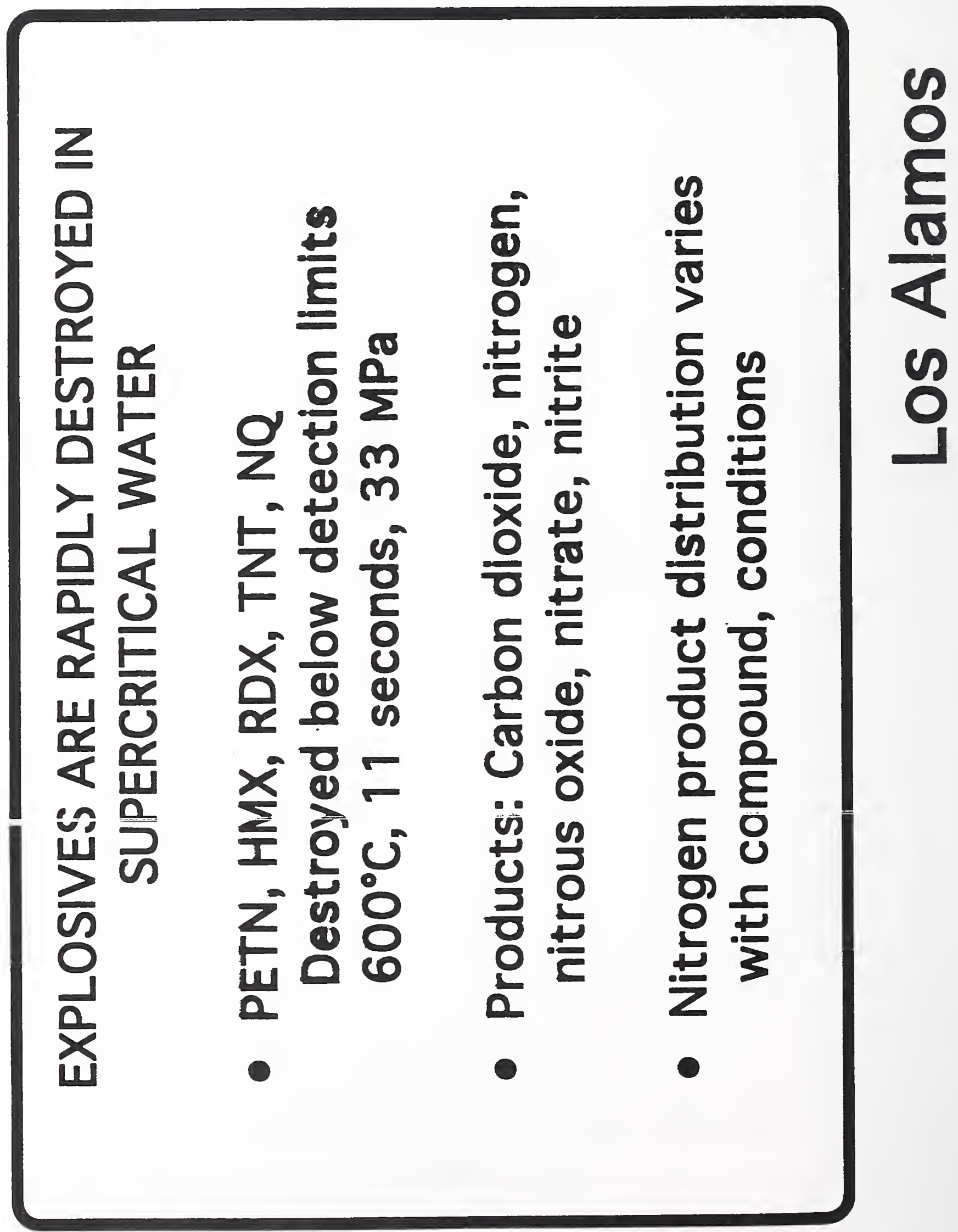




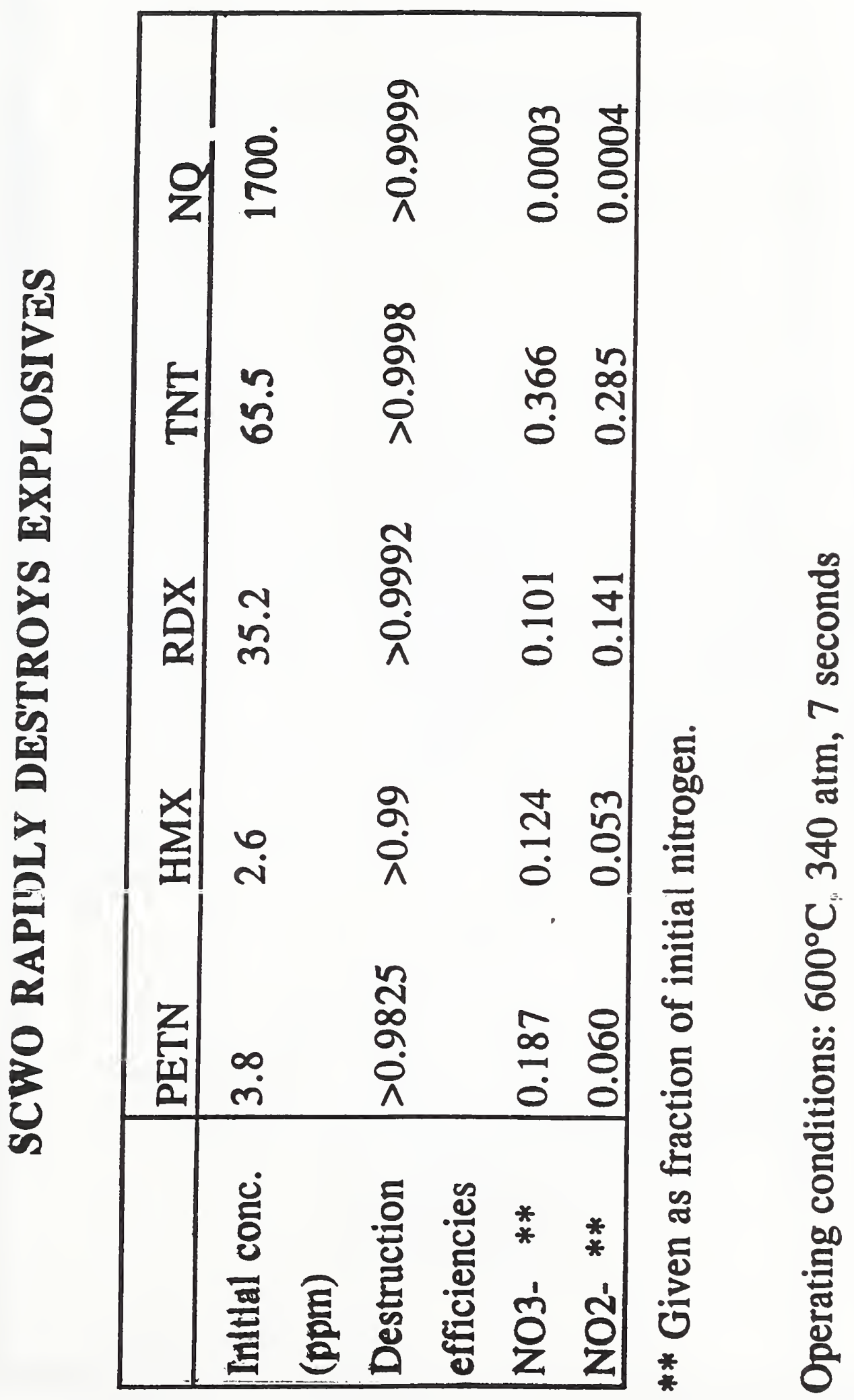




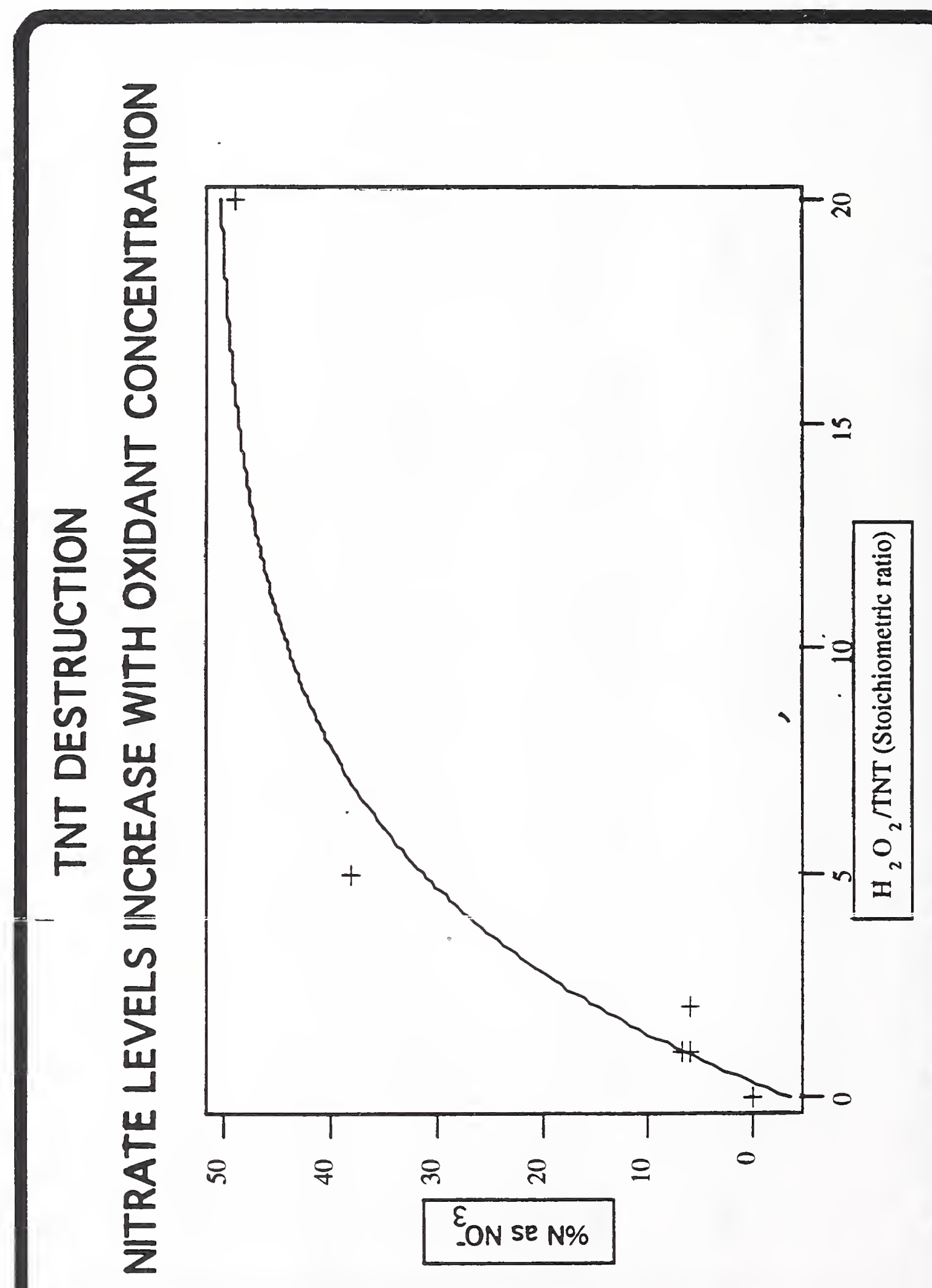




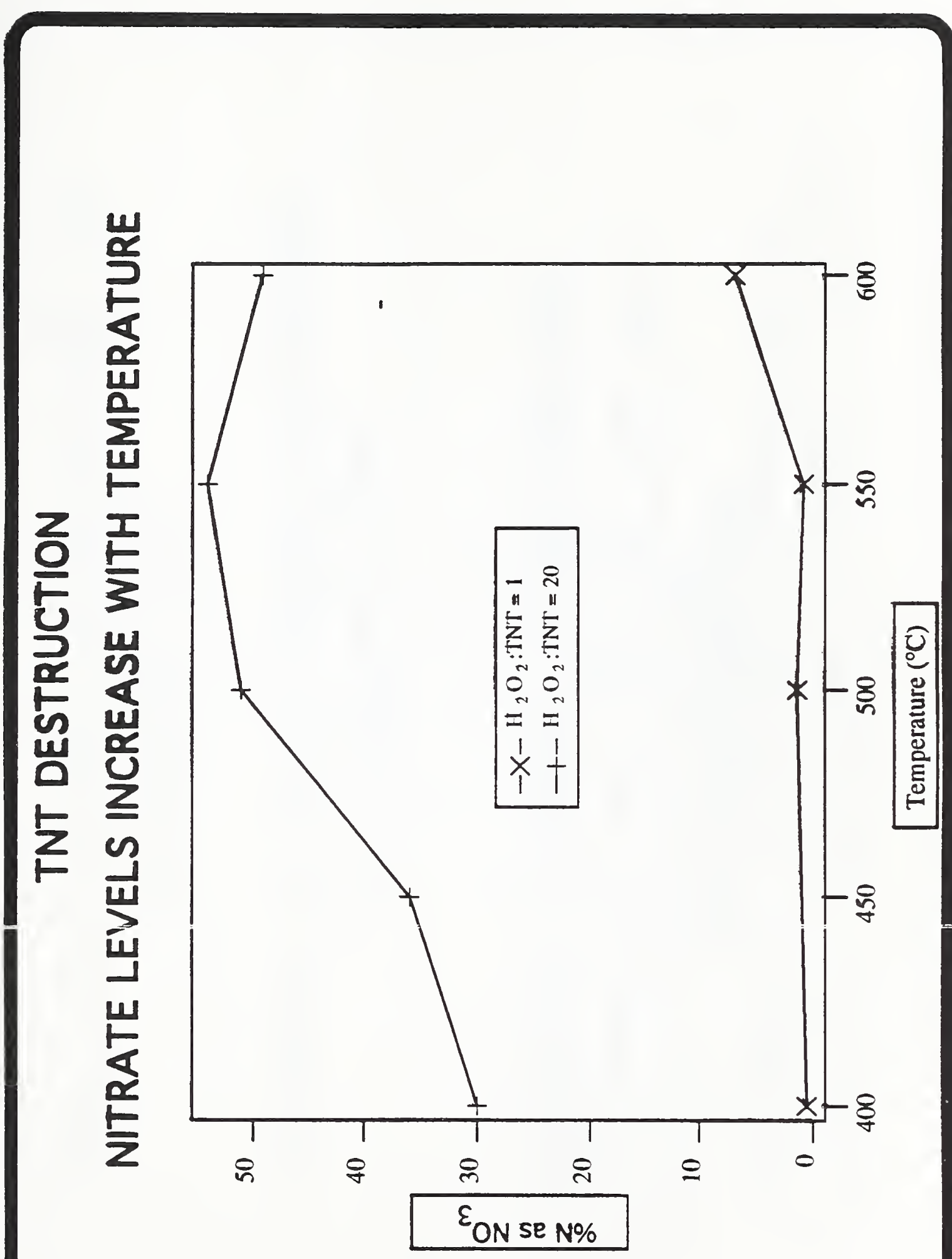




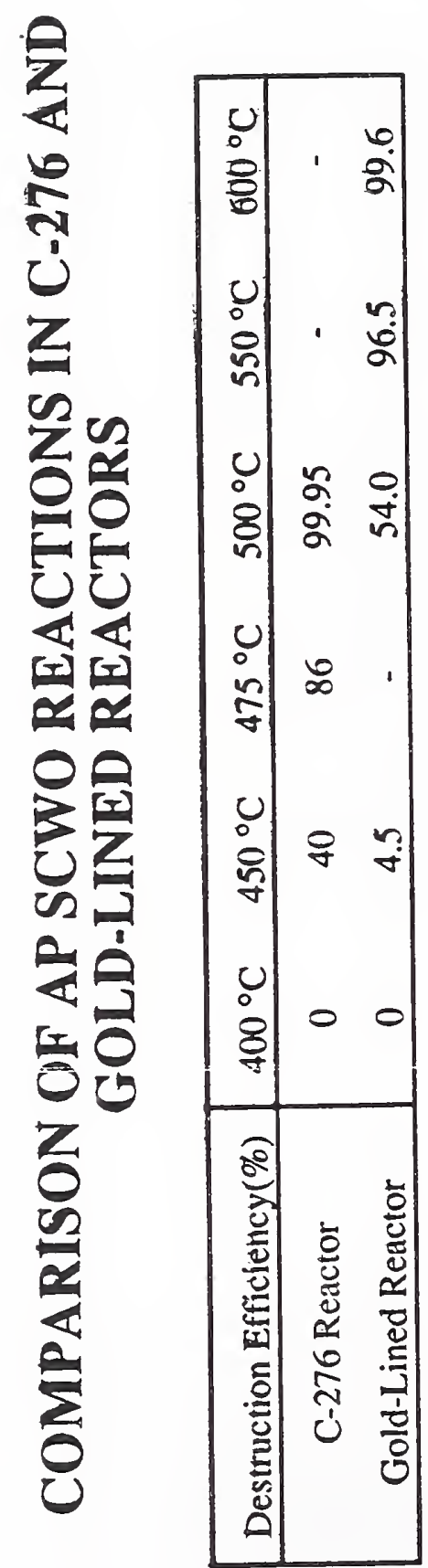




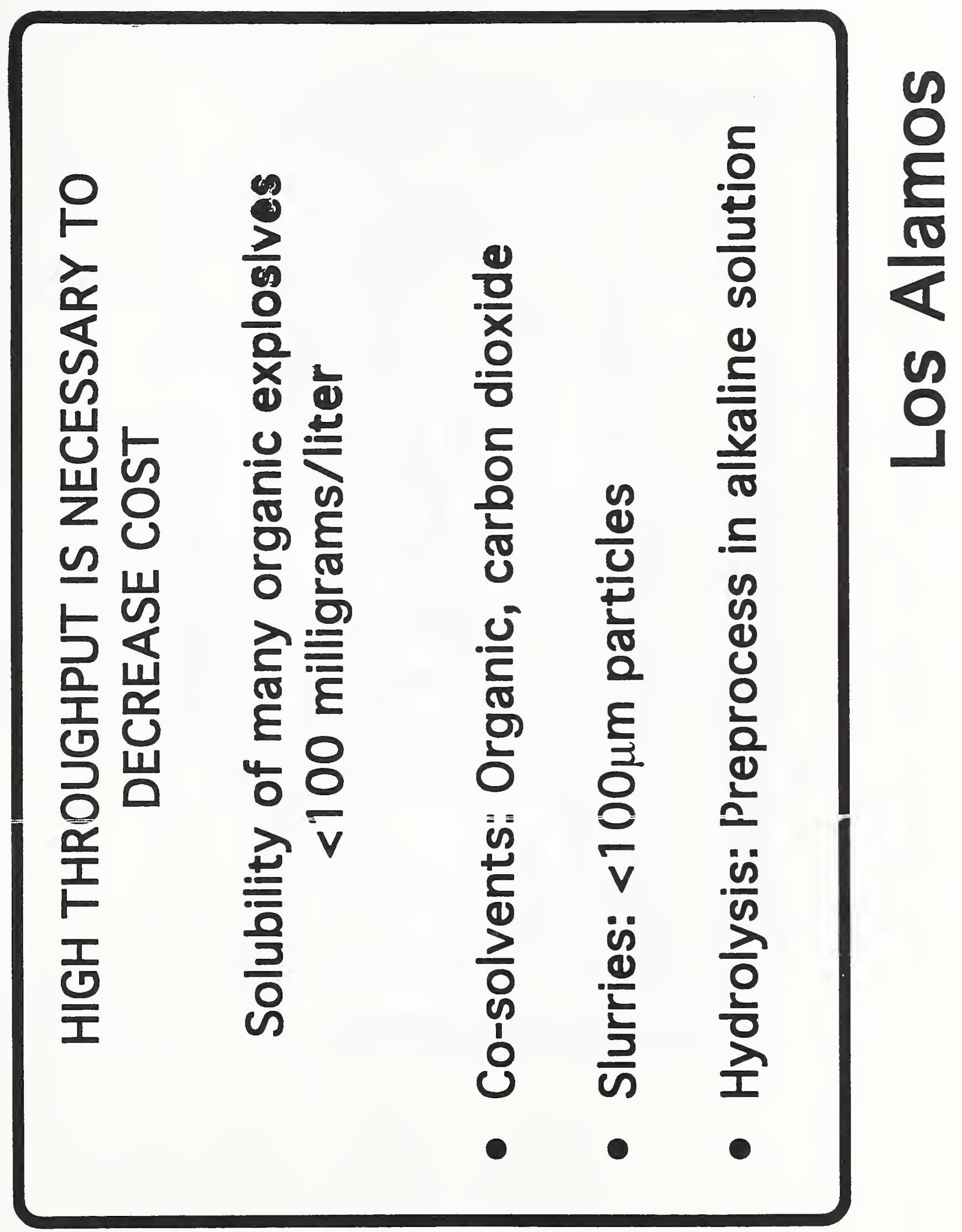




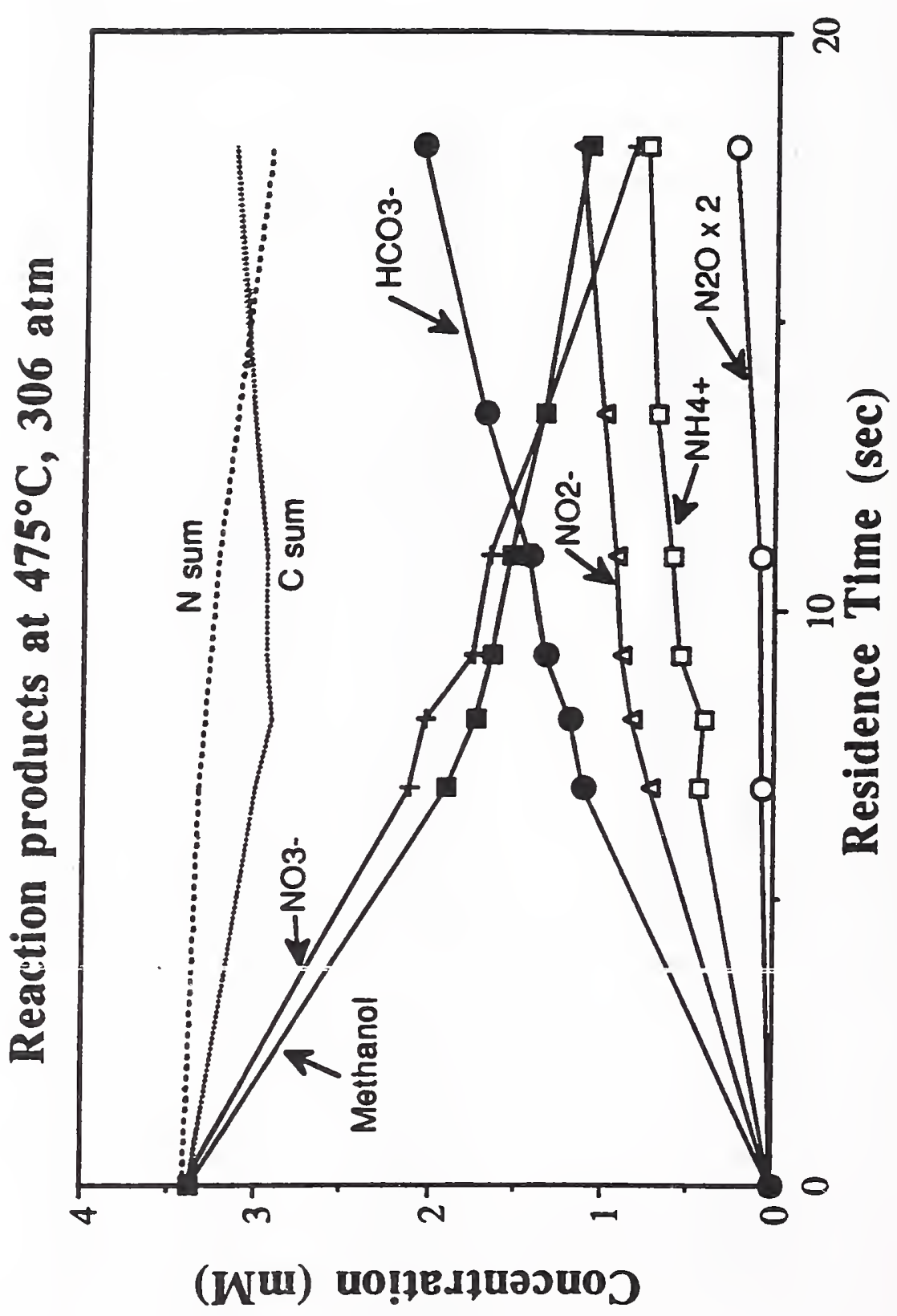


STOICHIOMETRIC EFFECTS ON

METHANOL REMOVAL AT $475^{\circ} \mathrm{C}$

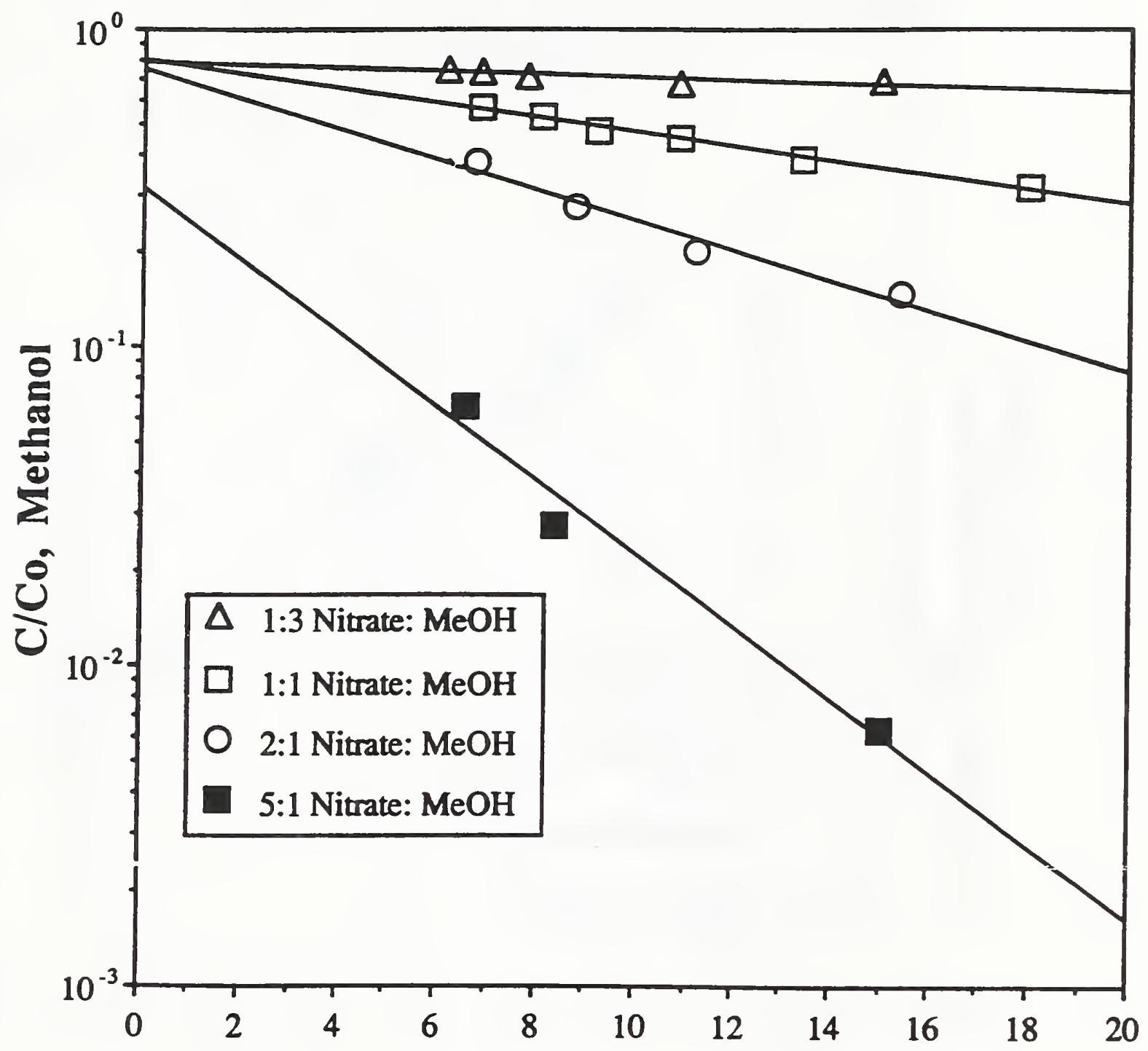

Residence Time (seconds) 


\section{STOICHIOMETRIC EFFECTS ON NITRATE REMOVAL AT $475^{\circ} \mathrm{C}$}

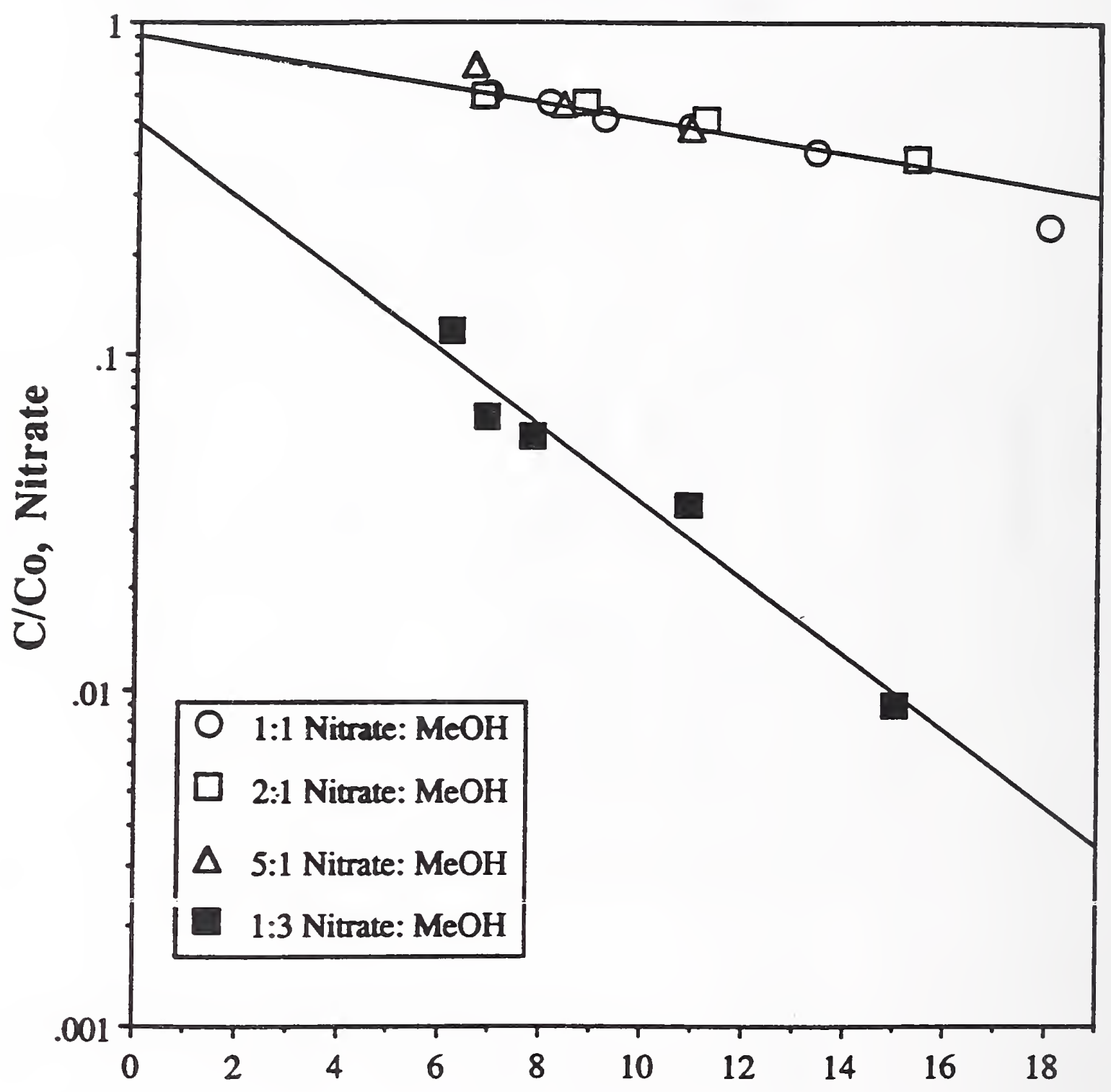

Residence Time (seconds) 


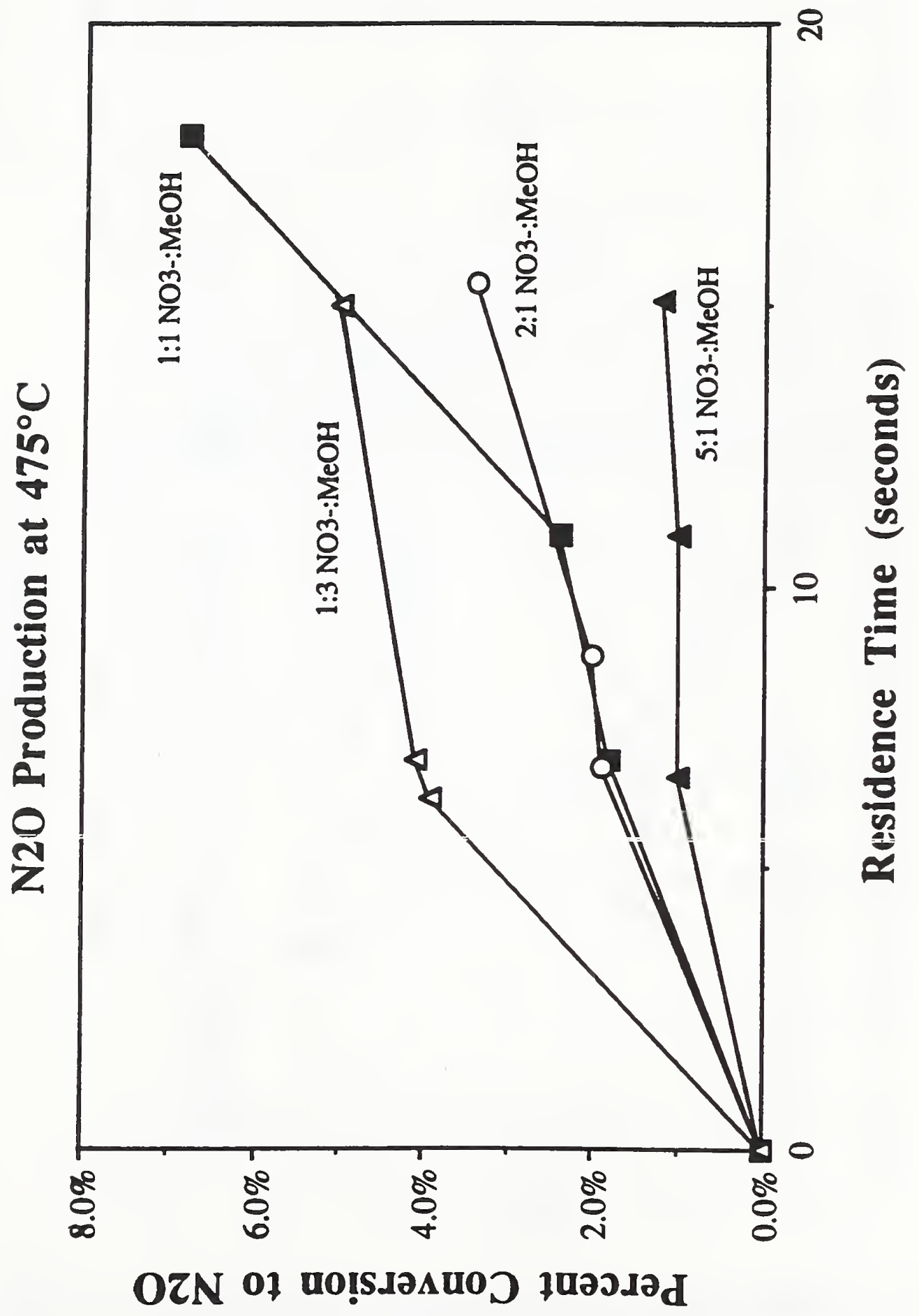




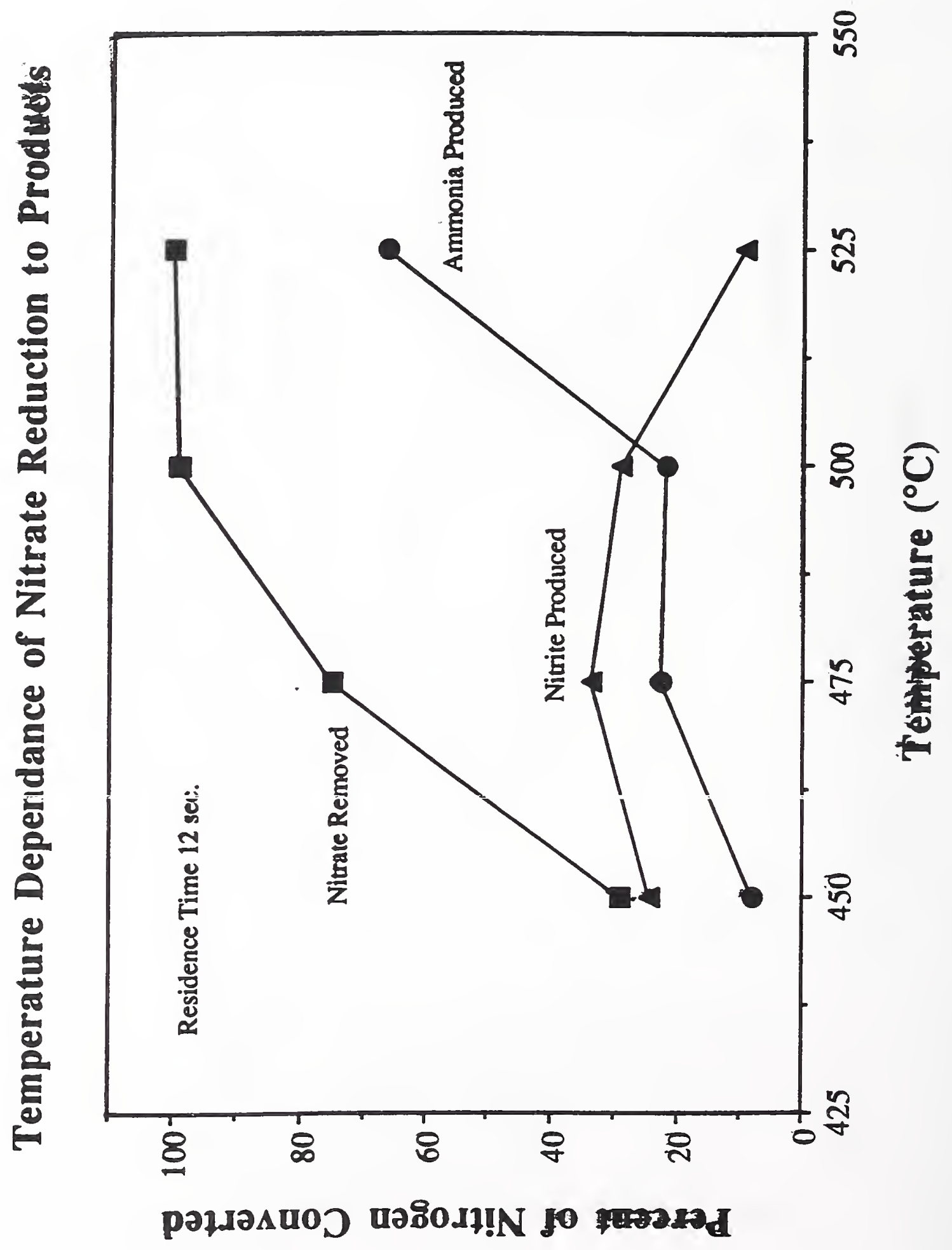




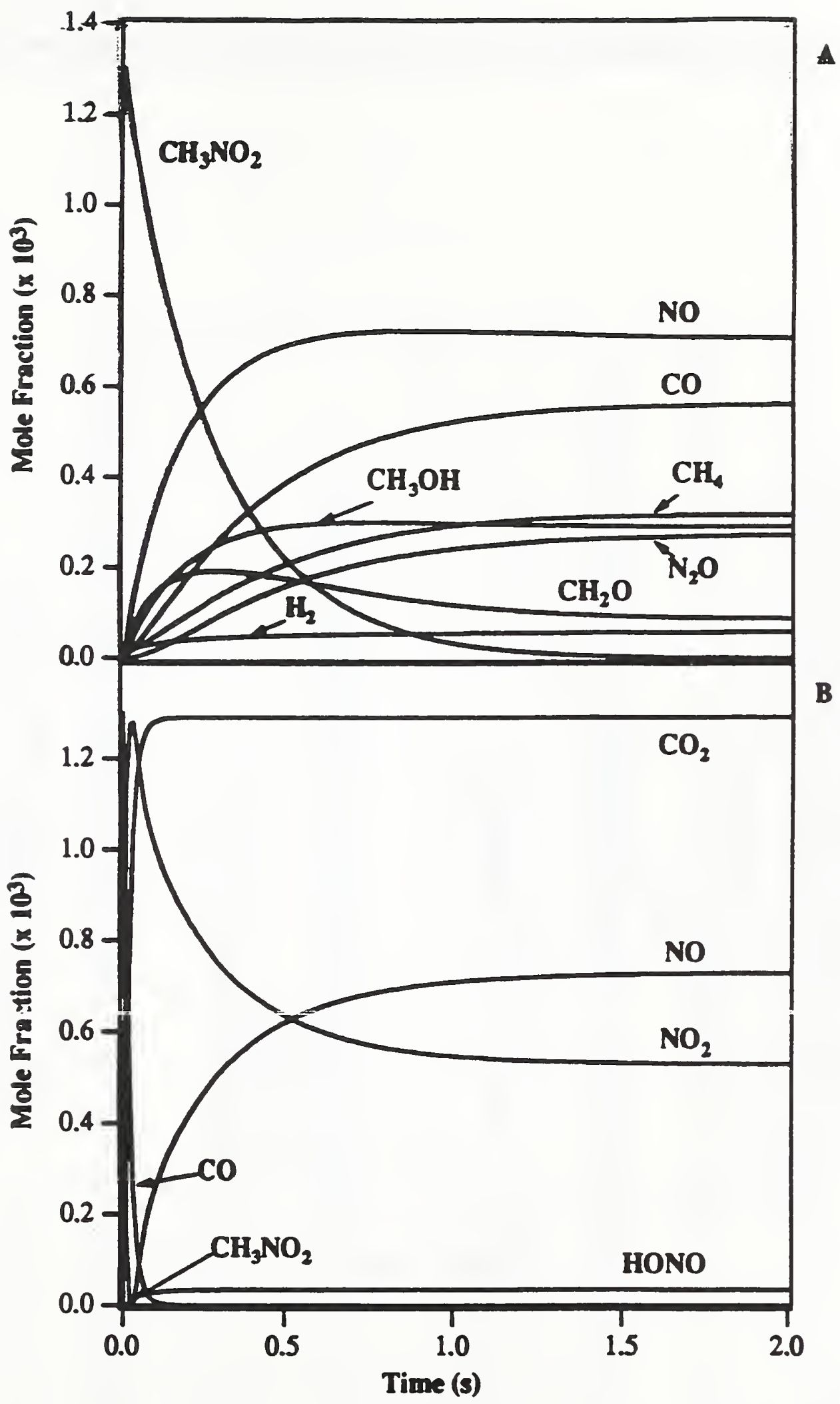

Figure 33 (A) Calculated species profiles for the decomposition of nitromethene in supercritical water at $550^{\circ} \mathrm{C}, 330$ atm and $0.4 \% \mathrm{v} / \mathrm{v}$ witromethane in water. (D) S-ree calculation with the addition of $\mathrm{H} 2 \mathrm{O} 2$ in a 10:1 motar ratio wo sidrounethene. 


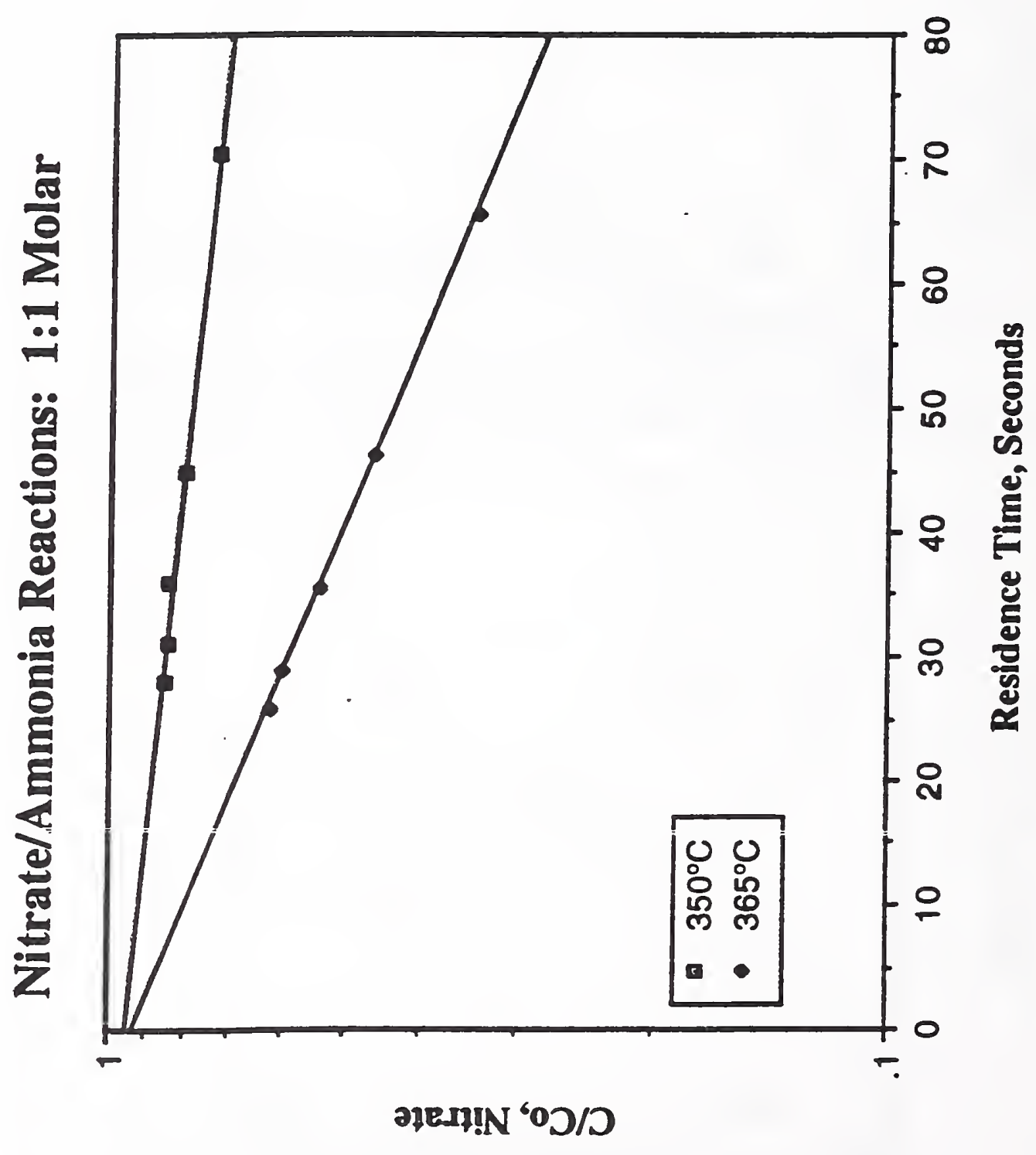




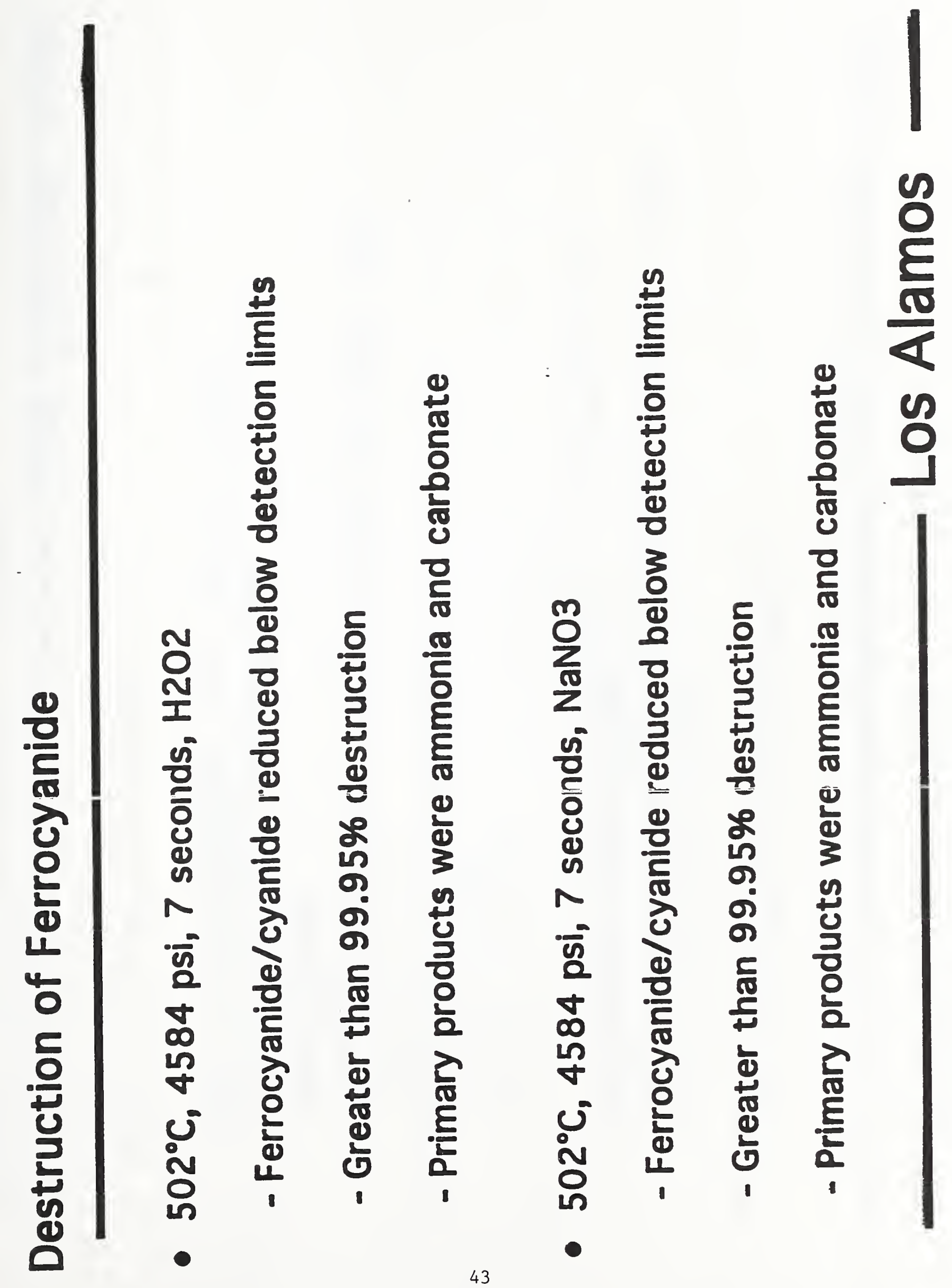




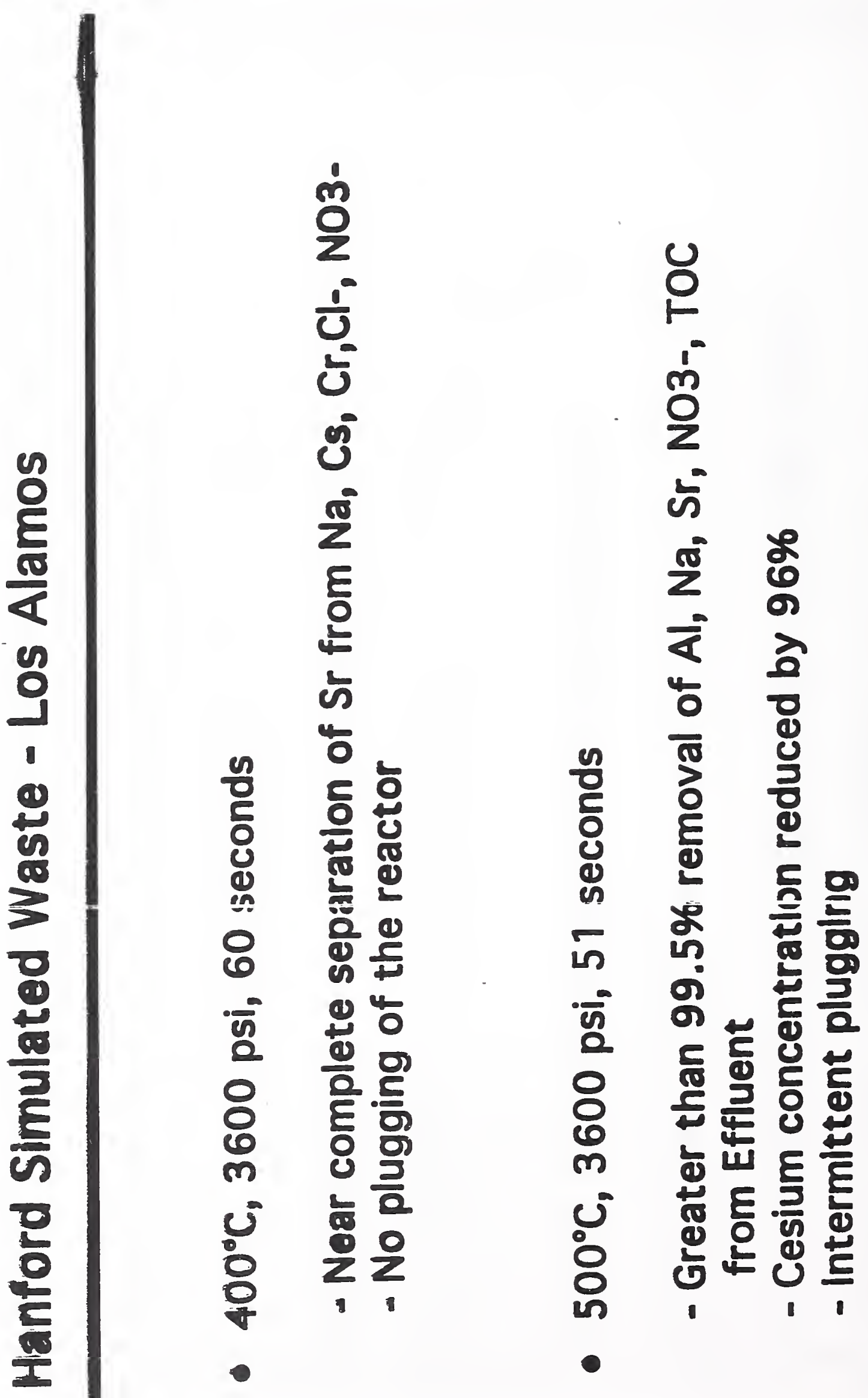




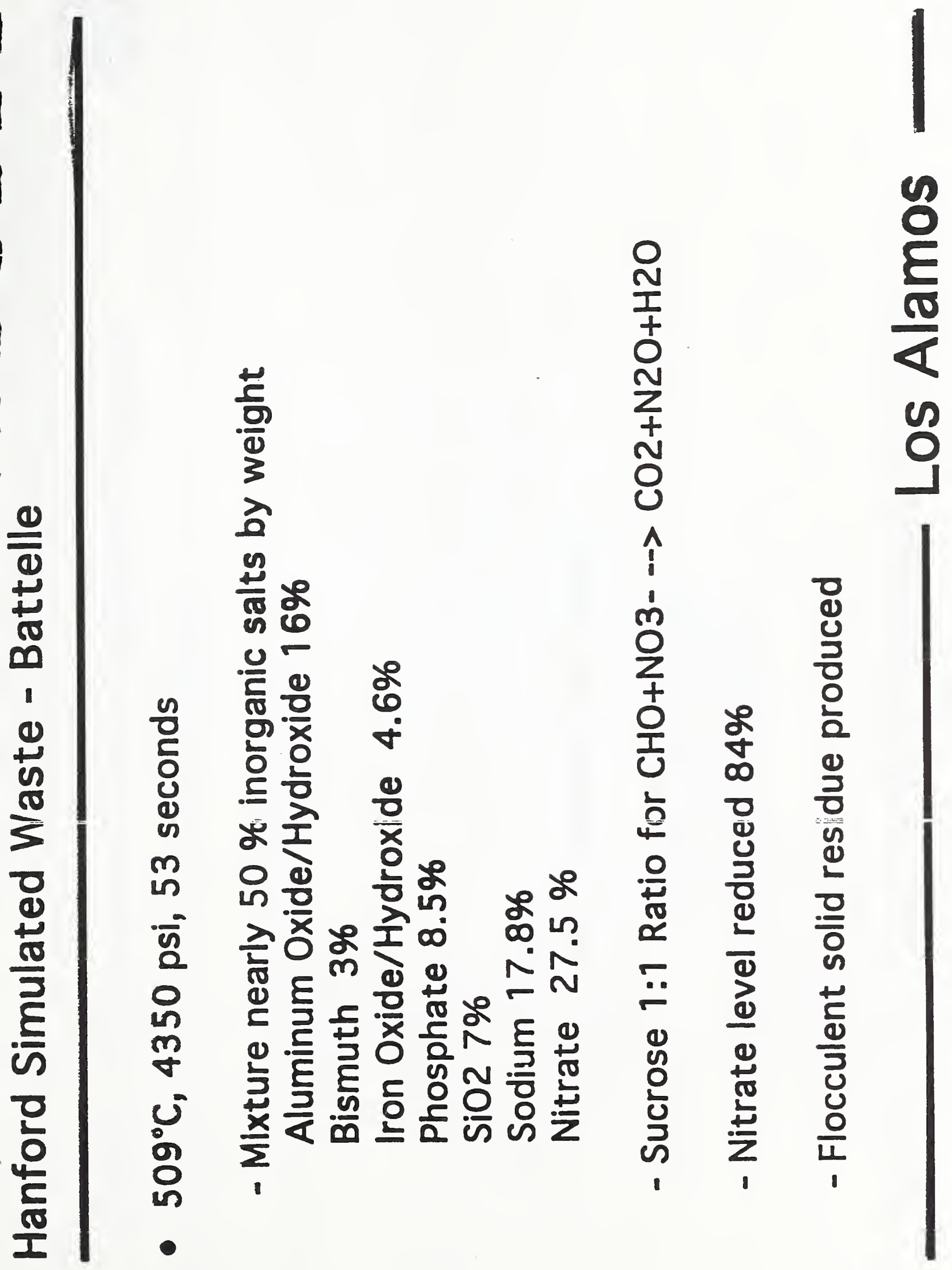




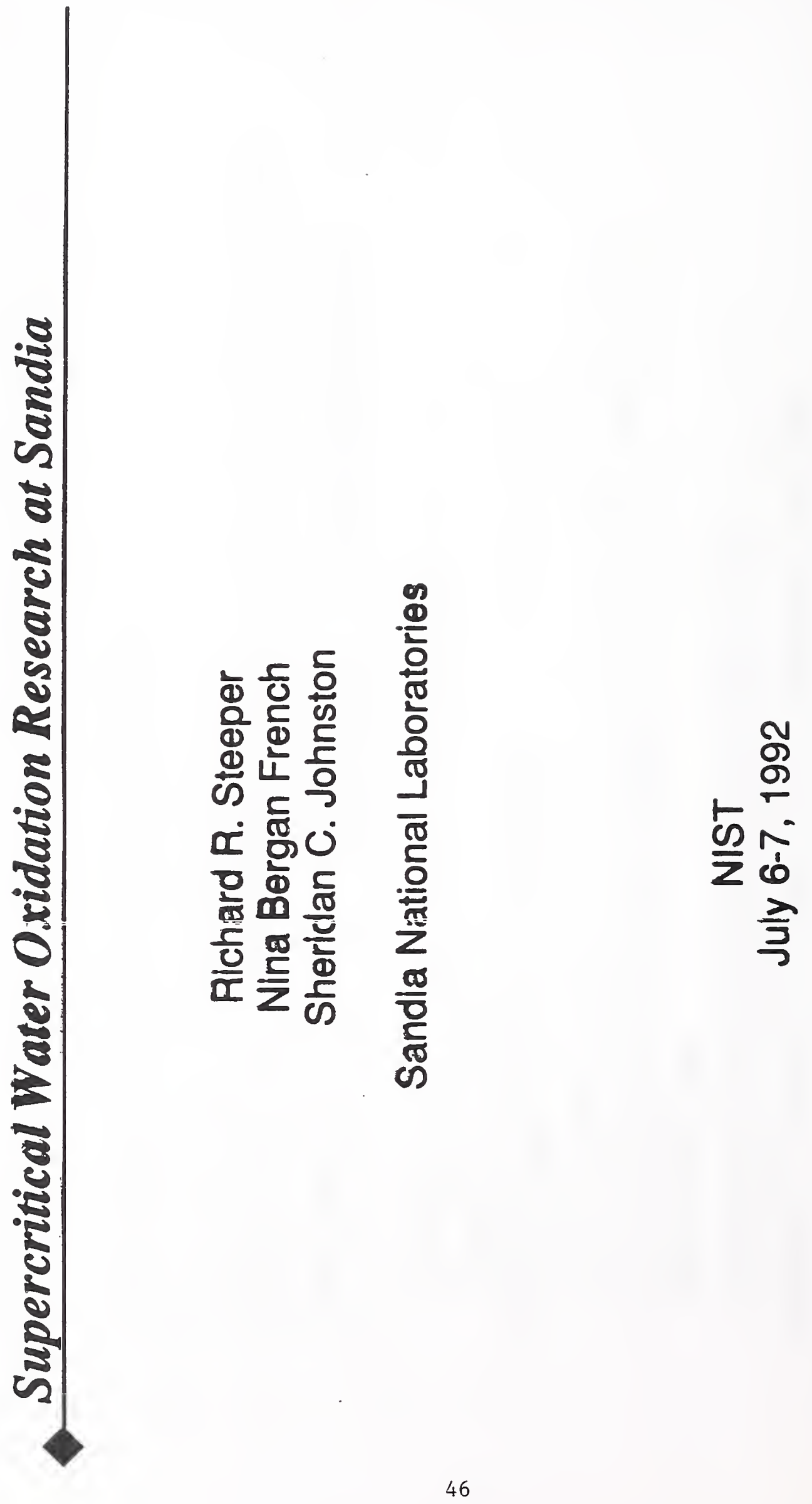




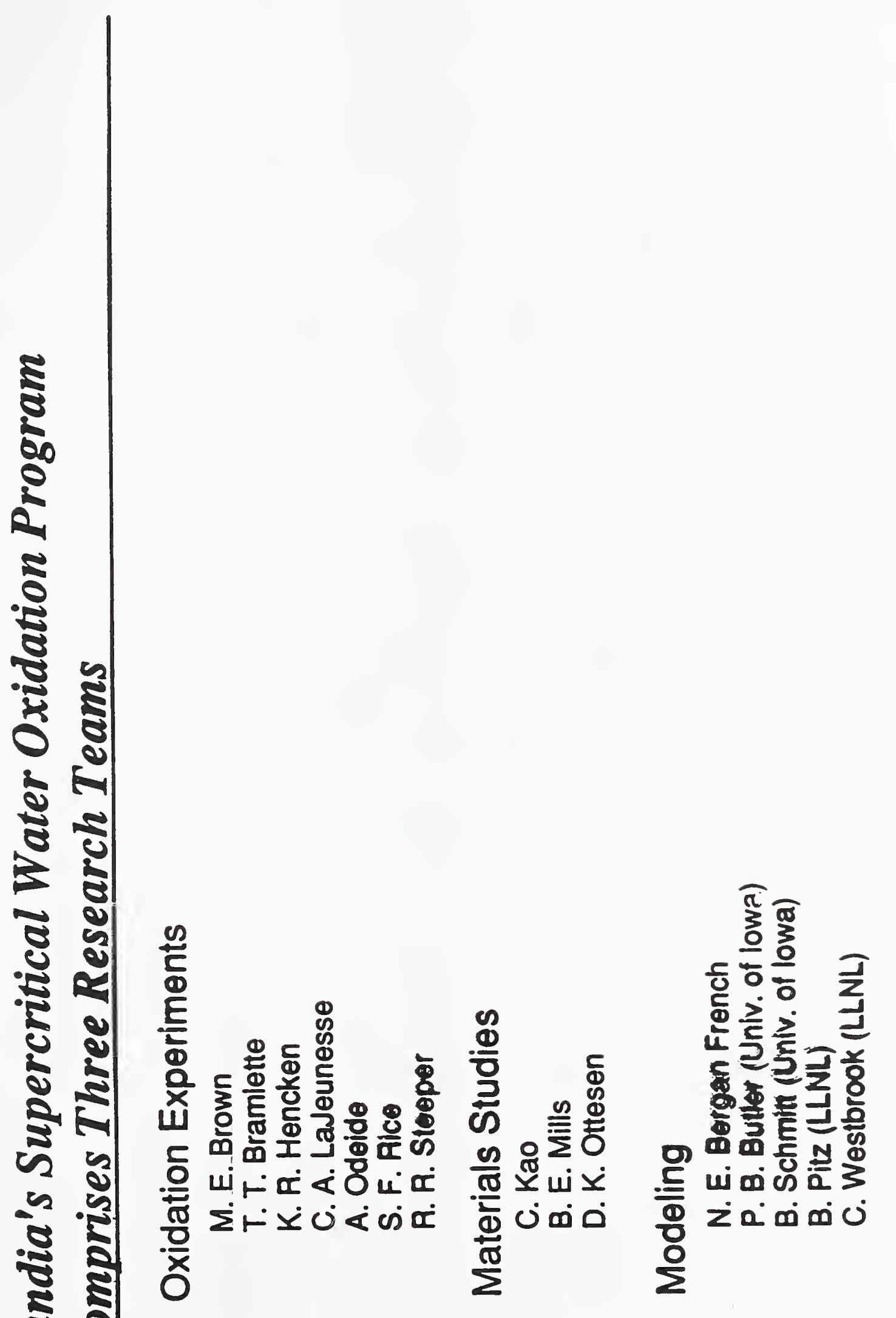




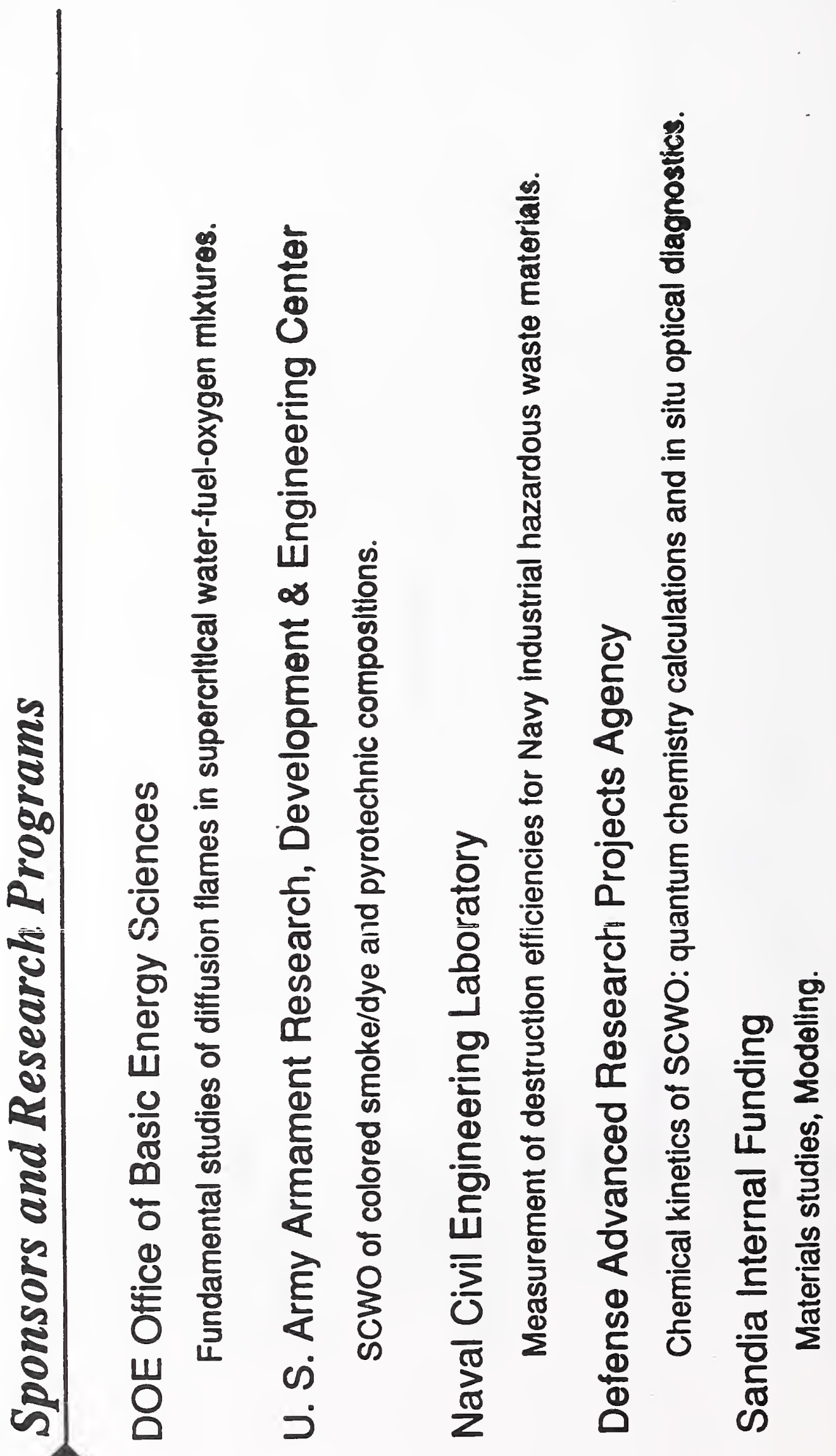




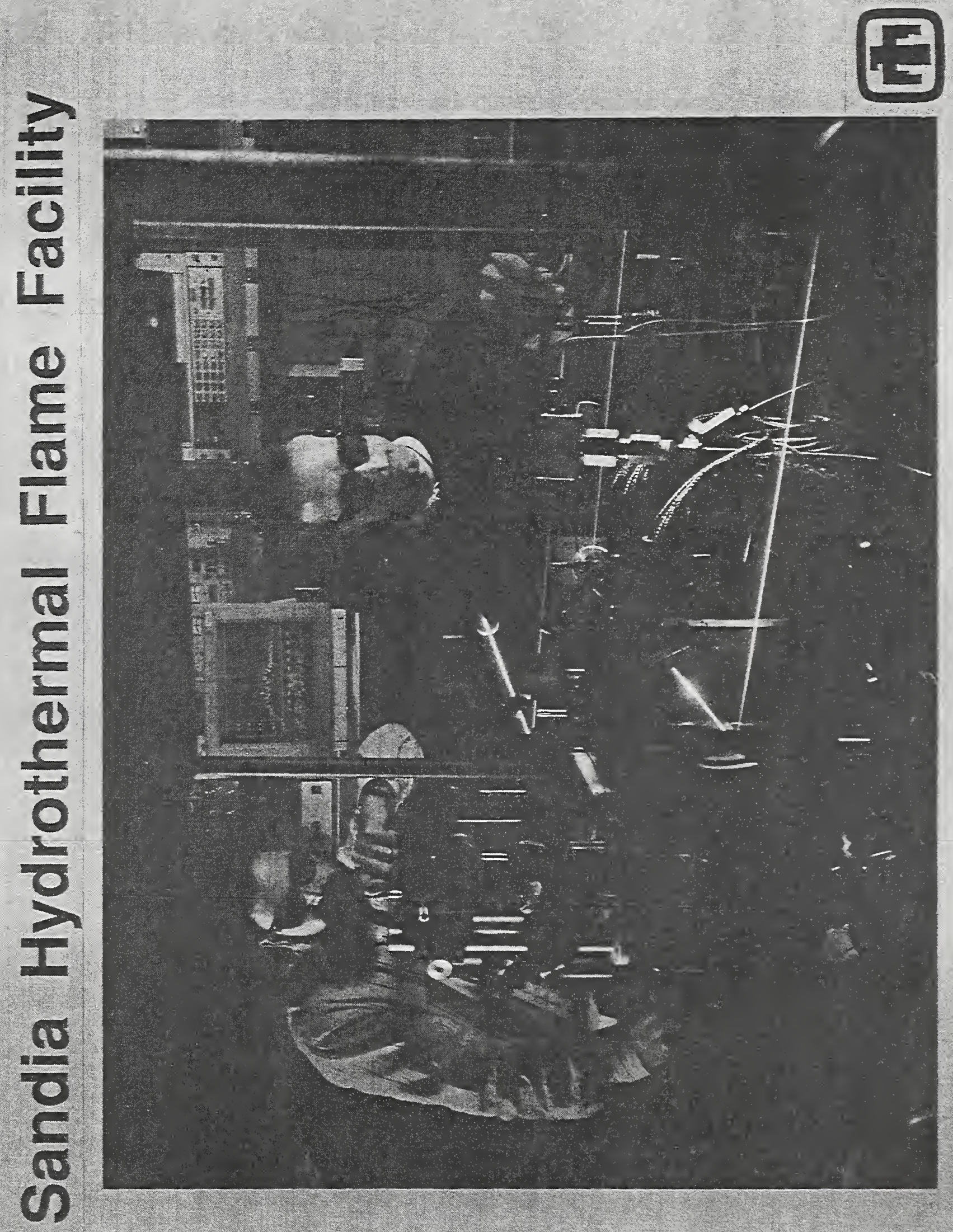




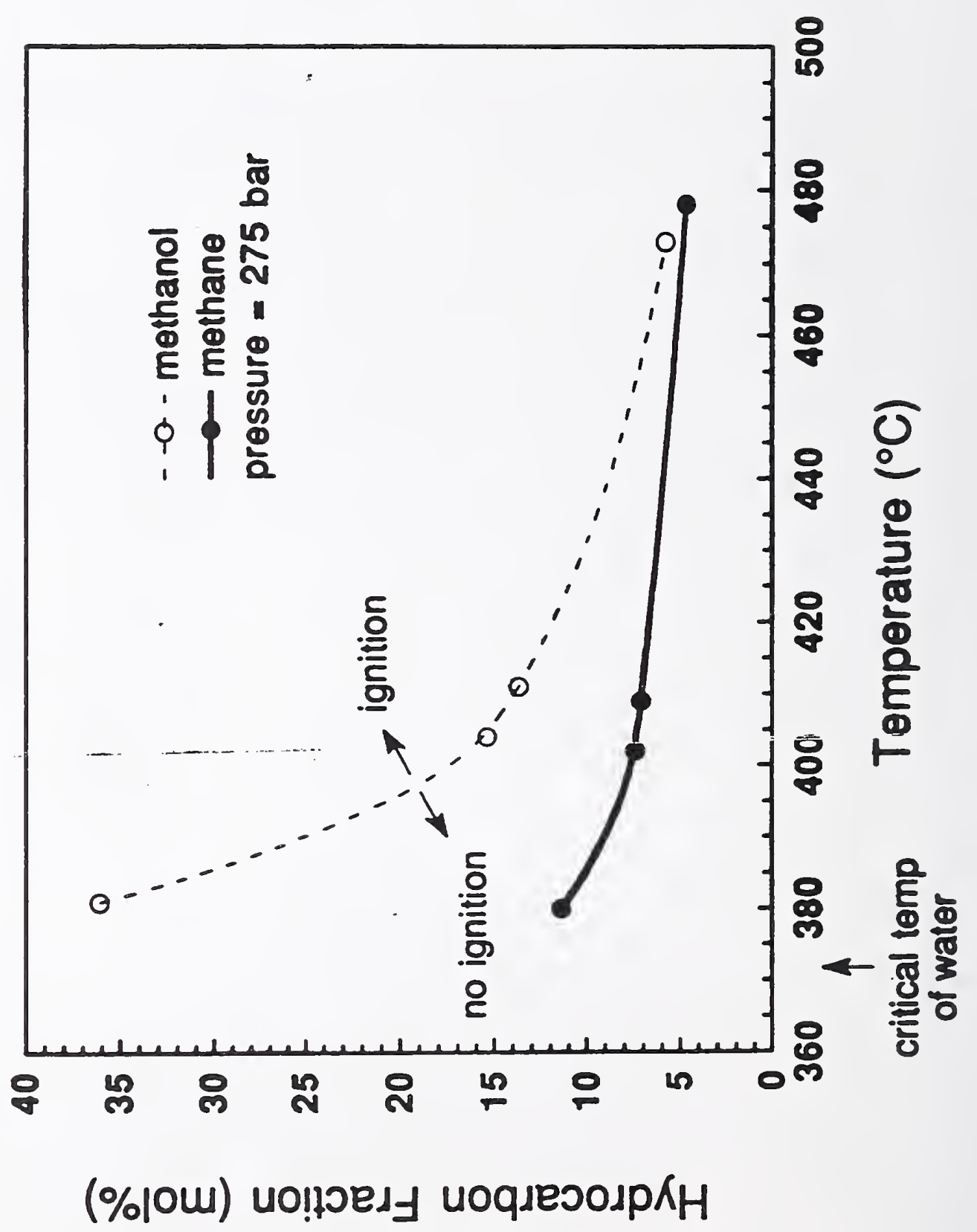



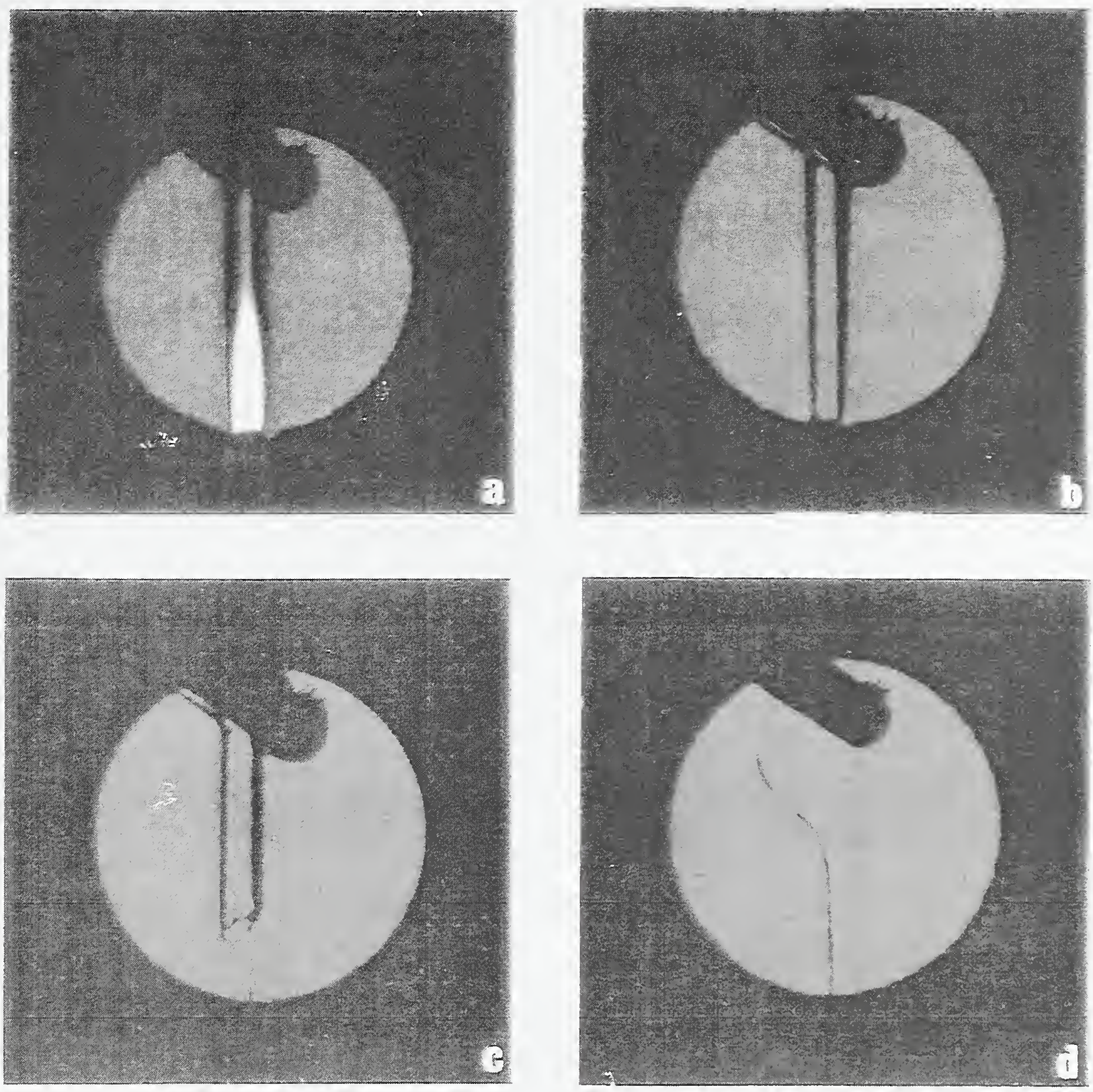


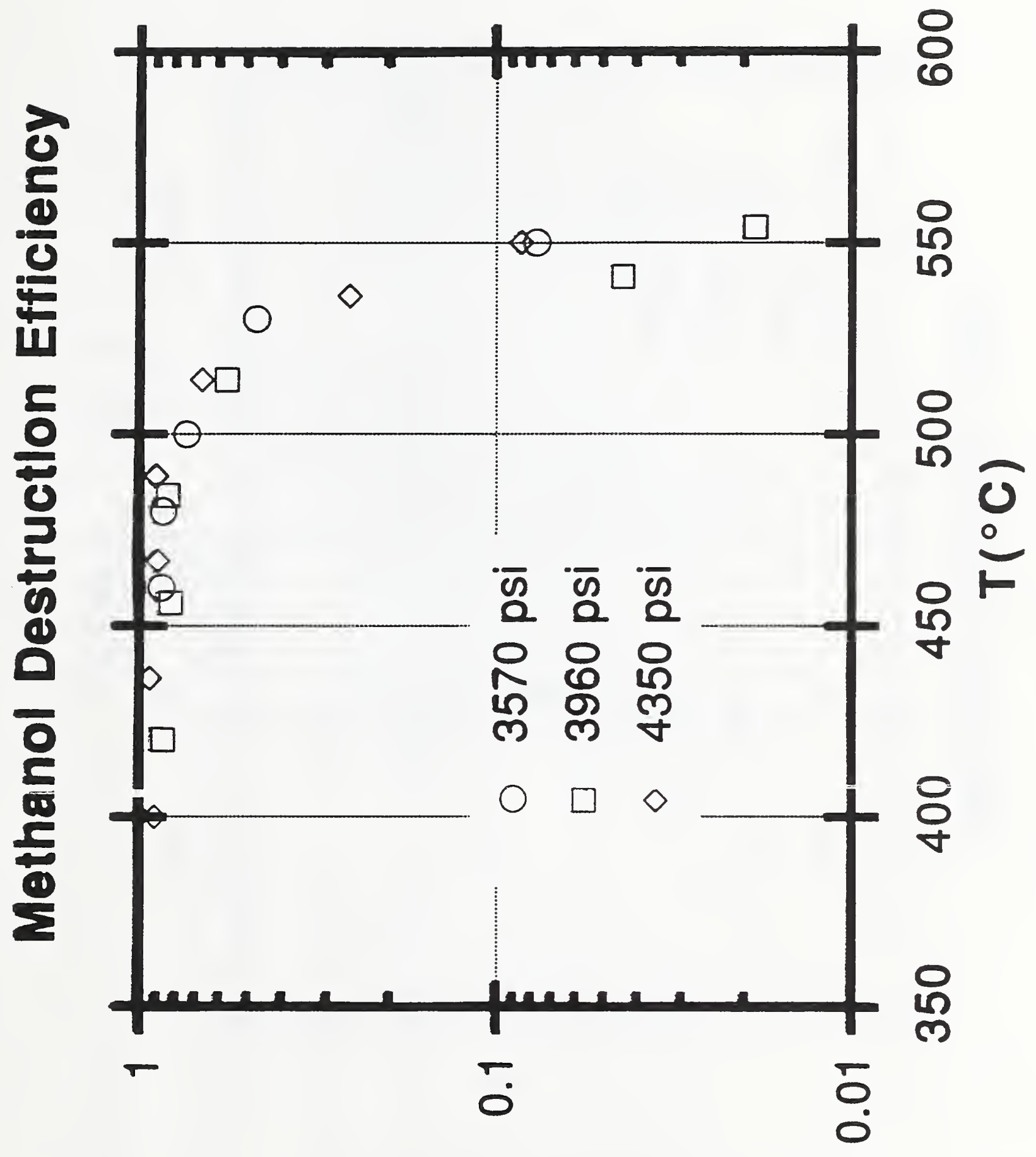

${ }^{\text {u! }}$ [HOәW] $/{ }^{\text {Ino }}$ [HOәW] 


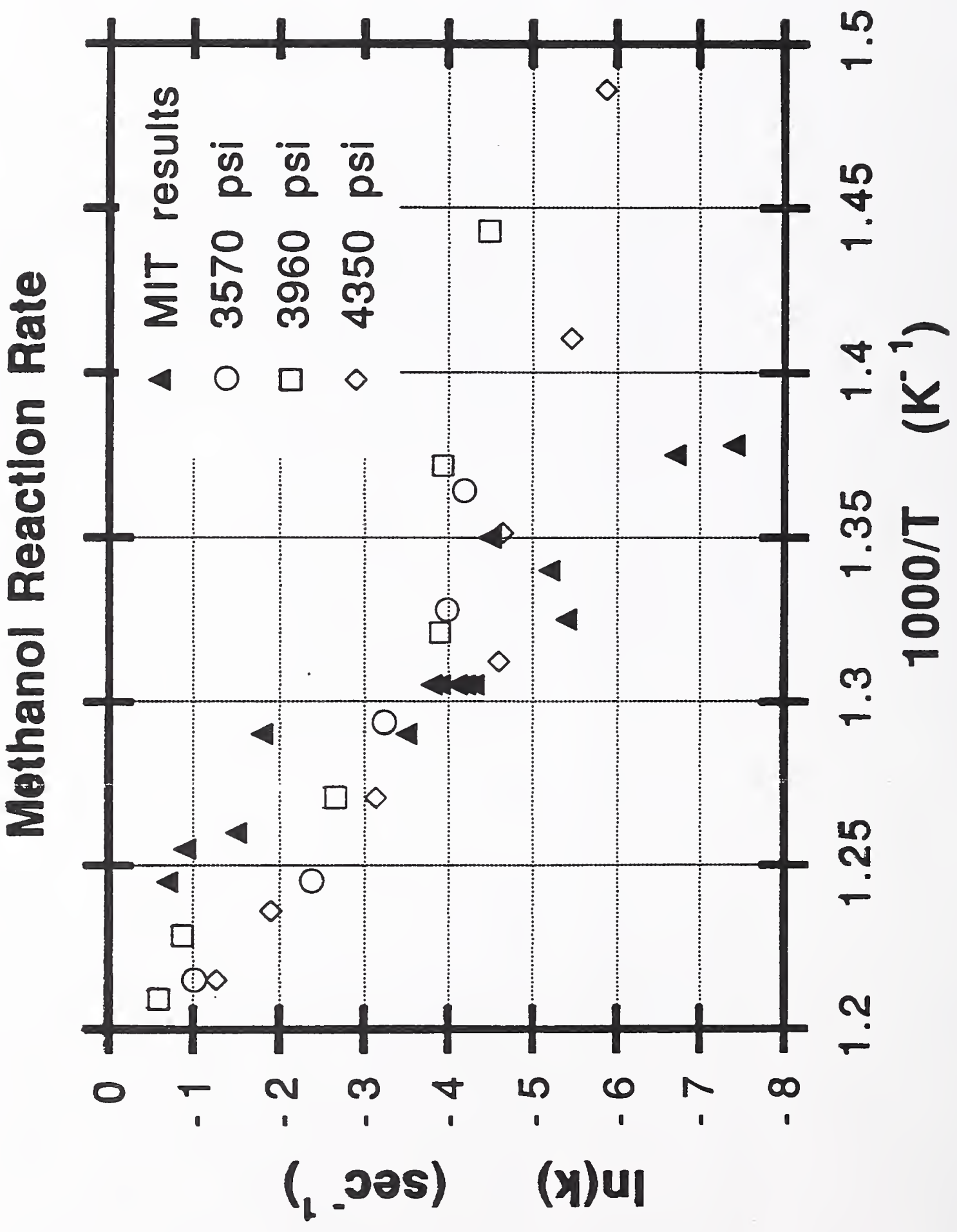




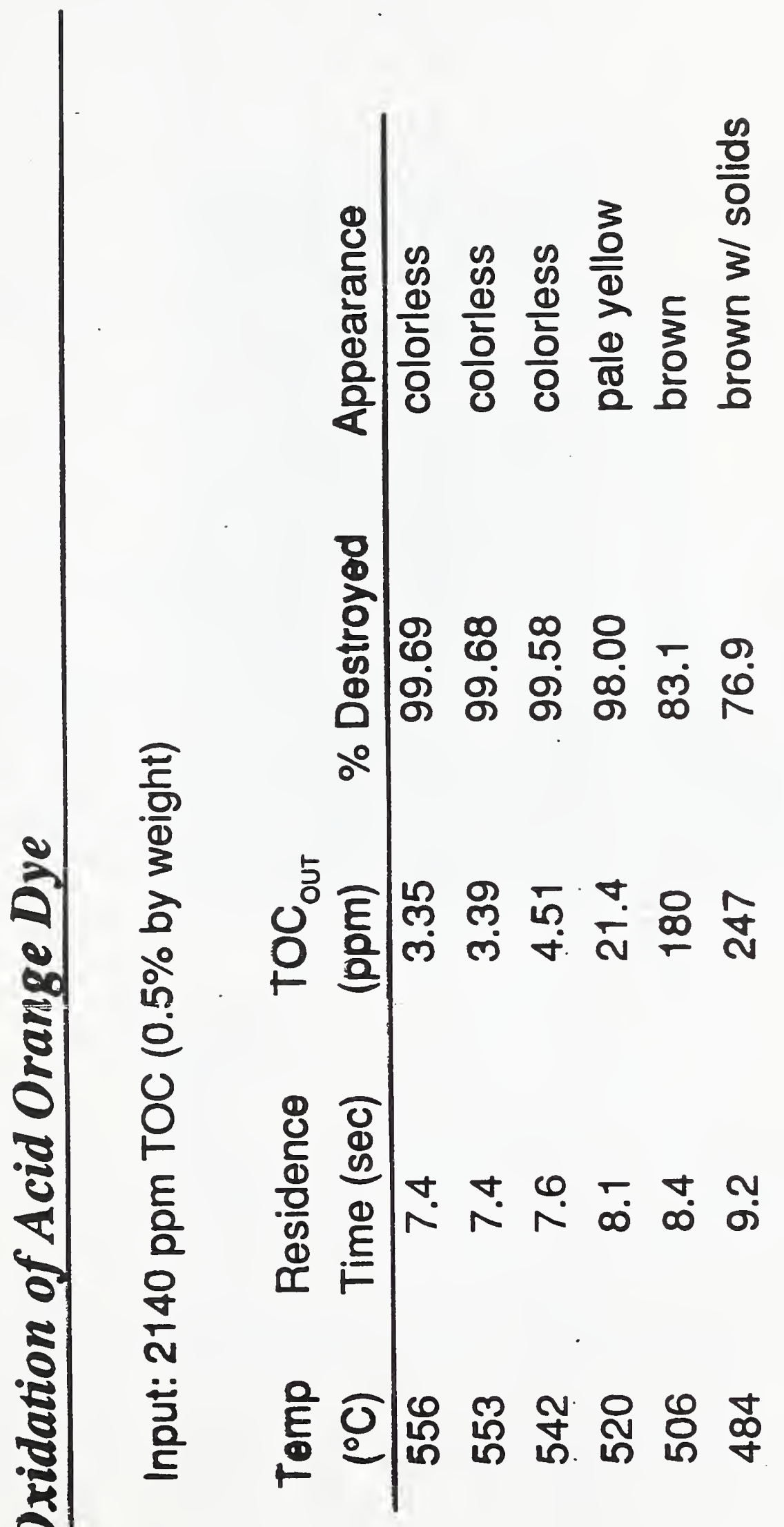




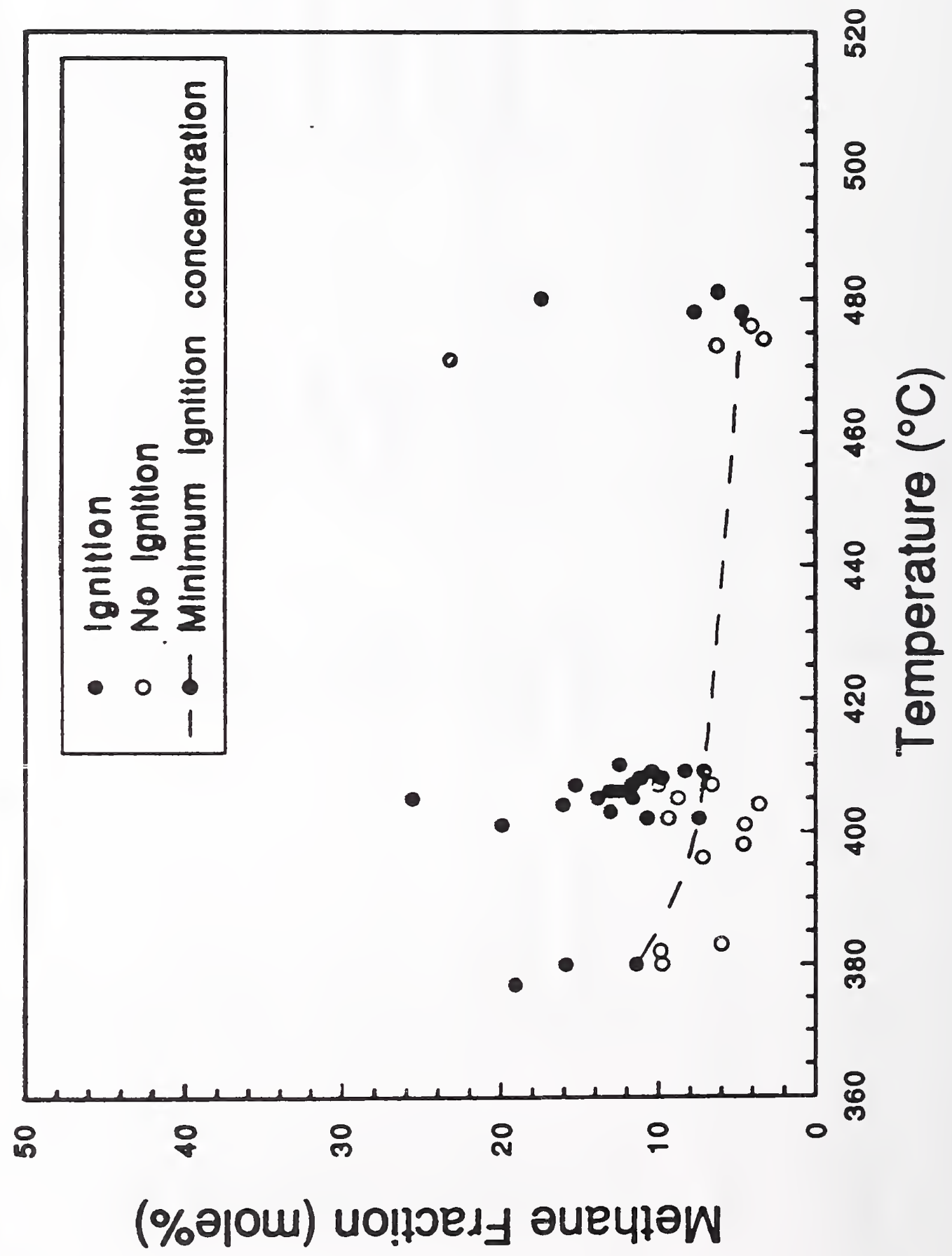




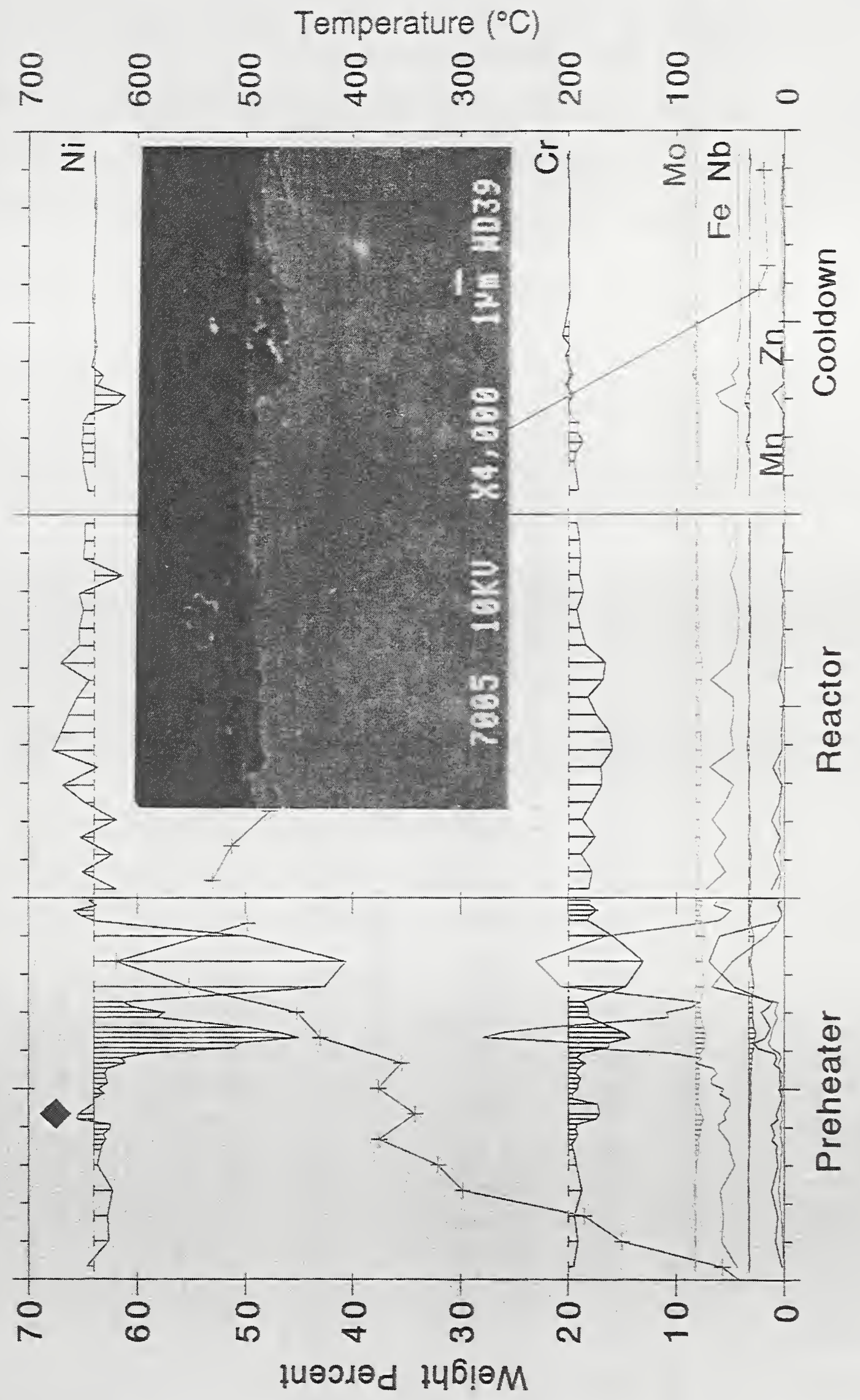




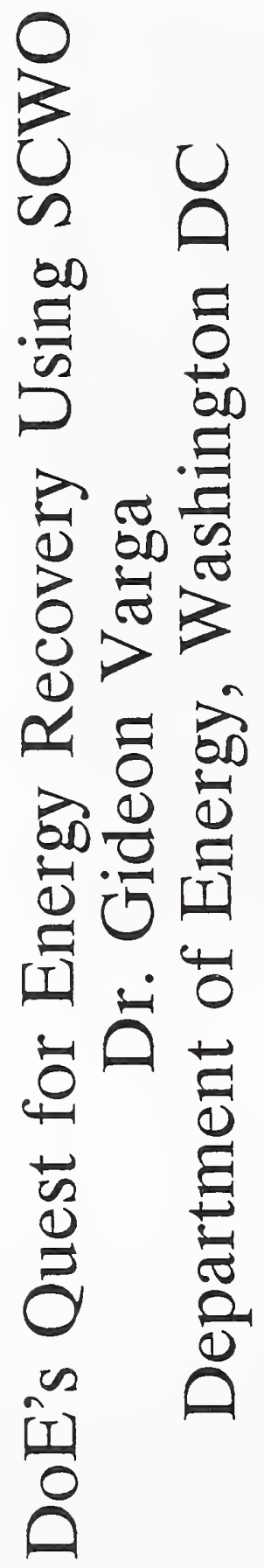




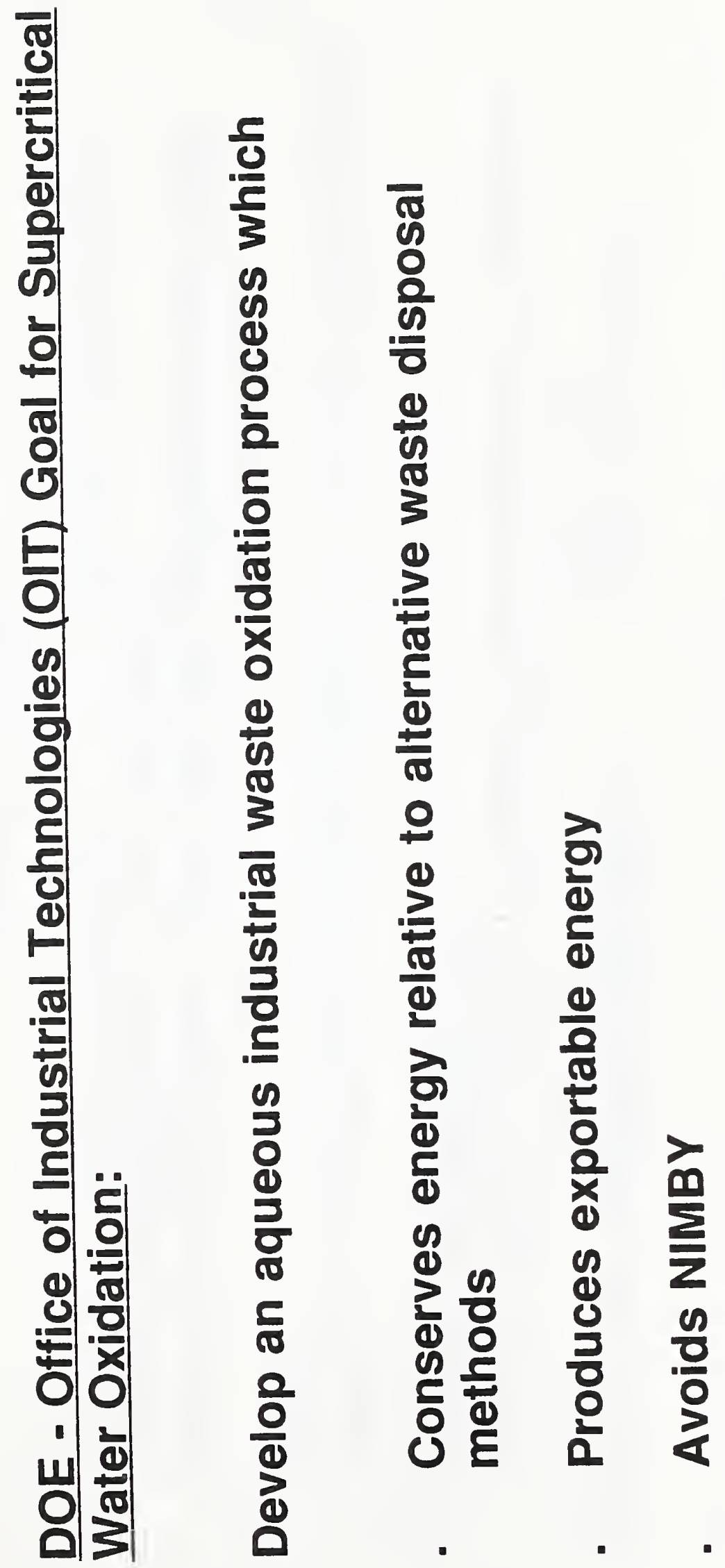




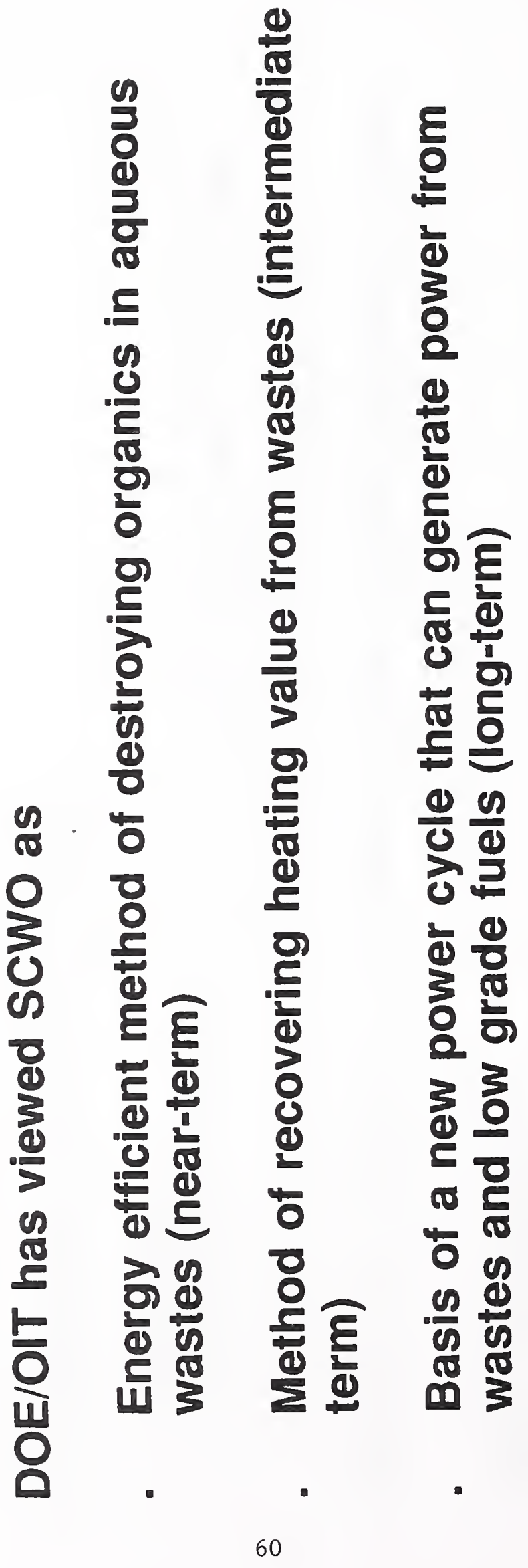




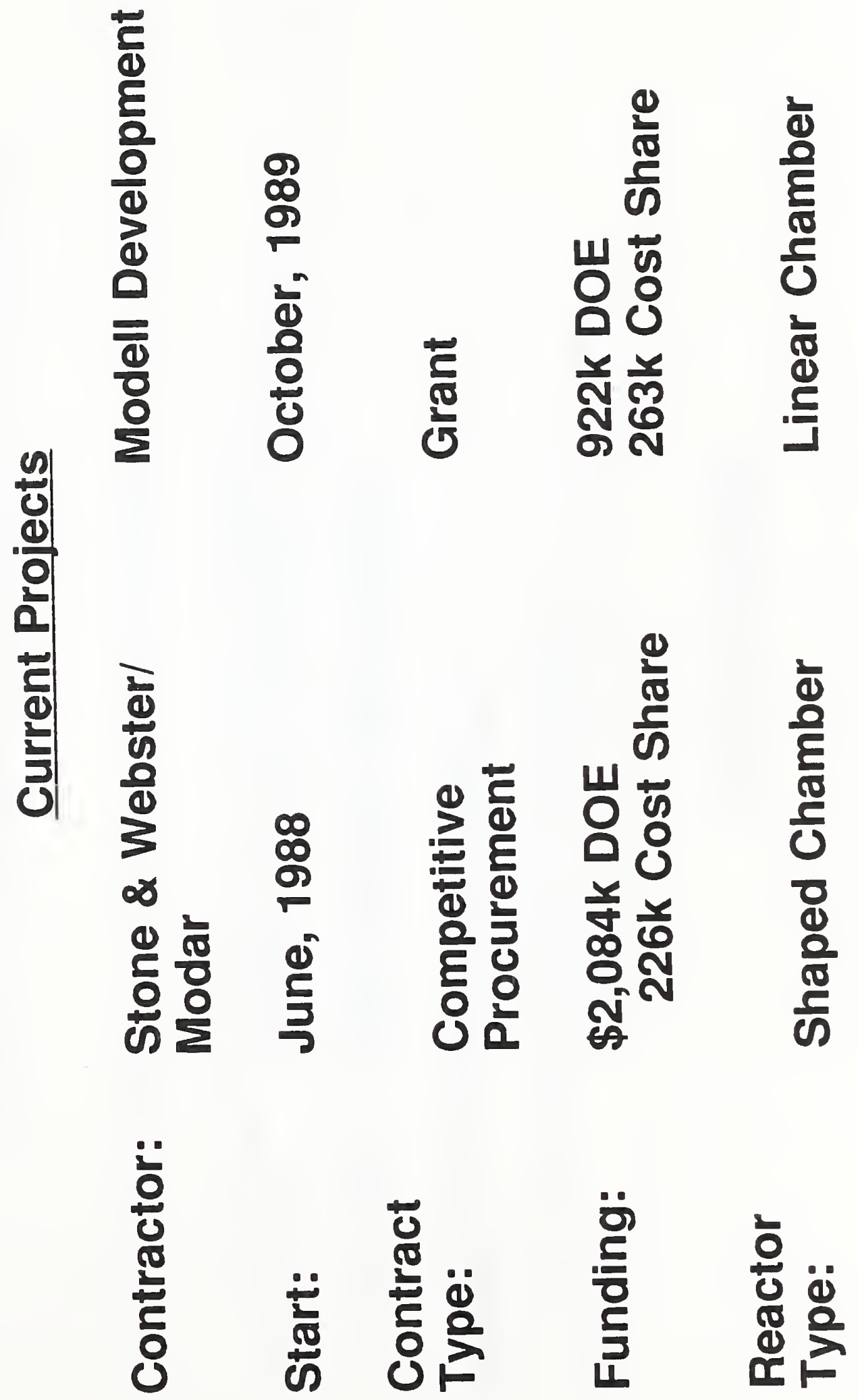




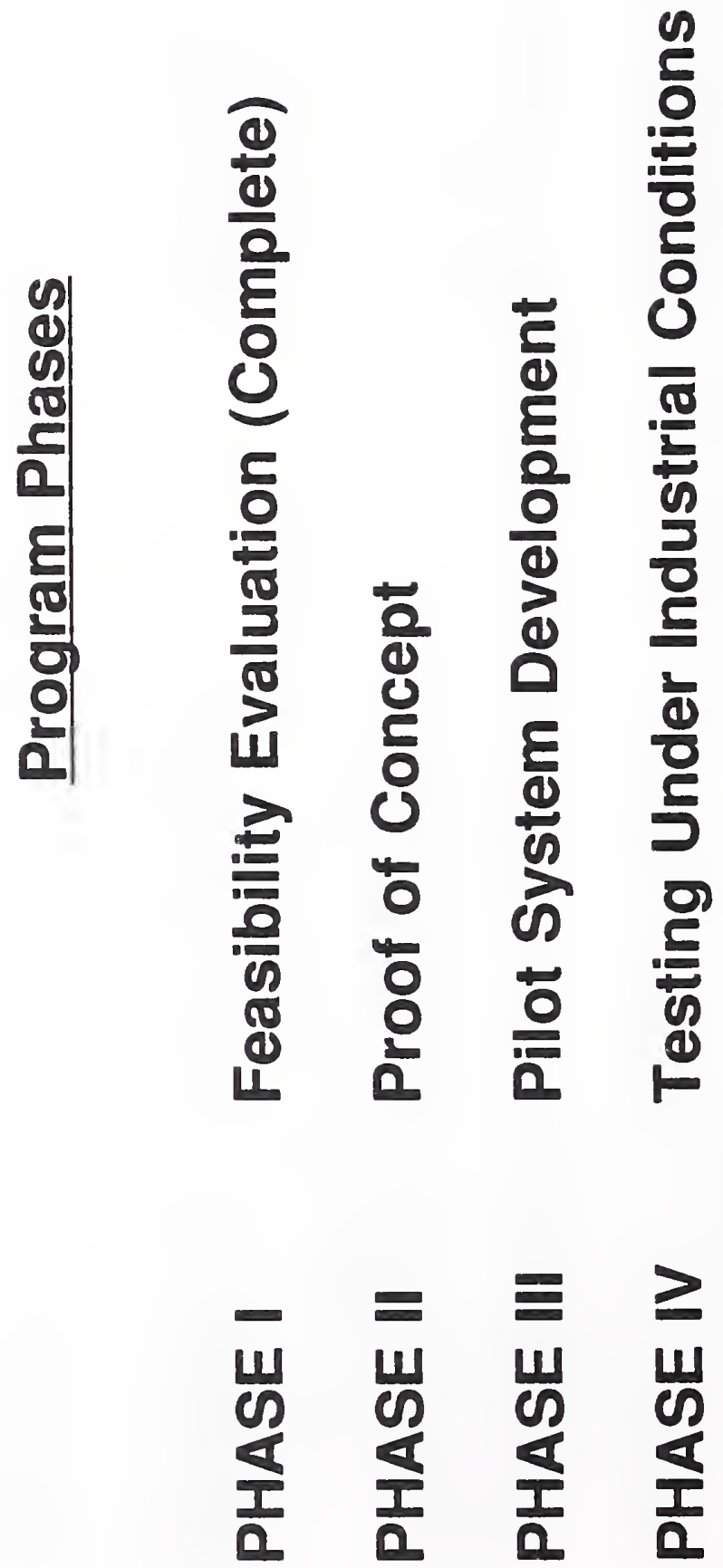




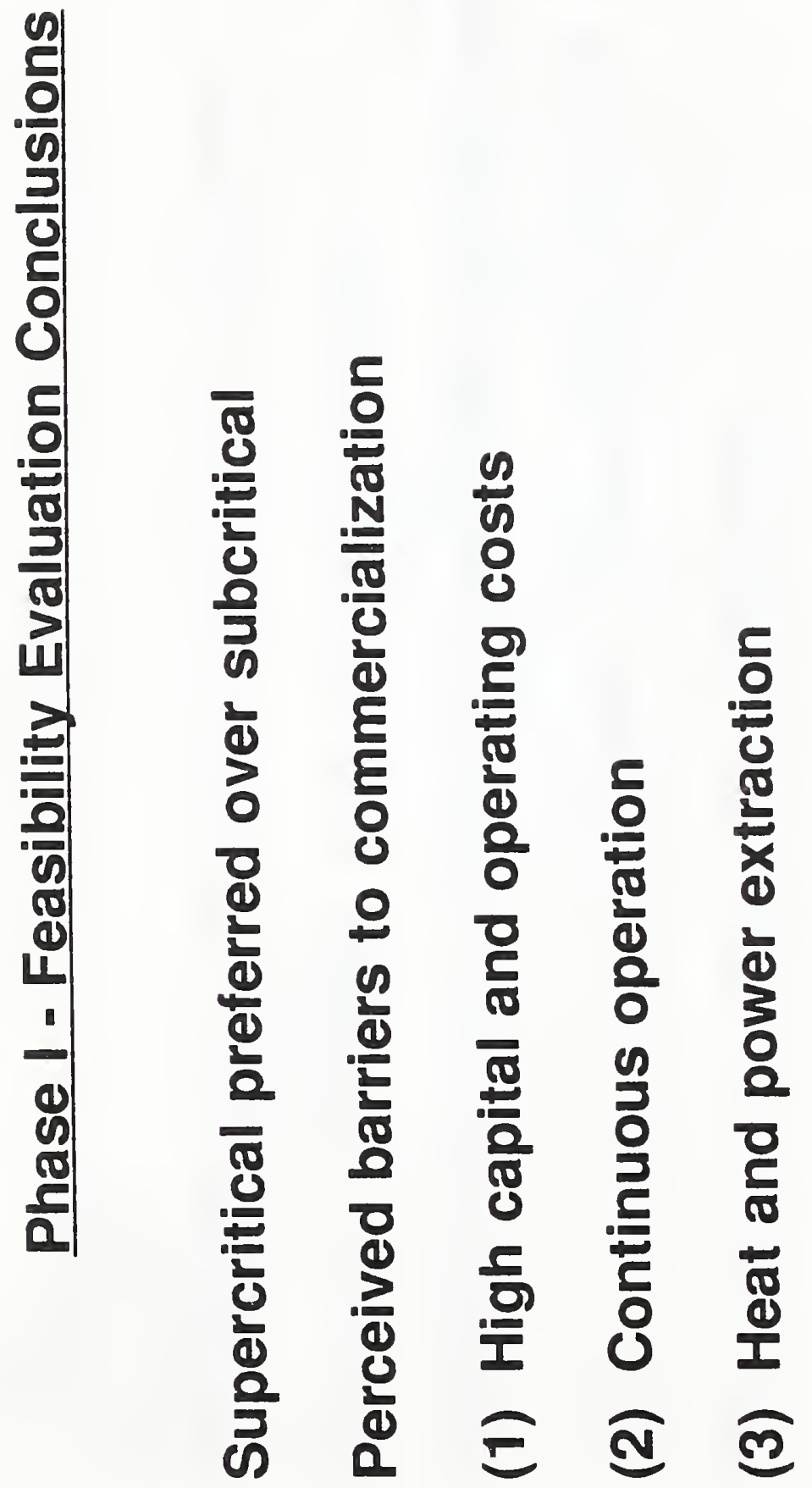




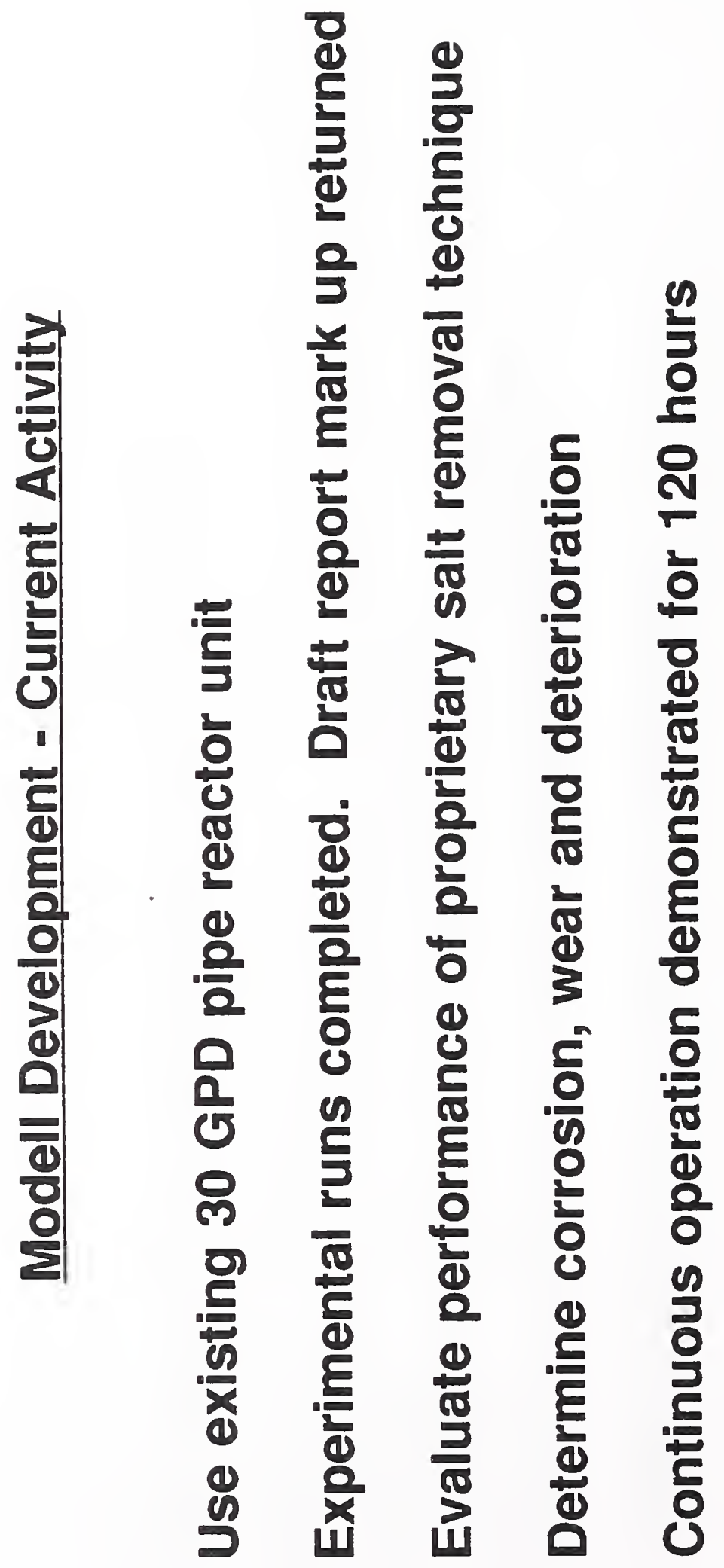



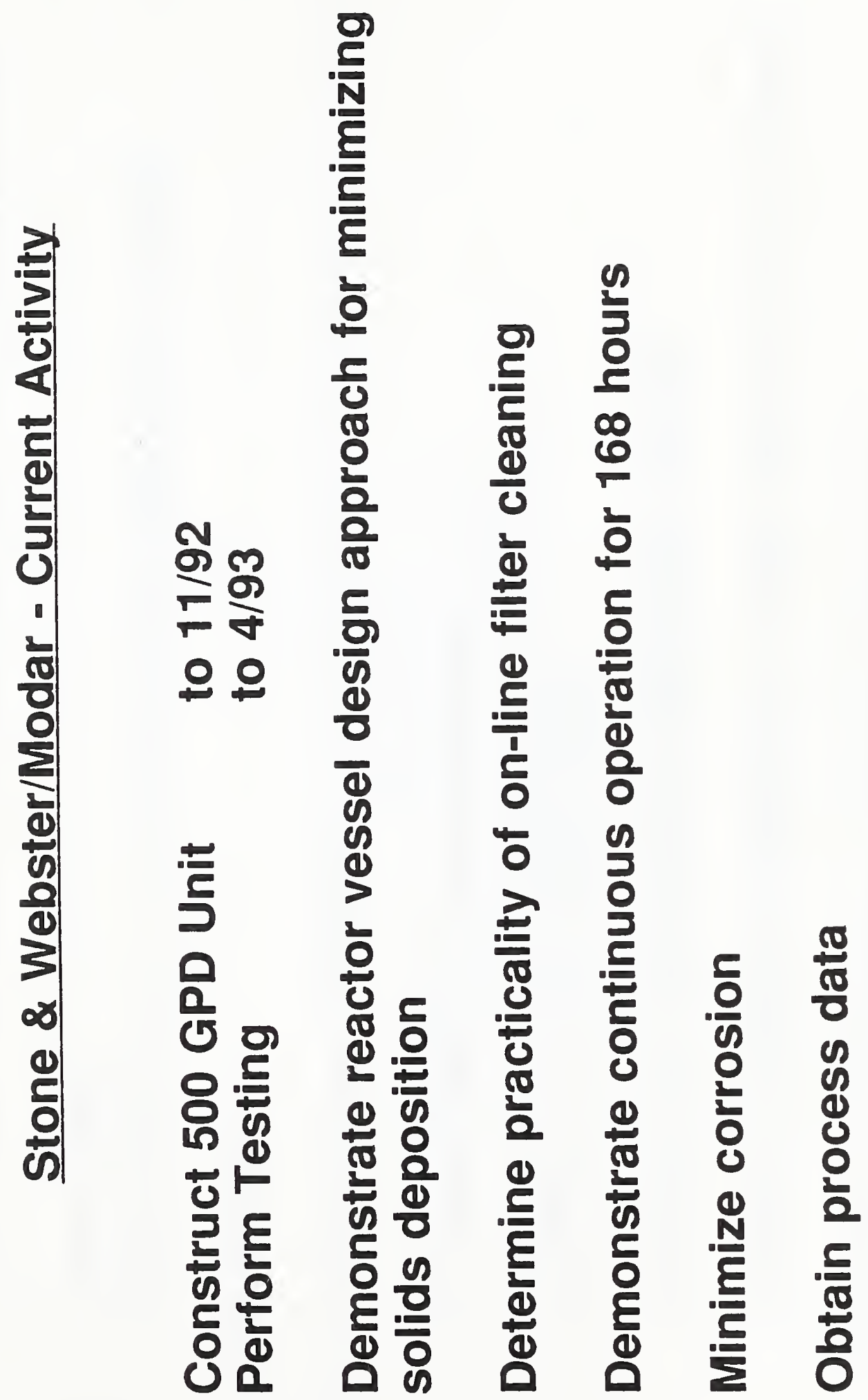


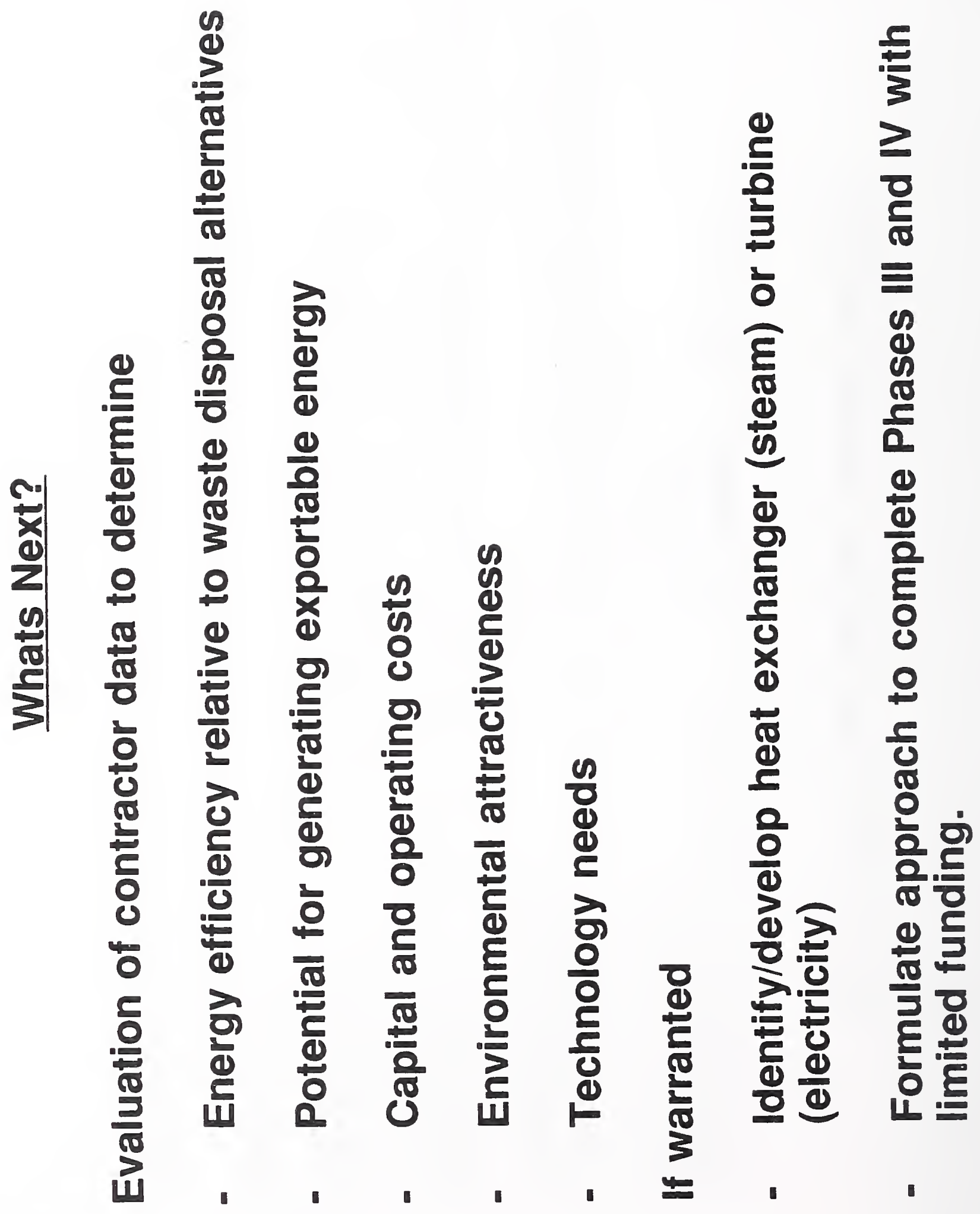




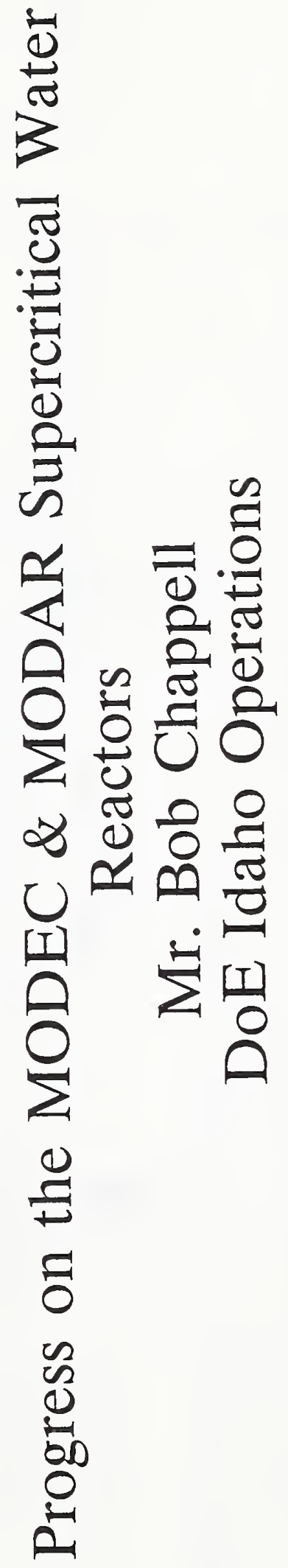



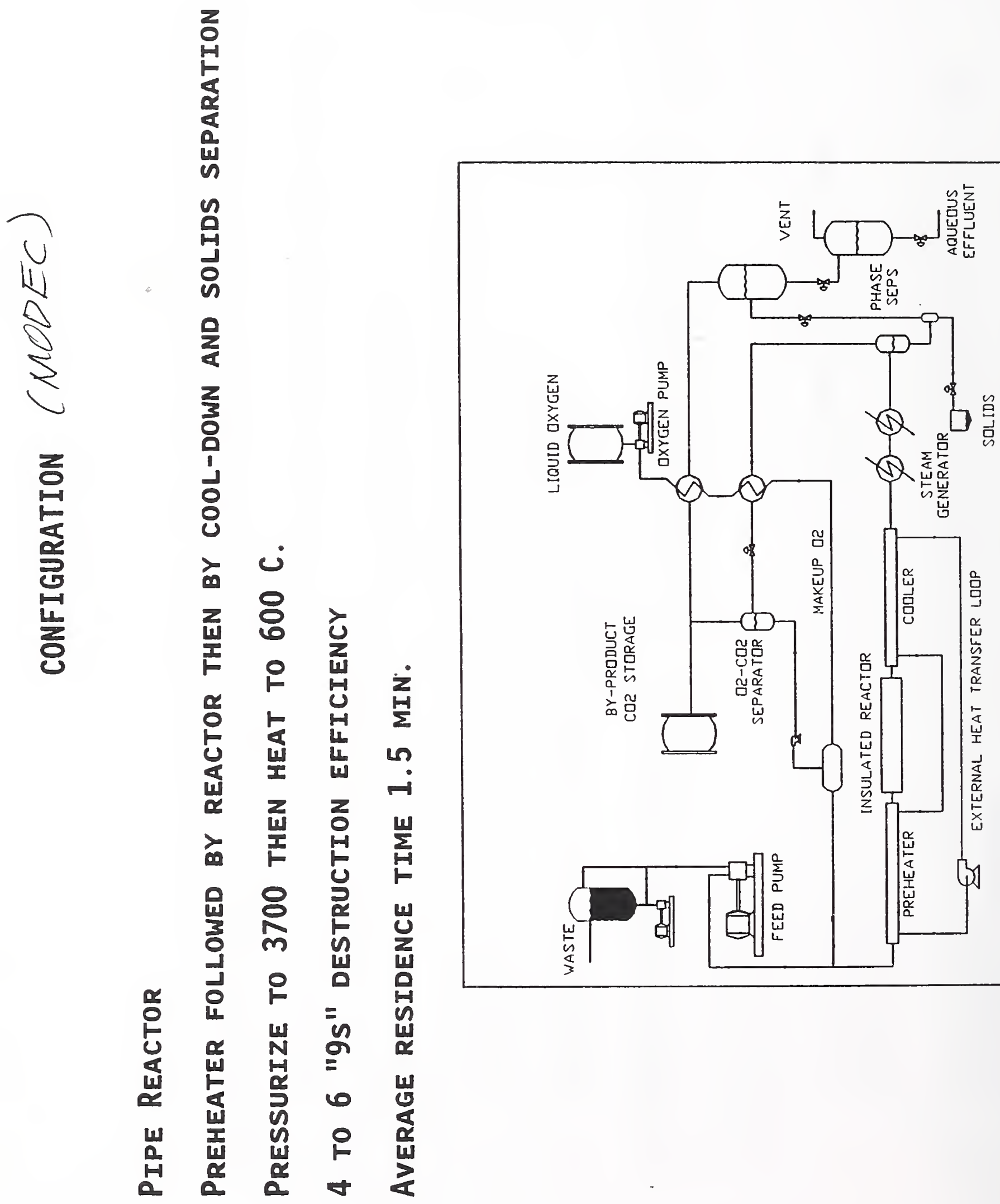


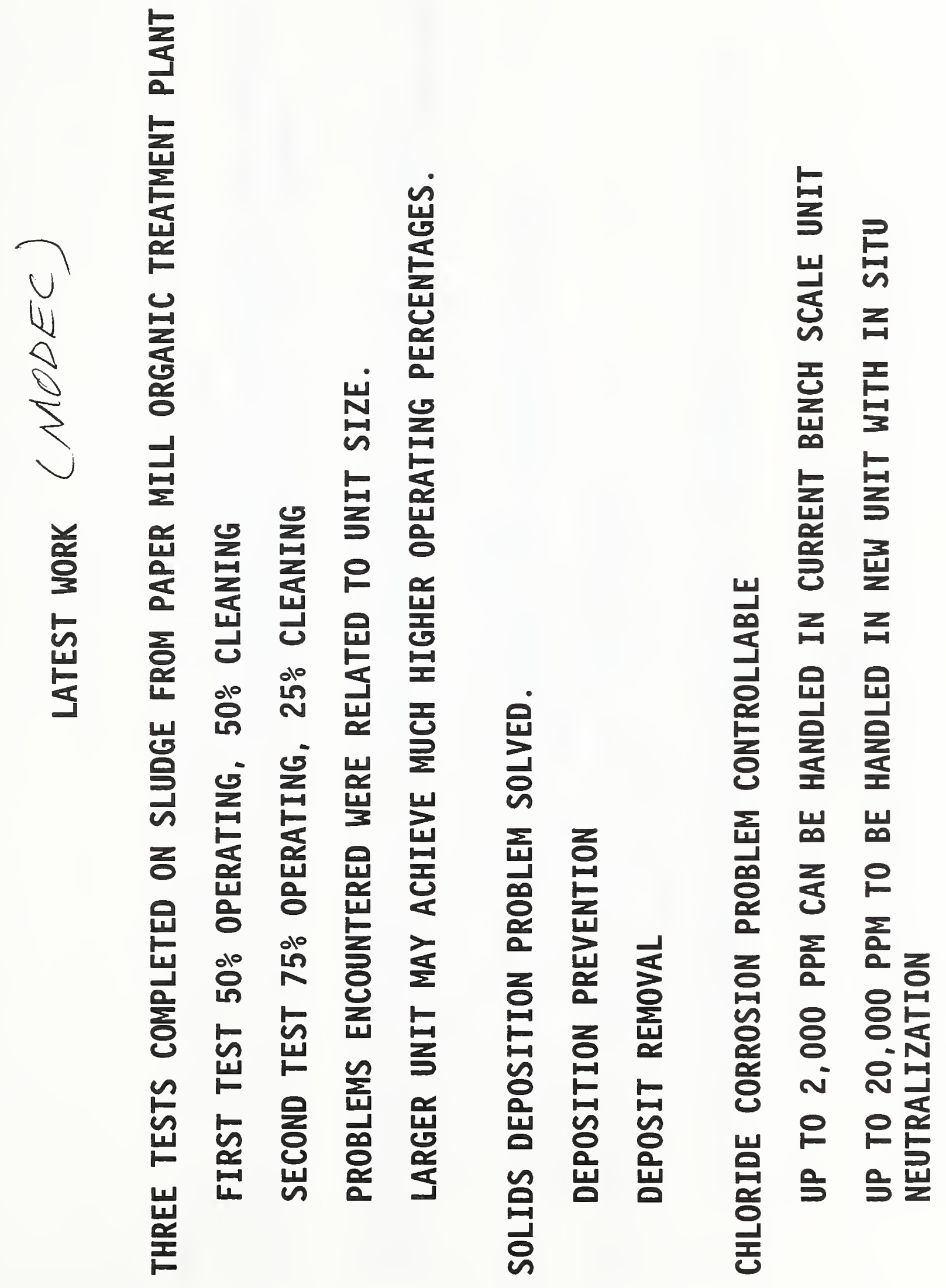



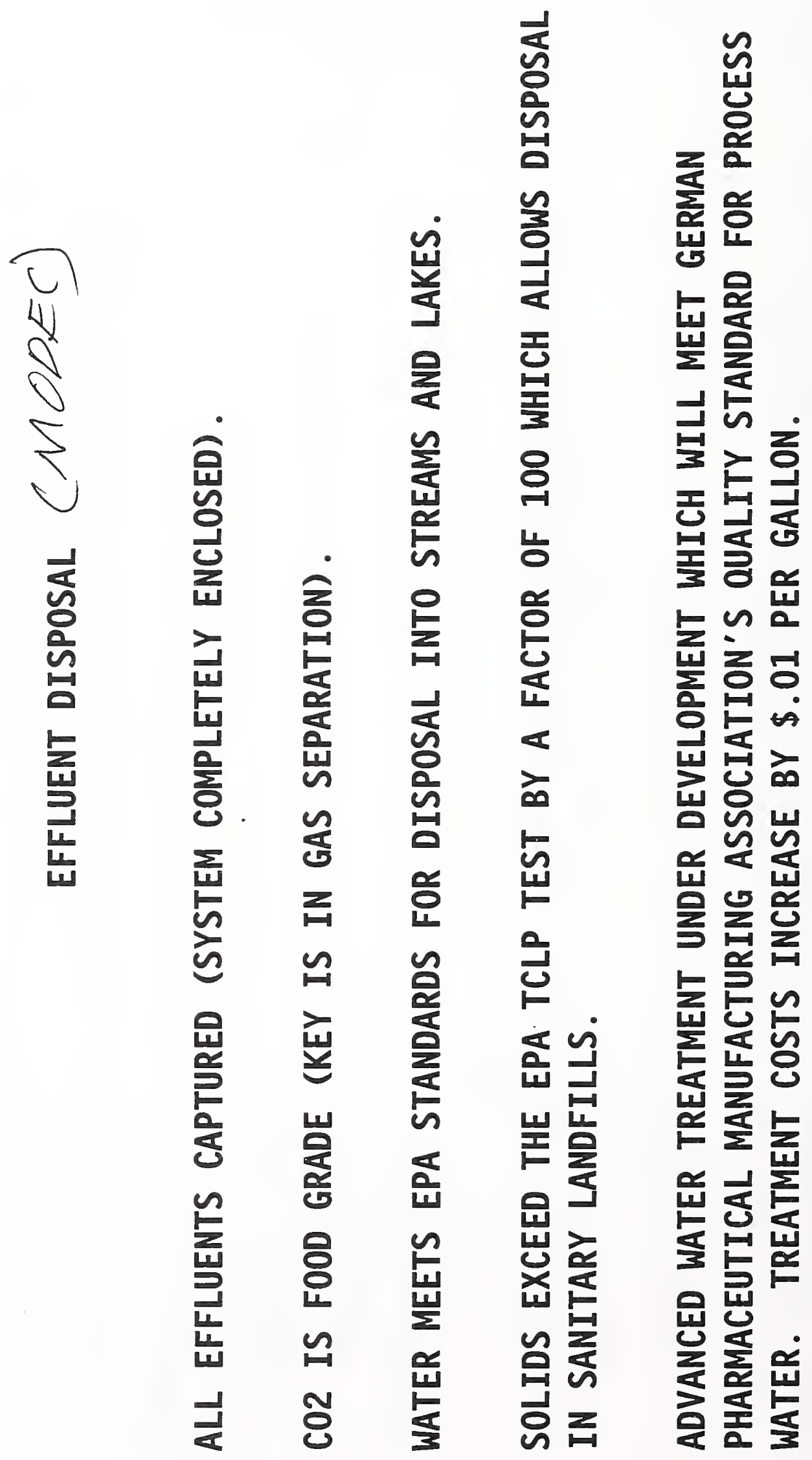


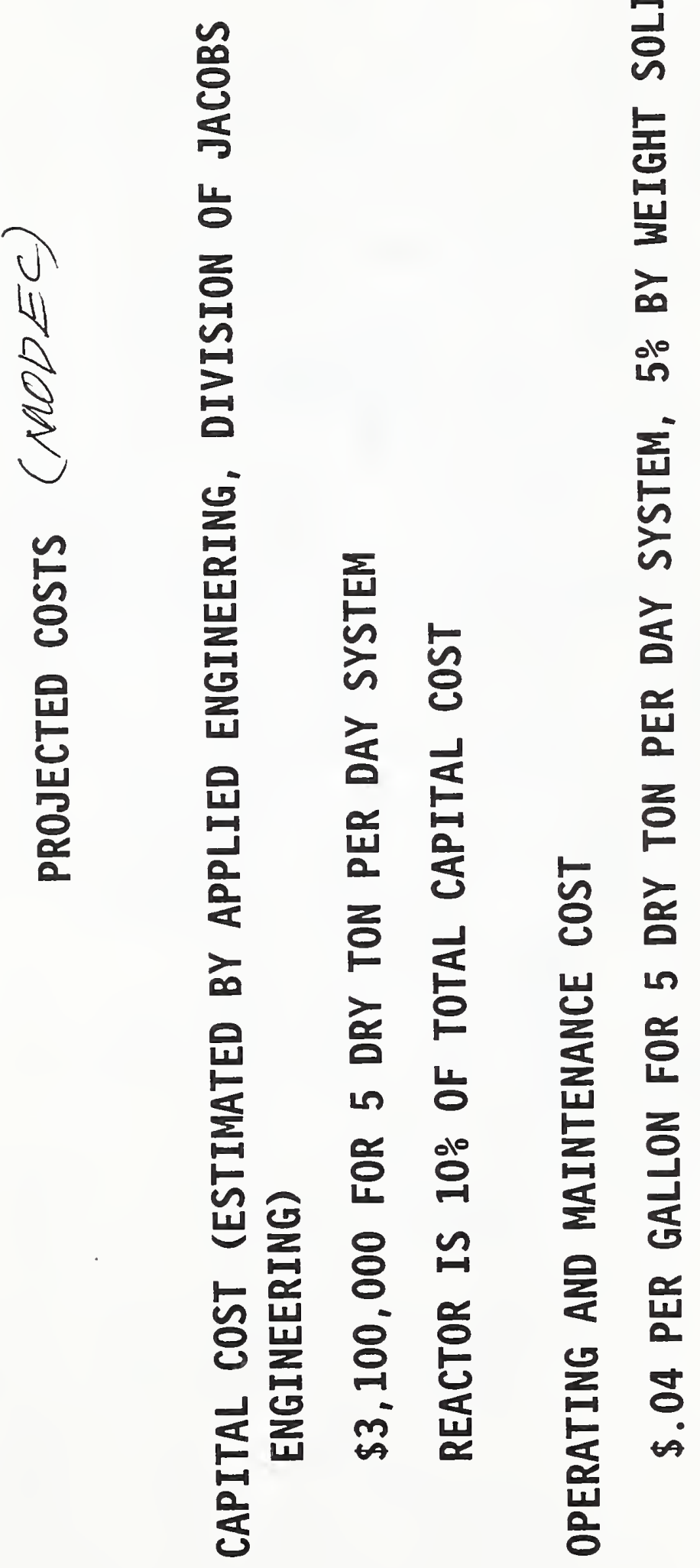




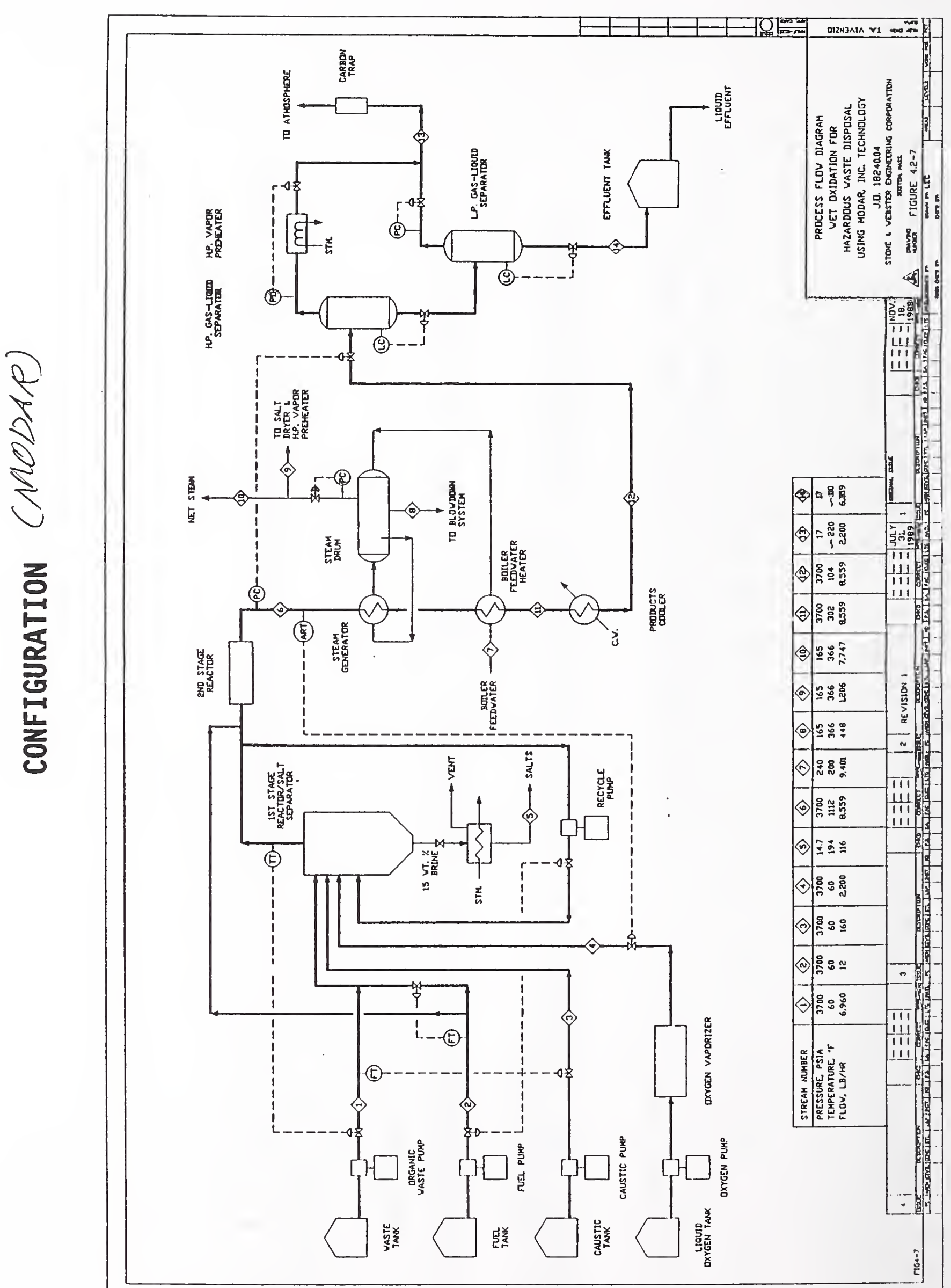


REACTOR DETAIL (MODAR)

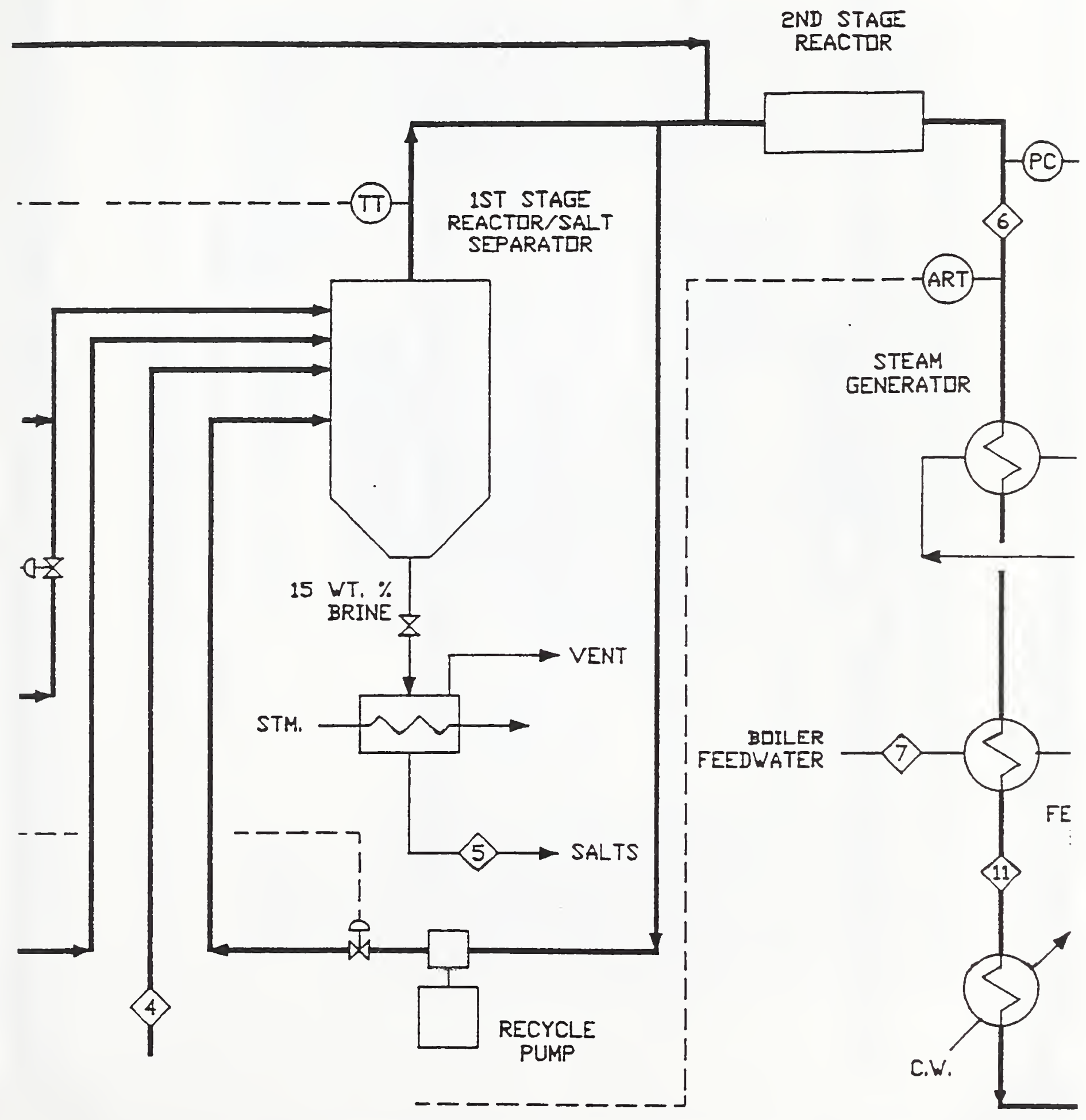




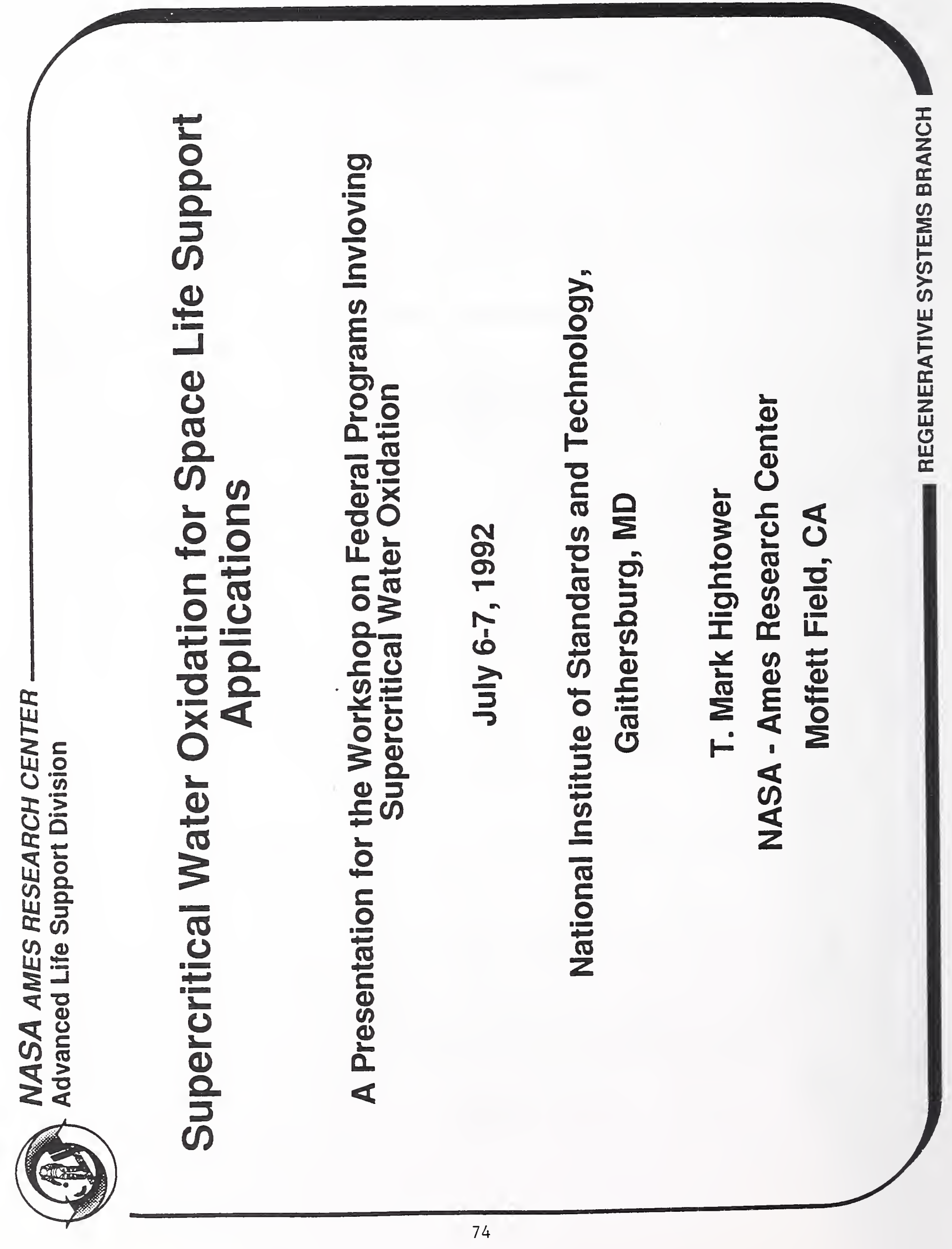




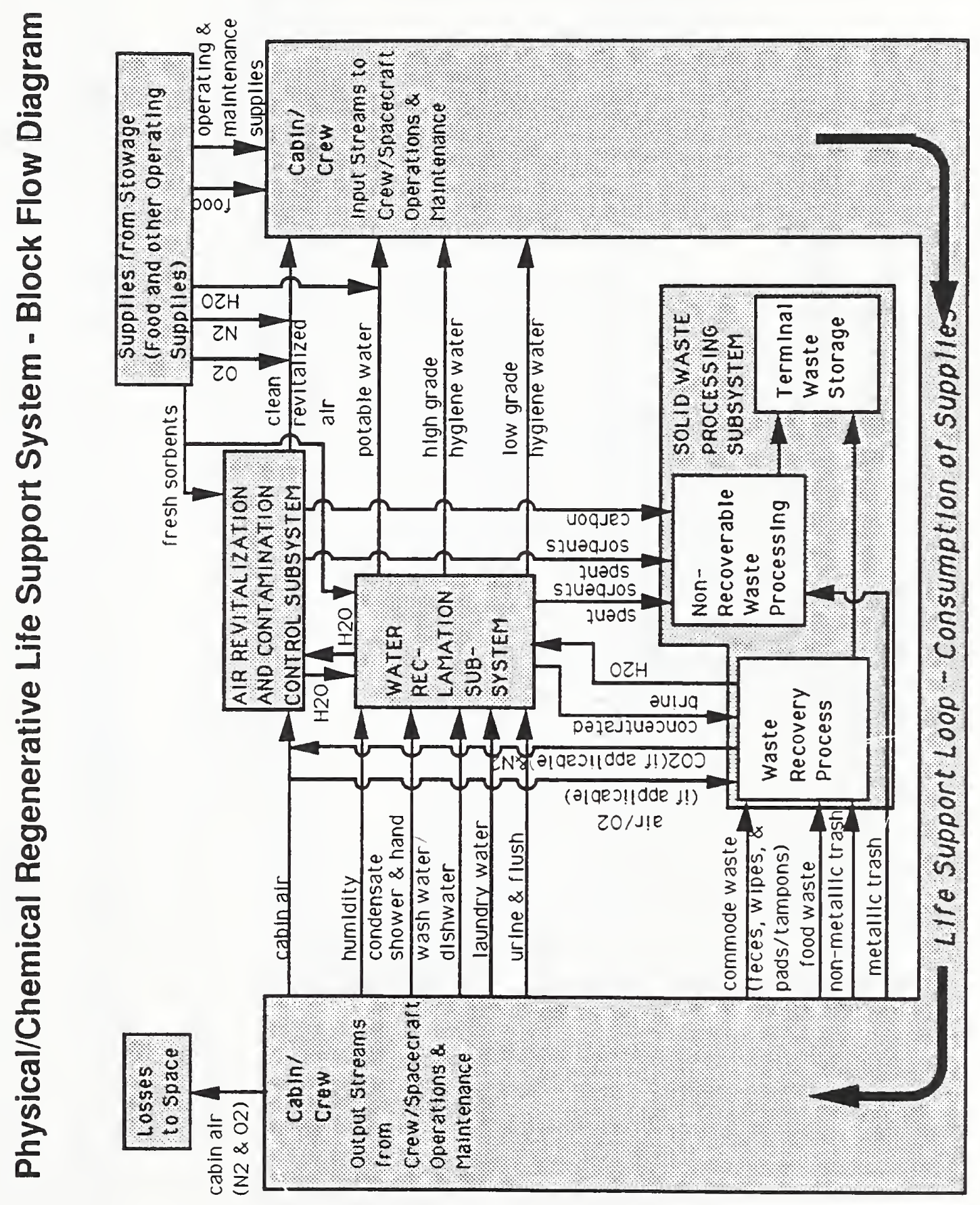



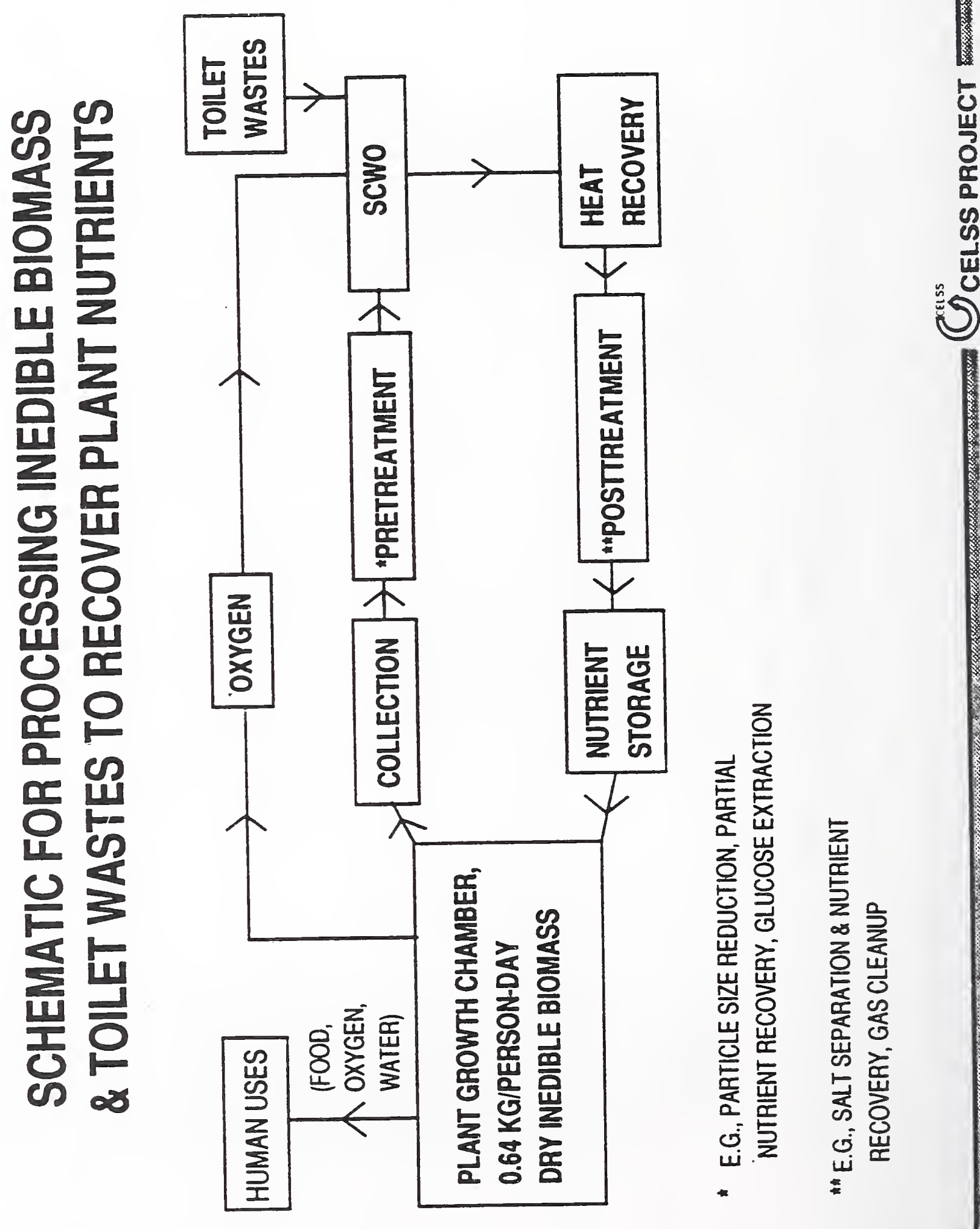

ac

4

능

U

บำ

둔

4 8

늘

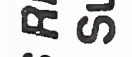

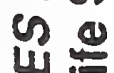

政

$<8$

$\rightarrow$ E

C)

$\sum \frac{8}{2}$

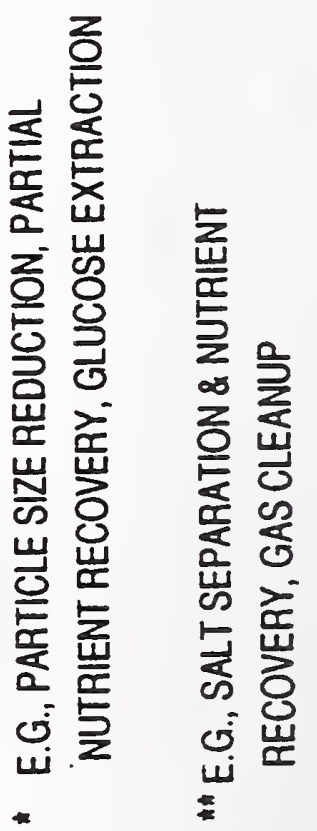




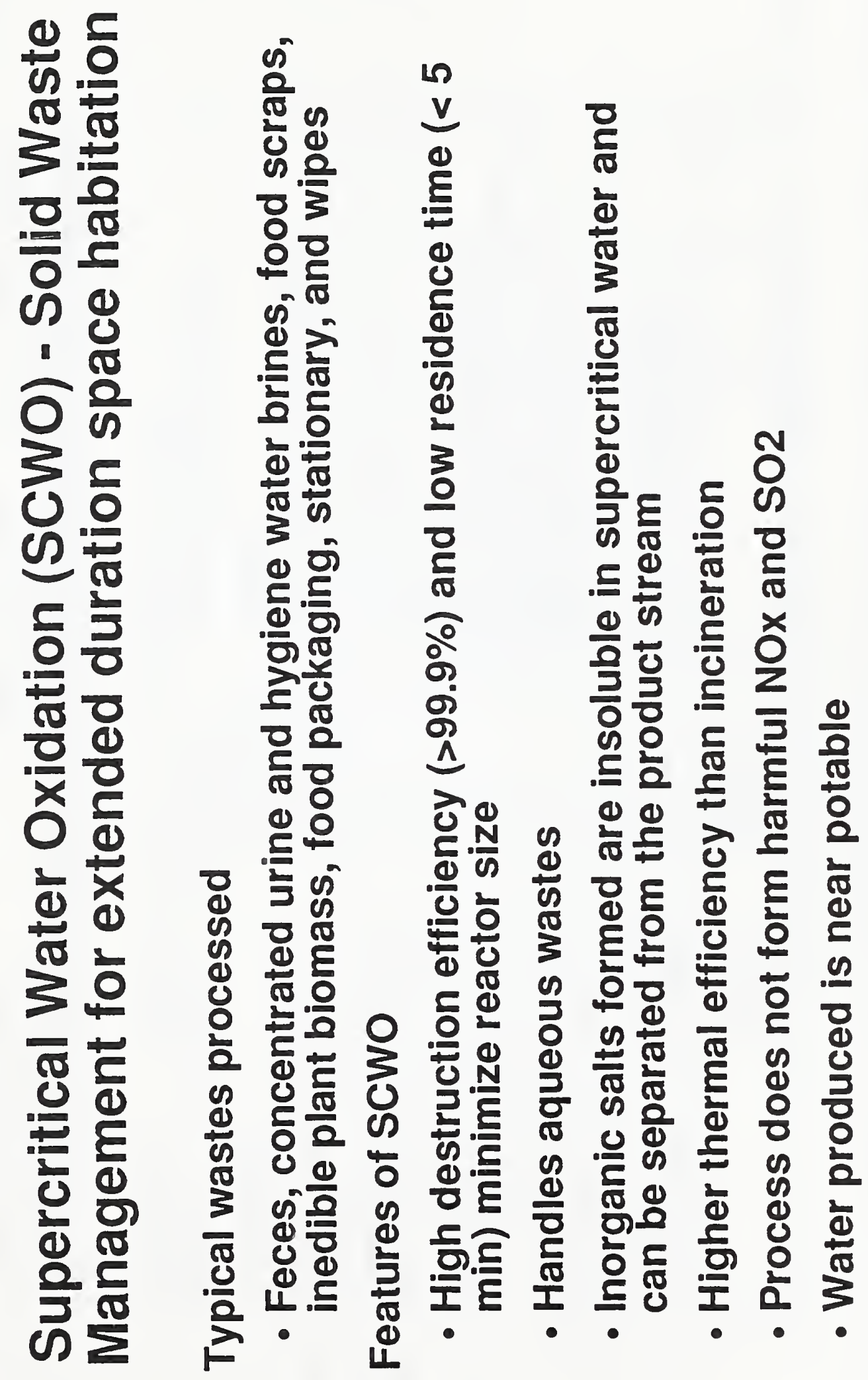

똔

$\sum$ 등

0 क

$\frac{1}{0}$

뚱

$+\frac{1}{2}$

48

늘

एक

C)

4

78

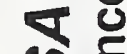

क है

$\sum 2$

을

00

03

0

a 10

E $\frac{2}{0}$

-

a)

$\pi$

5

(1)

드

글

())

(

- 2

\%

(1)

5

군

(1) 15

00

$\geqslant 0$

00

(3)

$+$

a)

20

(b) 90

20

$0 \geq$
$\Omega$

(1)

c

$\frac{5}{8}$

$\omega$ क 0

E

(1) 0

20 늘

0700

등 응

(1) 1 ह

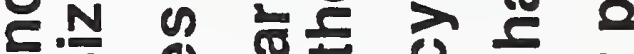

0

은 00 일

(1) 10400

인 3 (1) 0 .

a 0 क त

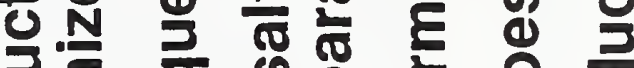

늘

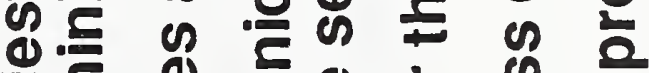

(1) 0001

두 0

$\underset{1}{\infty}$ 


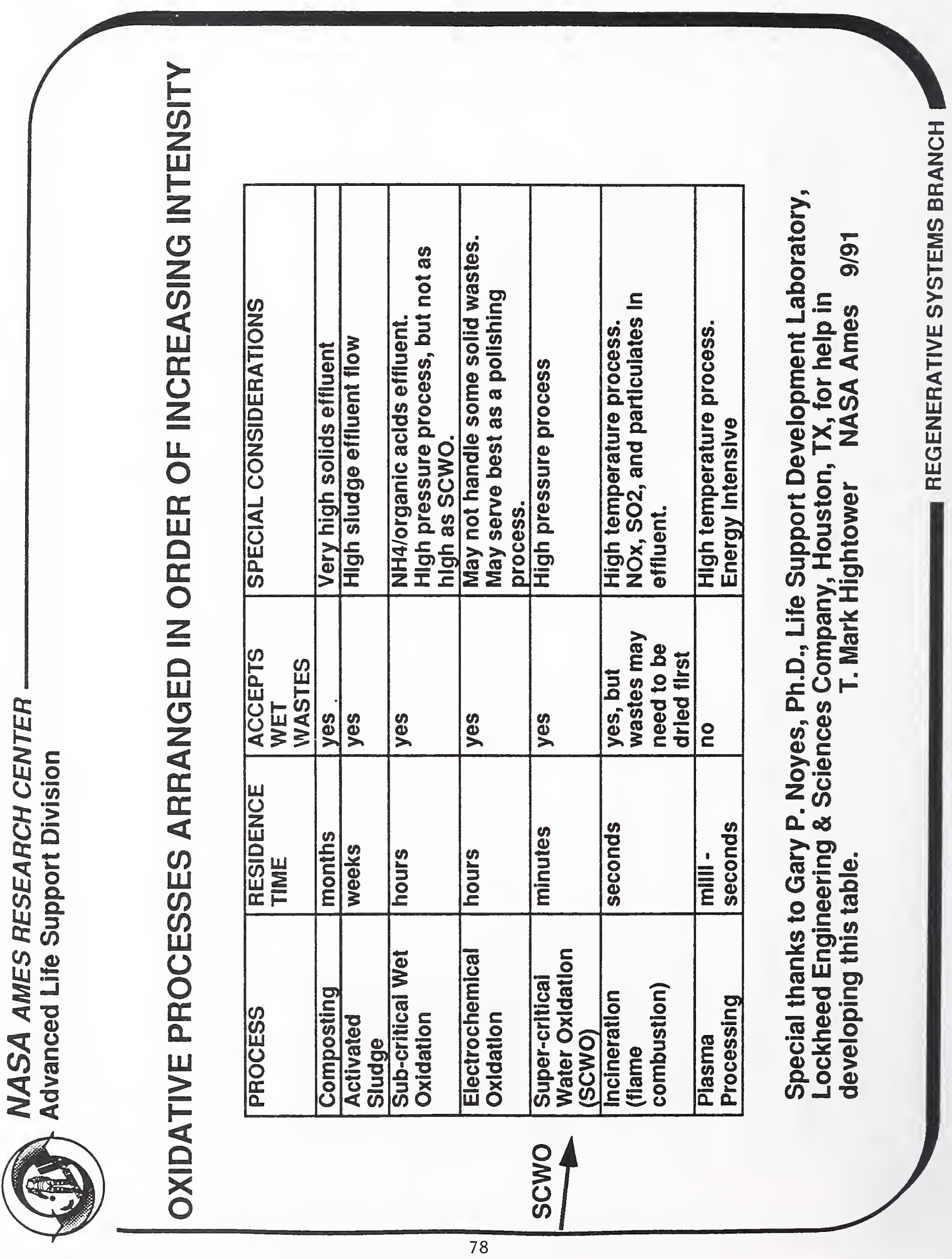




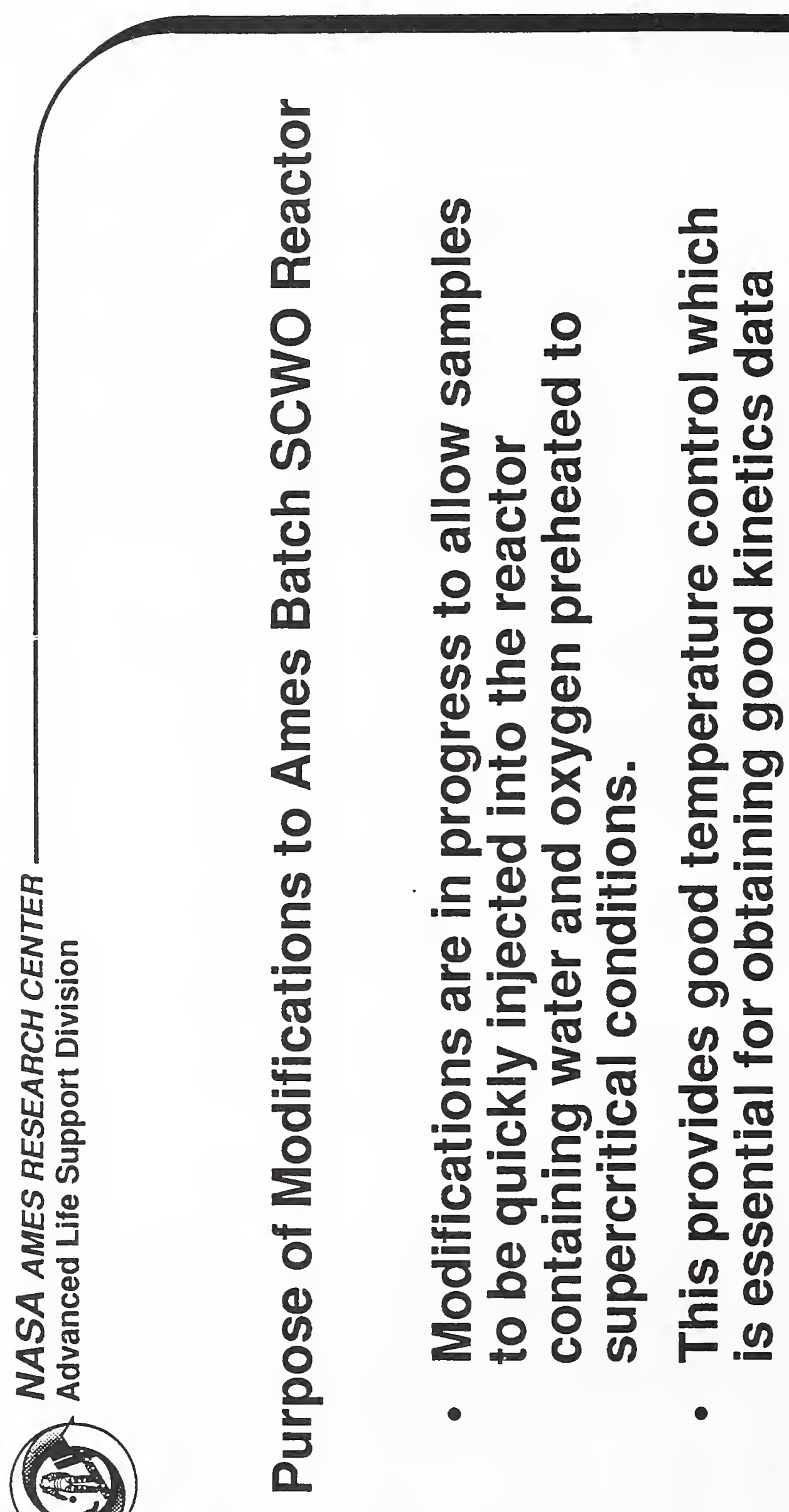


2ั0

딴

虫

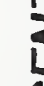

U. $\frac{0}{9}$

ป

둔

जै 응

쓴 윽

वै क

क

넨

$\sum_{5}^{2}$

$<\%$

$\nabla$

क त

$\leftarrow \geq$

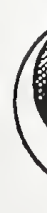

3

3

S

당

$\stackrel{0}{7}$

m

○

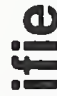

\%

을

$\stackrel{\Phi}{\rightleftarrows}$

항

일

흉

을 등

E 는

\& 5

드을

ब)

응.

ह 들

$+0$

(1) 20

ㄴำ

융

บ भ

을

은 이

¿

도

요응

$>\frac{0}{x}$

$\times$ 당

은

a

올

(⿻)

बำ

ठำ

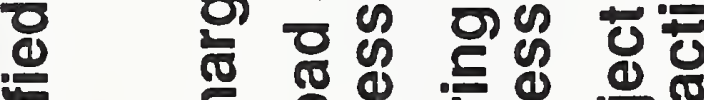

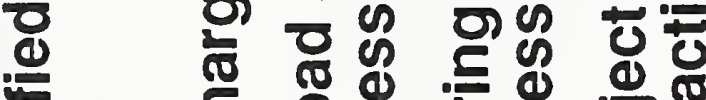

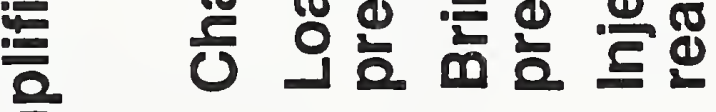

ह

ఏ을 흥

응 동 응

눈

(으워 엉

3 हN

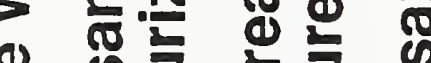

-

-
은 는

응

(1)을항

\%

을 중

भ त 0

d)

क ⿻ ㇒

() ह 응

응

을

क ले

ด ญ

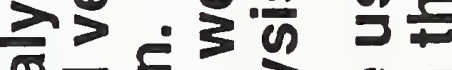

응

ส어유

तิ

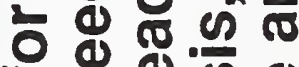

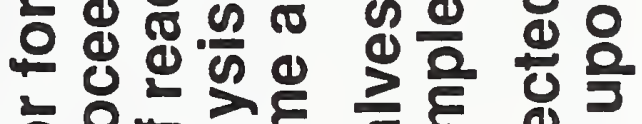

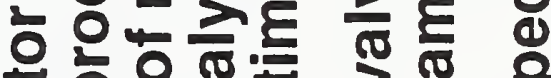

+

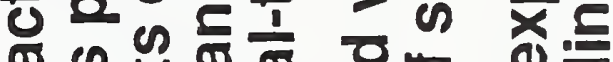

ब1

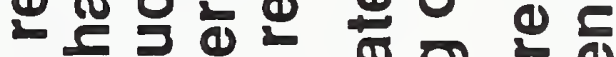

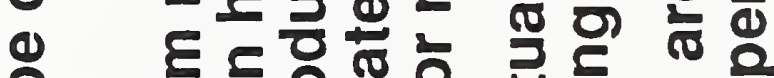

()

은은은 능로

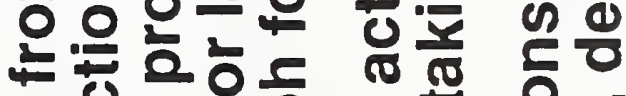

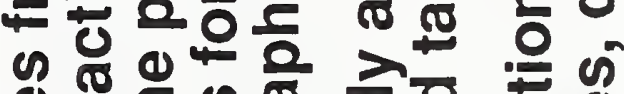

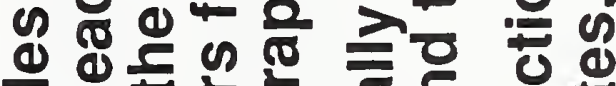

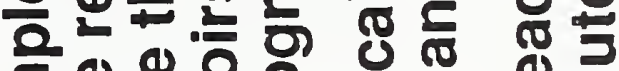

잉을 인

त

달

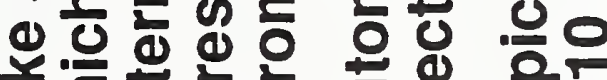

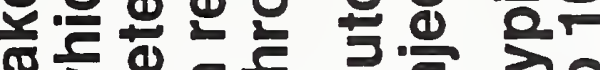

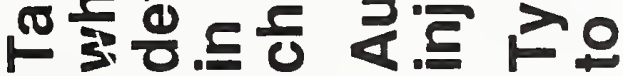

เ

$\omega^{\circ} N$ 


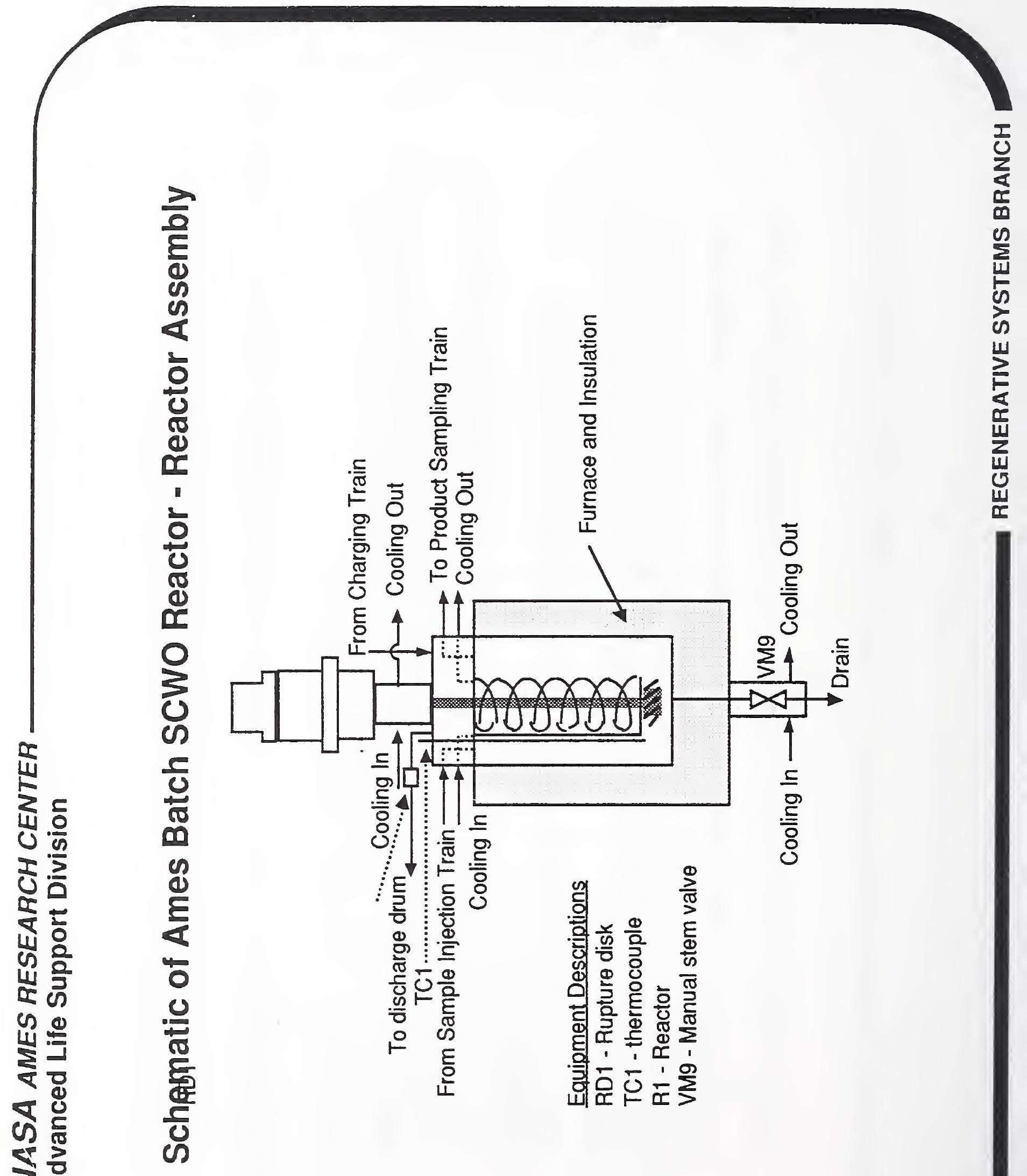




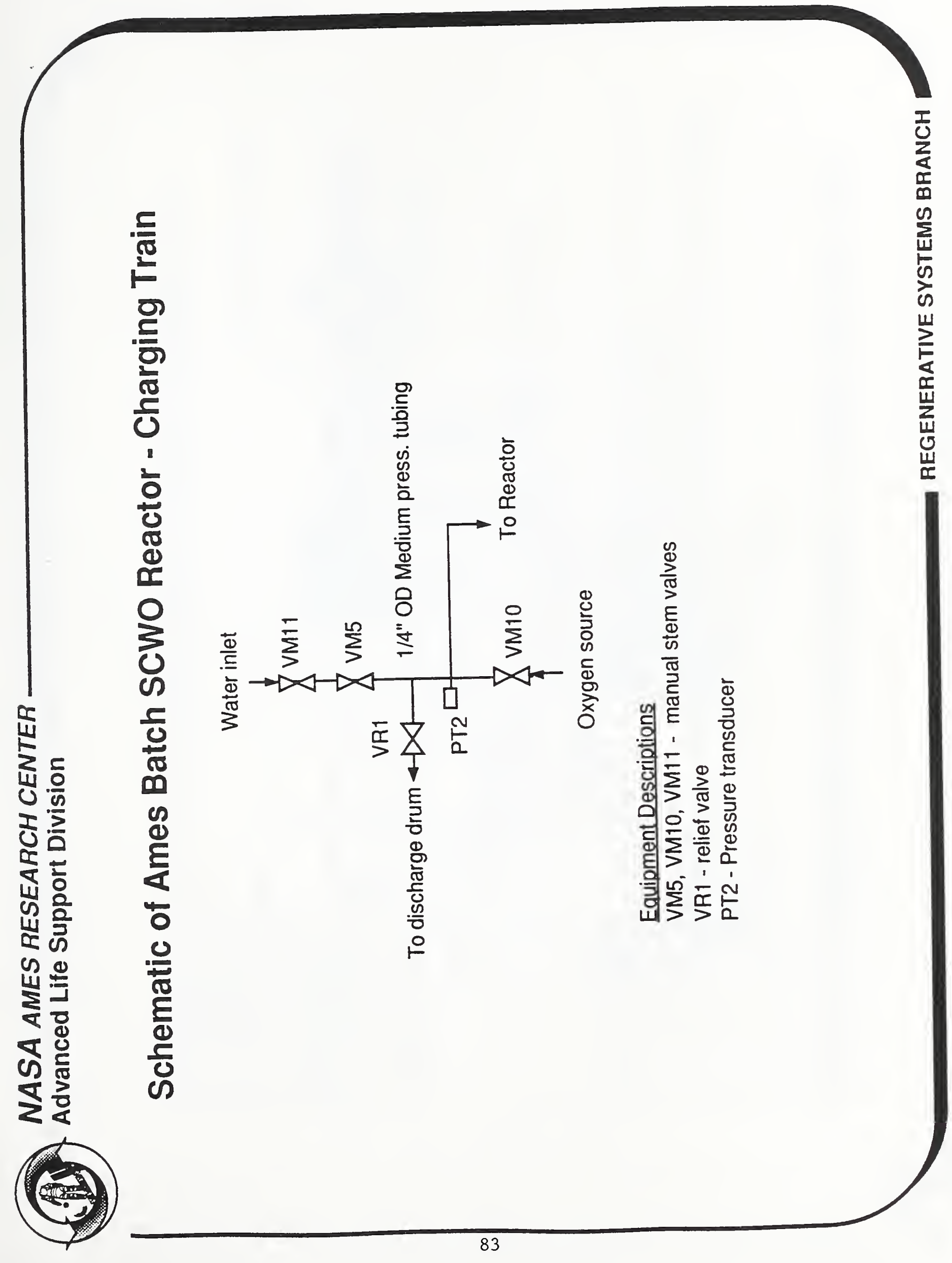




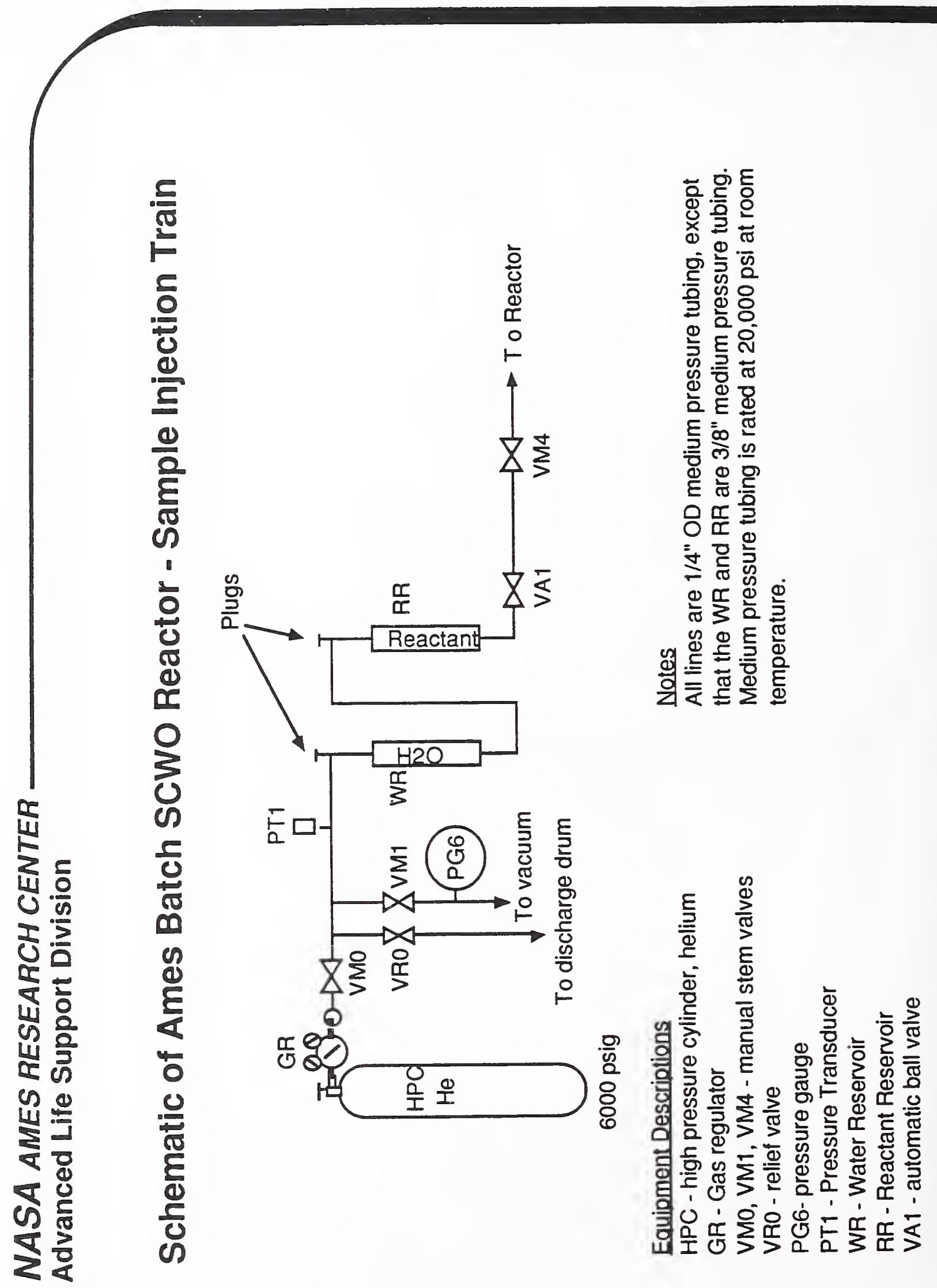




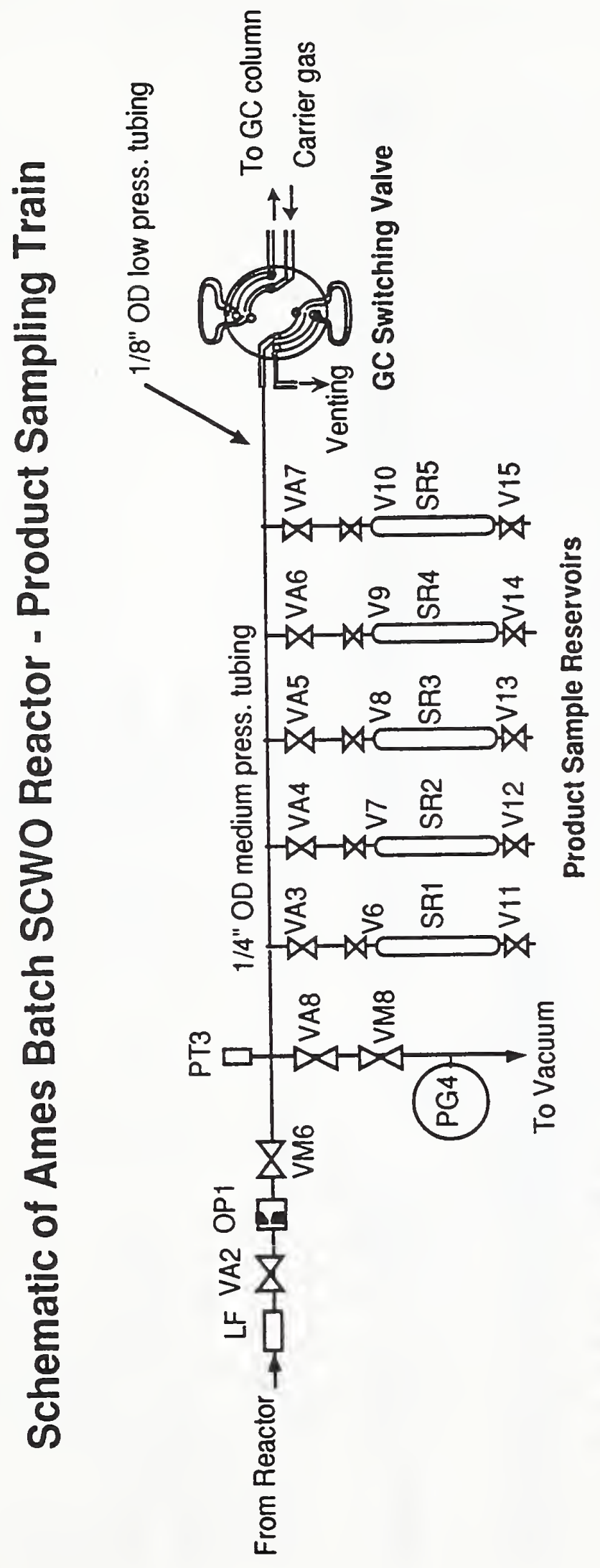

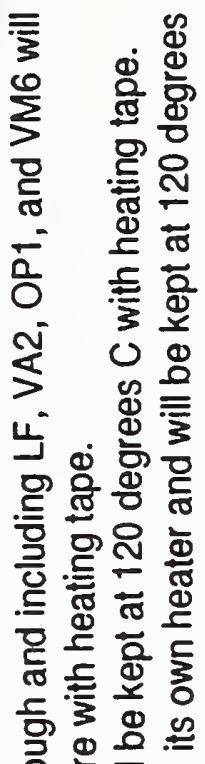

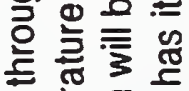

흥 웡

屯

흥

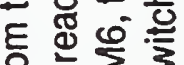

인

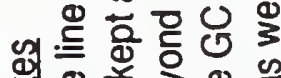

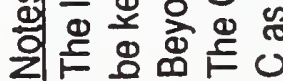

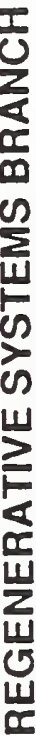

$x$

w

닌응

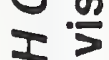

它

난

फ़ :

这

फ

$\sum$

궁

$\nabla 0$

क

$\leqslant \frac{1}{8}$

$<4$ 


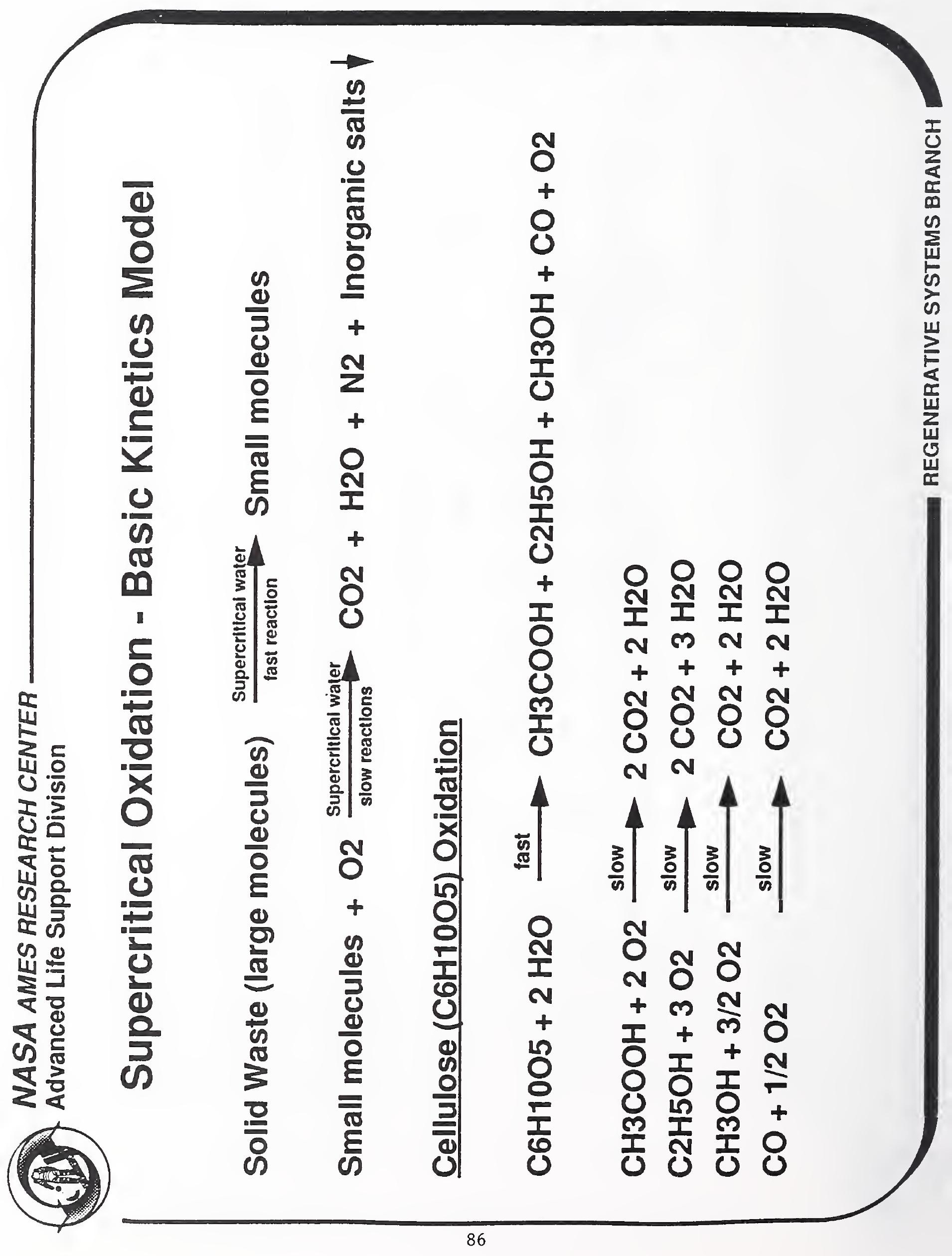




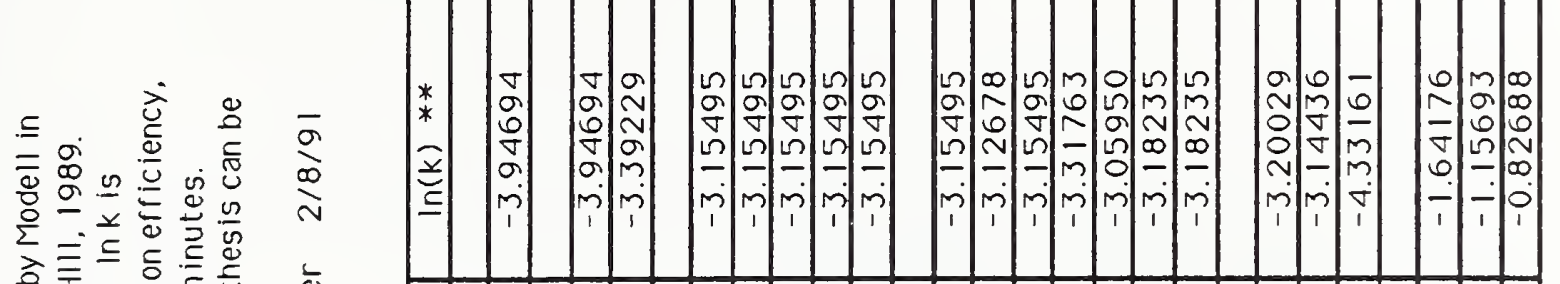
핑들ㅇㅇㅇ 당 ज行的

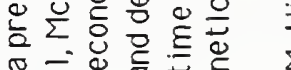
元 으원 흐능 잉

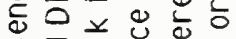
는 $= \pm \frac{0}{2} \frac{0}{2}=$ 응 능

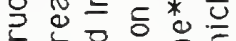
는은

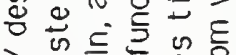

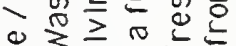

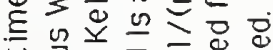
으으둔 는 능ㅇㅇ

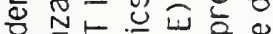

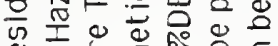
는잉ㄷㅇ

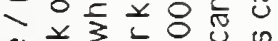

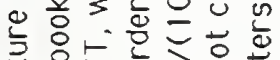

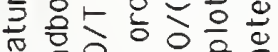
与 을

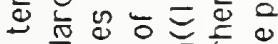

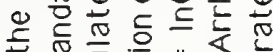

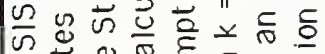
年

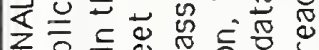

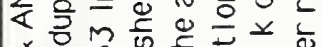

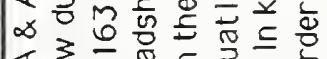

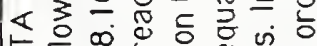
《市

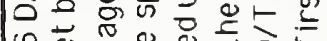
U⿺辶一 代-

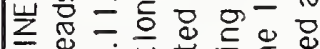
풍 은 응 引 岁岳完

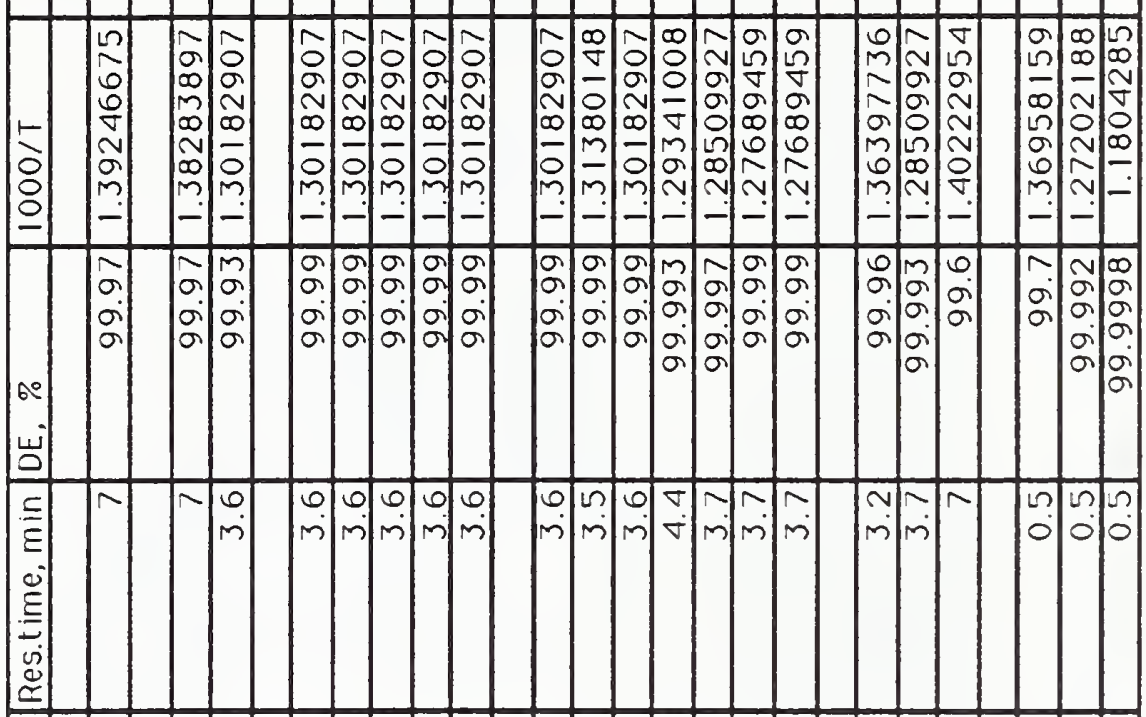

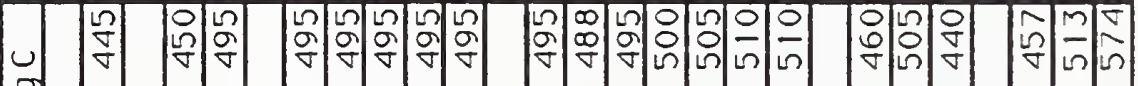
용.

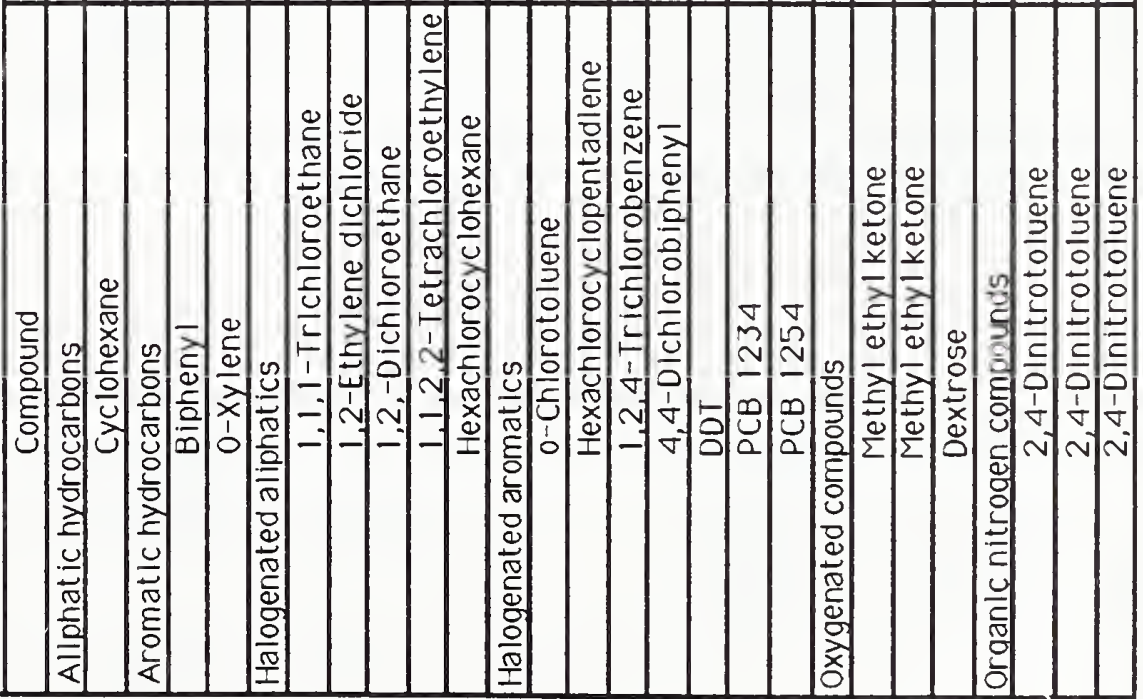




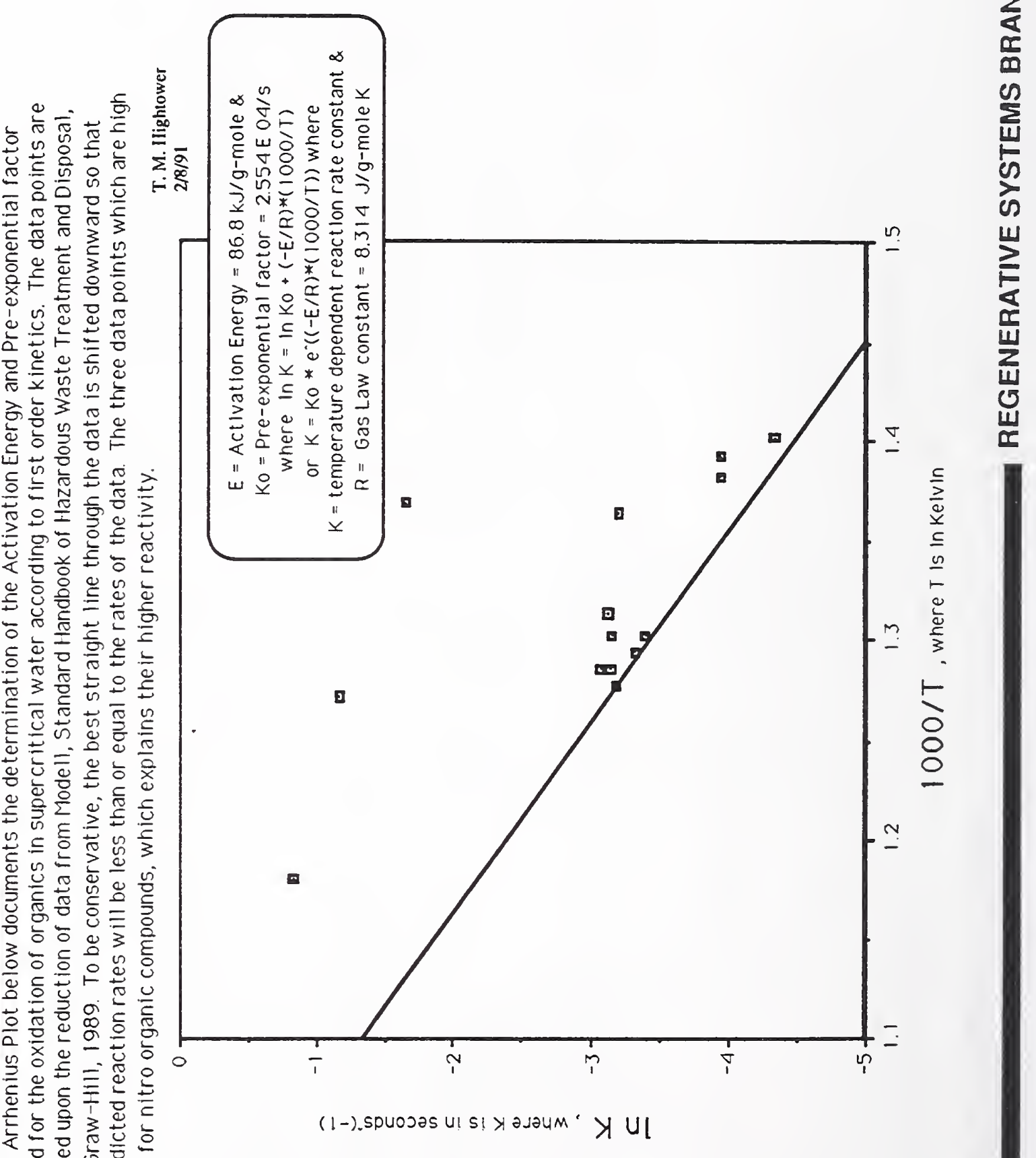




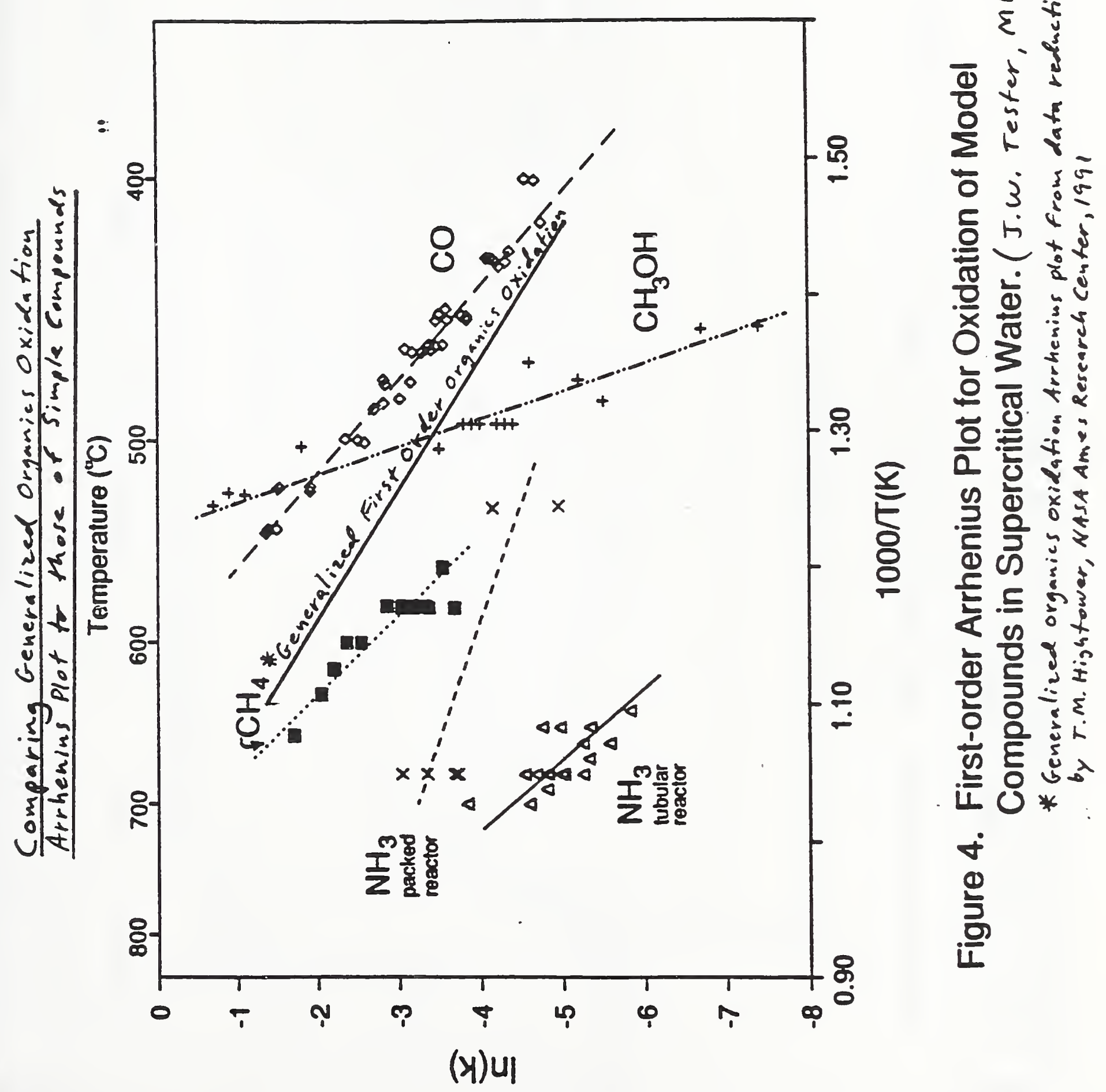




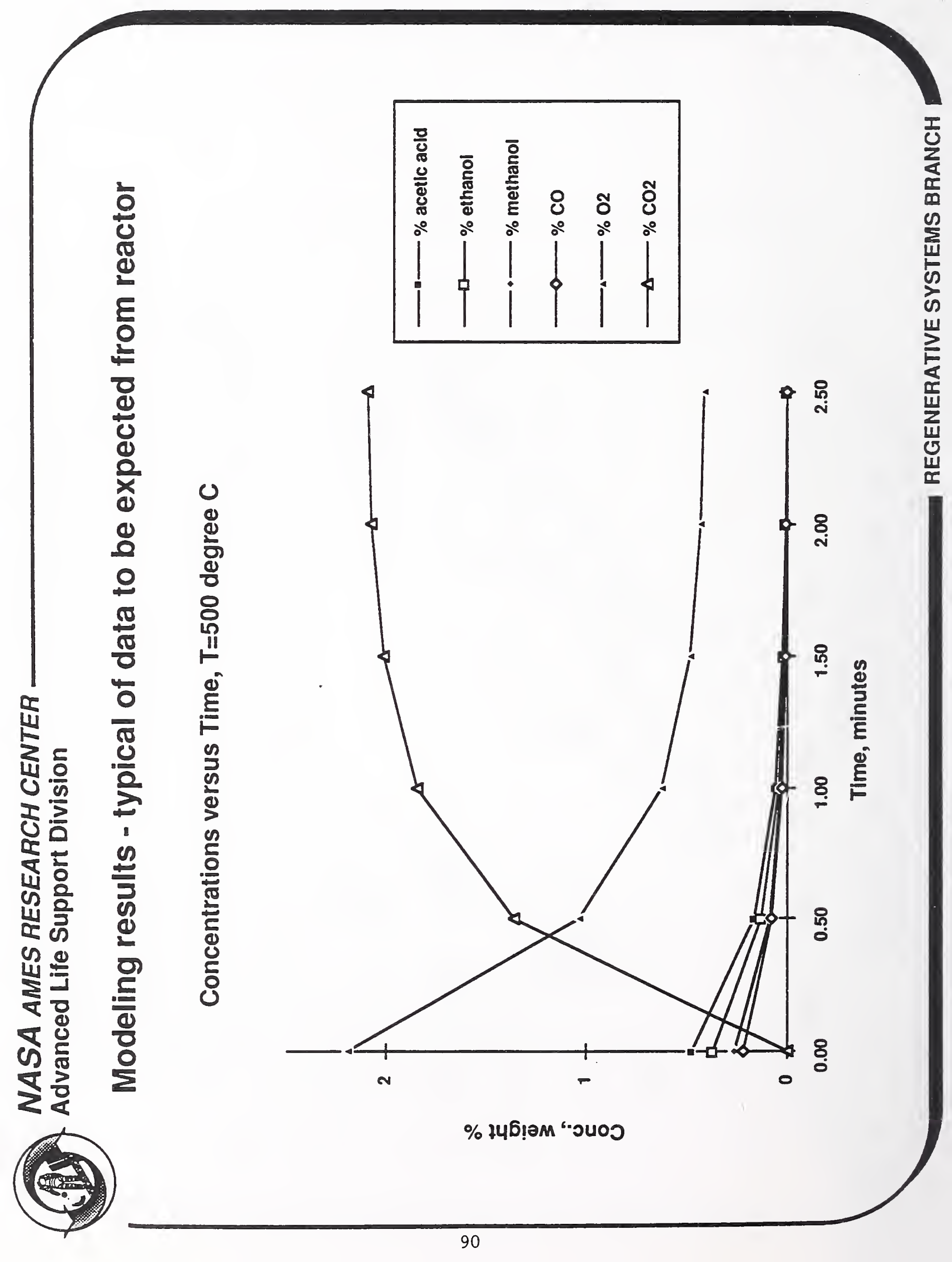




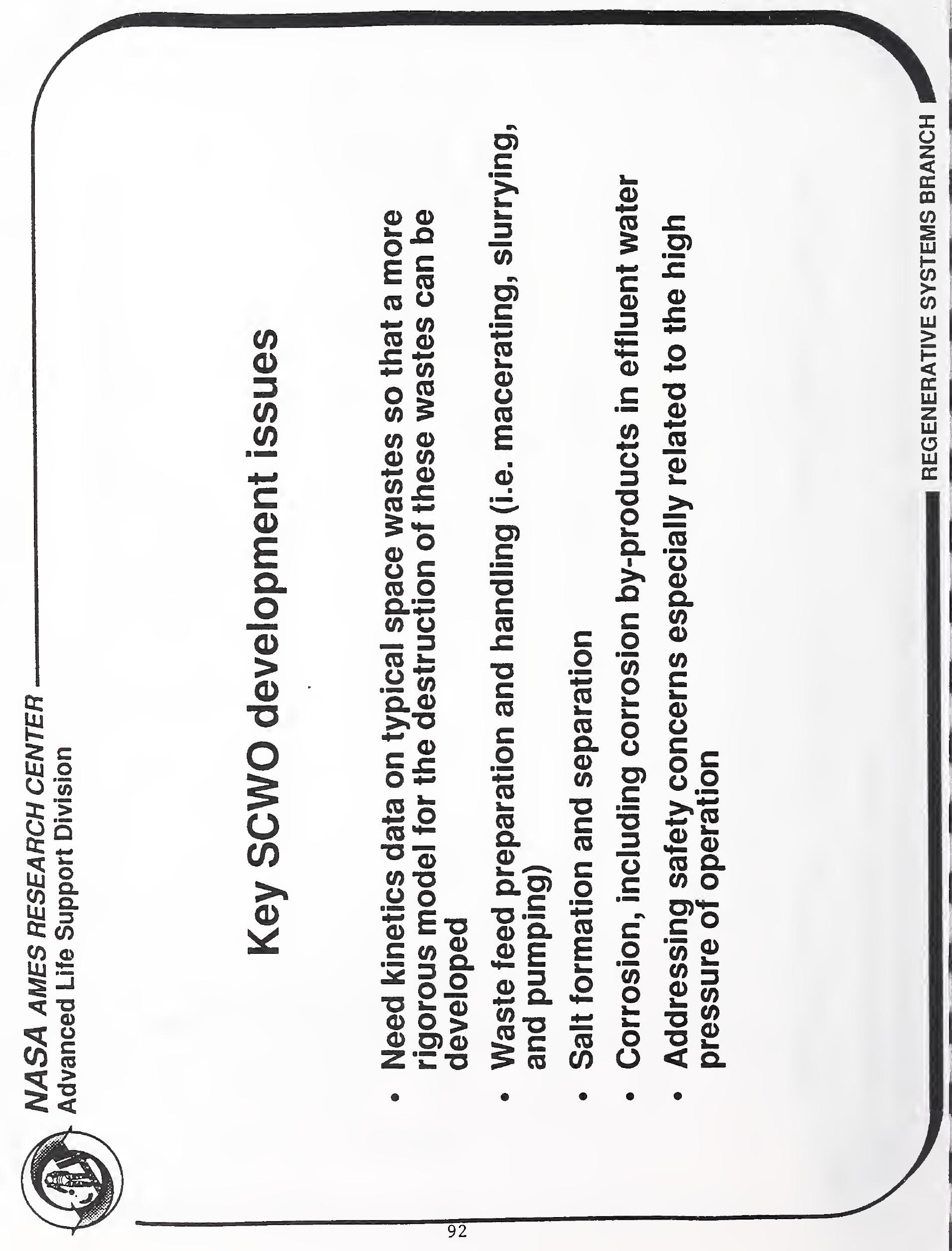




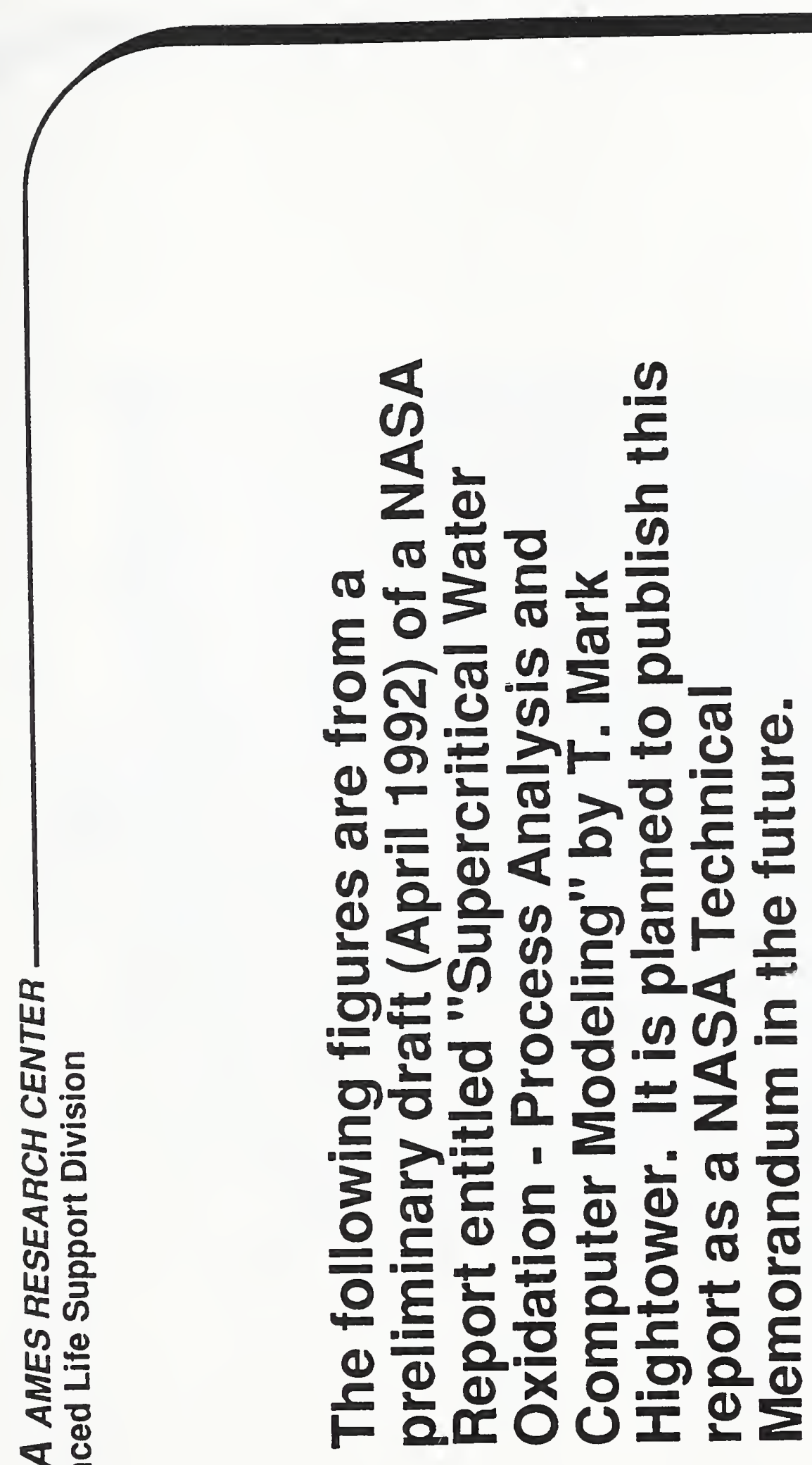




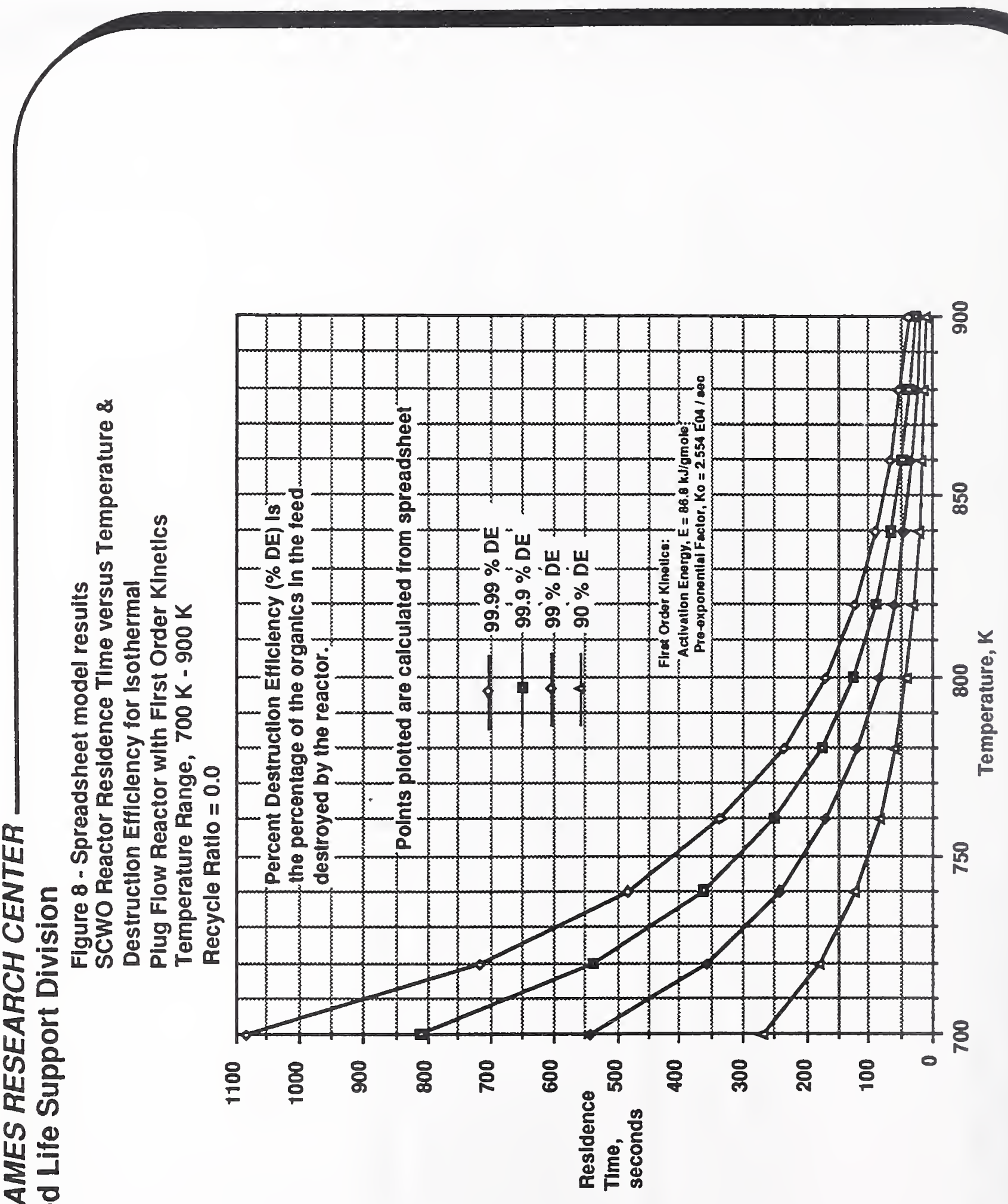




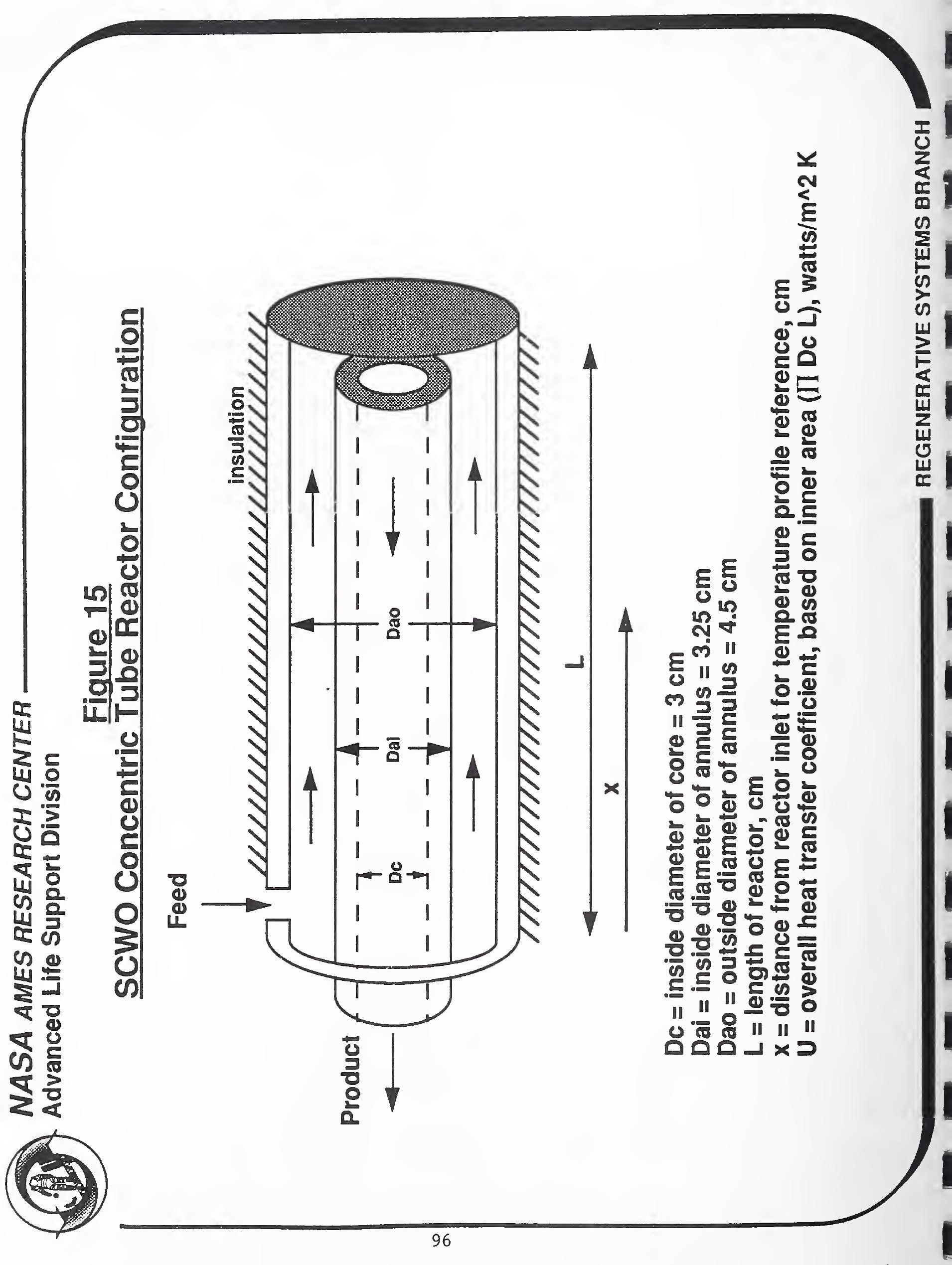




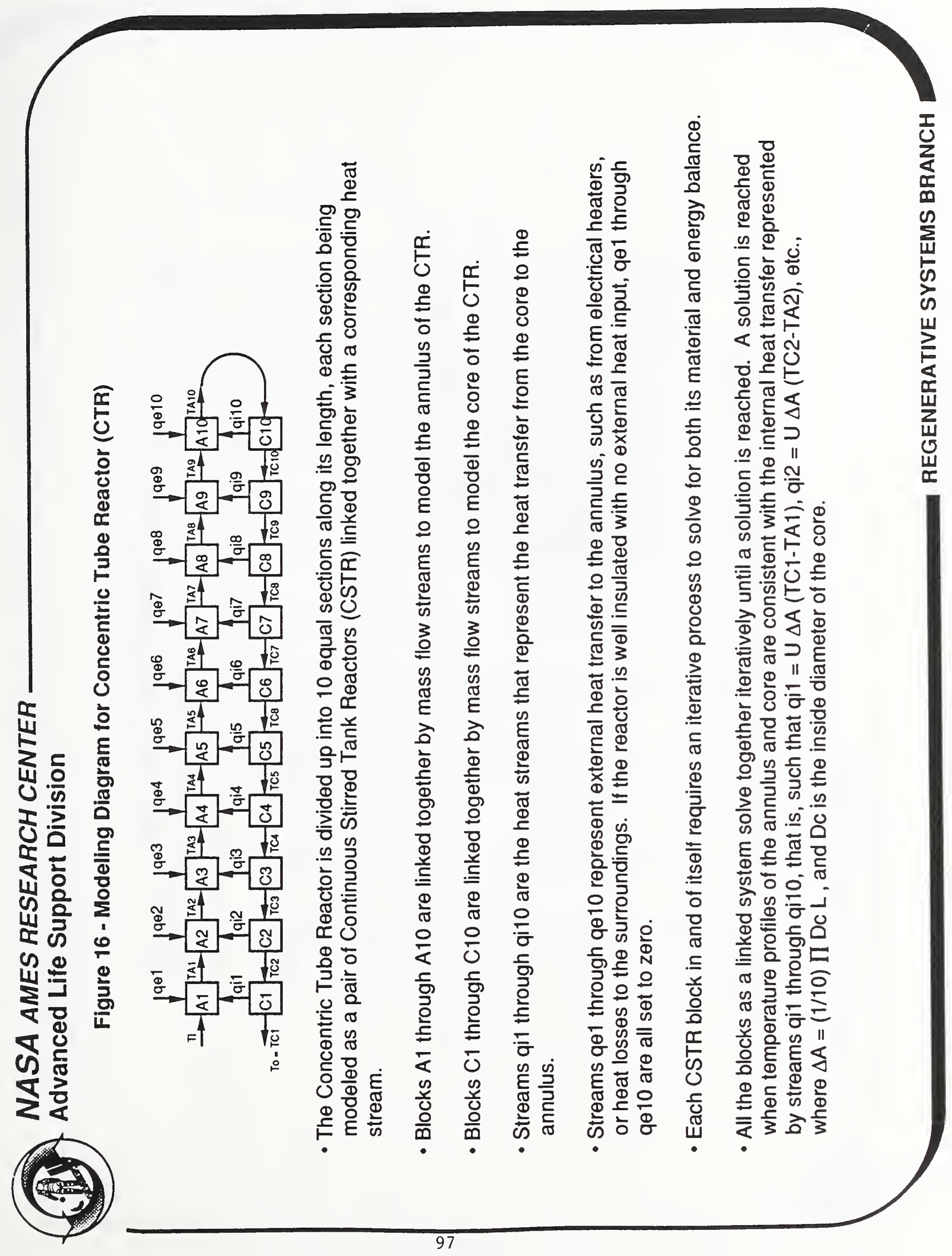




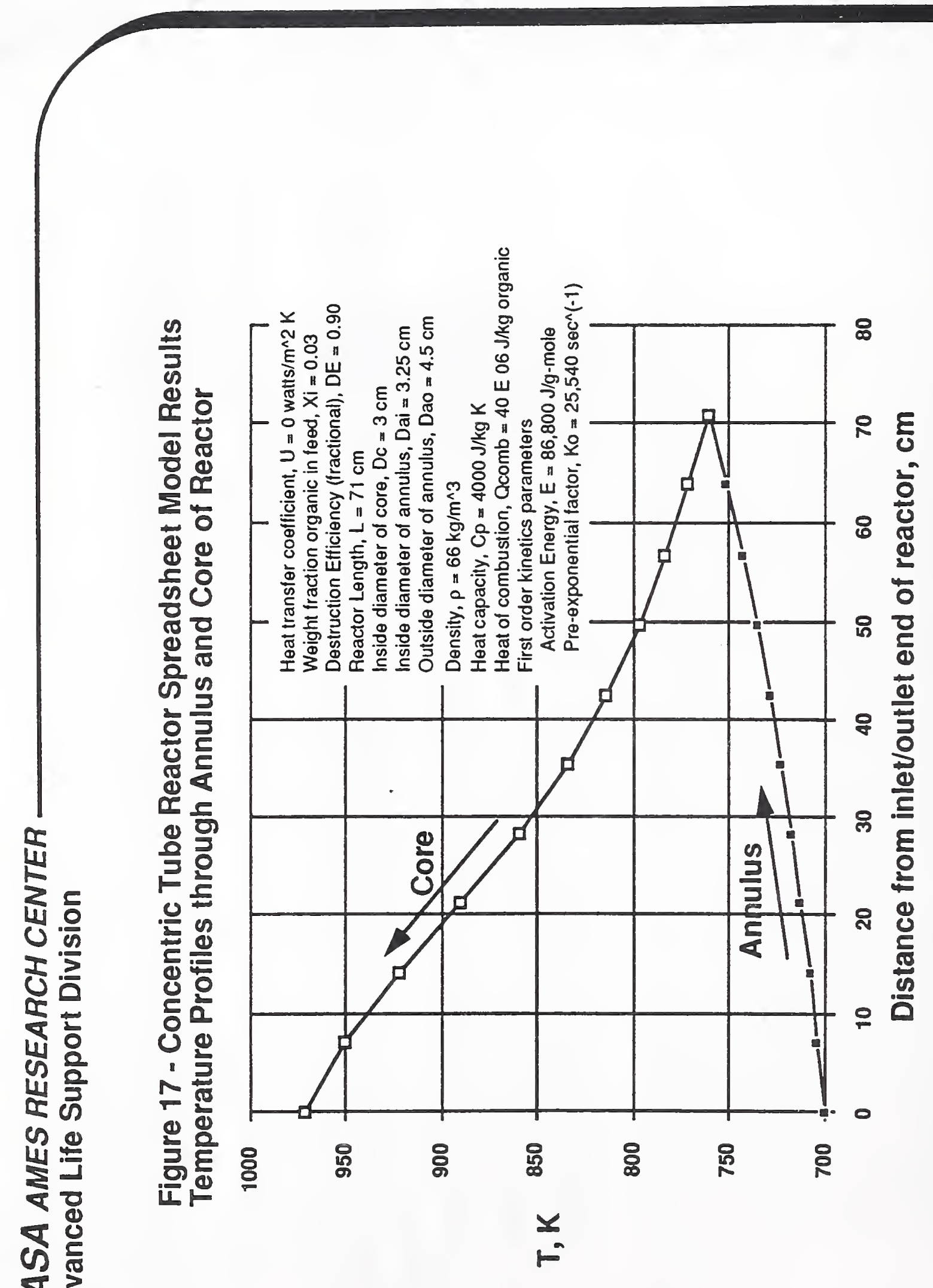




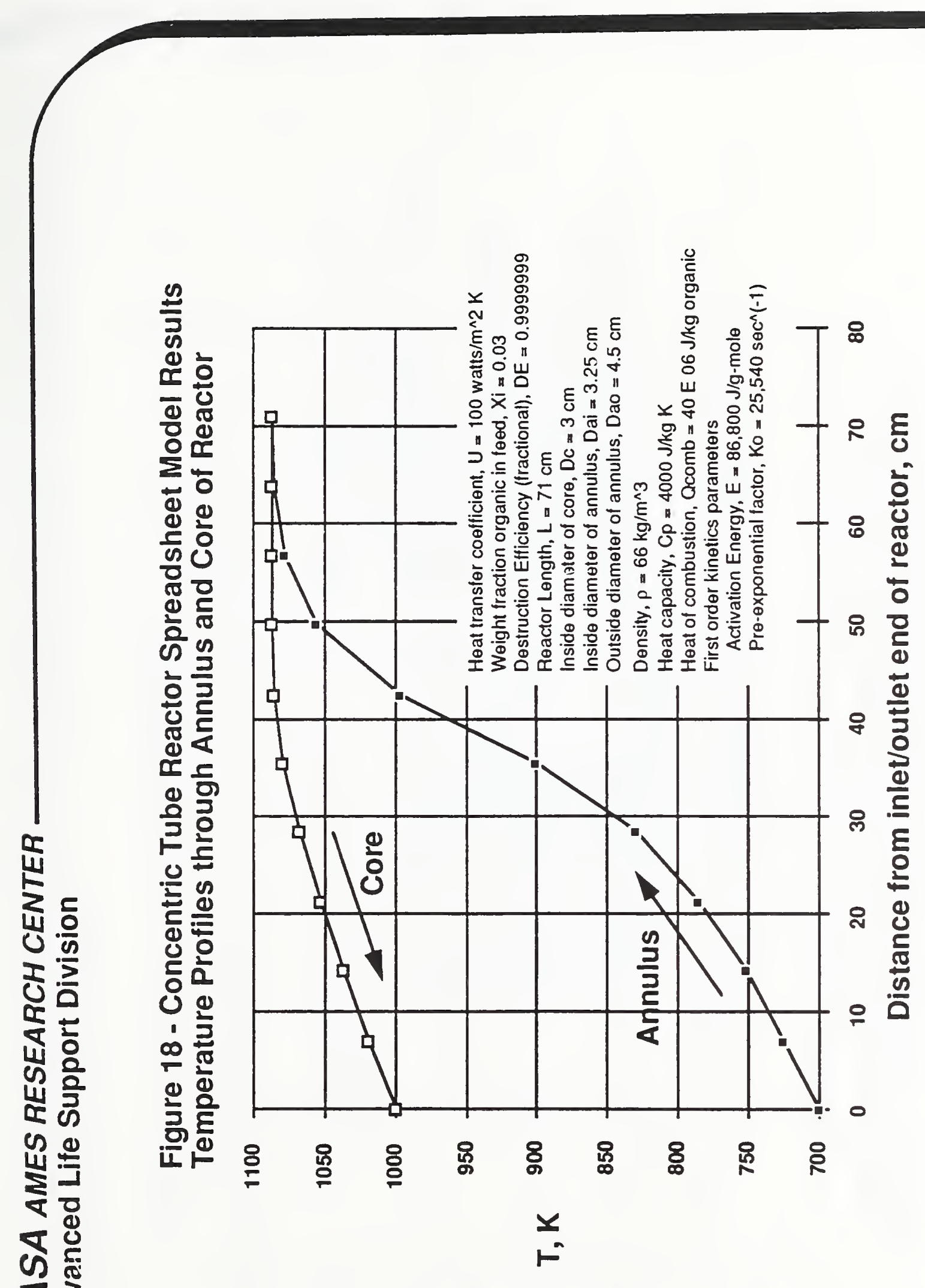

$\frac{1}{U}$
$\vdots$
$\frac{1}{0}$
$\frac{1}{0}$

en

es

os

W 


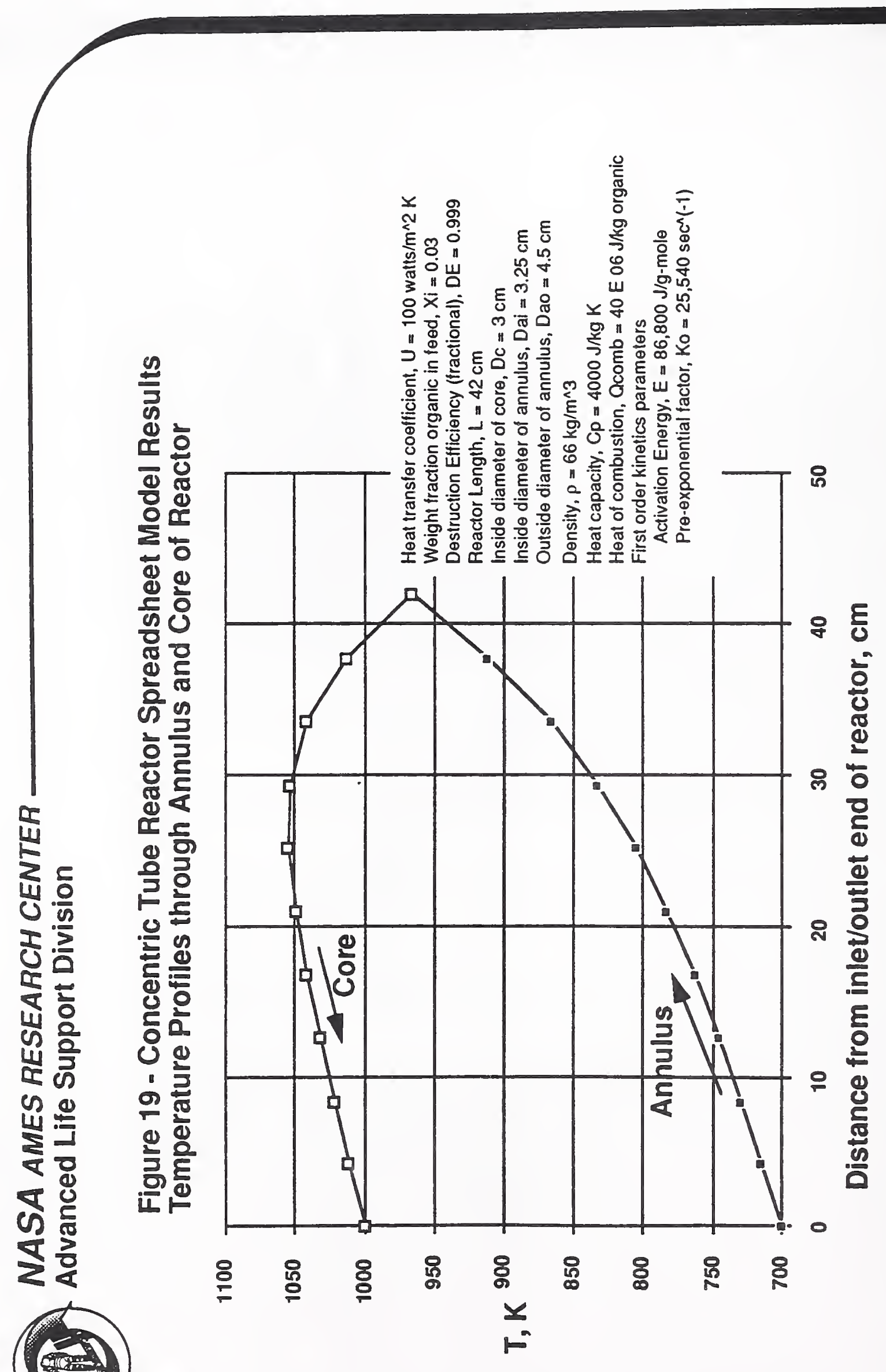




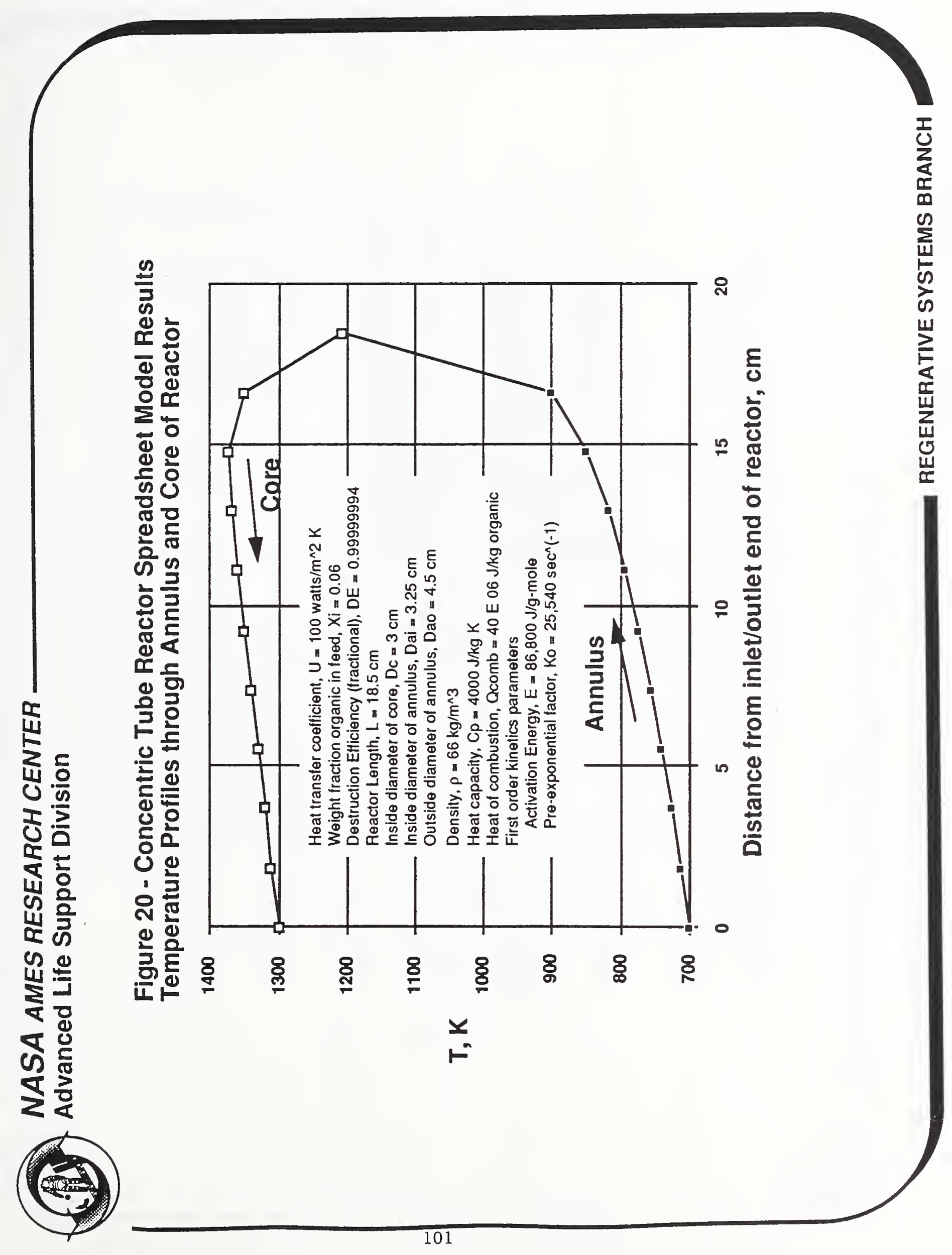




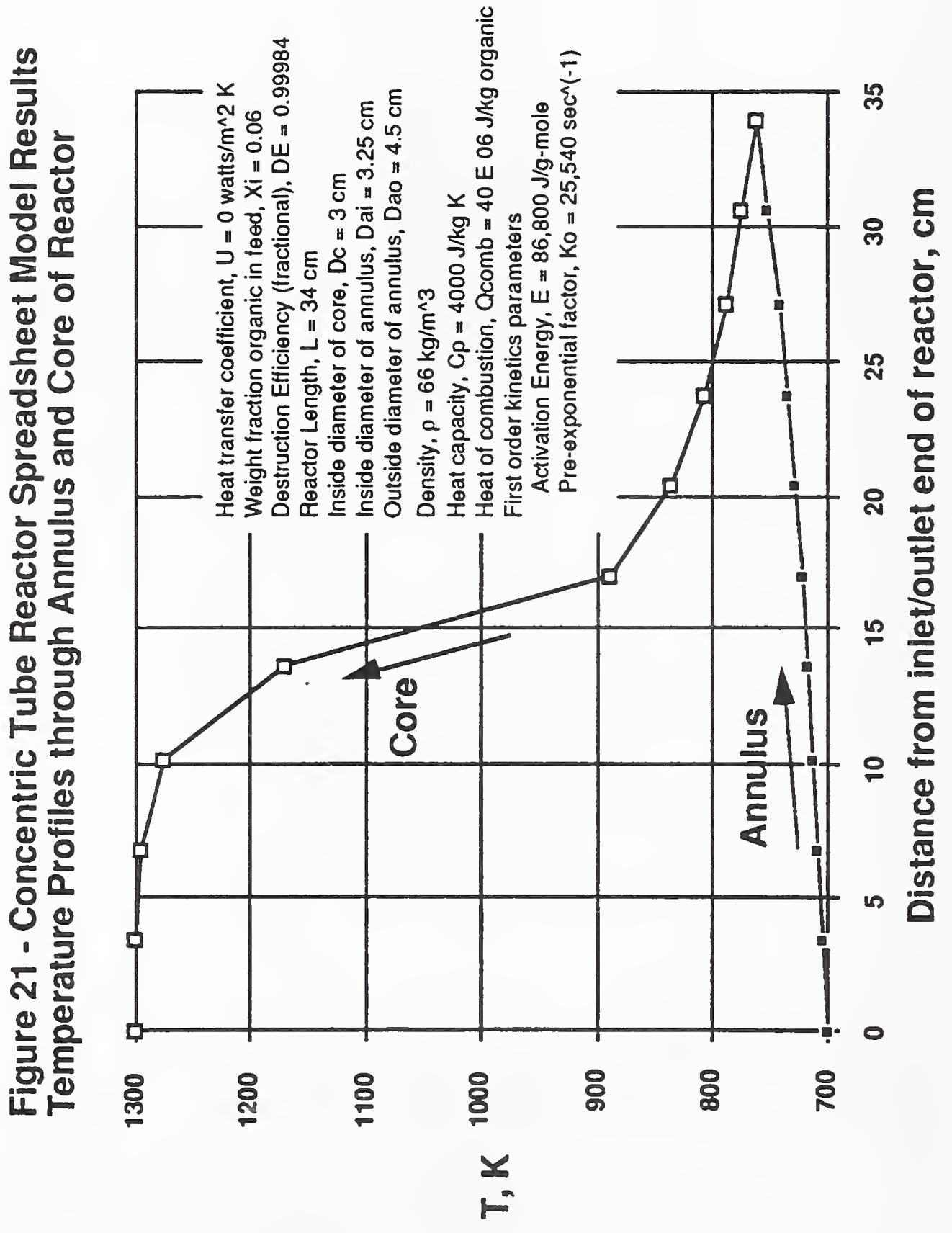




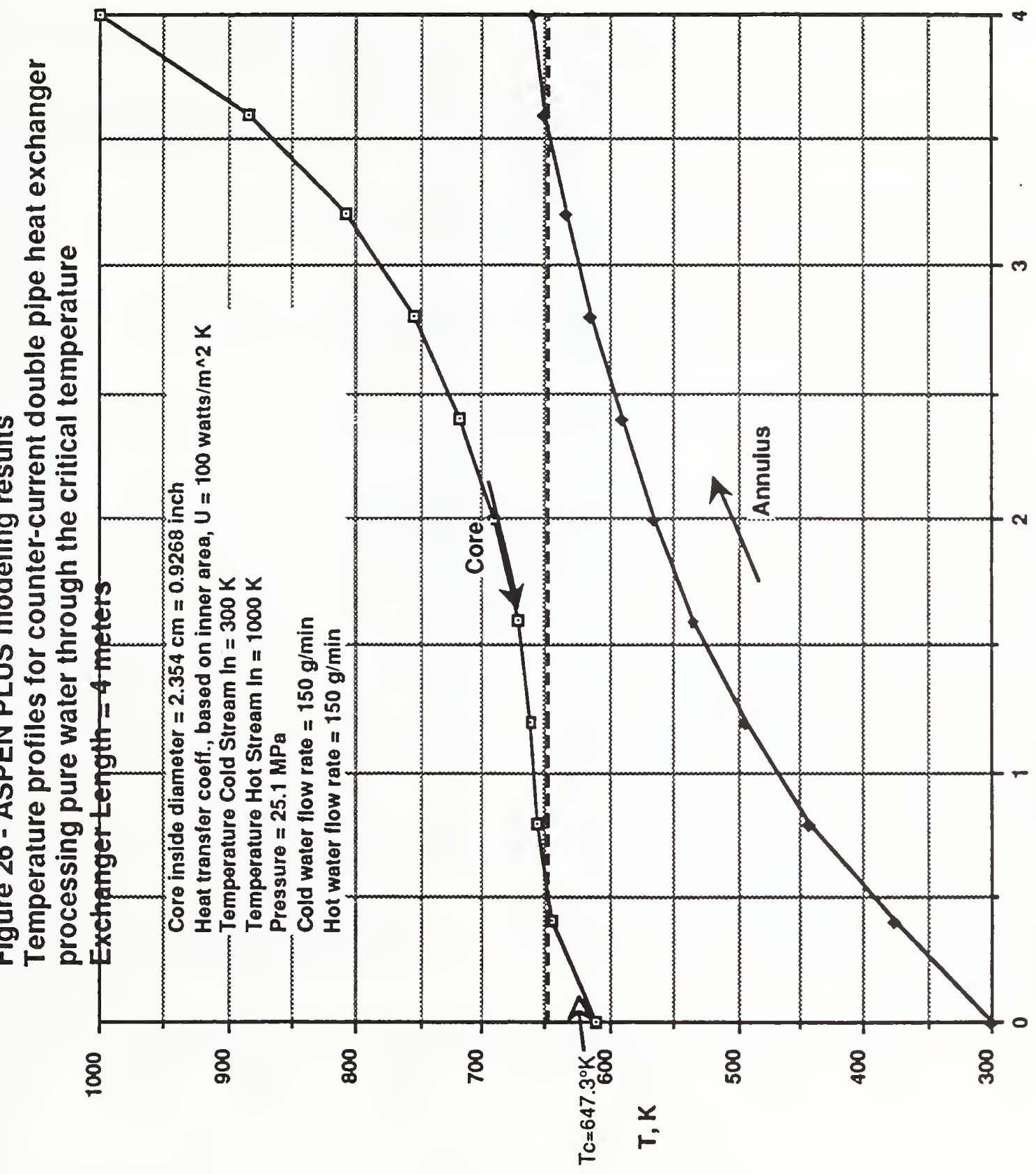

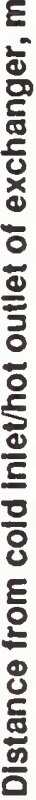




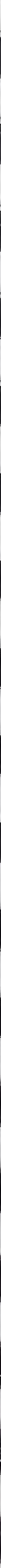




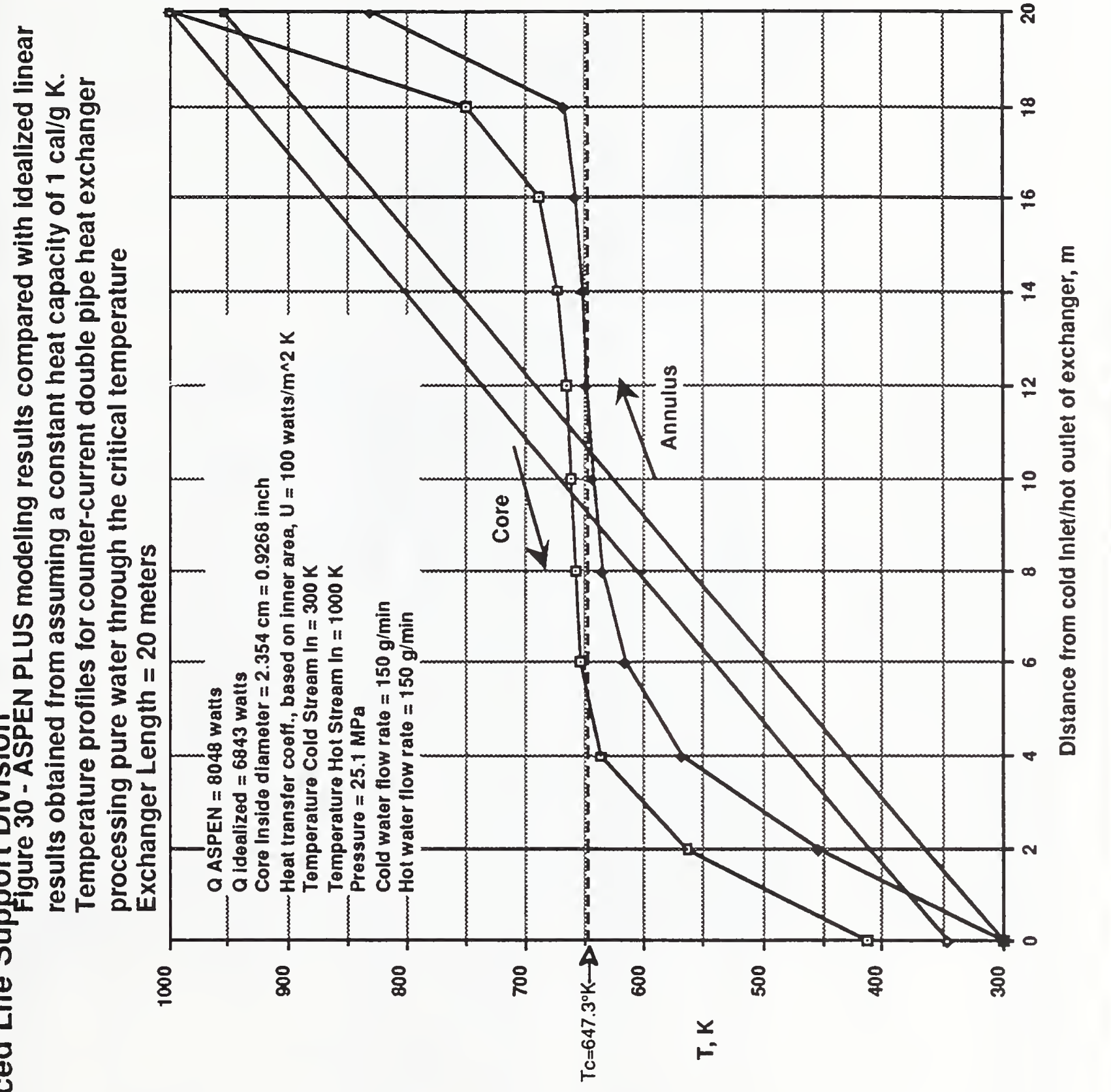


๕ัฐ

튼

앙

츠

항

i $=x$

응

동

उ०

용ㄹㄹ

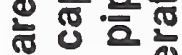

으류을

웅올 을

르응

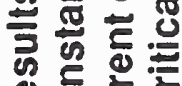

인 융 흔

일 0 웡

응 일ㄹ

홍 혼

ह 동ㅇㅇ을

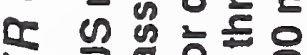

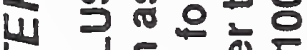

$\checkmark$ ह ह

य $\frac{5}{2} \stackrel{0}{2} \stackrel{0}{3}$

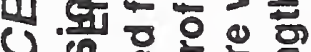

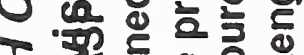

र 징

응 인

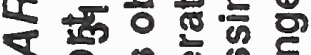

4 옹 क

क 둘 을 웡

u 동

एᄄ

(s)

世.

$\Sigma$

sิ

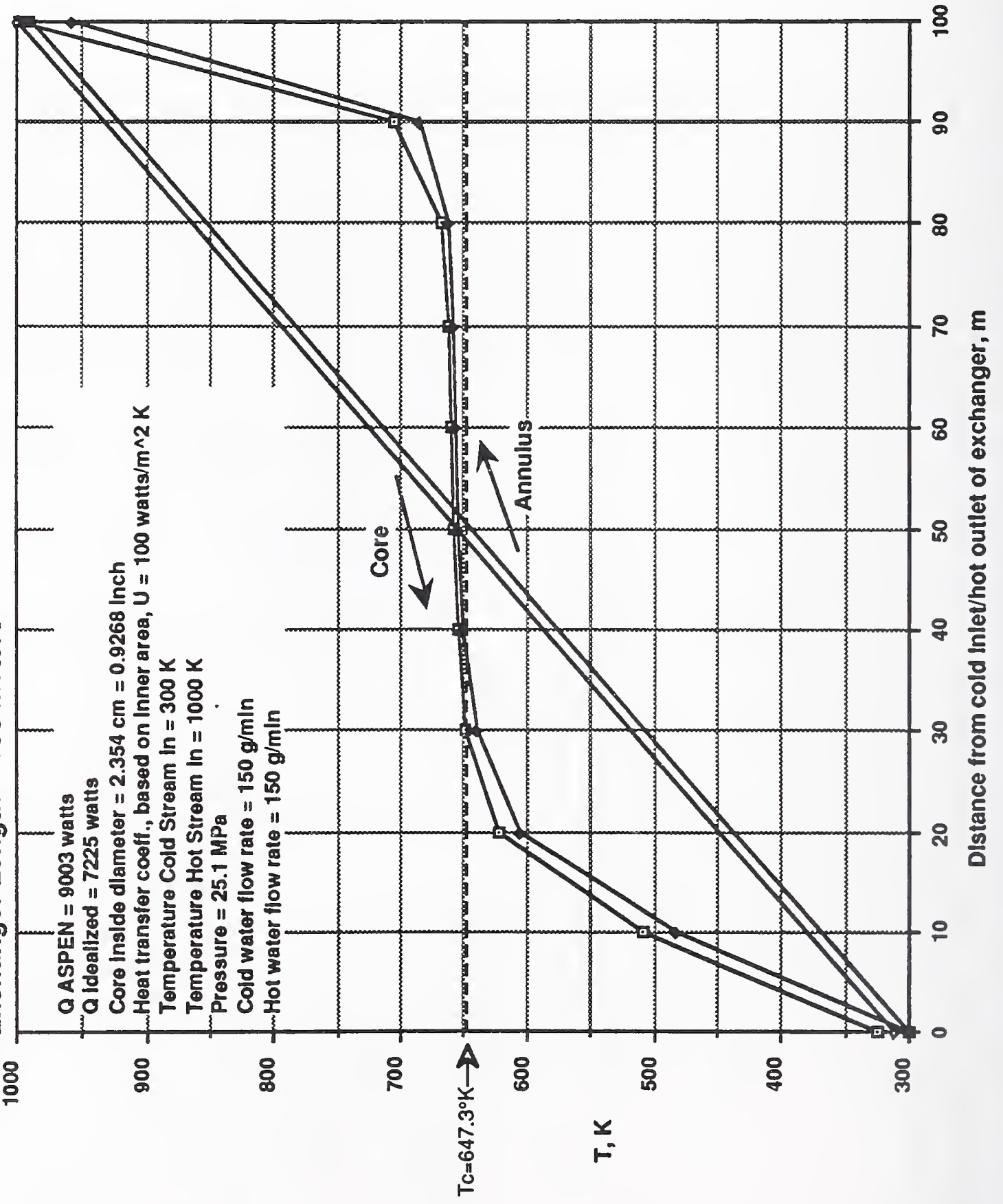

व

$\tau 0$

C

$+2$

$2 \frac{0}{4}$

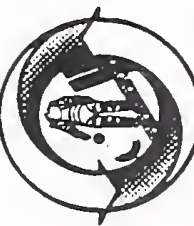

क क

ช

" 11 ธิ

乙

。등

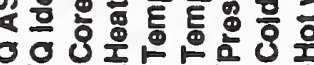




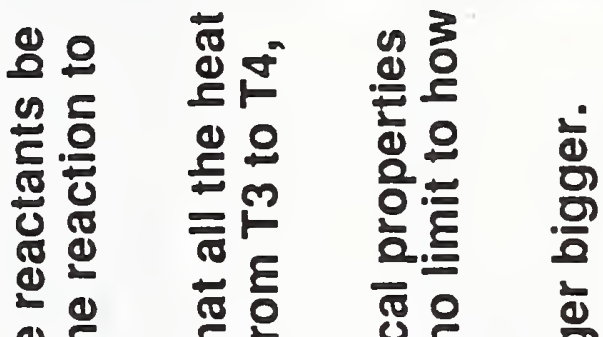

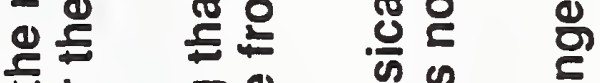

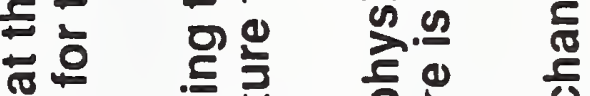

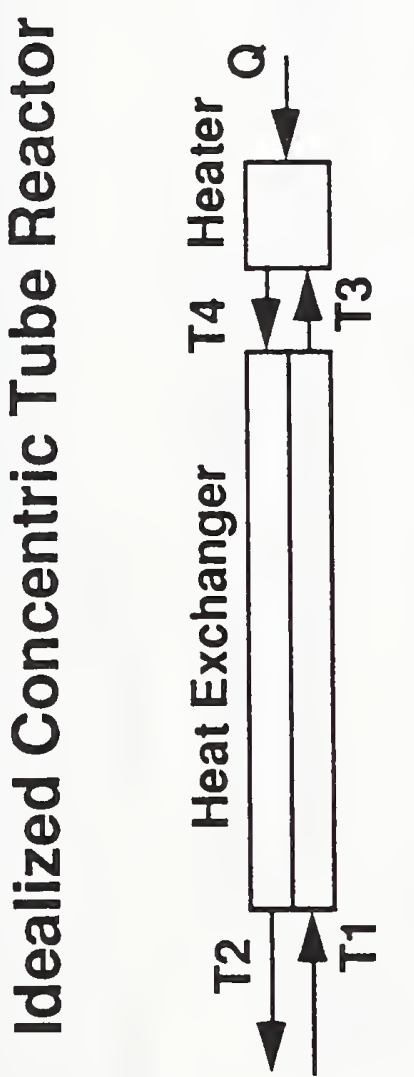

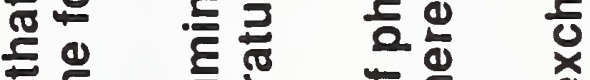

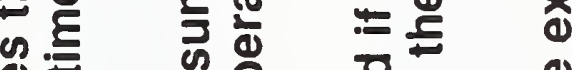

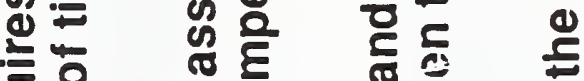

긍 >0

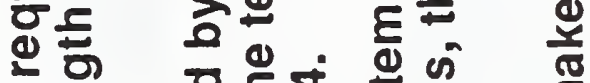

ธड

तٓ

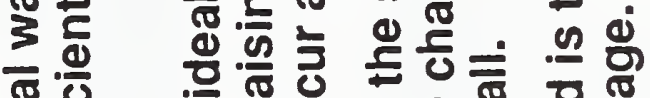

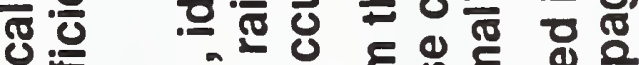

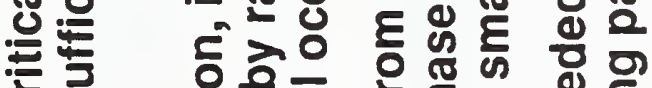

ㄴํㄴ क

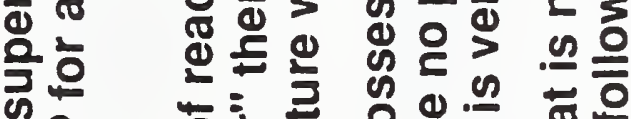

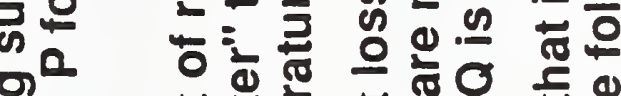

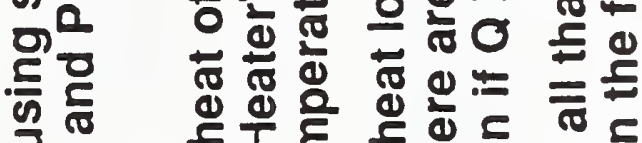

Ј

or

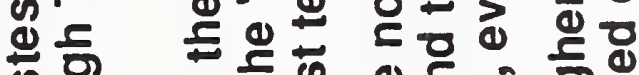

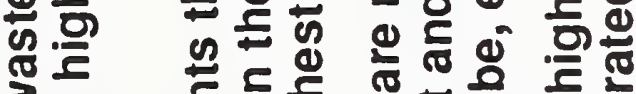

ऊ

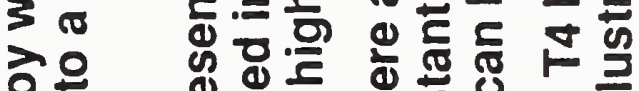

은 $\quad$ 屯.

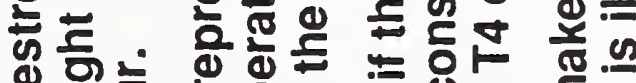

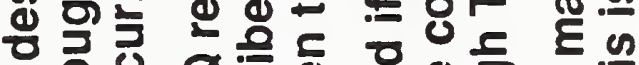

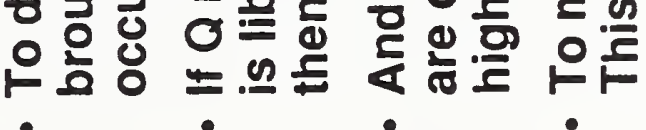

क

닌

$<8$

$\tau$ 


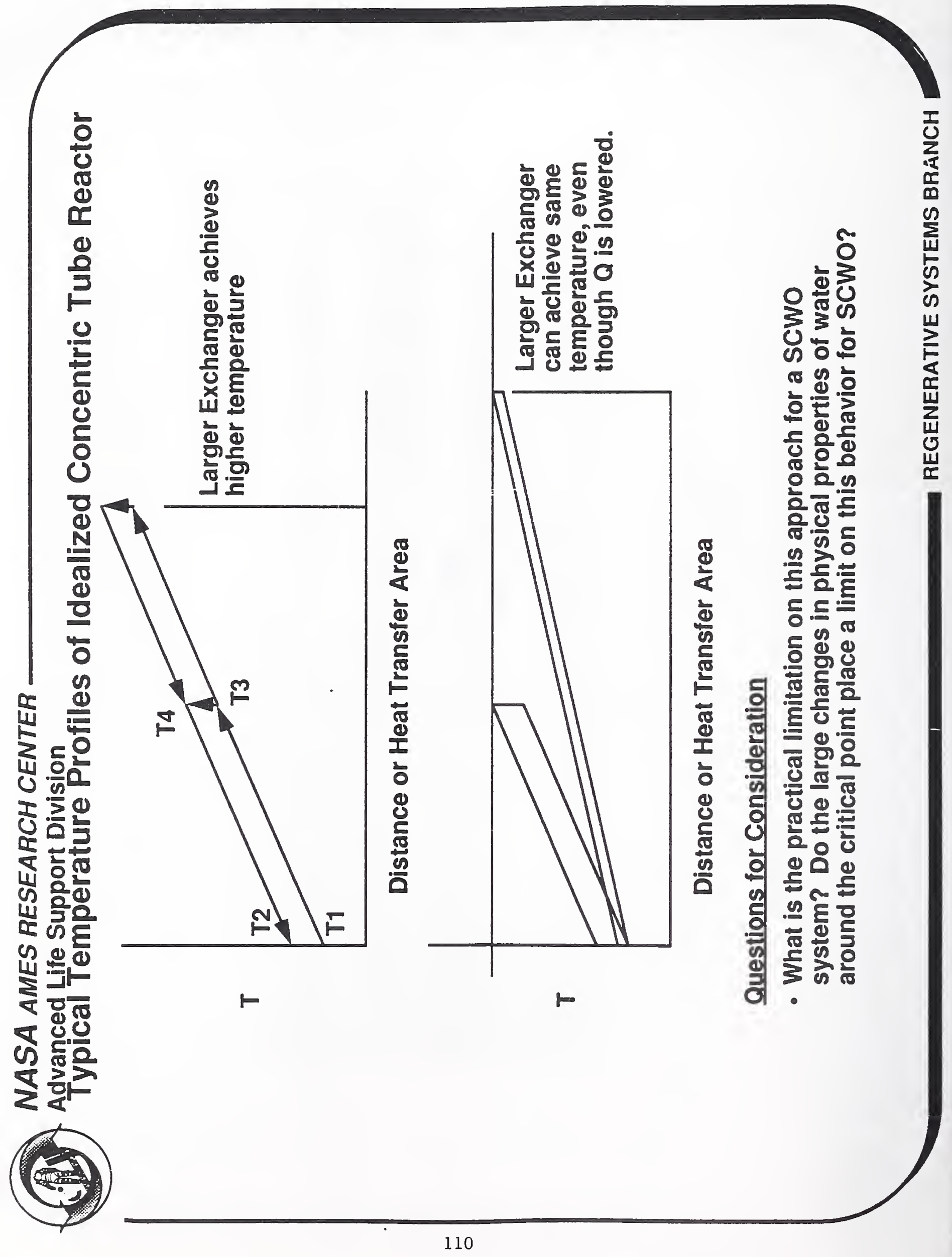




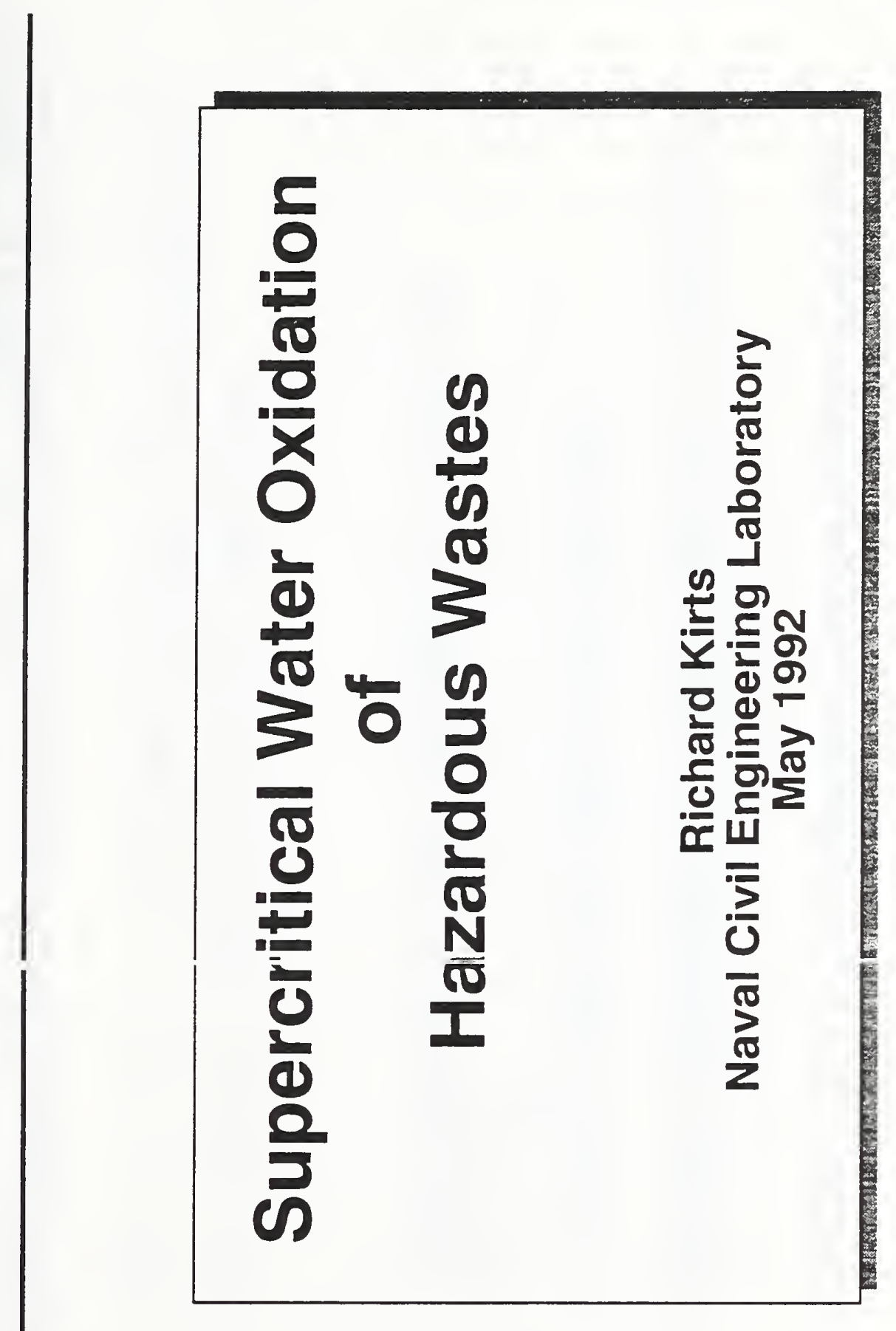

$\frac{5}{E}$

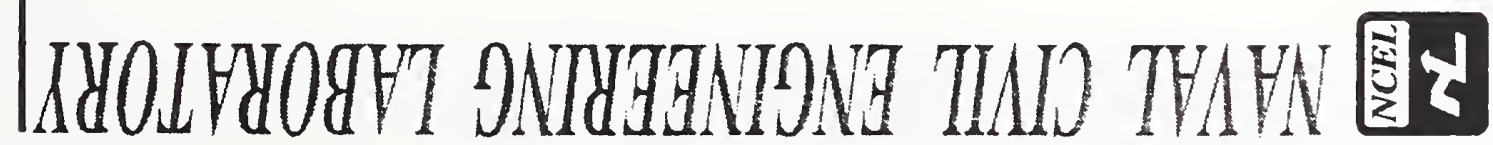




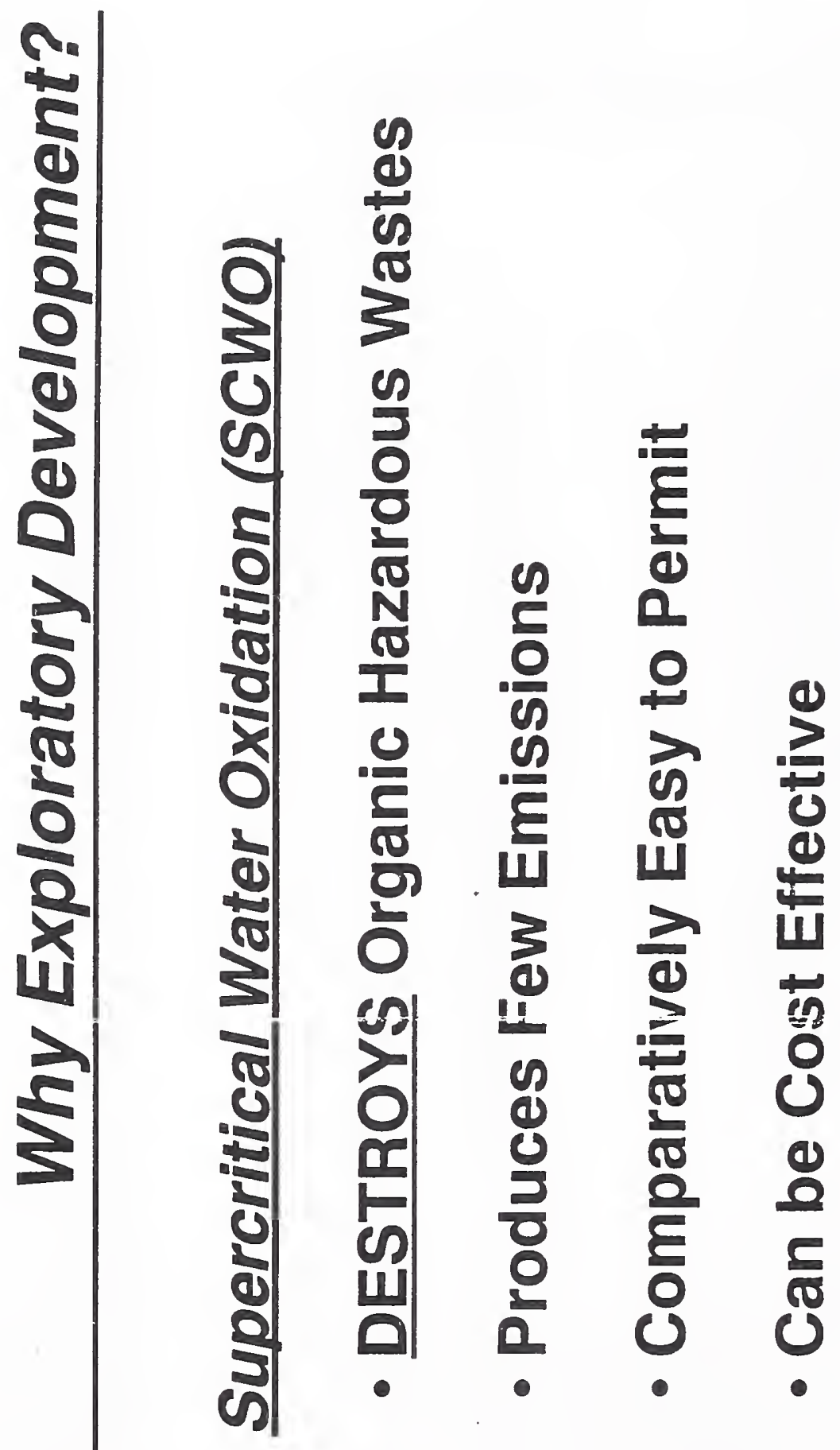




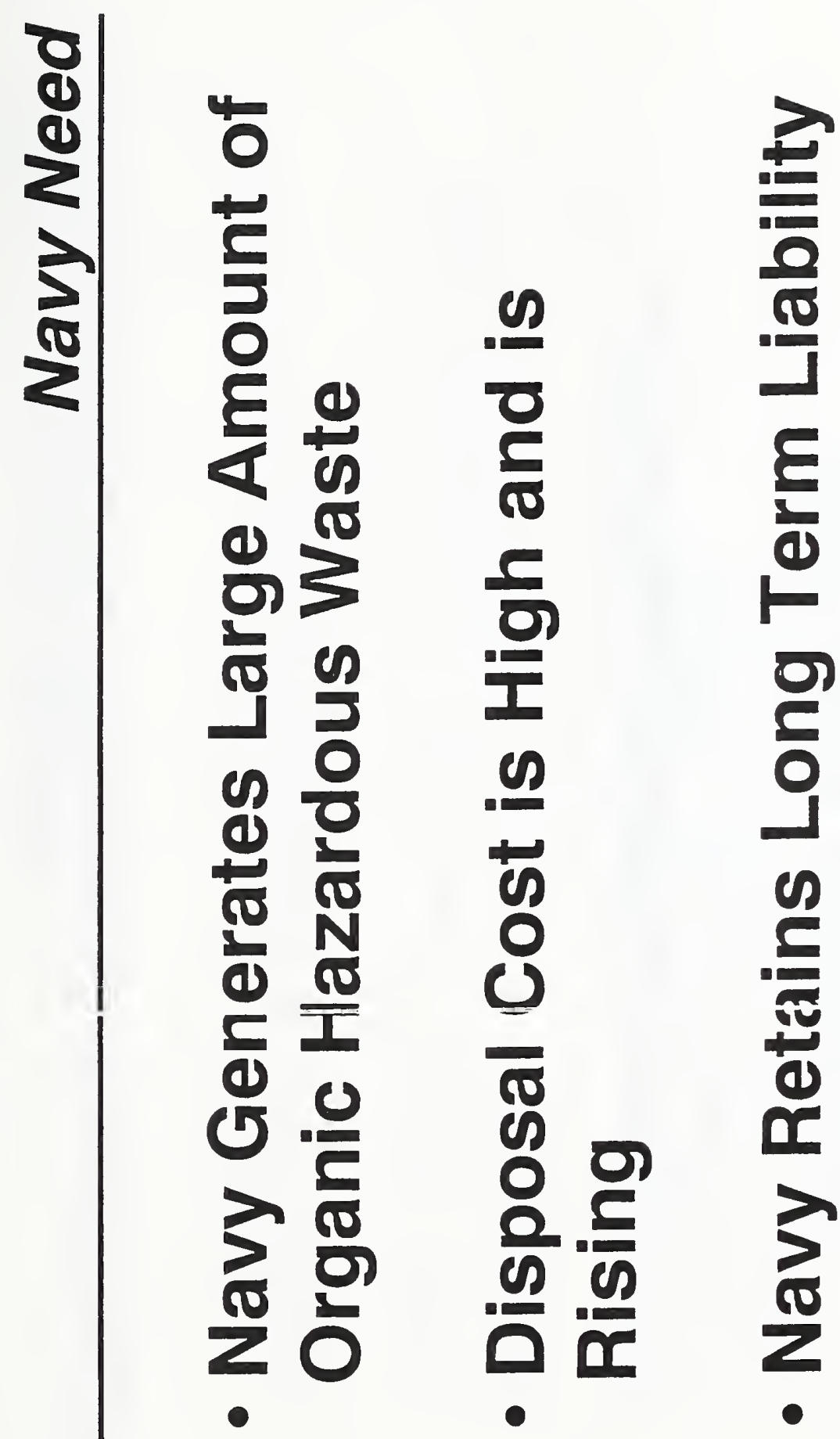

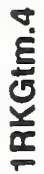

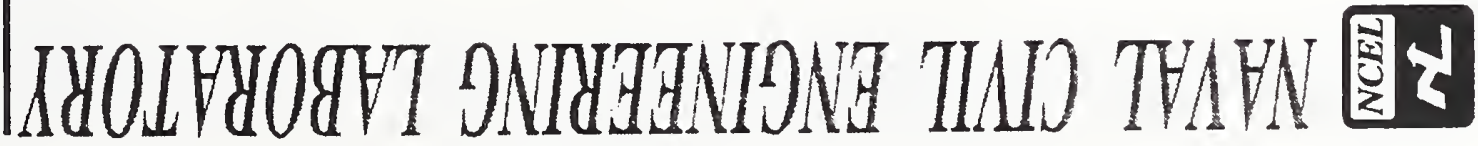




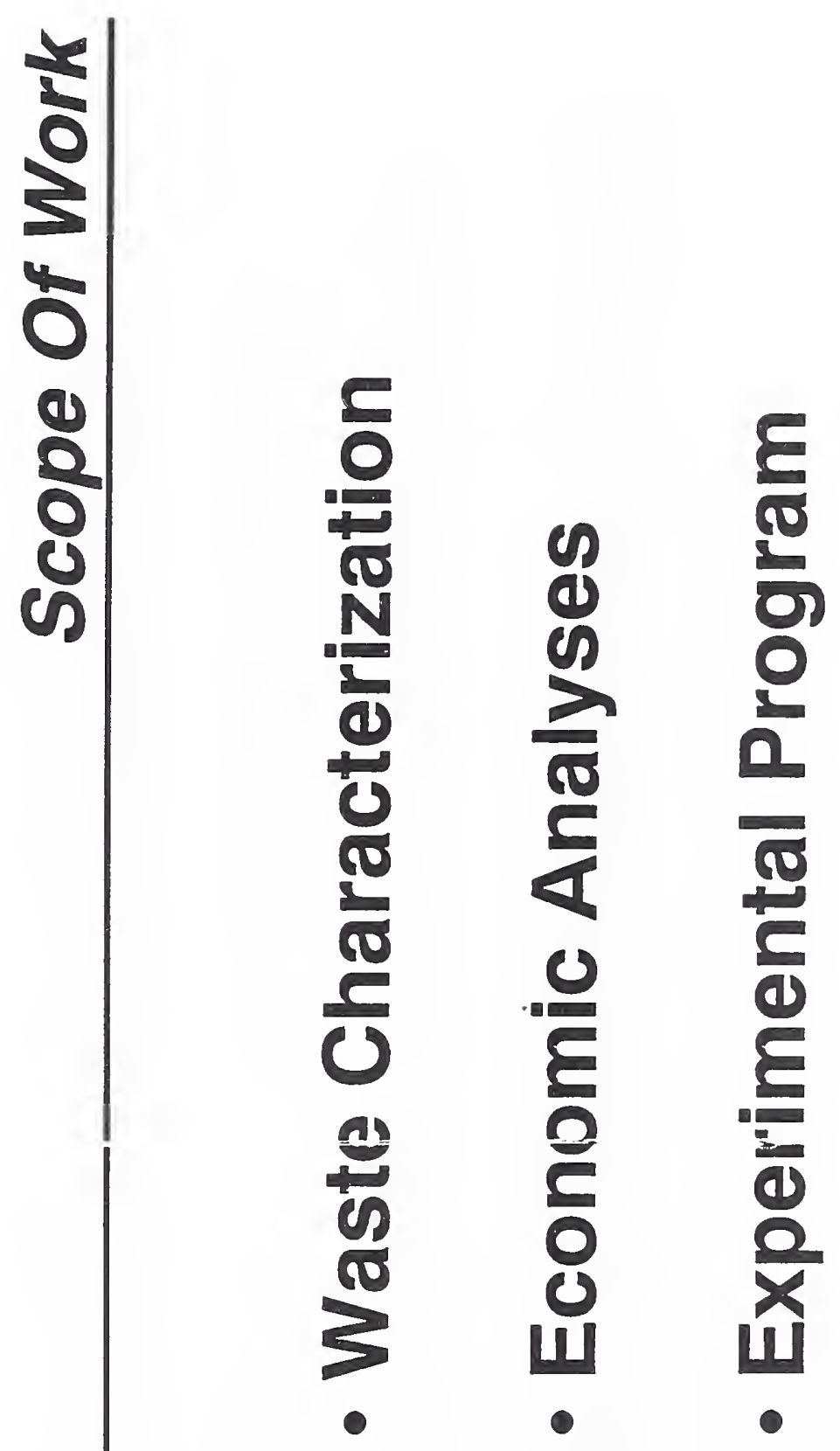

$\frac{\text { हैं }}{\frac{5}{0}}$

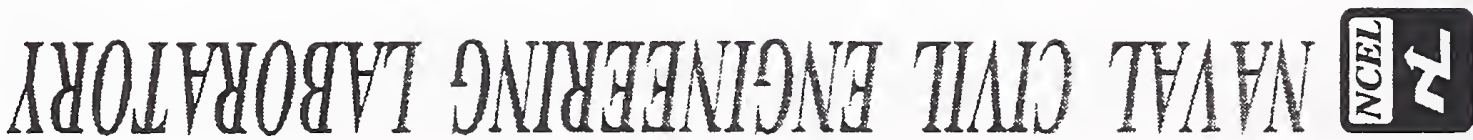




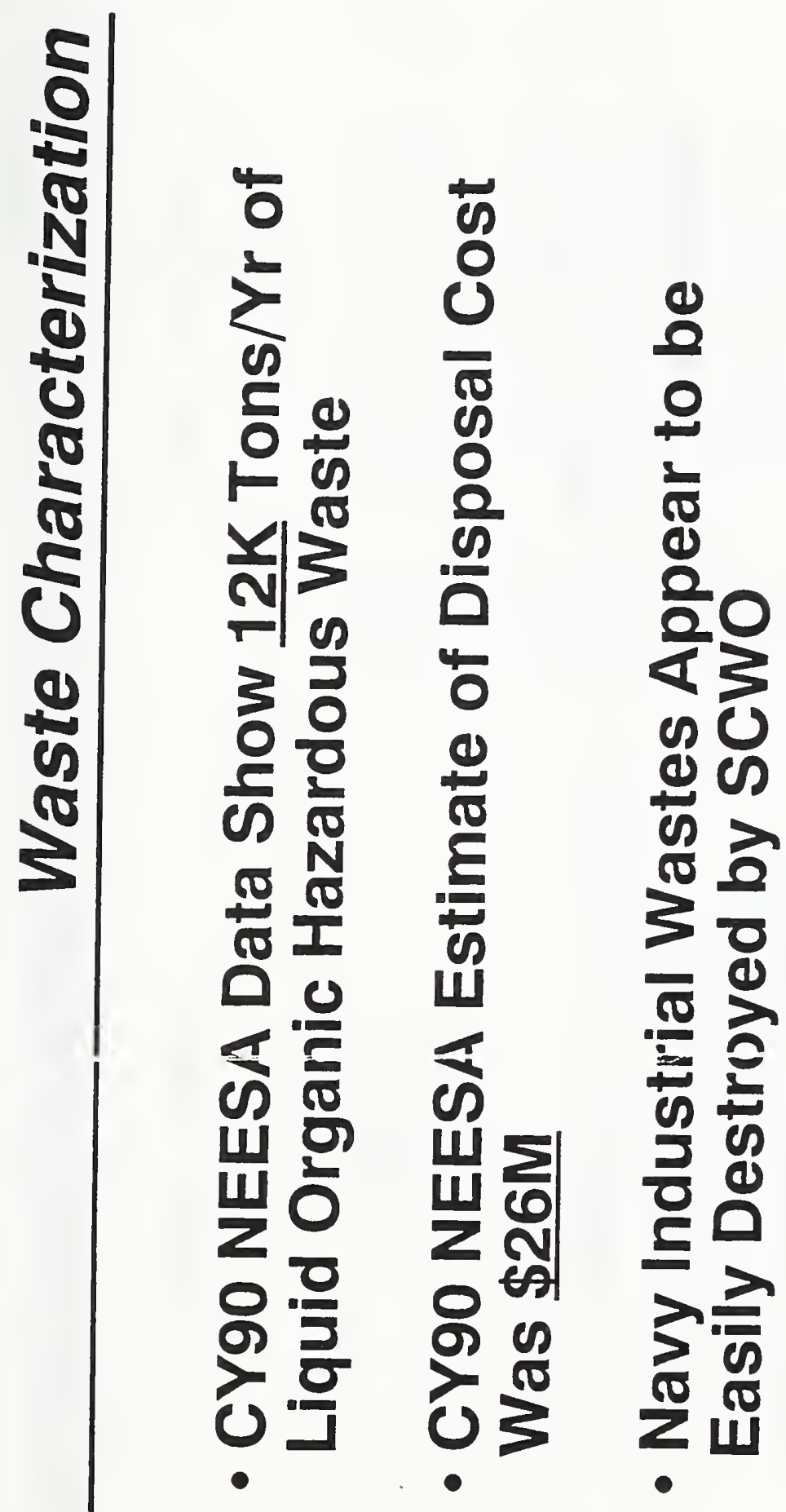

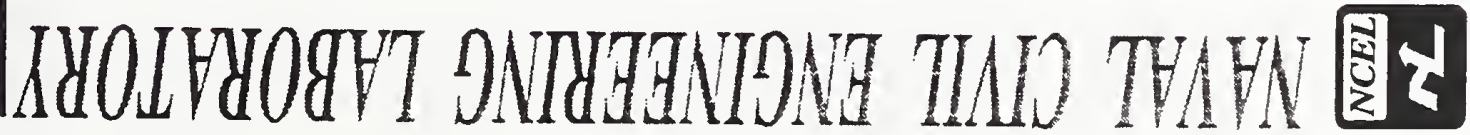


0
0
0
0
0
0
0
0
0
0
0
0
0
0
0
0
0
0

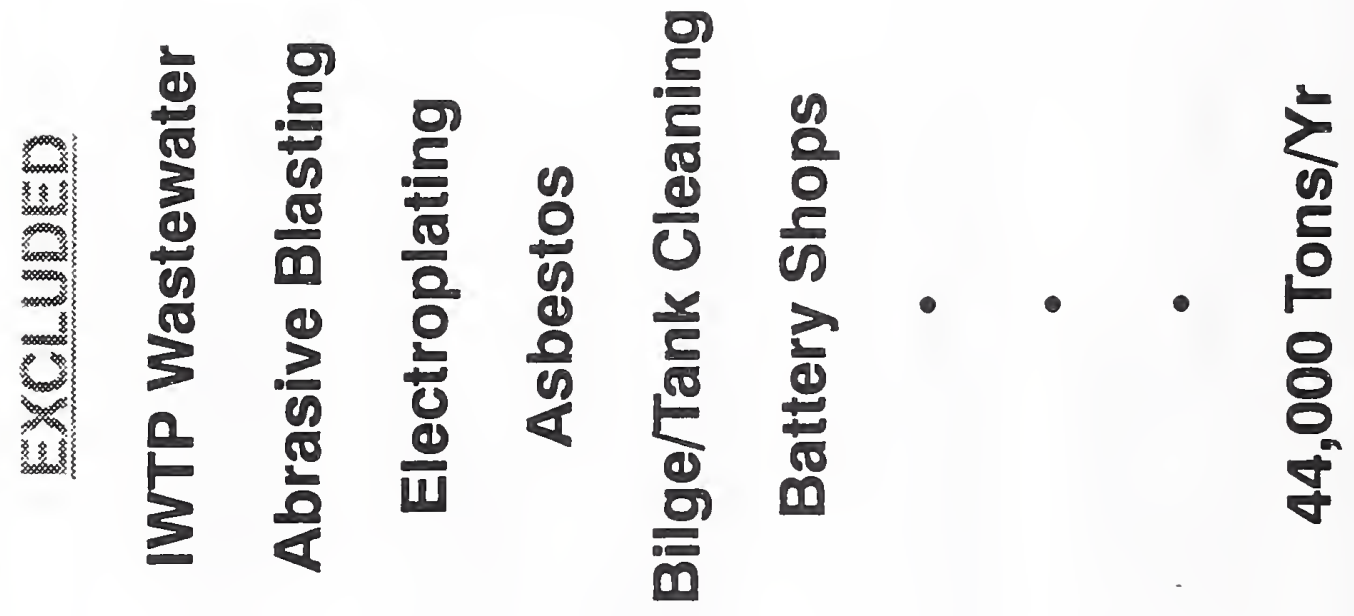

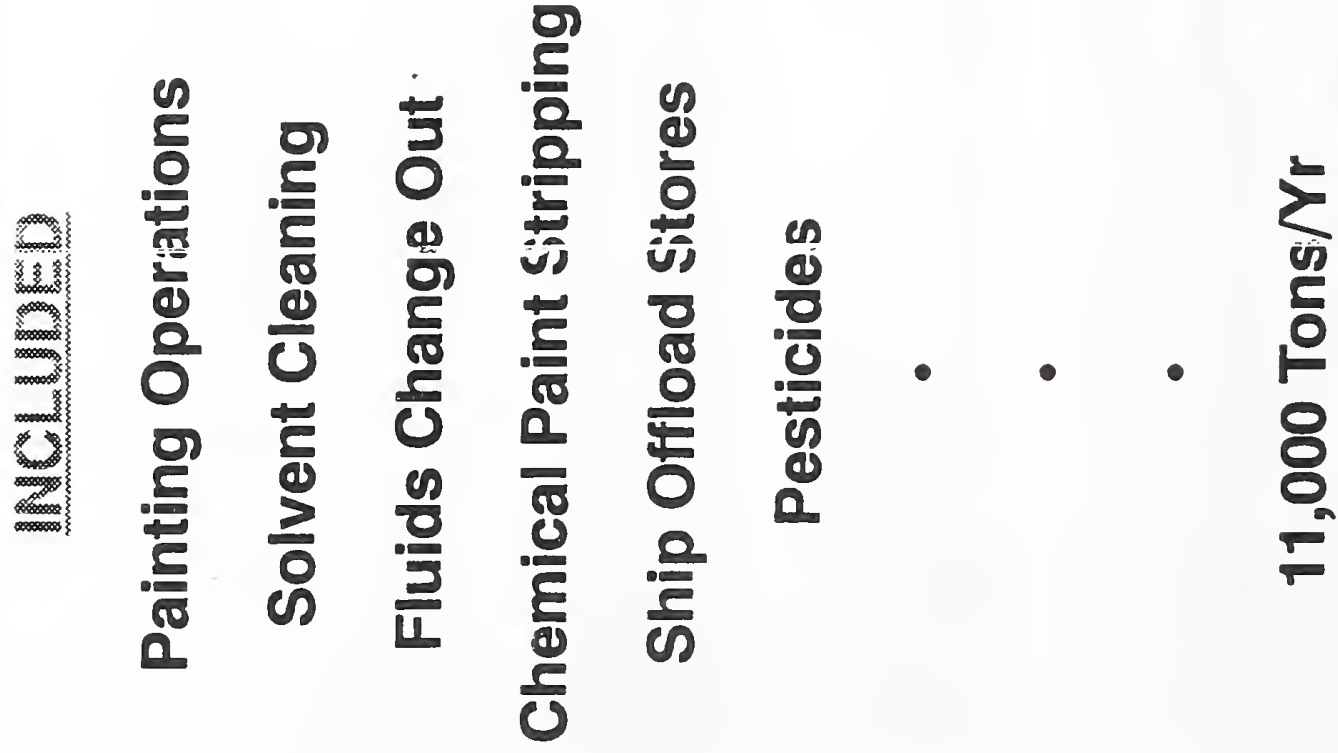




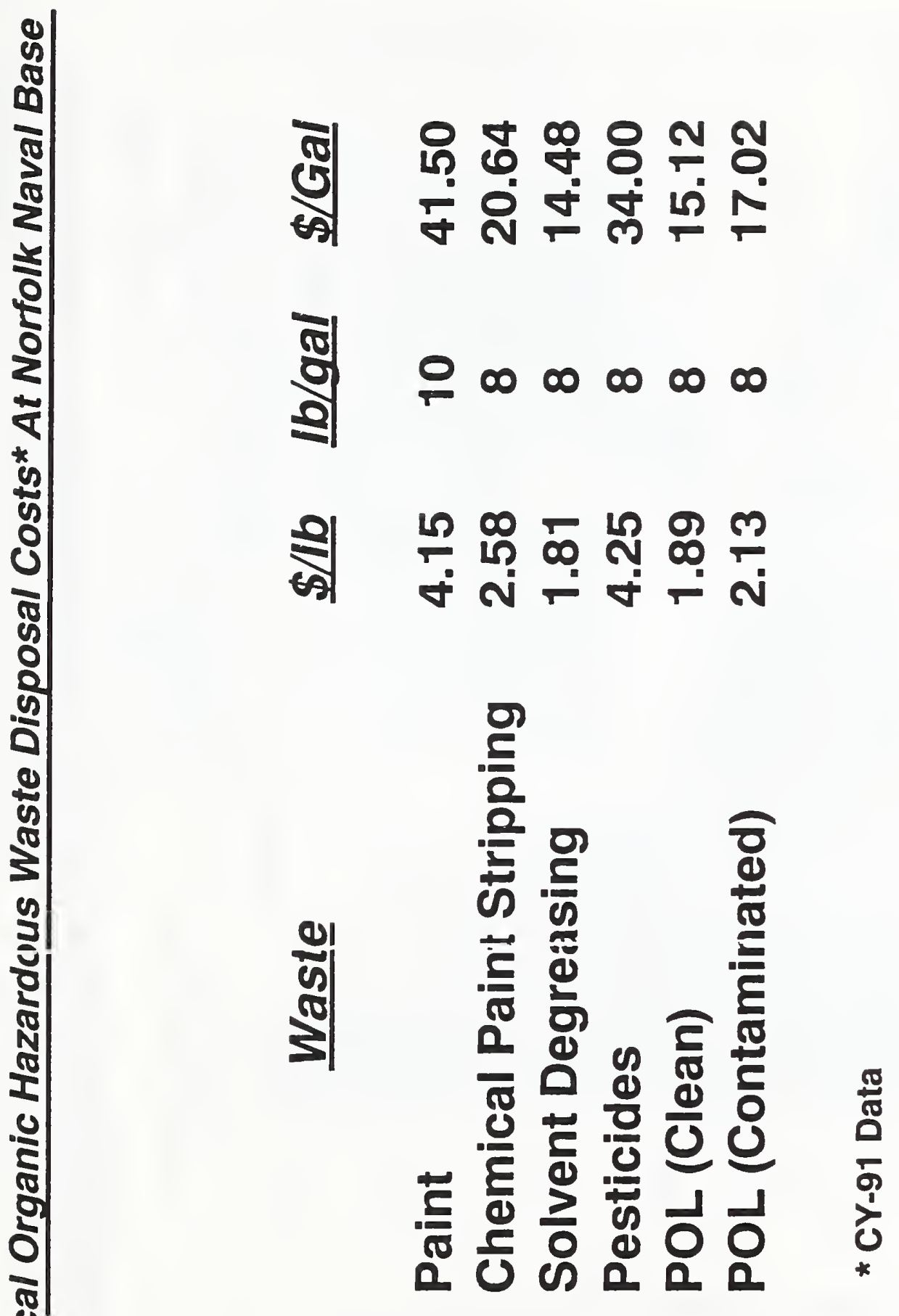

롤

요

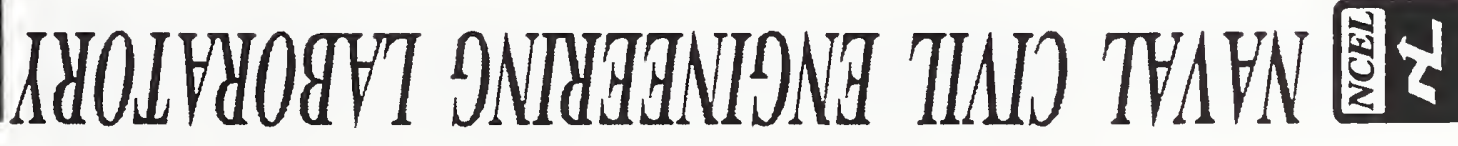




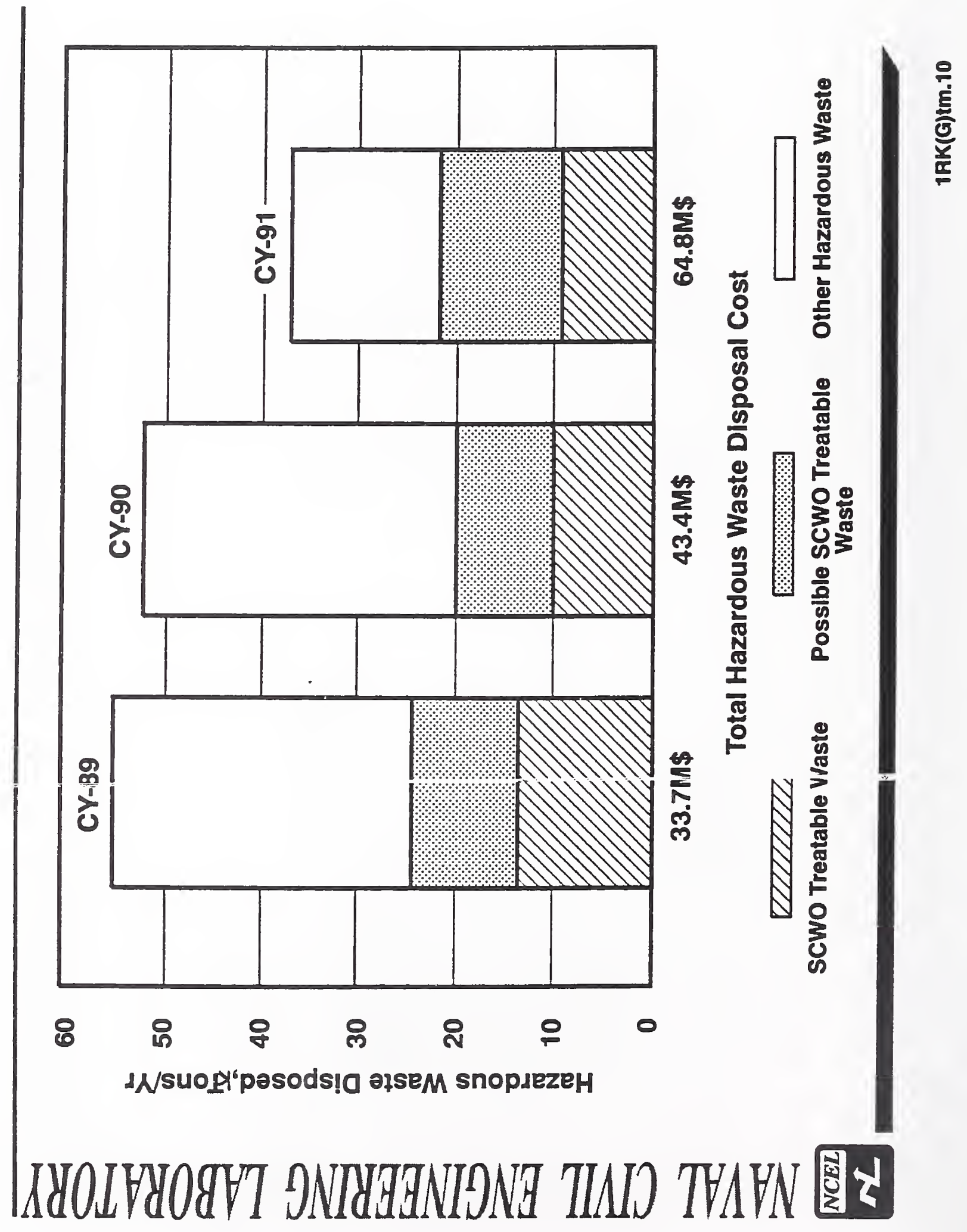




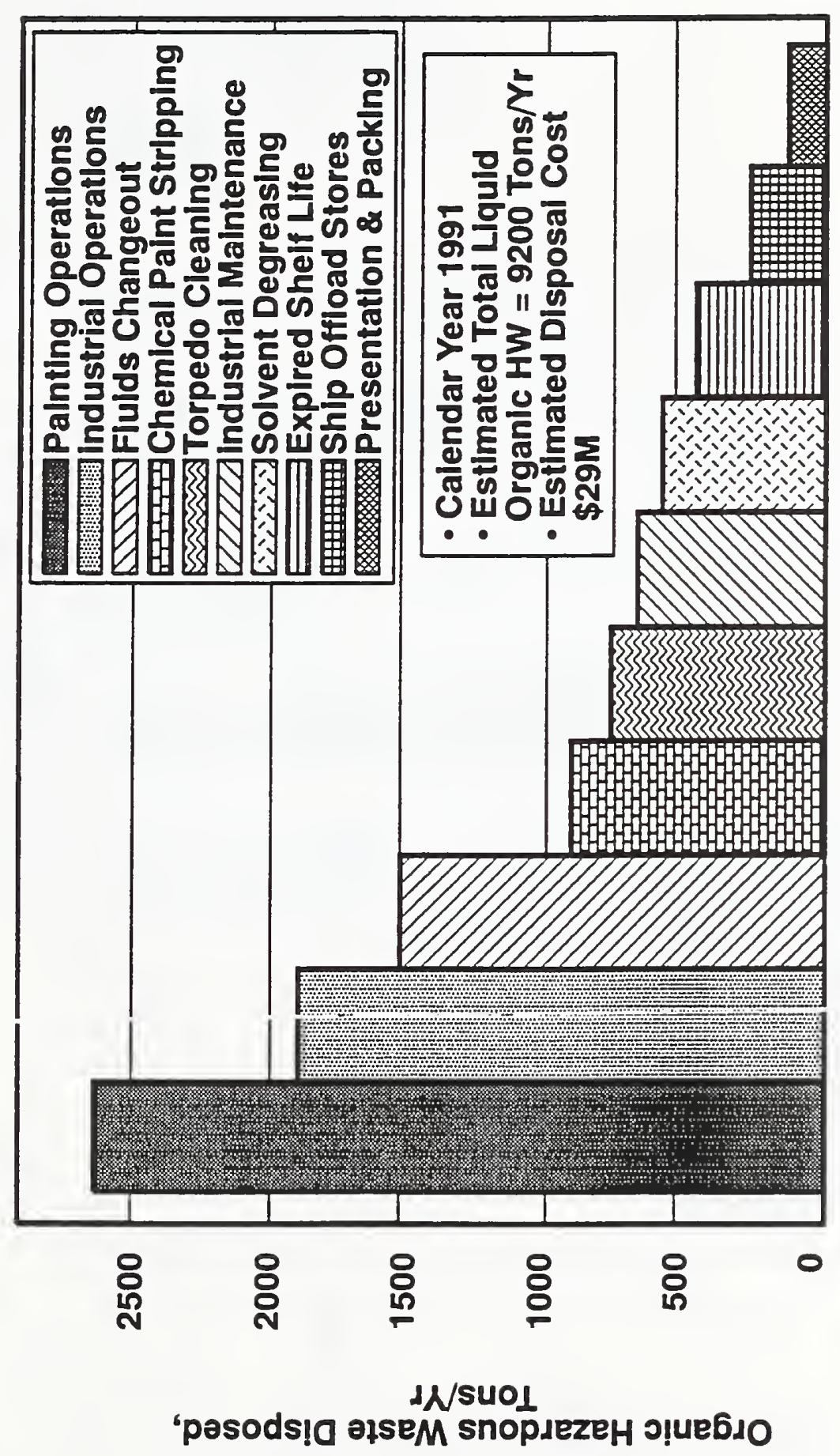

론 


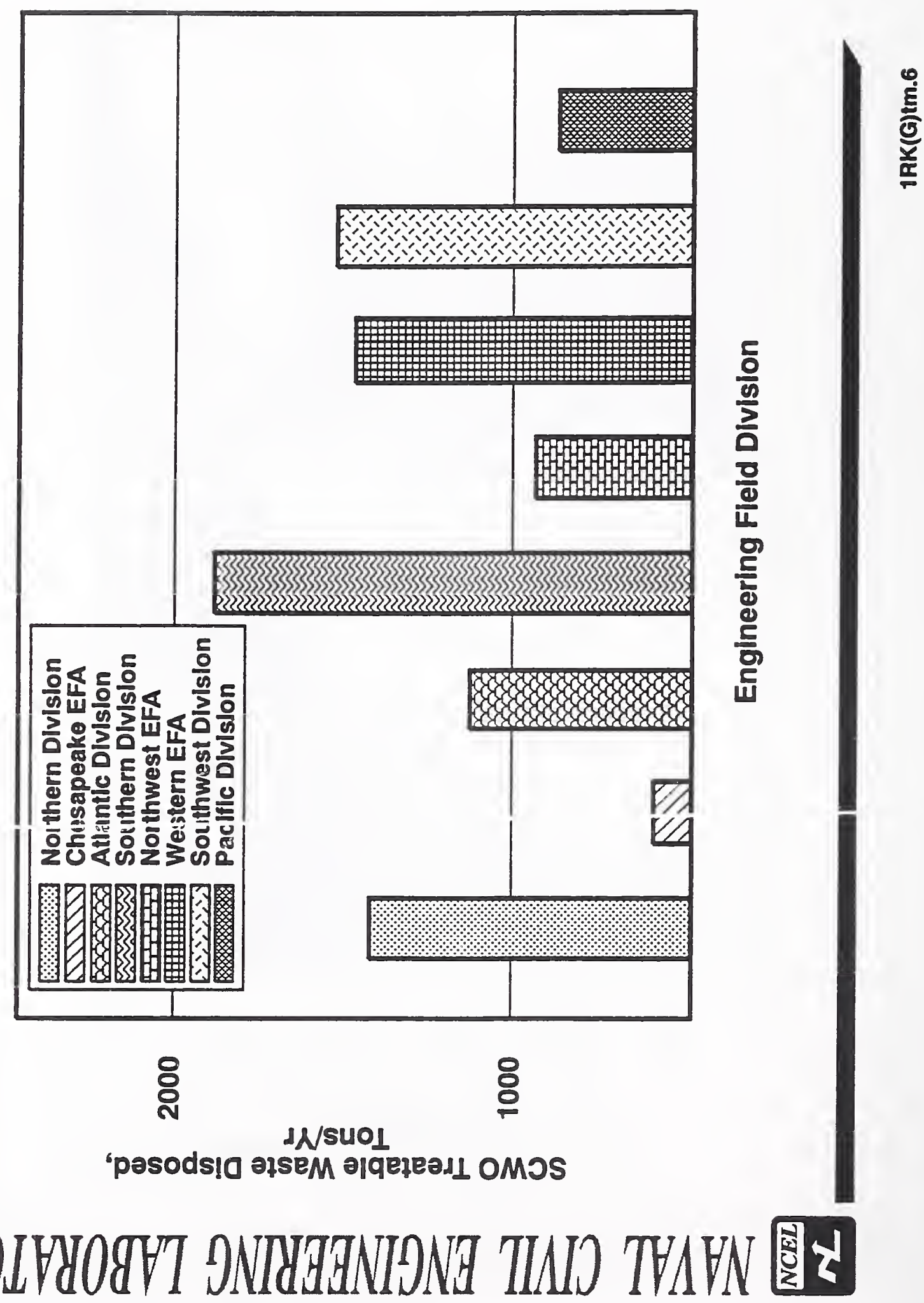




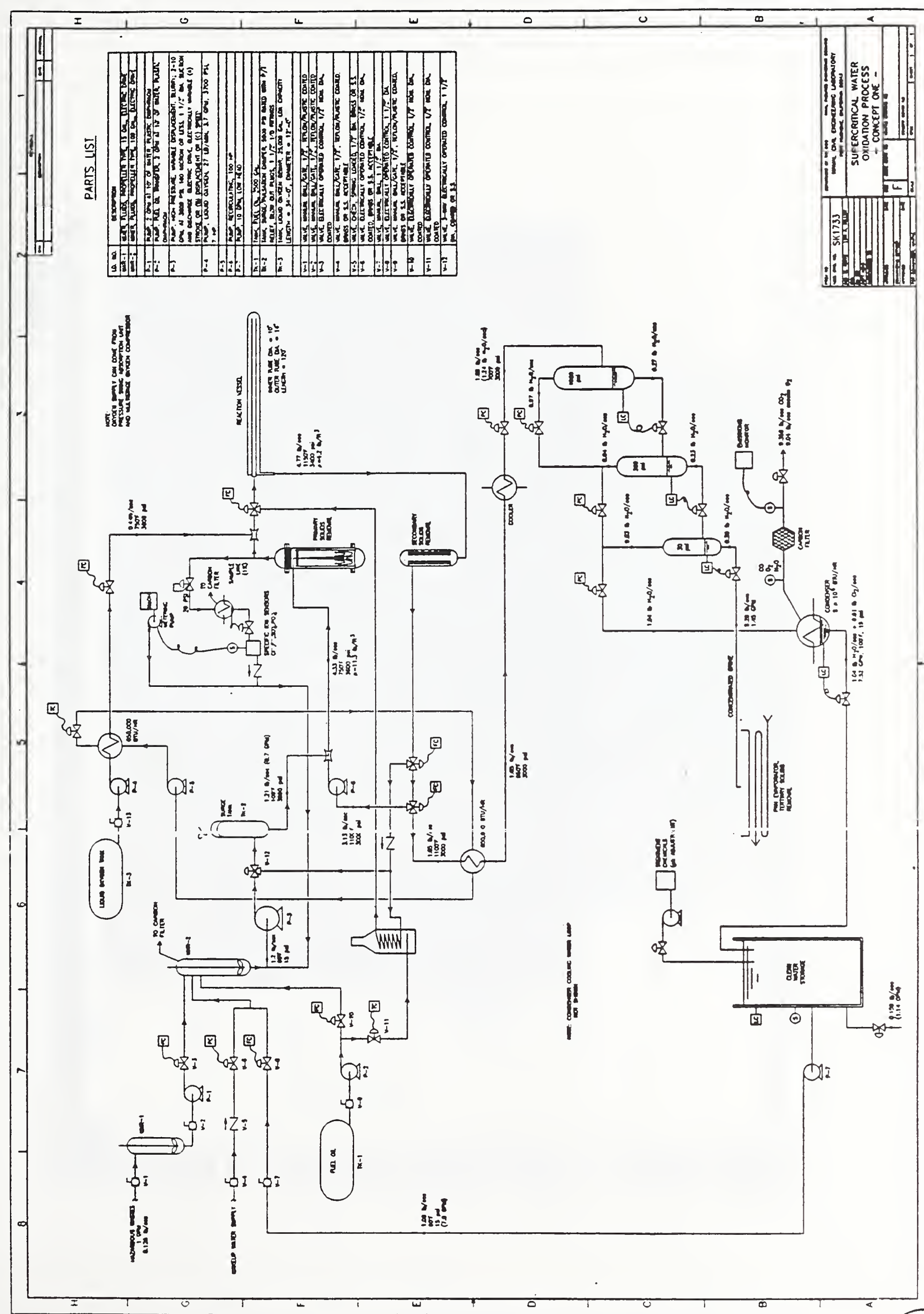




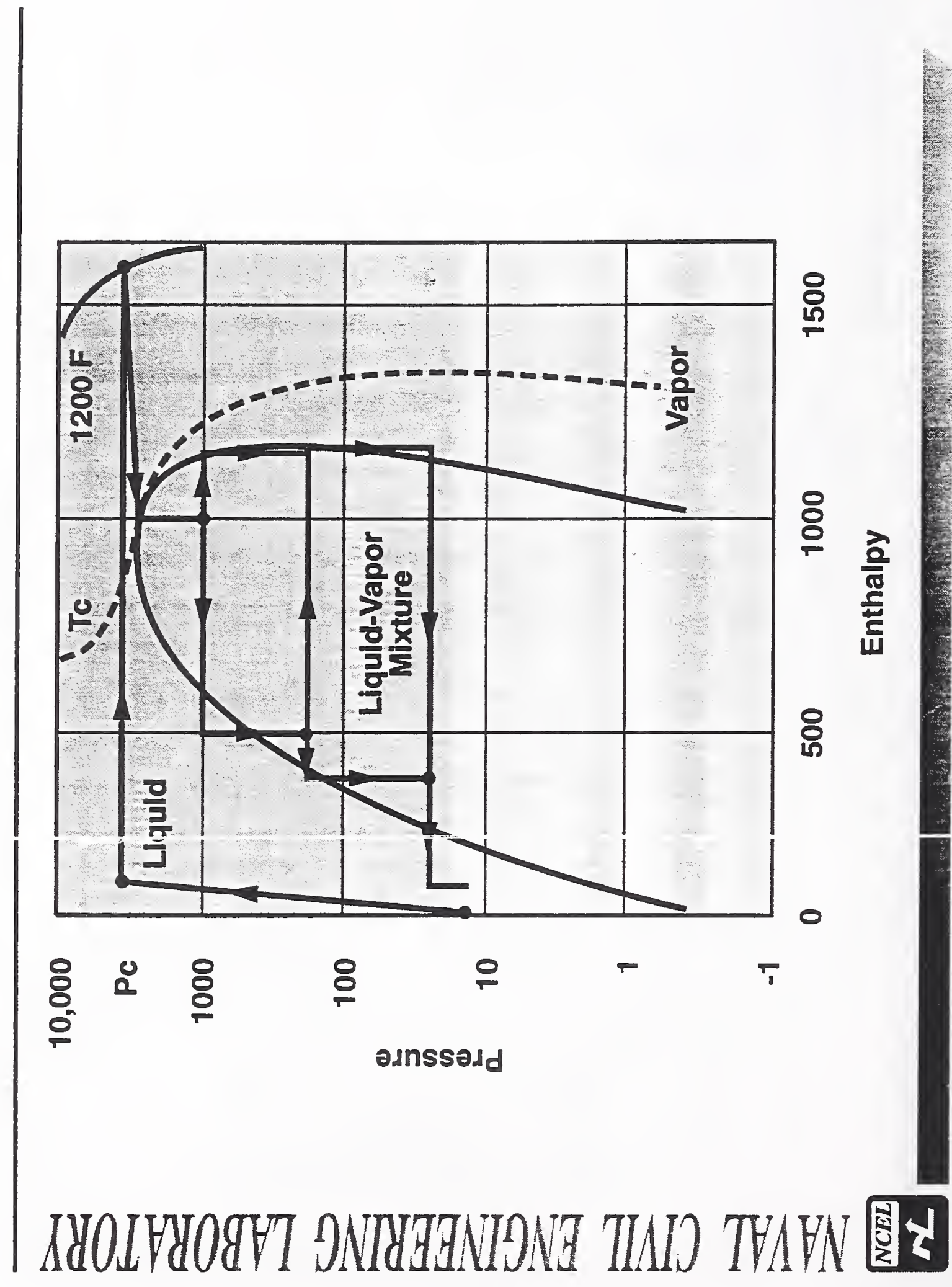




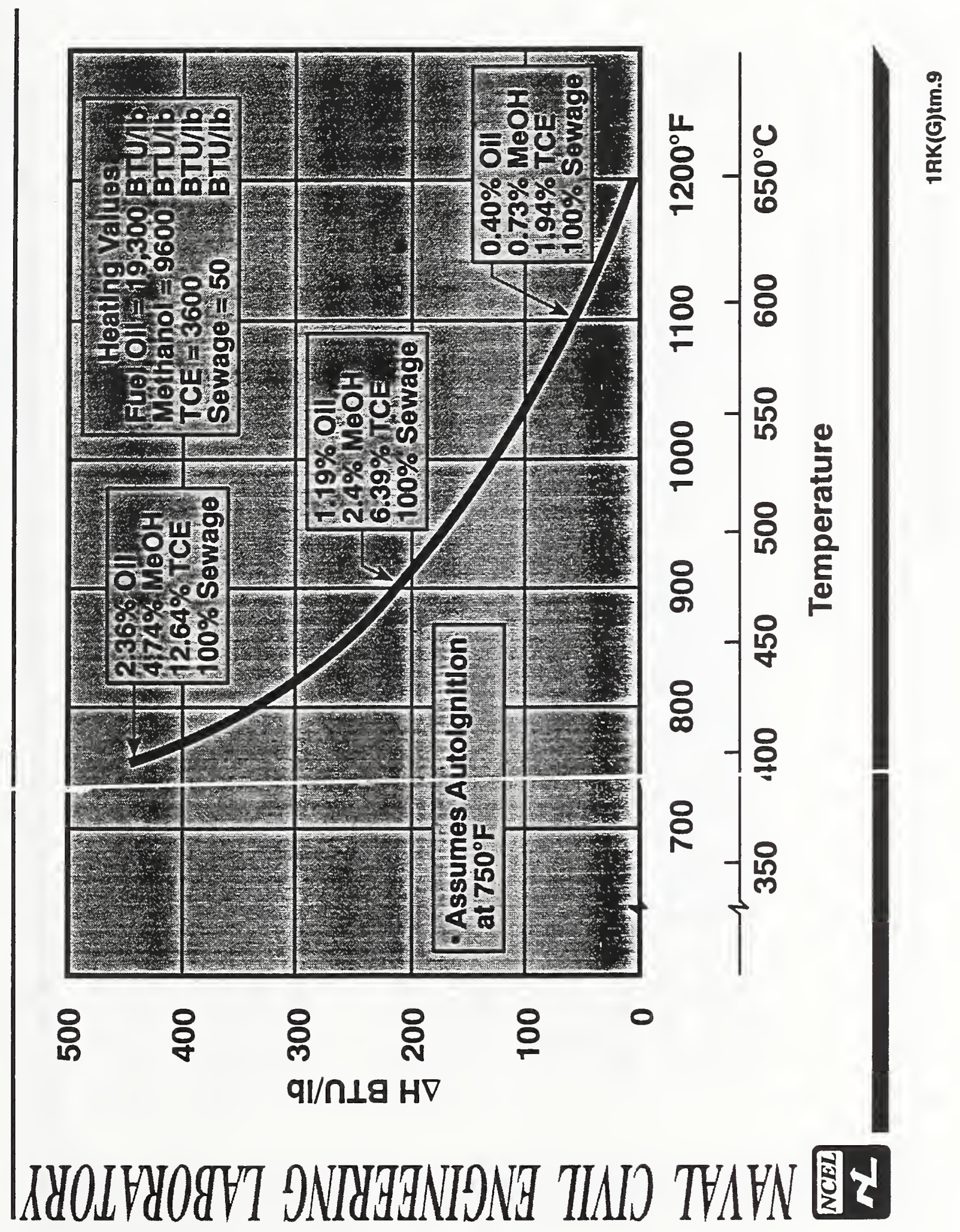




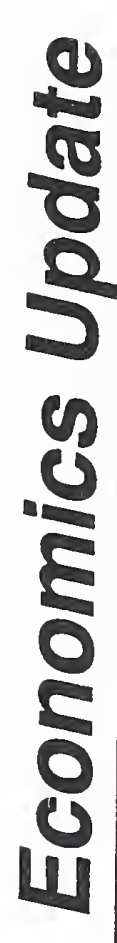

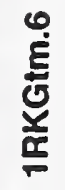

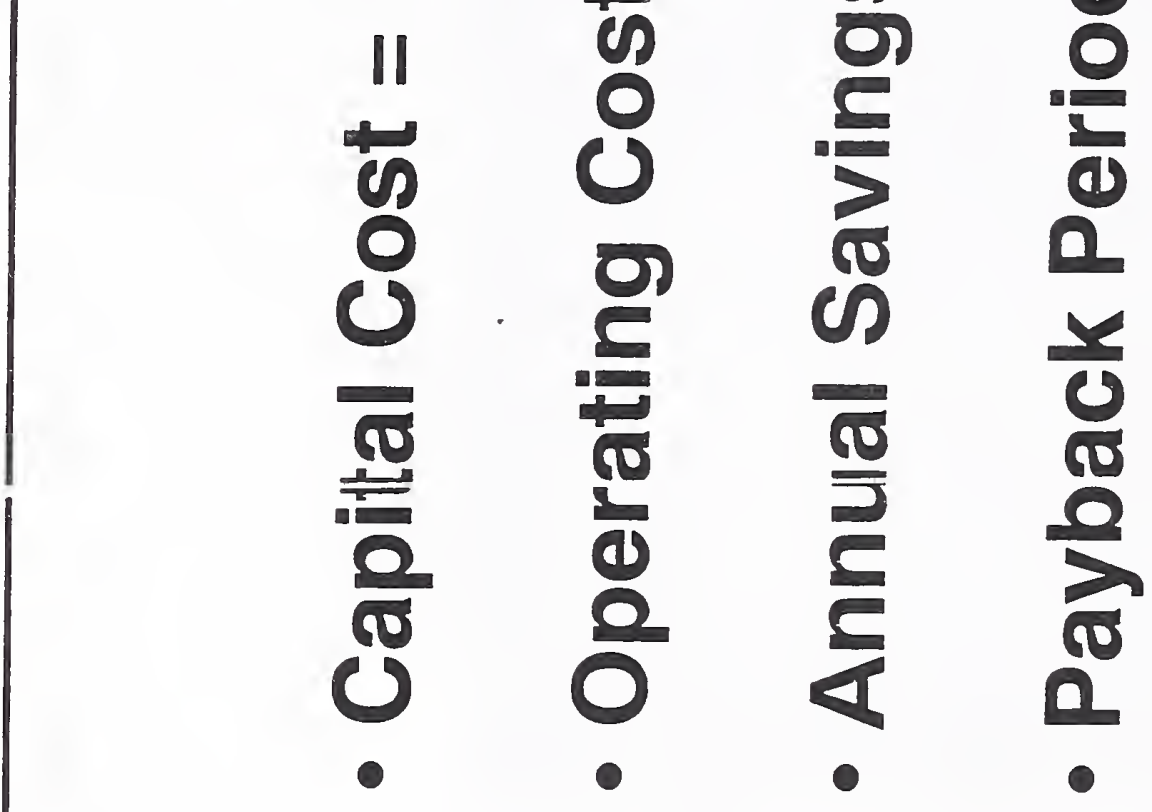

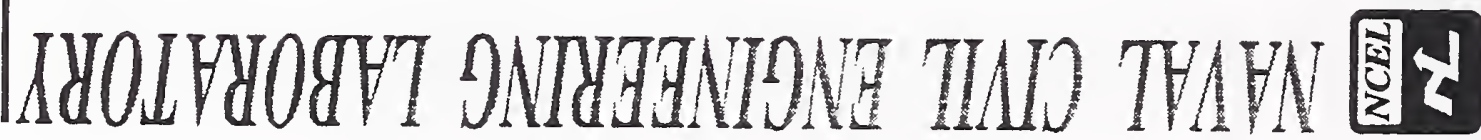




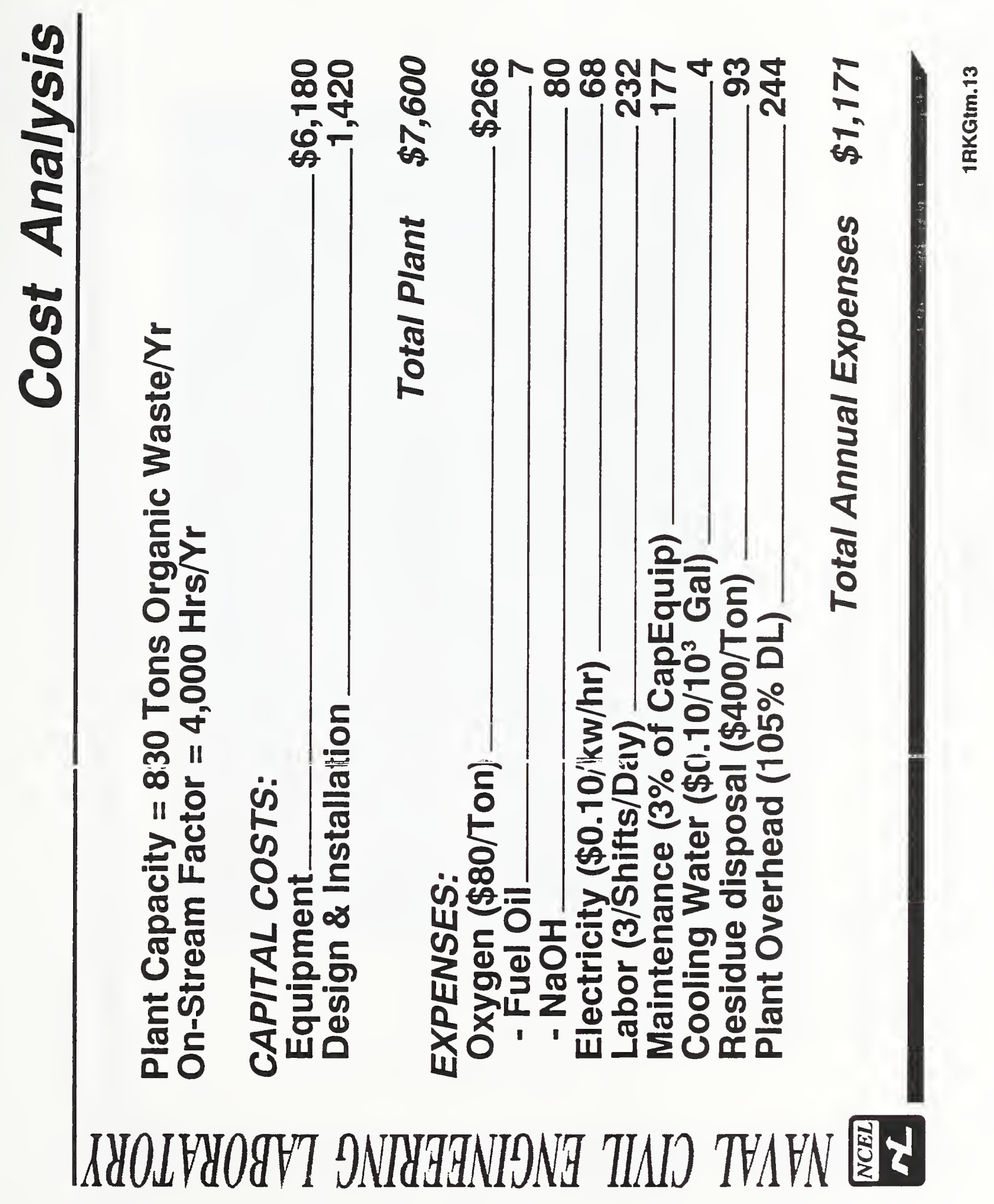




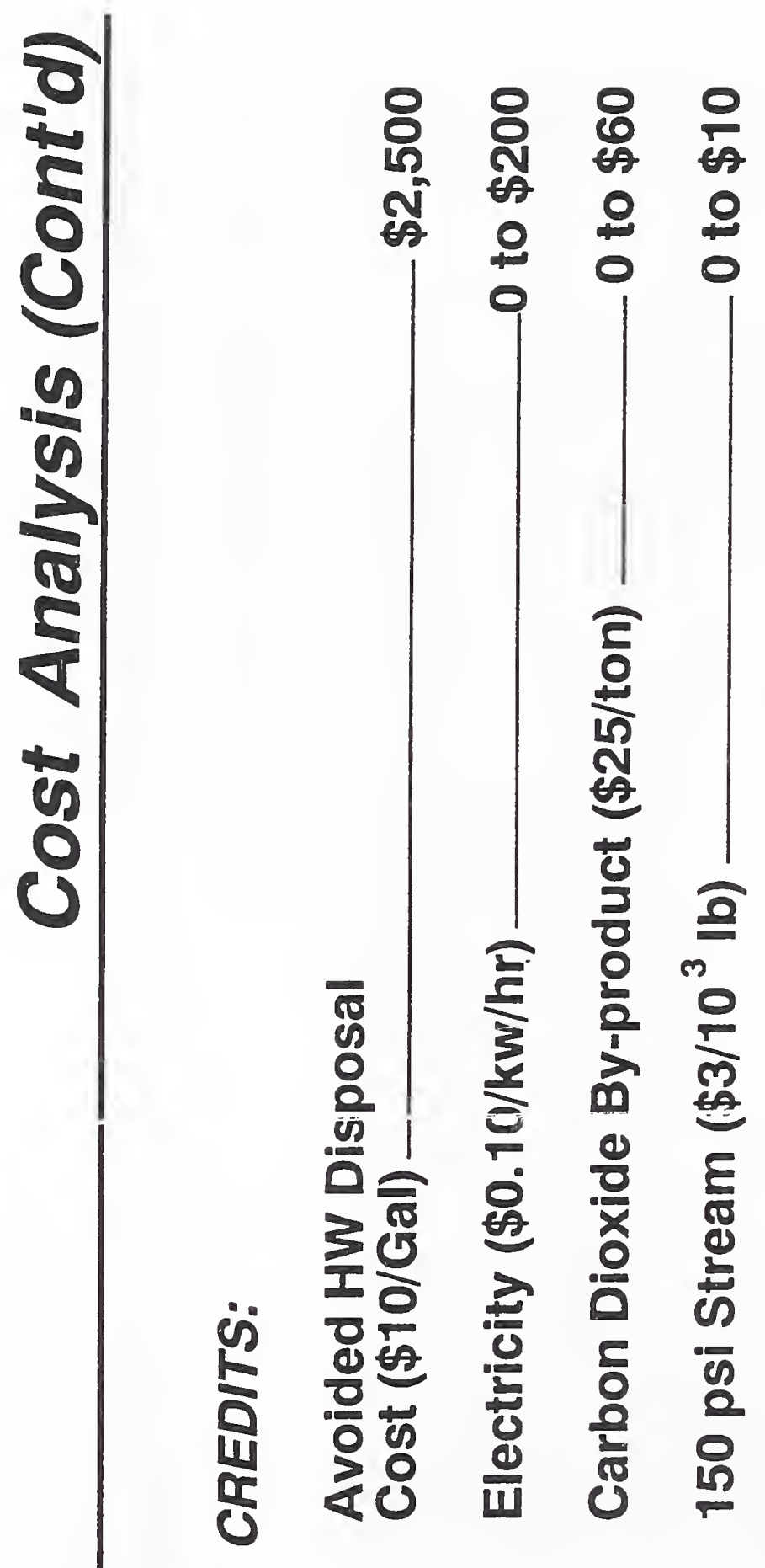

롱 


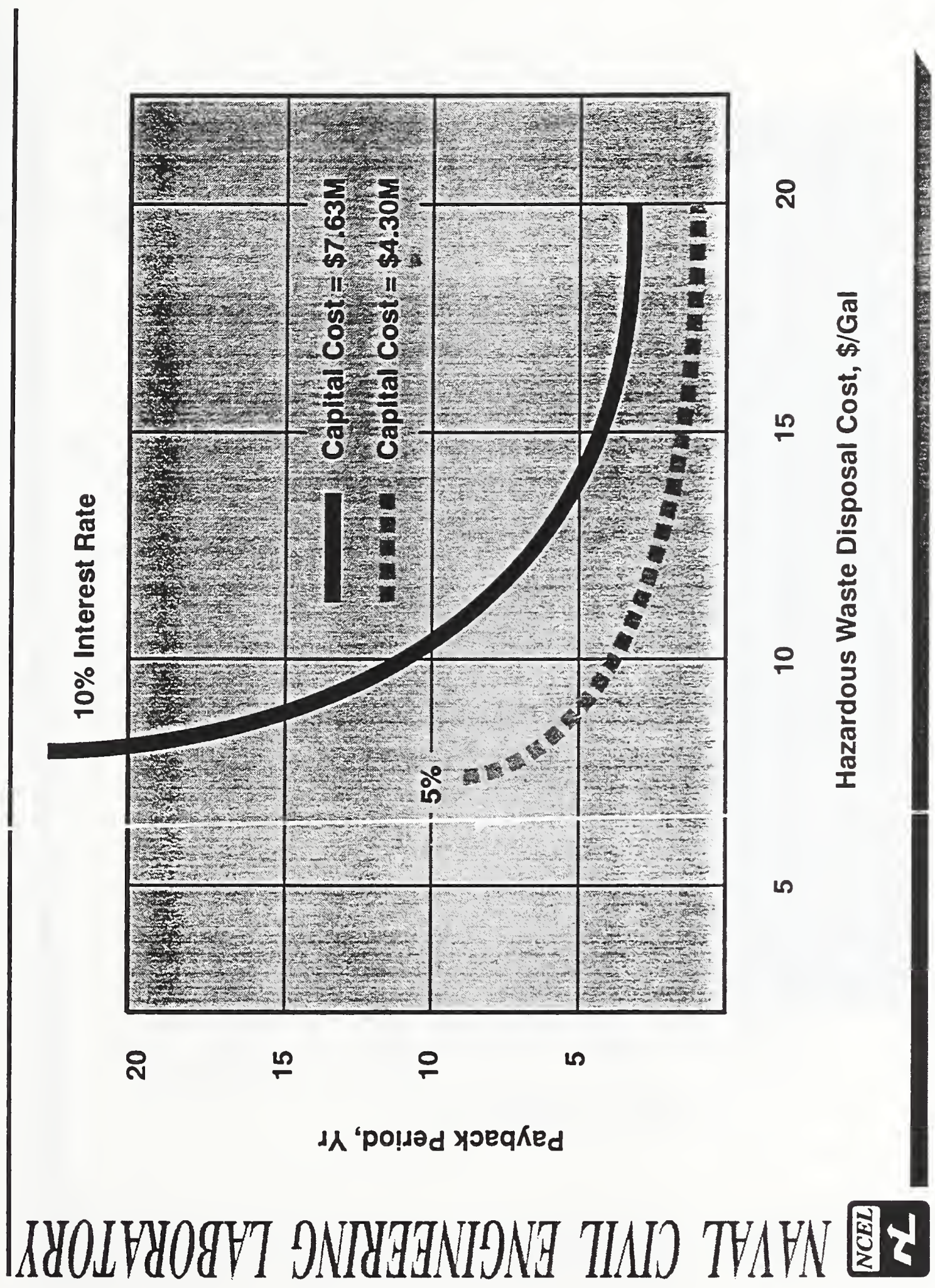




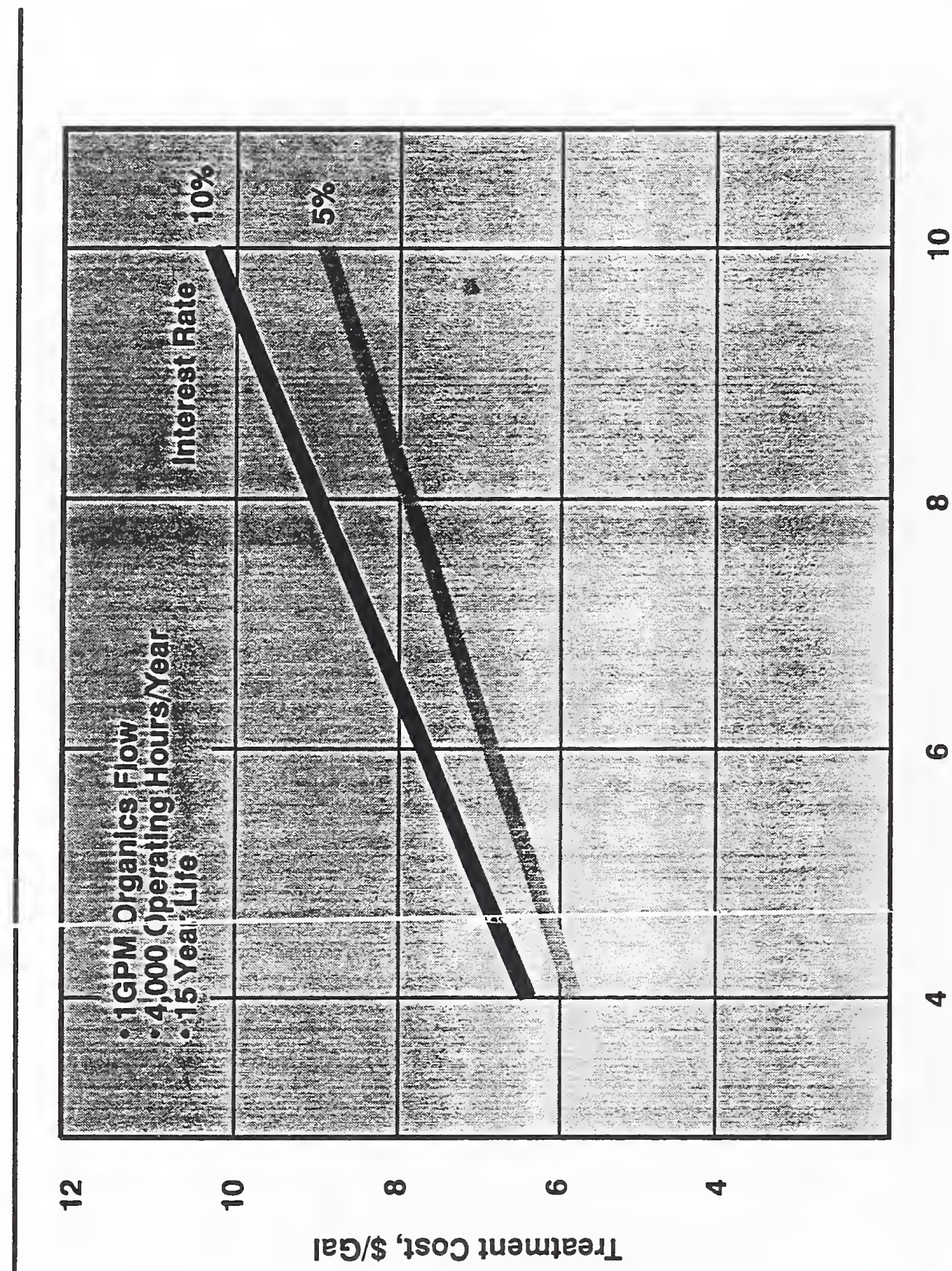

$n$
E⿱
$\frac{1}{\sigma}$

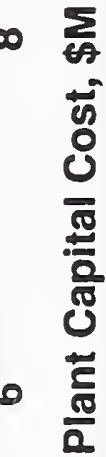

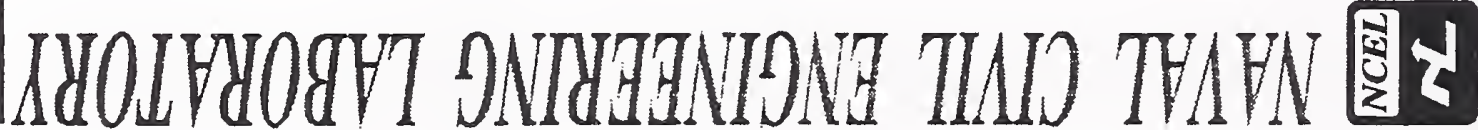




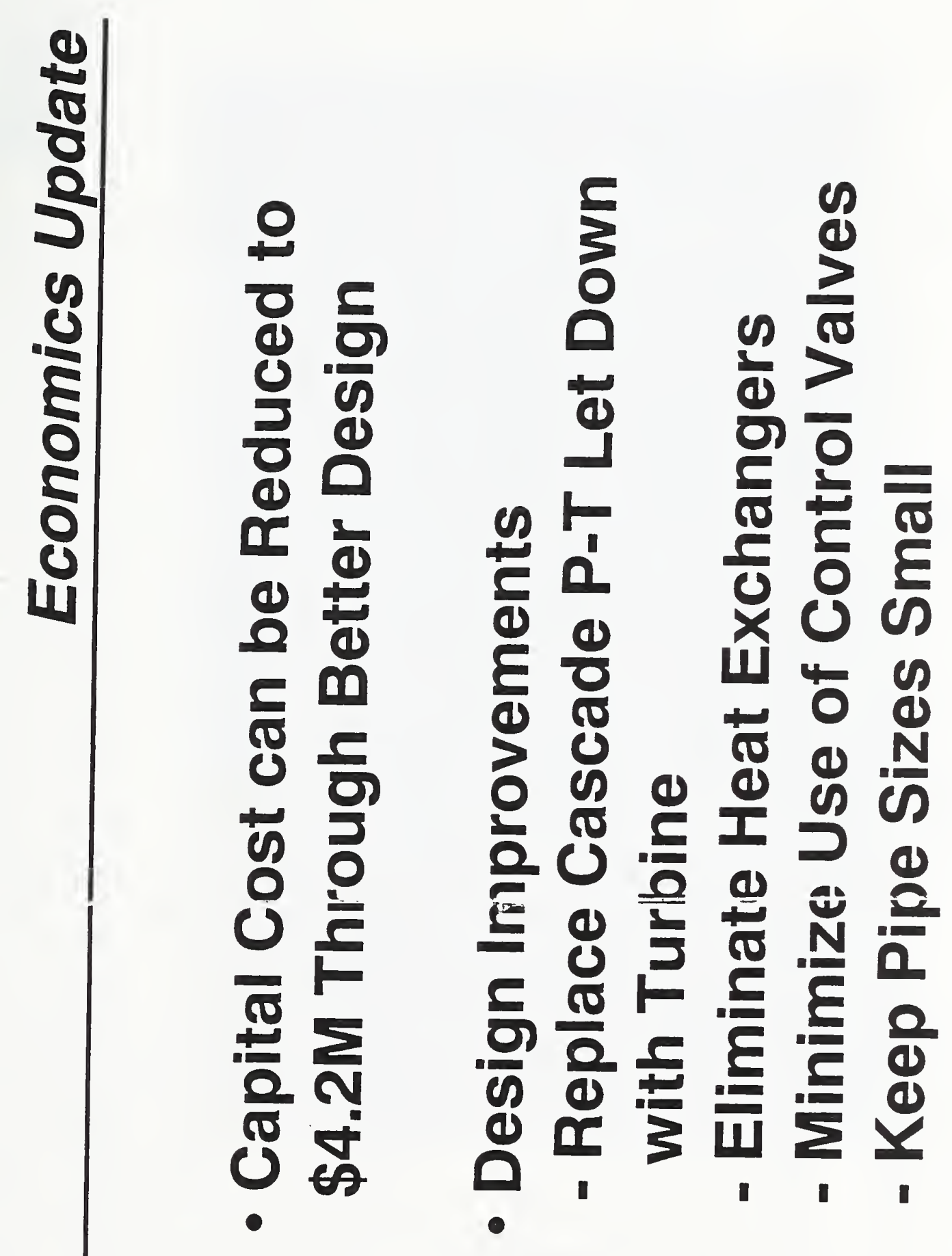

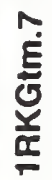




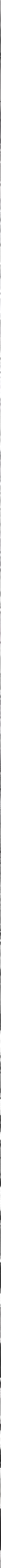




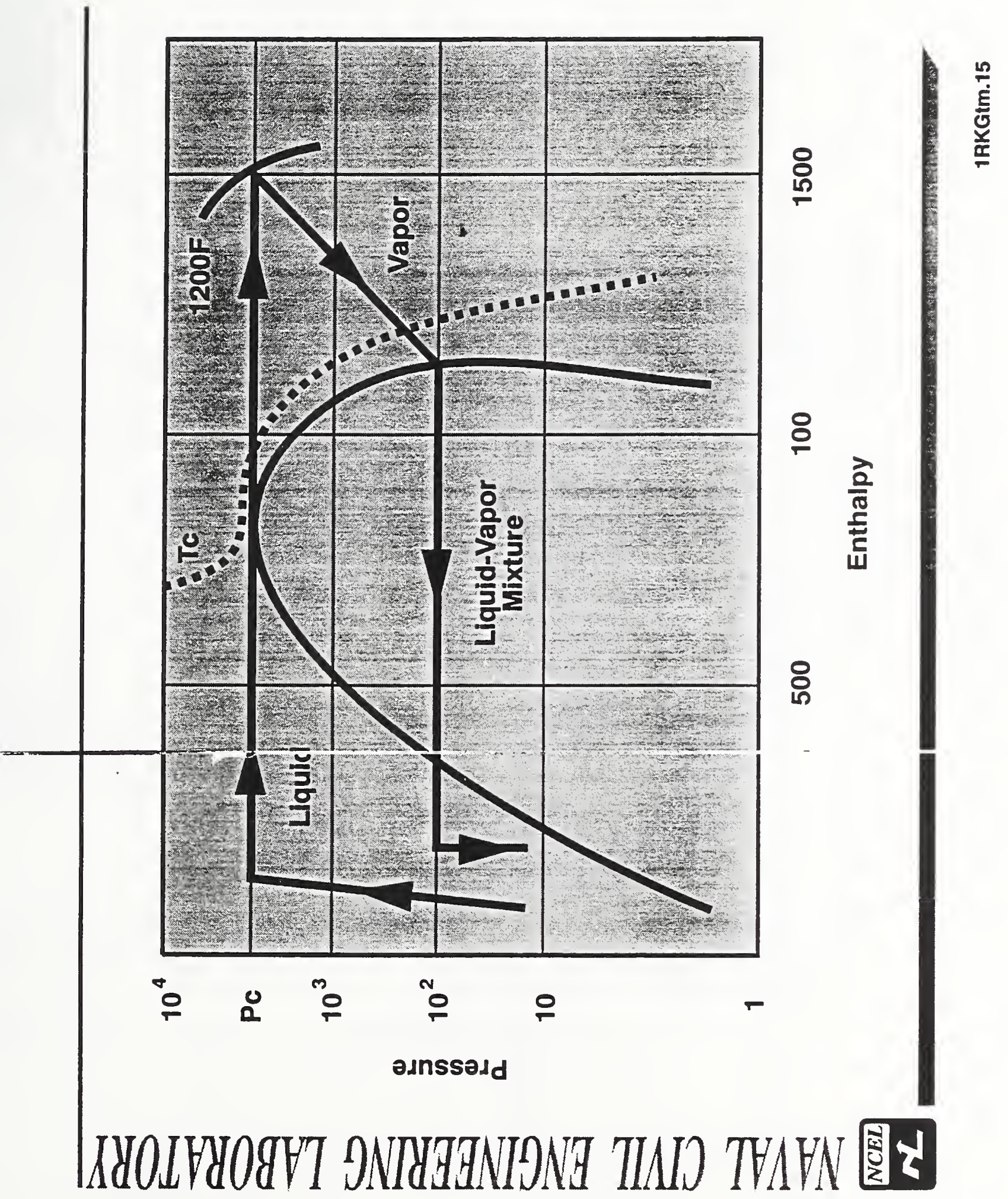




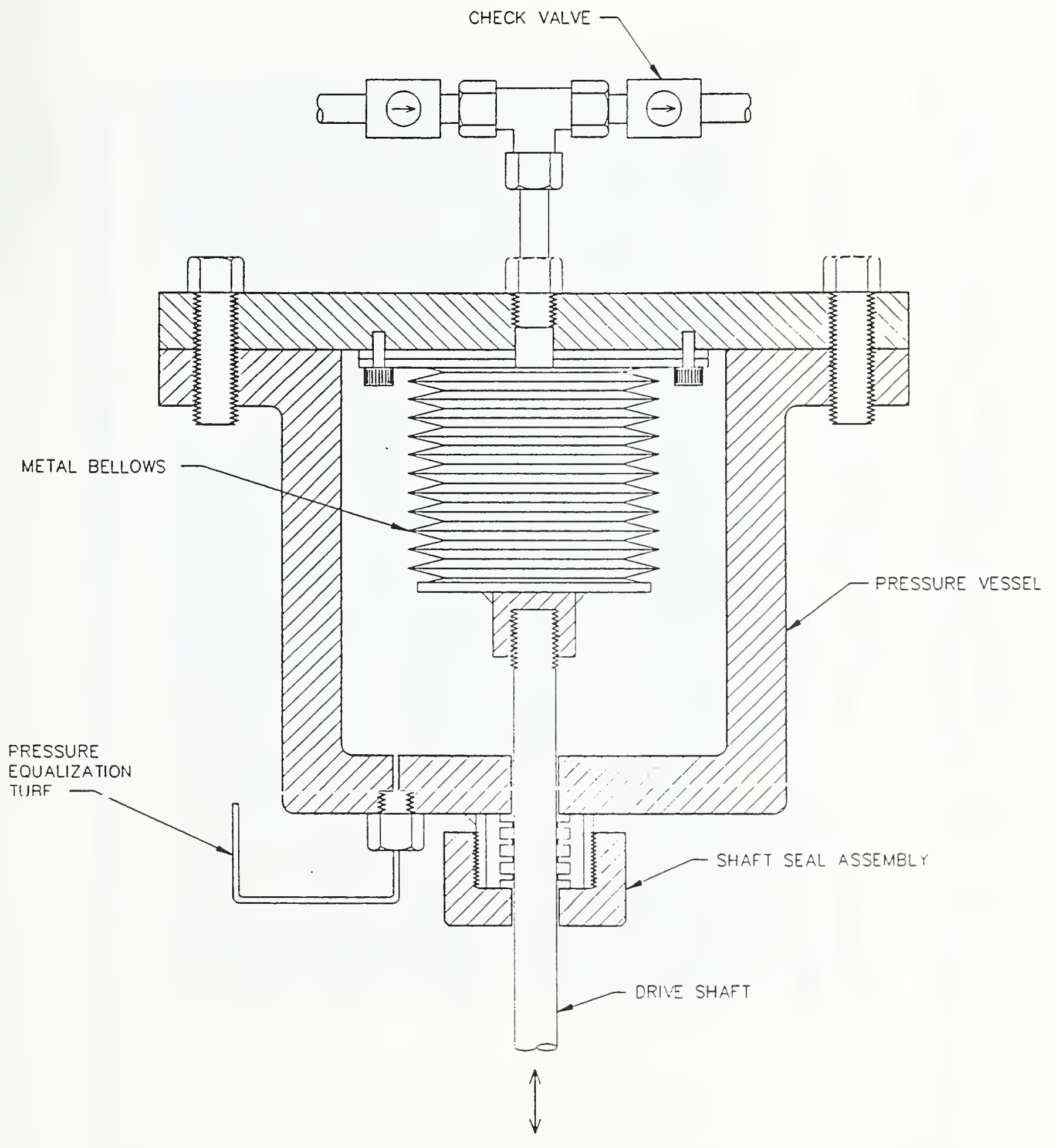

HOT GAS RECIRCULATION COMPRESSOR 


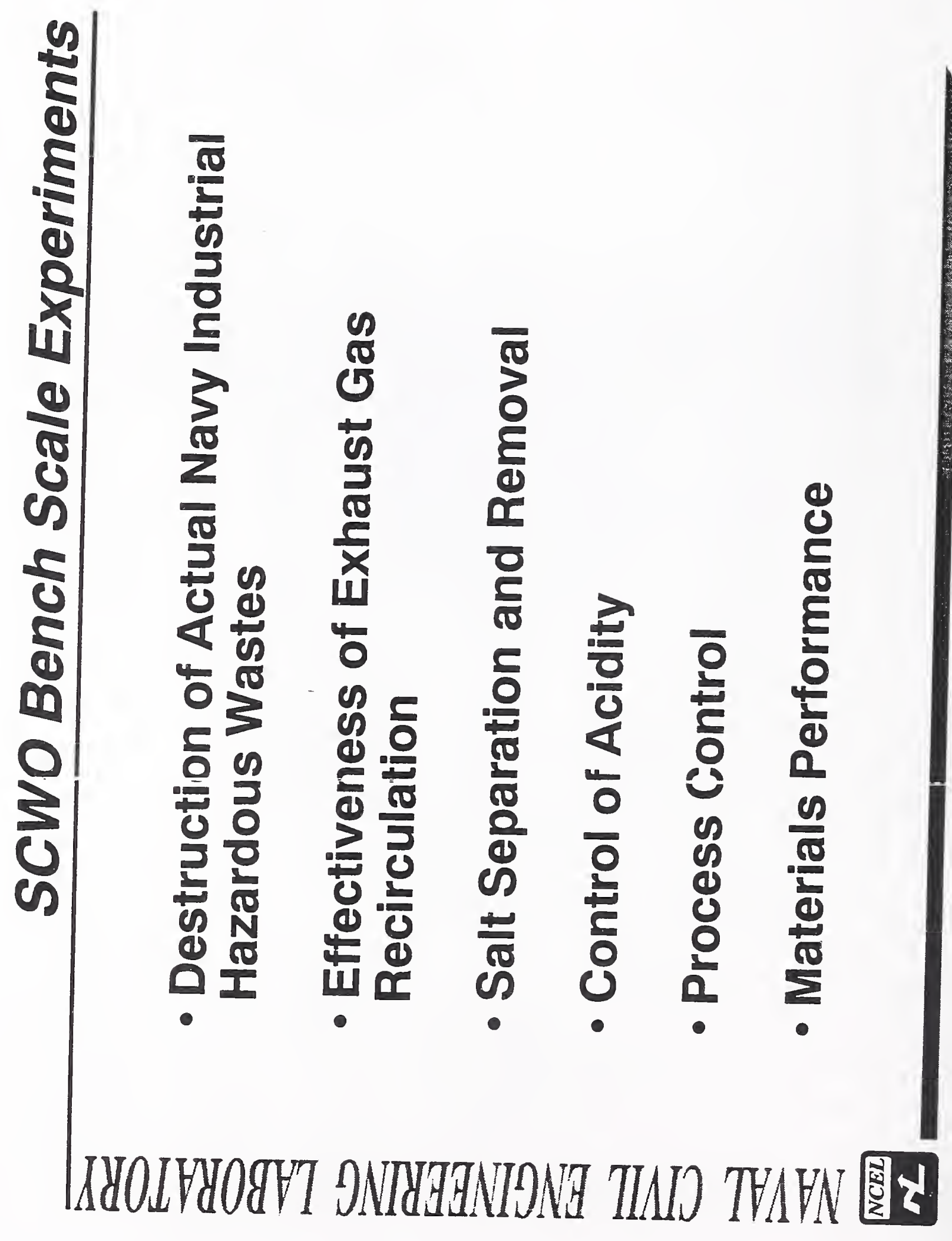




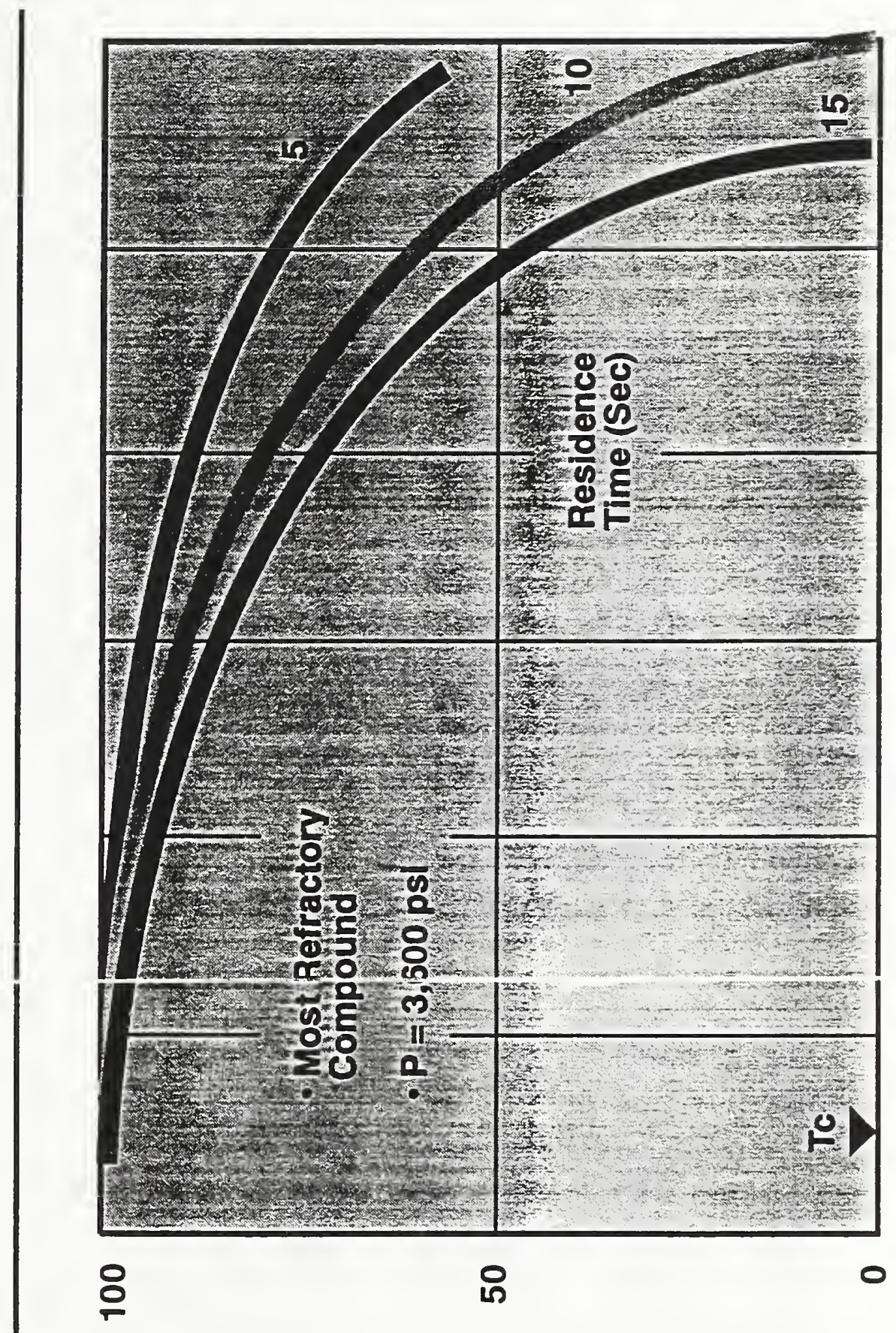

옹 $\frac{0}{\frac{5}{5}}$

유 0

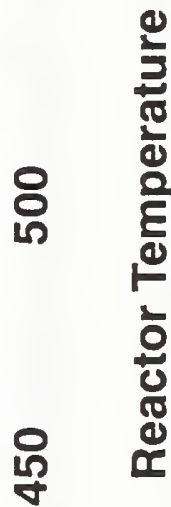

8

กิ

6u!̣ı̣eməy punodwos to uo!̣oedy

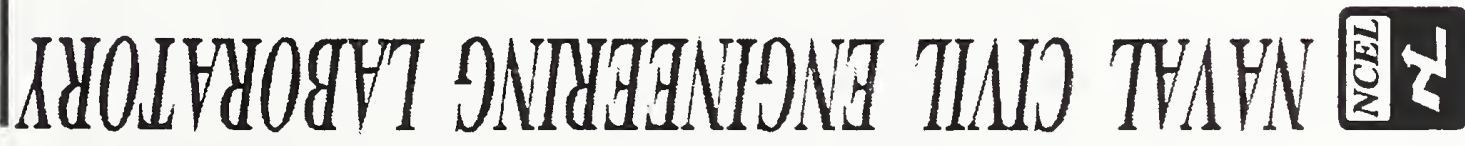




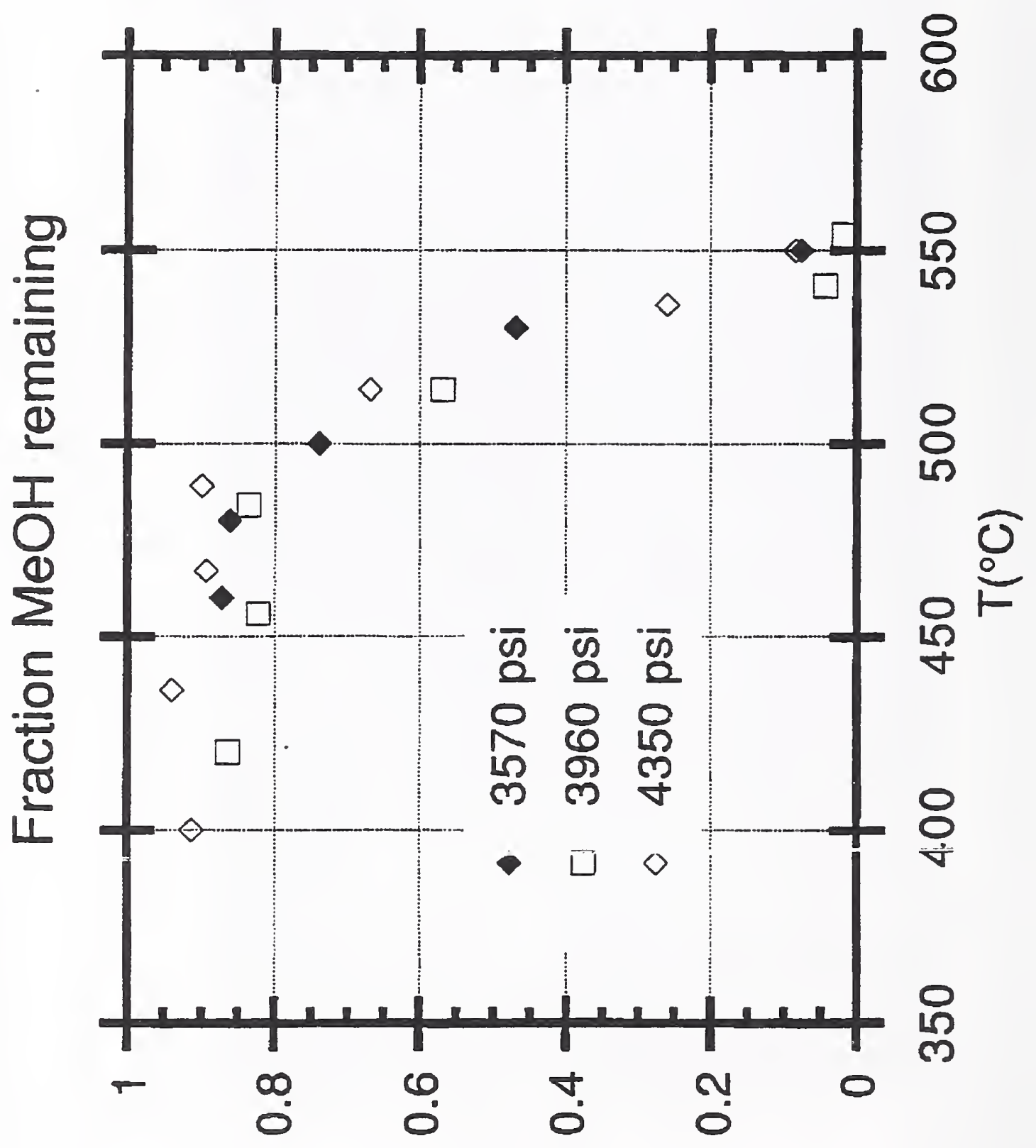




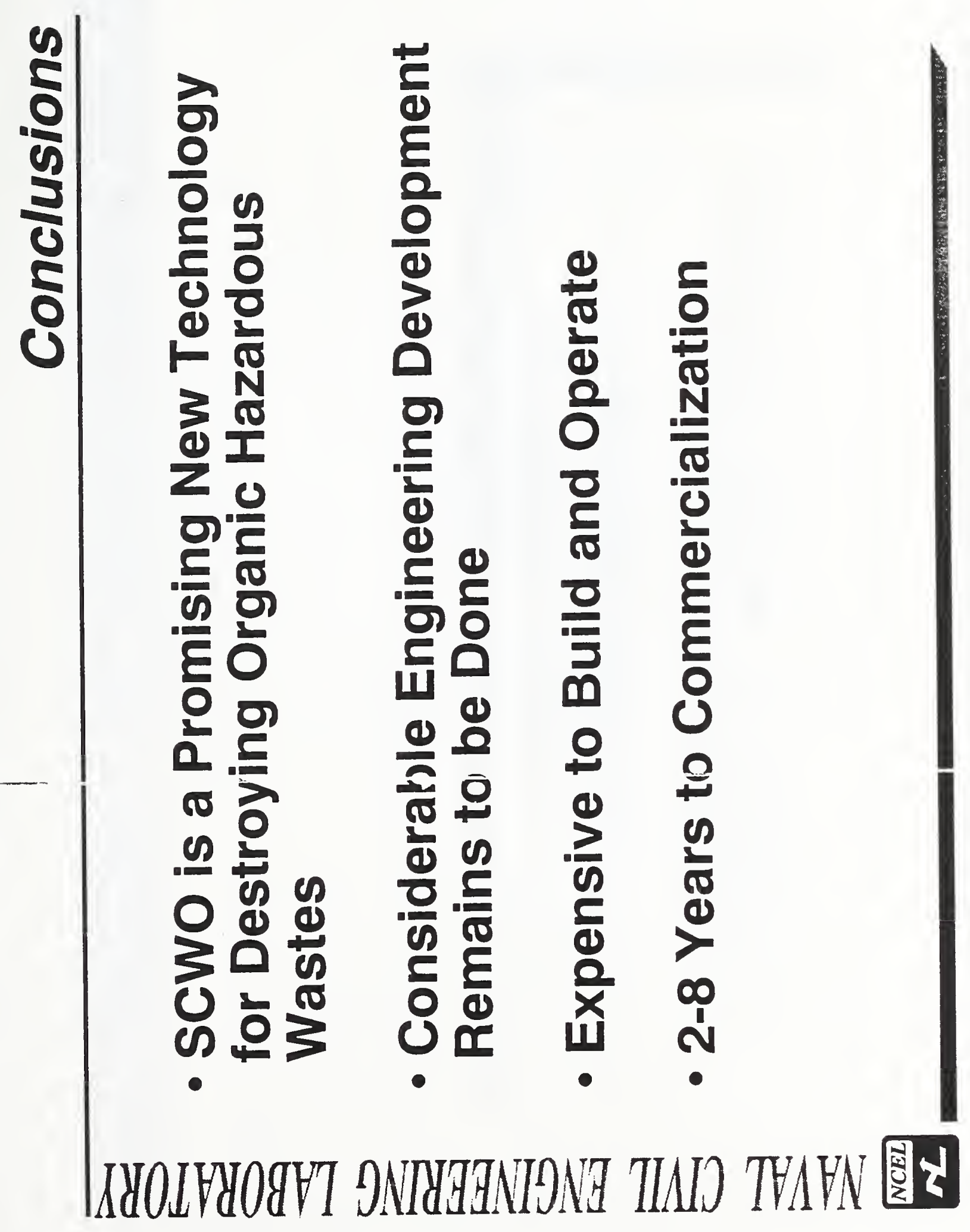




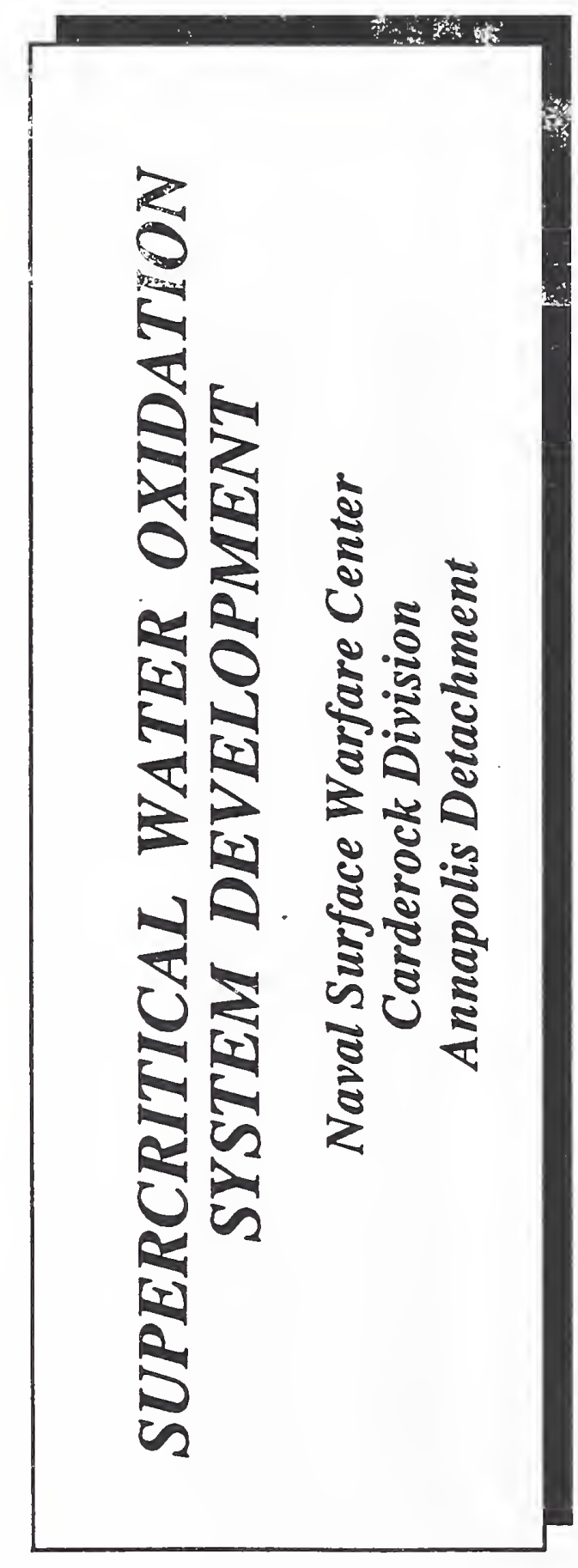




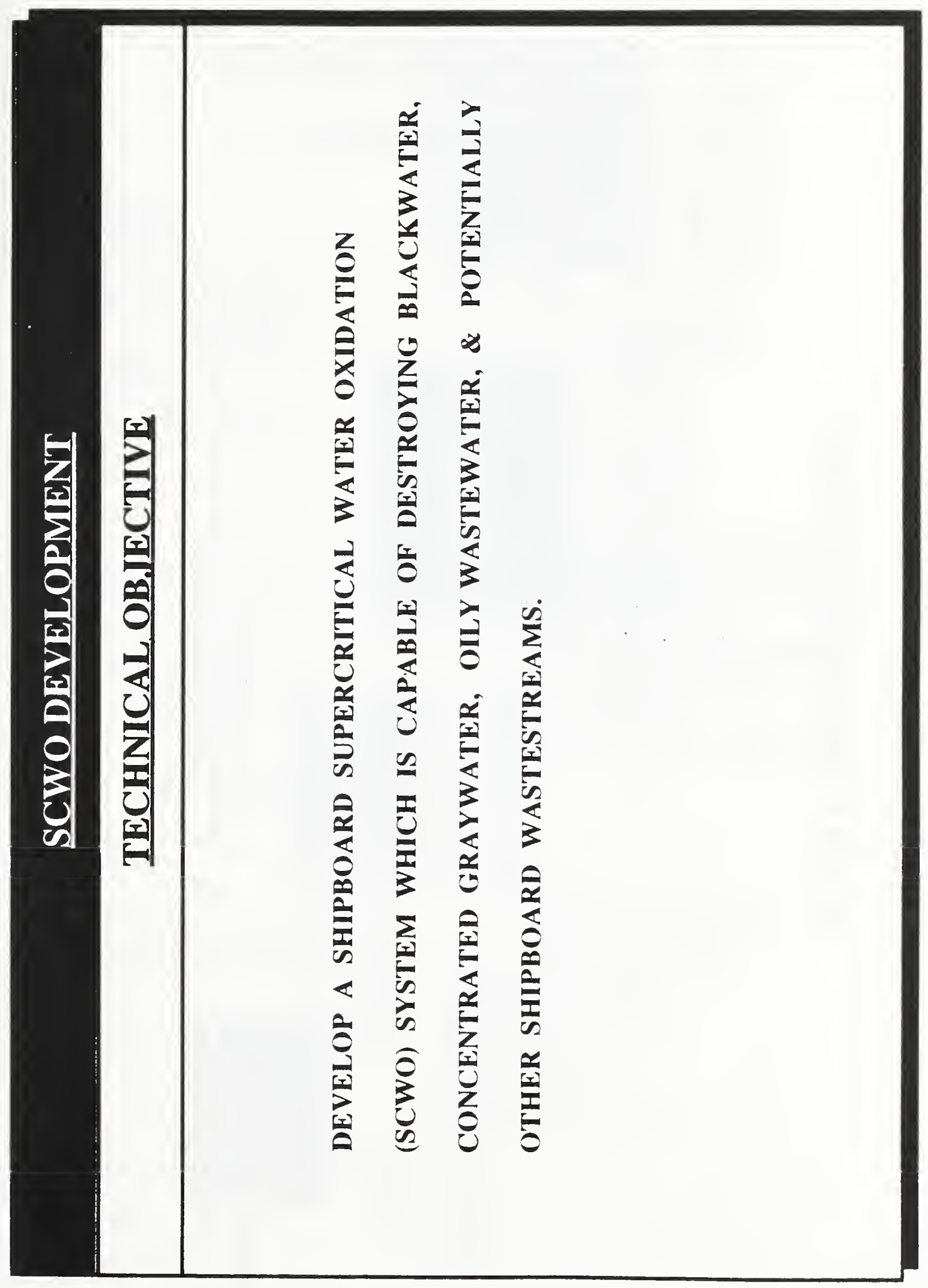




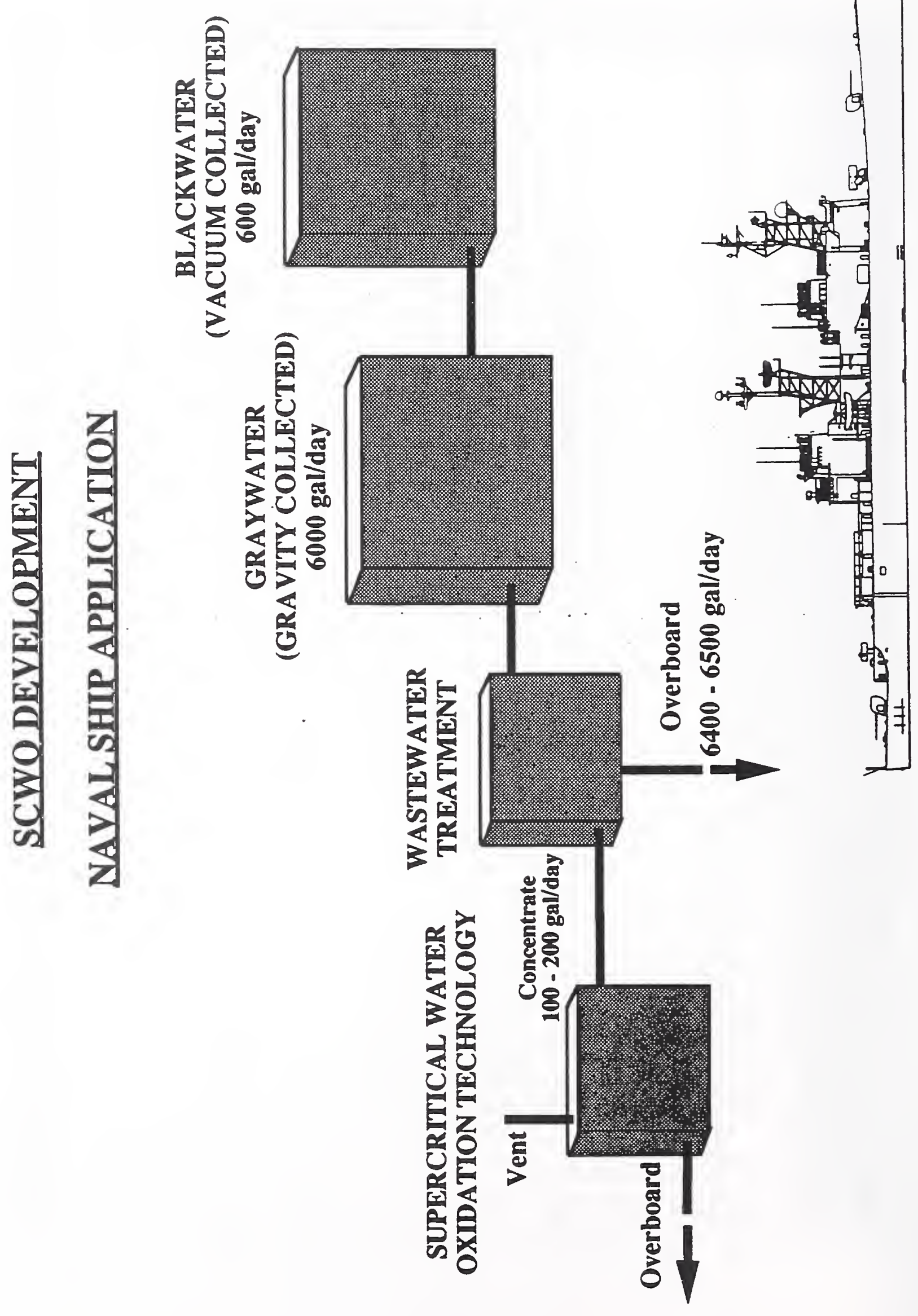




\section{SCWO DEVELOPMENT}

\section{APPROACH}

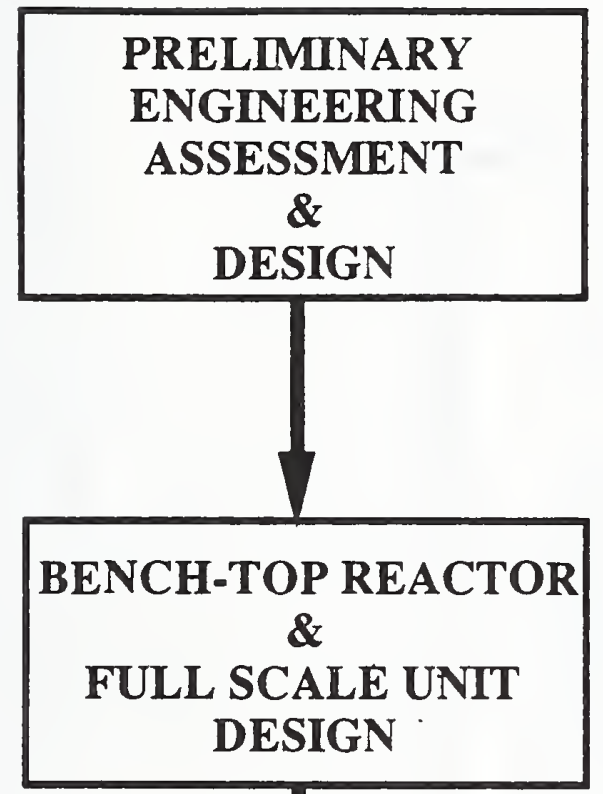

Decision Point

FULL SCALE UNIT DEVELOPMENT
Feasibility Study

BW Treatability Study

PFR Kinetic Study
Wastewater Kinetic Model Pretreatment \& Concentration Heat Transfer Model Preliminary Hazards Analysis Materials Demonstrations Scale-Up Factors Component Testing Platform
EDM

High Pressure Pump

High Pressure Oxidant

Reactor Materials

Safety Studies

SHIPEVAL

OPEVAL

TECHEVAL

Software Support 


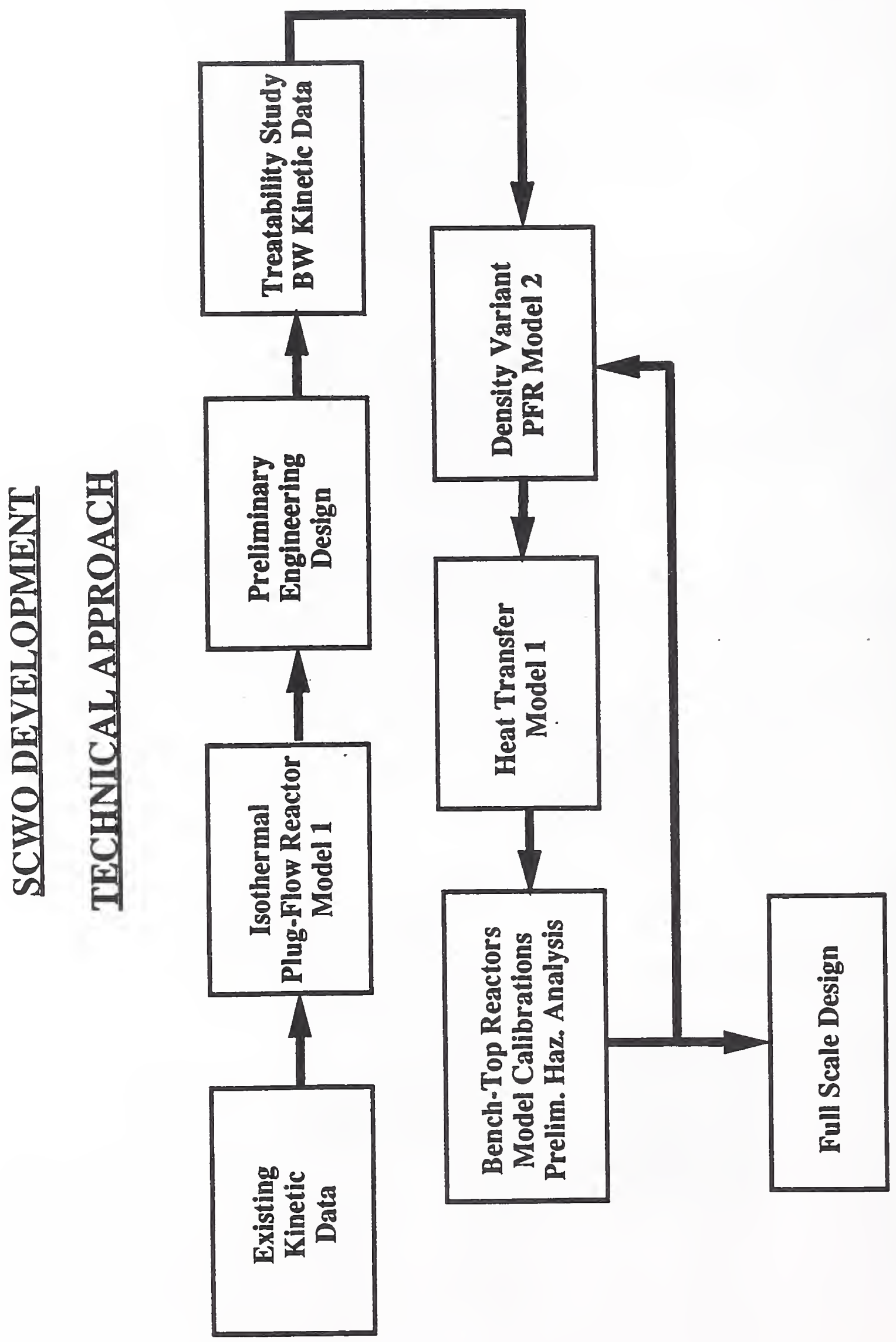




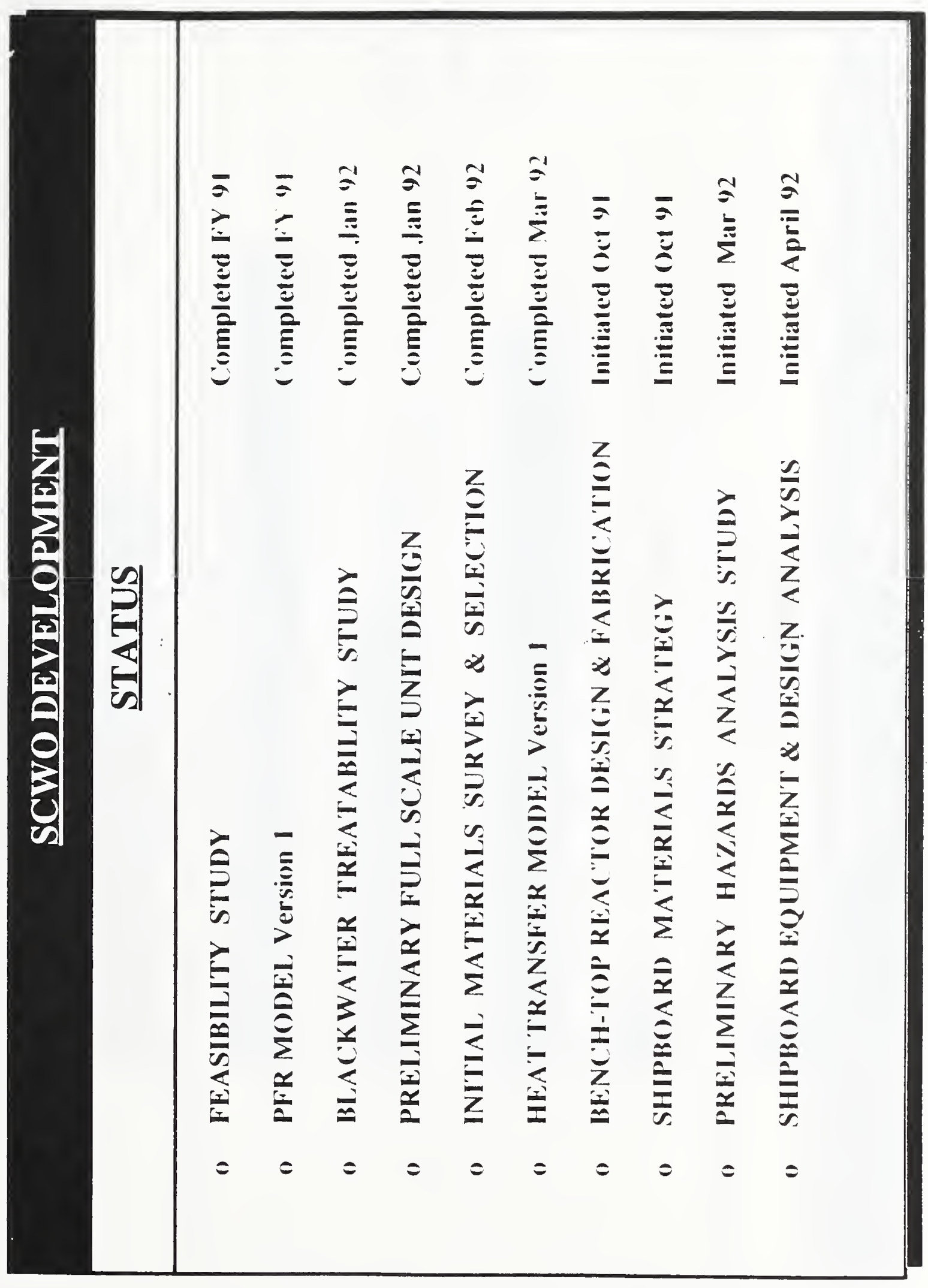




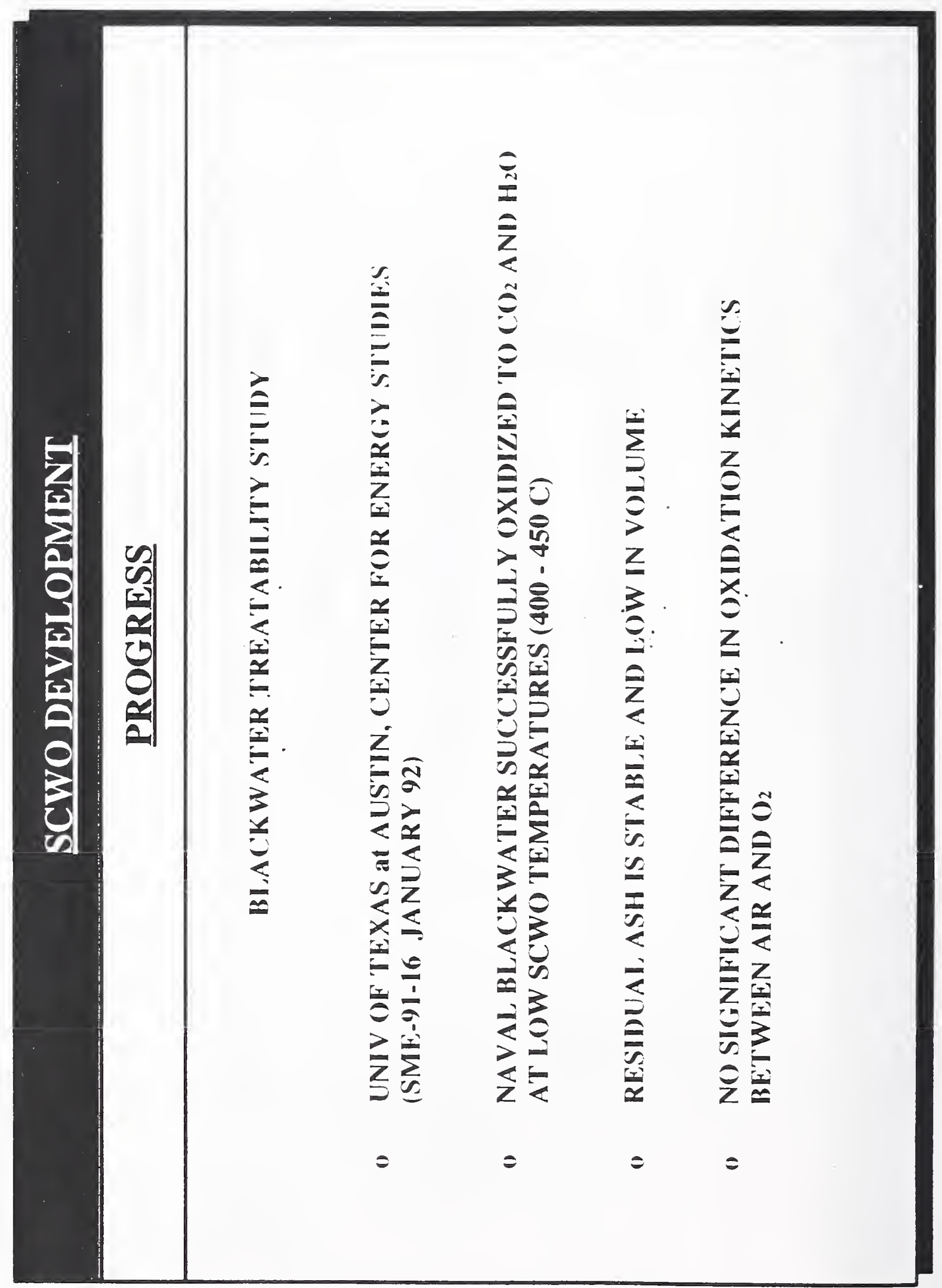




$$
\begin{array}{lll}
0 & 0 & 0 \\
8 & 0 & 8 \\
8 & 0 & 8 \\
m & 0 & 4 \\
& \square &
\end{array}
$$

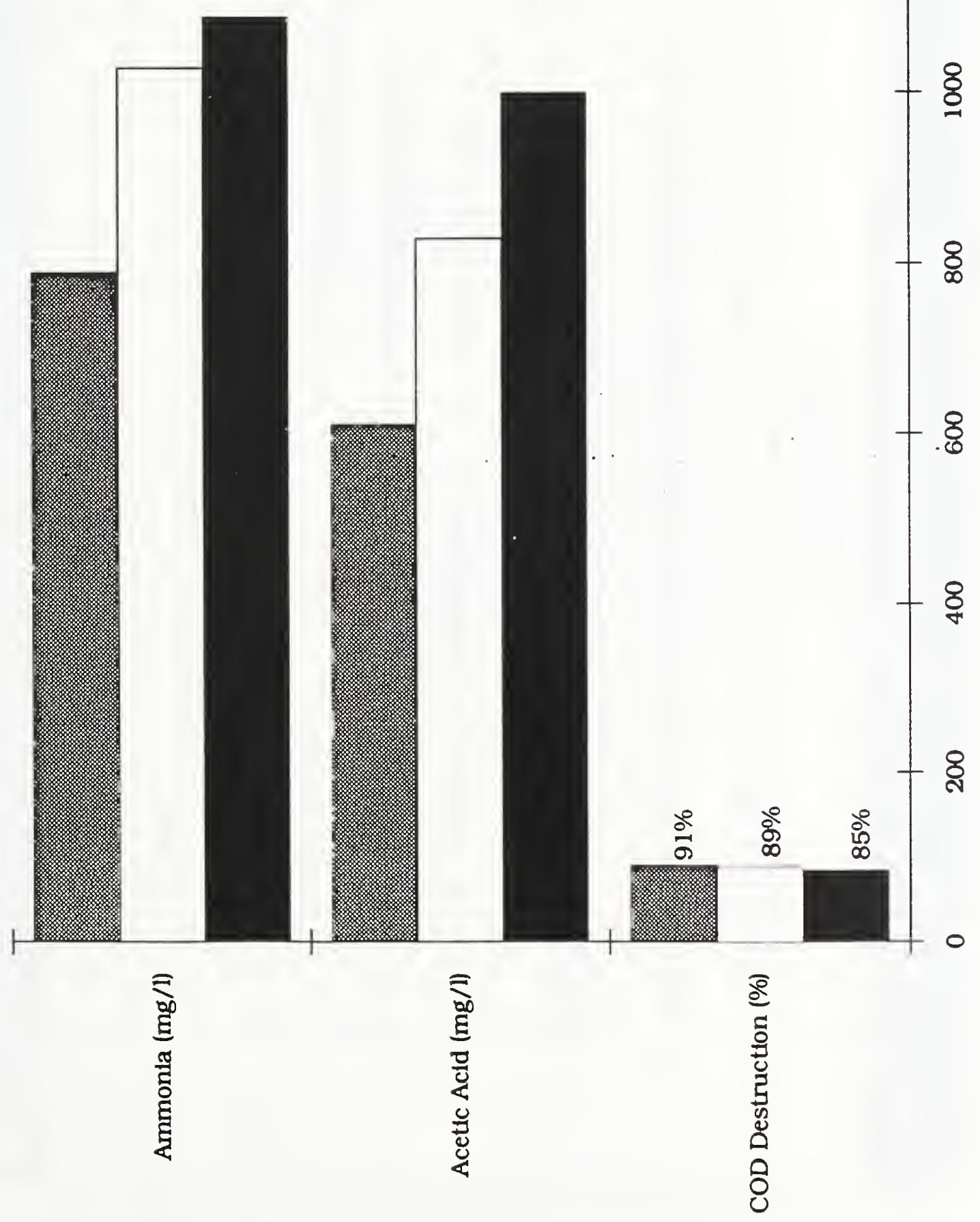




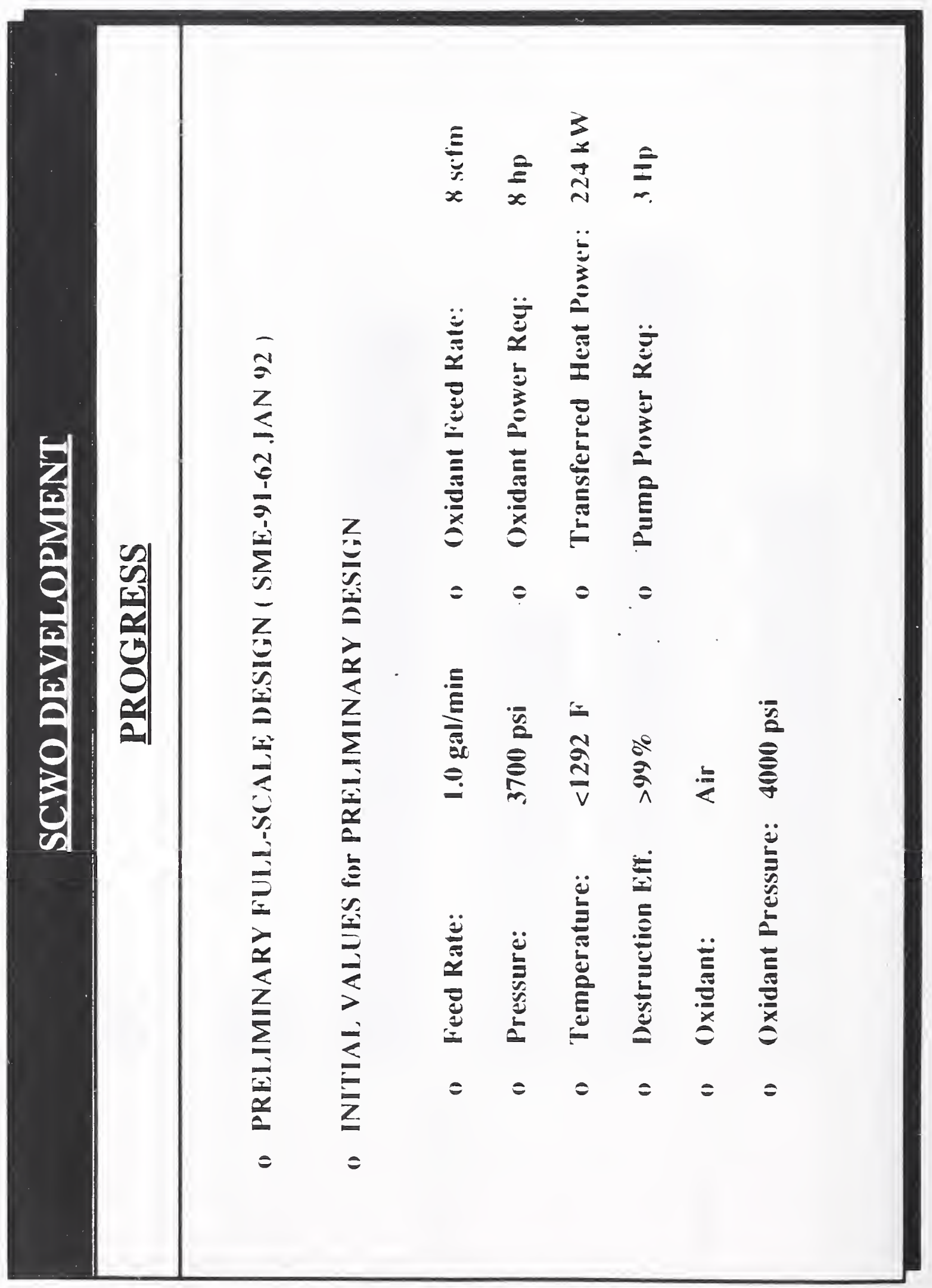




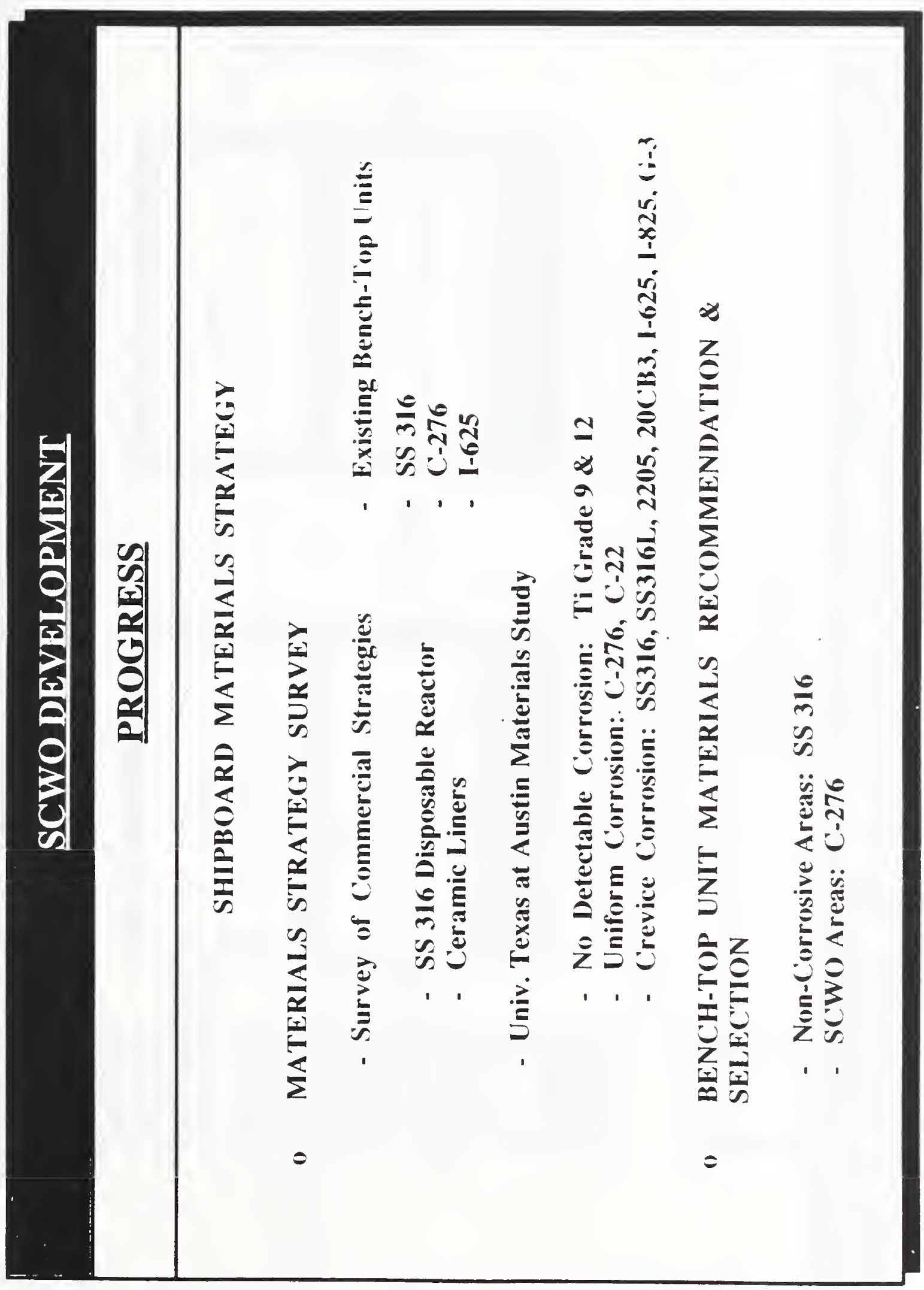




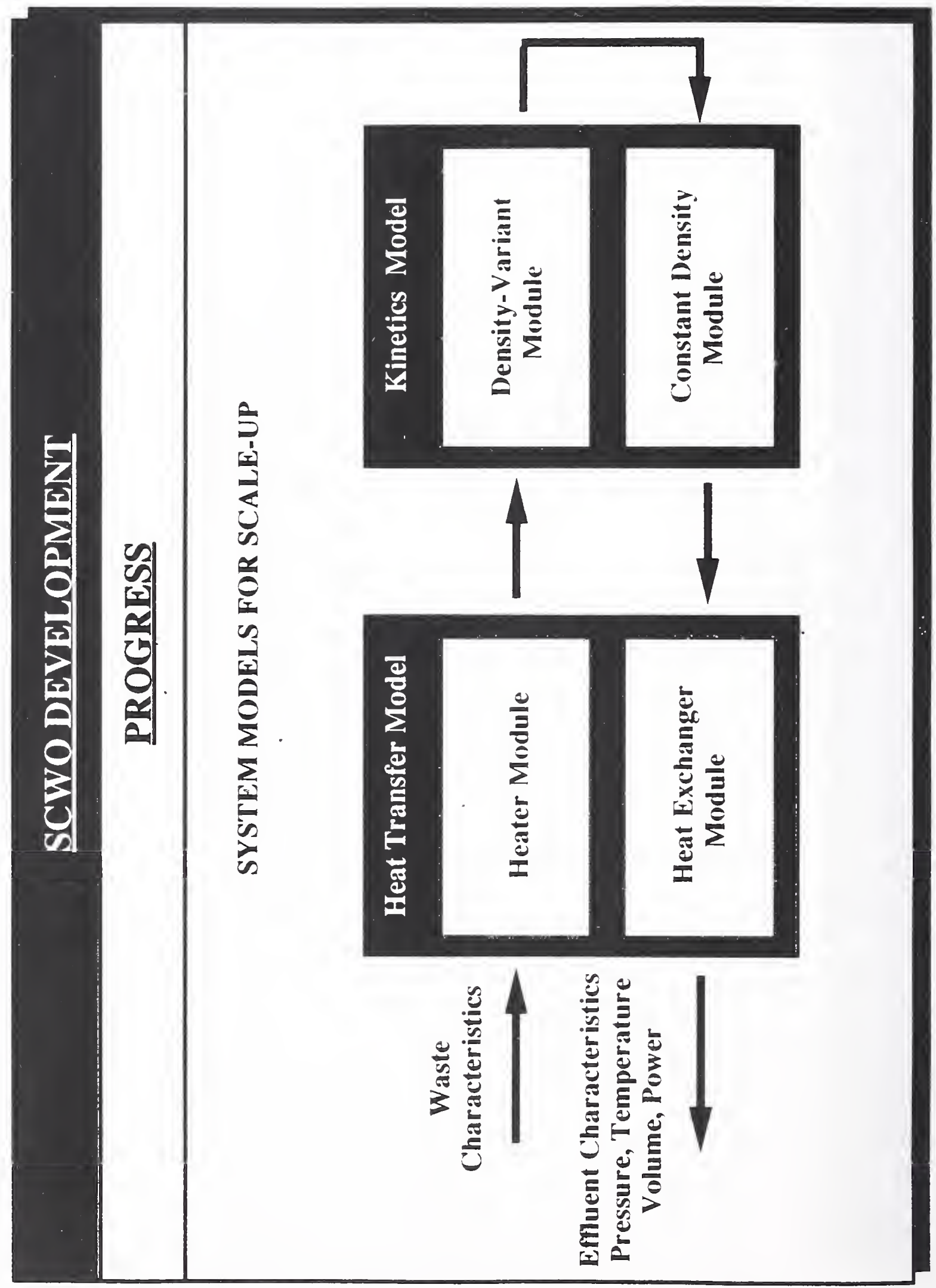




\section{PLUG-ELOW REACTOR MODEL}
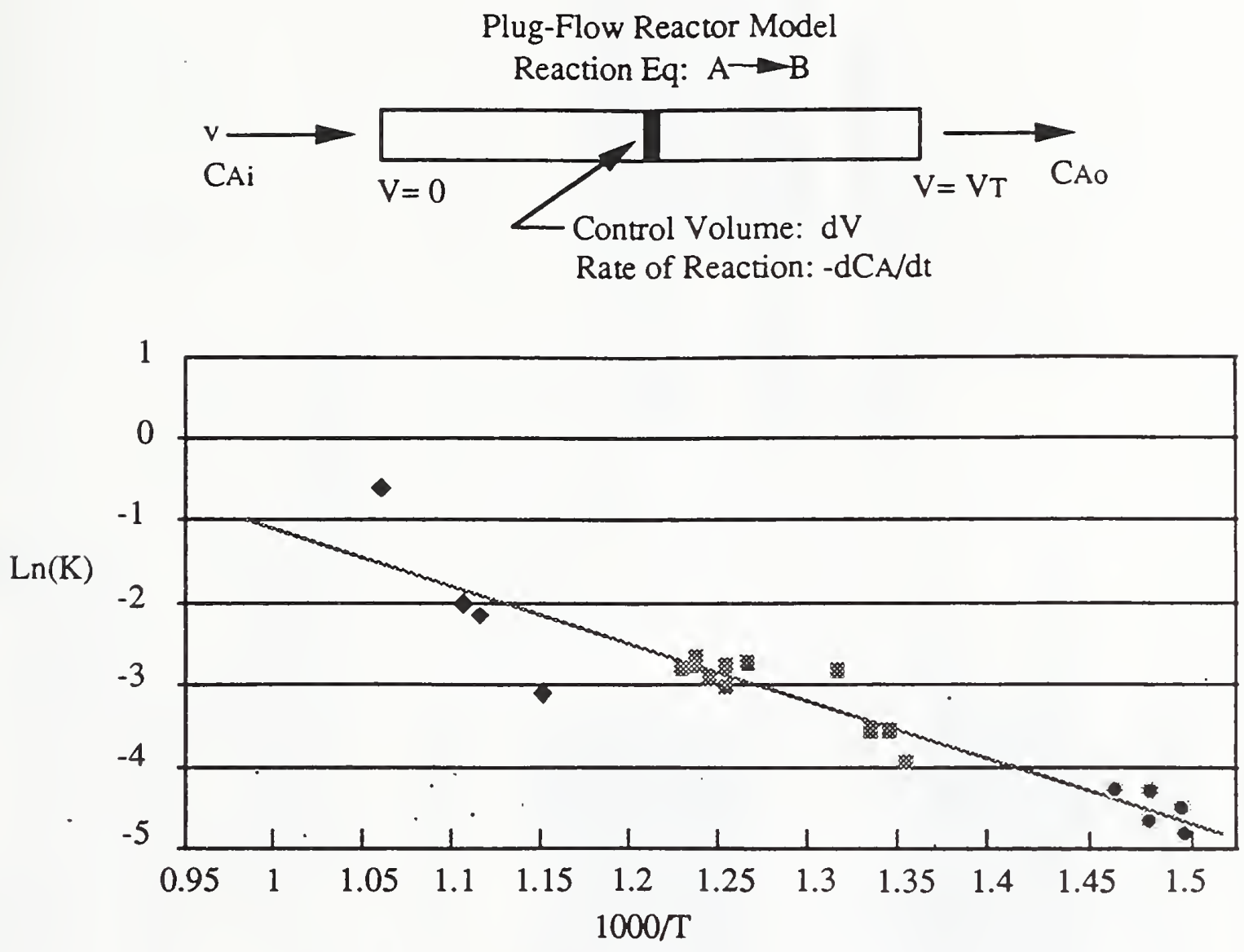

Modell Data Timblk. Data - DTRC Data

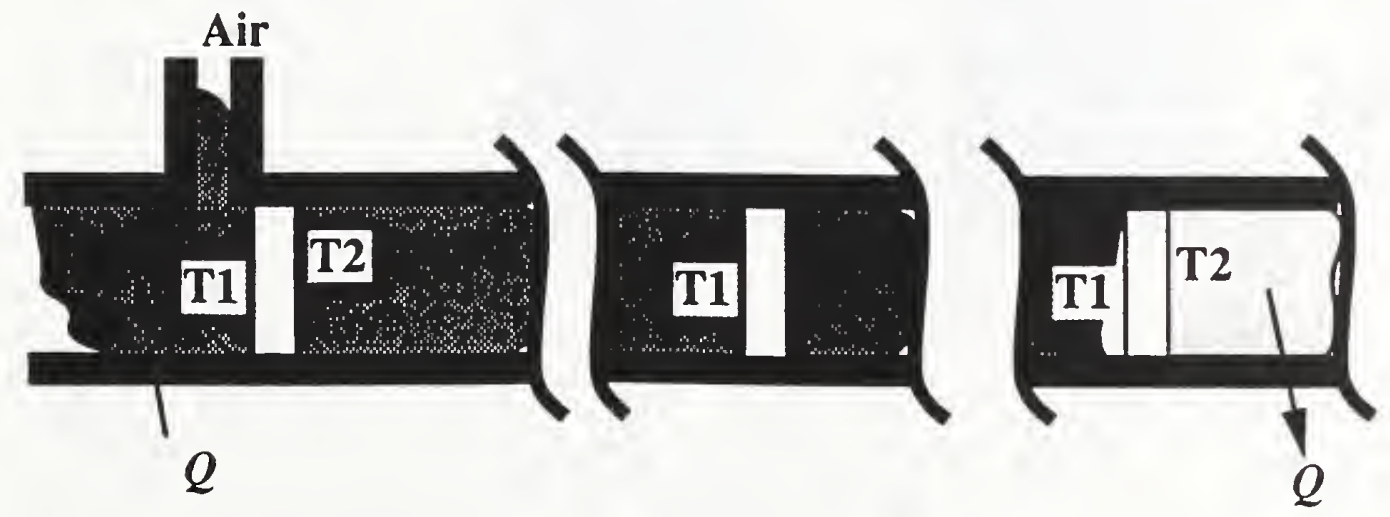




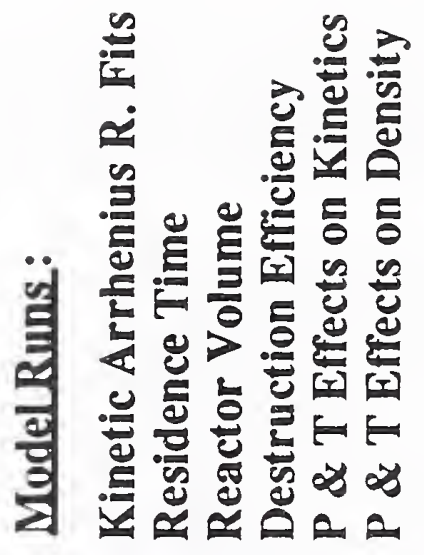

닐

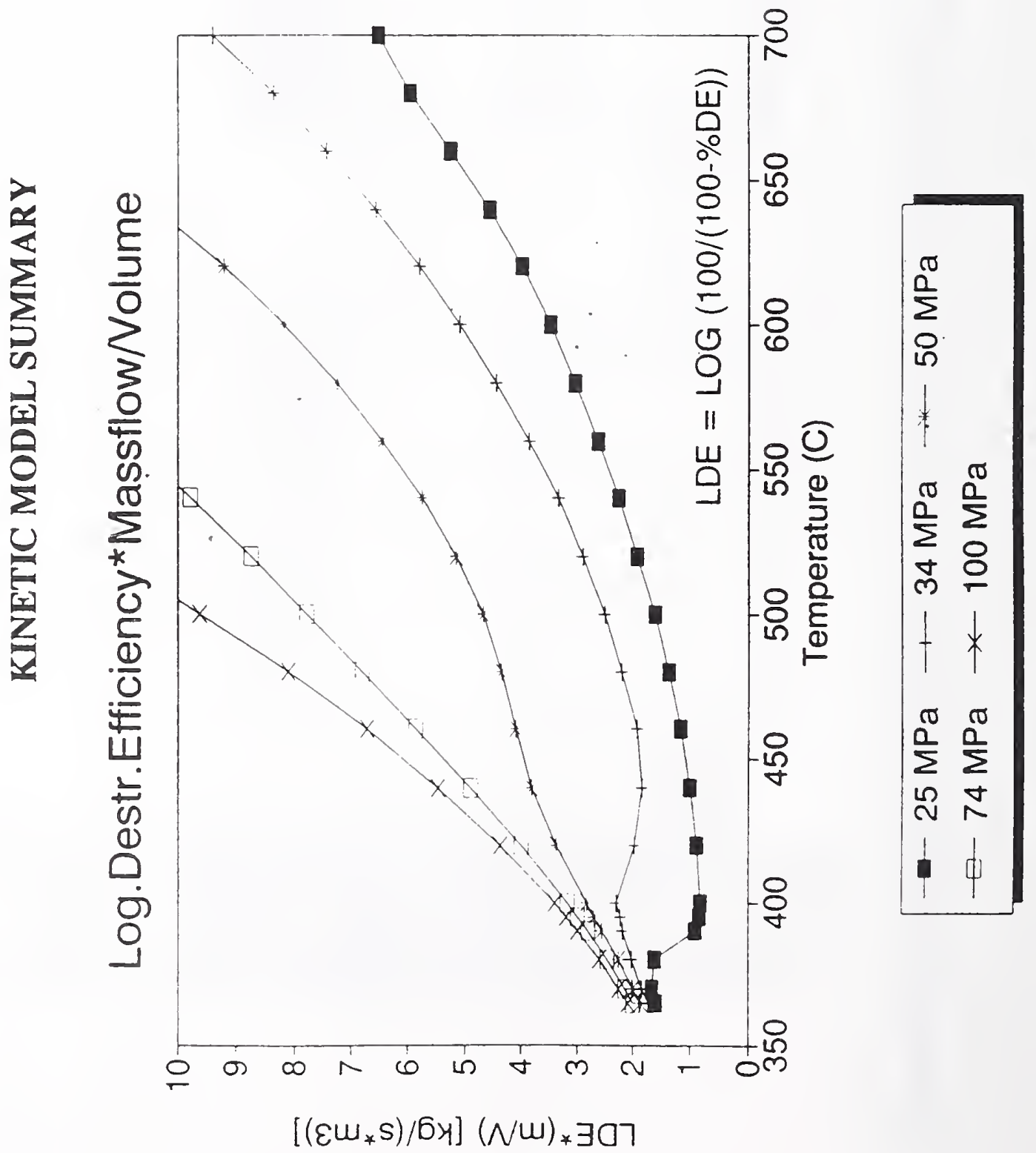




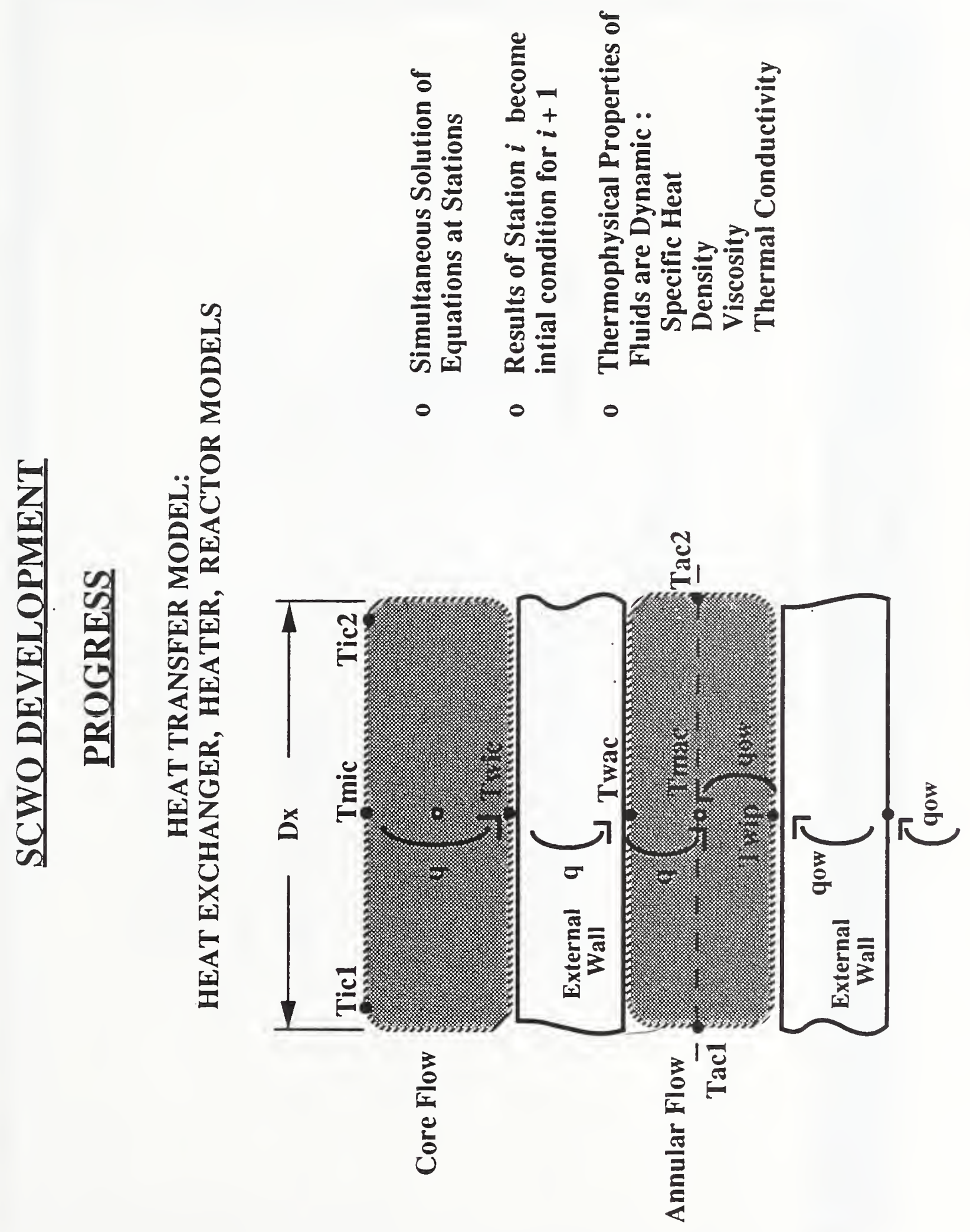




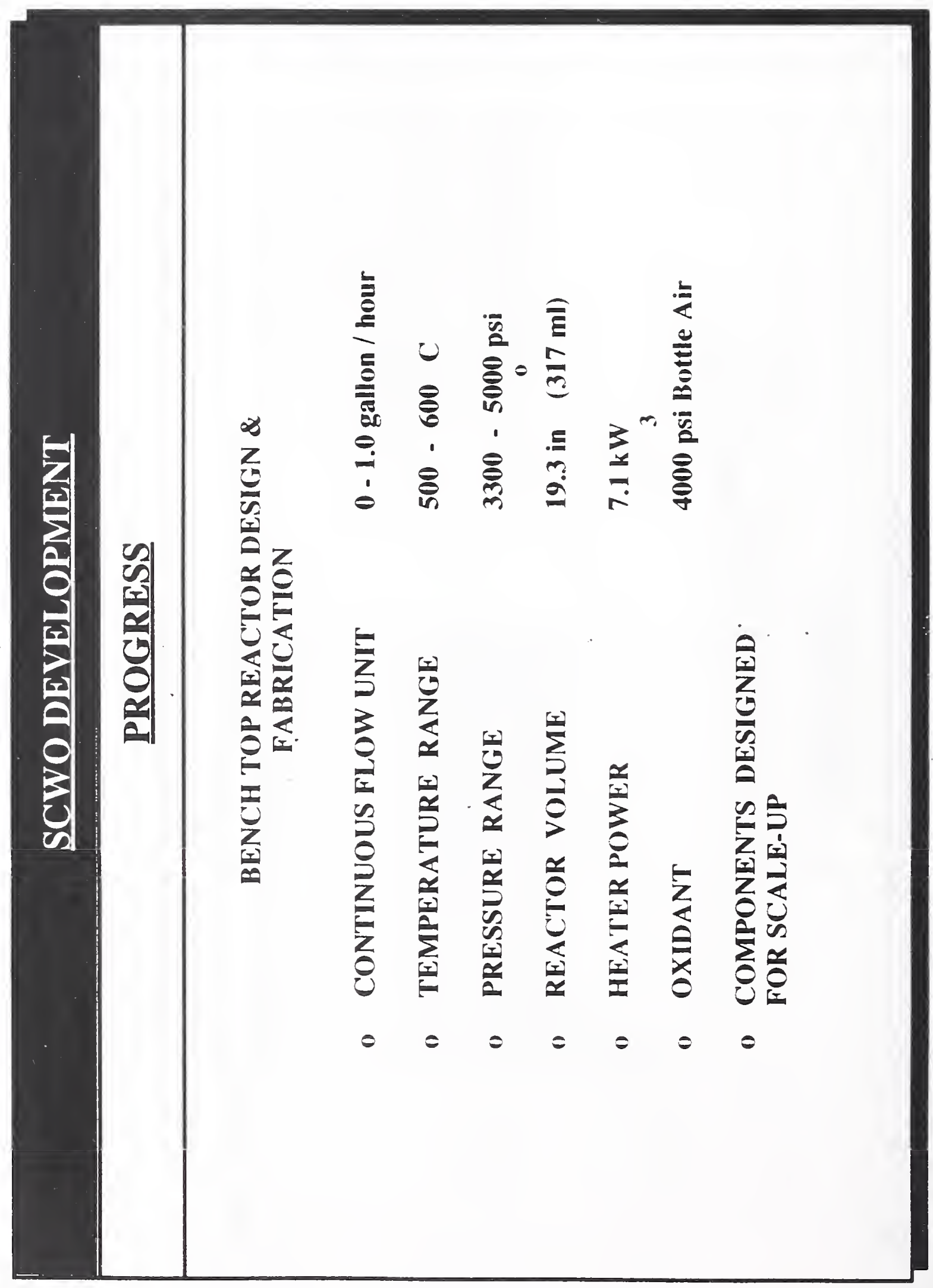




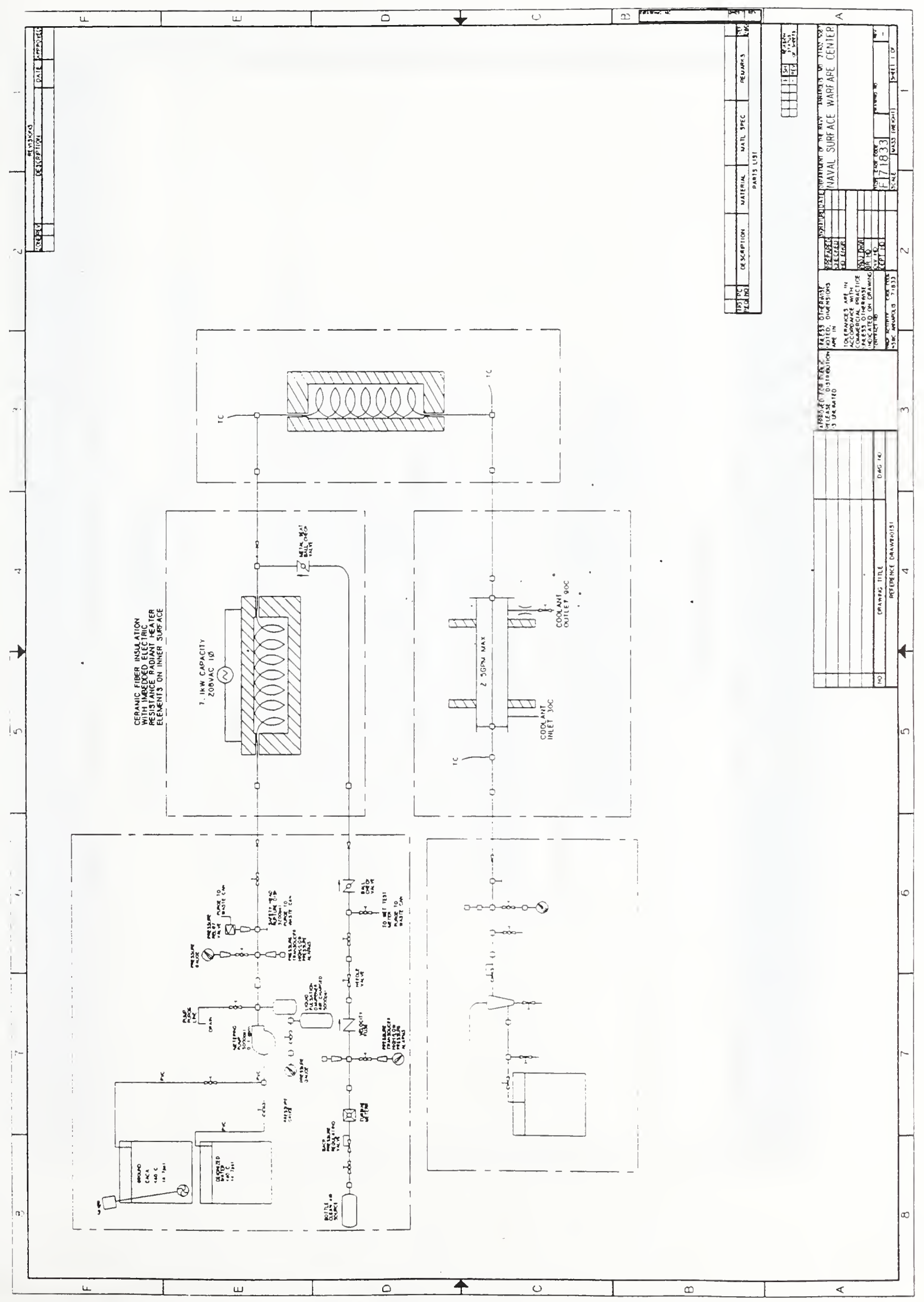




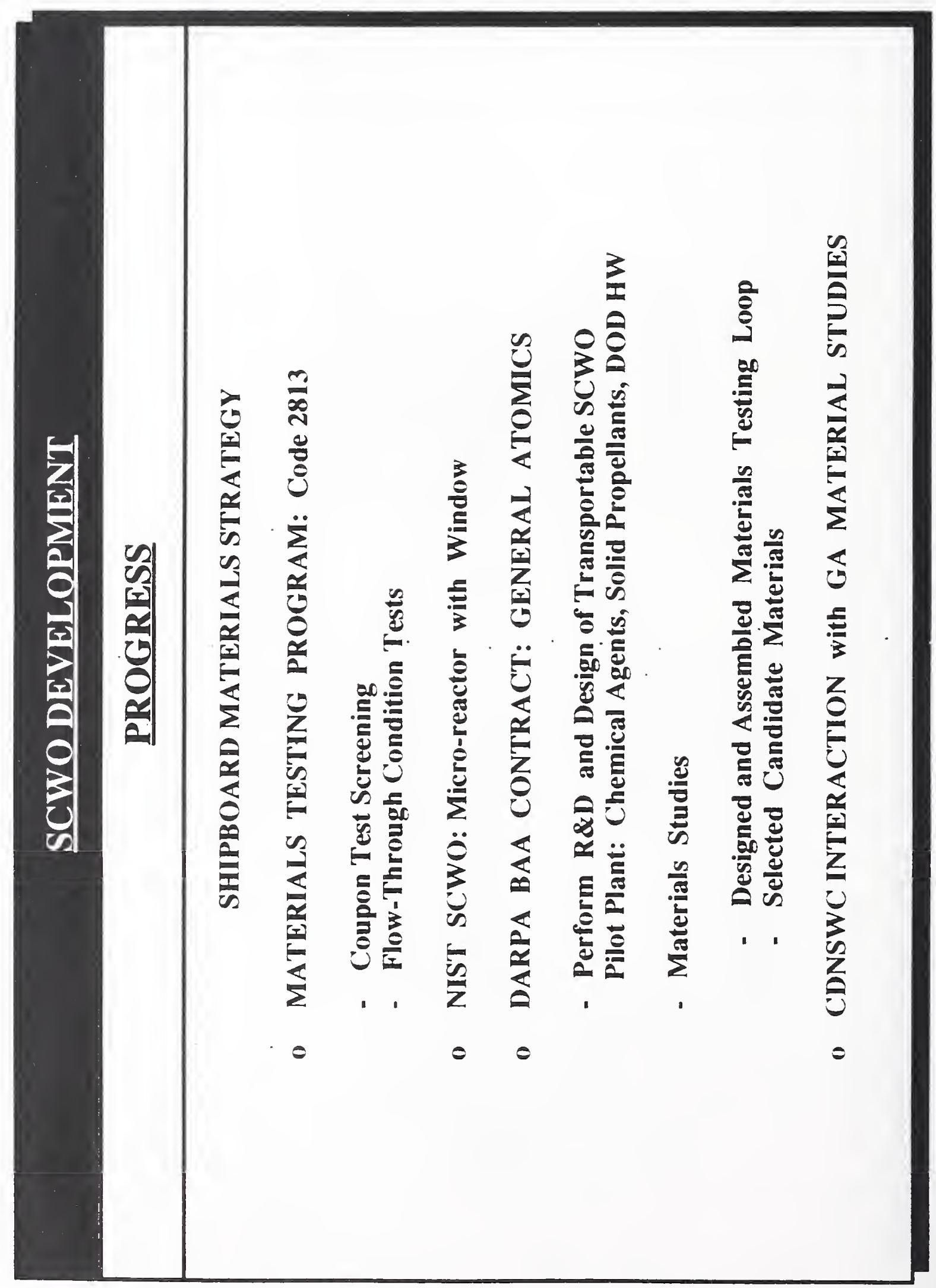




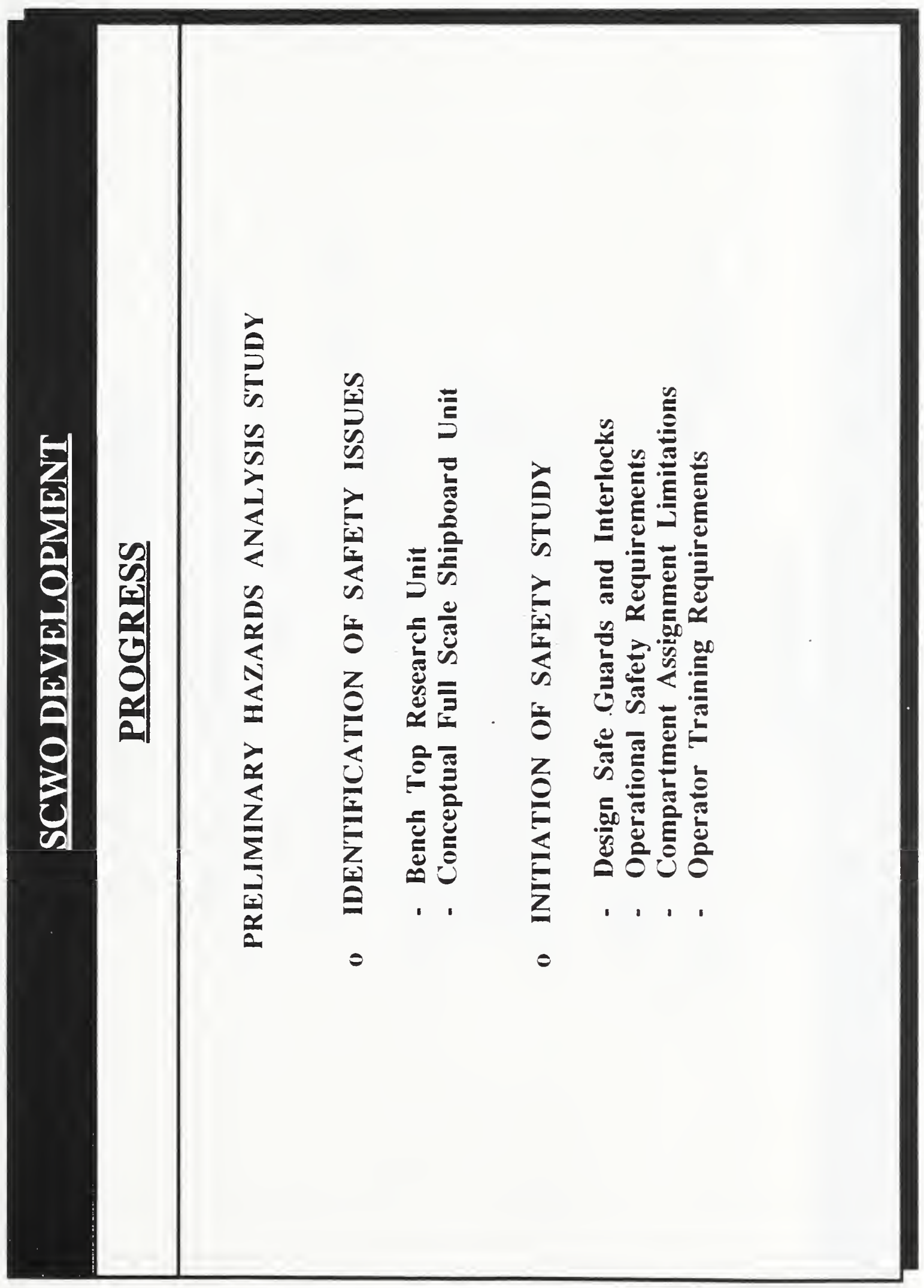




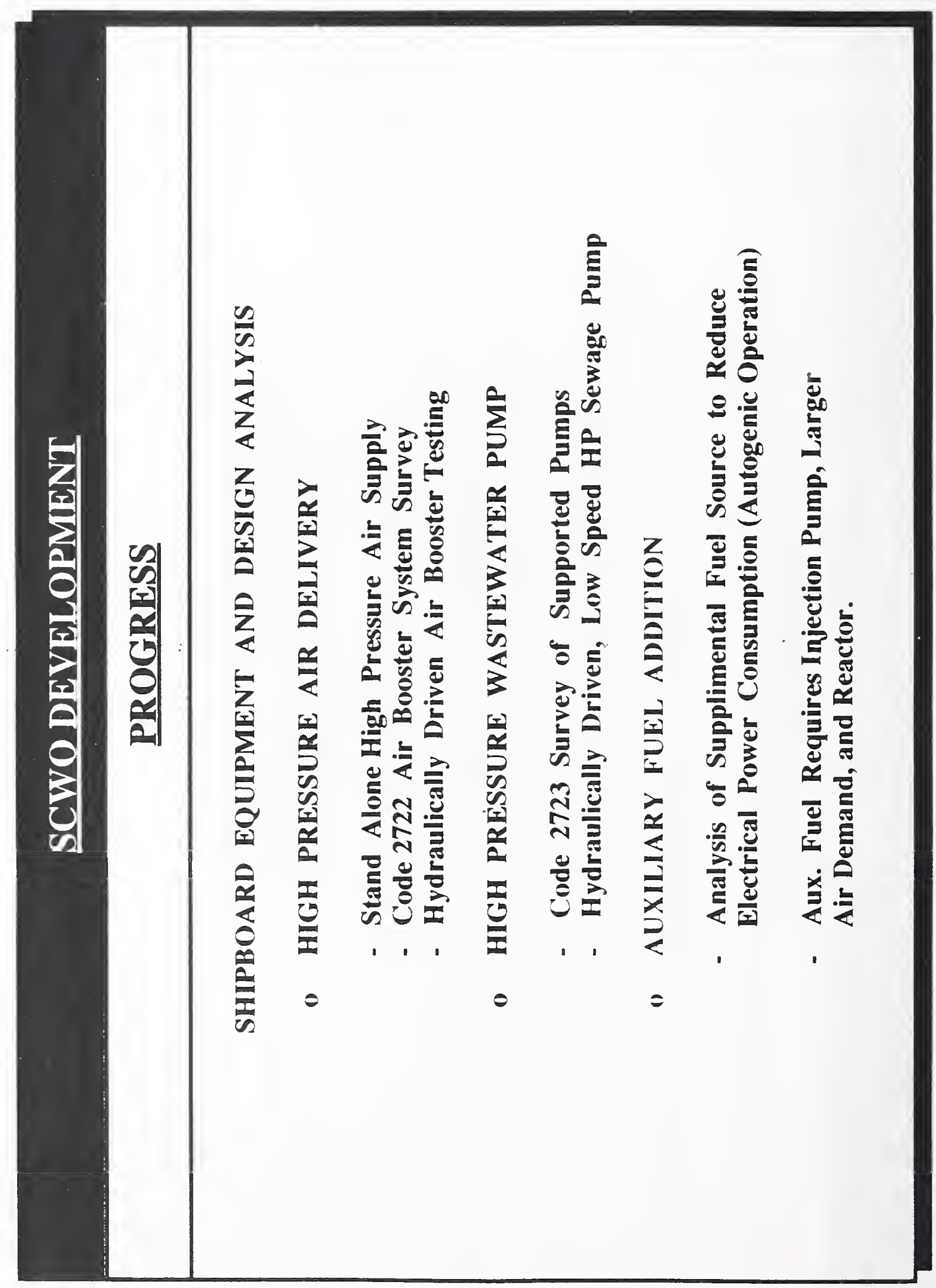




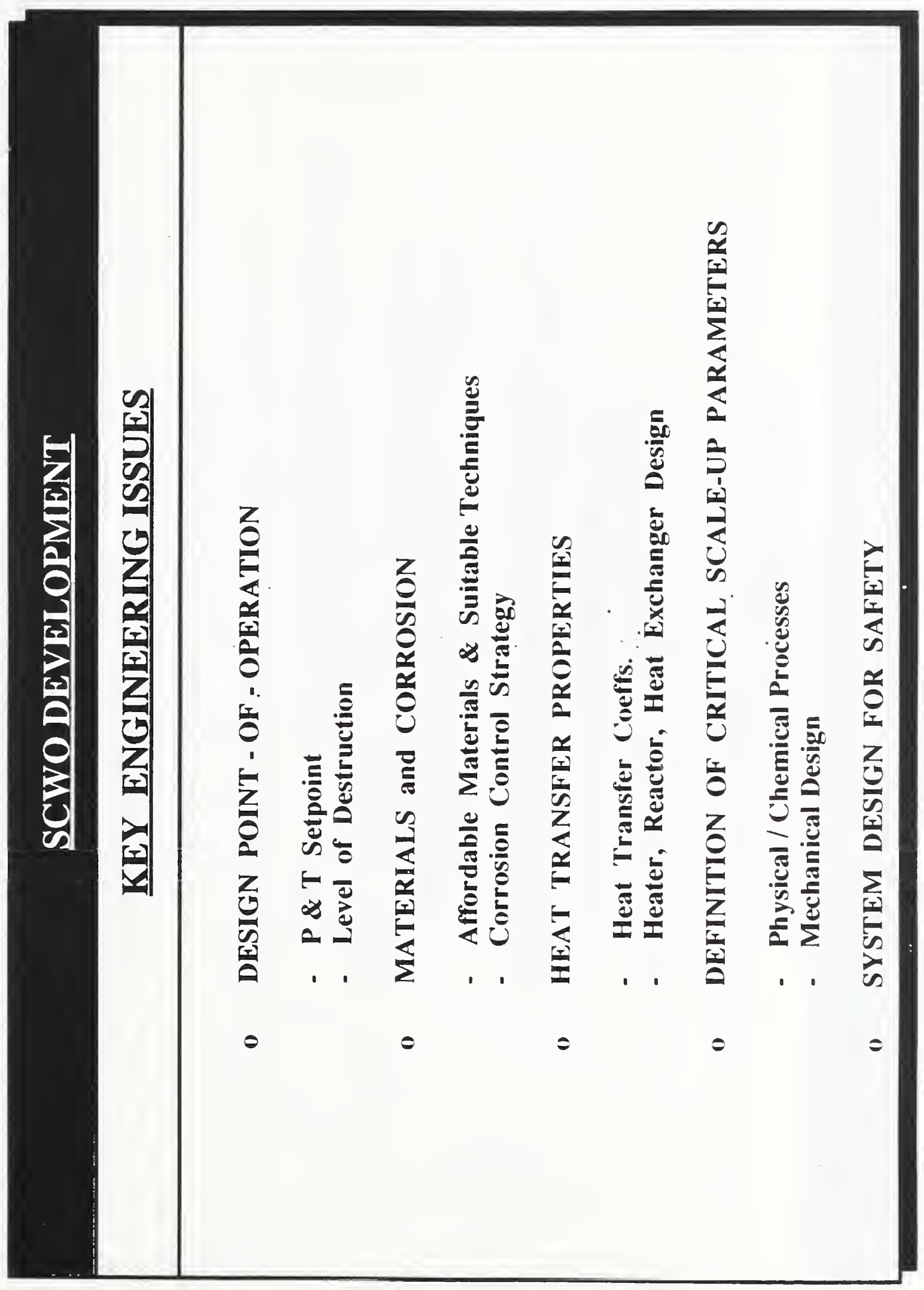




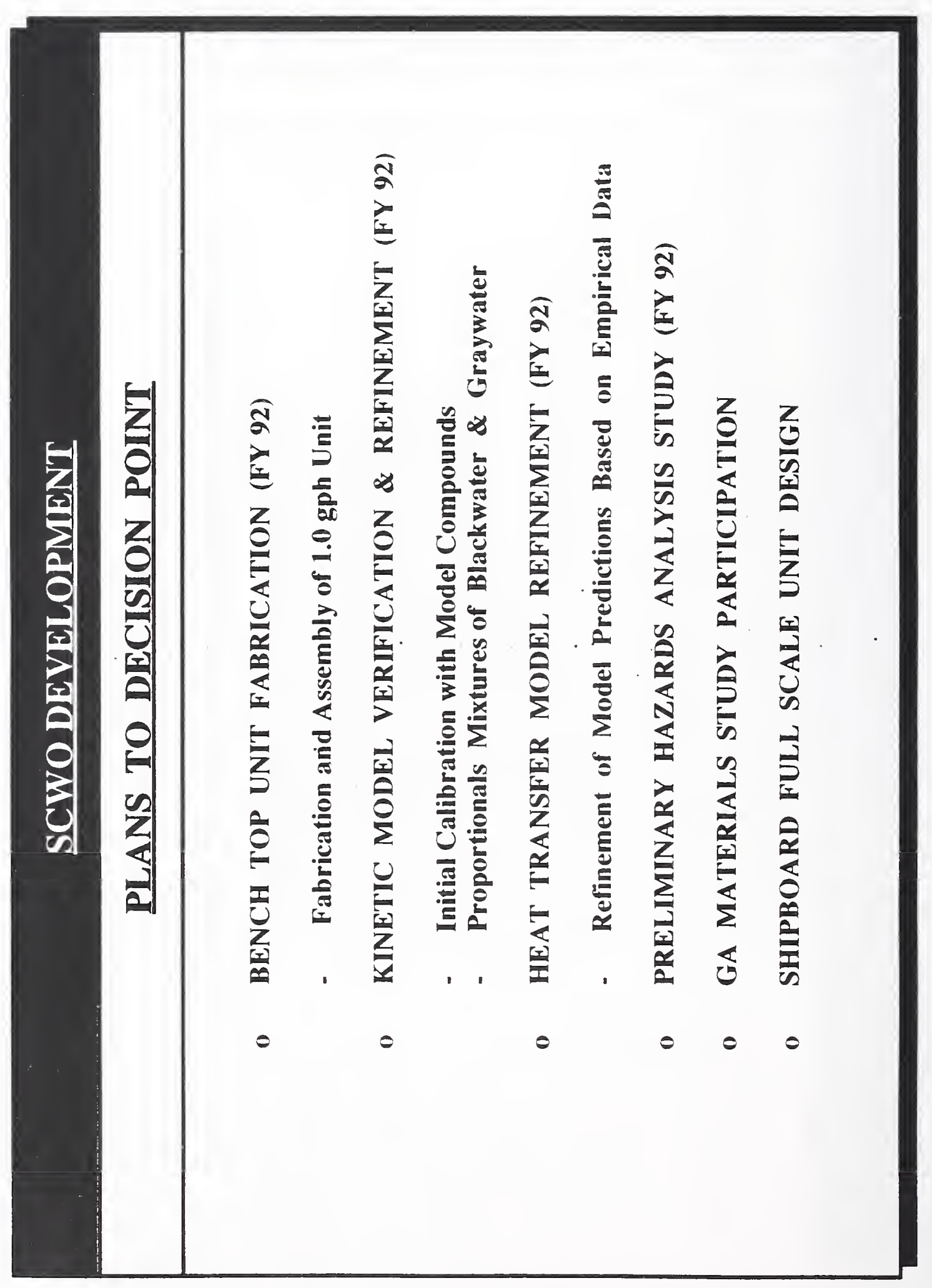




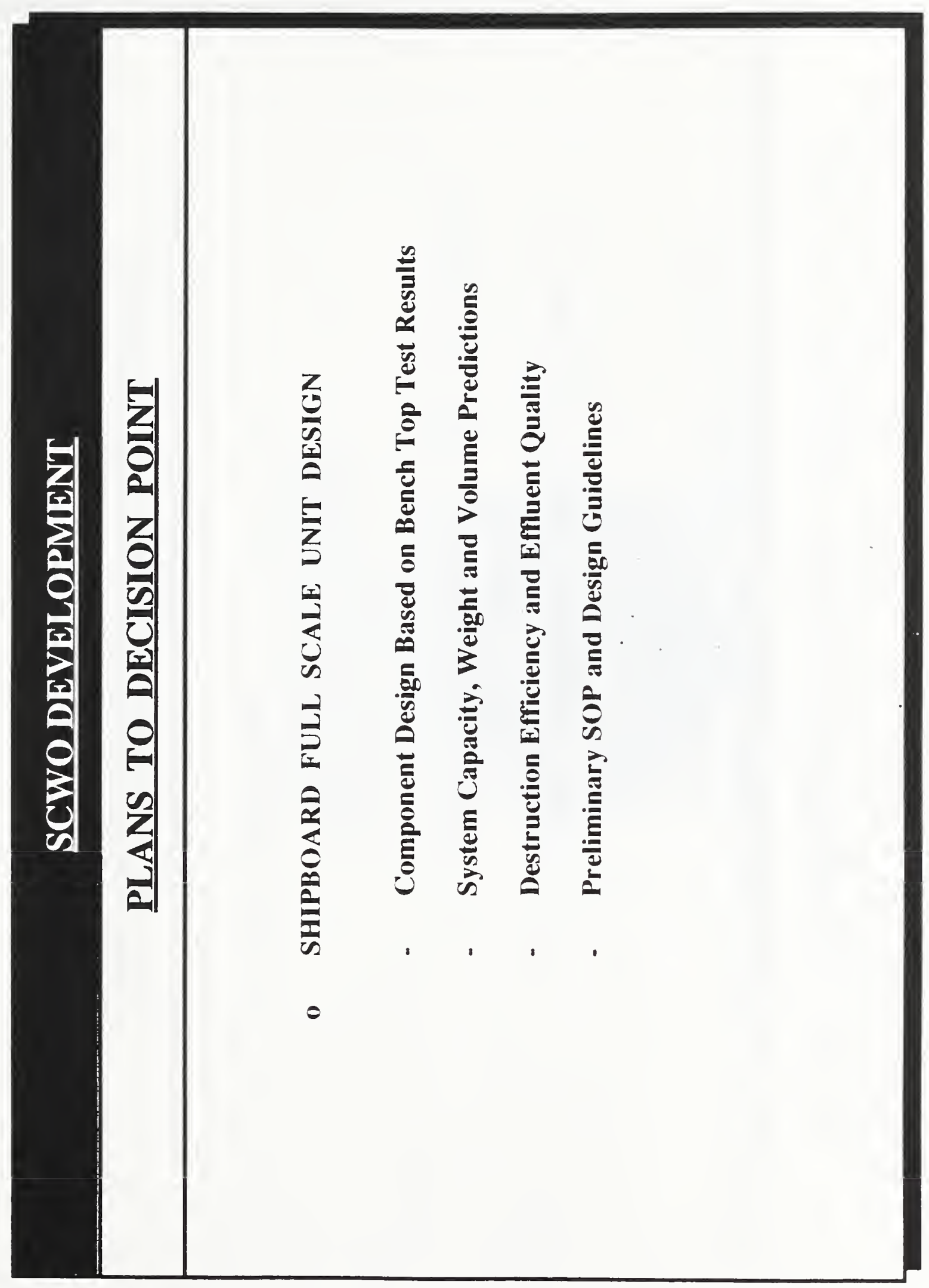




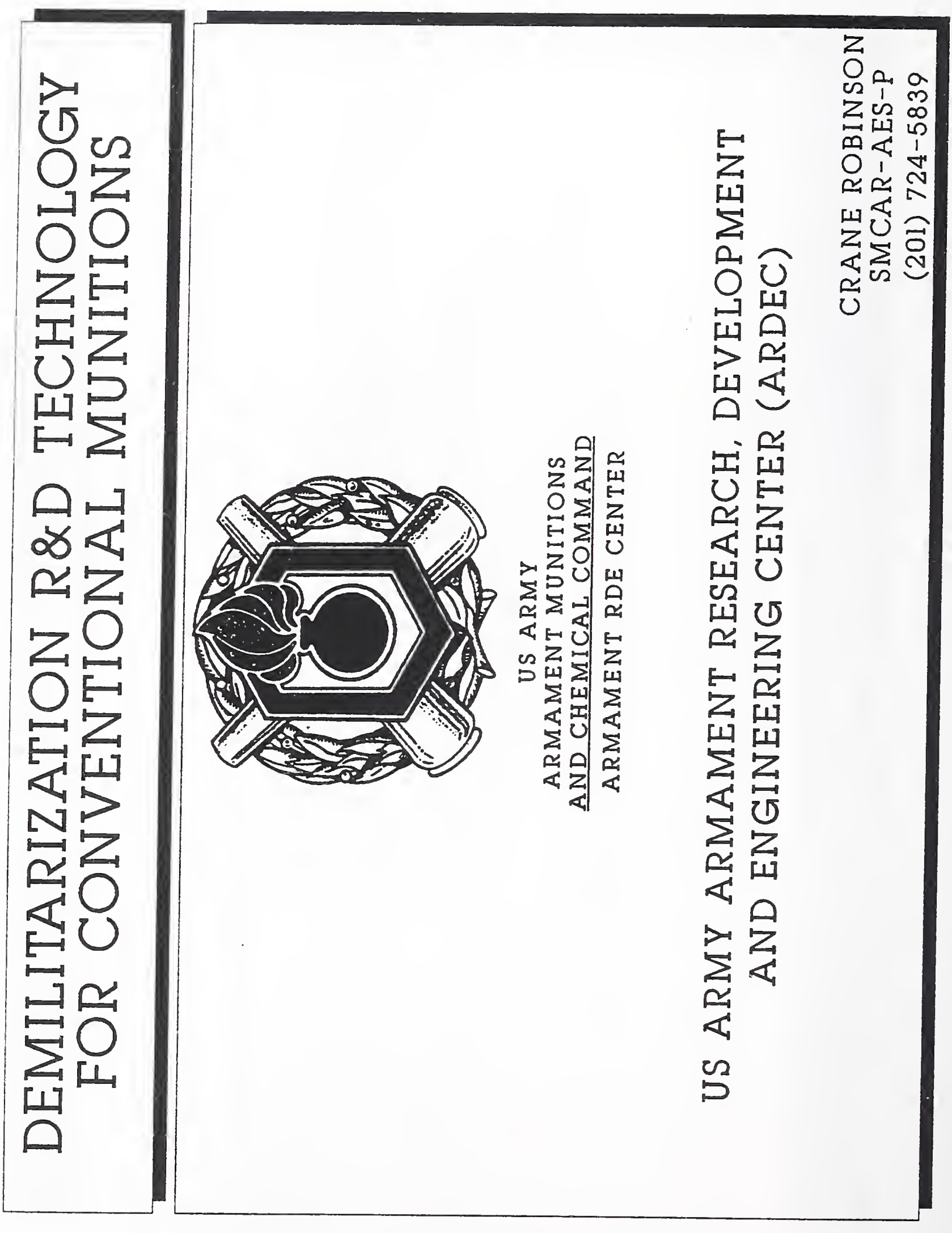




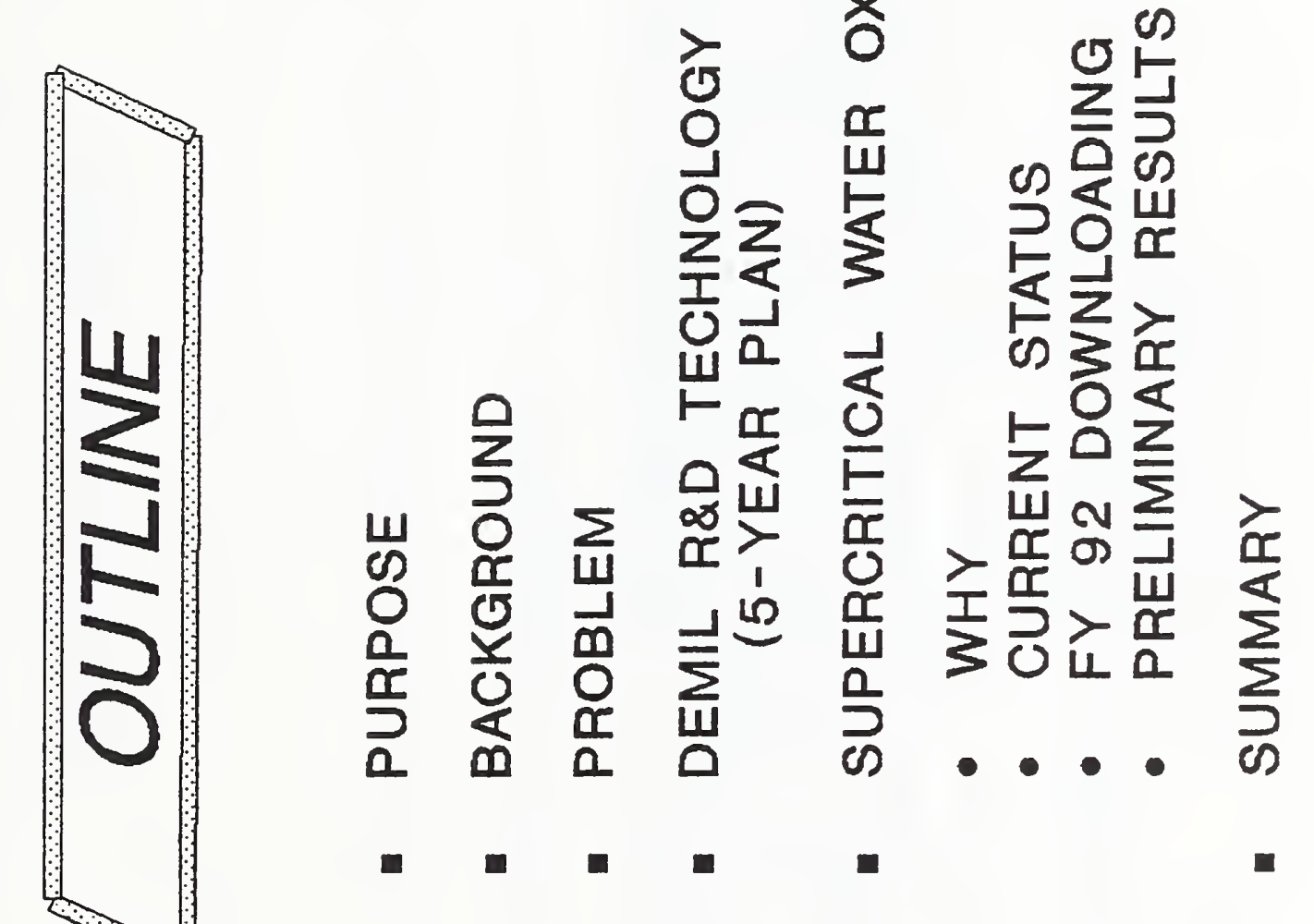



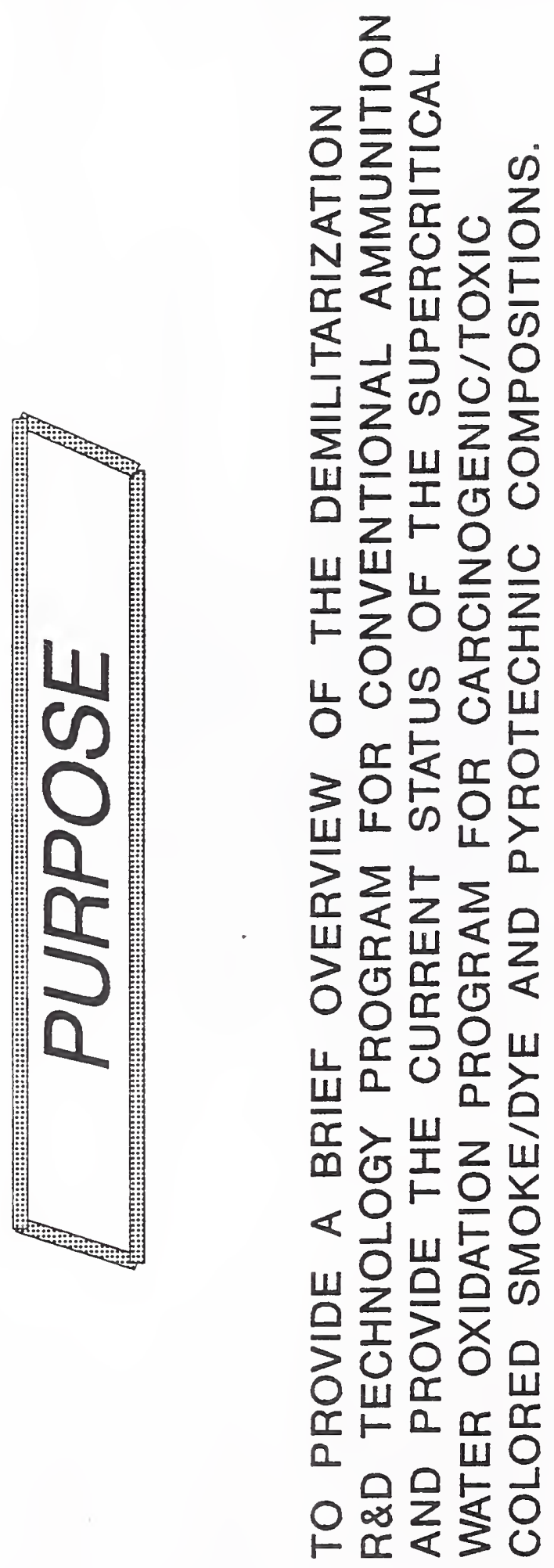


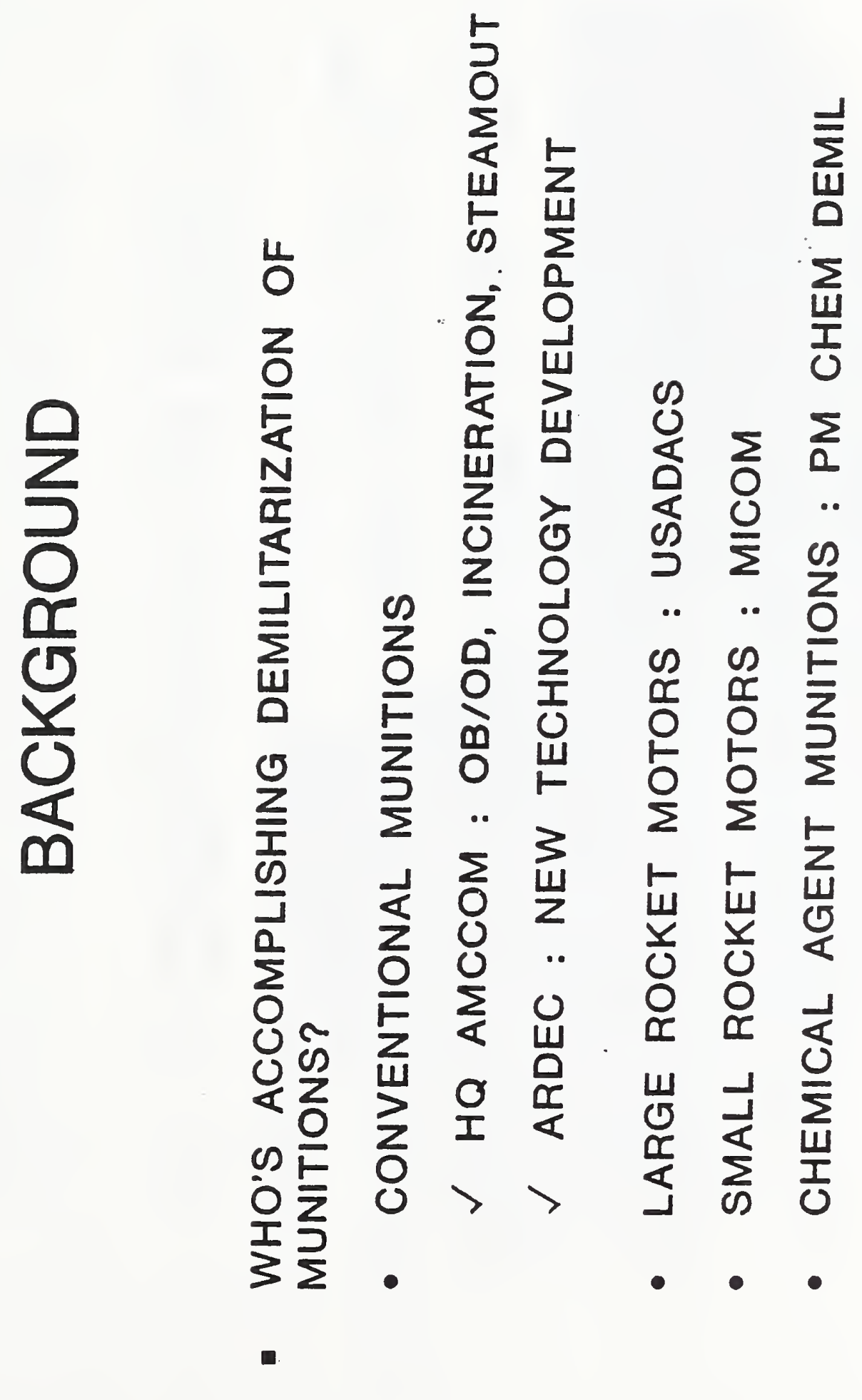




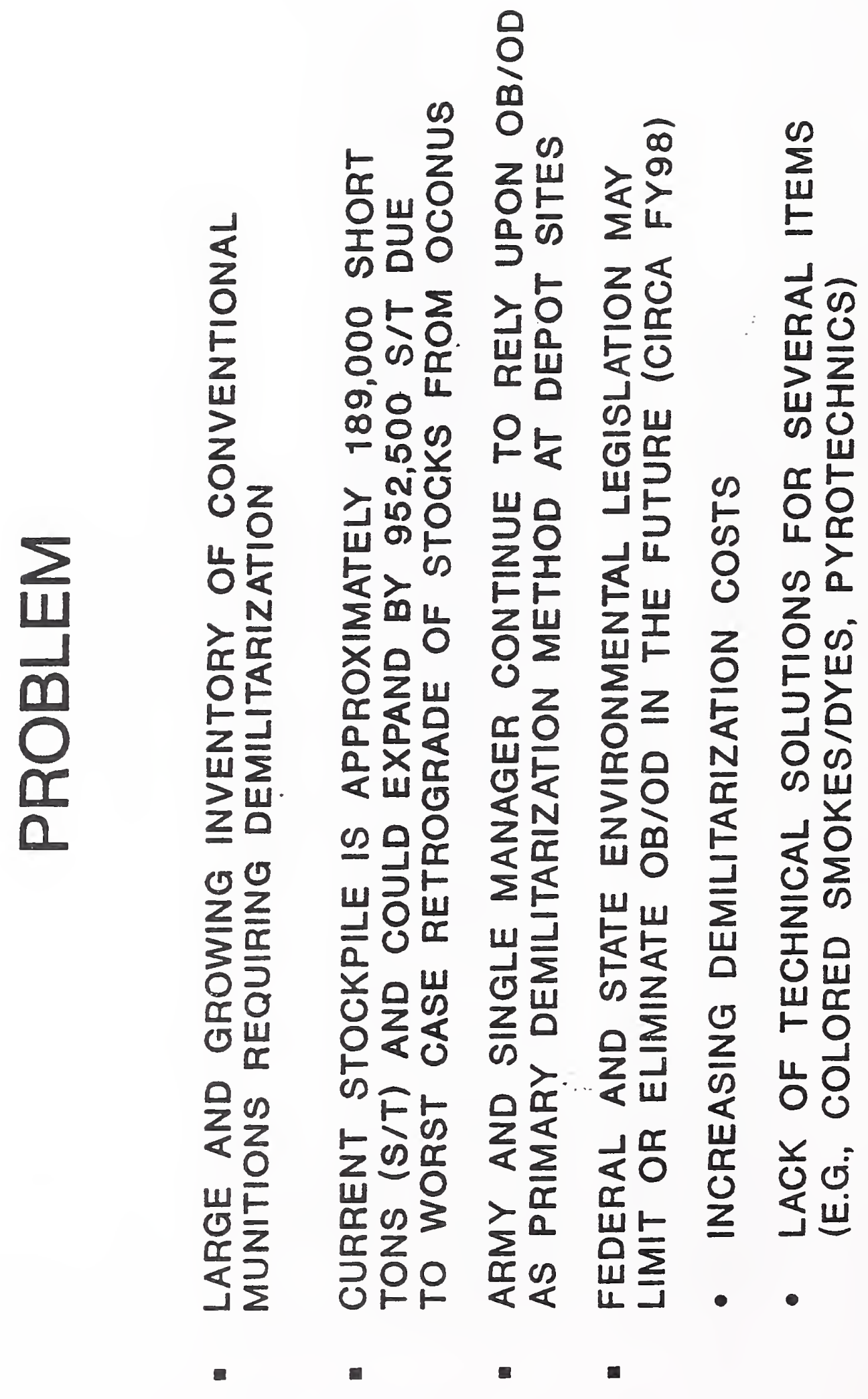



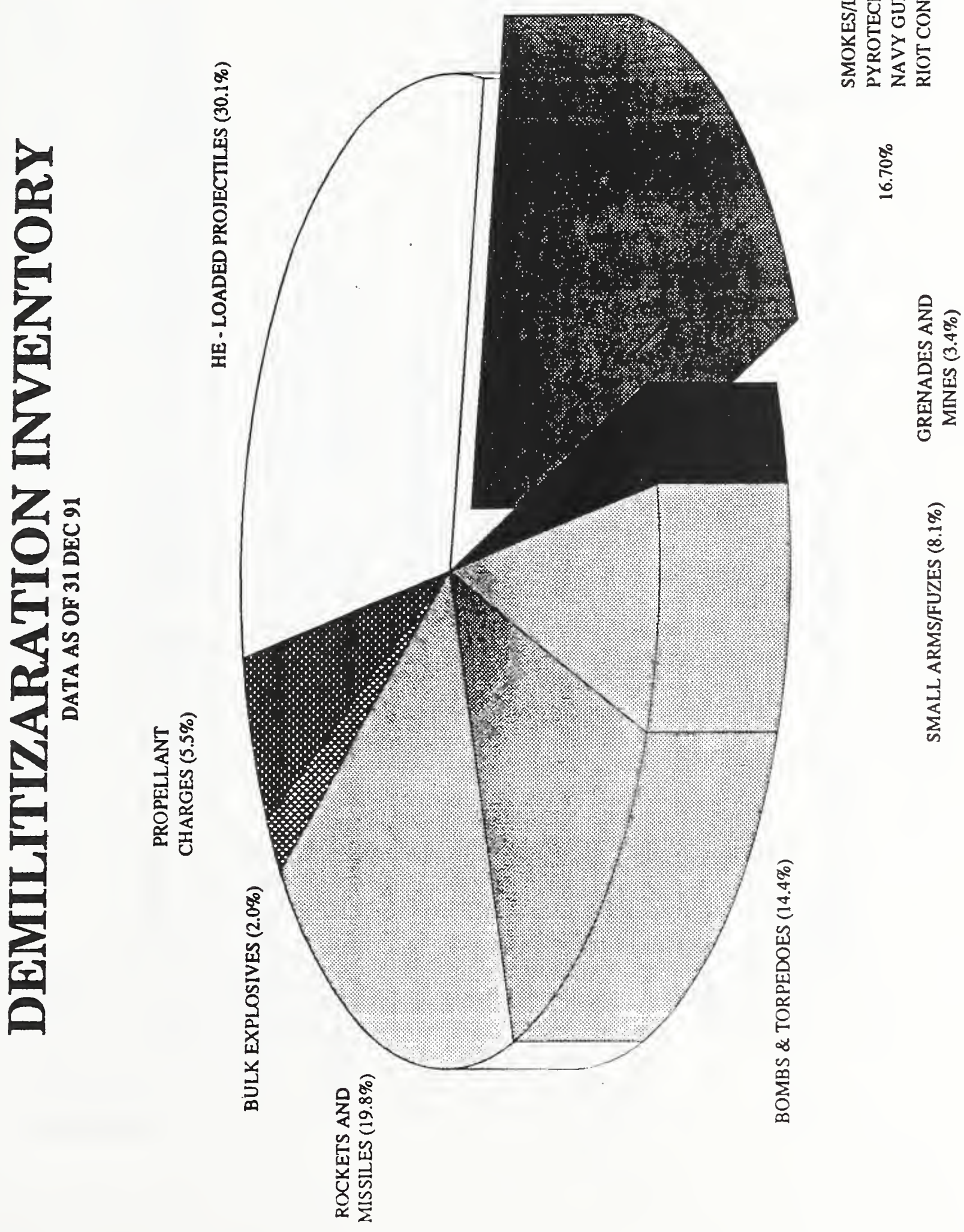


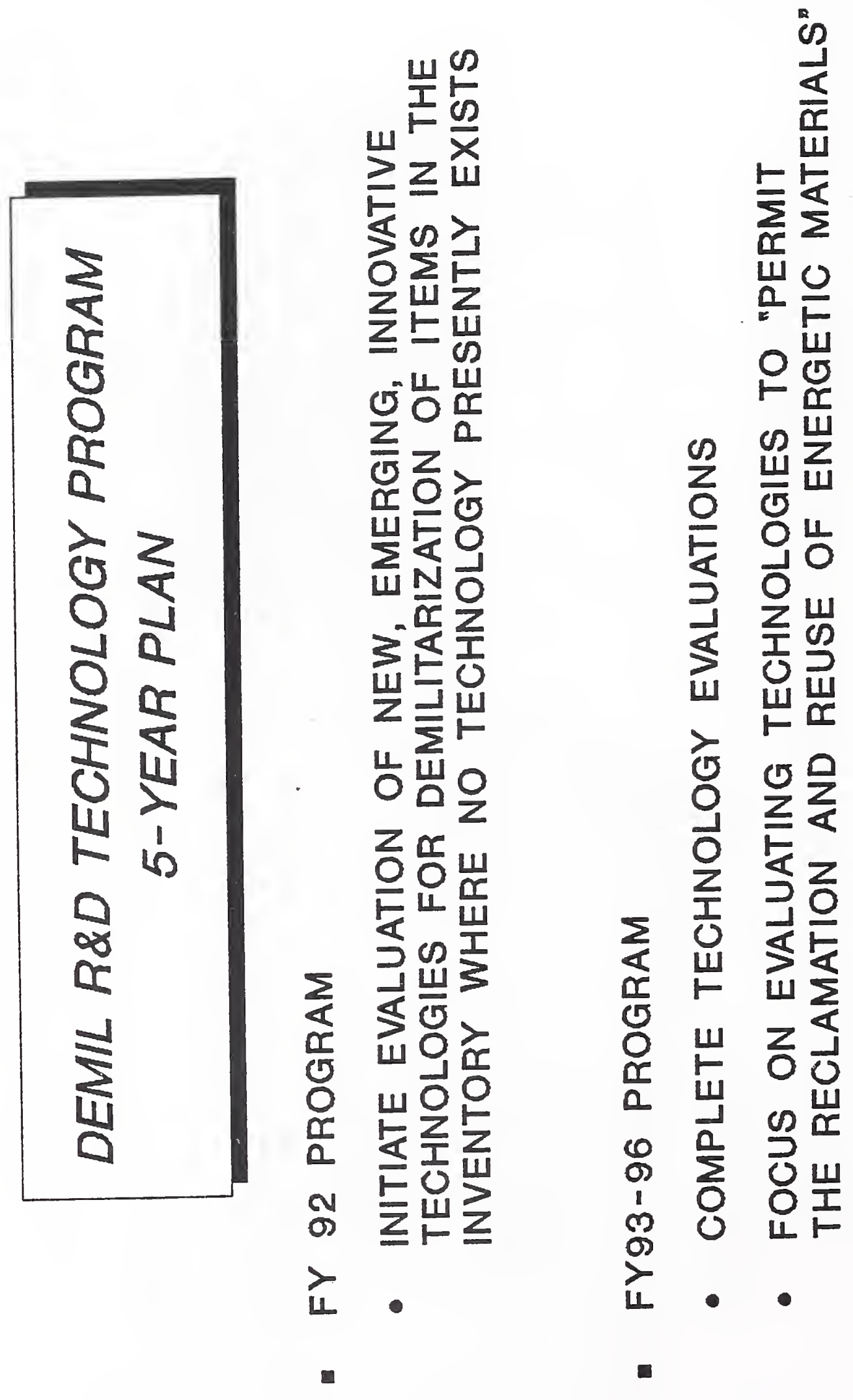



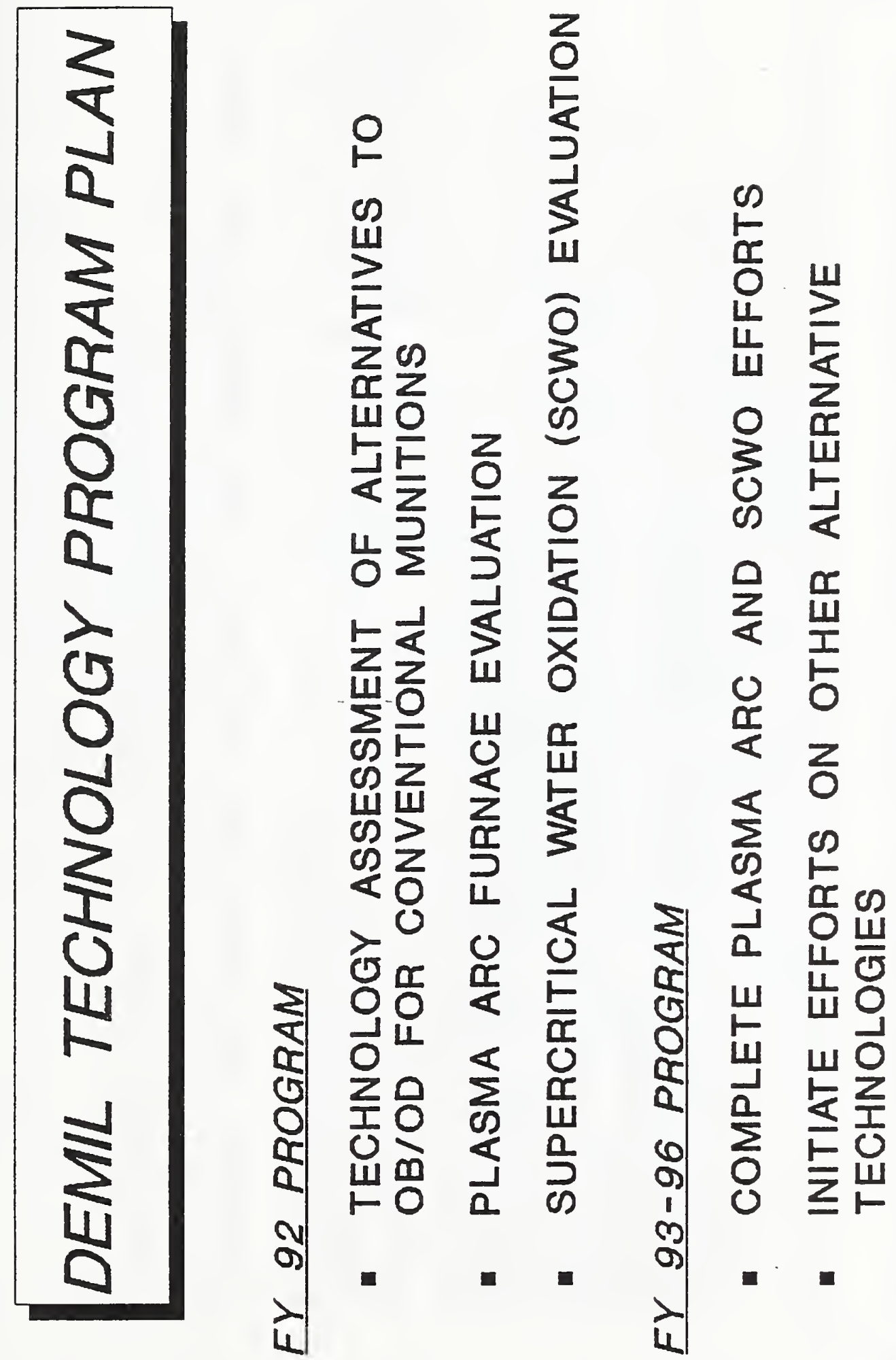

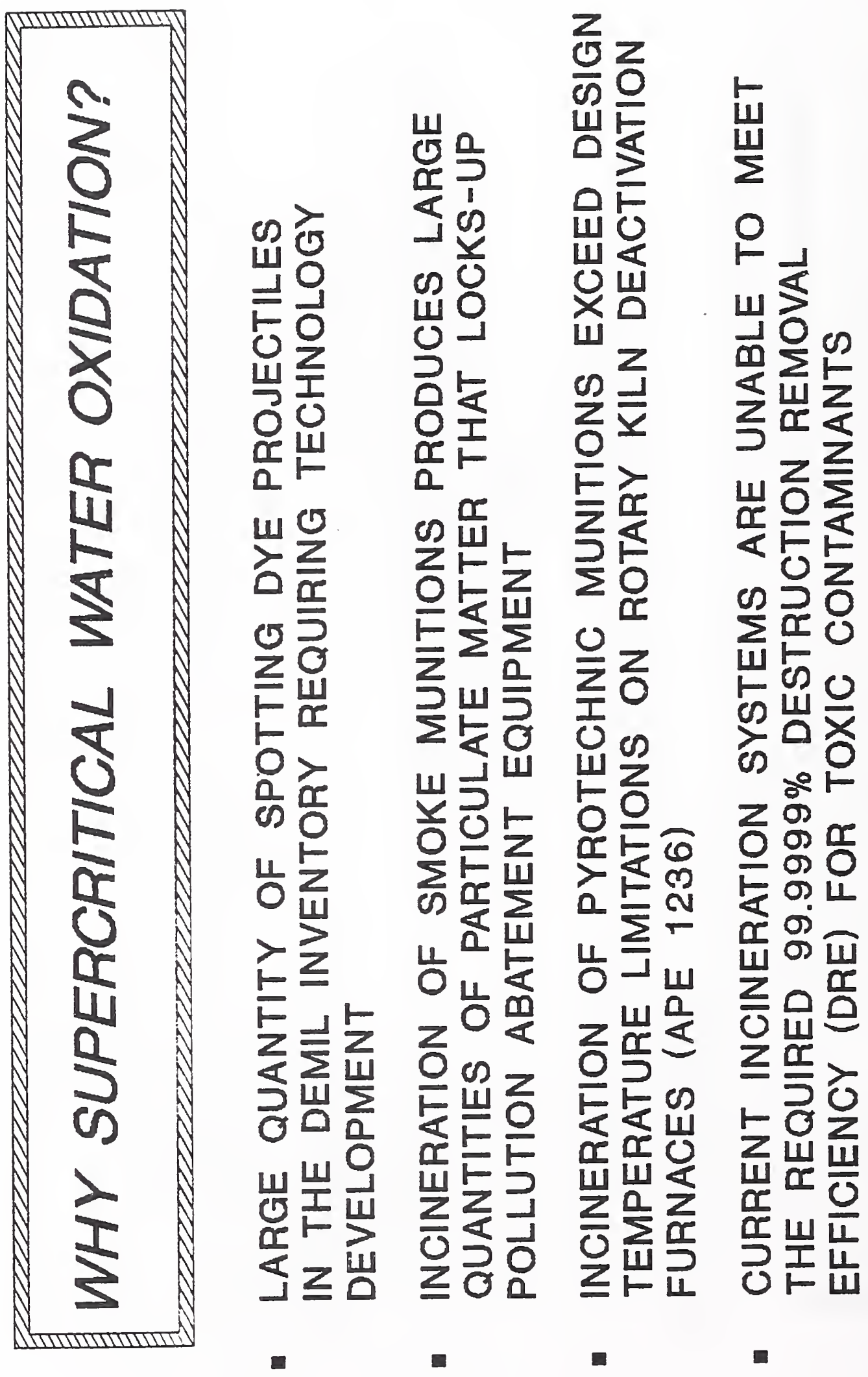


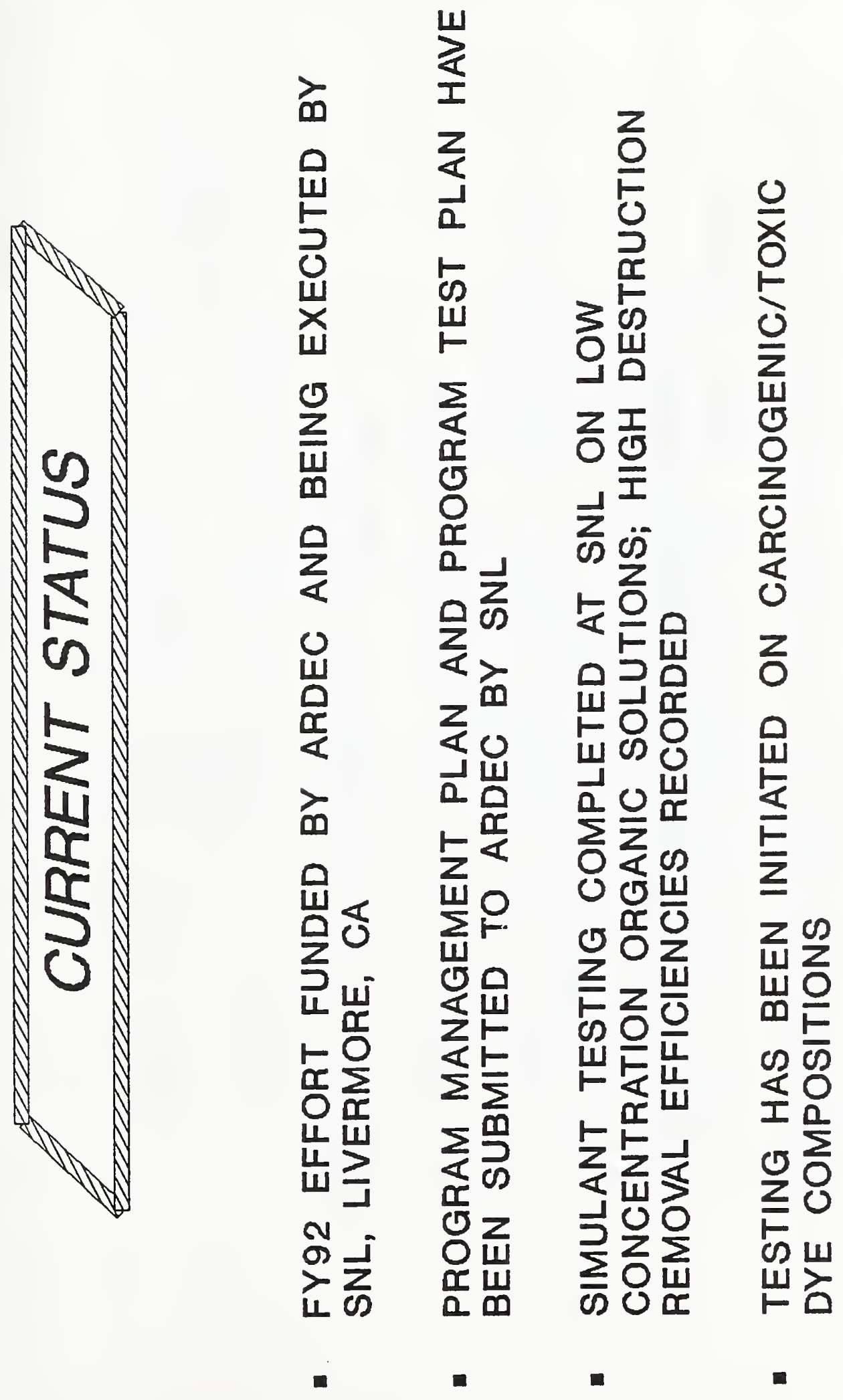




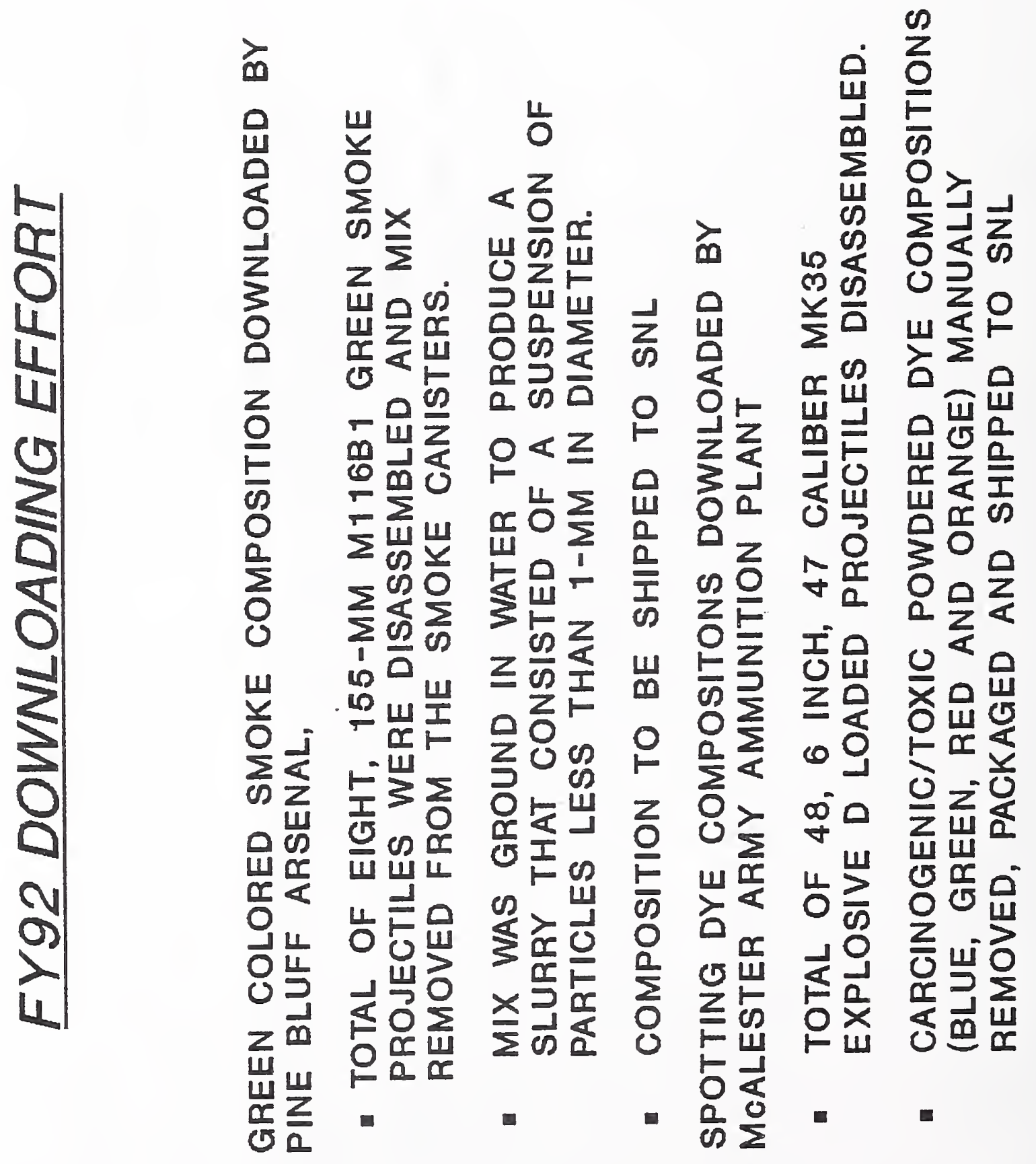




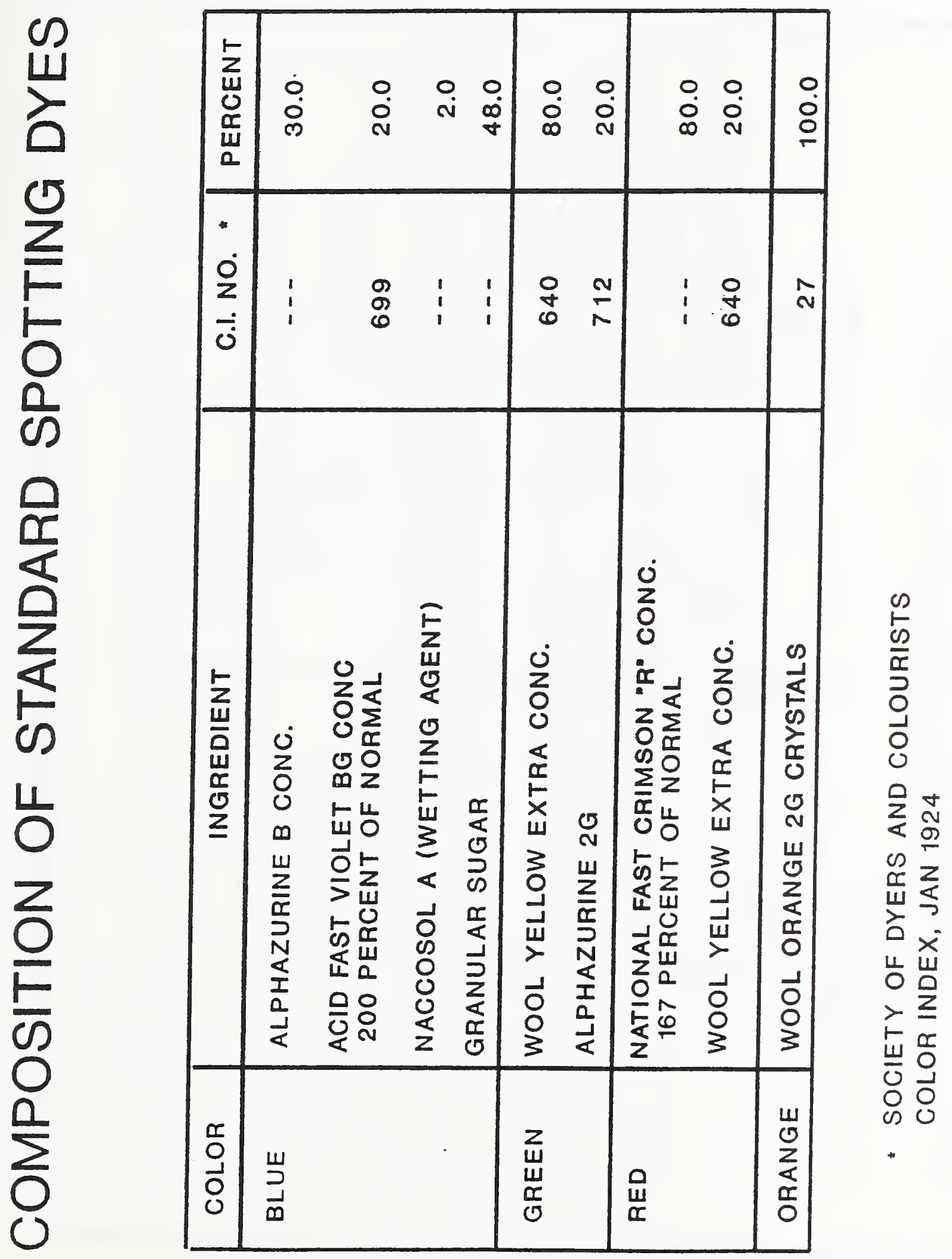




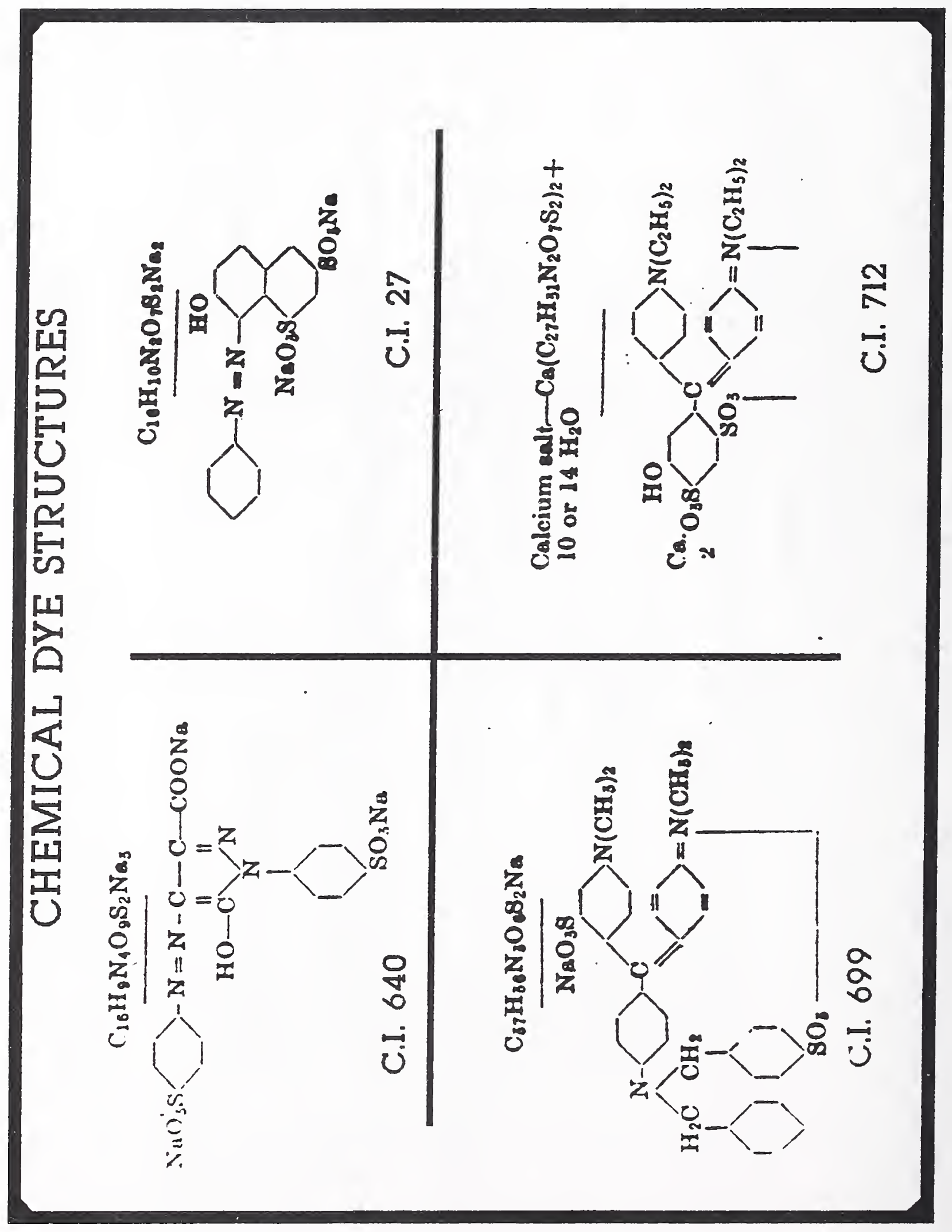




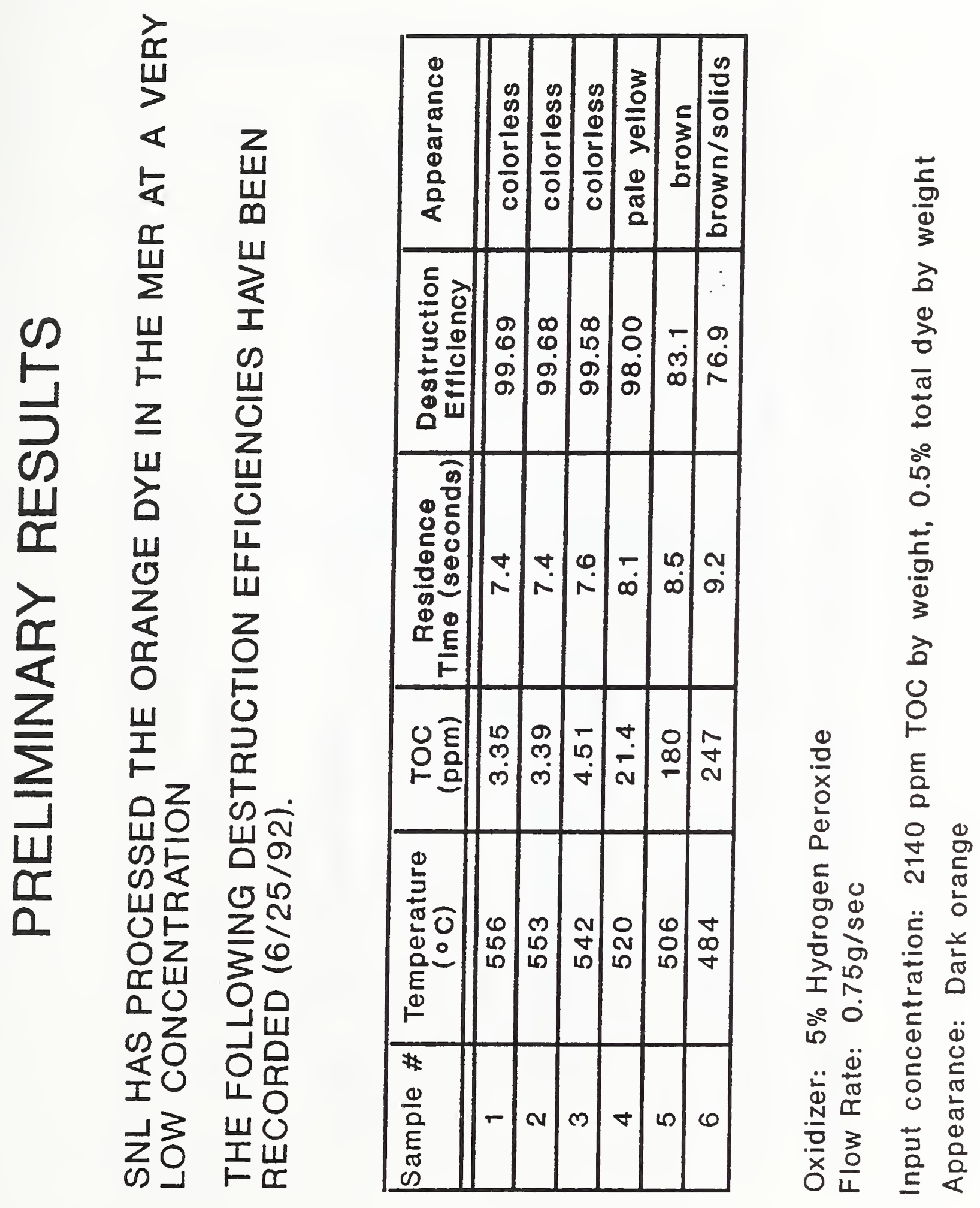




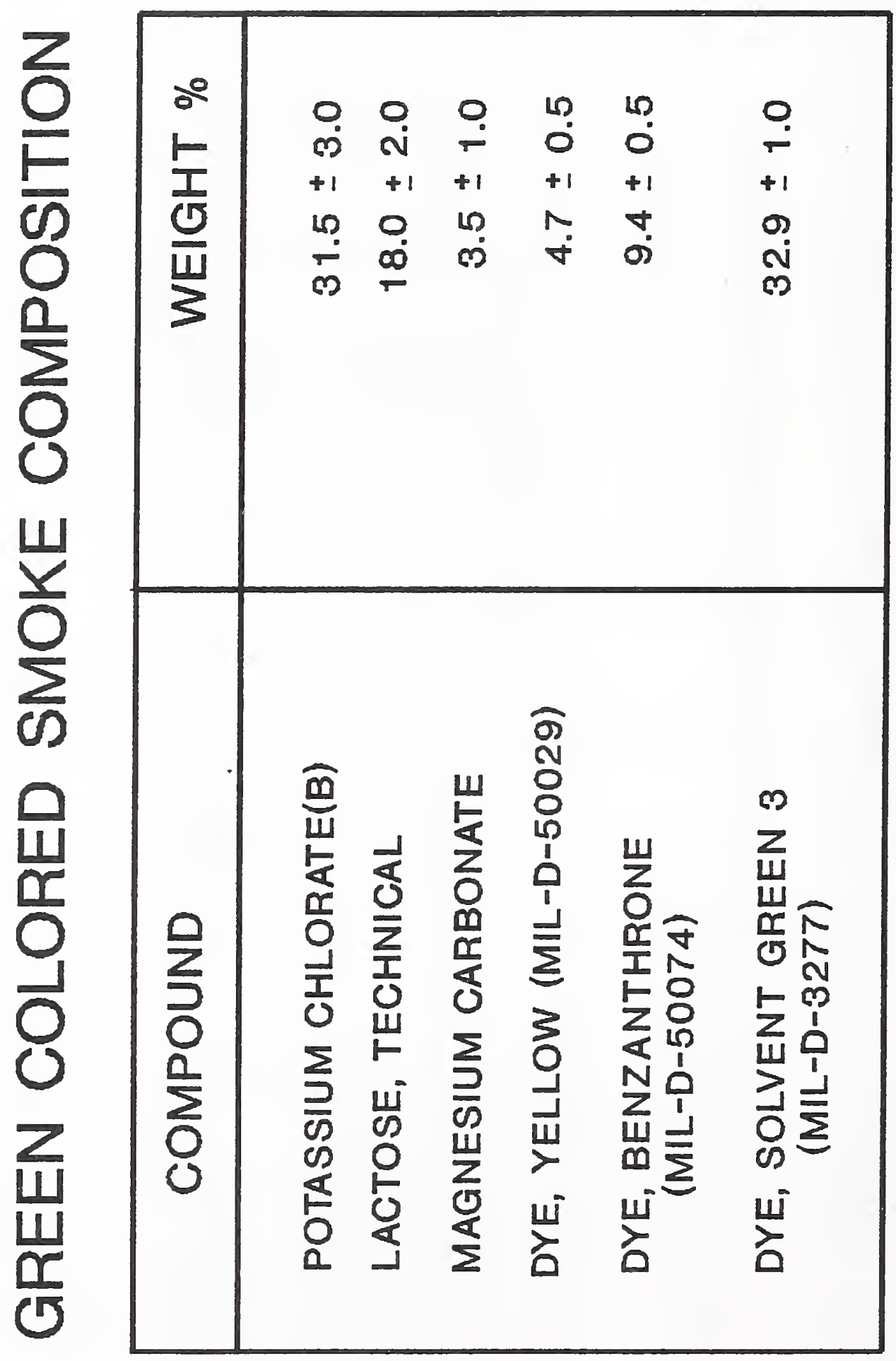



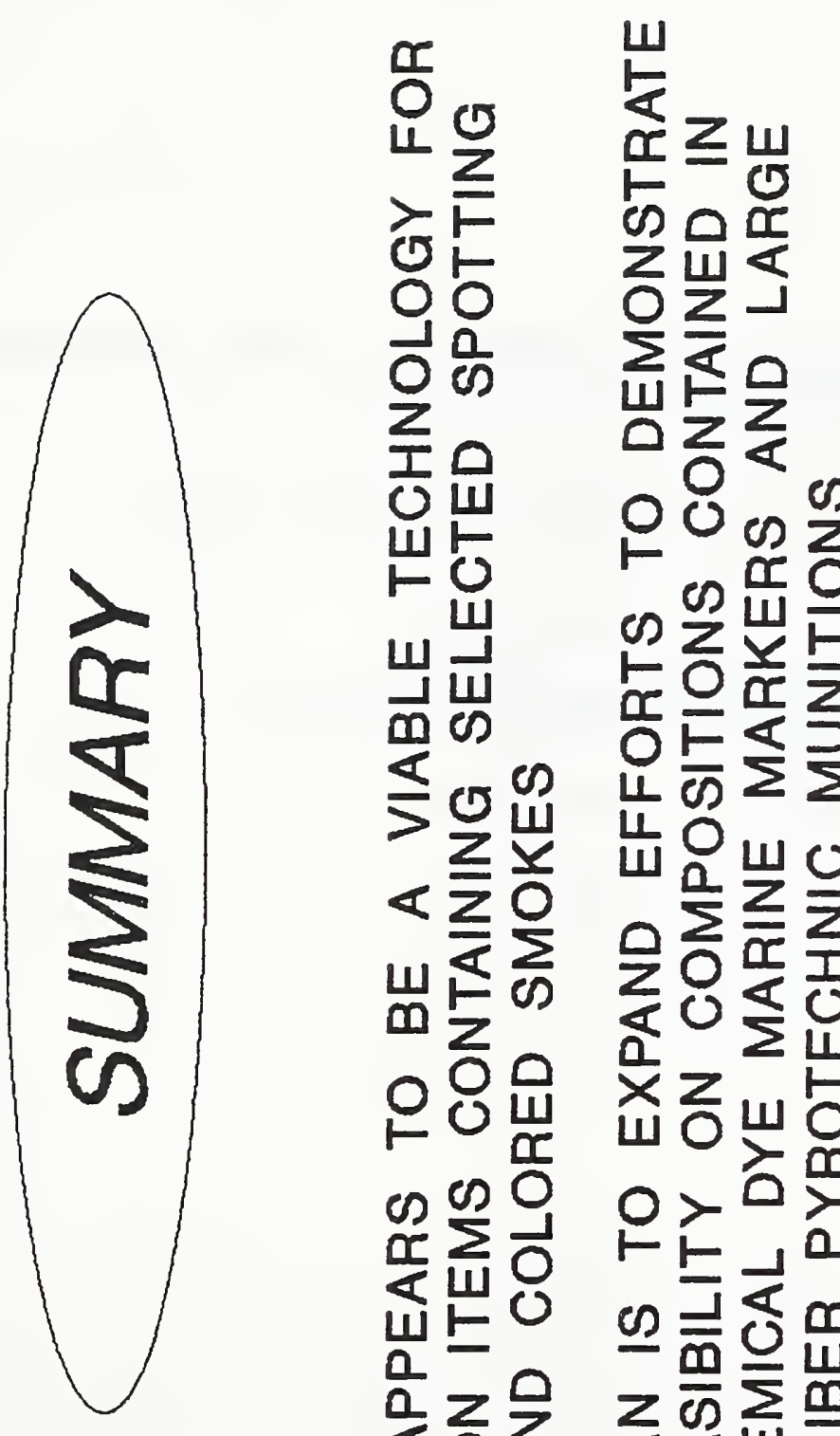

$\frac{1}{0} \frac{0}{\pi} \quad \sum \square$

I

Uய山

U $\quad \cos \frac{\alpha}{2}$

崩

$12 \frac{1}{2}$

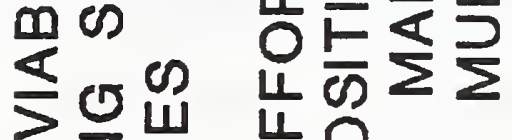

Z艾 $\frac{1}{0}$ 世

$<\overline{2} \quad$ 는

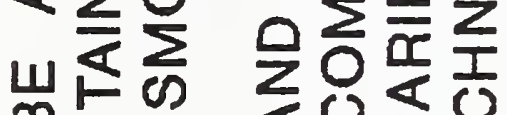

$m \sum \infty \leq 0 \leq u$

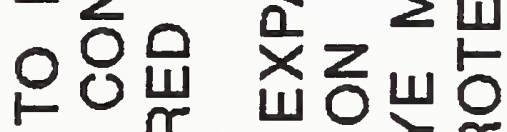

$\cos 0$ o>0

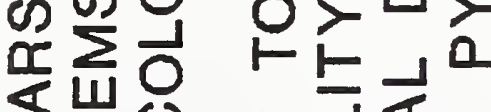

шएU

느을

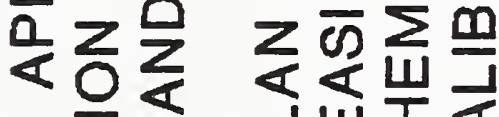

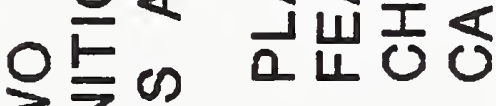

$0 \sum^{0}$

$\leq$

$\bar{m} \longleftarrow$

岁

는

ш

₹

ш

$\sum_{0} 0$

웅

$\sum_{U} \underset{\square}{a}$

0

خ

00

0

20

U

ш

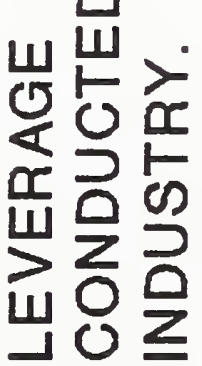




\author{
Roger L. Schneider \\ U.S. Army Corps of Engineers \\ Construction Engineering Research Laboratory
}

Champaign, IL 61824-9005 
IDENTIFICATION AND ASSESSMENT OF ENVIRONMENTALLY SAFE ALTERNATIVE TECHNOLOGIES

TO OPEN BURNING/OPEN DETONATION DESTRUCTION OF EXPLOSIVE AND PROPELLANT PRODUCTION WASTES

Roger L. Schneider and Bernard A. Donahue

\author{
U. S. Army Corps of Engineers \\ Construction Engineering Research Laboratory
}

Champaign, IL 61824-9005, 217-373-6733 


\section{ENERGETIC MATERIALS (PEP)}

Propellants

Explosives

Pyrotechnics 


\section{USA CERL \\ ENVIRONMENTAL ENGINEERING DIVISION}

Energetic Materials Research Programs include,

- Red Water / Pink Water

- Propellant Recycling (NC)

- Alternatives to $\mathrm{OB} / \mathrm{OD}$ for contaminated production wastes 


\section{ENERGETIC MATERIAL DISPOSAL}

$-\mathrm{OB} / \mathrm{OD}$

- Incineration

- Supercritical Water Oxidation (SCWO)

- Wet Air Oxidation (WAO)

- Biodegradation

- Electrochemical Oxidation / Reduction

- "Enclosed" Open Burning 


\section{EXPLOSIVE AND PROPELLANT WASTE CONTAMINANTS}

- Tramp Metals

- Cementitious Materials e.g., gravel

- Glass

- Wood and other Cellulosics

- Plastics and Composites

- "Off-Spec" 


\section{CONTAMINATED EXPLOSIVE AND PROPELLANT PRODUCTION WASTE PRETREATMENTS}

- Hydromilling

- High Pressure, 35,000 - 55,000 psig

- Lower Pressure, Abrasive Augmented, 2,000-10,000 psig

- Alkaline Hydrolysis

- Supercritical Fluid Extraction, e.g., $\mathrm{CO}_{2}$

- Solvents 


\section{M31A1E1 TRIPLE BASE PROPELLANT}

$\begin{array}{lc} & \% \mathrm{w} / \mathrm{w} \\ \text { nitrocellulose (NC) } & 21.50 \\ \text { nitroglycerin (NG) } & 18.00 \\ \text { nitroguanidine (NQ) } & 54.70 \\ \text { dibutylphthalate } & 3.00 \\ \text { ethyl centralite } & 1.50 \\ \text { potassium sulfate } & 1.25 \\ \text { carbon black } & 0.05\end{array}$



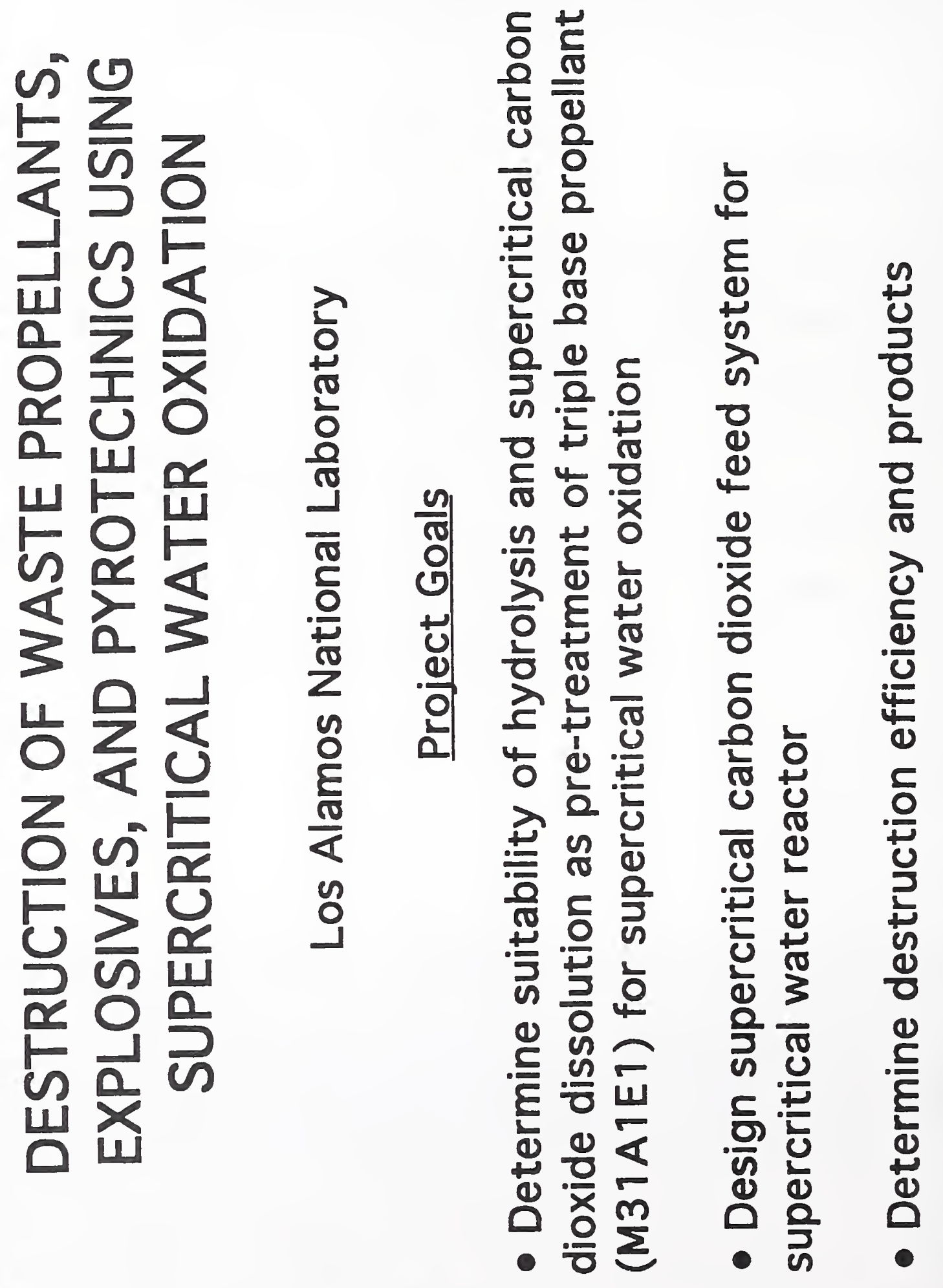

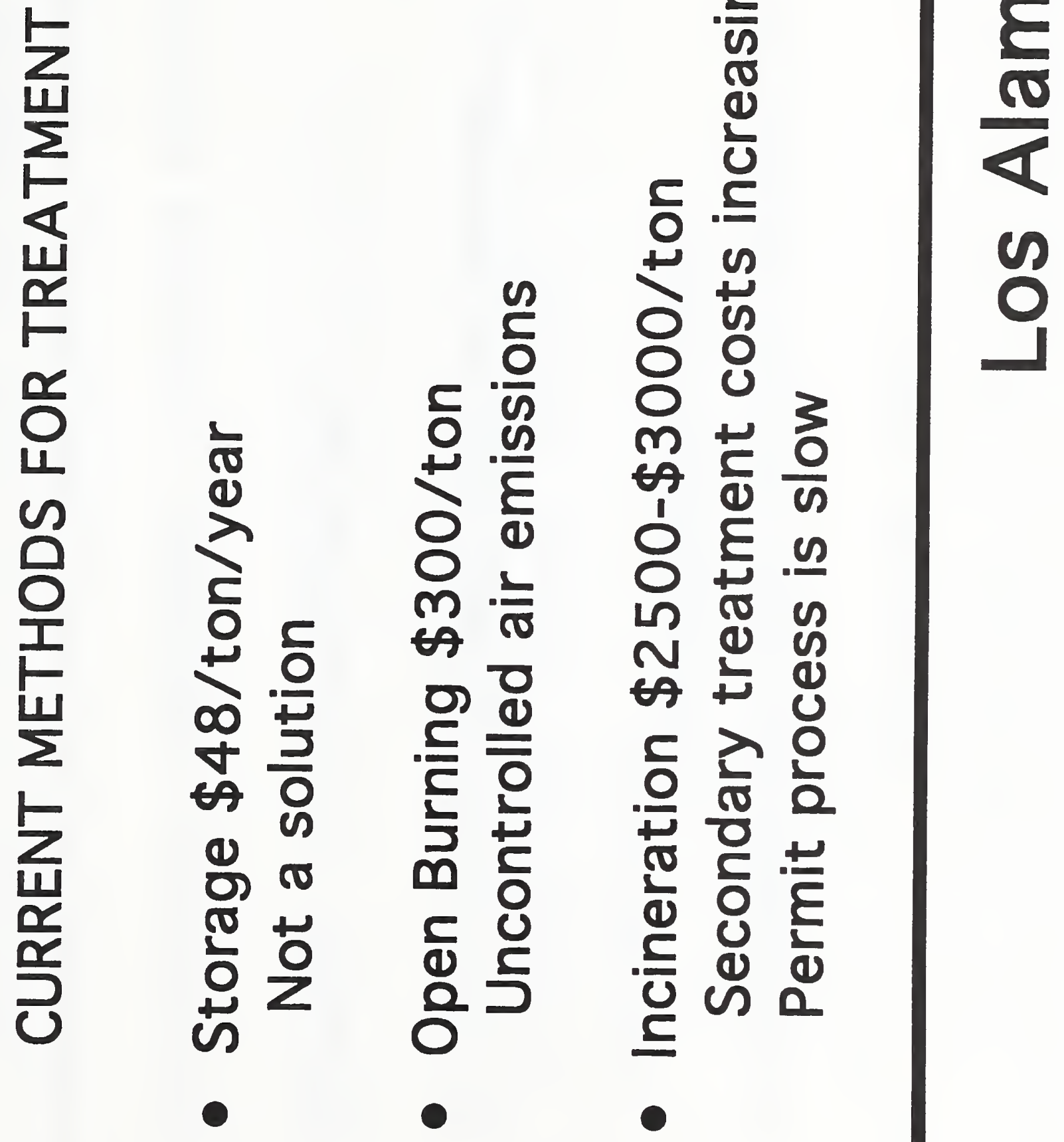

1

㭊

00

1

क)

0

$E$

$\infty$

1

8

$m+3$

\& $\frac{1}{\omega}$

0 ह

ก

ก 1

\& 10

용ํㅇ

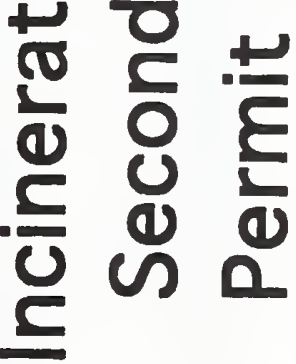

0 


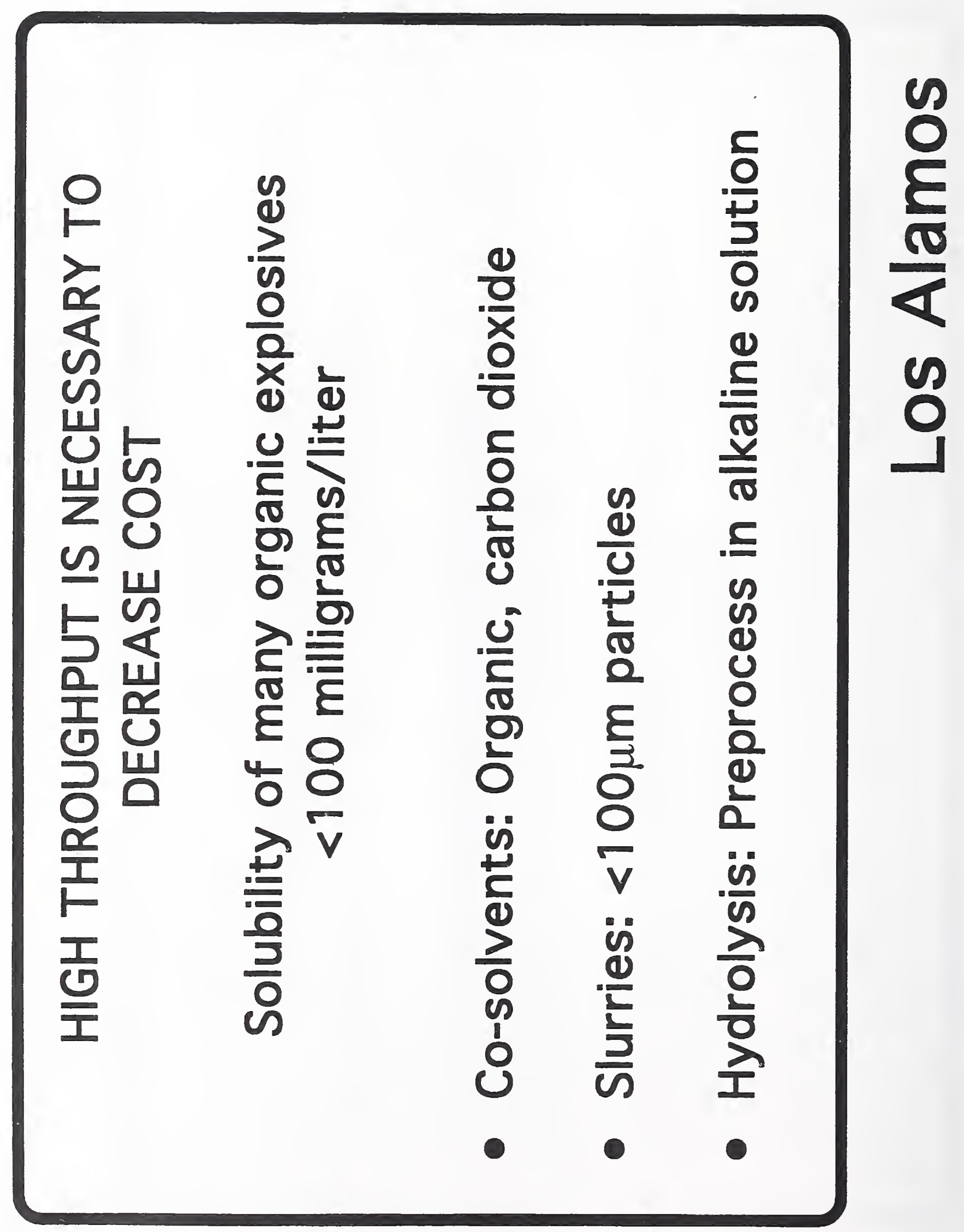



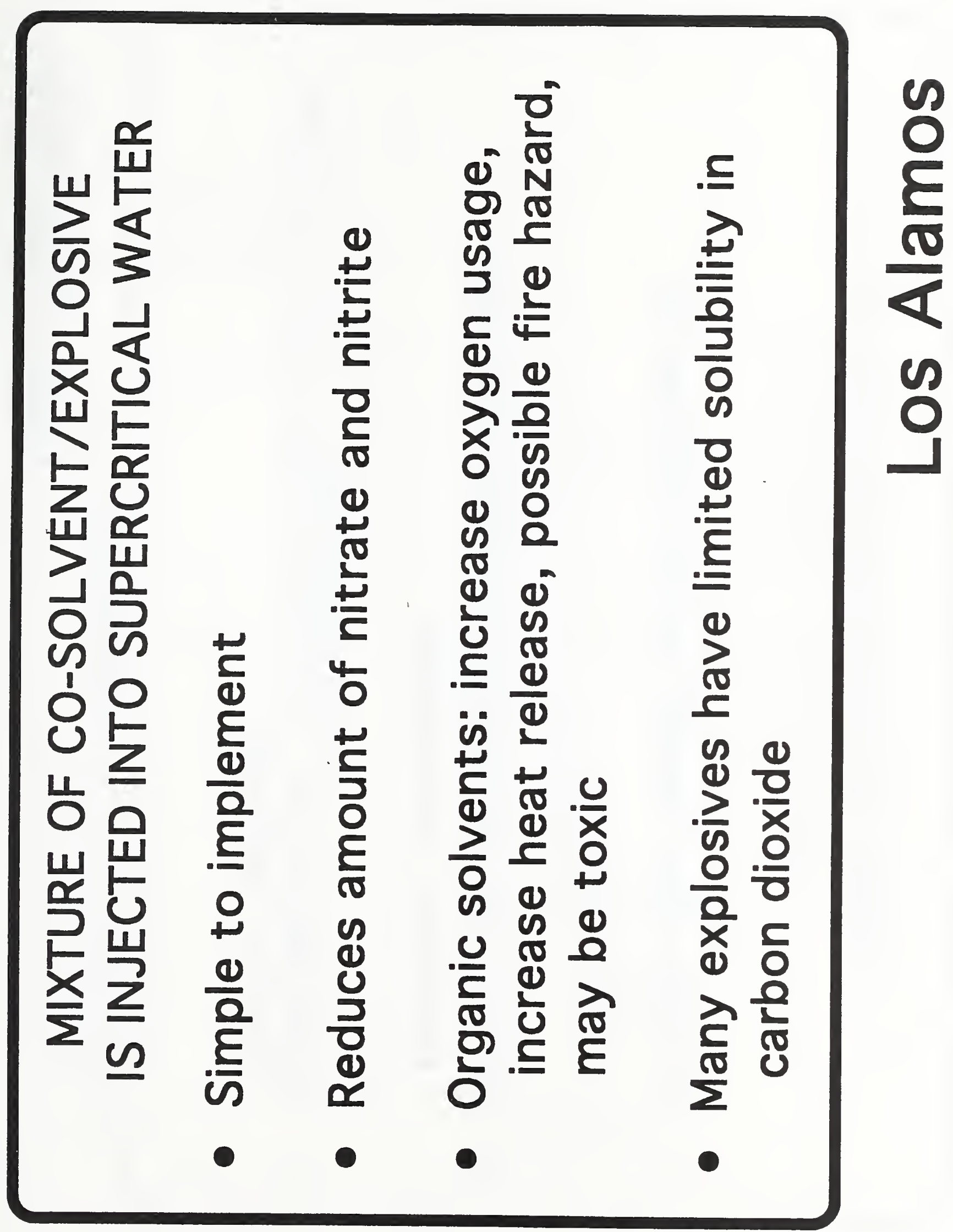


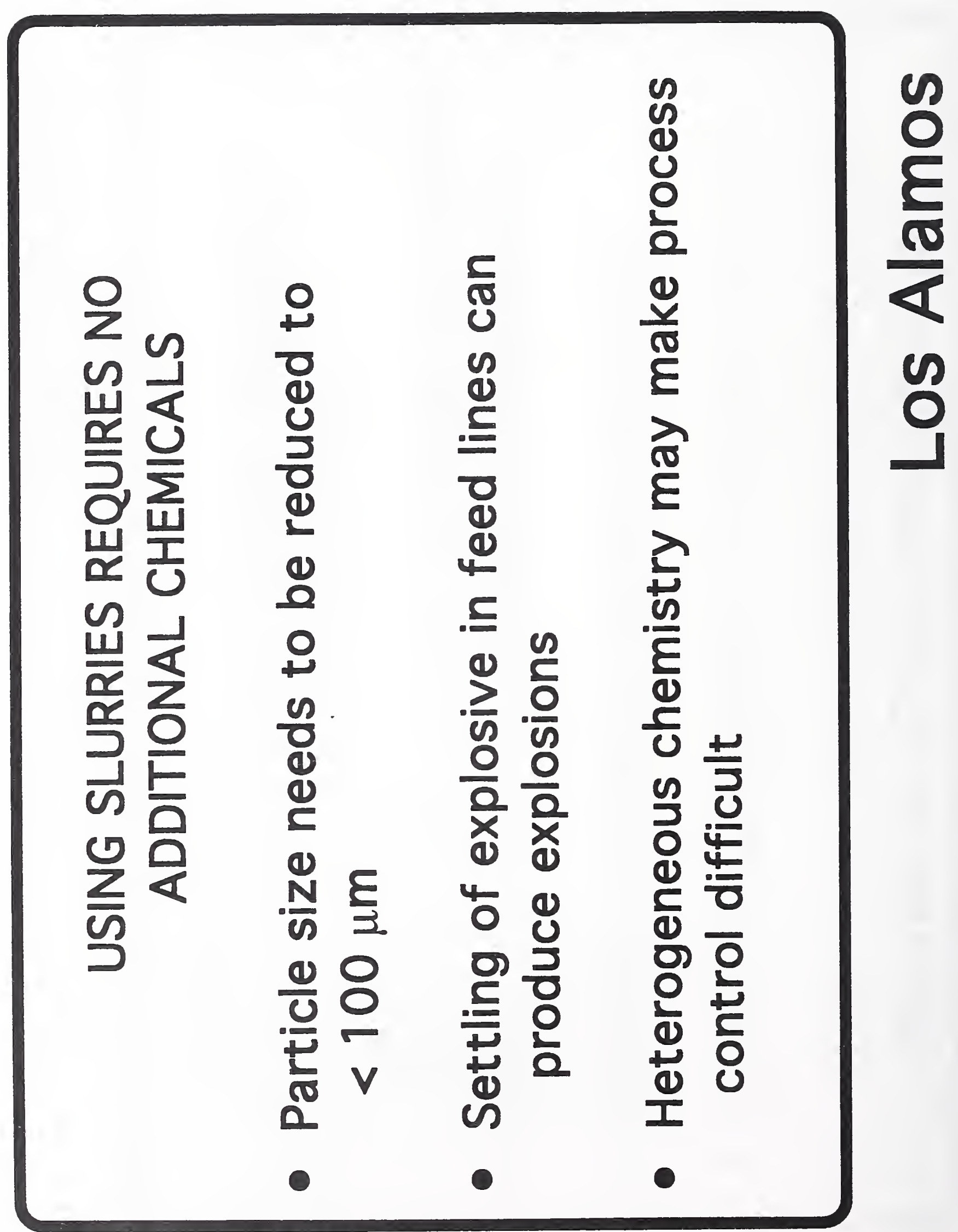




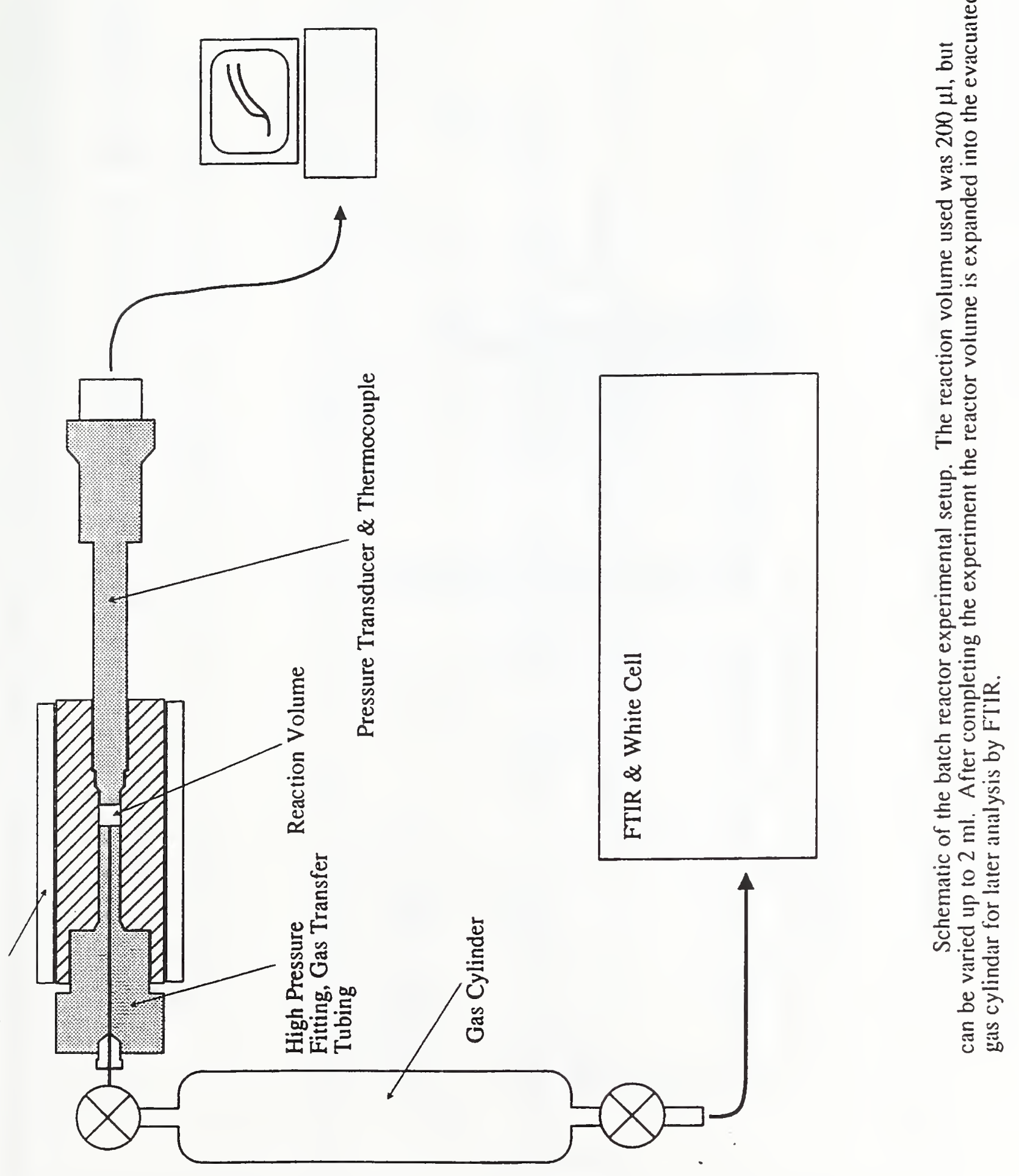


TATB

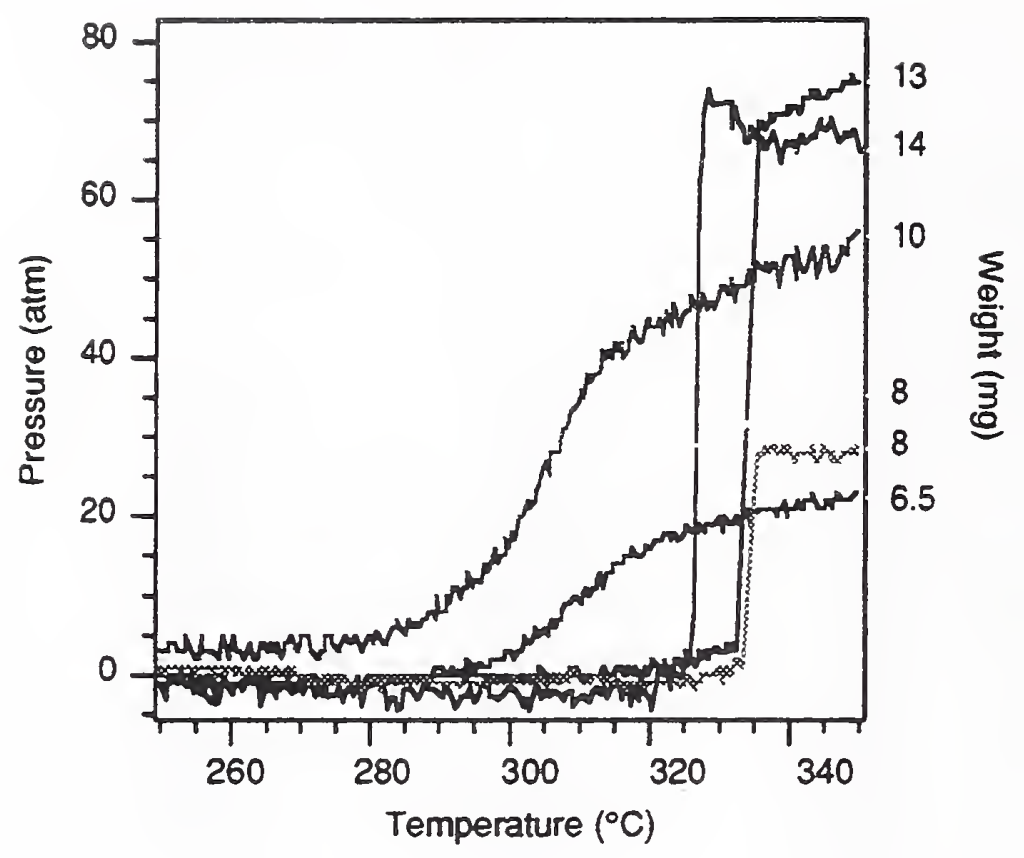

Double Base Propellent

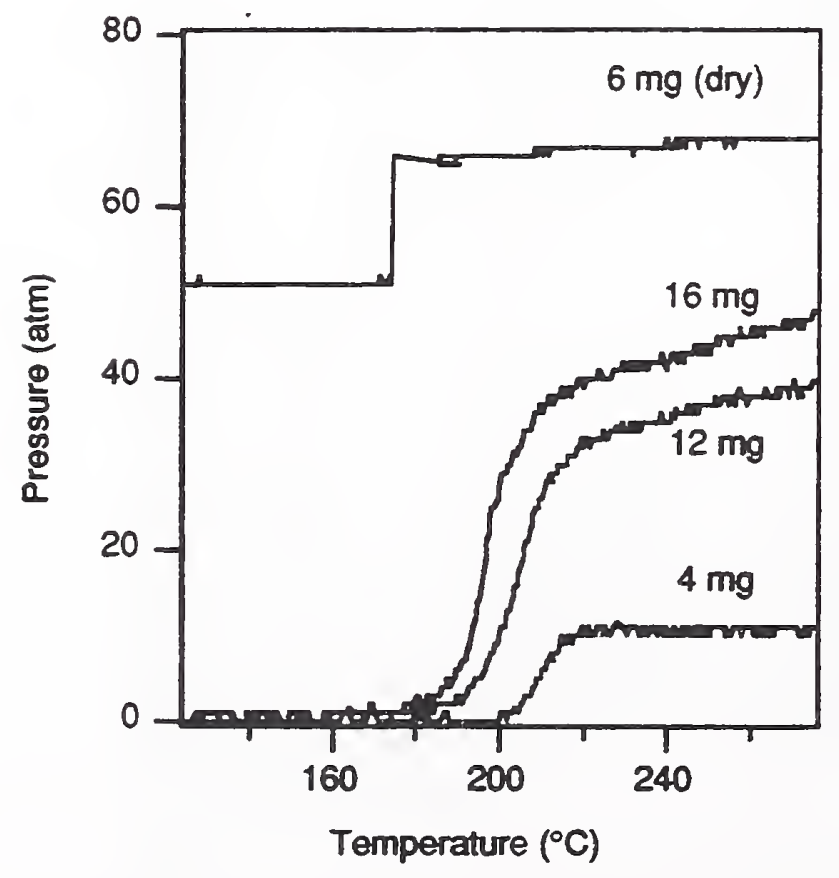




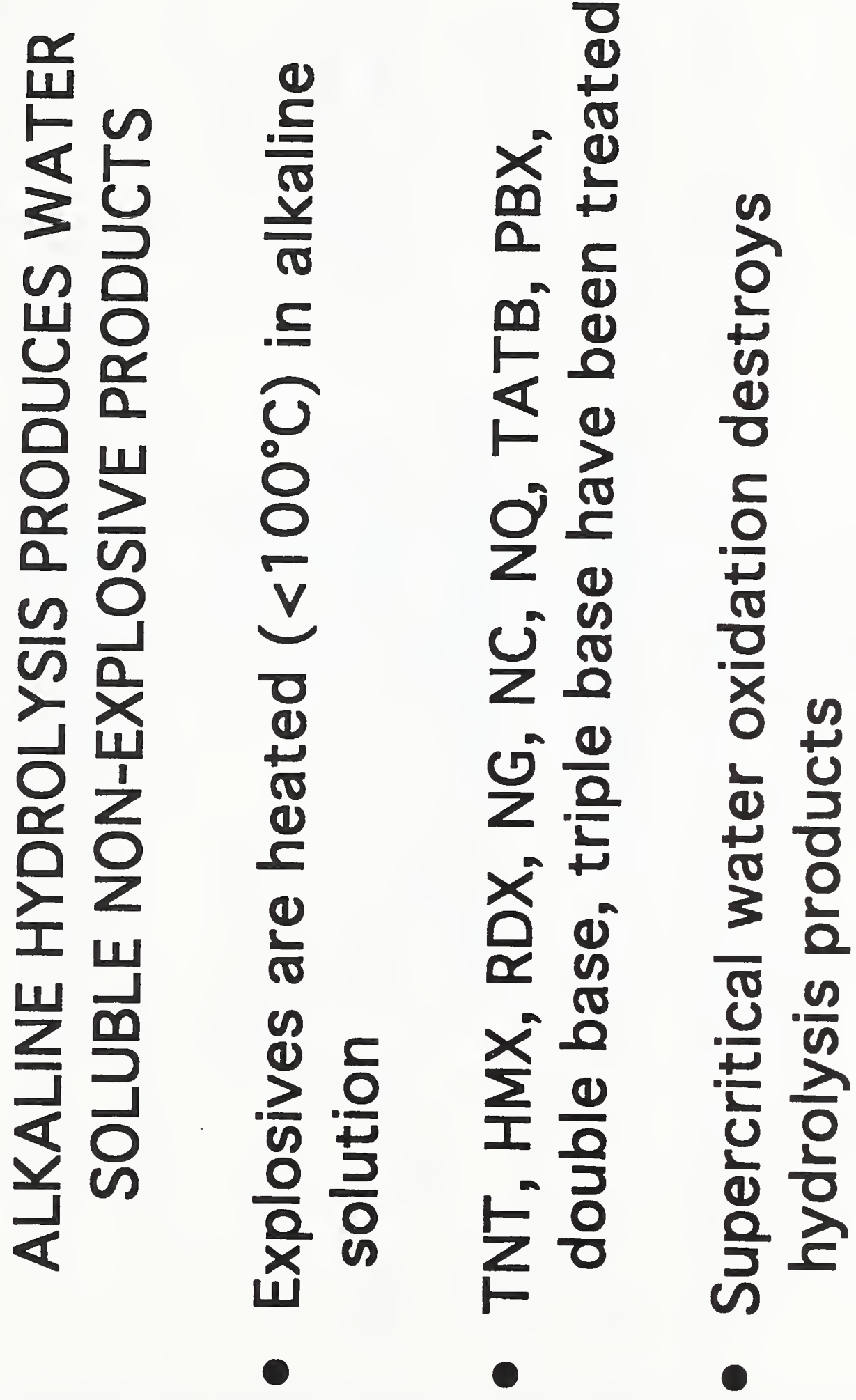

$\frac{8}{8}$

() 


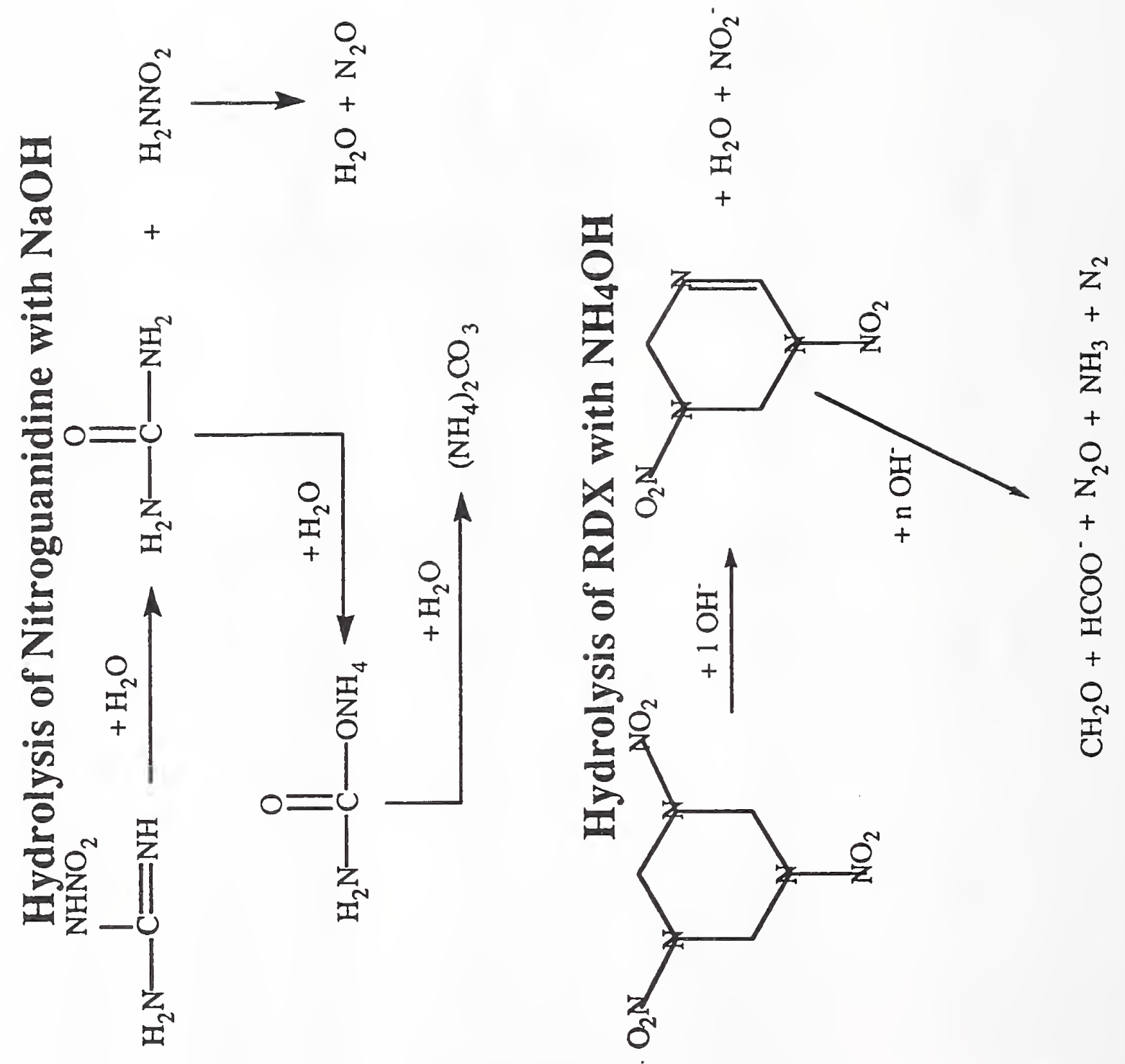


$\sum^{X}$

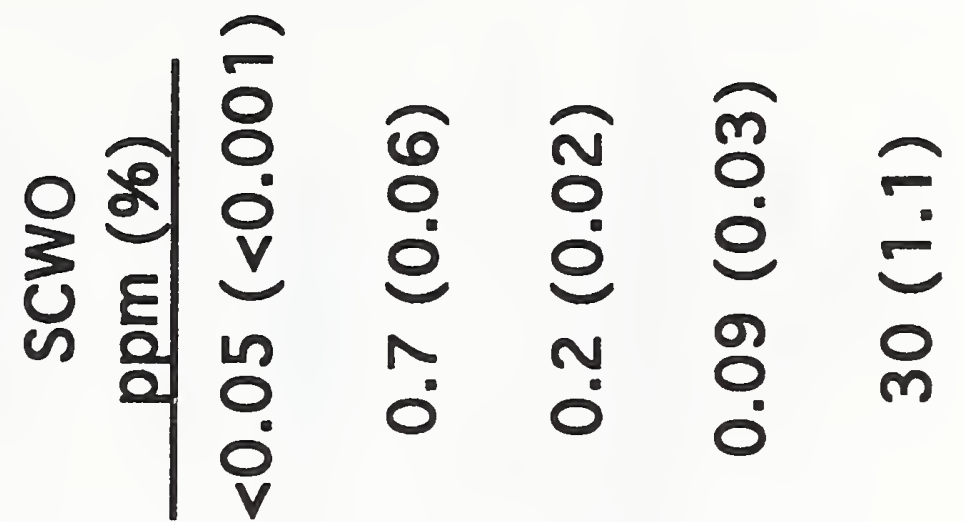

$\frac{8}{8}$

$\omega$

능

$\sum_{0}^{\circ}$

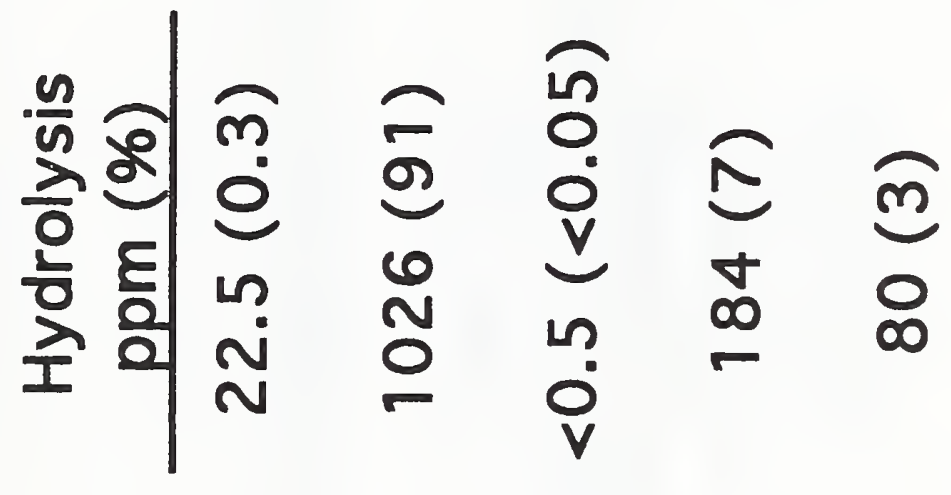

它

000

로

$\stackrel{N}{\sim} 0$

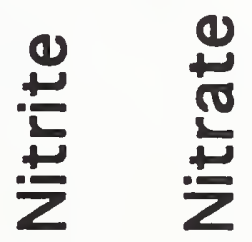

爻 
$\stackrel{⺊}{\mathbb{Z}}$

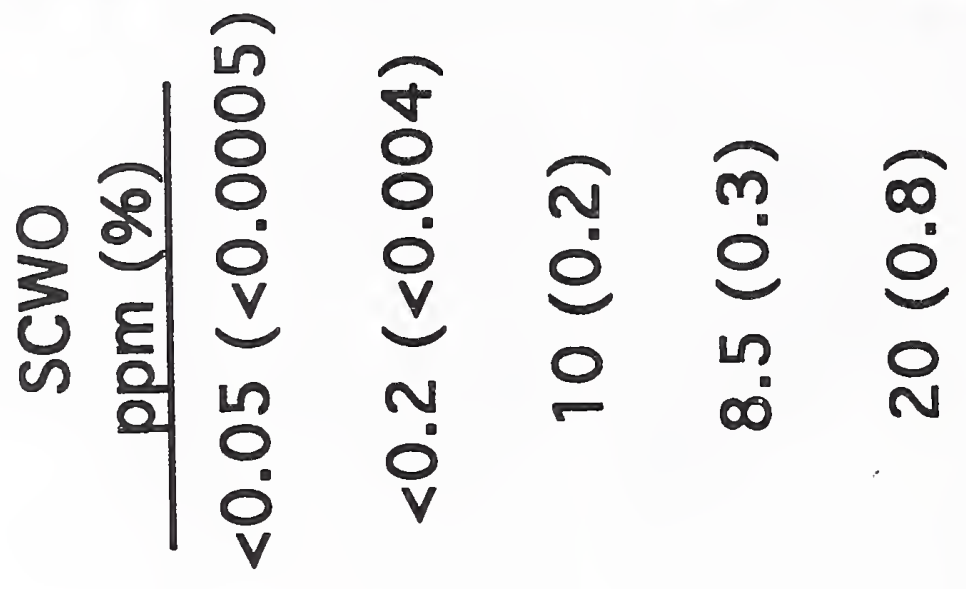

능

$\sum_{0}^{0}$

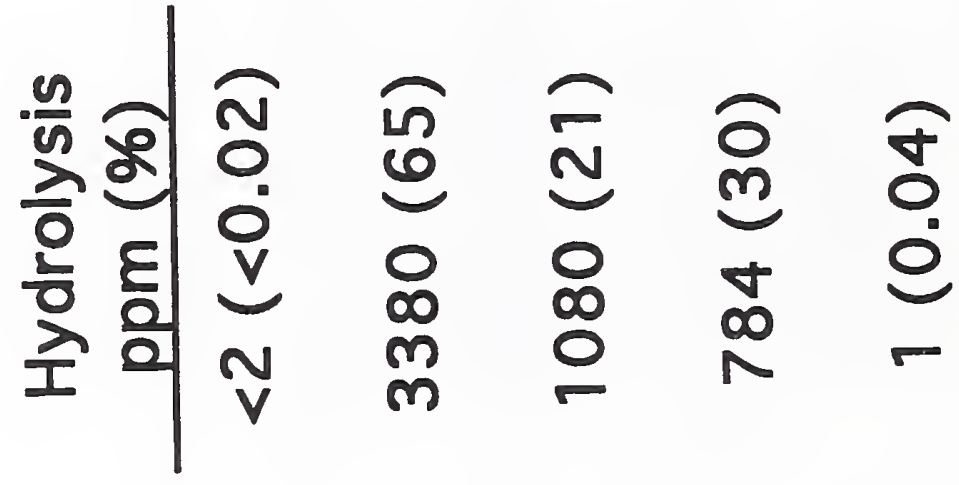

$\overline{4}$

$\frac{1}{2}$

물

I

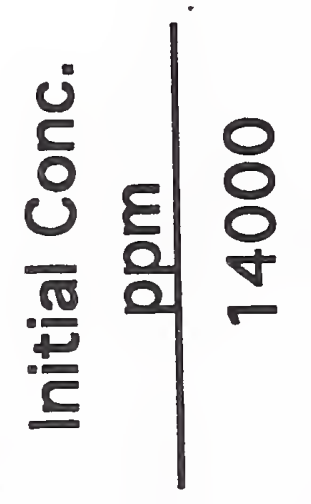

$\frac{5}{\infty}$

000

步

Ł 

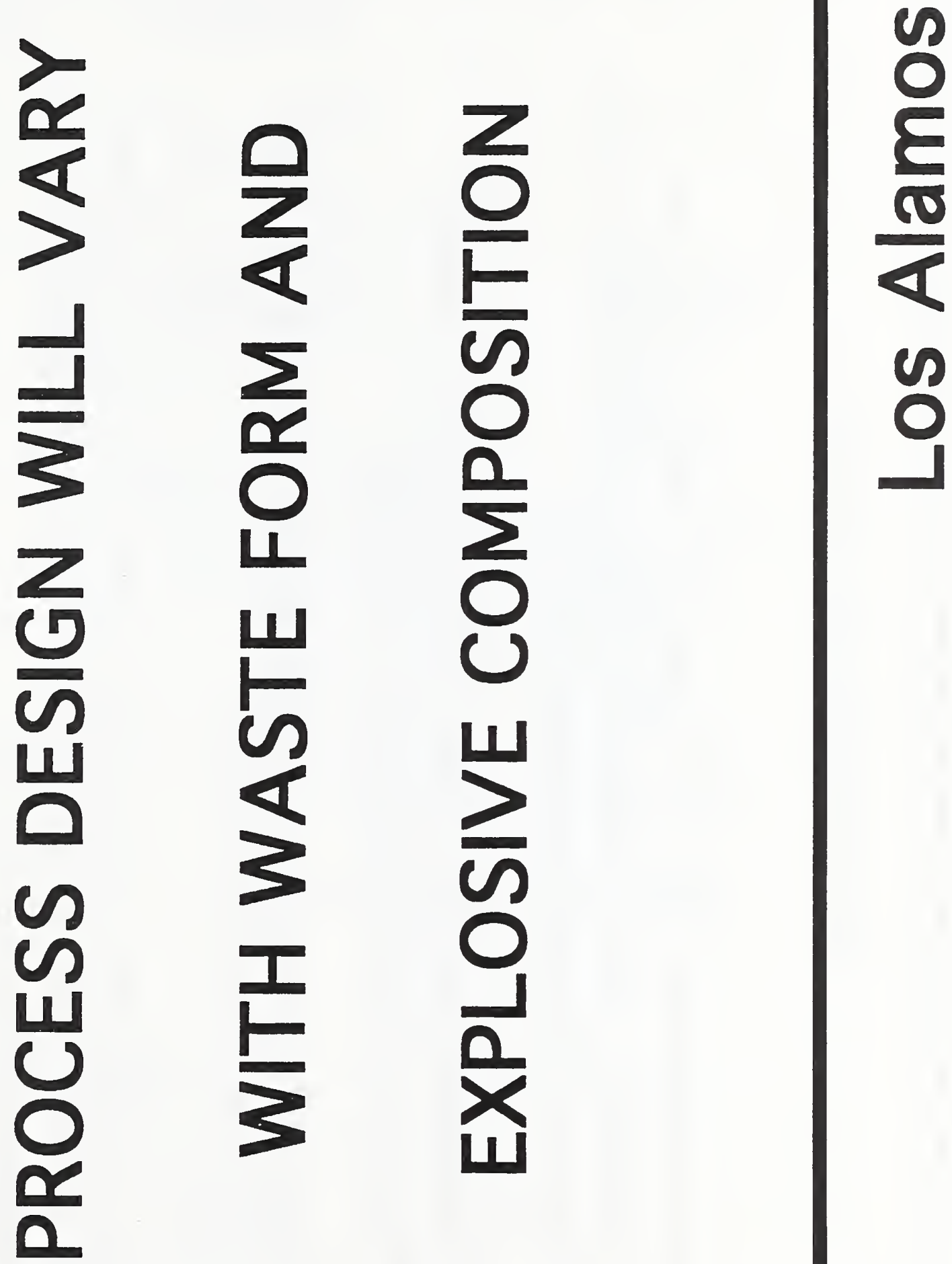

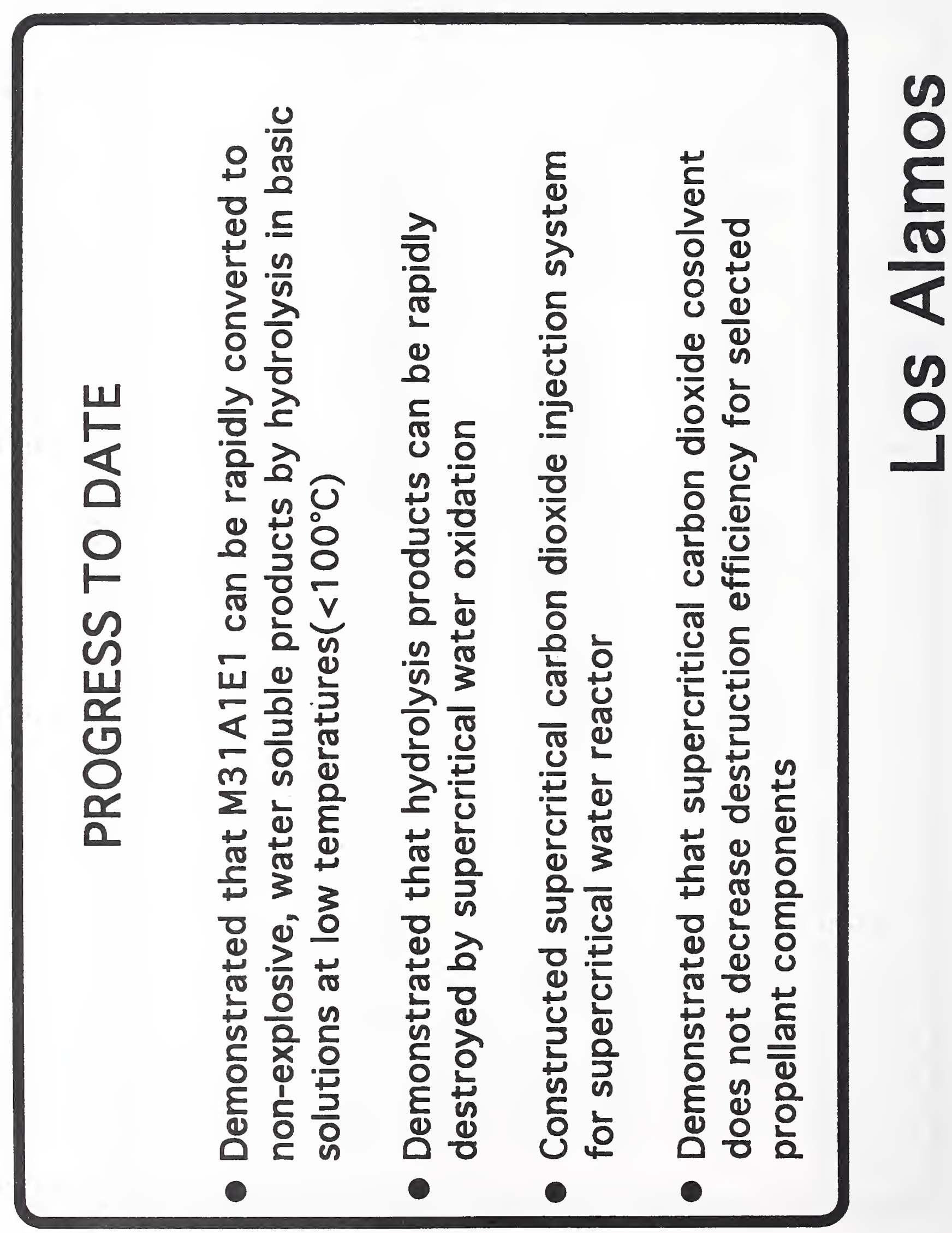


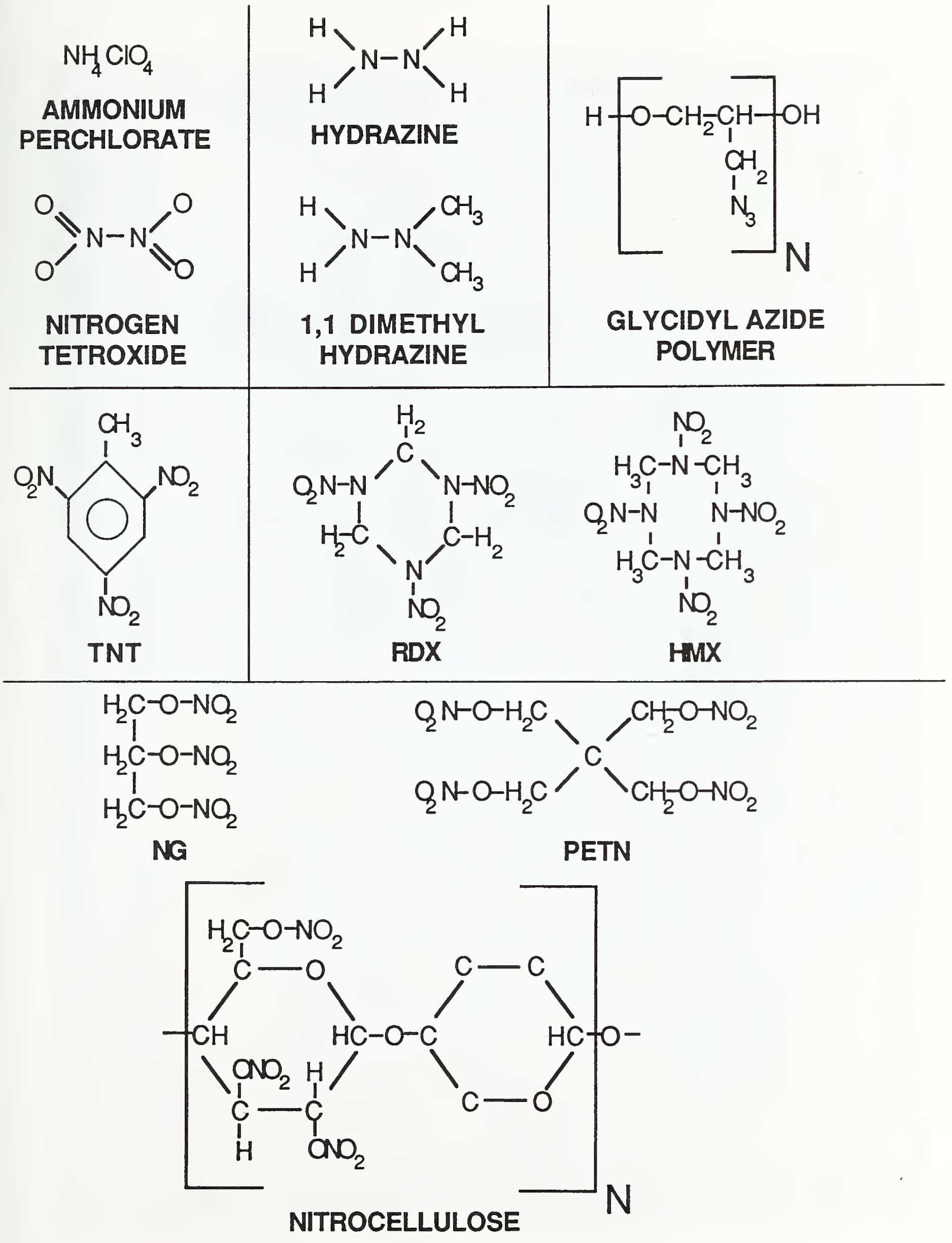



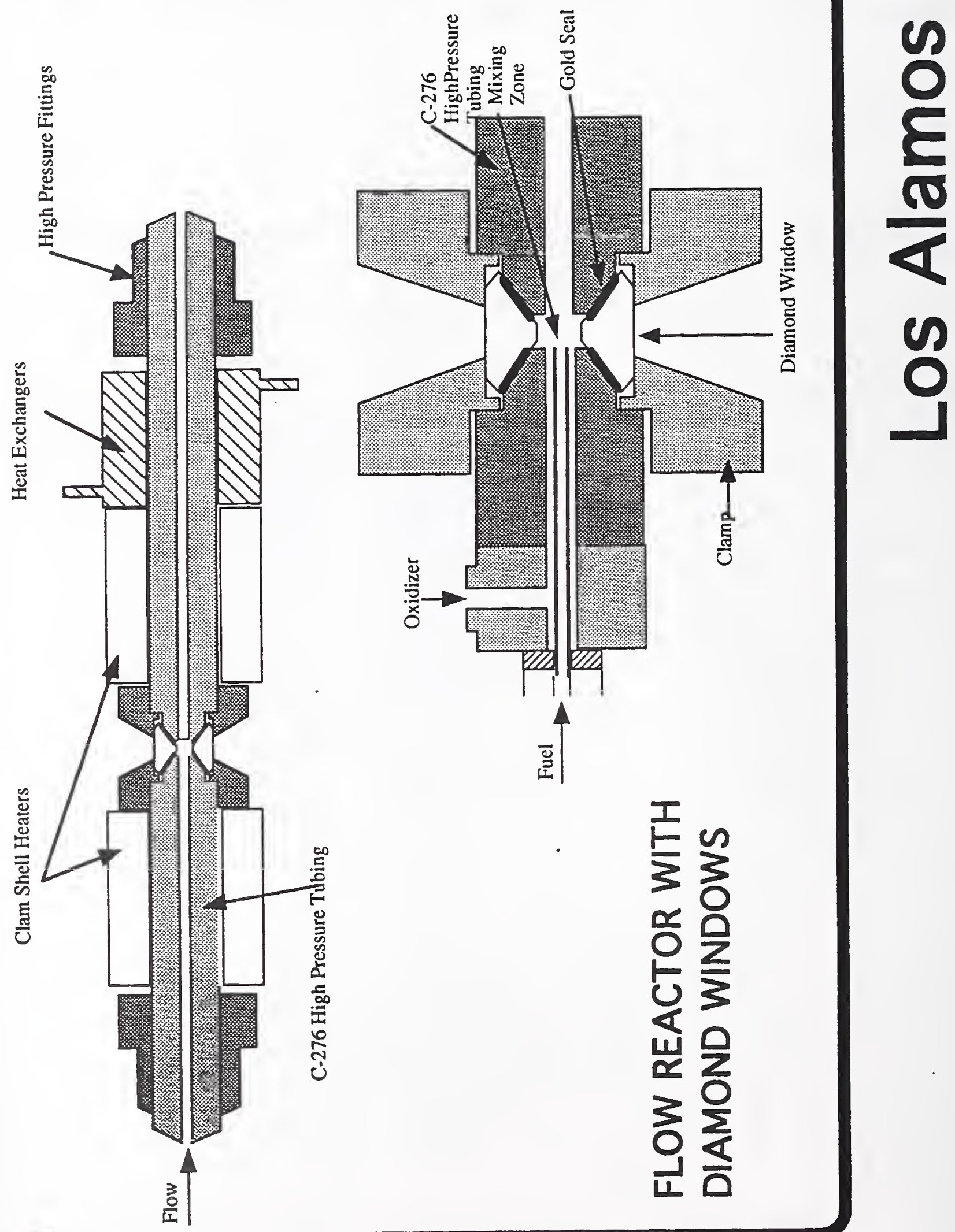


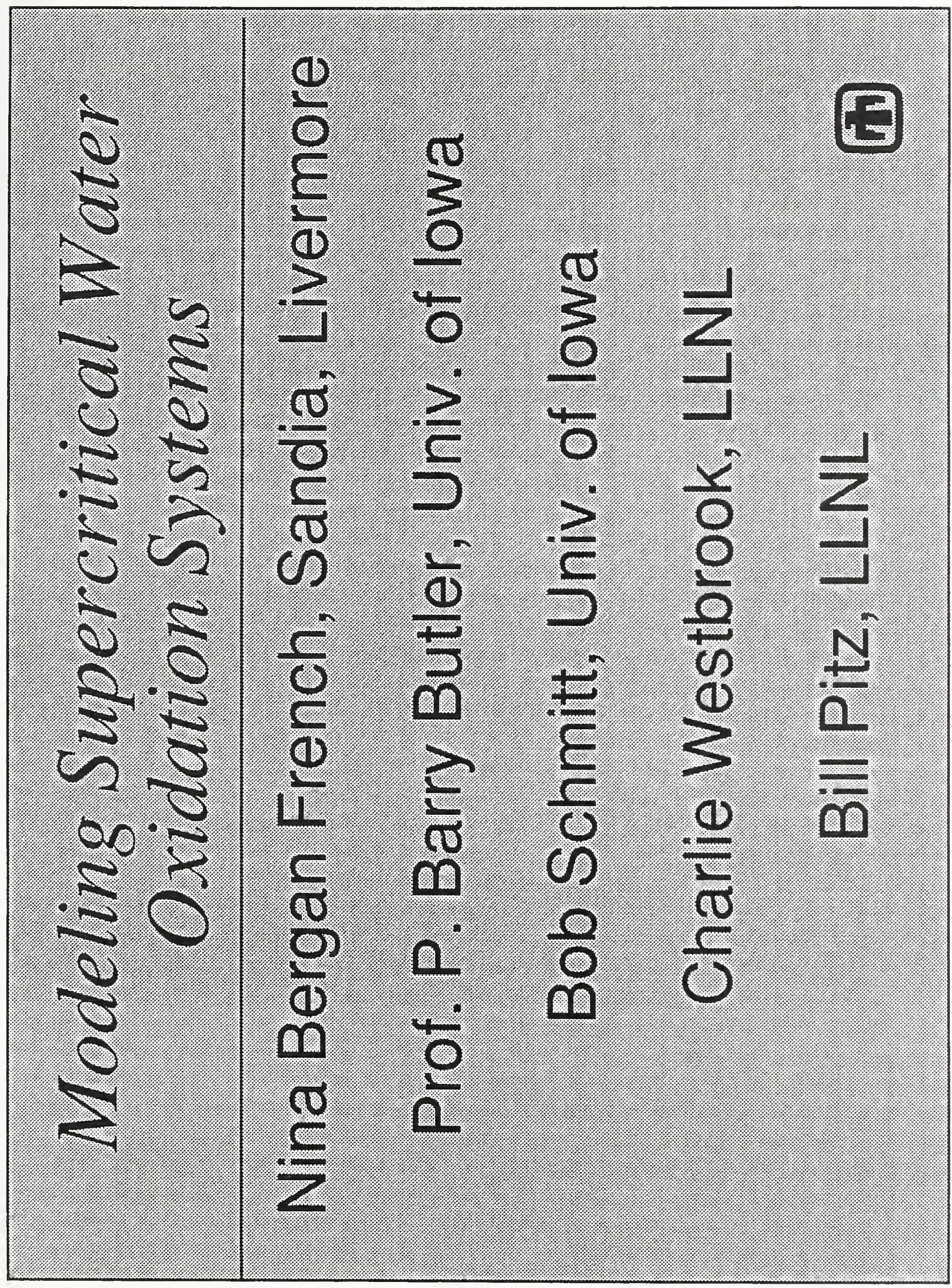




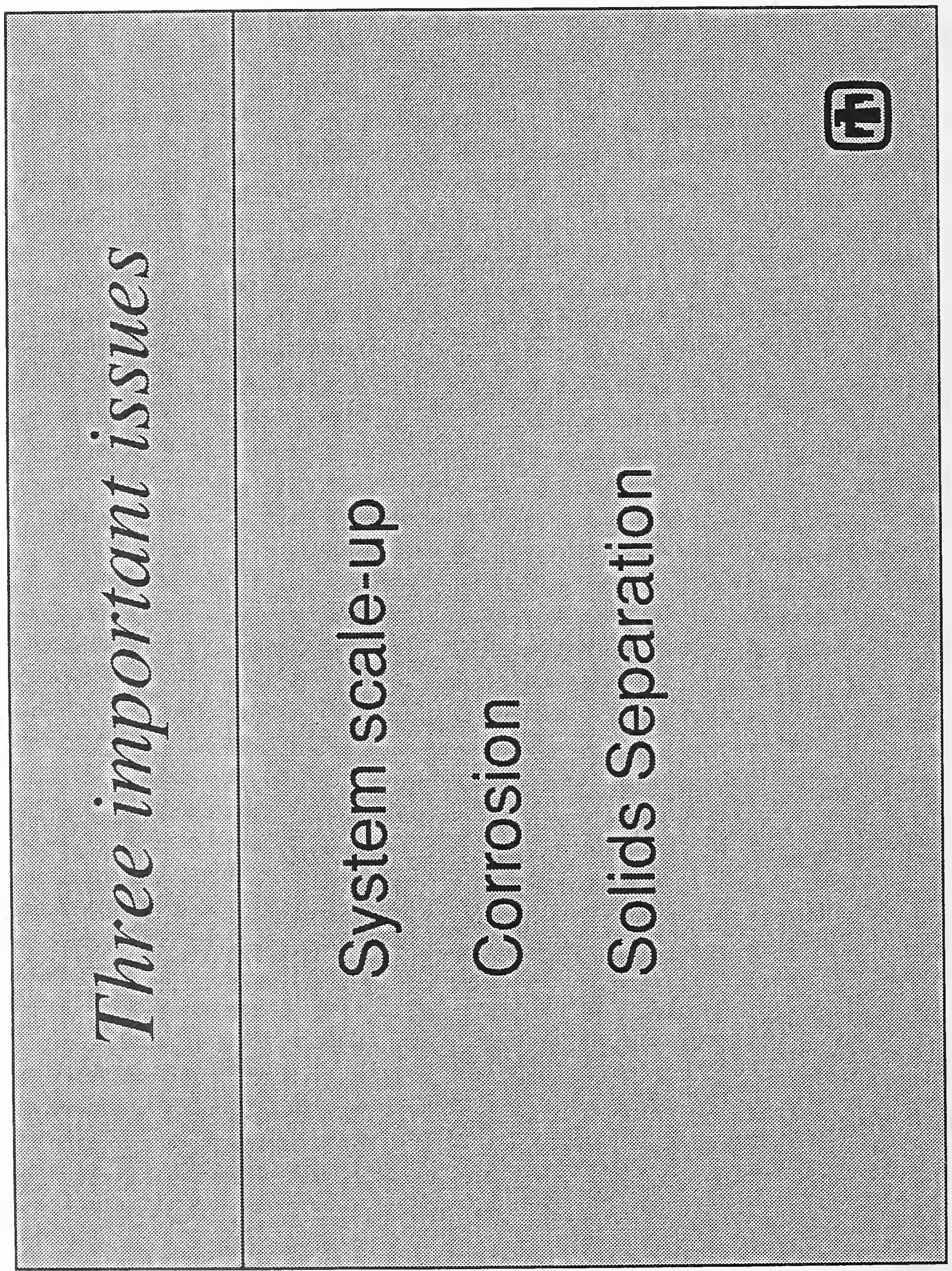




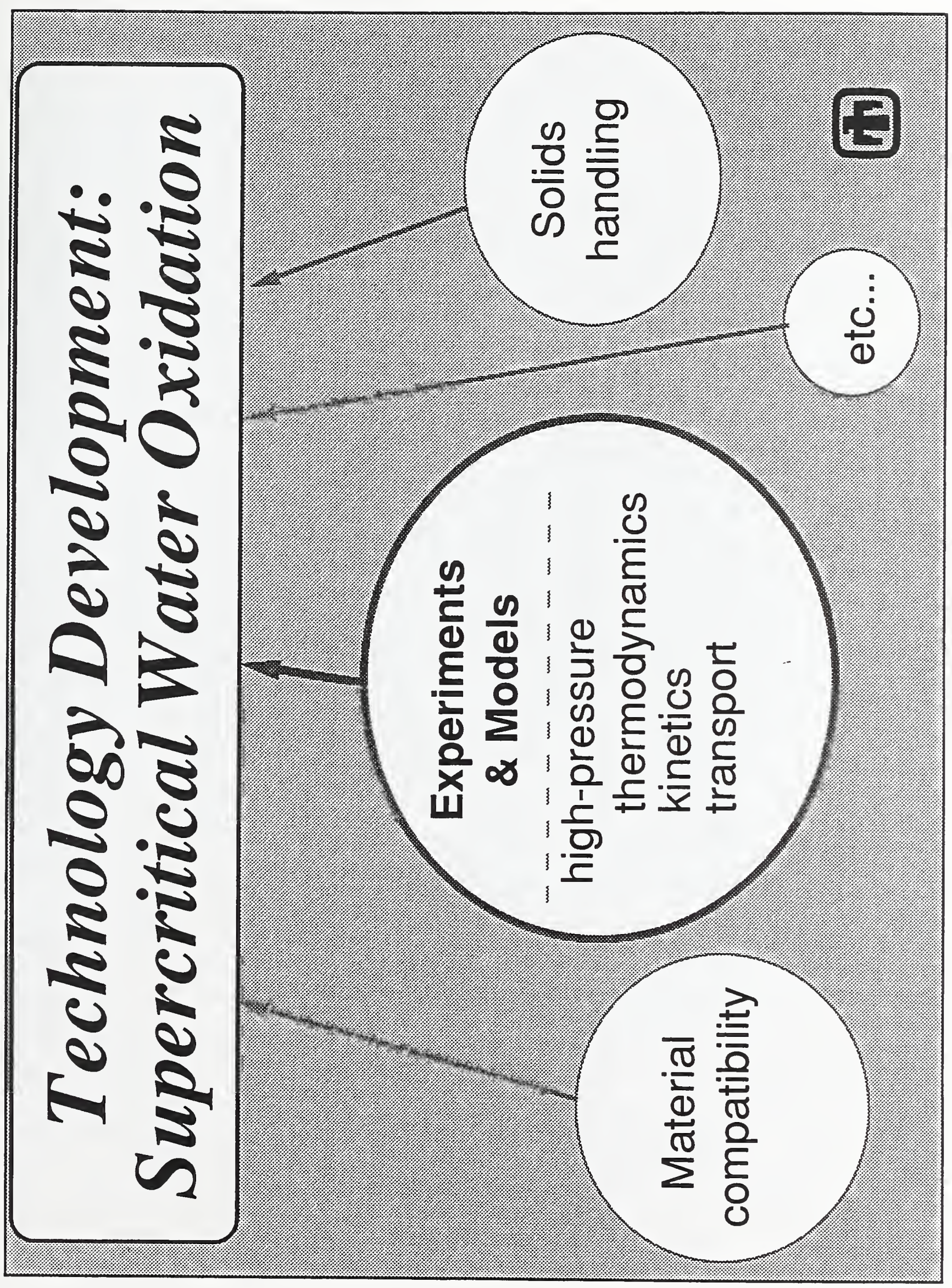




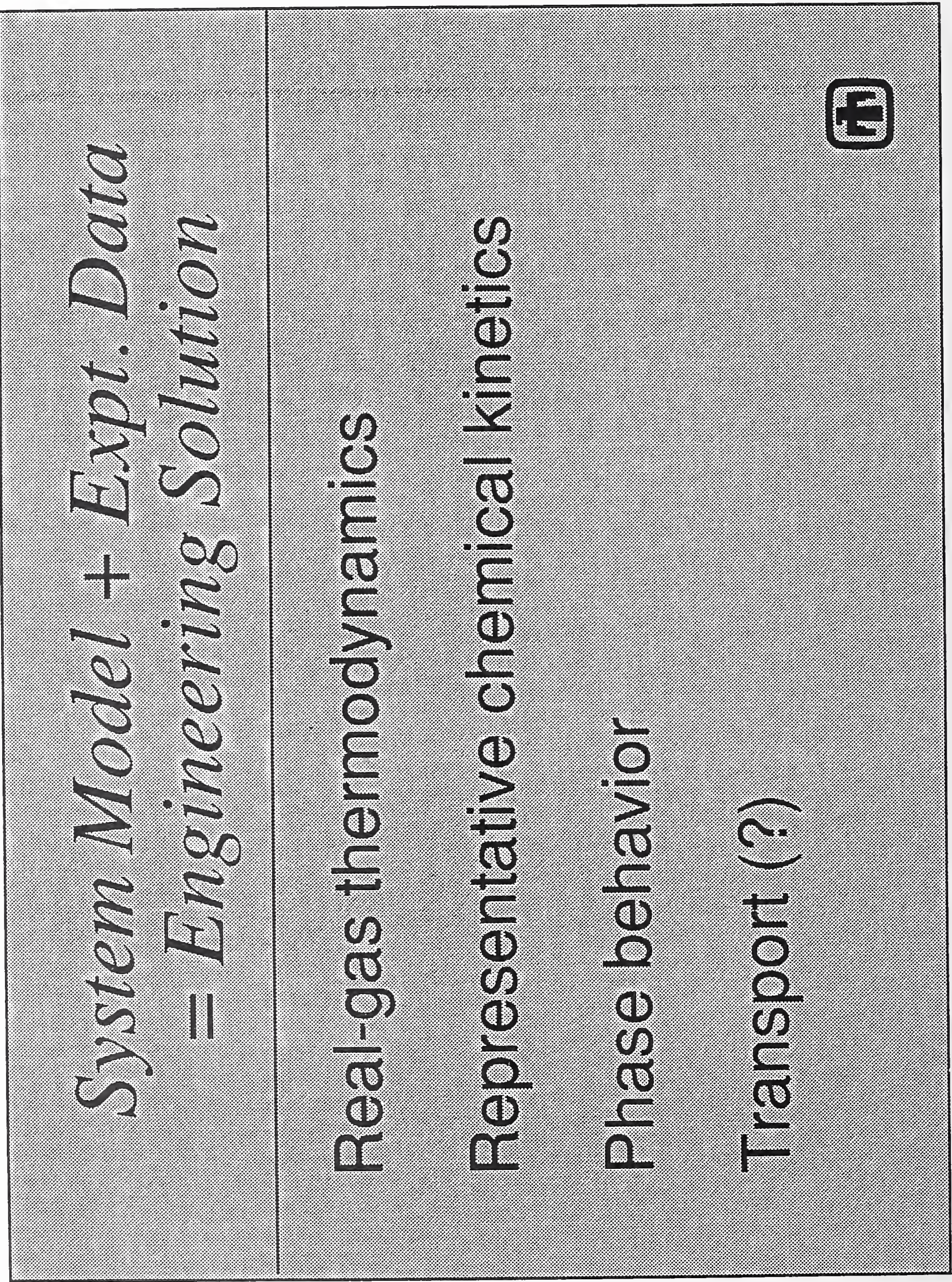




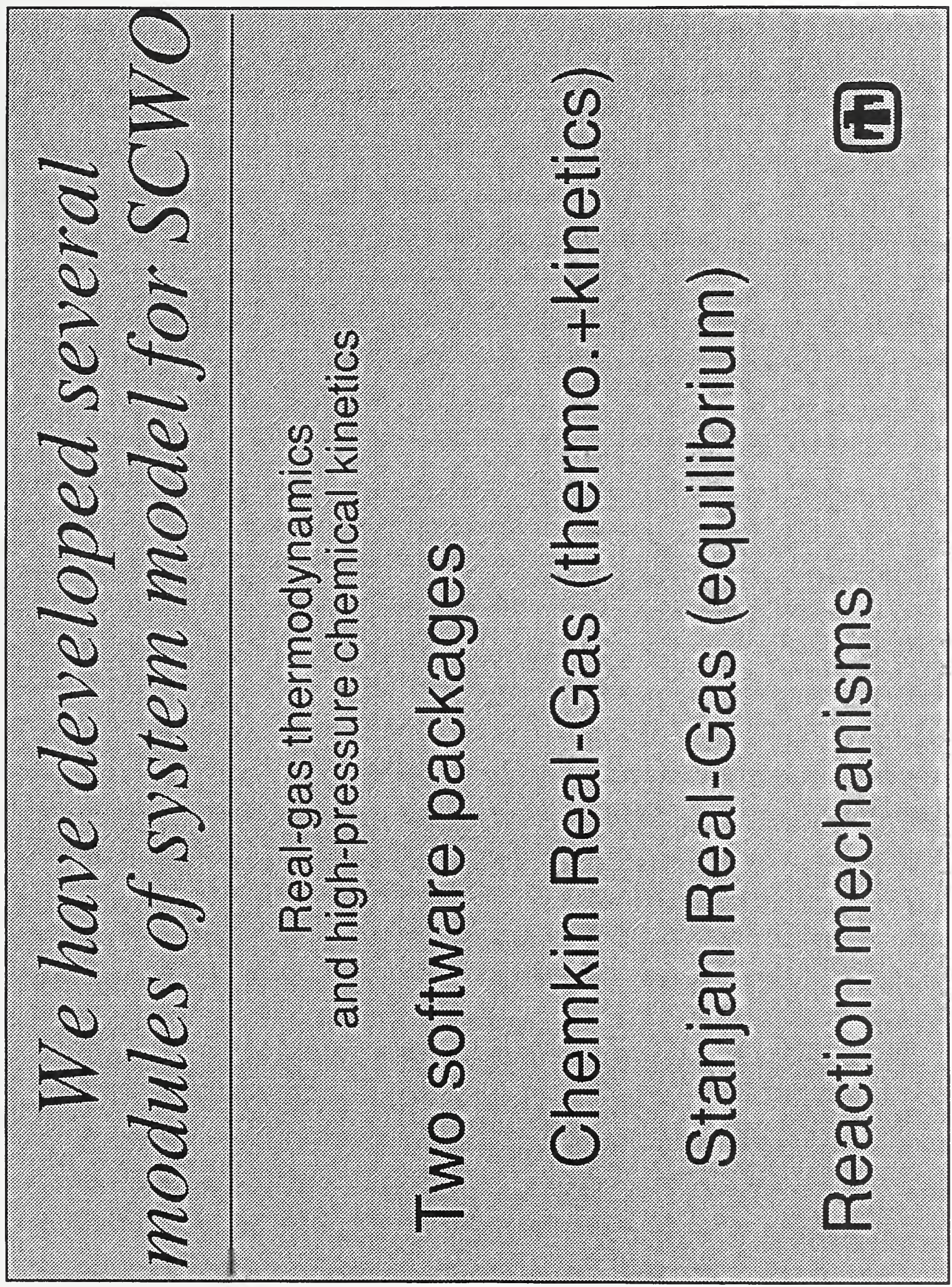




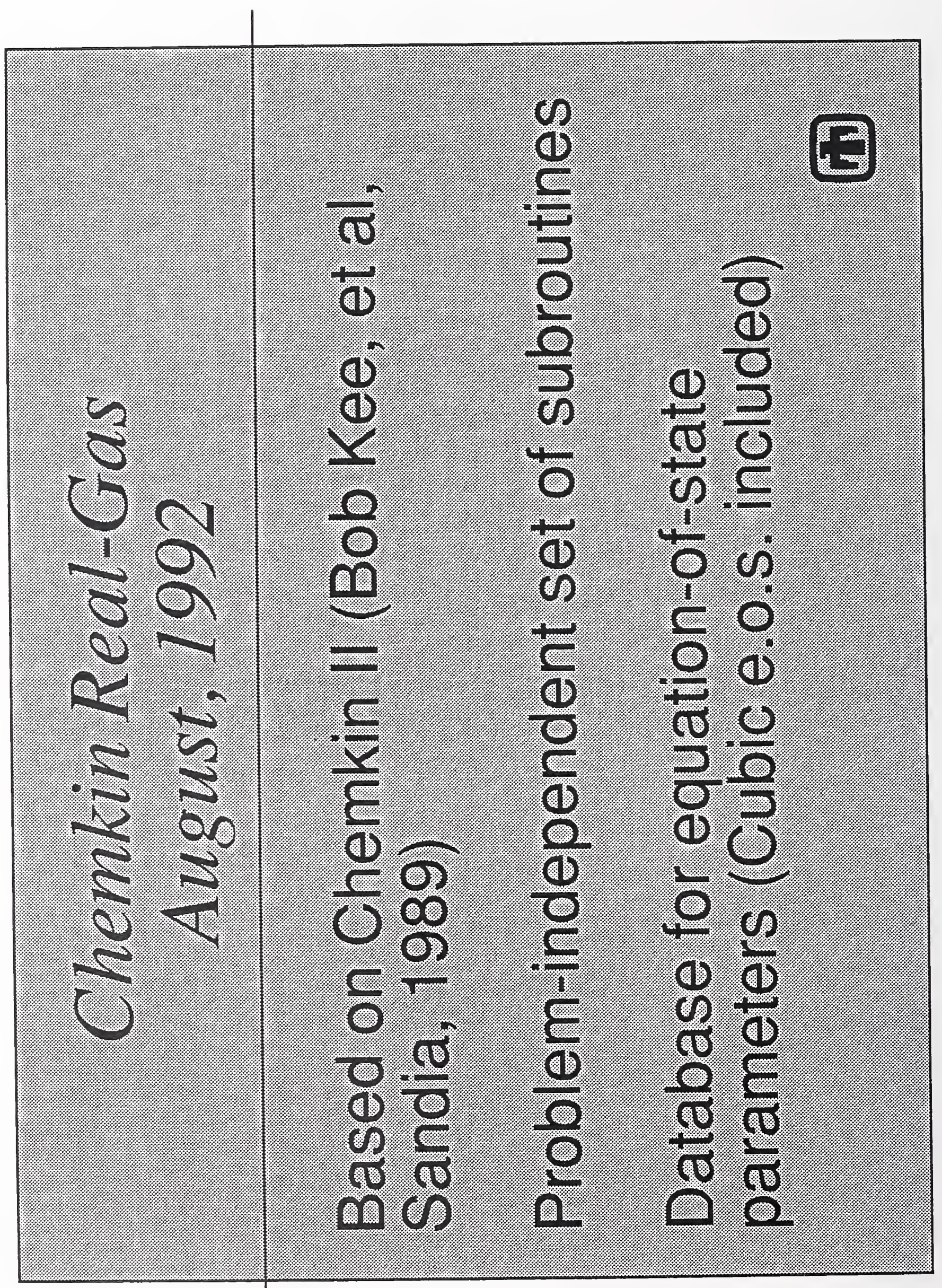




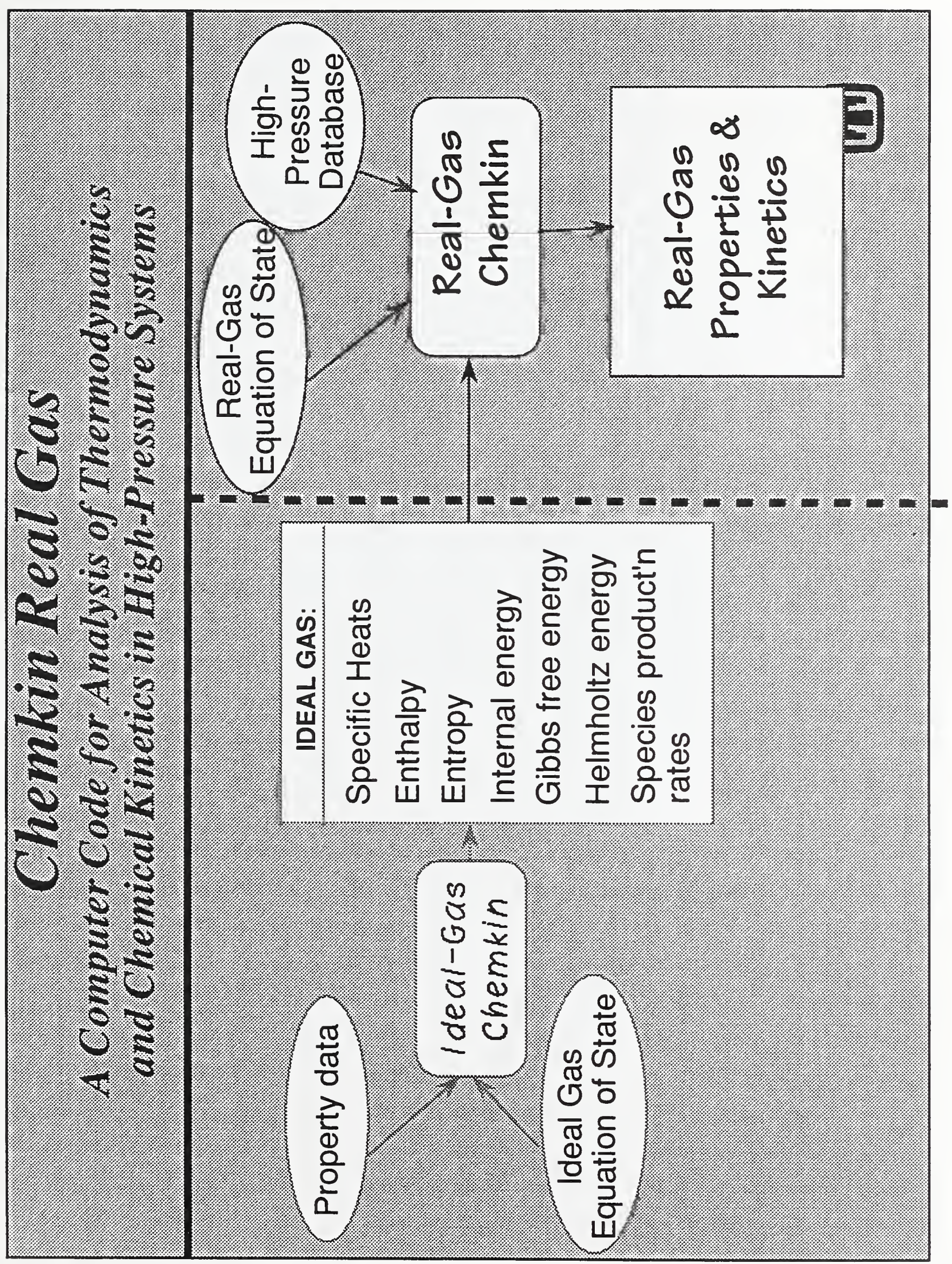




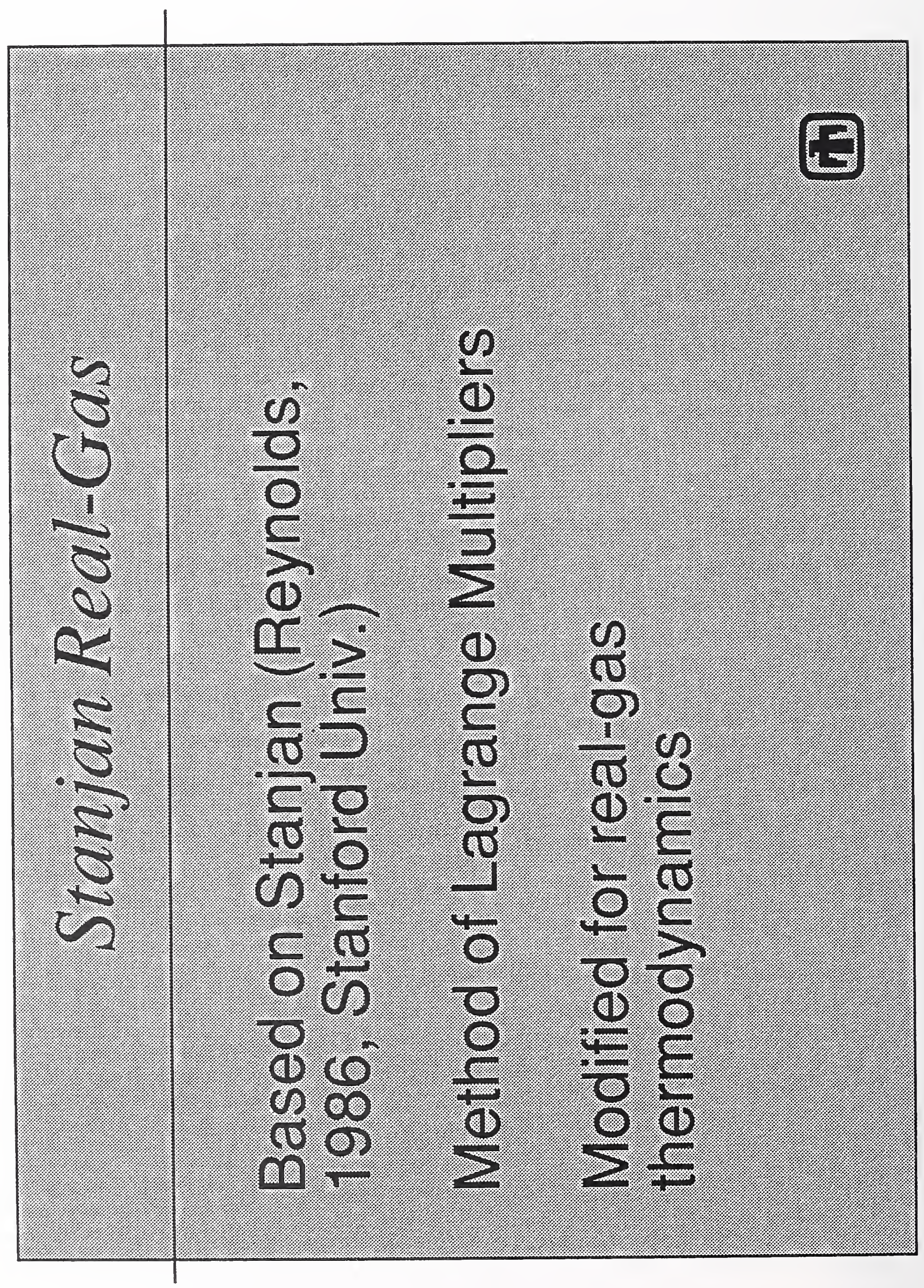




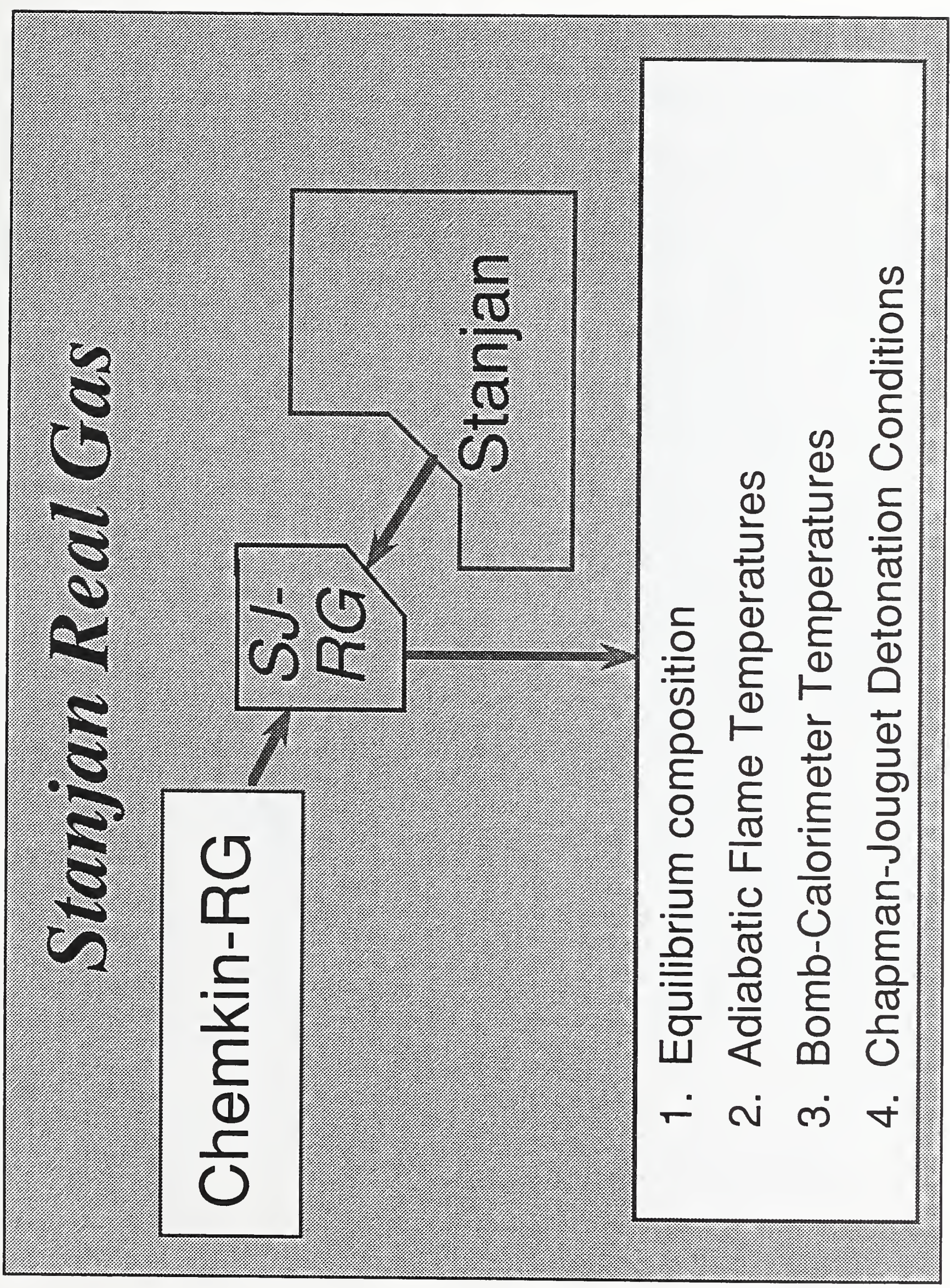




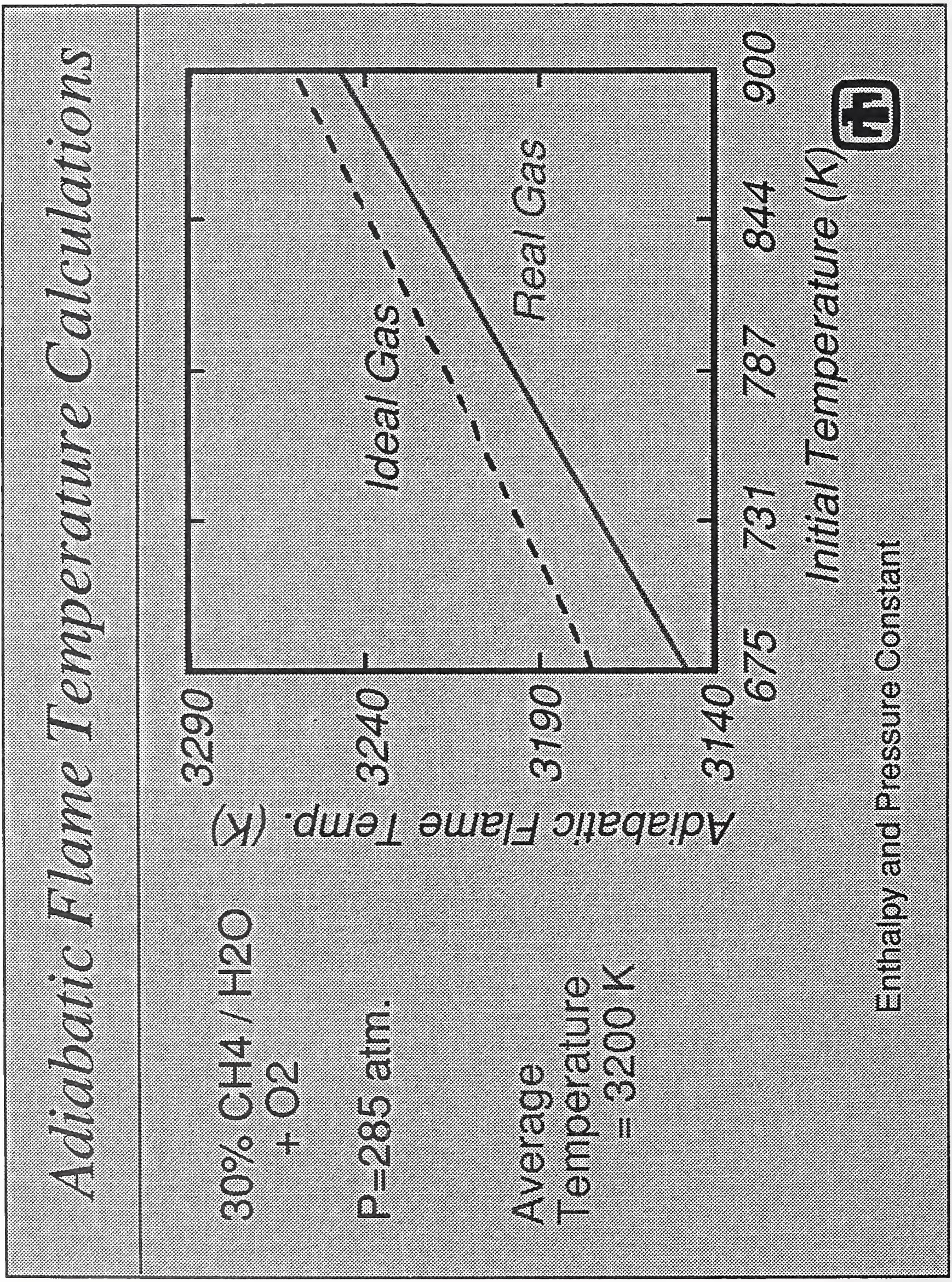




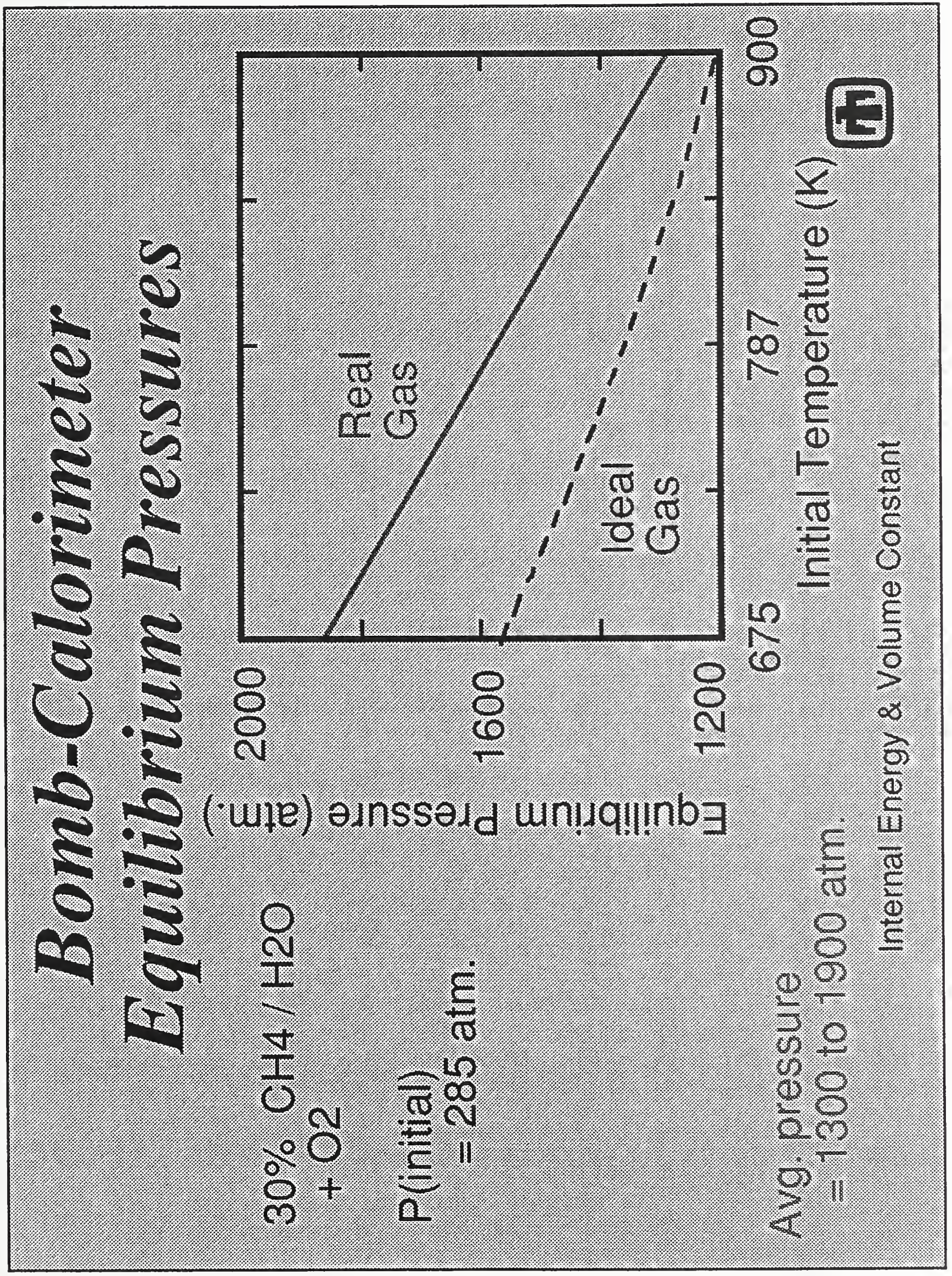




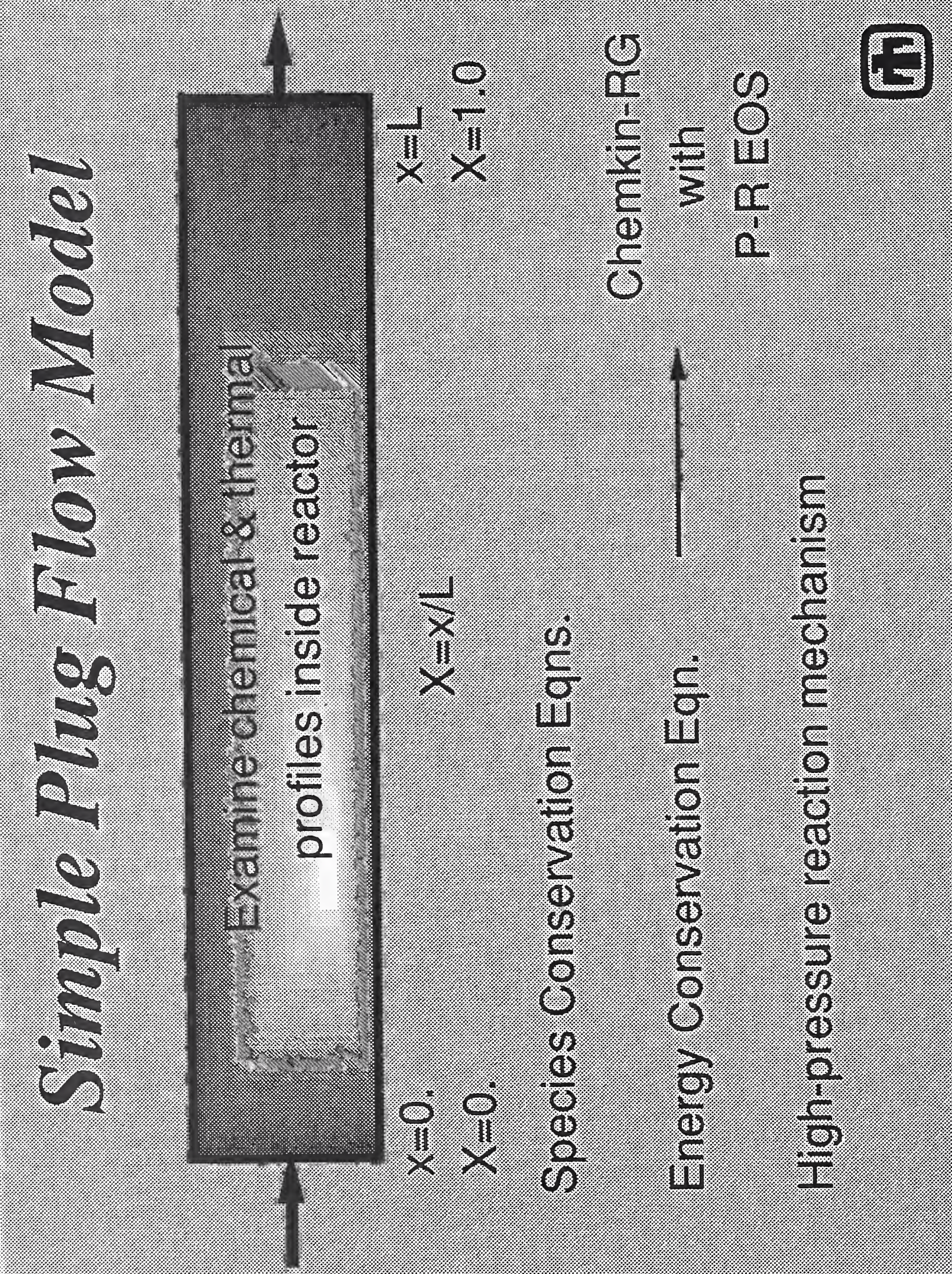




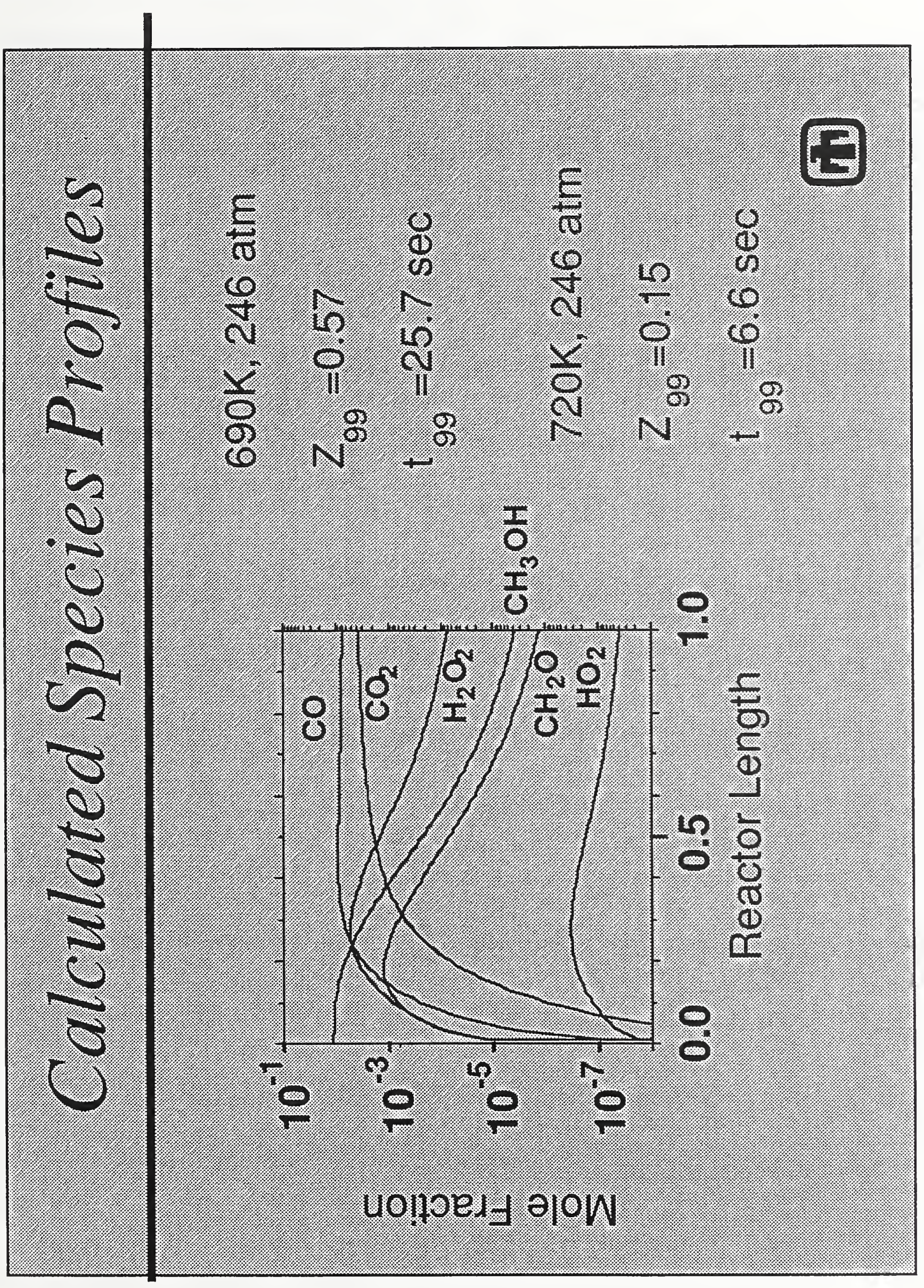




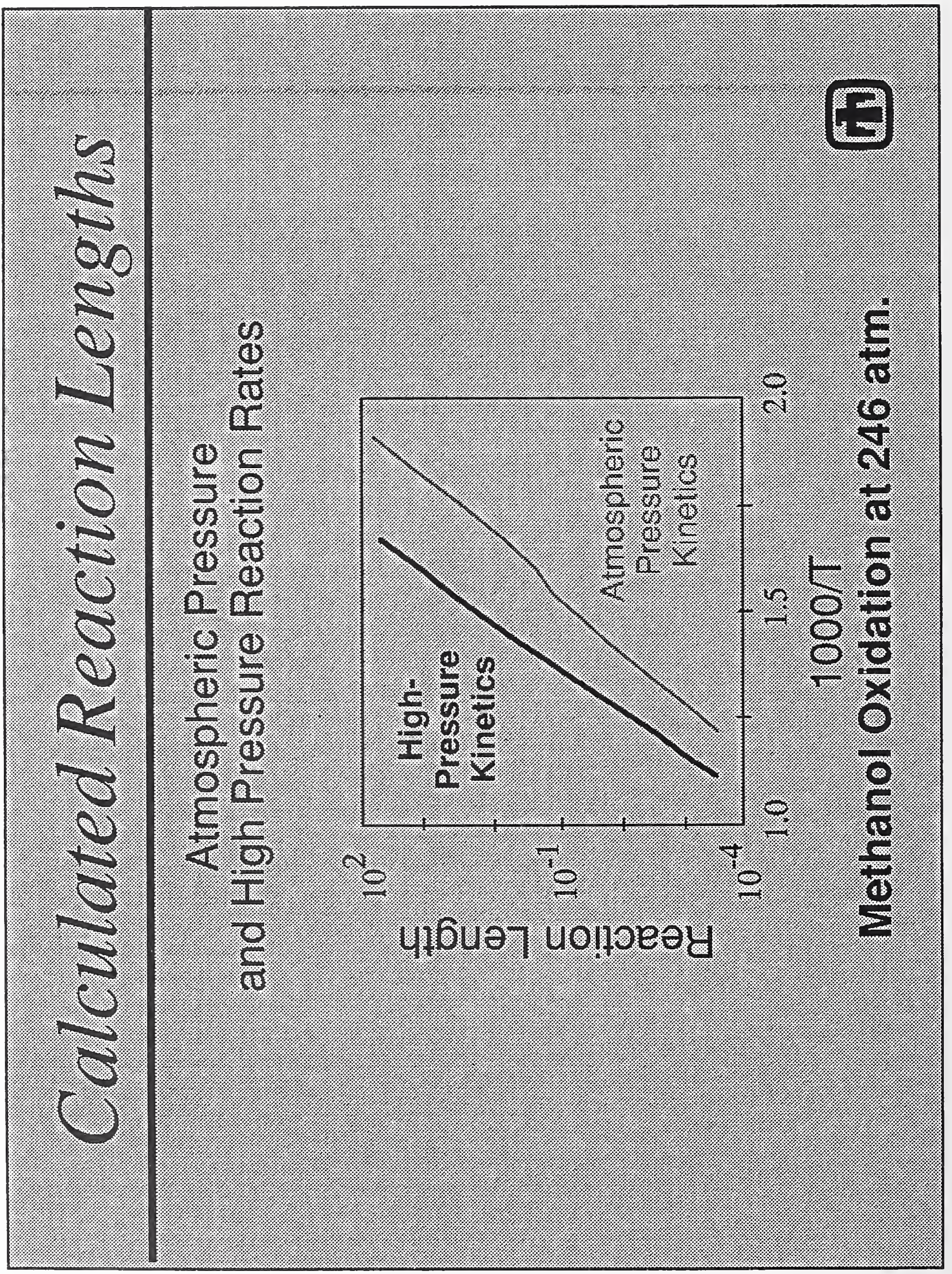




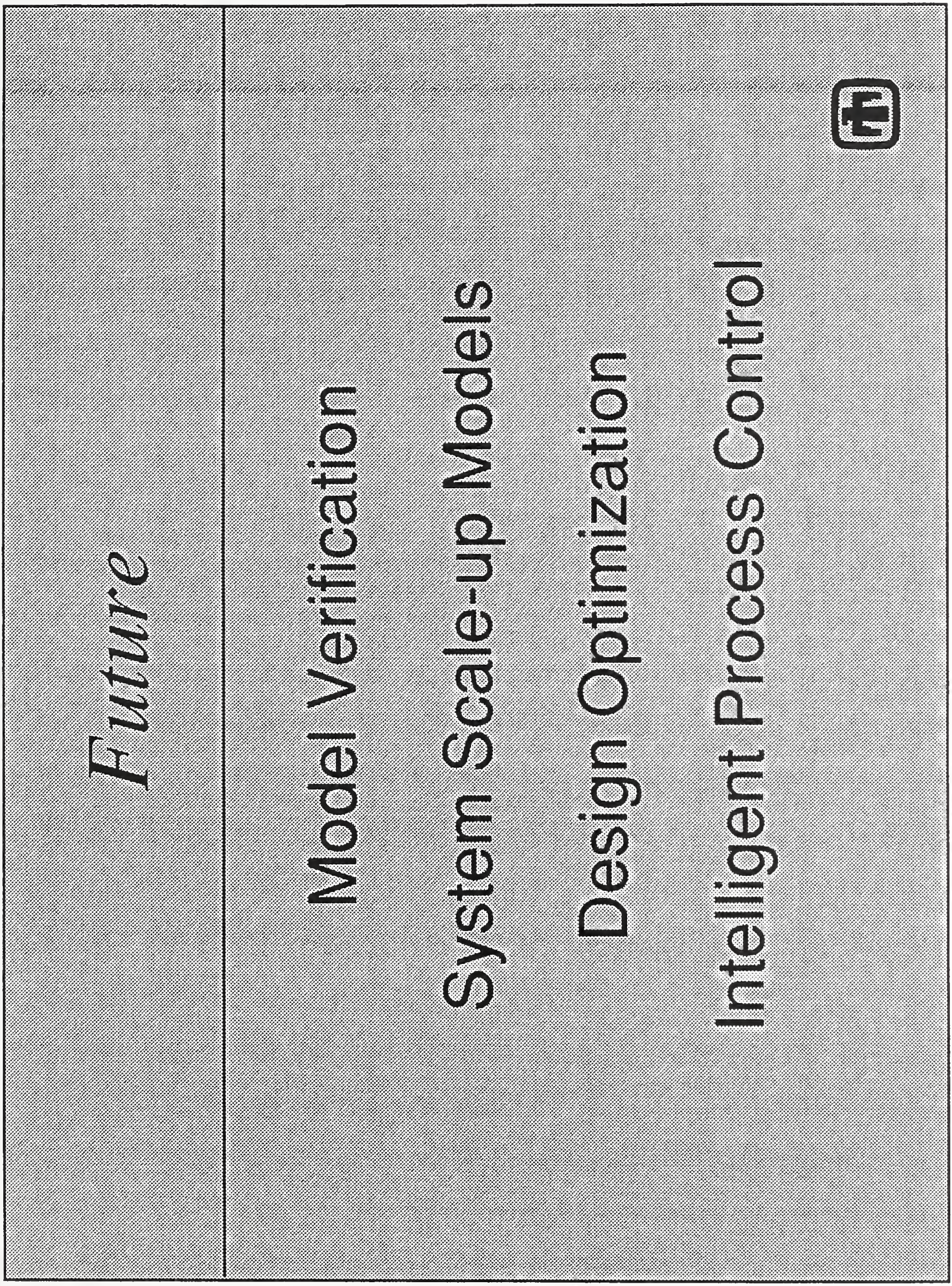


WORKSHOP ON FEDERAL PROGRAMS

INVOLVING SUPERCRITICAL WATER OXIDATION

July $6-7,1992$ at NIST

THERMOPHYSICAL PROPERTIES

OF SUPERCRITICAL MULTICOMPONENT AQUEOUS SYSTEMS

J.M.H. Levelt Sengers

Thermophysics Division, NIST, Gaithersburg

Part I

(Part II, by Dan Friend, on interactive computing, follows next)

QUESTIONS WE ARE BEING ASKED

\section{*Phase Boundaries}

Under what conditions does a solid salt separate out?

Under what conditions does a brine separate out?

Does the vapor phase contain salt?

What are the solubilities of gases such as $\mathrm{CO}_{2}, \mathrm{O}_{2}$, etc., in water at high temperatures and pressures?

*Thermophysical Properties for Reactor Design

Enthalpy, density, heat capacity

Viscosity, thermal diffusivity

*Chemical Reactions in Highly Nonideal Mixtures

Nonionic components

Ionic components 


\section{FACTS OF LIFE}

* Water is a Unique Fluid

Very wide coexistence curve

Very high $\mathrm{T}_{\mathrm{c}}$

Large variation of dielectric constant

*Phase Diagrams of Aqueous Systems are Complex and Varied

$$
\begin{aligned}
& \mathrm{NaCl}-\mathrm{H}_{2} \mathrm{O} \\
& \mathrm{O}_{2}-\mathrm{H}_{2} \mathrm{O} \\
& \mathrm{CO}_{2}-\mathrm{H}_{2} \mathrm{O} \\
& \text { Alkanes }-\mathrm{H}_{2} \mathrm{O}
\end{aligned}
$$

*Methods Developed for Liquid Mixtures Fail: in this Regime Large anomalies in excess properties

Partial molar properties of solute, and related standard states, are highly anomalous. 
COMPLEX PHASE DIAGRAMS OF AQUEOUS BINARY MIXTURES $\rightarrow$ 
S. Sourirajan and G. C. Kennedy
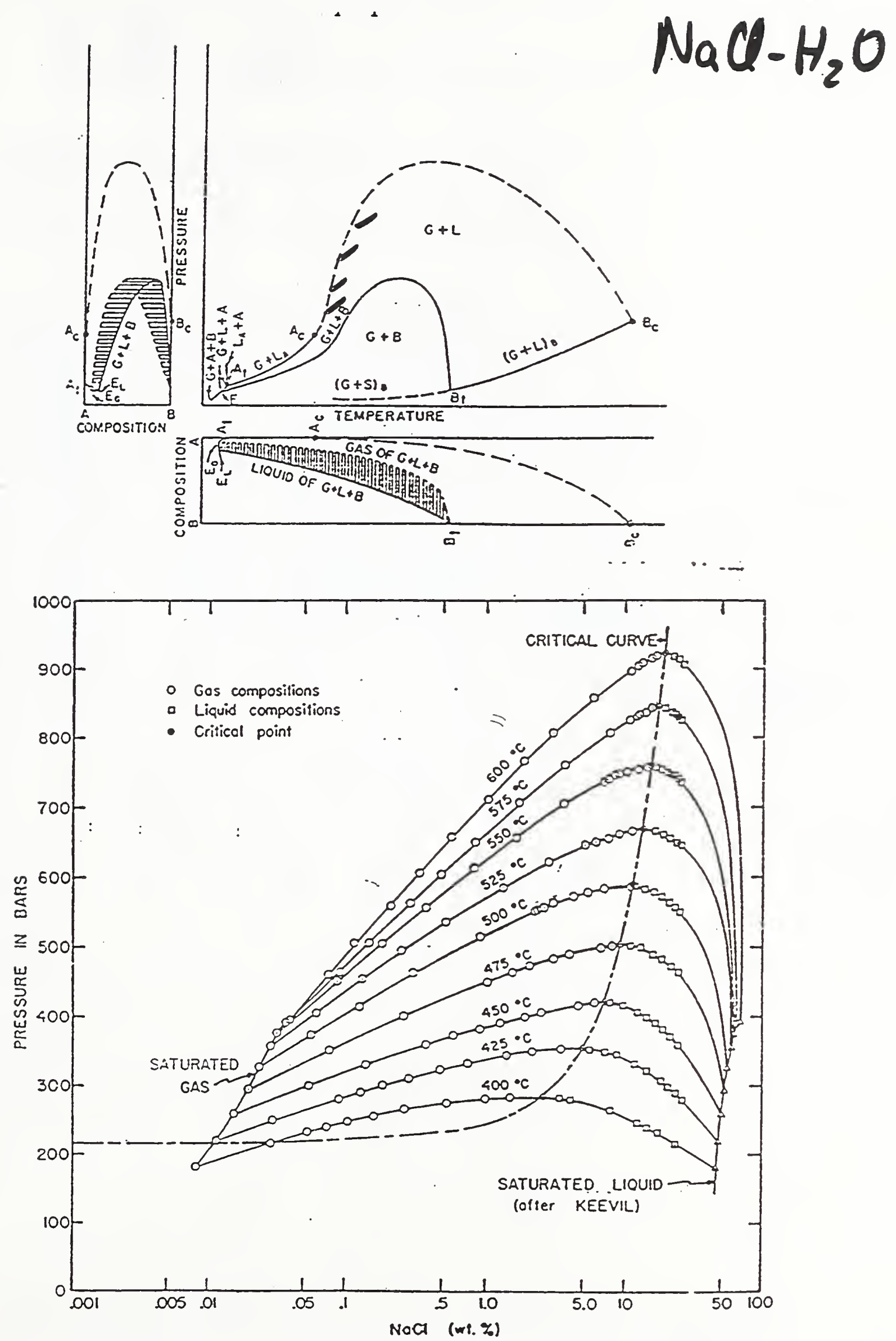

Fig. 13. Isotherms, $400^{\circ} \mathrm{C} \cdot 600^{\circ} \mathrm{C}$, showing compositions of coexisting gases and liquids. 
$\mathrm{O}_{2}-\mathrm{H}_{2} \mathrm{O}$

M. L. Japas and E. U. Franck

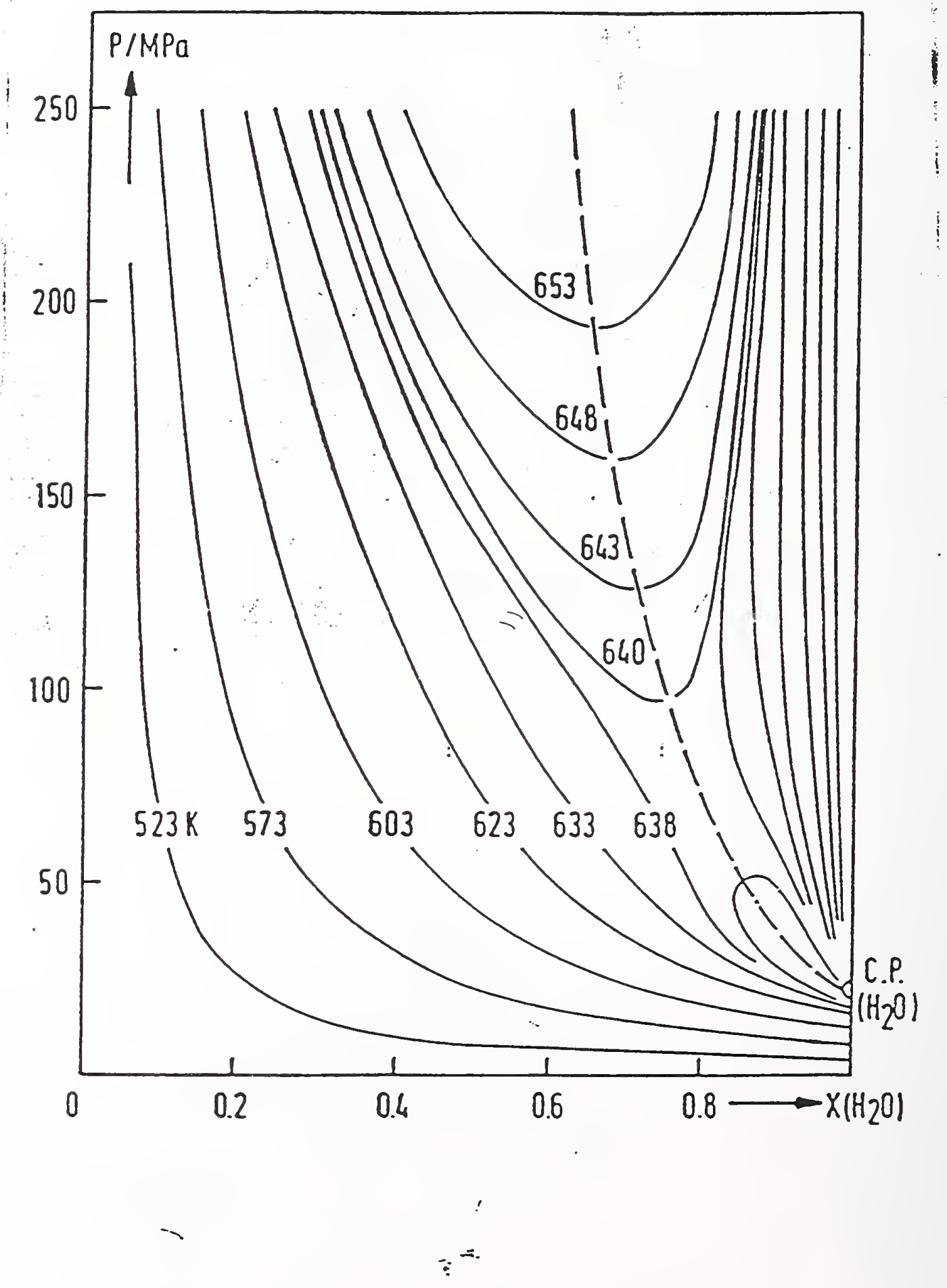



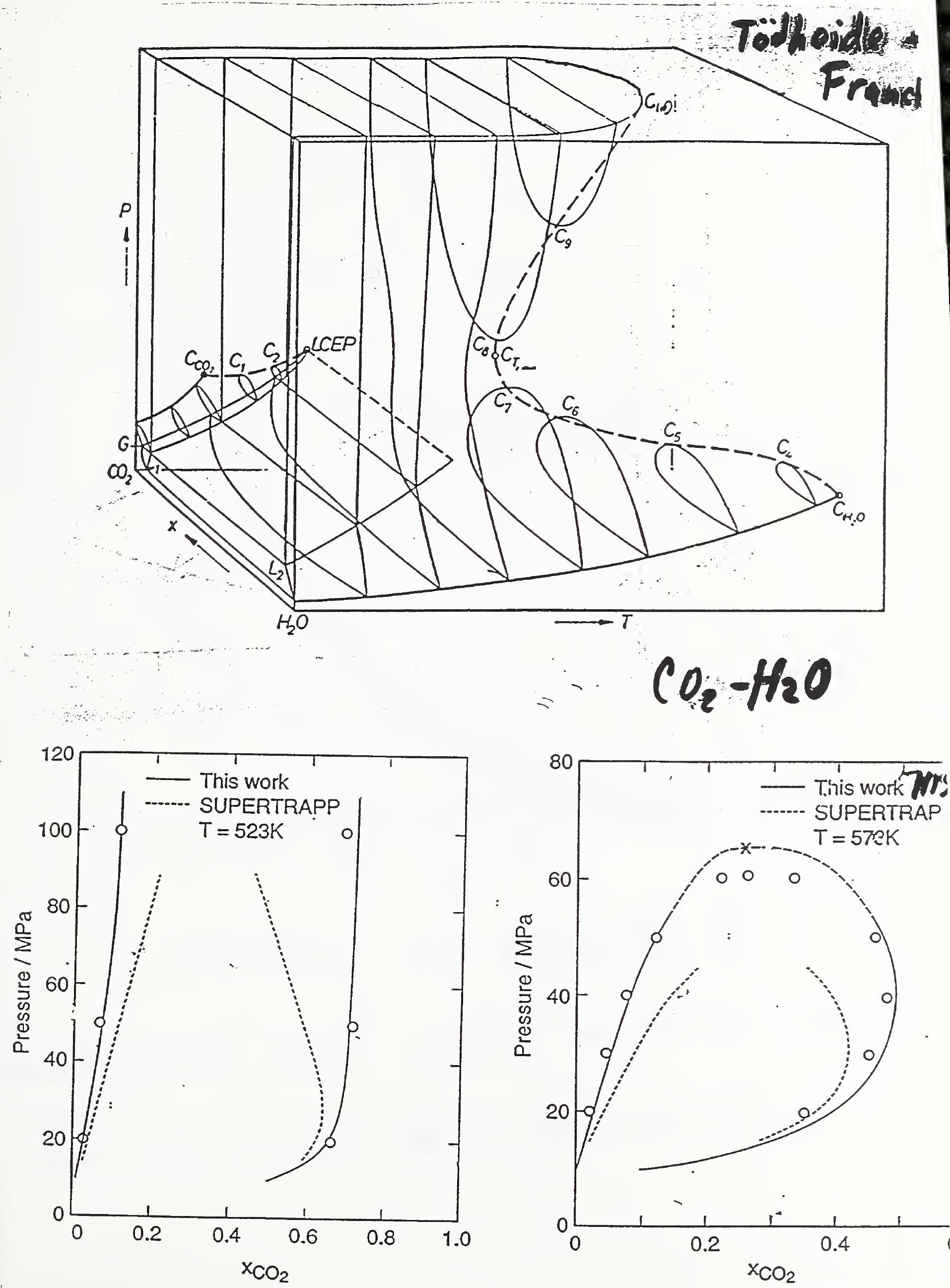
E. BRYNNER

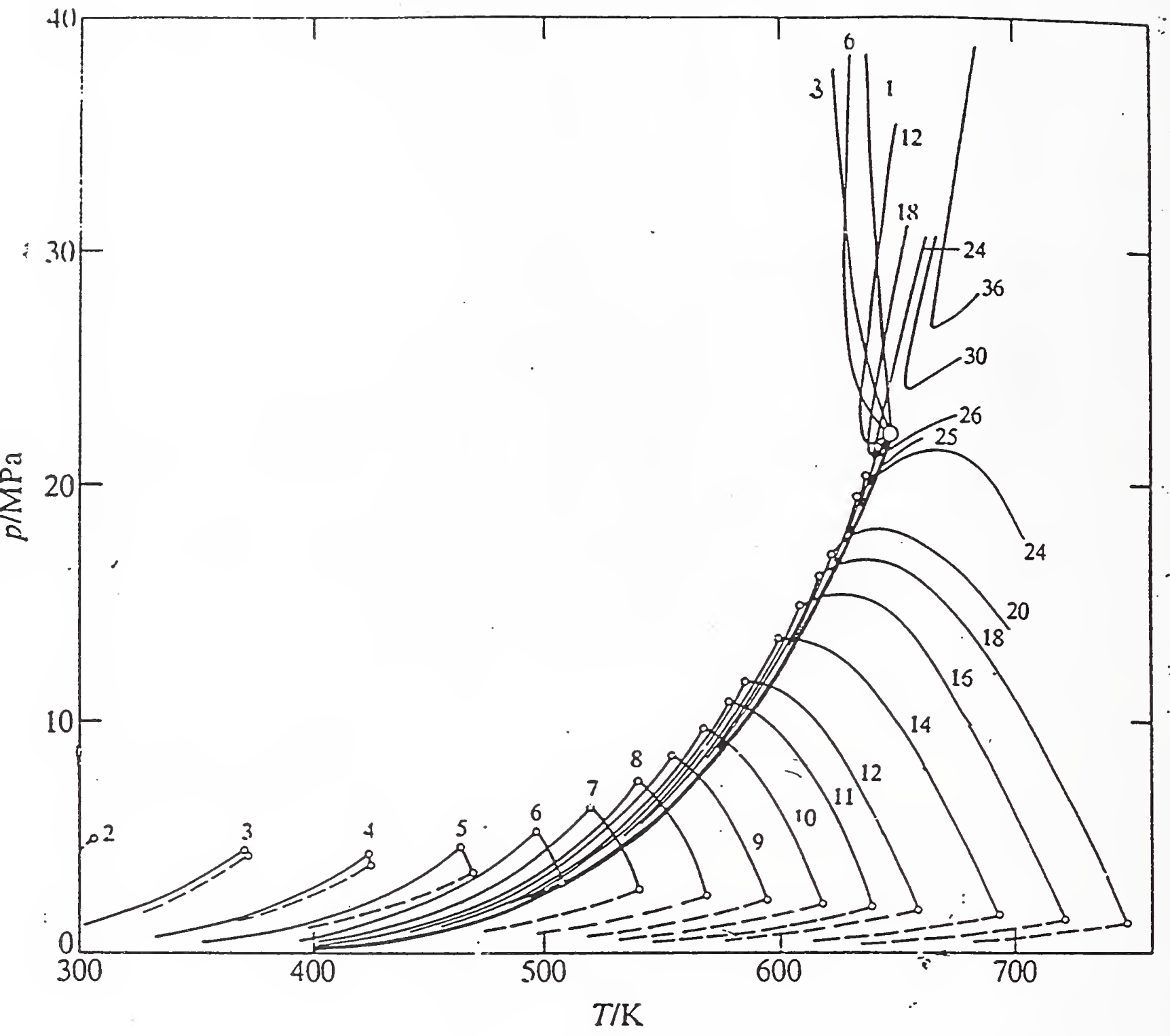

$n$-alkanes in water

critical lines and three -phase

lines

-... pure-component vapor pressure

220 
ANOMALOUS EXCESS ENTHALPIES

AND APPARENT MOLAR VOLUMES 
1322

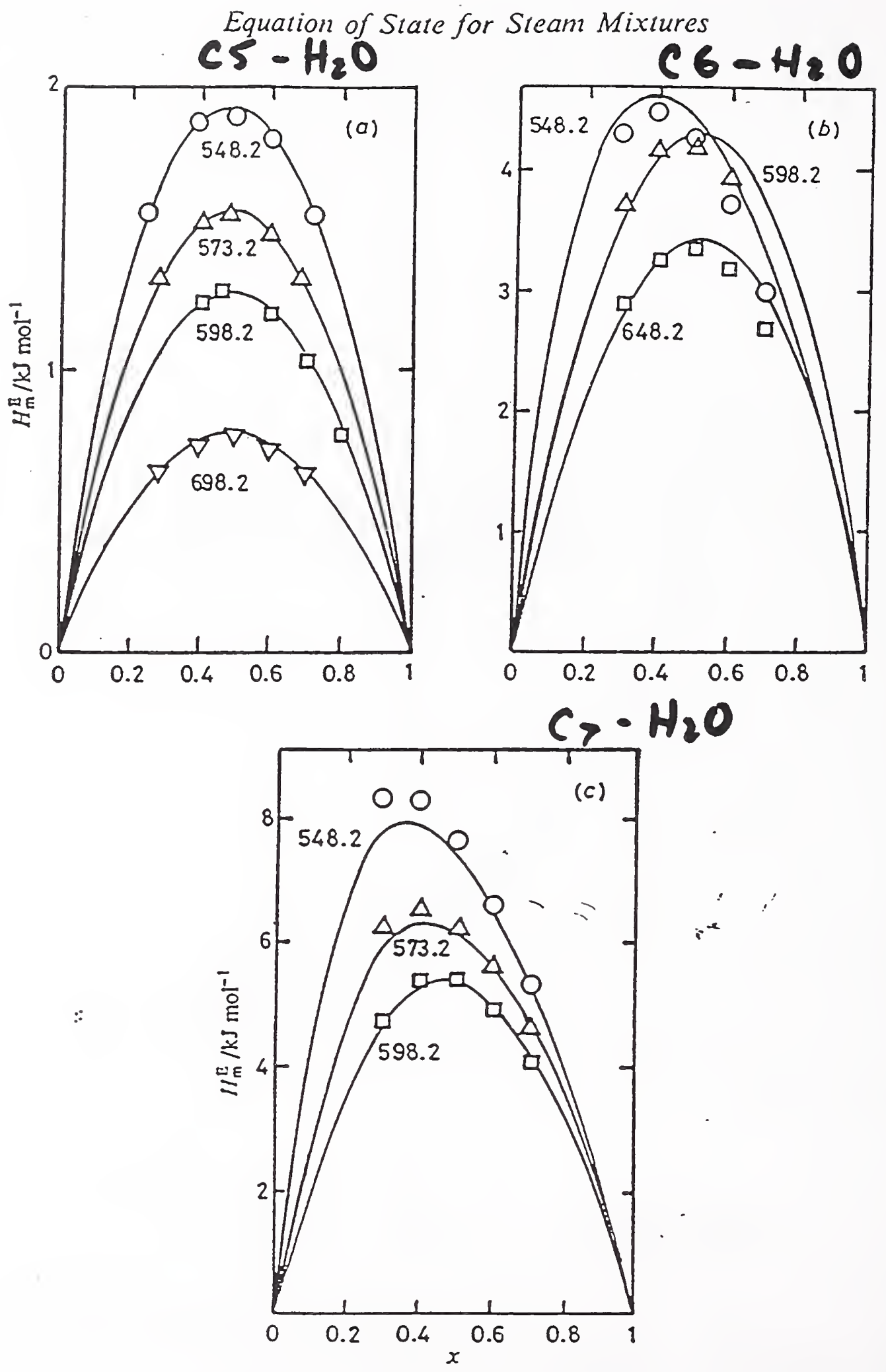

Fig. 3. Comparison of calculated and experimental excess molar enthalpies $H_{\mathrm{m}}^{\mathrm{F}}$ of $\left[x \mathrm{H}_{2} \mathrm{O}+(1-x) \mathrm{C}_{n} \mathrm{H}_{2 n+2}\right]$ for $n=5-7$. Solid curves were calculated from eq (27) as described in the text. (a) $\left[x \mathrm{H}_{2} \mathrm{O}+(1-x) \mathrm{C}_{5} \mathrm{H}_{12}\right]^{18}$ all measurements at $4.50 \mathrm{MPa}$, (b) $\left[x \mathrm{H}_{2} \mathrm{O}+(1-x) \mathrm{C}_{6} \mathrm{H}_{13}\right] .{ }^{23}$ measurements at $548.2 \mathrm{~K}$ and $4.93 \mathrm{MPa}, 598.2 \mathrm{~K}$ and $9.41 \mathrm{MPa}, 648.2 \mathrm{~K}$ and $11.48 \mathrm{MPa}$, (c) $\left.\left[x \mathrm{H}_{2} \mathrm{O}+(1-x) \mathrm{C}_{3} \mathrm{H}_{16}\right]\right]^{23}$ measurements at $548.2 \mathrm{~K}$ and $4.58 \mathrm{MPa}, 573.2 \mathrm{~K}$ and $6.00 \mathrm{MPa}$. $598.2 \mathrm{~K}$ and $7.68 \mathrm{MPa}$.

P $5-11 M P_{a}$

Wormald

222 


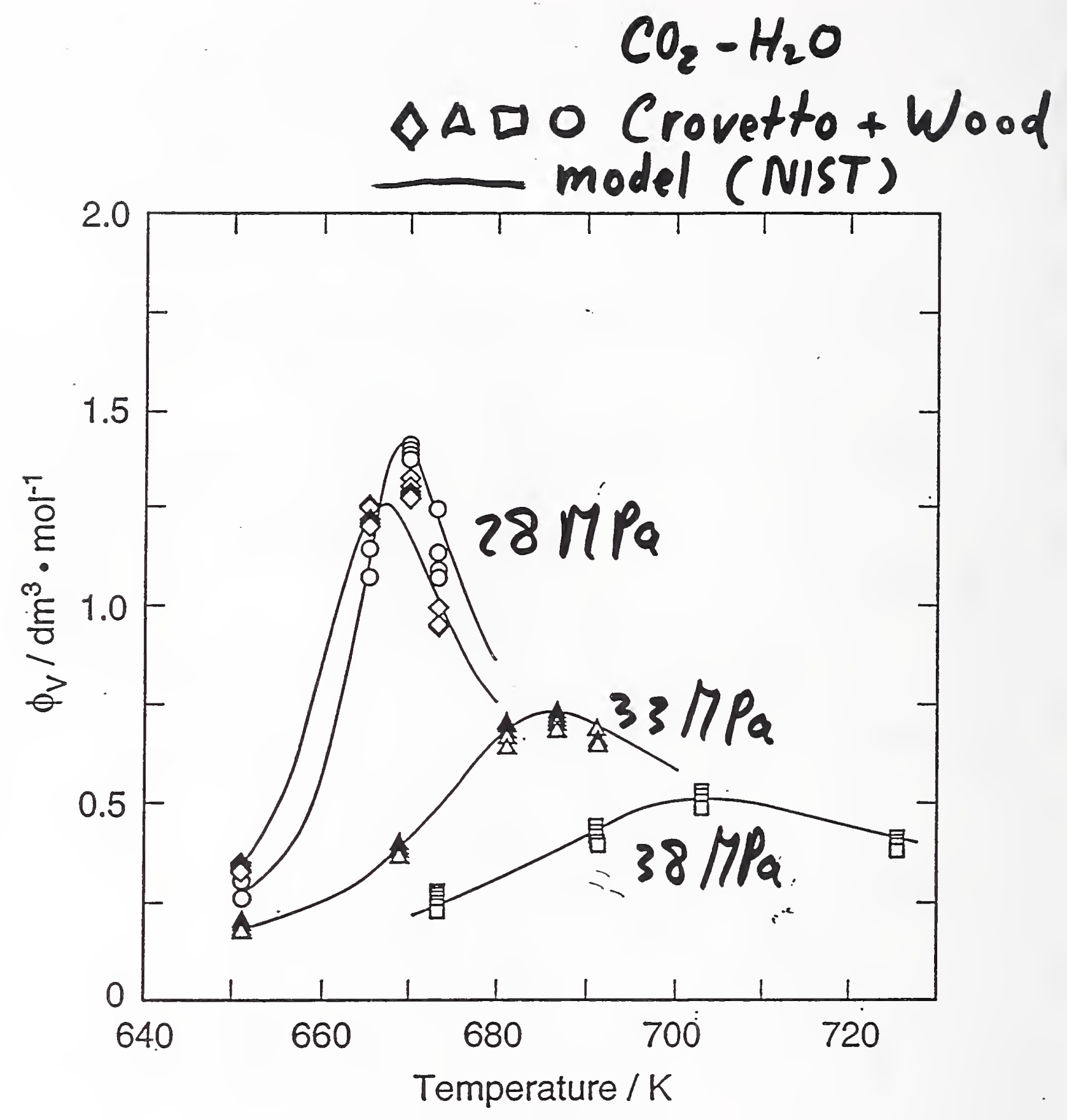

apparent molar volume near to dilution for $\mathrm{CO}_{2}$ 3 supercritical isobars

224 


\section{WHAT TO DO?}

*Use NBS/NRC Steam Tables, Hemisphere Publishers 1984, as a reference fluid

*Develop a Helmholtz free energy for multicomponent aqueous mixtures including phase boundaries, along the lines of the interactive programs to be discussed by Dan Friend.

\section{THIS MUST ALSO BE DONE}

*Predictive method for the viscosity of multicomponent aqueous systems

Incorporate nonionic chemical reactions

Extend to ionic components 
REFERENCES

THERMOPHYSICAL PROPERTIES OF WATER AND STEAM

L. Haar, J. S. Gallagher and G. S. Kell, NBS/NRC Steam Tables, Hemisphere Publishing Company, 1984.

Programs available on disk from the Standard Reference Data Program at NIST, ordering information included here.

International Association for the Properties of Water and Steam;ordering information on property formulations ("releases") included here.

Properties include: density, dielectric constant, enthalpy, entropy, heat capacities, transport properties, ion product, electrical conductivity.

PHASE DIAGRAMS SHOWN HERE

$\mathrm{NaCl}-\mathrm{H}_{2} \mathrm{O}$

S. Sourirajan and G.C. Kennedy, Am. J. Science 260, 115 (1962).

$\mathrm{O}_{2}-\mathrm{H}_{2} \mathrm{O}$

M.L. Japas and E.U. Franck, Ber. Bunsenges. Phyș. Chem.89, 1268, (1985).

$\mathrm{CO}_{2}-\mathrm{H}_{2} \mathrm{O}$

K. Toedheide and E.U. Franck, Zeitsch. Physik. Chem. NF 37, 387 (1963). (data).

J.S. Gallagher, R. Crovetto and J.M.H. Levelt Sengers, submitted to

J. Phys. Chem. Ref. Data. (model).

Alkanes - $\mathrm{CO}_{2}$

E. Brunner, J. Chem. Thermodynamics 22, 335 (1990).

EXCESS ENTHALPIES

Alkanes - $\mathrm{H}_{2} \mathrm{O}$

C.J. Wormald and N.M. Lancaster, J. Chem. Soc. Faraday Trans. 85, 1315 (1989)

$\mathrm{CO}_{2}-\mathrm{H}_{2} \mathrm{O}$

C.J. Wormald, N.M. Lancaster and A.J. Sellars, J. Chem.

Thermodynamics 18, 279 (1987). 


\section{APPARENT MOLAR VOLUMES}

R. Crovetto, R.H. Wood and V. Majer, J. Chem. Thermodynamics 23, 1079 (1991)

J.S. Gallagher, R. Crovetto and J.M.H. Levelt Sengers, submitted to J. Phys. Chem. Ref. Data. (model).

\section{FAILURE OF STANDARD METHODS}

J.M.H. Levelt Sengers, C.M. Everhart, G. Morrison and K.S. Pitzer, Chem. Eng. Comm. 47, 315 (1986).

J.M.H. Levelt Sengers, A.H. Harvey, R. Crovetto and J.S. Gallagher, accepted, Fluid Phase Equilibria. 


\title{
IAPWS NEWS
}

International Association for the Properties of Water and Steam

\author{
President: J.M.H. Levelt Sengers \\ Vice President: J.R. Cooper \\ Executive Secretary: R.B. Dooley
}

May 1992

\section{President's Remarks}

I would like to draw attention to an issue that has been repeatedly stressed by Professor Franck, namely the possibility of destroying hazardous waste and toxic products in oxygenated supercritical steam.

In the United States there is currently much interest in exploring this issue. Several universities and many Government agencies are doing research on the topic, while a number of companies are gearing up to build reactors. The December issue of Chemical and Engineering News is devoted to this topic, and IAPWS member Prof. Franck is one of the five authors of the feature article

In my own organization, NIST, a team headed by Dr. Rosasco is building a prototype reactor with windows permitting spectroscopic studies. In my consulting activities with this team, I am becoming very aware of the similarities between this work and the principal concerns of IAPWS's working groups on Physical Chemistry of Aqueous Systems and on Chemistry in Power Cycles. The proposed reactors operate in a range comparable to that of supercritical power cycles, namely at about $250 \mathrm{bar}$ and $400-600^{\circ} \mathrm{C}$. Contrary to power plants, the water in the reactor is quite contaminated, but the compounds present are similar (oxygen, carbon dioxide, organics, salts) and the materials problems will overlap at least in part those encountered in the power industry. On the other hand, the research that is currently initiated in the field of supercritical aqueous hazardous waste destruction could indirectly benefit the power industry. Thus, for instance, the NIST reactor under construction will permit insitu Raman studies of metal surfaces exposed to the supercritical water stream. Also, strenuous efforts are underway in many groups in the USA to model the chemical reactions taking place in the presence of excess water in a very nonideal state.

It is my hope that no opportunity will be missed for mutual fertilization between the field of power plant chemistry and this emerging technology. More may be needed than individual efforts of two or three IAPWS members happening to be active in both fields. I encourrage the chairmen and members of PCAS and PCC to do some creative thinking about possible roles for IAPWS related to this new technology.

\section{Russia Hosts 1992 IAPWS Meeting}

Professor Alexandrov and his Russian National Committee are organizing the 1992 meetings for the executive committee and the working groups in St. Petersburg from Sunday, September 6 - Saturday, September 12, 1992. The program will start on Monday, September 7 with the Plenary Session at 9:00 AM. There will be a Symposium on Tuesday, September 8 covering the wide range of IAPWS activities and including presentations from Russian scientists, who do not usually attend the IAPWS meetings, and a number of IAPWS international members. The four working groups will meet throughout the week and the executive committee will meet all day Friday. Two visits are planned. The first on Wednesday will be to the Central Turbine and Boiler Institute (TsKTD) in St. Petersburg; this will be regarded as optional so that some working group members could also have a relaxed working session instead. The second visit, on Saturday, September 12, will be to the St. Petersburg Nuclear
Plant which is about a two hour drive from the city; lunch will be provided.

All the meetings will be at the St. Petersburg Mining Institute. The Executive Secretary had the opportunity to visit with Professor N. Proskuryakov, the Rector, during a visit to St. Petersburg in March. The Institute is ideally situated on the Neva River and has all the required facilities to hold the IAPWS annual meetings. The Institute has its own Hotel, which is located about $2 \mathrm{~km}$ away and transportation will be provided for the delegates. The rates are $\$ 95$ for a single room or $\$ 130$ for a double with all meals being provided. Alternatively, accommodation is available at the Pribaltiiskaya Hotel, (\$160 single, no meals) which is located very close to the Institute's own hotel. Professor Proskuryakov also indicated that there will be some cultural activities included in the events.

We hope to see you in the magnificent city of St. Petersburg in September.

For further information on LAPWS or to request guidelines and releases, contact:

Dr. R.B. Dooley, Executive Secretary, EPRI, 3412 Hillview Ave., Palo Alto, Califomia 94304, USA

Telephone: $415-855-2458$

Fax: 415-855-8759

Telex : 82977 EPRI UF 


\section{Current LAPWS Guidelines and Releases}

- "Release on the Refractive Index of Ordinary Water and Steam as a Function of Wavelength, Temperature and Pressure". (September 1991).

- Electrolytic Conductivity (Specific Conductance) of Liquid and Dense Supercritical Water from $0^{\circ} \mathrm{C}$ to $800^{\circ} \mathrm{C}$ and Pressures up to $1000 \mathrm{MPa}$ ". (May 1990).

- "Solubility of Sodium Sulfate in Aqueous Mixtures of Sodium Chloride and Sulfuric Acid from Water to Concentrated Solutions, from $250^{\circ} \mathrm{C}$ to $350^{\circ} \mathrm{C}$. (May 1990).

- Release on the Pressure along the Melting and Sublimation Curves of Ordinary Water Substance". (September 1989).

- "Surface Tension of Heavy Water Substances $\left(\mathrm{D}_{2} \mathrm{O}\right)$ ". (September 1985).

- "LAPS Skeleton Tables 1985 for the Thermodynamic Properties of Ordinary Water Substarce". November 1985).

- IAPS Formulation 1985 for the Thermal Conductivity of Ordinary Water Substances". (November 1985).

- "IAPS Formulation 1985 for the Viscosity of Ordinary Water Substances". (November 1985$).$

- "IAPS Formulation 1984 for the Thermodynamic Properties of Heavy Water Substance". (December 1984).

- The IAPS Formulation 1984 for the Thermodynamic Properties of Ordinary Water Substance for Scientific and General Use". (December 1984).

- "Viscosity and Thermal Conductivity of Heavy Water Substance". (February 1984).

- 7983 IAPS Statement, Values of Temperature, Pressure and Density of Ordinary and Heavy Water Substances at their Respective Critical Points". (1983).

- "Ton Product of Water Substance". (May 1980).

- "Static Dielectric Constant of Water Substance". (1977).

- "Surface Tension of Water Substance". (1976).

- The 1967 IFC Formulation for Industrial Use".

- LAPS Supplementary Release: "Saturation Properties of Ordinary Water Substance". (September 1986. Rev. November 1986) 


\section{NATIONAL INSTITUTE OF STANDARDS AND TECHNOLOGY STANDARD REFERENCE DATABASES}

\section{PRICE SCHEDULE}

Databases of physical and chemical properties prepared by NIST's Standard Reference Data Program are available in magnetic tape, diskette, and CD ROM format under a license agreement. There are three types of licenses, applicable to an Individual User, to a Distributor, and to a Subscription Search Service.

\section{\# NIST STANDARD REFERENCE DATABASE}

\begin{tabular}{|c|c|c|}
\hline 1. & NIST/EPA/MSDC Mass Spectral & $\$ 3,300$ \\
\hline 1A. & NIST/EPA/MSDC Mass Spectral: PC Version 4.0 & 1,200 \\
\hline 1B. & NIST Mass Spectral Library of Common Compounds & 350 \\
\hline 2. & NIST Chemical Thermodynamics (NBS Tech Note 270) & 1,000 \\
\hline 3. & NIST Crystal Data Identification File ${ }^{1}$ & 2,000 \\
\hline 9. & NIST Thermophysical Properties of Hydrocarbon Mixtures & 490 \\
\hline 9. & NIST Electron and Positron Stopping Powers of Materials & 350 \\
\hline 9. & NIST X-Ray and Gamma-Ray Attenuation Coefficients and Cross Sections & 400 \\
\hline 9. & NIST Activity and Osmotic Coefficients of Aqueous Electrolyte Solutions & 200 \\
\hline 10. & NIST Thermophysical Properties of Water & 300 \\
\hline 18. & DIPPR Data Compilation of Pure Compound Properties²: Version 7.0 & 4,000 \\
\hline 11A. & Student DIPPR & 75 \\
\hline 18. & NIST Thermophysical Properties of Fluids & $4 \cong 0$ \\
\hline 13. & NIST JANAF Thermochemical Tables & 1,200 \\
\hline 13. & NIST Mixture Property Program² & 400 \\
\hline 18. & NIST/Sandia/ICDD Electron Diffraction ${ }^{l}$ & 3,000 \\
\hline 16. & NIST Corrosion Performance ${ }^{3}$ & 240 \\
\hline 18. & NIST Chemical Kinetics: PC Version 4.0 & 390 \\
\hline 18. & NIST Estimation of the Thermodynamic Properties for Organic Compounds at $298.15 \mathrm{~K}$ & 215 \\
\hline $19 \mathrm{~A} \& \mathrm{~B}$. & NIST Positive and Negative Ion Energetics & 130 \\
\hline 20. & NIST X-Ray Photoelectron Spectroscopy & 495 \\
\hline 21. & NIST/CARB Biological Macromolecule Crystallization Version 2.0 & 390 \\
\hline 22. & Tribomaterials $I^{4}$ & 350 \\
\hline 23. & NIST Thermodynamic Properties of Refrigerants and Refrigerant Mixtures: Version 3.0 & 390 \\
\hline 24. & NIST Atomic Transition Probabilities Data File & 215 \\
\hline 25. & NIST Structures and Properties Database and Estimation Program & 240 \\
\hline
\end{tabular}

'Available from the JCPDS-International Centre for Diffraction Data, 1601 Park Lane, Swarthmore, PA 19081. Phone (215) 328-9400.

${ }^{2}$ Discount available to members of DIPPR. Discount available to GPA members for DDMTX.

${ }^{3}$ Available from NACE, P.O. Box 218340. Houston, TX 77218. Phone (713) 492-0535.

${ }^{4}$ Available from ACTTS Inc., 1118 Highgate Rd., Wilmington, DE 19808. Phone (302) 998-8240. 


\begin{tabular}{|r|r|r||}
\hline \multicolumn{1}{|l}{ NIST STANDARD REFERENCE DATABASE } & FEE \\
\hline 26. & NIST Vibrational Electronic Energy Levels of Small Polyatomic Transient Molecules Version 2.0 & 390 \\
\hline 27. & NIST Molten Salts Version 2.0 & 240 \\
\hline 30. & NIST Structural Ceramics & 495 \\
\hline 35. & NIST/EPA Gas Phase Infrared & 415 \\
\hline 36. & NIST/NIH Desktop Spectrum Analyzer Program and X-Ray Database & 790 \\
\hline 38. & NIST Spectroscopic Properties of Atoms and Atomic Ions & 190 \\
\hline
\end{tabular}

\# NIST SPECIAL DATABASES

FEE

\begin{tabular}{|r|l|r|}
\hline \hline 1. & NIST Binary Images of Printed Digits, Alphas, and Text & $\$ 895$ \\
\hline 2. & NIST Structured Forms Reference Set of Binary Images & 250 \\
\hline 3. & NIST Binary Images of Handwritten Segemented Characters & 895 \\
\hline
\end{tabular}

\section{INDIVIDUAL USER}

An individual PC user makes use of a database solely on one machine on an in-house basis. Magnetic tape or source code use is discussed on an individual basis. Please call (301) 975-2208 for further details.

As an individual PC user, a single payment at the initiation of the license permits use under the specified restrictions without limit of time. Updates and revisions will be offered to each' licensee from time to time at prices to be determined; the restrictions on use given in the original license will apply to each update or revision that is delivered.

\section{DISTRIBUTOR}

A Distributor refers to a licensee who provides copies of the database to other individual users on a fee basis.

A distributor must pay NIST one half of the NIST price for each copy made. Different rates are available for the Mass Spectral

Database and the DIPPR Database. Please call (301) 975-2208 for further details.

\section{SUBSCRIPTION SEARCH SERVICE (ON-LINE)}

A license arrangement is available to organizations that distribute data to third parties via an on-line network. Royalty fees to NIST are based on connect time and/or other measures of usage. Please call (301) 975-2208 for further details.

\section{FURTHER INFORMATION}

Please contact:

Standard Reference Data

National Institute of Standards and Technology

221/A320

Gaithersburg, MD 20899

Telephone: (301) 975-2208

FAX: (301) 926-0416 


\section{THERMOPHSYICAL PROPERTIES OF FLUIDS: RESEARCH AT NIST}

Thermophysics Division:

Dr. R. F. Kayser, Chief

-Gaithersburg, MD

-Boulder, CO

-Internal, OA, commercial sponsorship

-Standard reference data

-Liaison with industry for fluid properties 


\section{STANDARD REFERENCE DATA PROGRAM}

Dr. M. W. Chase, Chief

Dr. J. Rumble, Jr., Program Manager

-Fluid Mixtures Data Center D.G. Friend

-Properties of Polar Fluids Project R.F. Kayser 


\section{COMPUTERIZED DATABASES FROM THE}

THERMOPHYSICS DIVISION

-NIST Thermophysical Properties of Hydrocarbon Mixtures Database (SUPERTRAPP, SRD 4)

-NIST Mixture Properties Database (DDMIX, SRD 14)

-NIST Thermophysical Properties of Pure Fluids Database (MIPROPS, SRD 12)

-NIST Thermodynamic Properties of Refrigerant and Refrigerant Mixtures Database (REFPROP, SRD 23)

-NIST Thermophysical Properties of Water Database (NBS/NRC Steam Tables, SRD 10) 


\section{GENESIS OF DDMIX}

-NIST (NBS) Supercritical Fluid Properties Consortium

-14 companies primarily interested in supercritical $\mathrm{CO}_{2}$ processes

-4 year project

Experimental measurements

Theory

Modeling

Computer package

-Distribution by SRD 


\section{SUPERTRAPP (SRD 4)}

\section{Dr. Marcia L. Huber}

-Extended corresponding states (ECS)

-Transport property corresponding states

-Propane reference fluid

-116 Fluids in database

alkanes, alkenes, aromatics, cycloalkanes, common impurities

-Excess and residual properties easily calculated

-Ability to add new fluids

-User friendly 


\section{Thermophysical properties calculated by SUPERTRAPP}

phase boundaries

density

$\mathrm{C}_{\mathrm{p}}$

Joule-Thomson coefficient enthalpy

$\mathrm{C}_{\mathrm{p}} / \mathrm{C}_{\mathrm{v}}$

viscosity

entropy

sound speed

thermal conductivity

compressibility factor

molecular mass 


\section{Component database in SUPERTRAPP}

methane

ethane

propane

n-butane

isobutane

n-pentane

isopentane

neopentane

2,2-dimethylbutane

2,3-dimenthylbutane

3-methylpentane

2-methylpentane

n-hexane

2,2,3-trimethylbutane

3,3-dimethylpentane

2,4-dimethylpentane

2,3-dimethylpentane

2,2-dimethylpentane

3-ethylpentane

3-methylhexane

2-methylhexane

n-heptane

2,2,3,3-tetramethylbutane

2,3,4-trimethylpentane

2,3,3-trimethylpentane

2,2,4-trimethylpentane

2,2,3-trimethylpentane

3-methyl-3-ethylpentane

2-methyl-3-ethylpentane

3,4-dimethylhexane

3,3-dimethylhexane

2,5-dimethylhexane

2,4-dimethylhexane

2,3-dimethylhexane

2,2-dimethylhexane

3-ethylhexane

4-methylheptane

3-methylheptane

2-methylheptane n-octane

2,3,3,4-tetramethylpentane

2,2,4,4-tetramethylpentane

2,2,3,4-tetramethylpentane

2,2,3,3-tetramethylpentane

2,2,5-trimethylhexane

2,2-dimethylheptane

2-methyloctane

n-nonane

2,2,5,5-tetramethylhexane

2,2,3,3-tetramethylhexane

3,3,5-trimethylheptane

n-decane

n-undecane

n-dodecane

n-tridecane

n-tetradecane

n-pentadecane

$\mathrm{n}$-hexadecane

n-heptadecane

n-octadecane

n-nonadecane

n-eicosane

n-heneicosane

n-docosane

n-tricosane

n-tetracosane

ethylene

propylene

2-methylpropene

cis-2-butene

trans-2-butene

1-butene

2-methyl-2-butene

2-methyl-1-butene

3-methyl-1-butene

cis-2-pentene

trans-2-pentene

1-pentene 1-hexene

1-heptene

1-octene

1 -nonene

1-decene

propadiene

1,3-butadiene

1,2-butadiene

cyclopropane

cyclopentane

methylcyclopentane

ethylcyclopentane

cyclohexane

methylcyclohexane

ethylcyclohexane

benzene

toluene

ethylbenzene

ortho-xylene

meta-xylene

para-xylene

propylbenzene

isopropylbenzene

butylbenzene

isobutylbenzene

t-butylbenzene

naphthalene

1-methylnapthalene

2-methylnapthalene

biphenyl

hydrogen

nitrogen

oxygen

water

carbon monoxide

carbon dioxide

sulfur dioxide

hydrogen sulfide 


\section{AQUEOUS SYSTEMS IN ECS MODELS}

-Need Haar-Gallagher-Kell equation for reference states

-Refinement of phase boundary routines

-Further development of transport property corresponding states

-Structurally based ECS parameters

-Dielectric constant (or H-bonds) as ECS parameter

-Ionic components

-Chemically reacting systems 


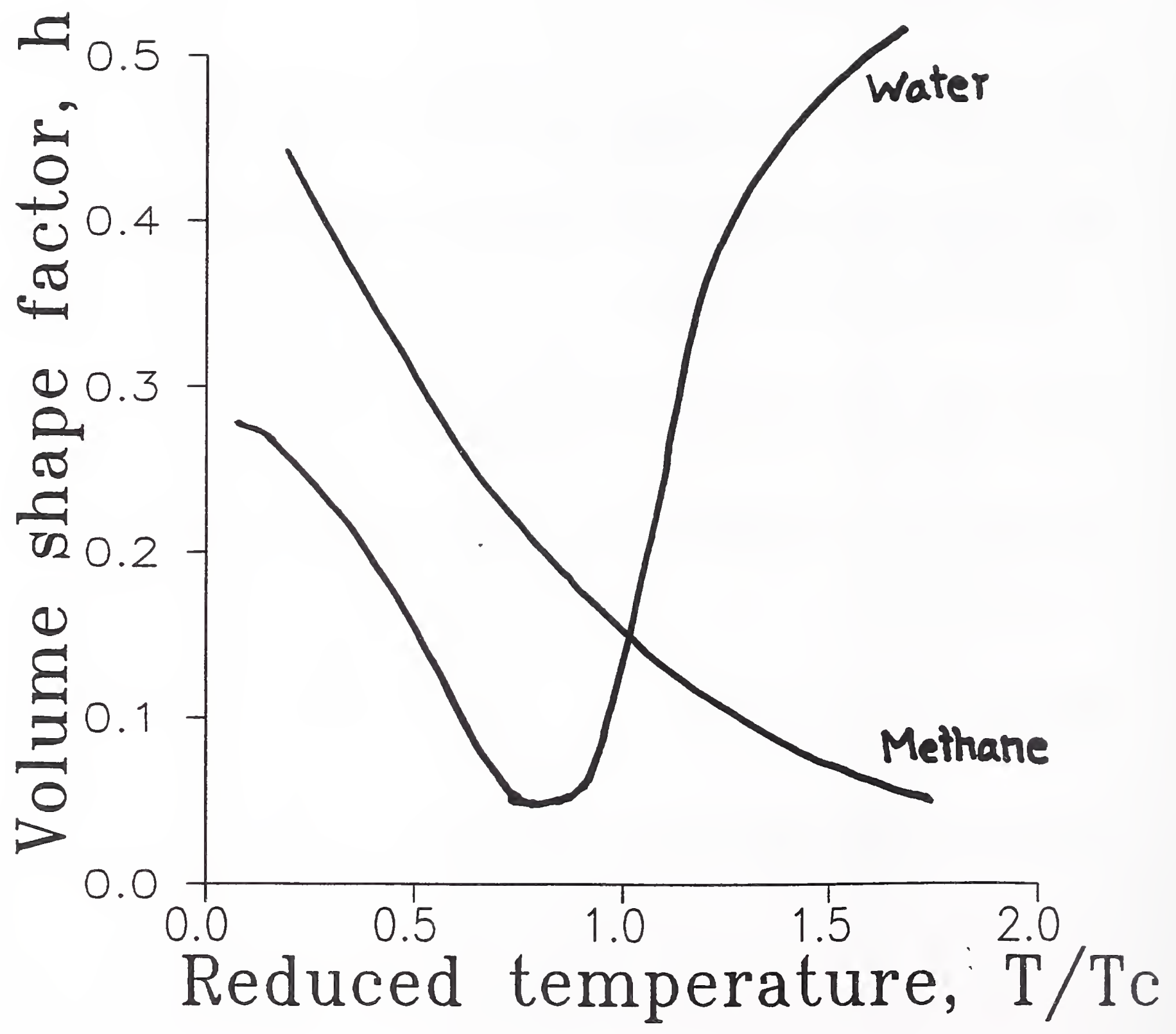





\section{CONCLUSIONS}

- There is a need for thermophsyical properties (phase diagrams, densities, viscosities, enthalpies, etc.) for a variety of supercritical aqueous systems:

-accurate

-thermodynamically consistent - easy to use

-Existing extended corresponding states models and computer packages can form the basis for fulfilling these needs. 


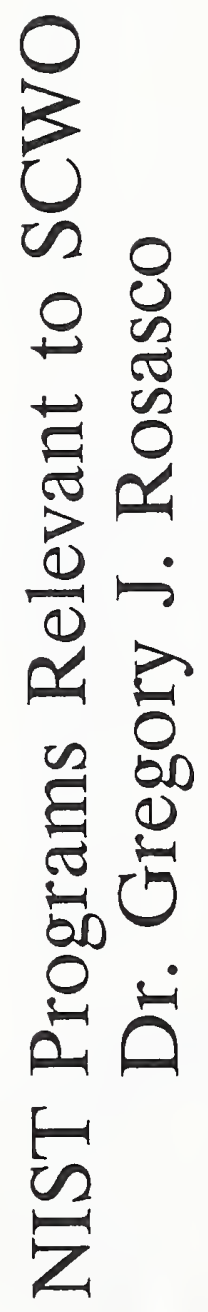




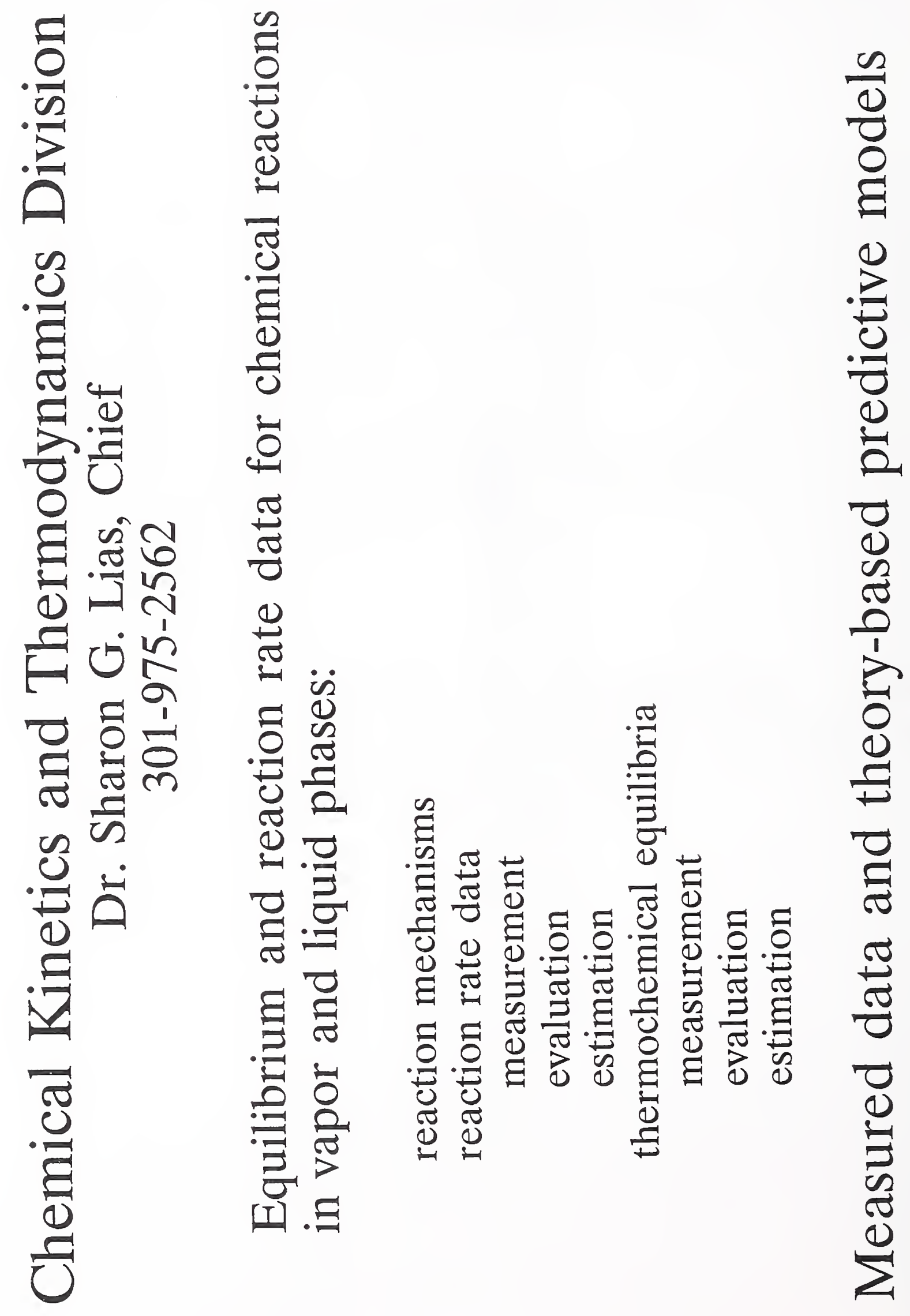




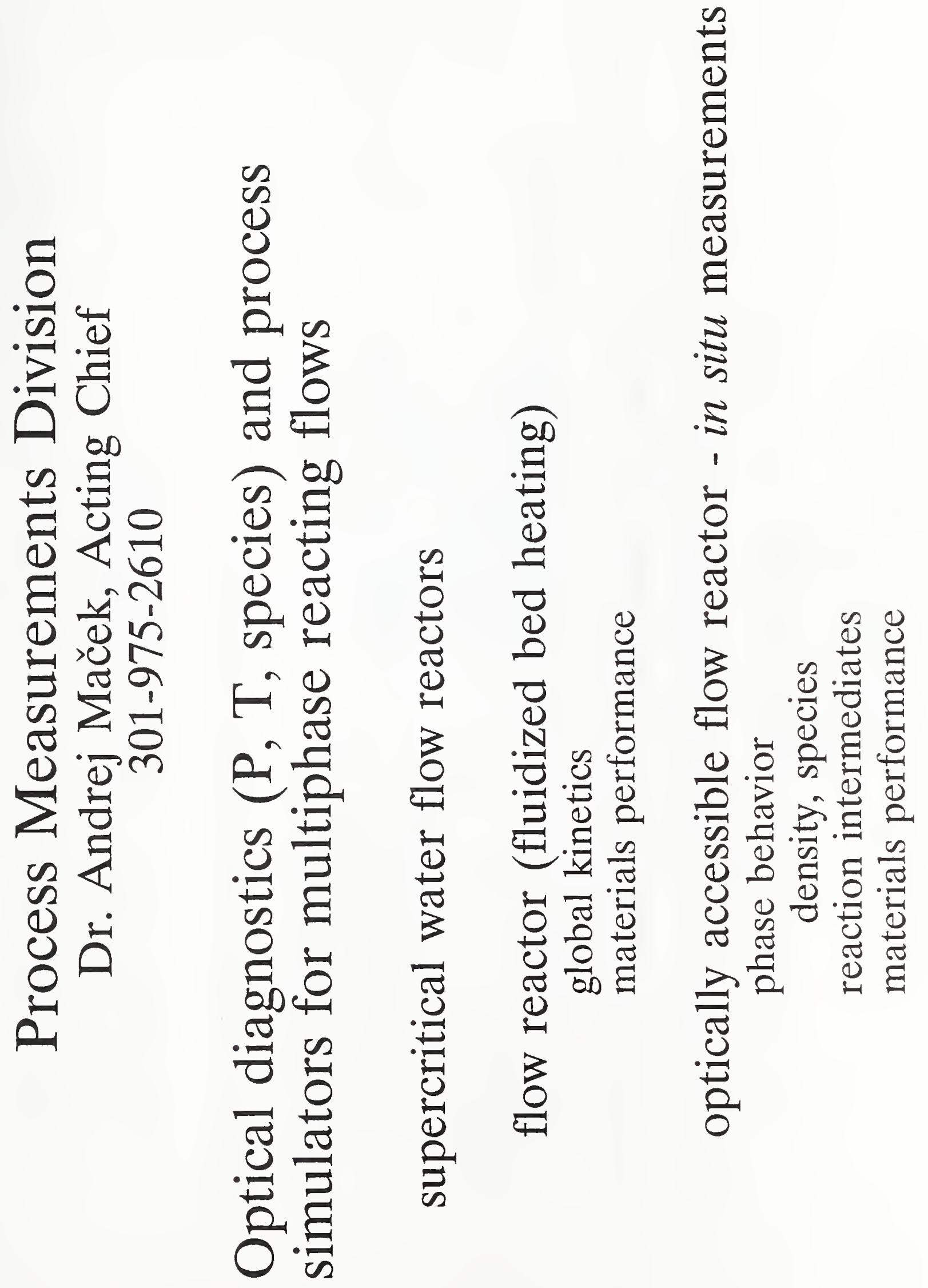


$\cdot \frac{a}{0} \cdot \frac{\pi}{2}$

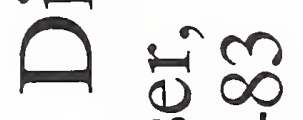

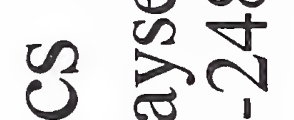

- 毞

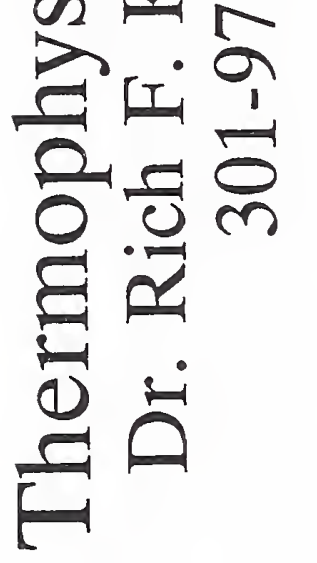

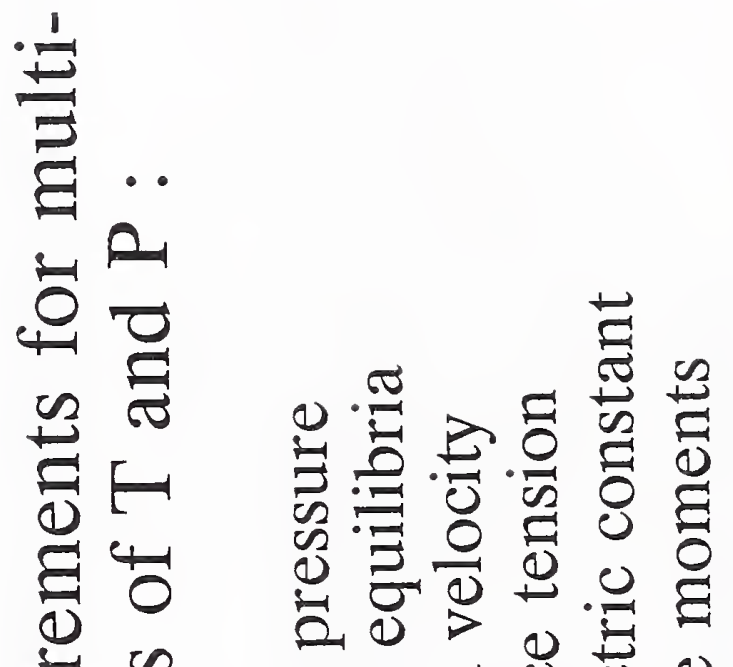

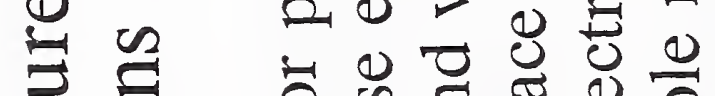

蛋.

证

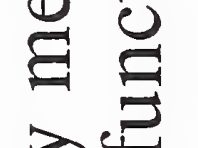

$\sum_{0}^{\infty}$

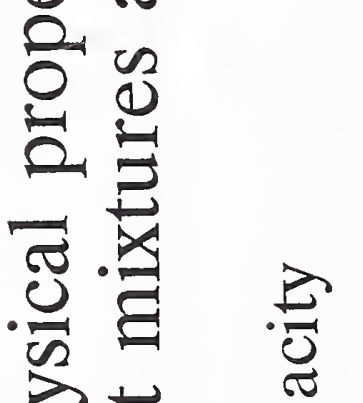

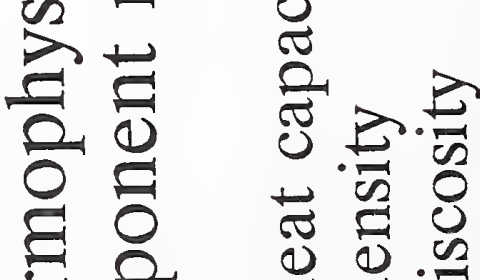

용 $\frac{2}{0}$

$\frac{i}{0} \quad \frac{\tilde{L}}{2}$

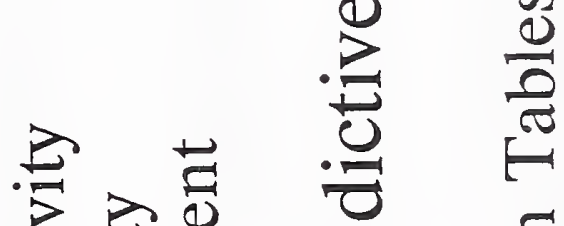

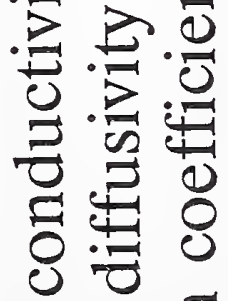

(1) E

말

(1) $\mathscr{\sim}$

$\sum_{0}^{\infty} \quad \frac{1}{2}$

.

E 

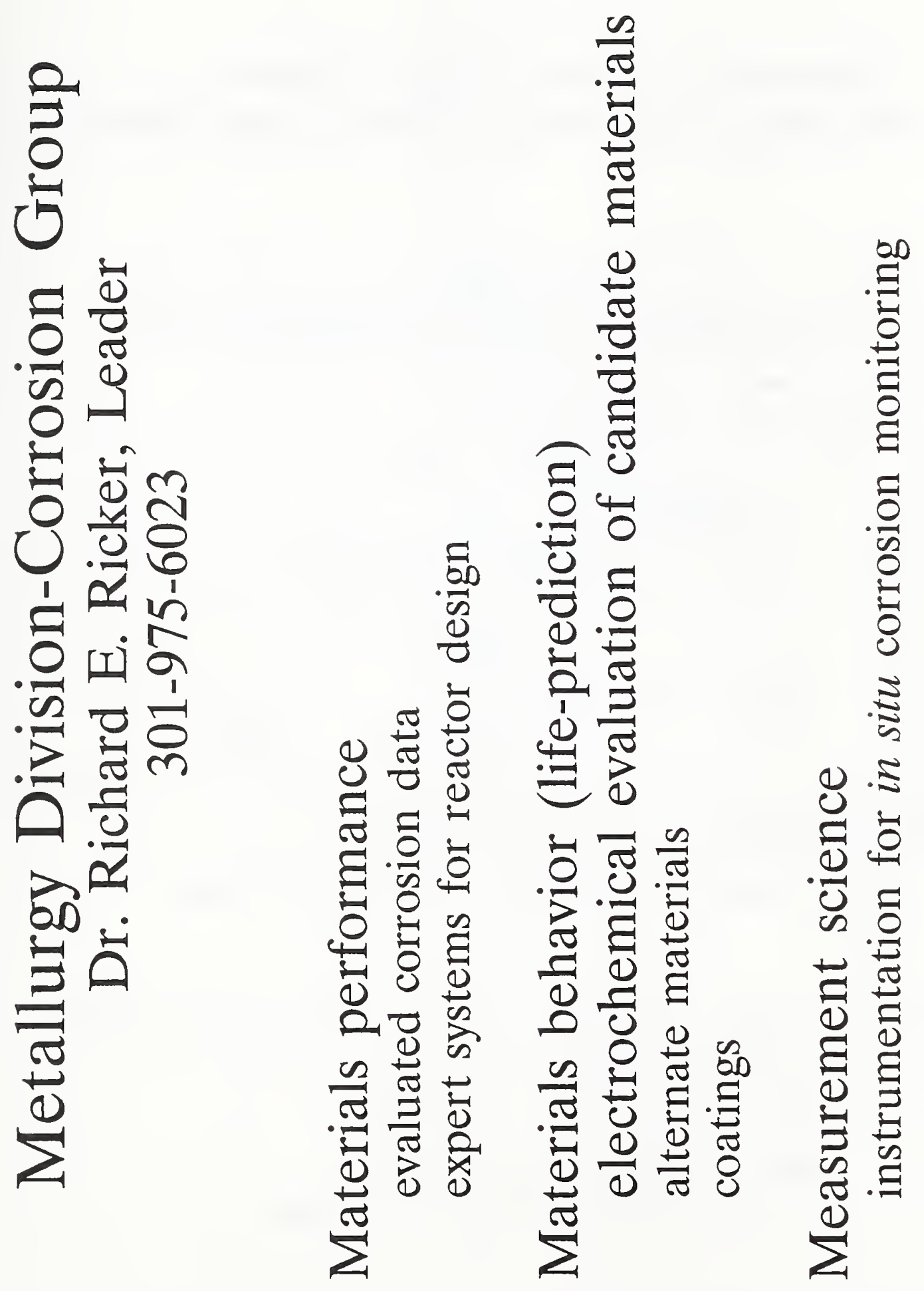


\section{NACE-NIST Corrosion Data Program}

\section{Materials Advisory Expert System for the Handling and Storage of Hydrogen Chloride}

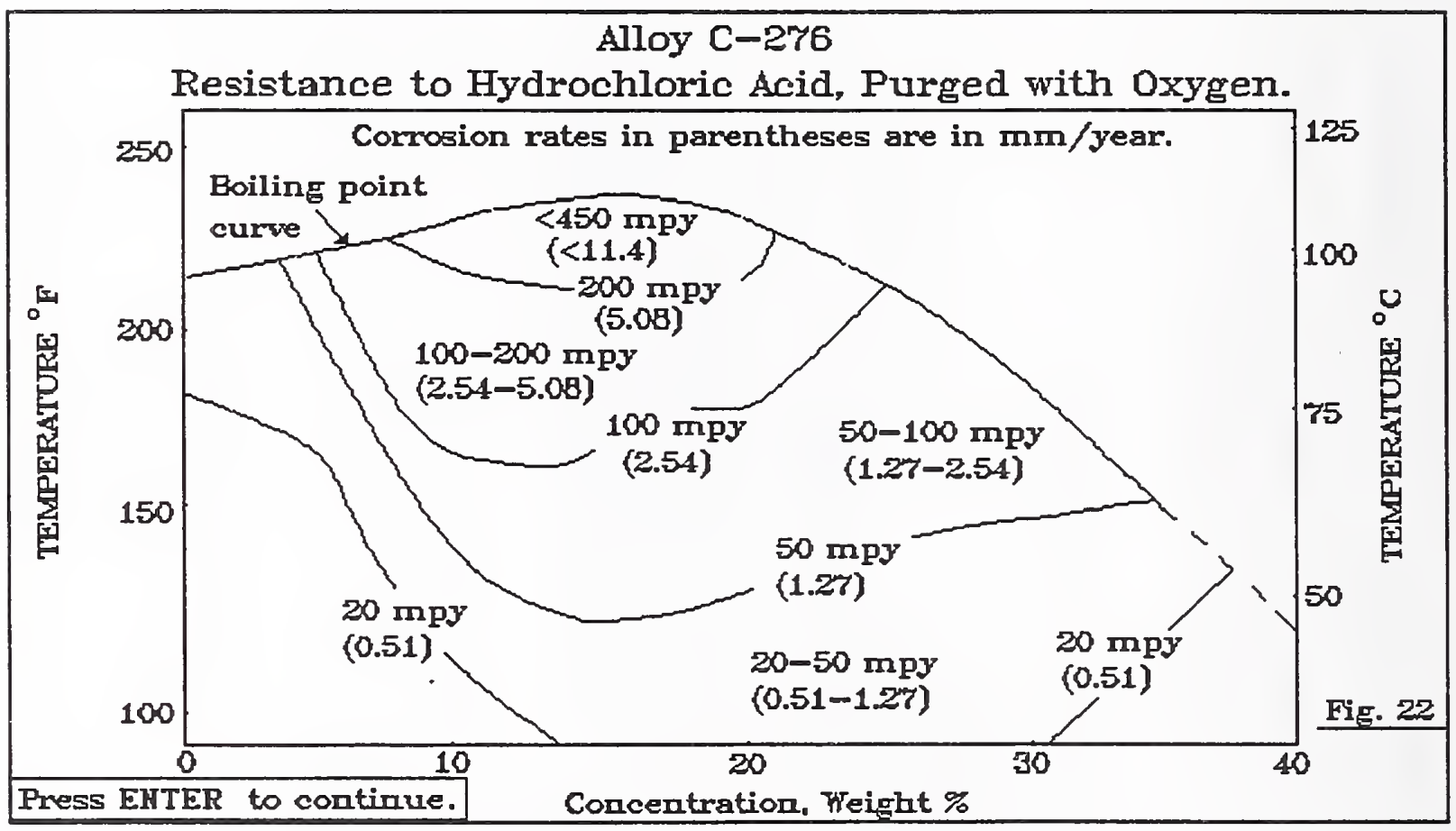

Fig. 22: Alloy C-276, resistance to $\mathrm{HCl}$, purged with oxygen

Rule: If $\mathrm{HCl}$ concentration $<=12 \%$ or $>=20 \%$ and Oxygen purge $=$ true and Temperature $<=50^{\circ} \mathrm{C}$ then consider Alloy C-276 and Corrosion Rate $<=50 \mathrm{mpy}$ 


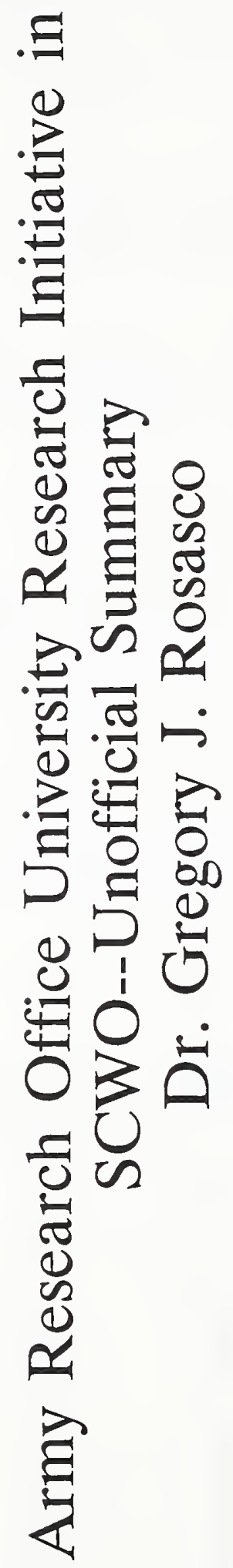




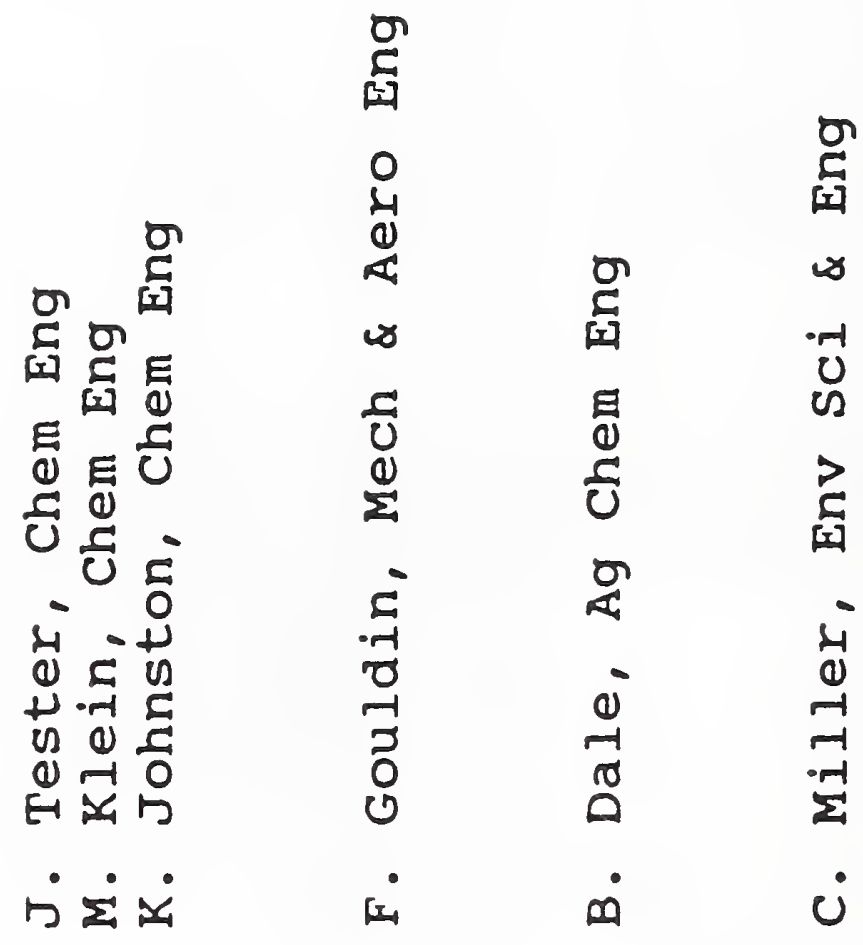

要

o i

a

$\infty$

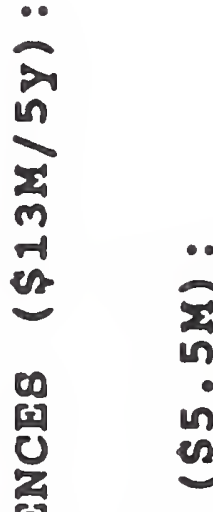

ar

E1

है

$\infty$

E E气̆

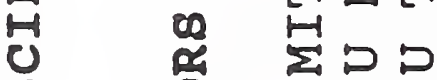

$\infty$

है

过

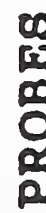

롤

( 1

- 3 in

iv

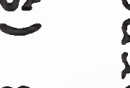

我

Z्द

究

$H$

EH

$\infty$

a

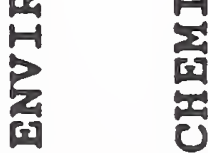

誉

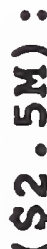




\section{ARO-University Research Initiative}

\section{Chemical Reactors}

Massachusetts Institute of Technology

J. E. Tester

H. J. Herzog

J. B. Howard

R. M. Latanision

W. A. Peters

A. F. Sarofim

University of Delaware

M. T. Klein

T. B. Brill

University of Texas-Austin

K. Johnston

M. A. Fox

A. Bard 


\section{CHEMICAL REACTORS: SUPERCRITICAL WATER OXIDATION \\ Massachusetts Institute of Technology:}

Experiments: Global Kinetics of Destruction

Salts - Nucleation and Phase Separation Corrosion

Modeling: Reactor Engineering

Process Simulation

Target Compounds:

Malathion

DiMethyl Sulfoxide

$\mathrm{H}_{3} \mathrm{CO}$
$\mathrm{H}_{3} \mathrm{CO}^{\mathrm{S}}$
$\mathrm{P}-\mathrm{S}-\mathrm{CHCOOC}_{2} \mathrm{H}_{5}$
$\mathrm{CH}_{2} \mathrm{COOOC}_{2} \mathrm{H}_{5}$<smiles>C[AsH2](C)C</smiles>

Ammonium Perchlorate $\quad \mathrm{NH}_{4} \mathrm{ClO}_{4}$

Nitroglycerine

Nitrocellulose<smiles>O=[N+]([O-])CC(CO[N+](=O)[O-])O[N+](=O)[O-]</smiles>

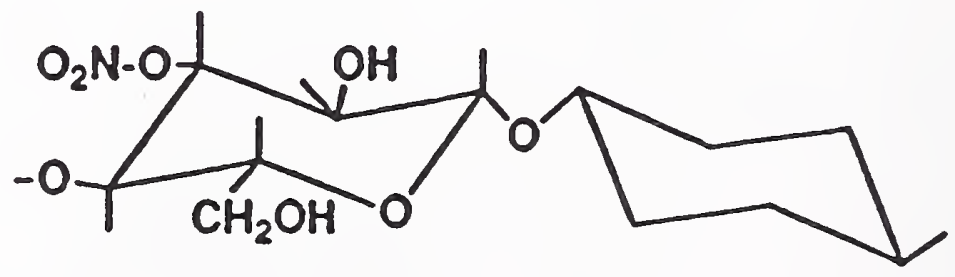




\section{CHEMICAL REACTORS: SUPERCRITICAL WATER OXIDATION}

University of Delaware:

Experiments: Identify Reactive Intermediates - FTIR, Modeling: Detailed Reaction Kinetics

Target Compounds:

Nitrates

Nitrate Esters

Nitramines

Nitrate, Perchlorate Salts

High N Compounds - Azides

$$
\begin{aligned}
& \text { R-C-NO } \\
& \text { R-C-O-NO } \\
& \text { R-C-N-NO }
\end{aligned}
$$

$\mathrm{NH}_{4} \mathrm{NO}_{3}, \quad \mathrm{NH}_{4} \mathrm{ClO}_{4}$

$\mathrm{Pb}\left(\mathrm{N}_{3}\right)_{2}$ 


\section{CHEMICAL REACTORS: \\ SUPERCRITICAL WATER OXIDATION \\ University of Texas-Austin:}

Experiments: Spectroscopy of charge transfer reactions Redox Reactions with Metal Oxides Electrochemistry

Modeling:

Equation of State including $\mathrm{H}$ bonding Solvent effects on Reaction 
3

$\sum_{0}^{\infty}$ ह

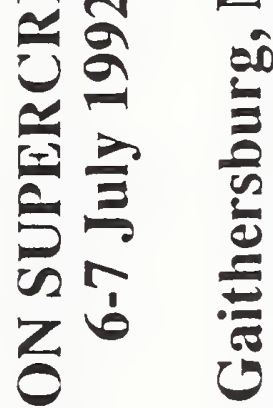

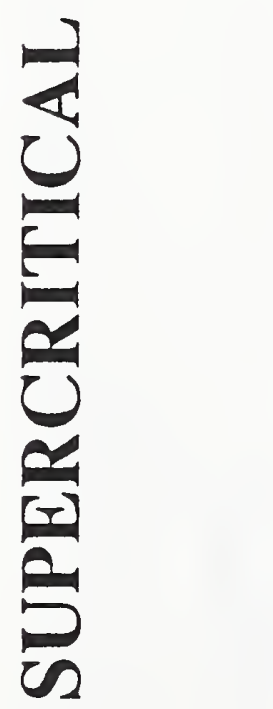

2

$\frac{2}{2}$ 
\%

되를

른

.



อิ

$\sum^{0} \cup$ 


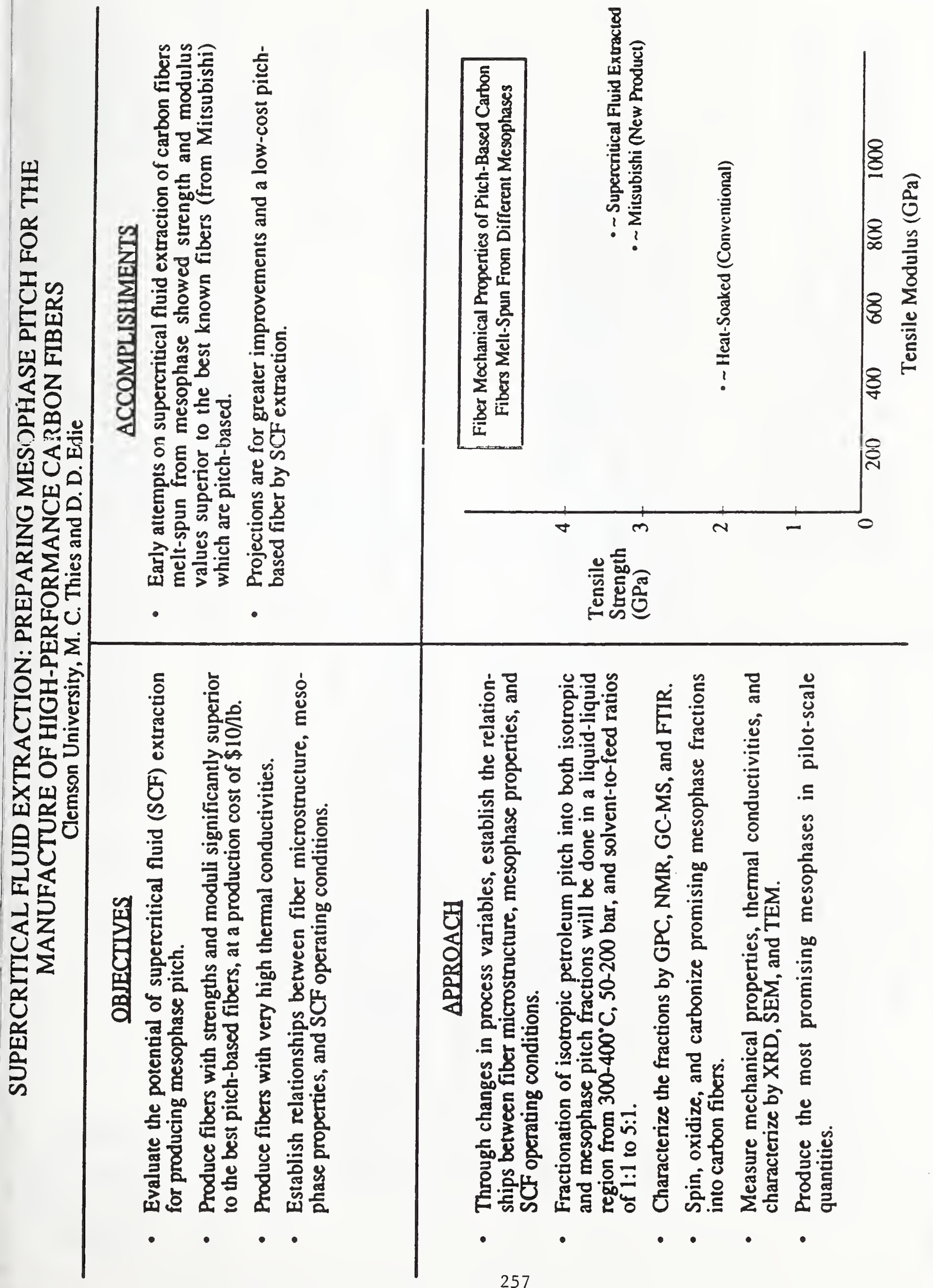




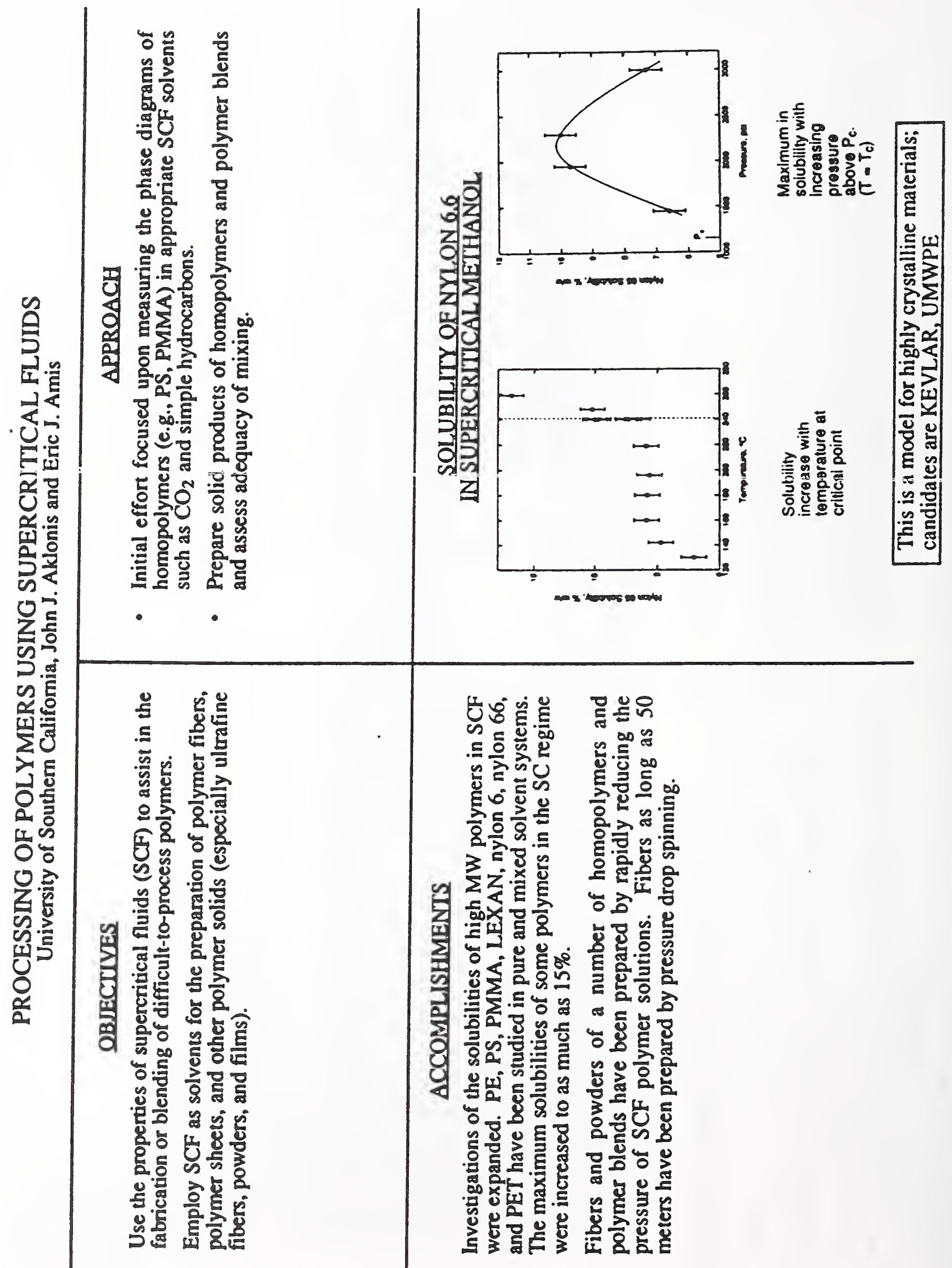




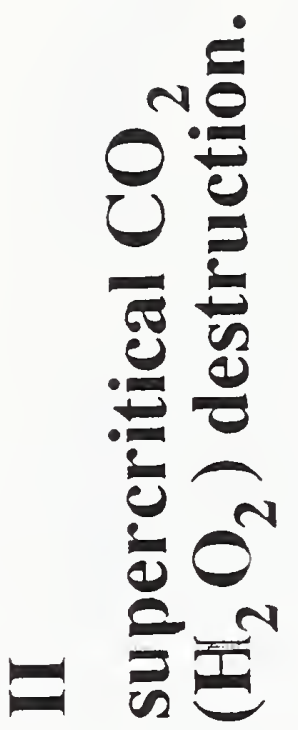

\& 0 일

ซ

(T)

등

5

5

ก료

$\dot{2}$

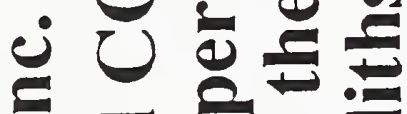

- 을 $00 \overline{0}$

ฆิ

2

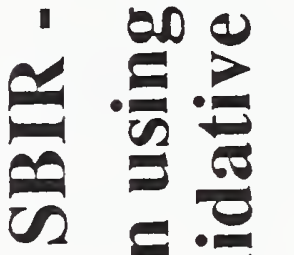

,

욜 을

ป

ह

$\sum \stackrel{\sigma}{m} 0^{m}$

$\circ=E$ ह

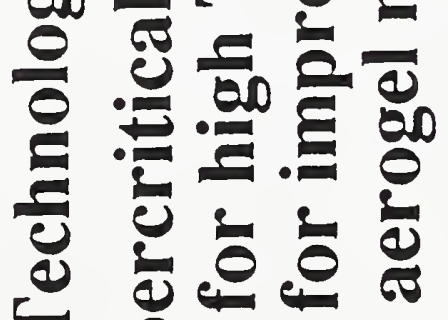

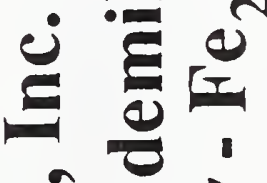

थै

क人 00

응 00

弟苞

\& 2

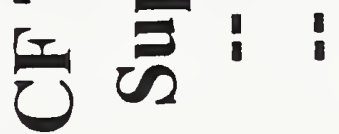

$\forall$ 을

U 


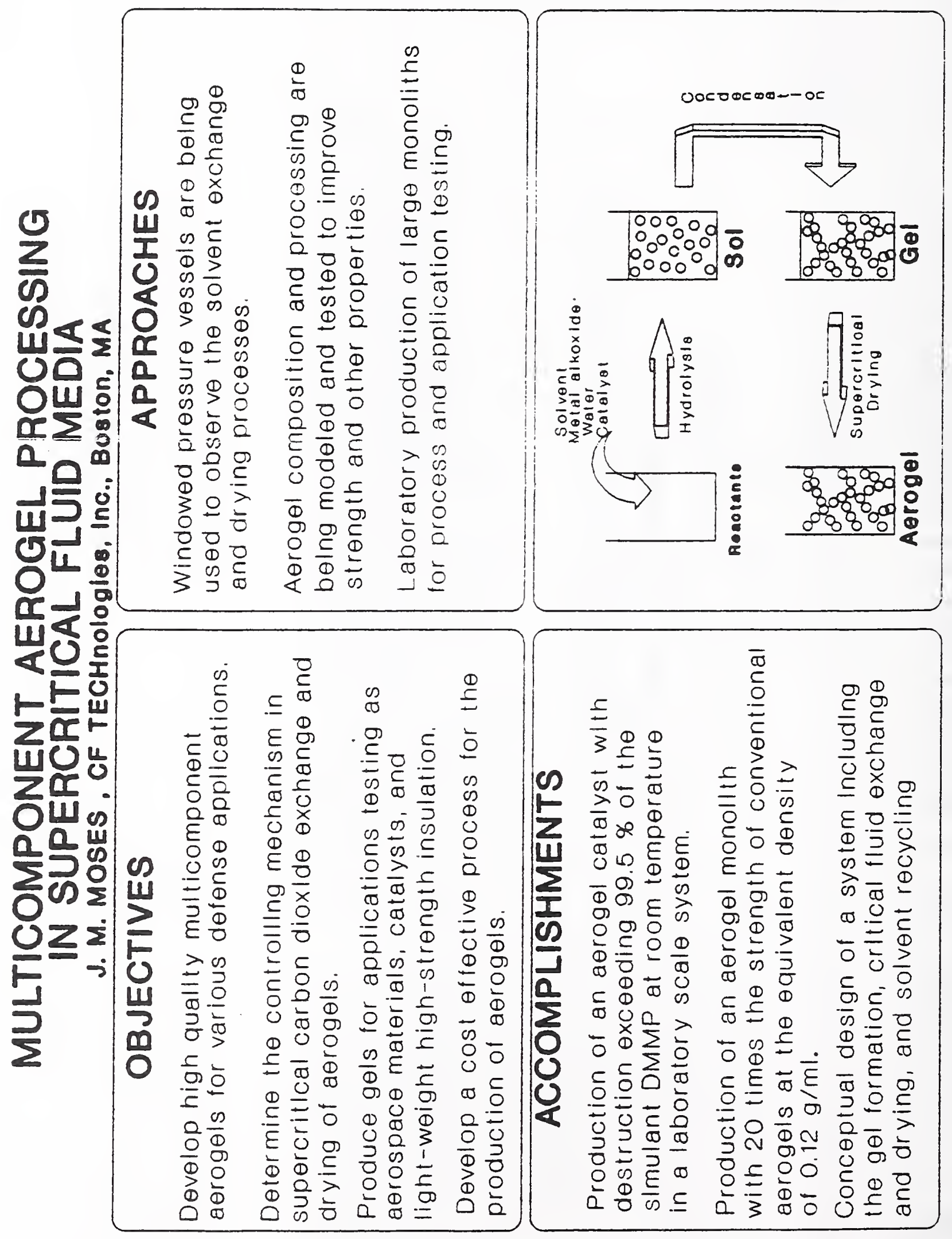




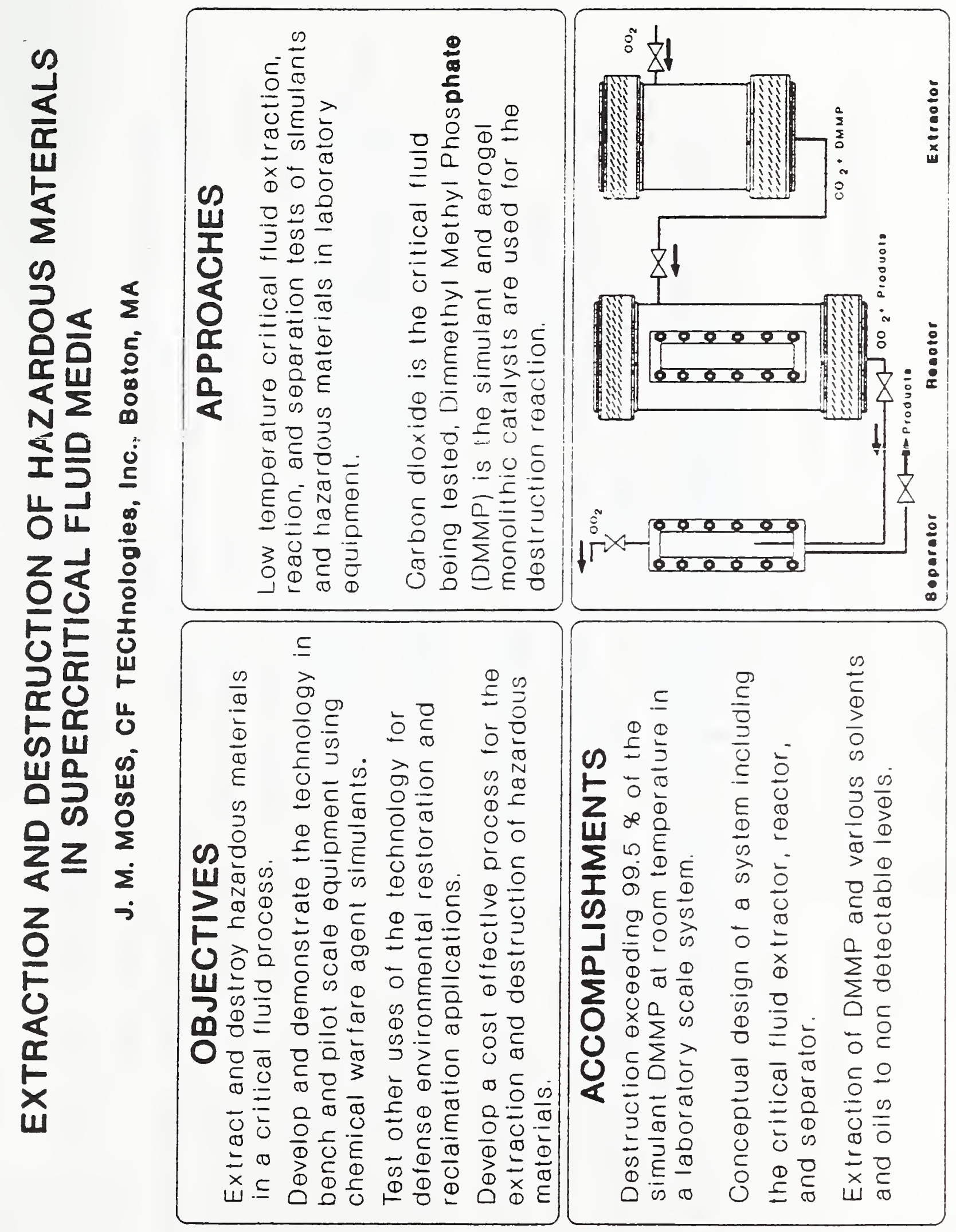




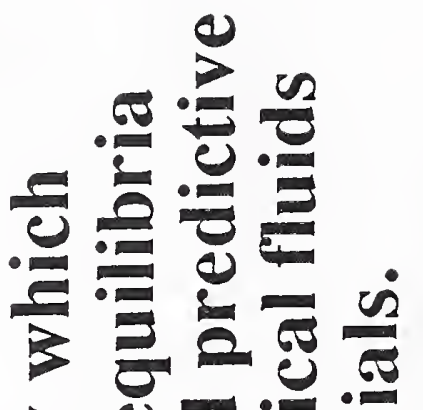

$\Rightarrow 00$ ह

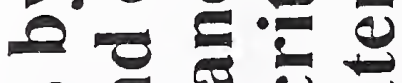

थ 60 है

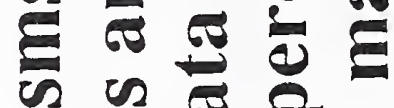

- 200

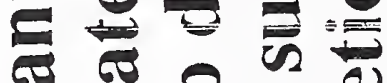

ธิ

$\checkmark$

2

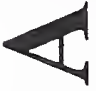

E

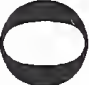

$\rightarrow$

$\infty$

$\Gamma$

2

$\theta \quad 2=00$

$\rightarrow$ e c 0 .

a (1) ह (2) थ 4

a) $\geqslant \geqslant$

$\therefore$ a $\theta$ a 0 e

$\because 0$ e $\because 0$

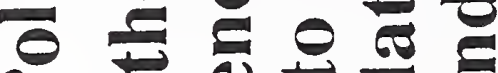

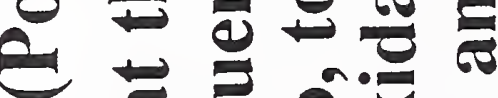

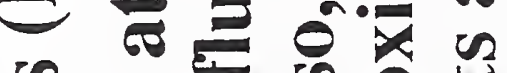

थ $ए$ थ $0+$

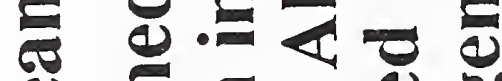

() E $E$ a 00

ए

$\theta 000$

$B$ Q थ 0 घ

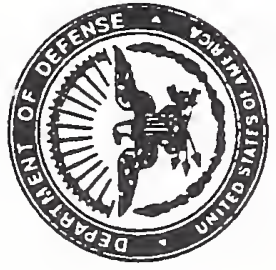

a) 0 है

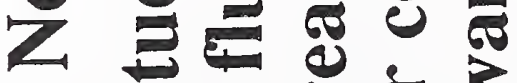

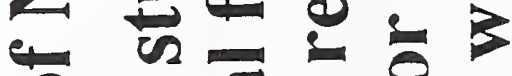

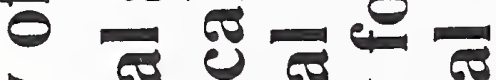

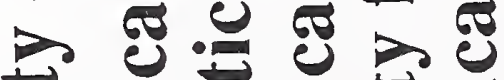

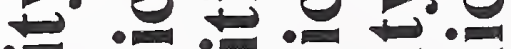

एव $\vec{a}$ ह $E$

(2) 0 ह 0 ह

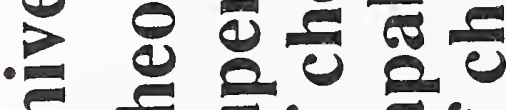

$\boxminus E$ है 0 है

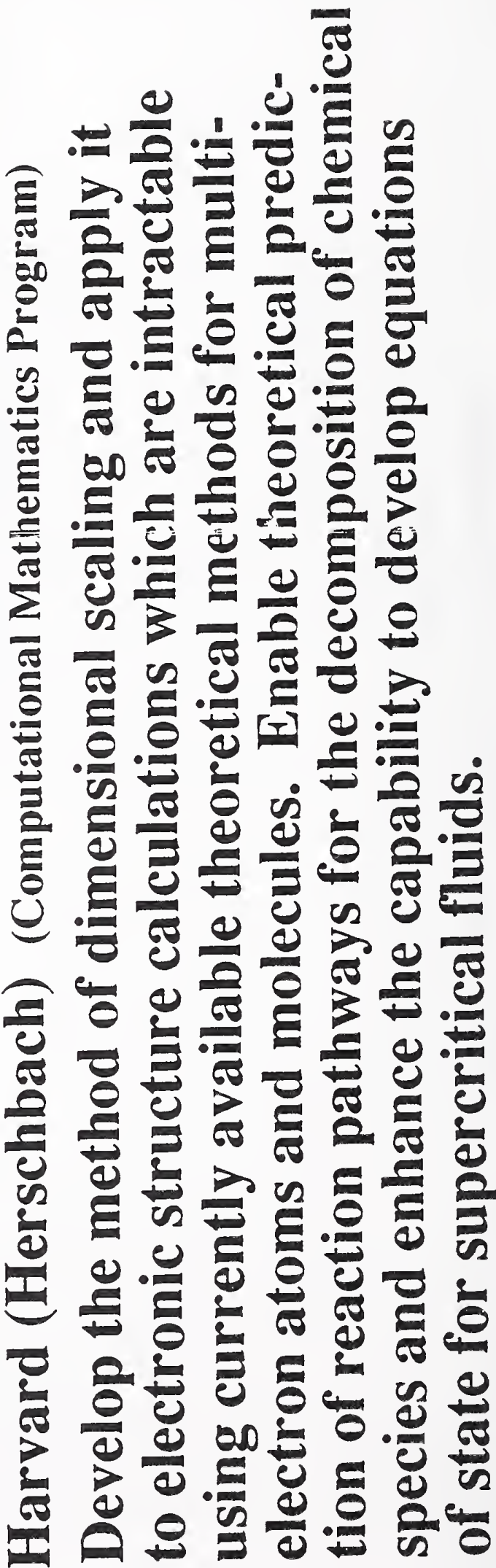




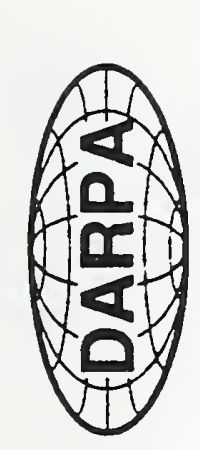

U2

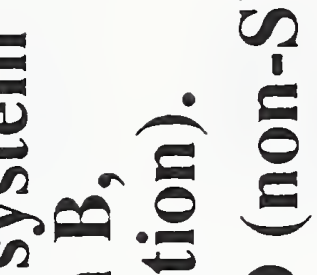

2

กำ

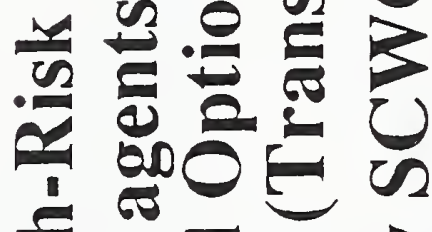

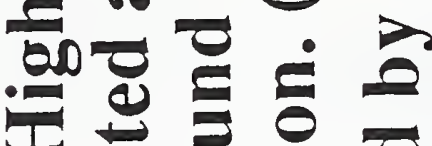

플

훙

$\pm$

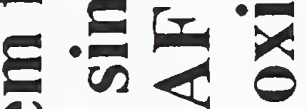

2

范

$E$

क

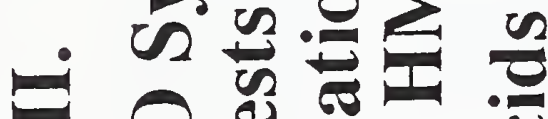

0

8

0 \&

$\frac{9}{2}$

- 2

$\therefore$.

U.ㅇำ

*

0

导

U

as

률유

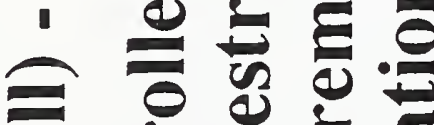

()

응휴

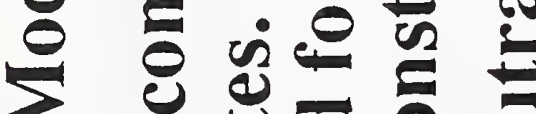

2

8 于

U बे है

될 흥

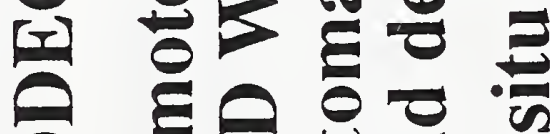

ํํㄴ을

궁

I

苞

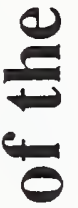

a

5

을

5

QD 3

-

它

¿

$\geq$.

․ㅡㄹ

.

$\overline{5}$

$\stackrel{0}{\circ}$

틀

항

E

.

ब)

드.

종으.

(2)

$\frac{\Xi}{3}$

(4)

=

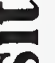

E

를

(5)

.0

कृ

ํㅡㅇ

8

E.

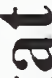

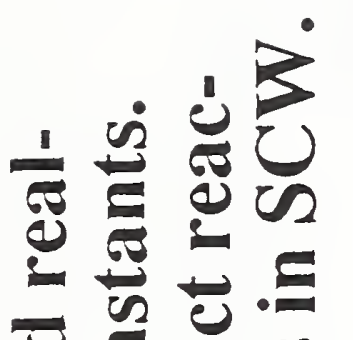

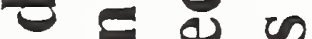

ह

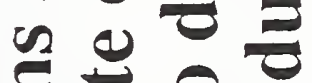

플응

为

일

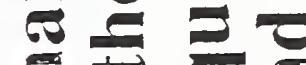

$\approx$ 들

政

อ

$=$

=

อ

$3 \cdot=$

(

¿ 0 인

-

บ.

.

ซอ

.

다음

i 

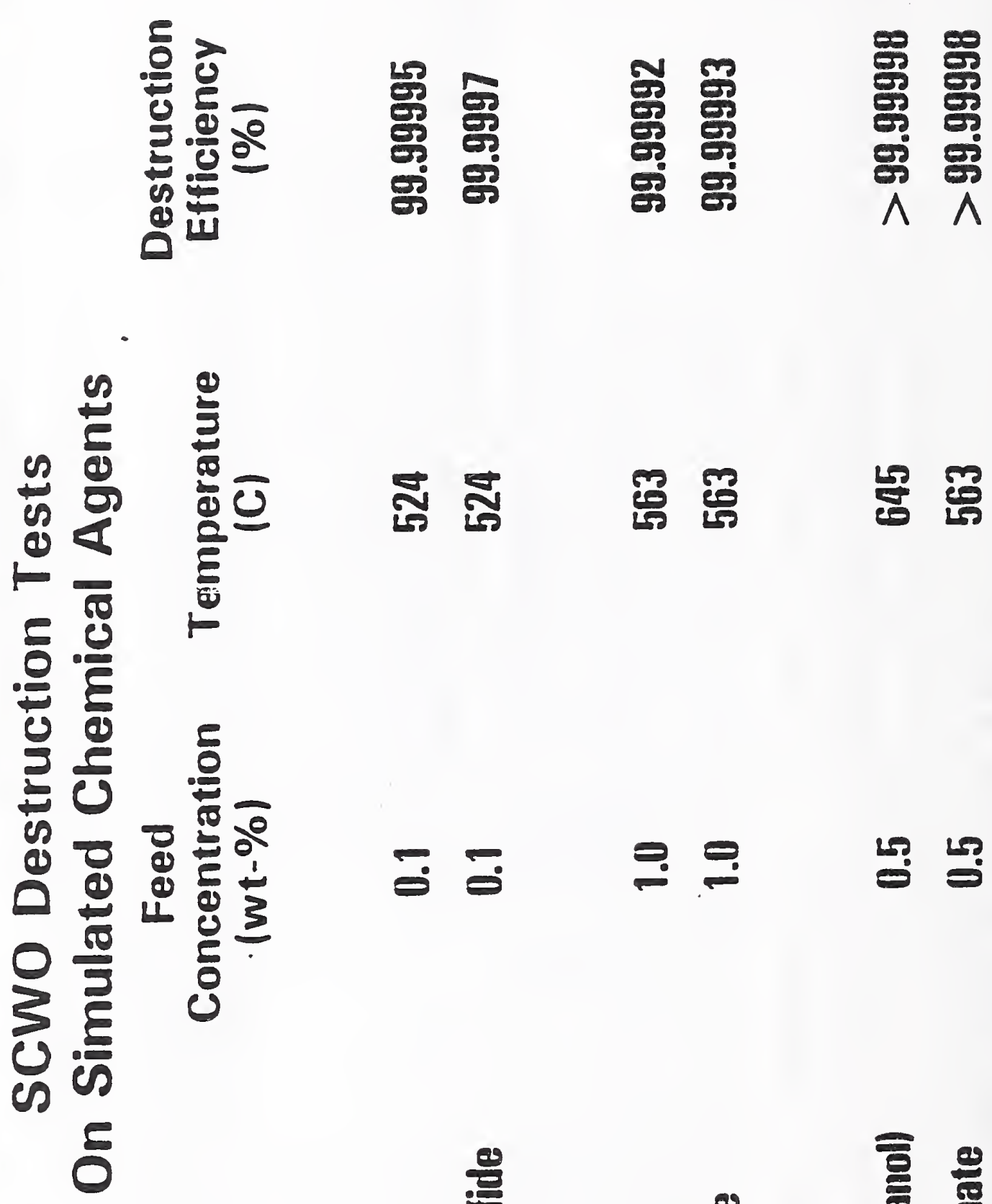

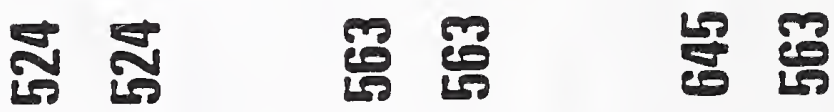
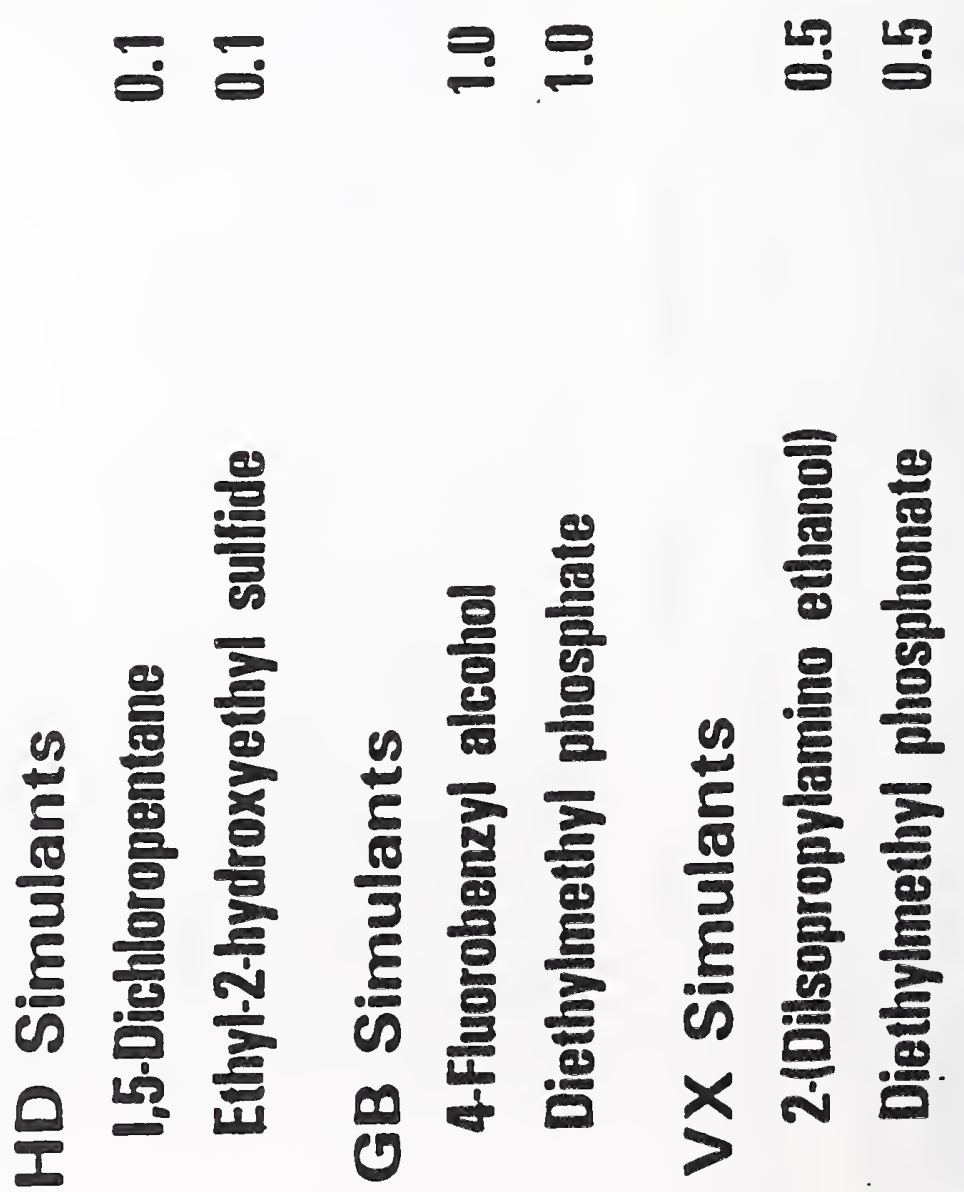
ย

(c) $N$

$\theta \rightleftarrows$

e

(2) 눙

$\theta+\infty$

$\stackrel{b}{\omega} \equiv$

- 60

E $\equiv$

${ }_{1}$

(4) 0 คิ

$\rightarrow \frac{a}{a}$

(1)

A.

$E 2$

2

2

$\theta$

요

응

를을

4 F

$\rightarrow 5$

a

02

$\theta$ ह

$\Rightarrow$ 잉

$0+0$

20

U $\vec{\sigma}$

¿

$<$ 을

드를

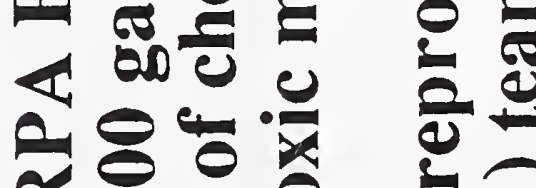

อ

02

능요을 는

尺 $\wedge$ 을

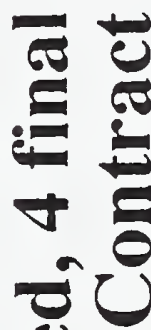

$\stackrel{0}{2}$

(8)

巳

ช

in

9

N

2

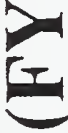

范

ㄴ.

है

$\stackrel{0}{2}$

$\nabla$

0

బ

$\sigma$

2

이

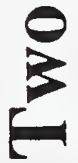



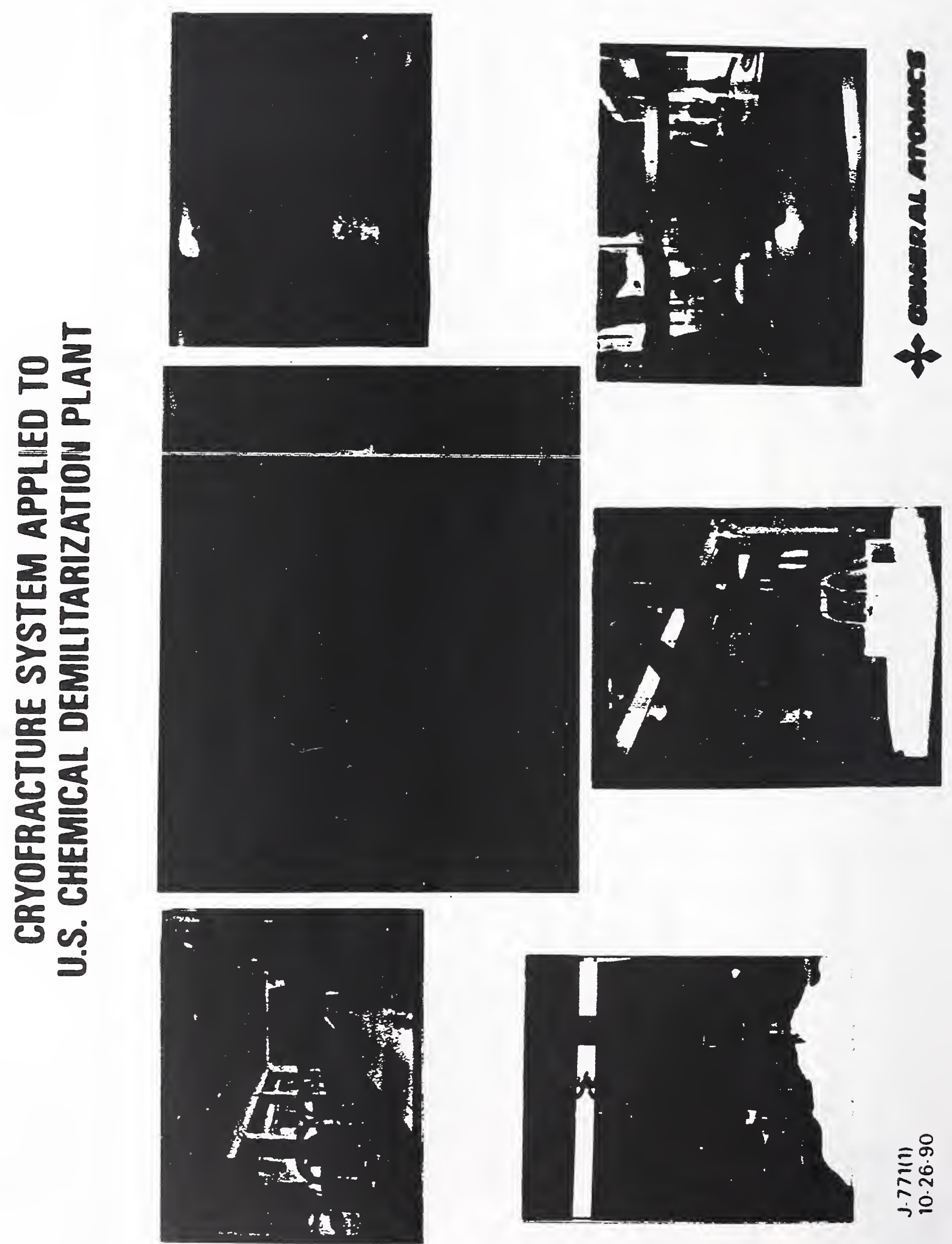
出

ш

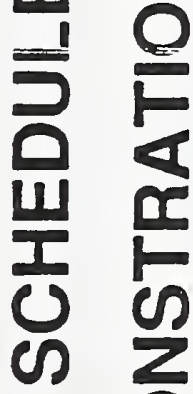

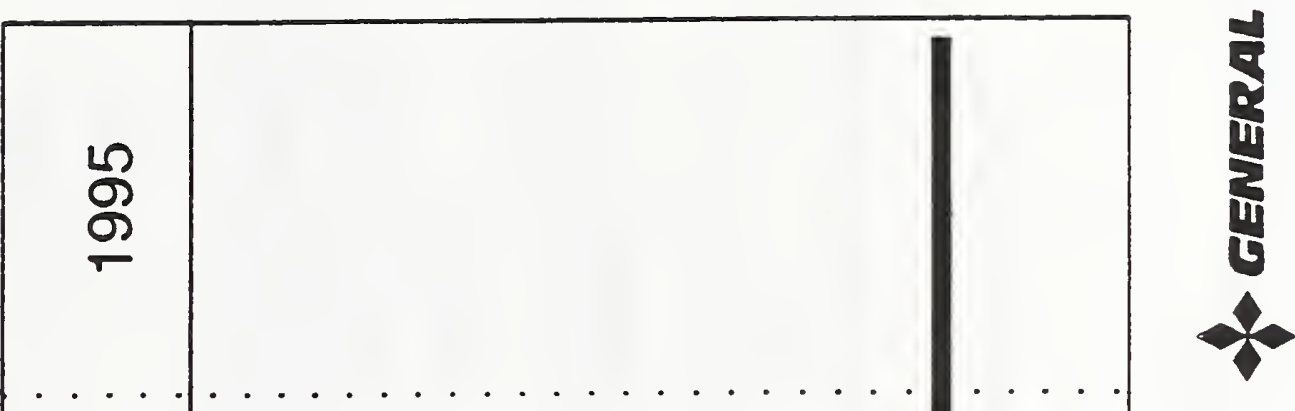

$\sum 0$

뜬

ज

는

品

음

文 5

क 1

$<\overline{\bar{\alpha}}$

$\frac{0}{\frac{1}{a}}$

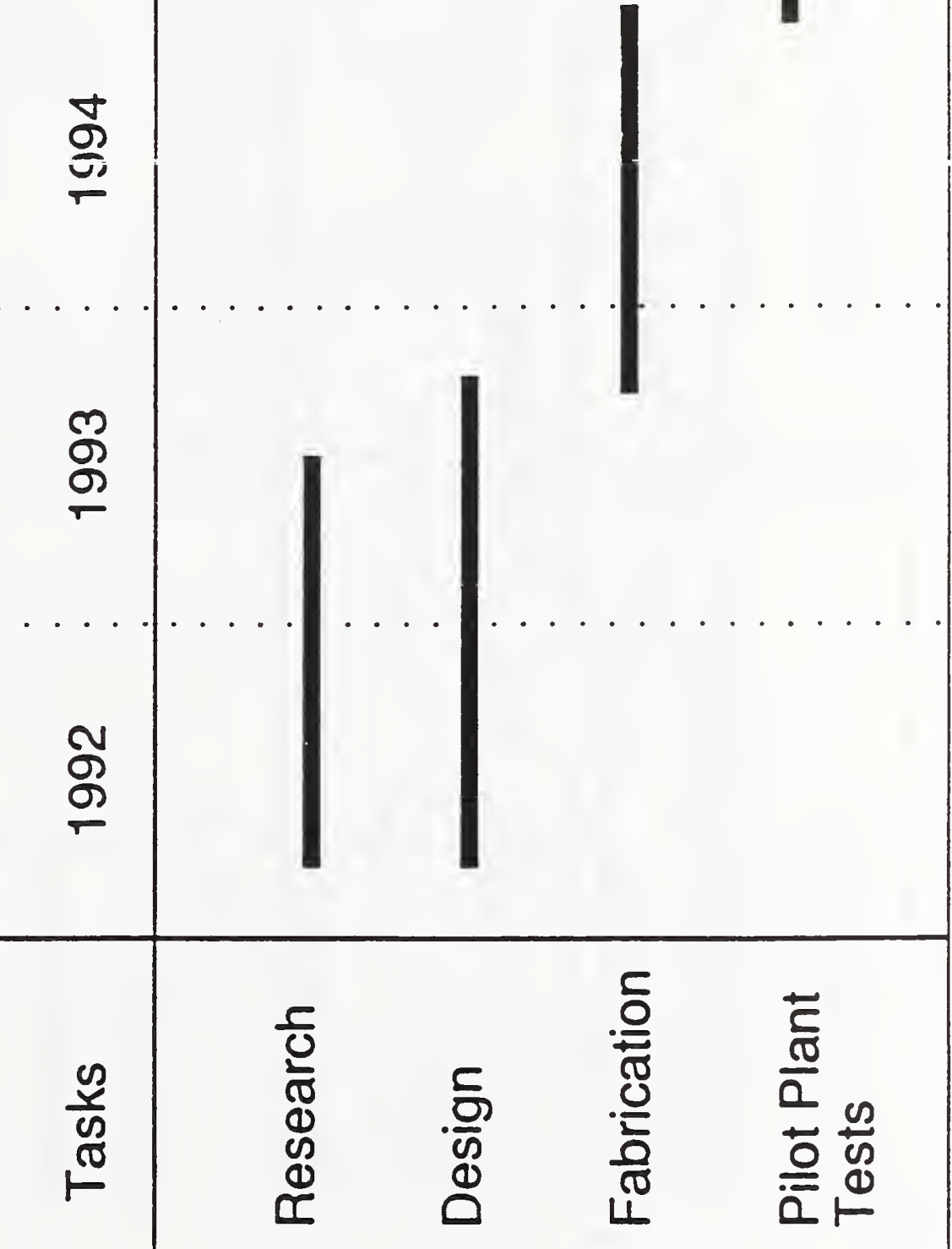



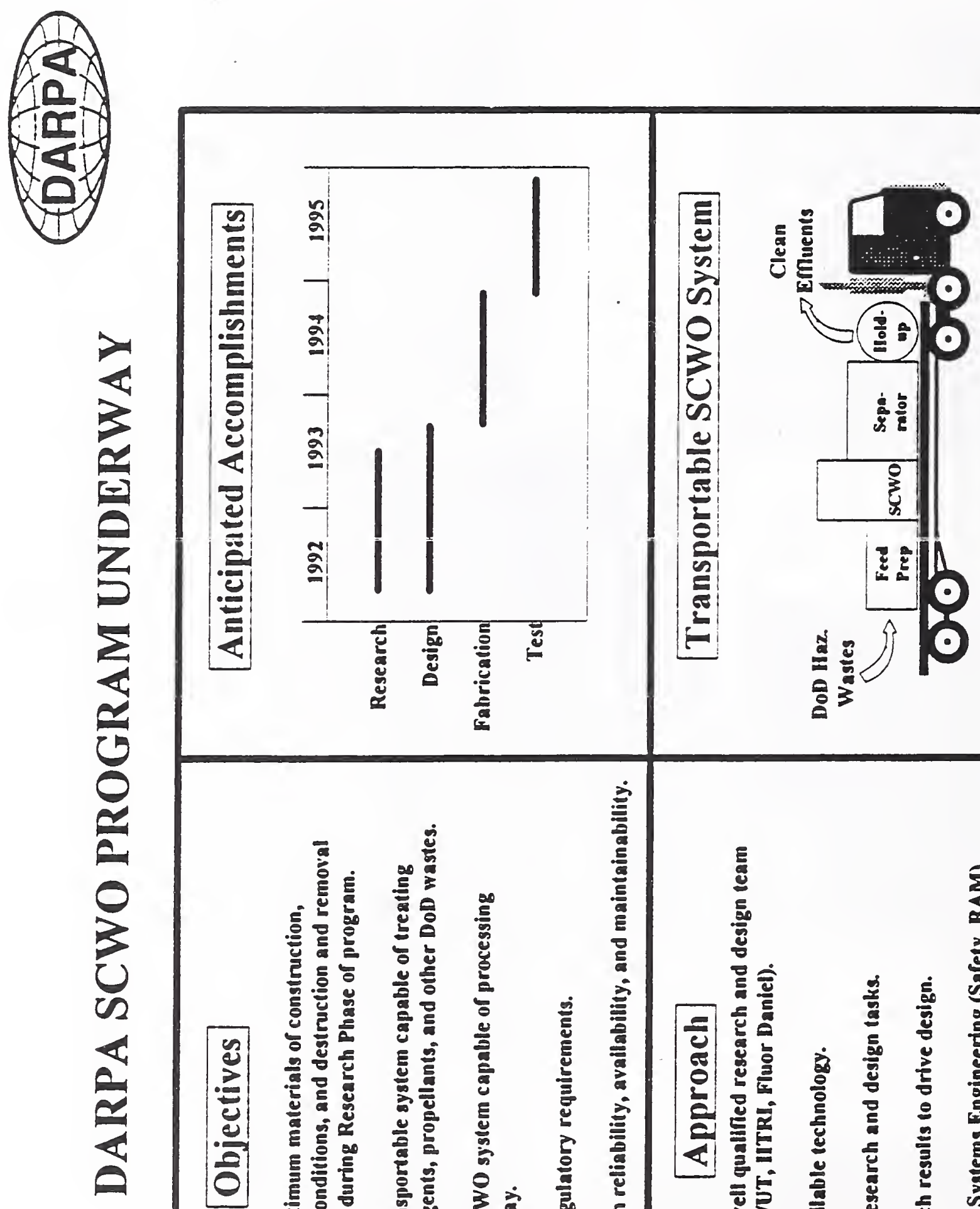

U
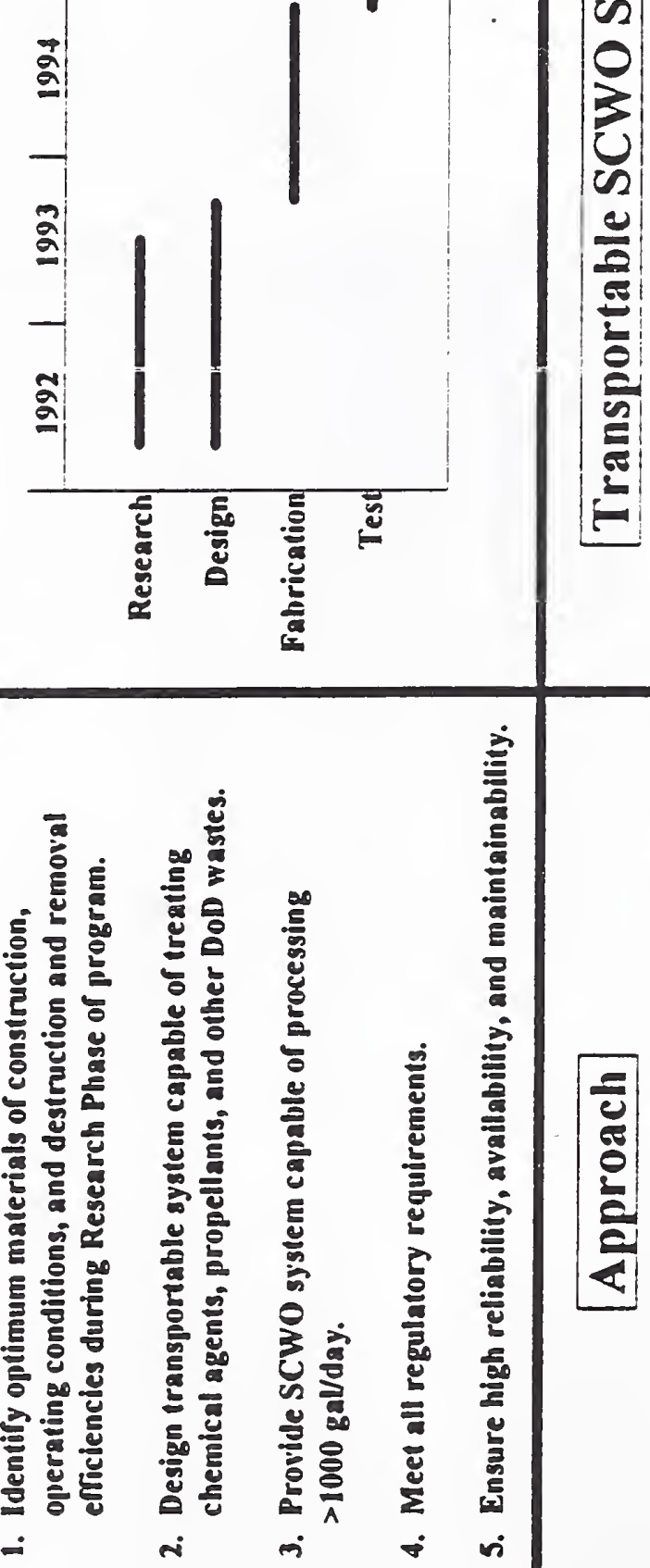


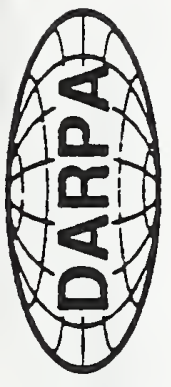

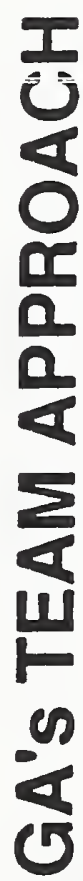

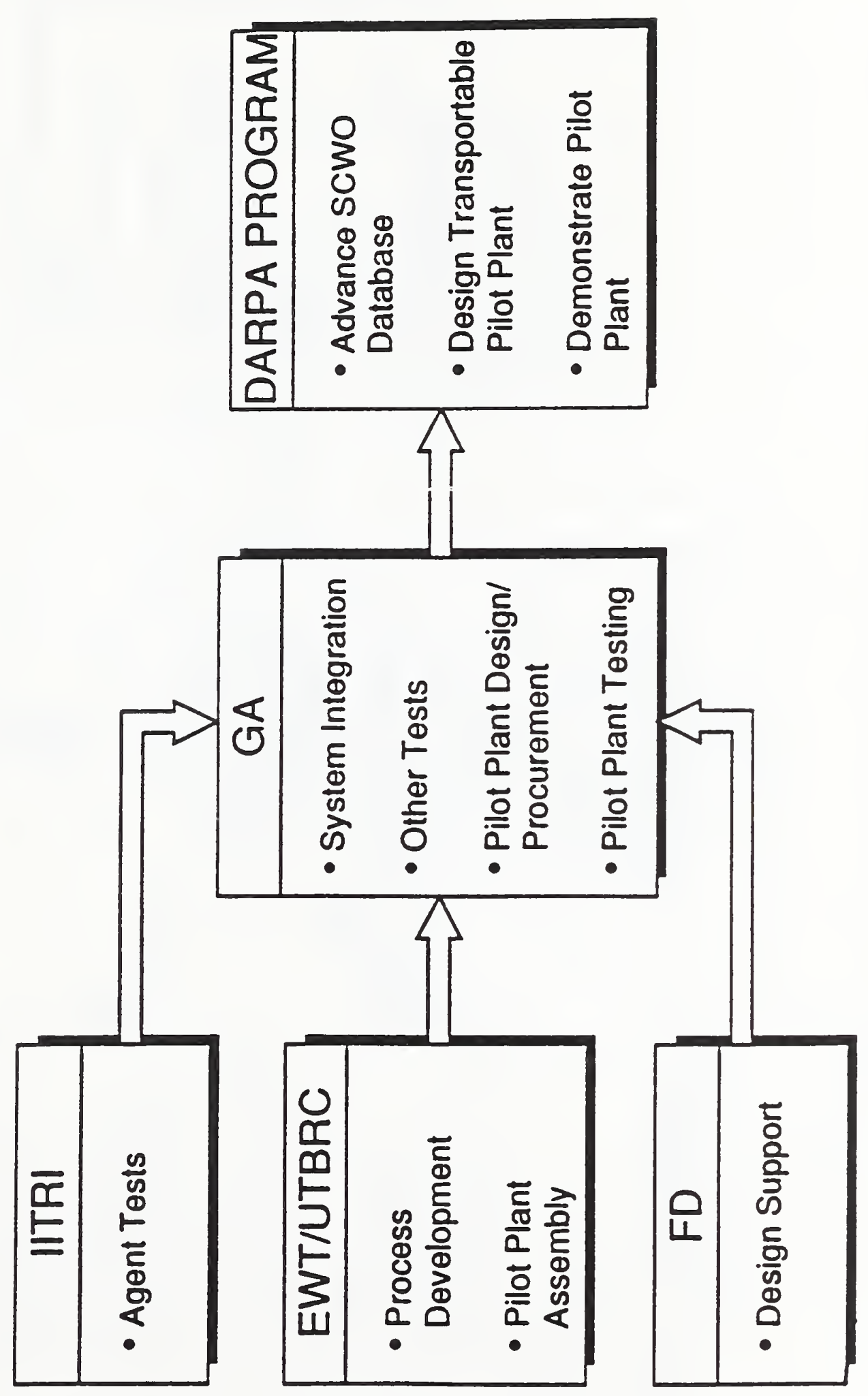



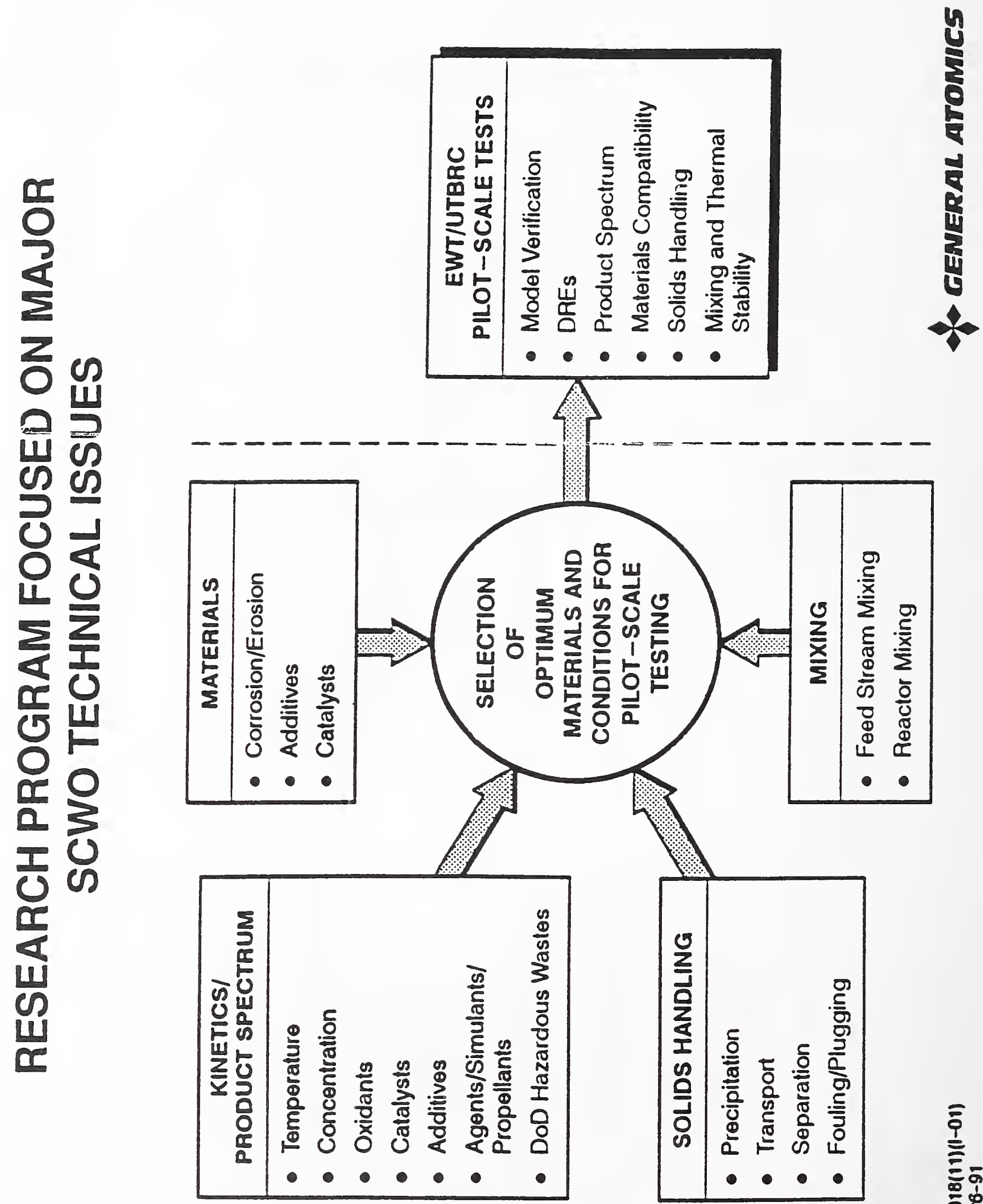

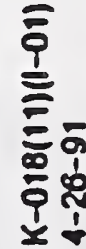



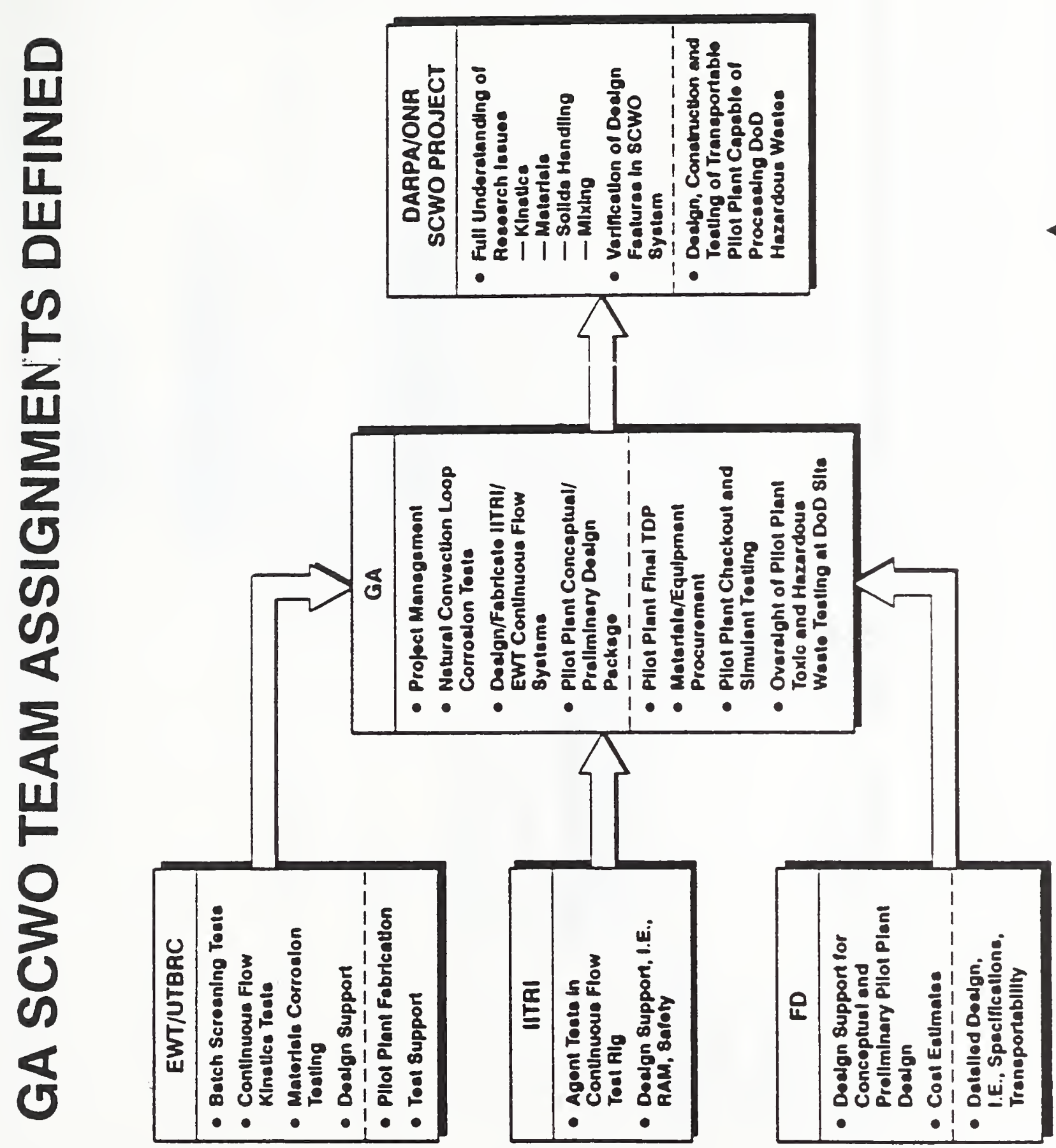


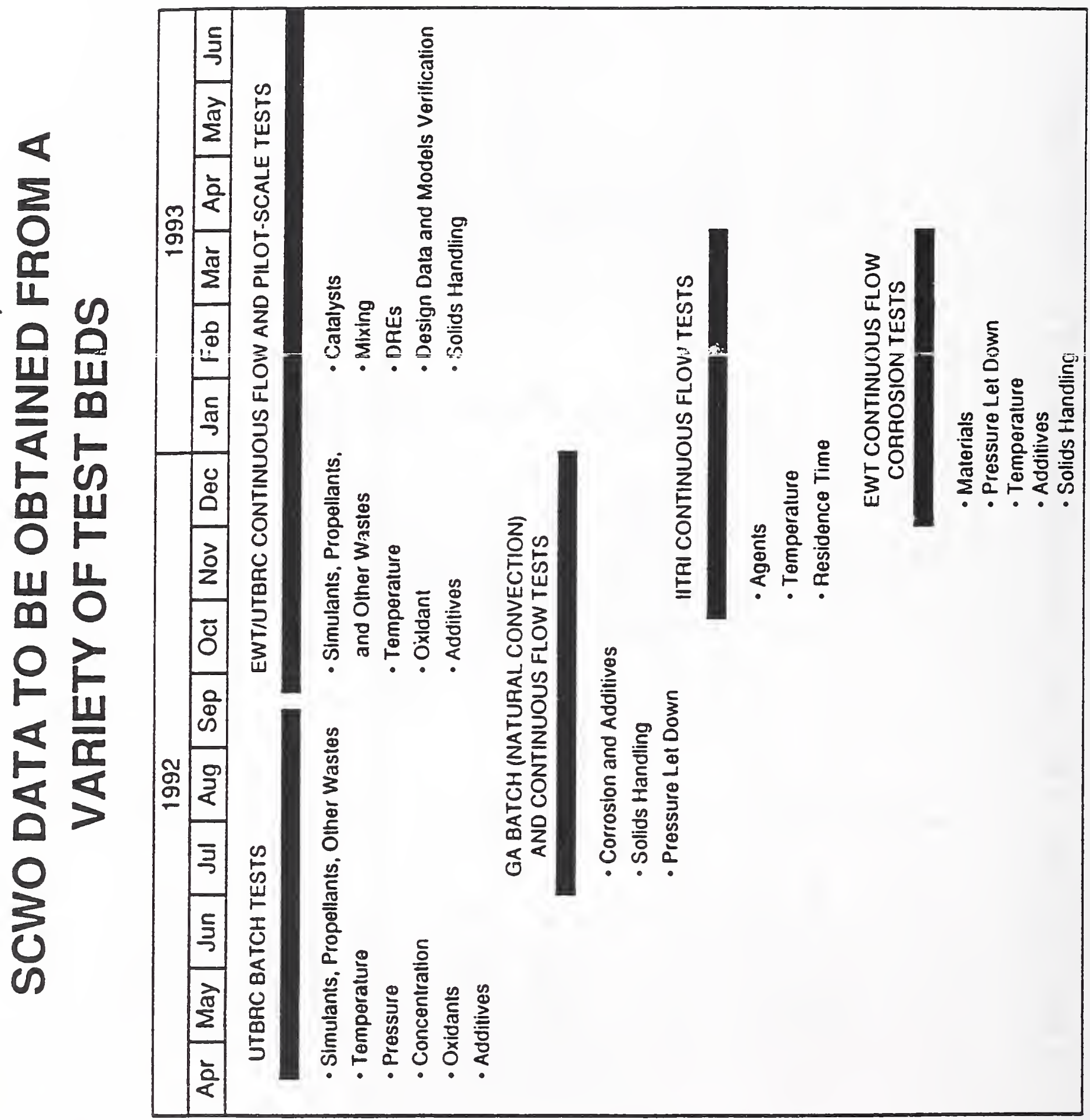




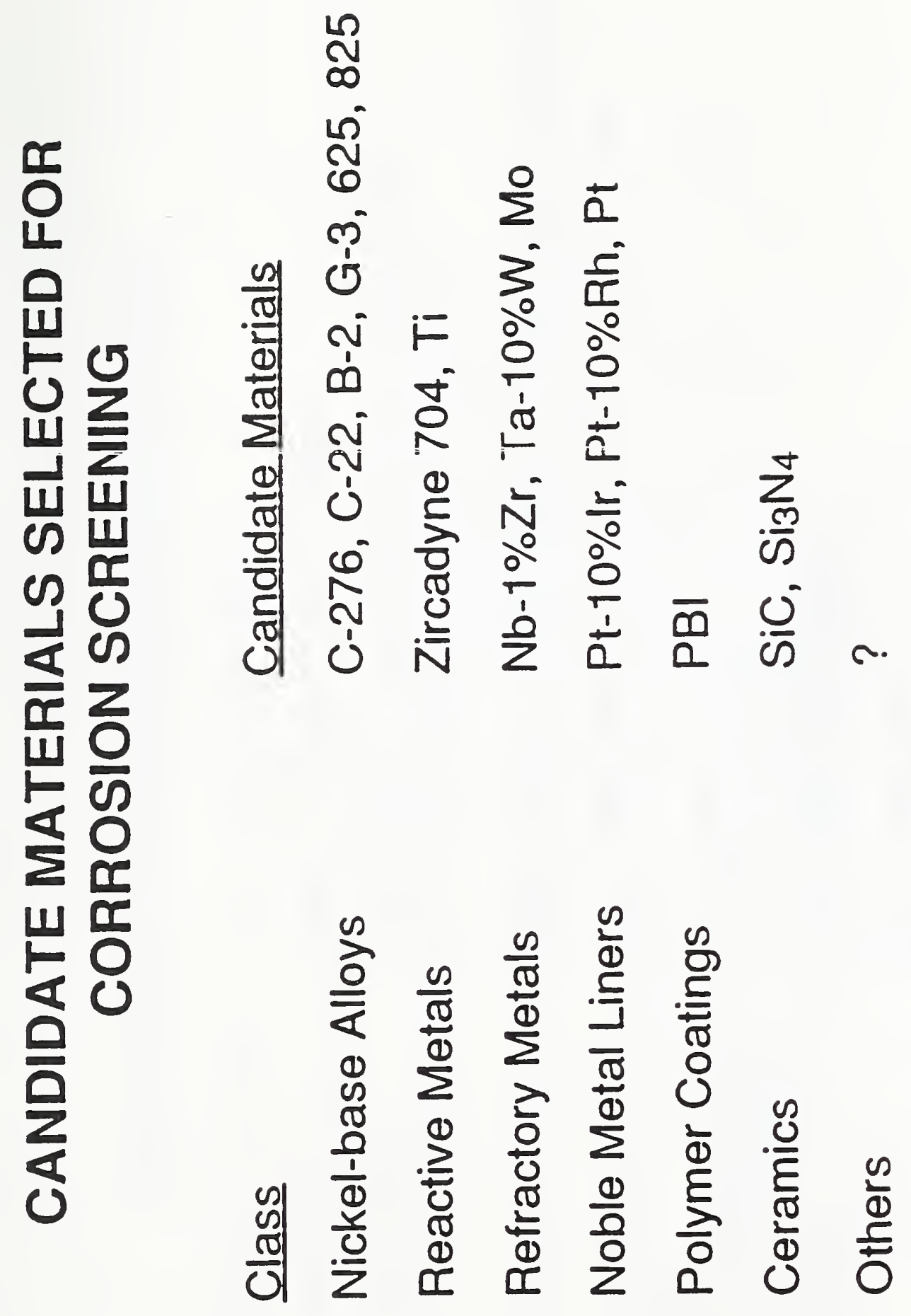




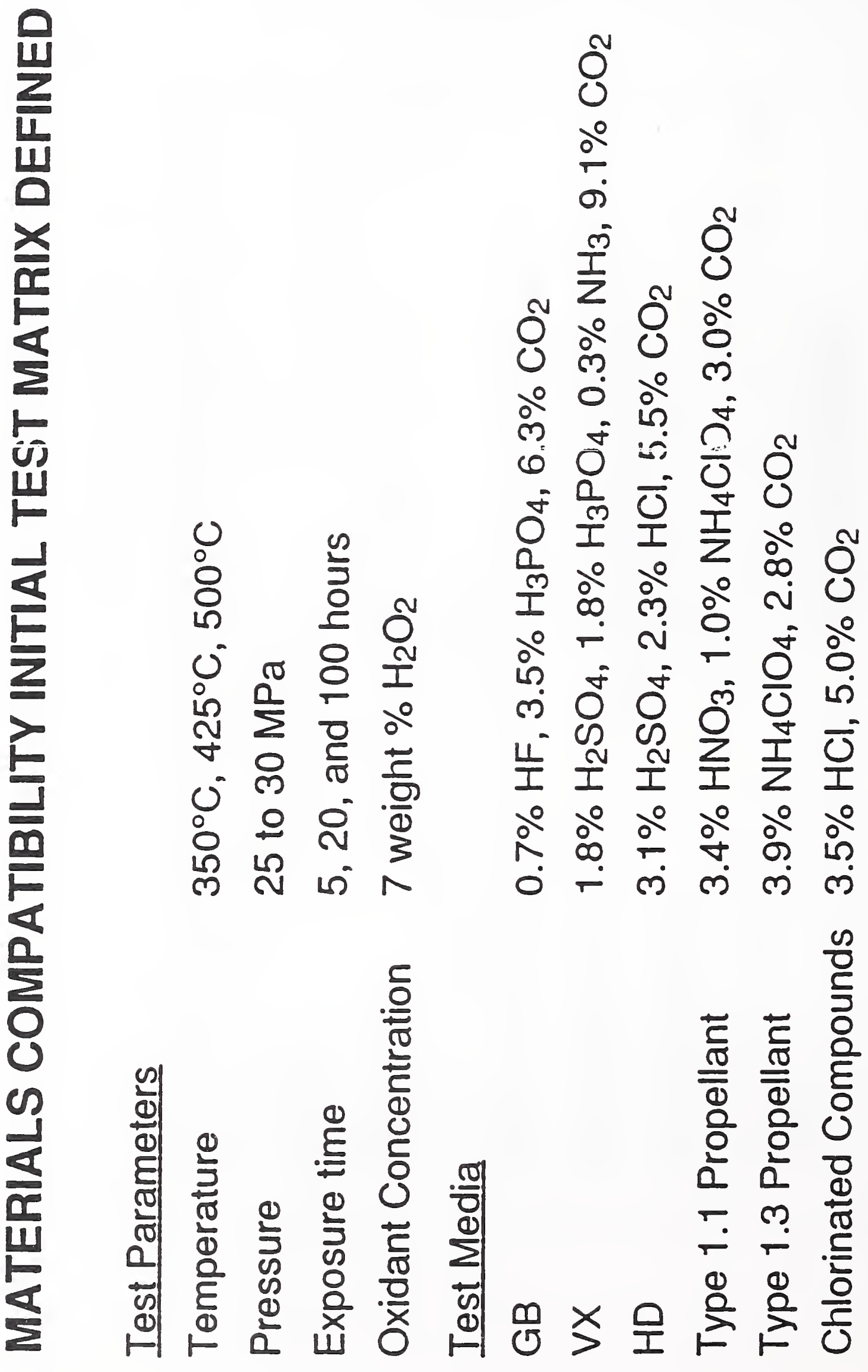


出

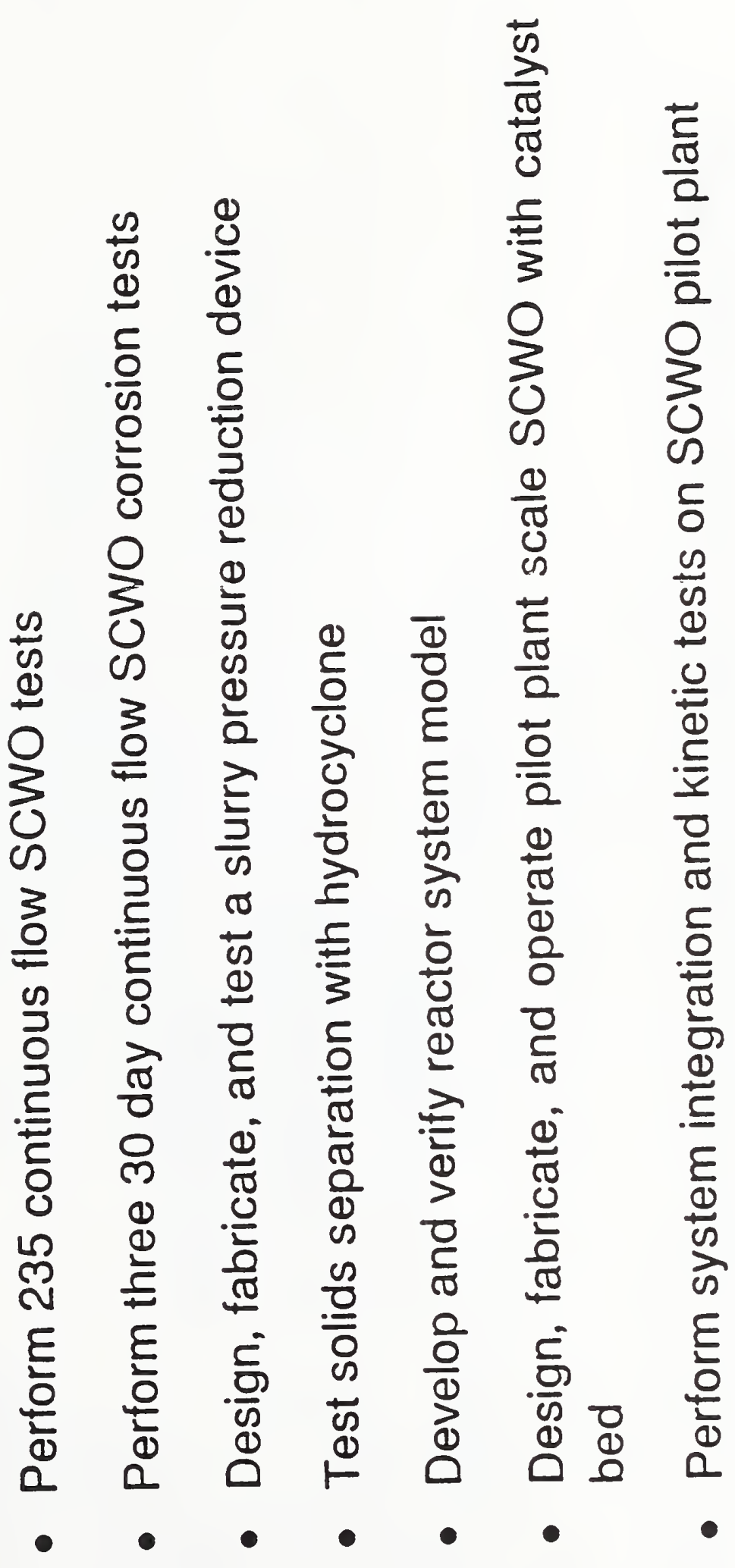




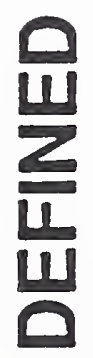

$\frac{\infty}{\frac{\omega}{E}}$

$\frac{9}{\infty}$

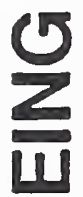

3

o

$\stackrel{\bar{\varpi}}{\frac{1}{\sigma}}$

N

$\frac{x}{\underline{x}}$

$\varepsilon$ \&

U

을 ญ्य

$\frac{1}{U}$

m

$\frac{1}{5}$ กั่

$\frac{\stackrel{0}{0}}{\frac{10}{0}}$

N

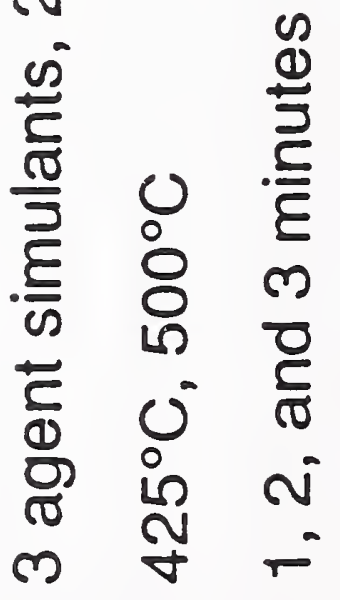

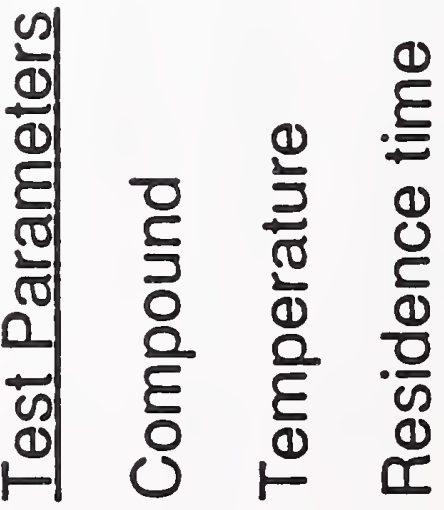




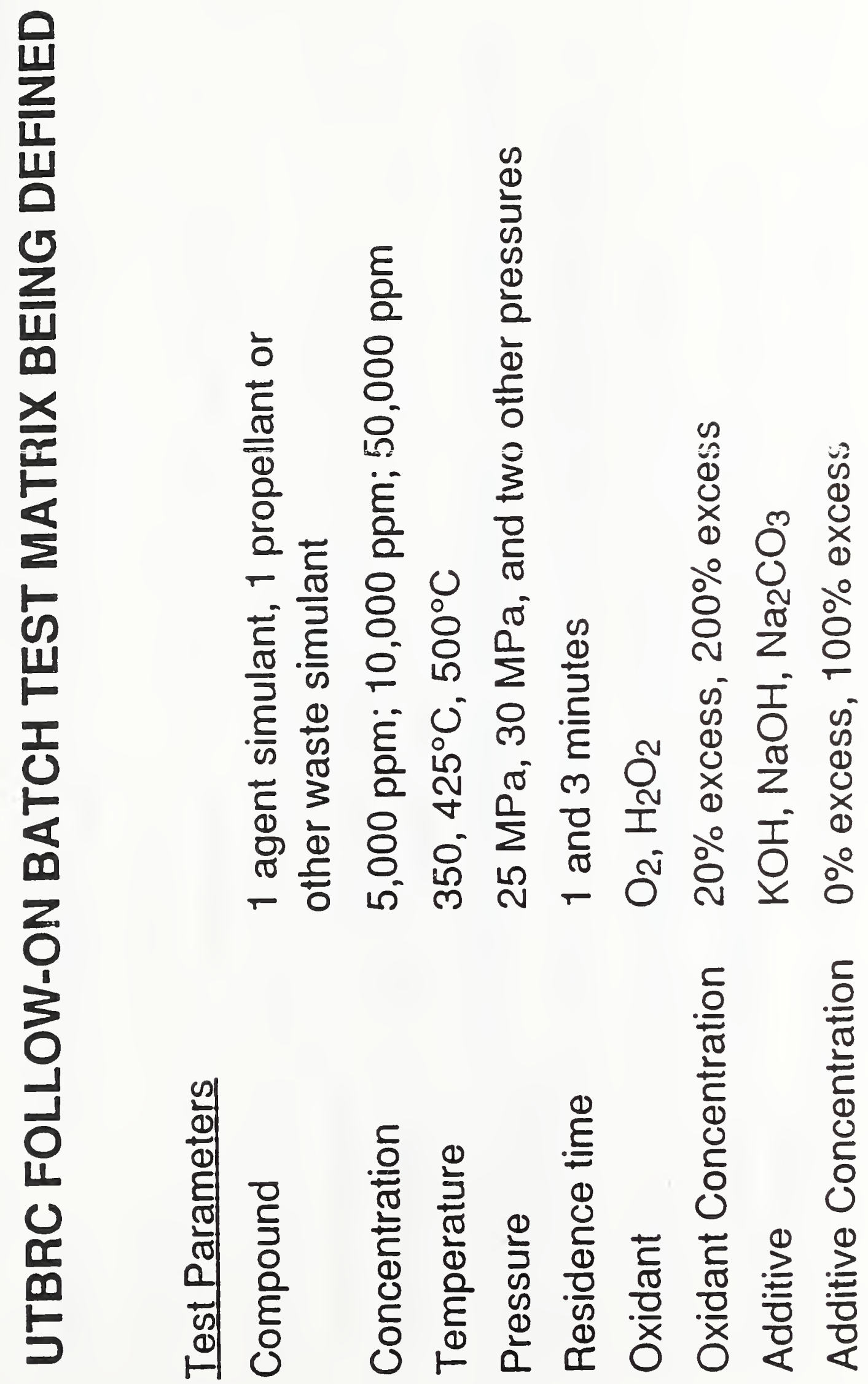




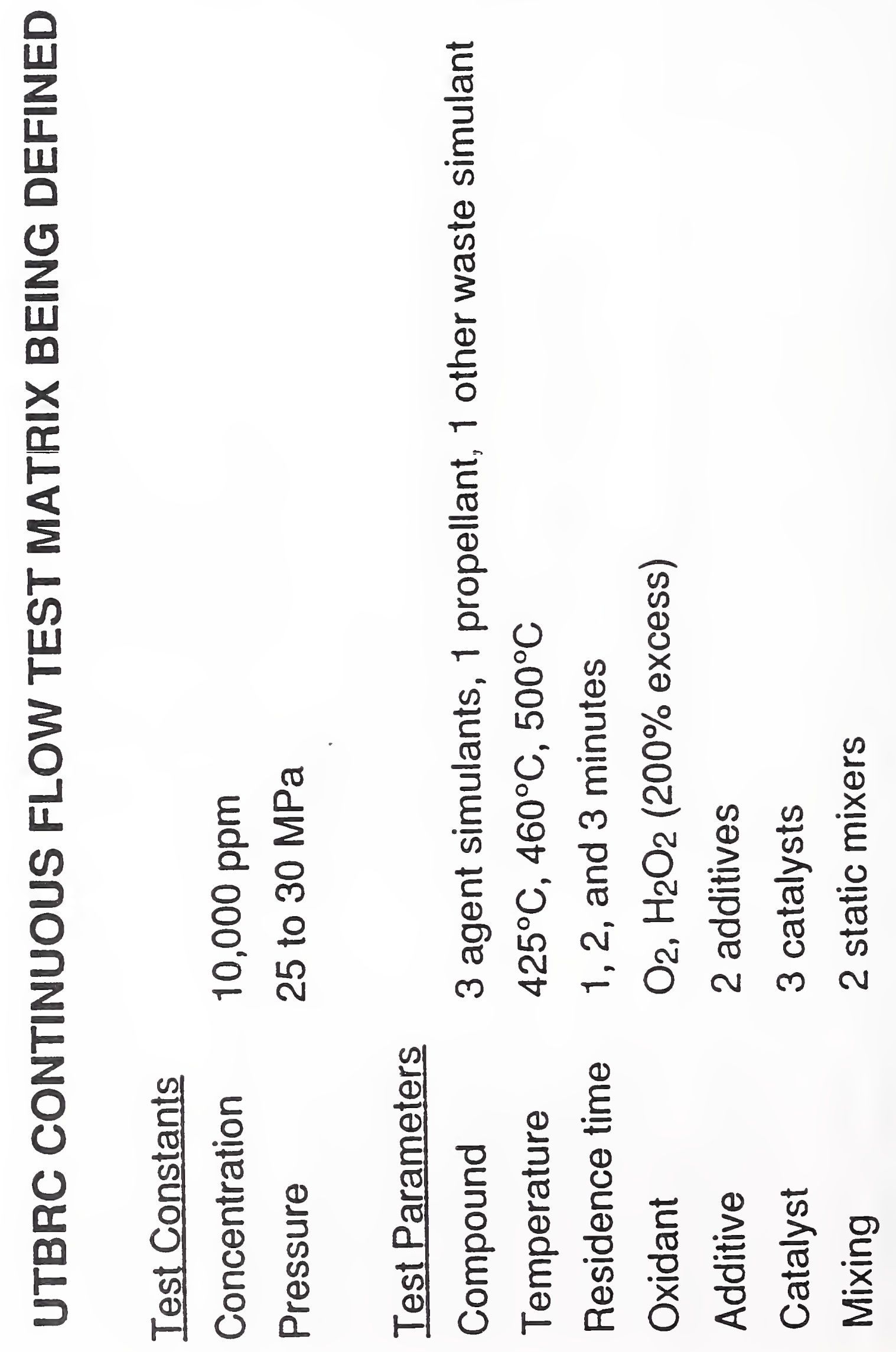




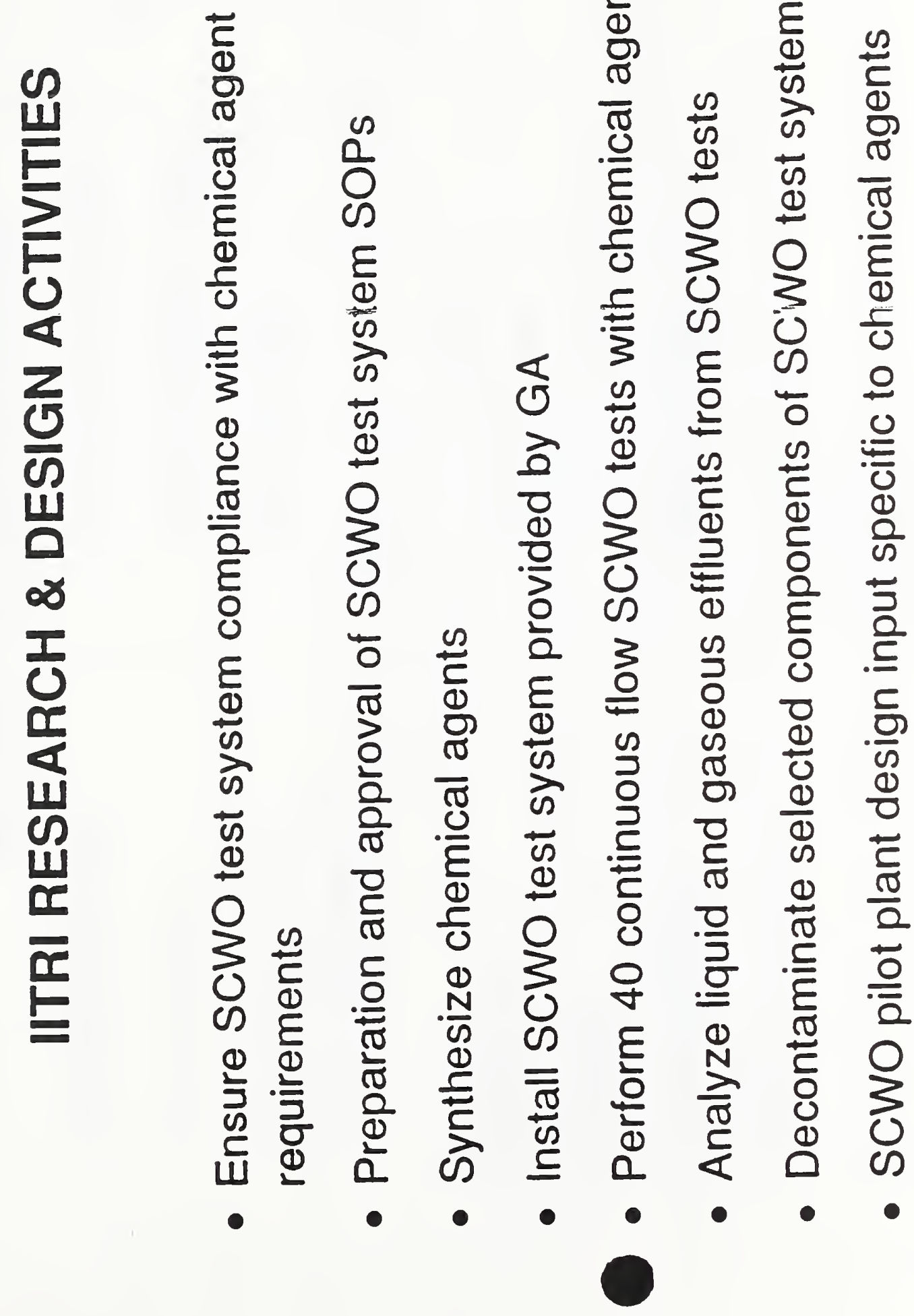


$\frac{Q}{4}$

5

$z$

UI

es

$x$

마

$F$

$<$

$\sum$

1

क

픈

5

핀

5

4

$\frac{1}{6}$

0

I

u

I

0

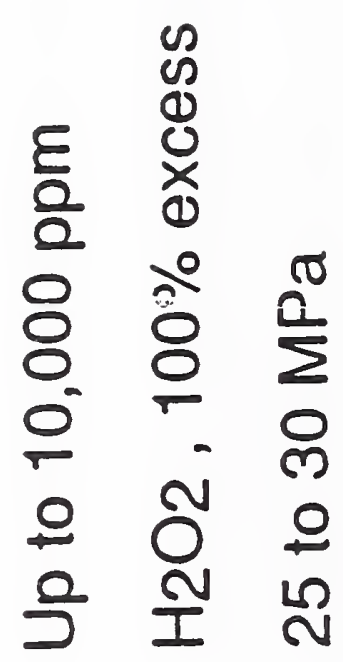

है

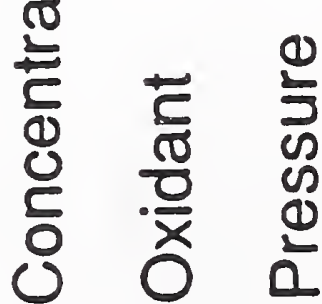

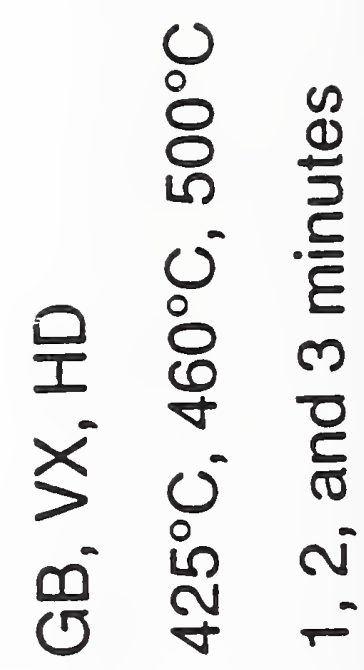

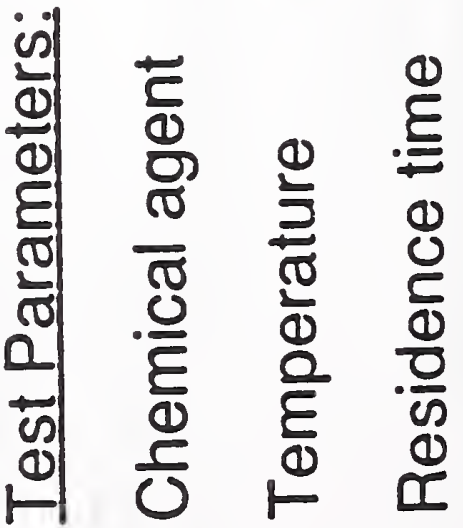




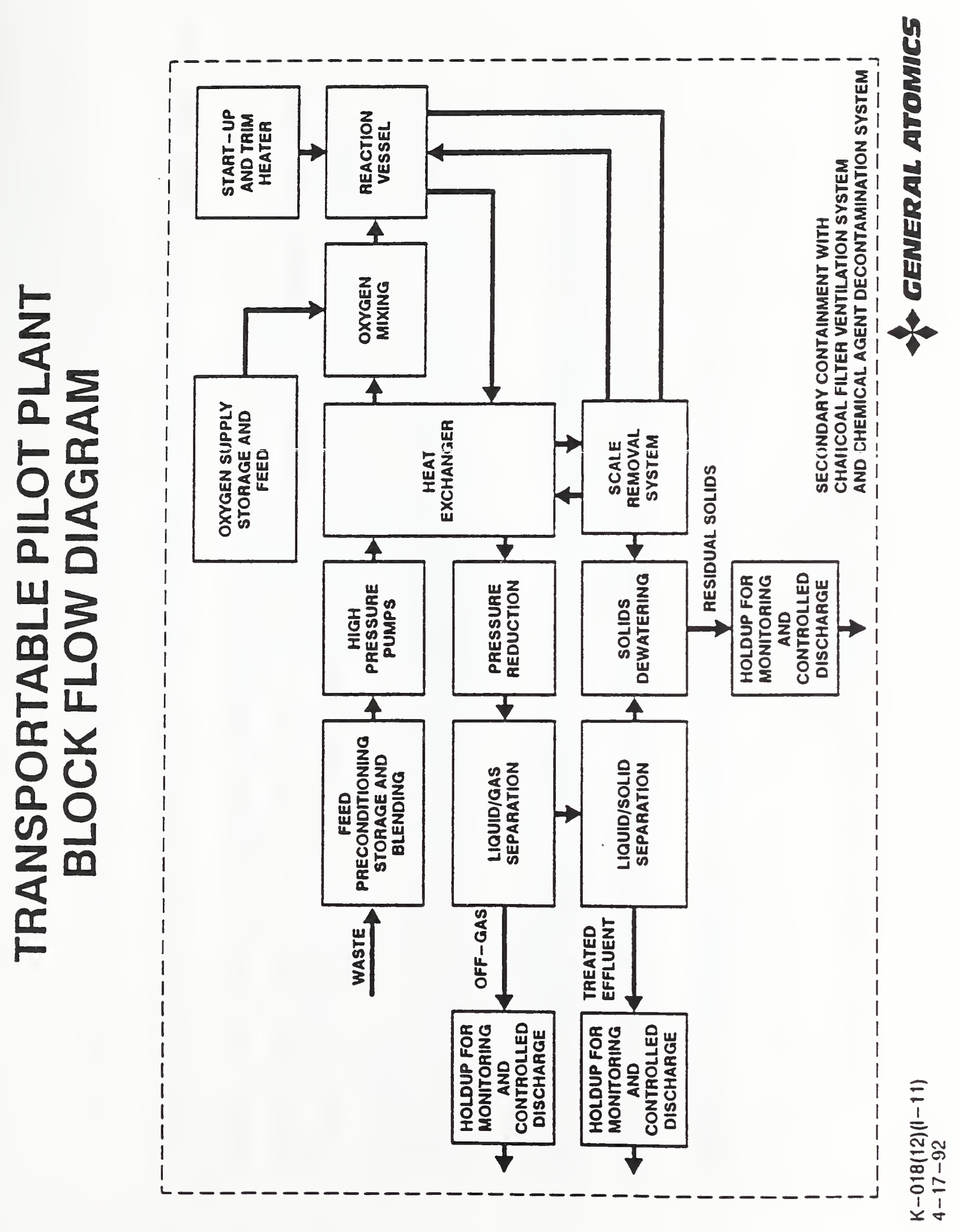




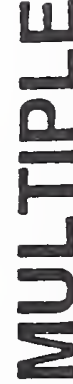

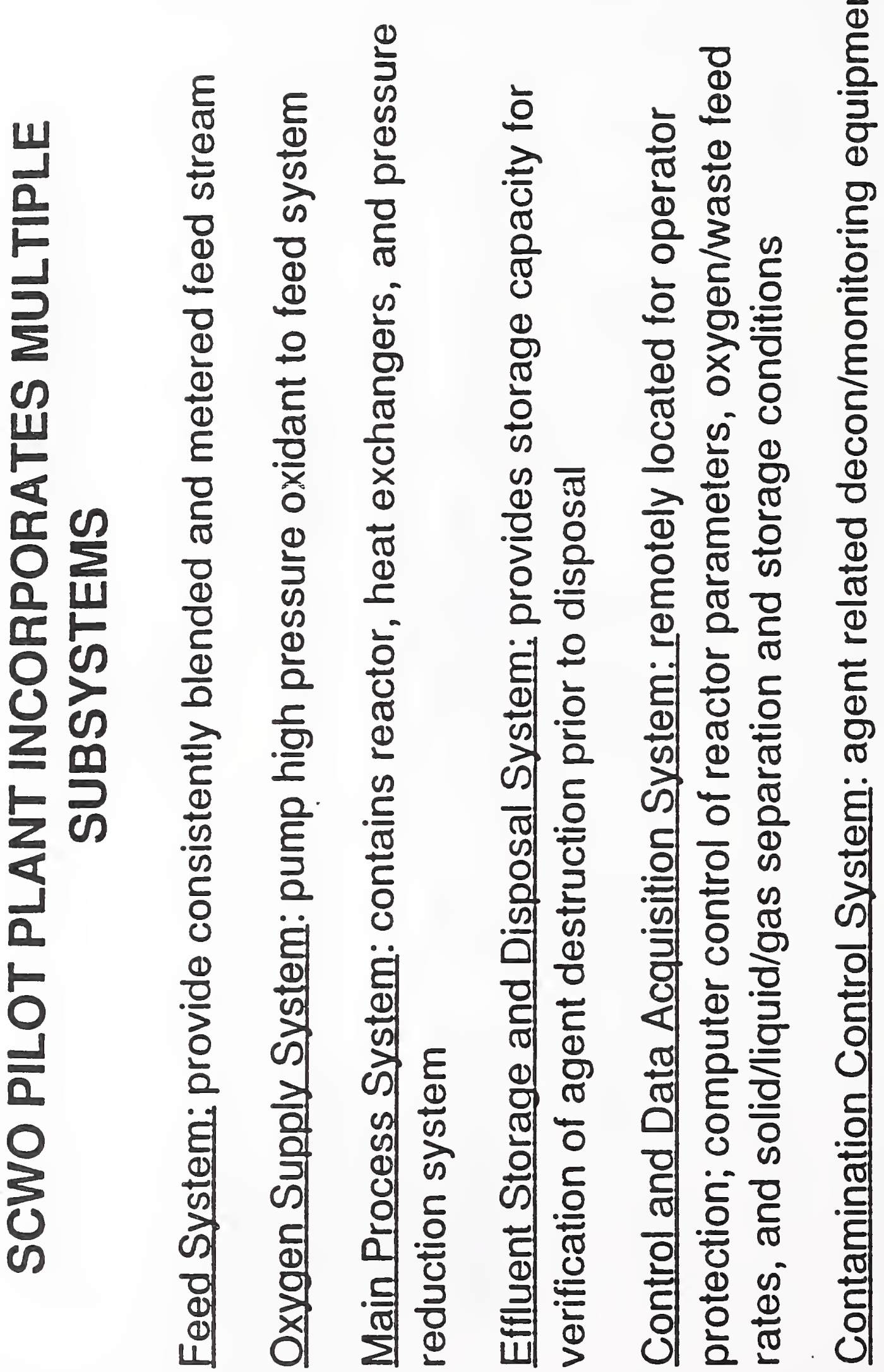




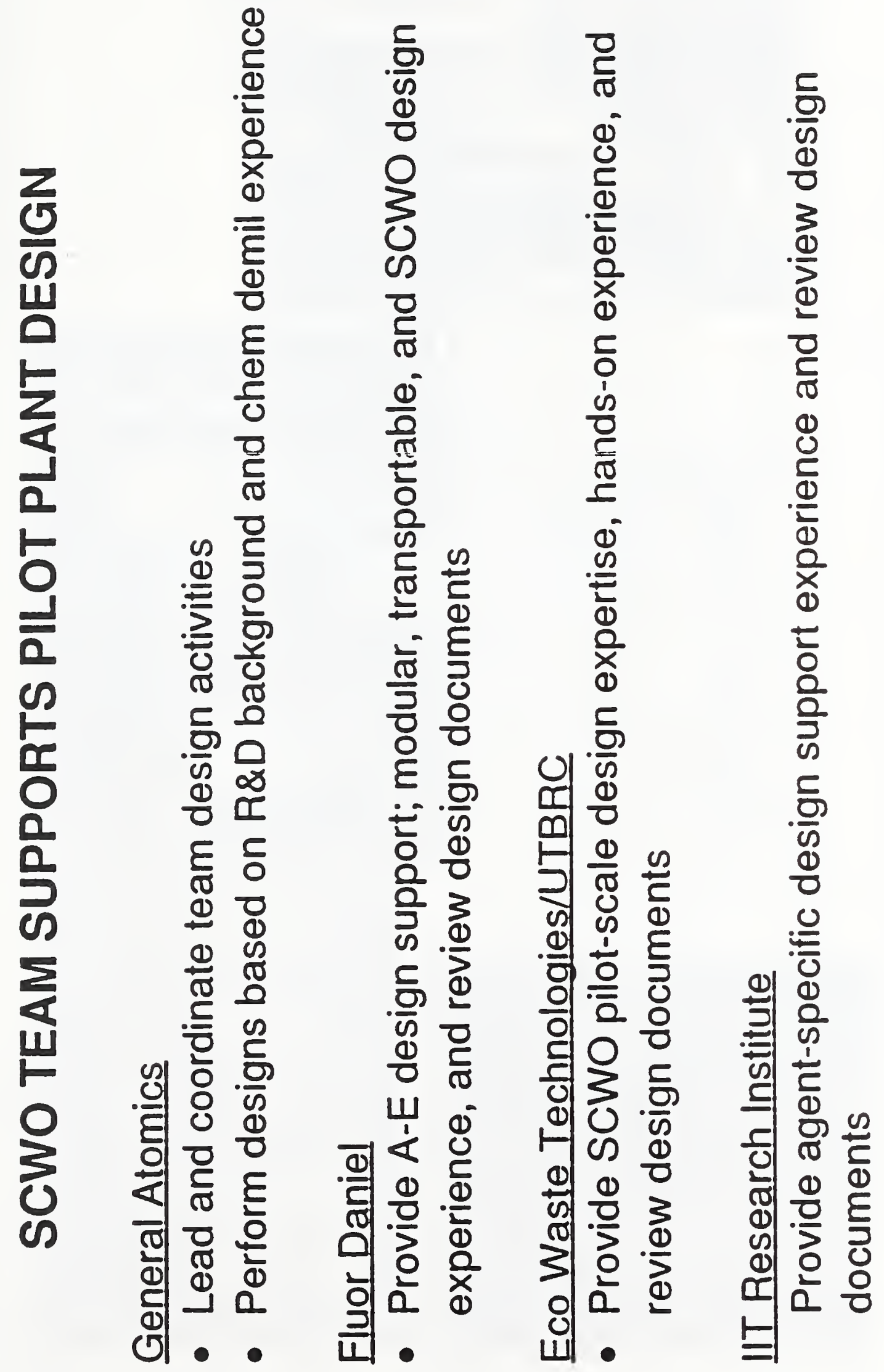




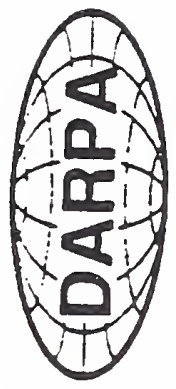

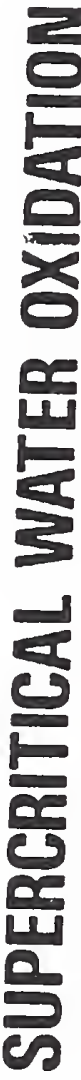

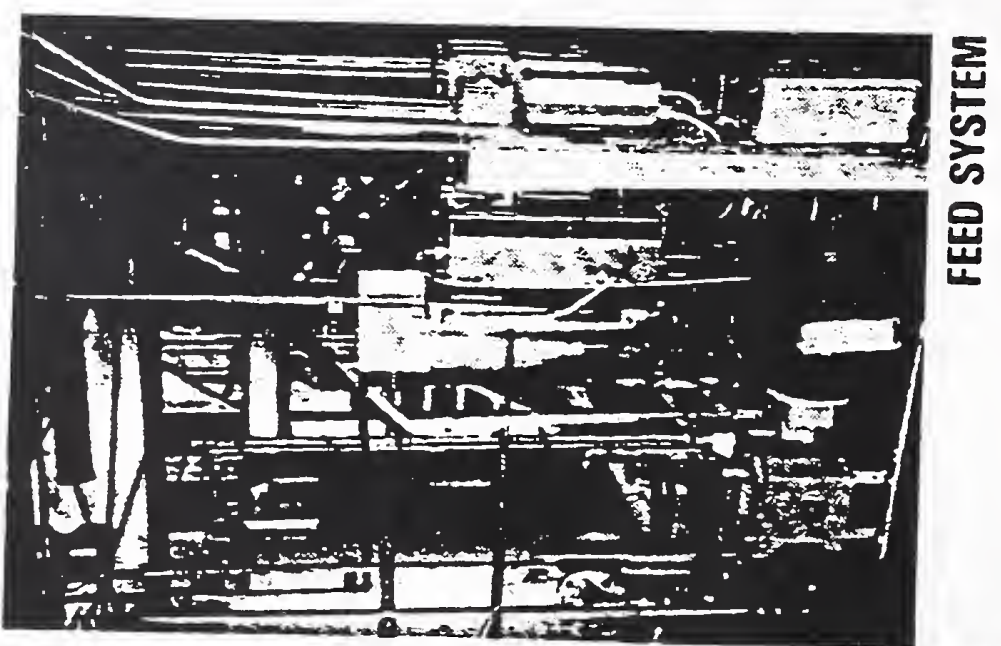

点
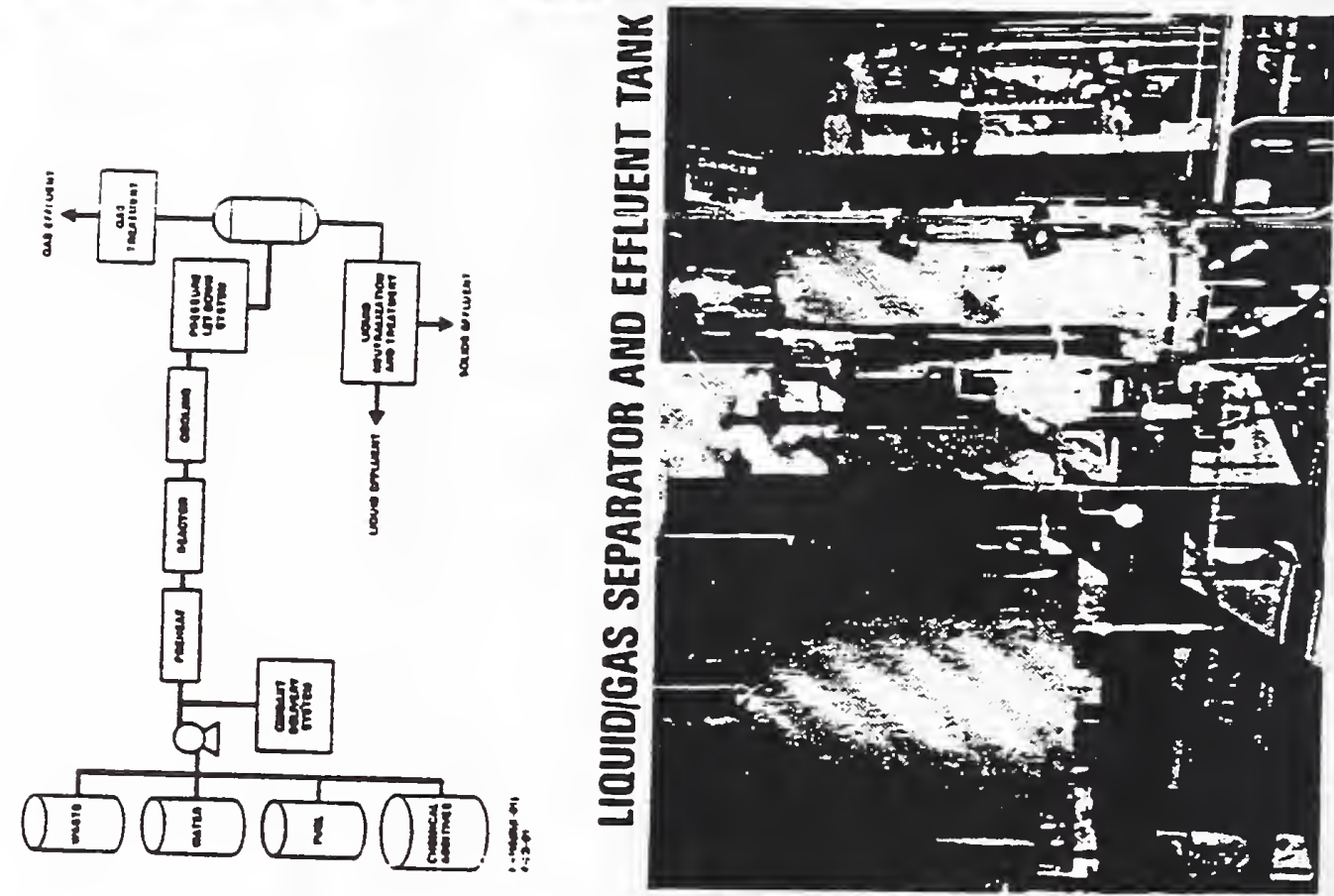
政

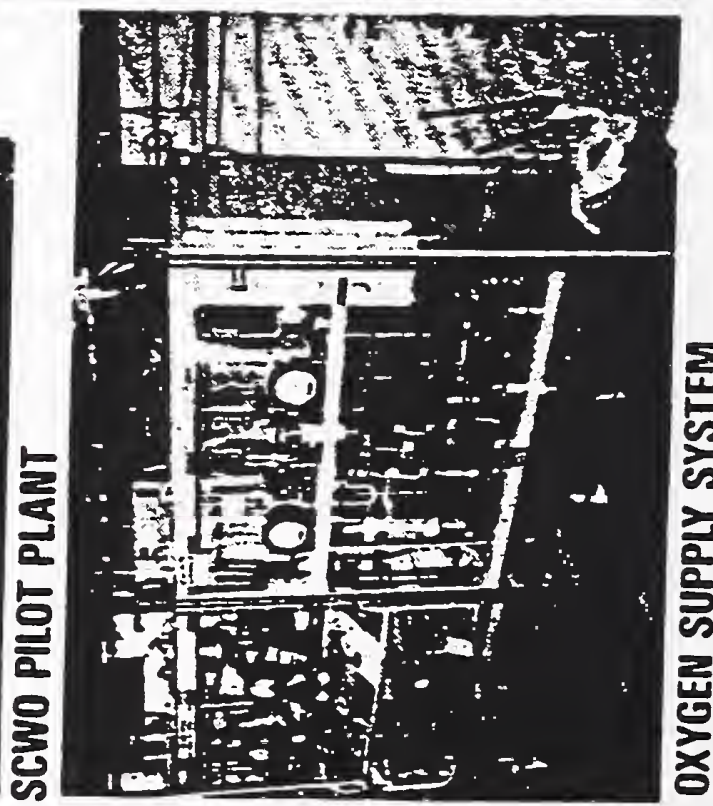

్ㅎ

$=0$

○ فنا 


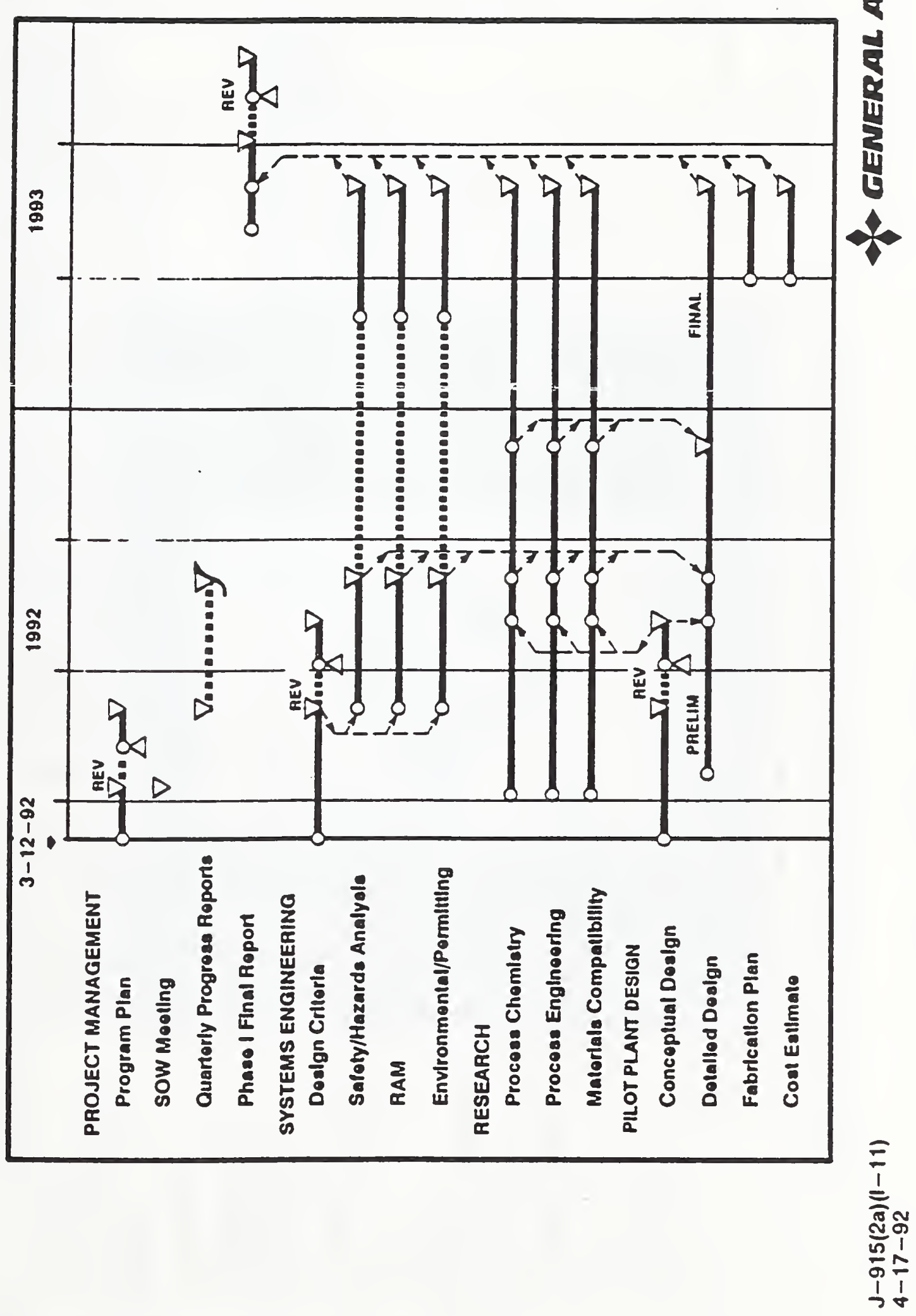




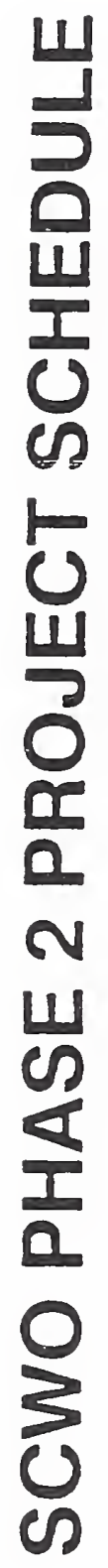

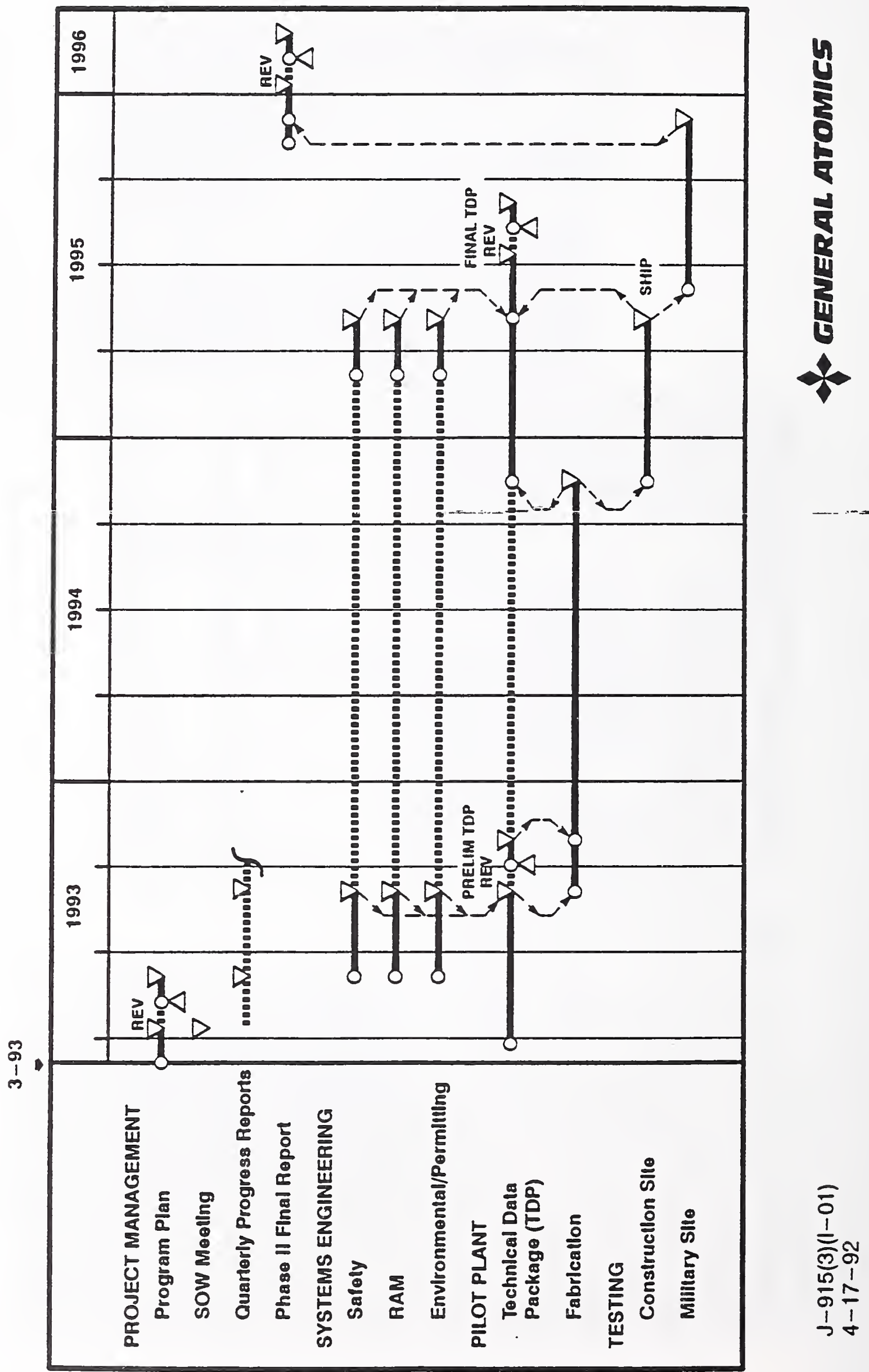



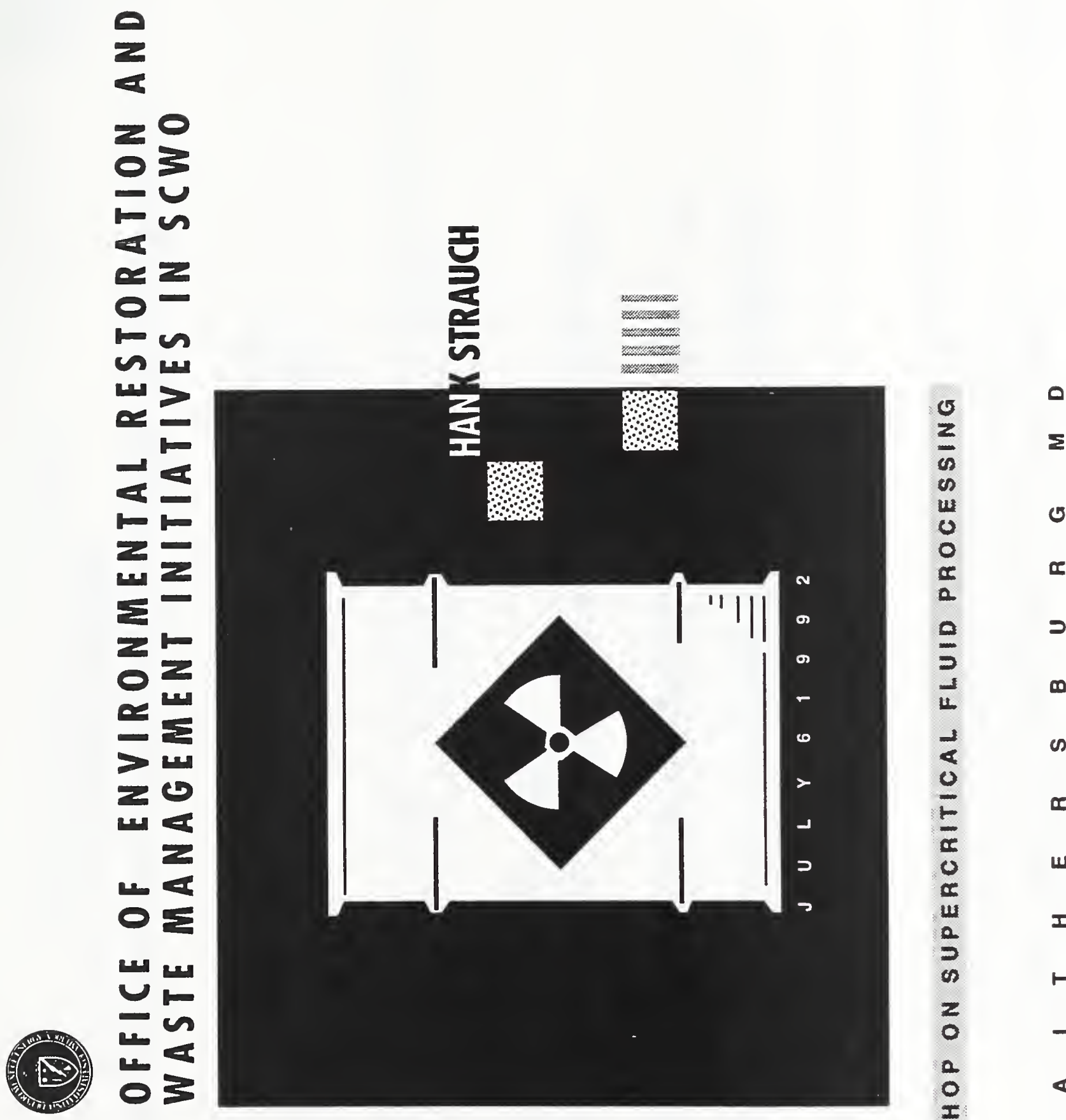

a

a

5

$\overrightarrow{4}$

$\boldsymbol{\infty}$

$\frac{1}{2}$

1

F

c

o

c

II

a.

$\infty$

2

$a$

I

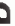




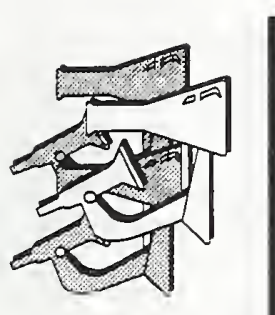

울
$\frac{2}{2}$
$\frac{2}{0}$

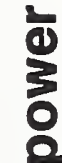

3

6

4

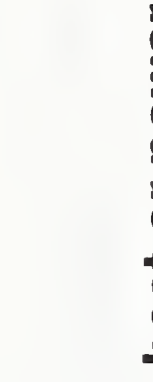

ㅎ

(c)

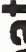

동

๑ 8 है

(0)

응

(1)

5

$+$

\&

를

다 0

$\Phi$

82

르 ㅎํ

0 응

으 ह

2

(1) 5

잉 을 을

क

$\rightarrow$ 10

$\infty$

o) 0

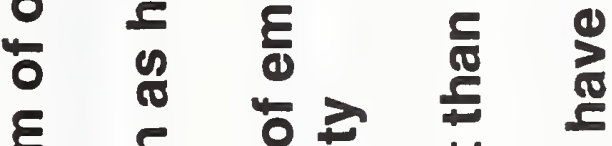

른 을 $\frac{0}{2}=$

$\frac{0}{9} \quad \frac{1}{3}$

응

을 을

क

(1) क ह

응 응

\% है क है

๘

(1)

ㅇ.

เ 40

\& क ल

다을

요 을

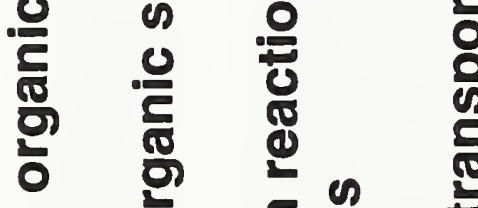

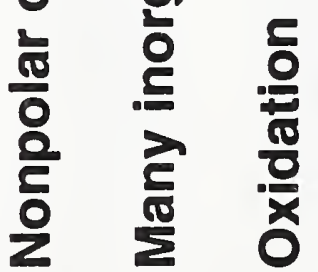

$4 \quad \frac{0}{0}$

응

क के

20

复

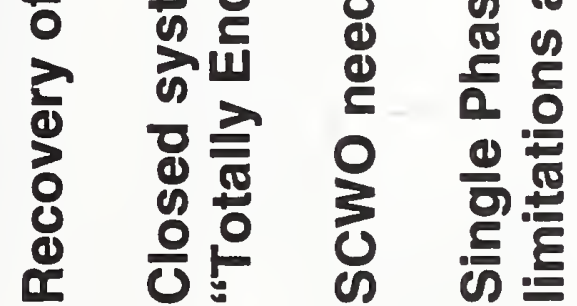




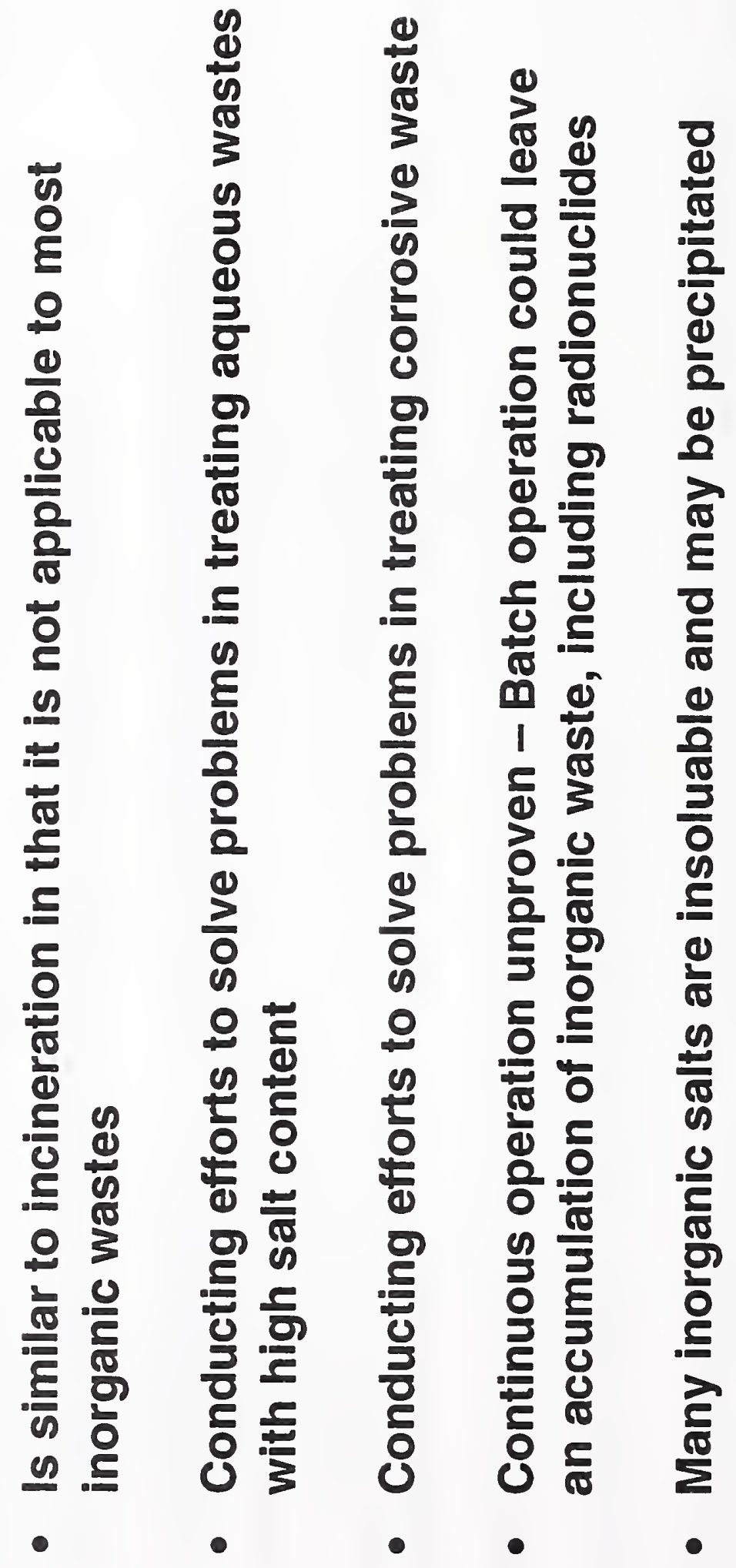




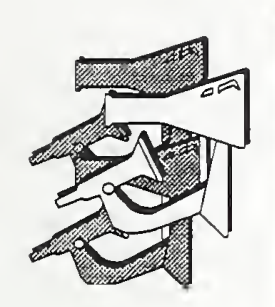

$\frac{0}{0}$

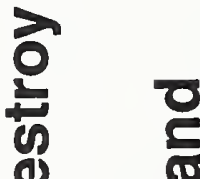

(1)

을

을

잉

$\sum_{0}^{0}$

0

3

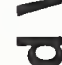

8

2

ㄴํㄴ 응

응

d)

I

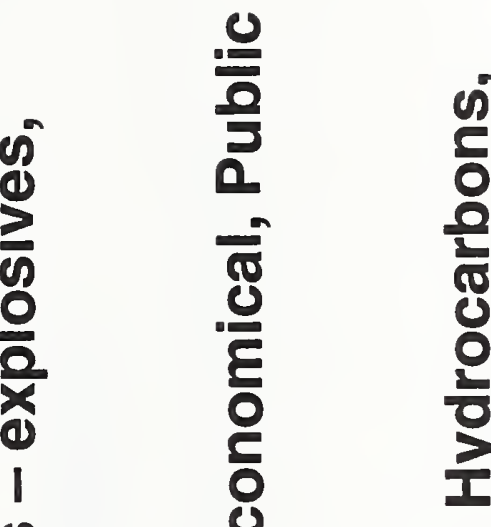

$+\quad+2$

(])

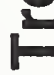

車

$\rightarrow$

in

$-\infty$

을

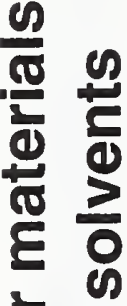

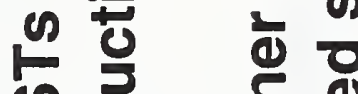

동

$+2$

(1)

1 过

응

- 0 \% 0

$3 \geqslant \frac{2}{10}$

30

क

क

tᄒ

$\geqslant \geqslant$ 른

를 응

응 응

음 0

응 은 든

궁ㅎㅇ 중

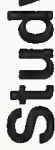

क के क

$\geq \%$

3

$\infty \%$

$4 \frac{0}{\circ}$

$\geqslant x$

U

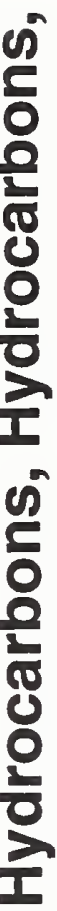

등

$\Phi \omega$

ํㅜㅇ

ఏ)

$\circ 0$

कृ

I

is

오

$\frac{1}{0}$

ㅇำ

흥

엉

0

路

\%

$+$

e

- Q ह प

등 ڤ

은 흥 을

흥 웡 잉

30

(1)

ț

党 


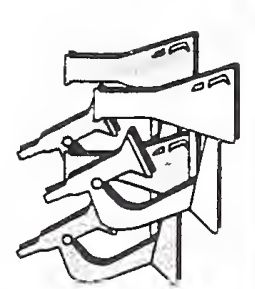

0
0
0

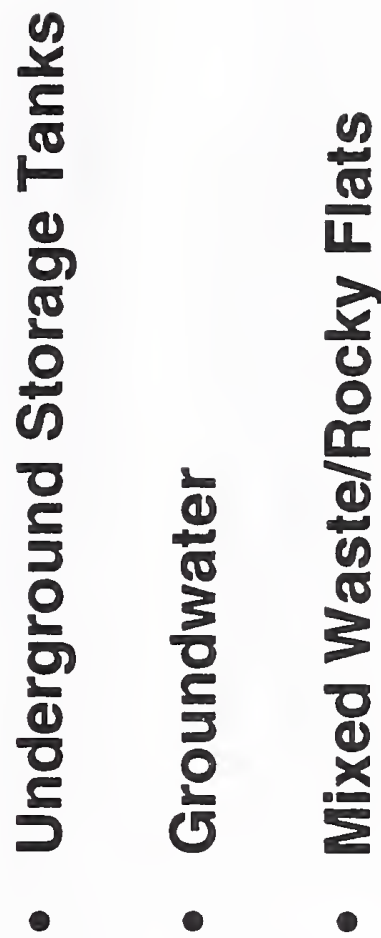




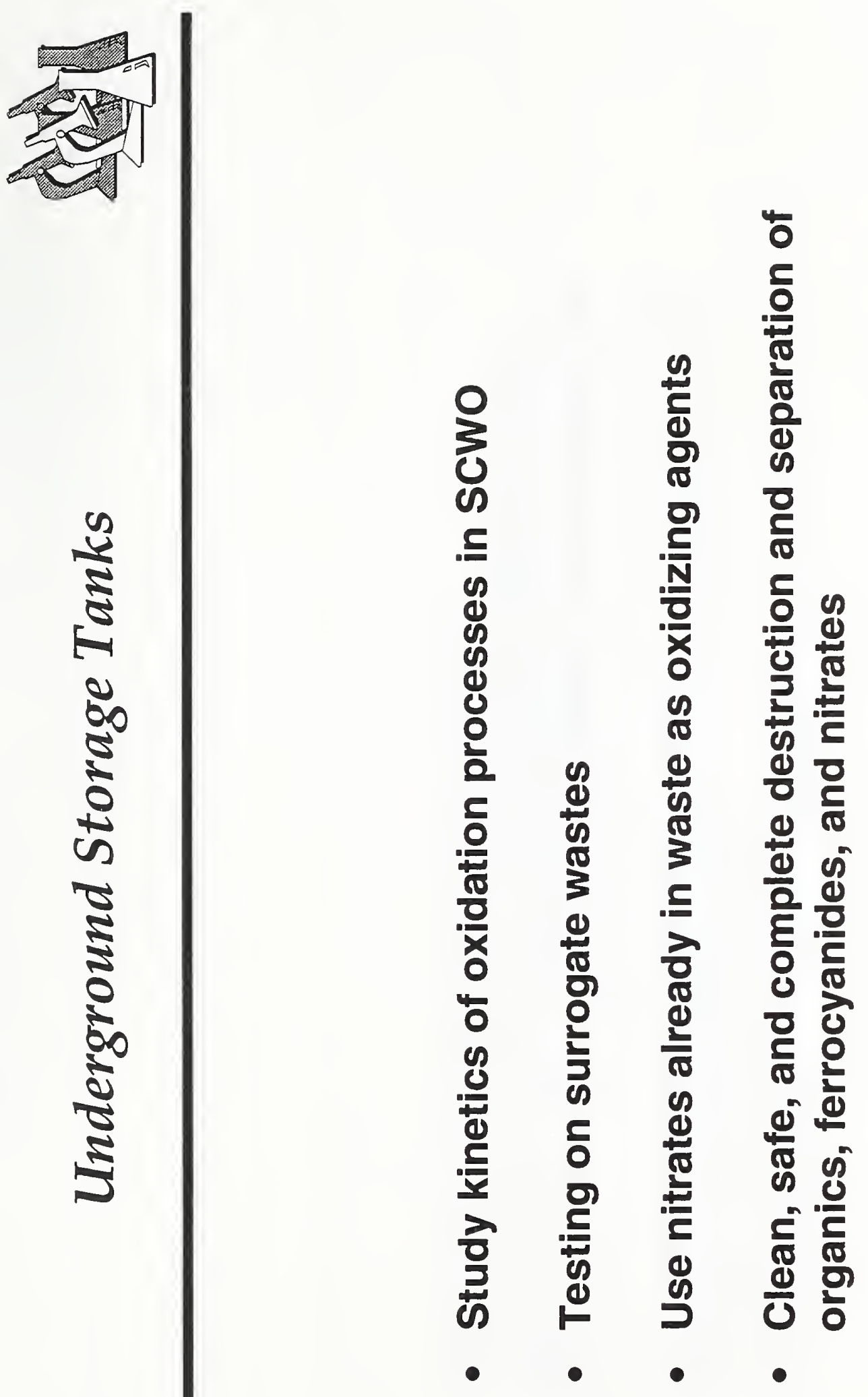




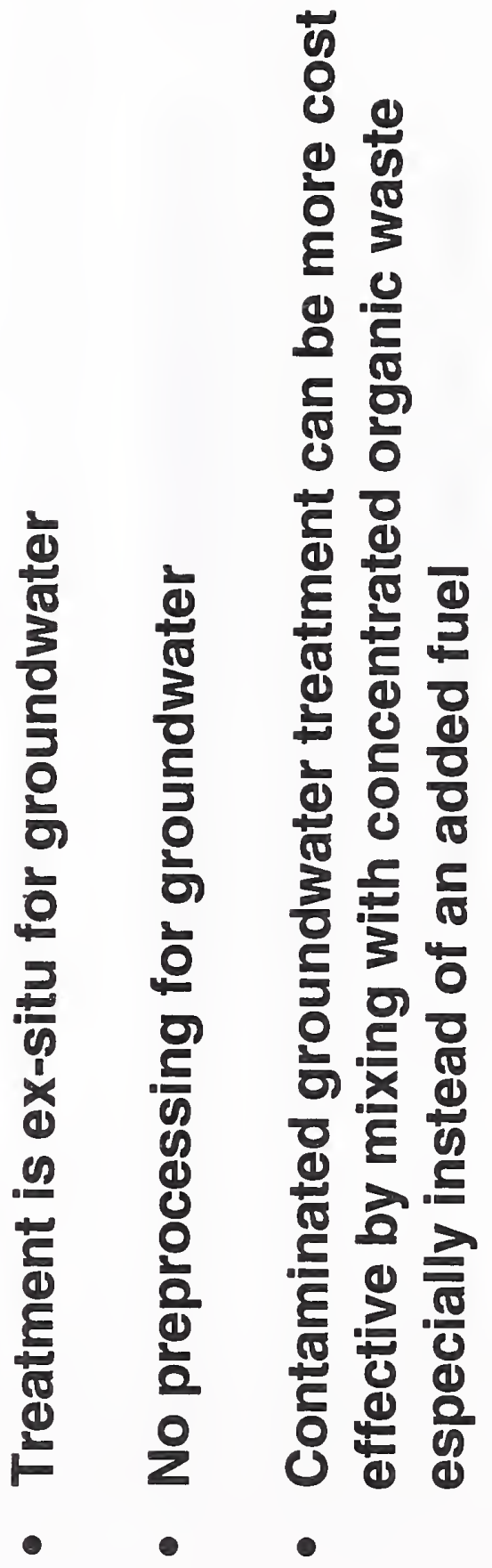




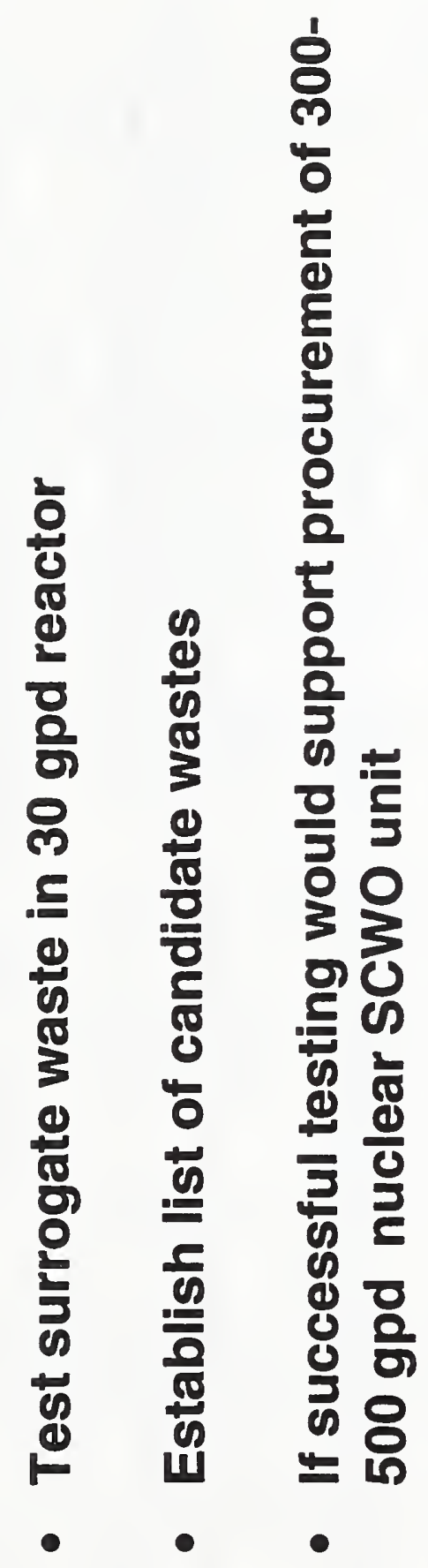



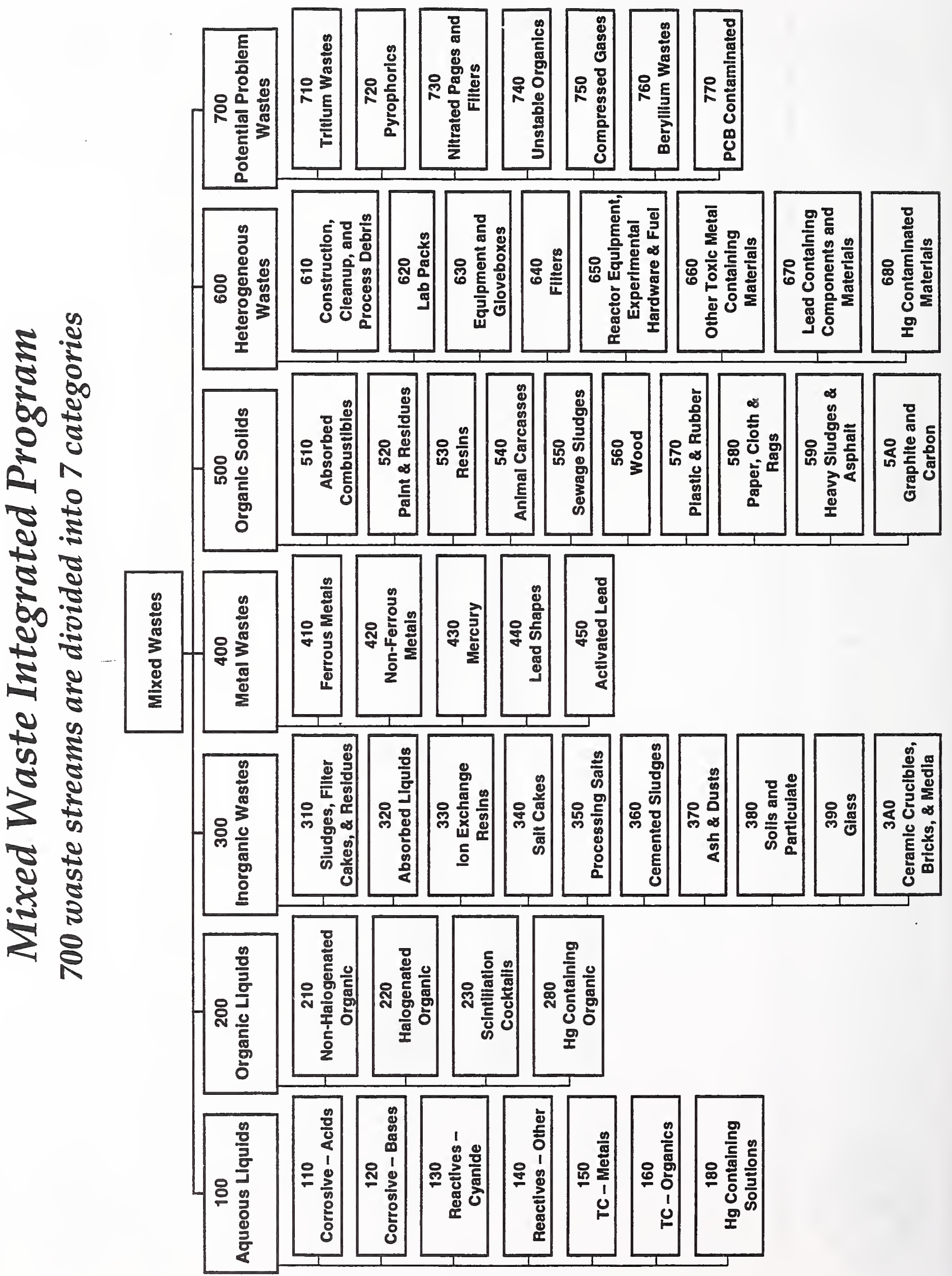


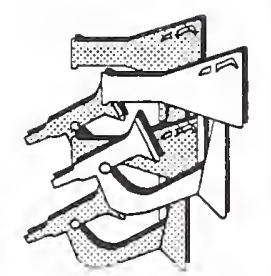

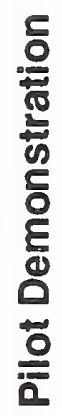
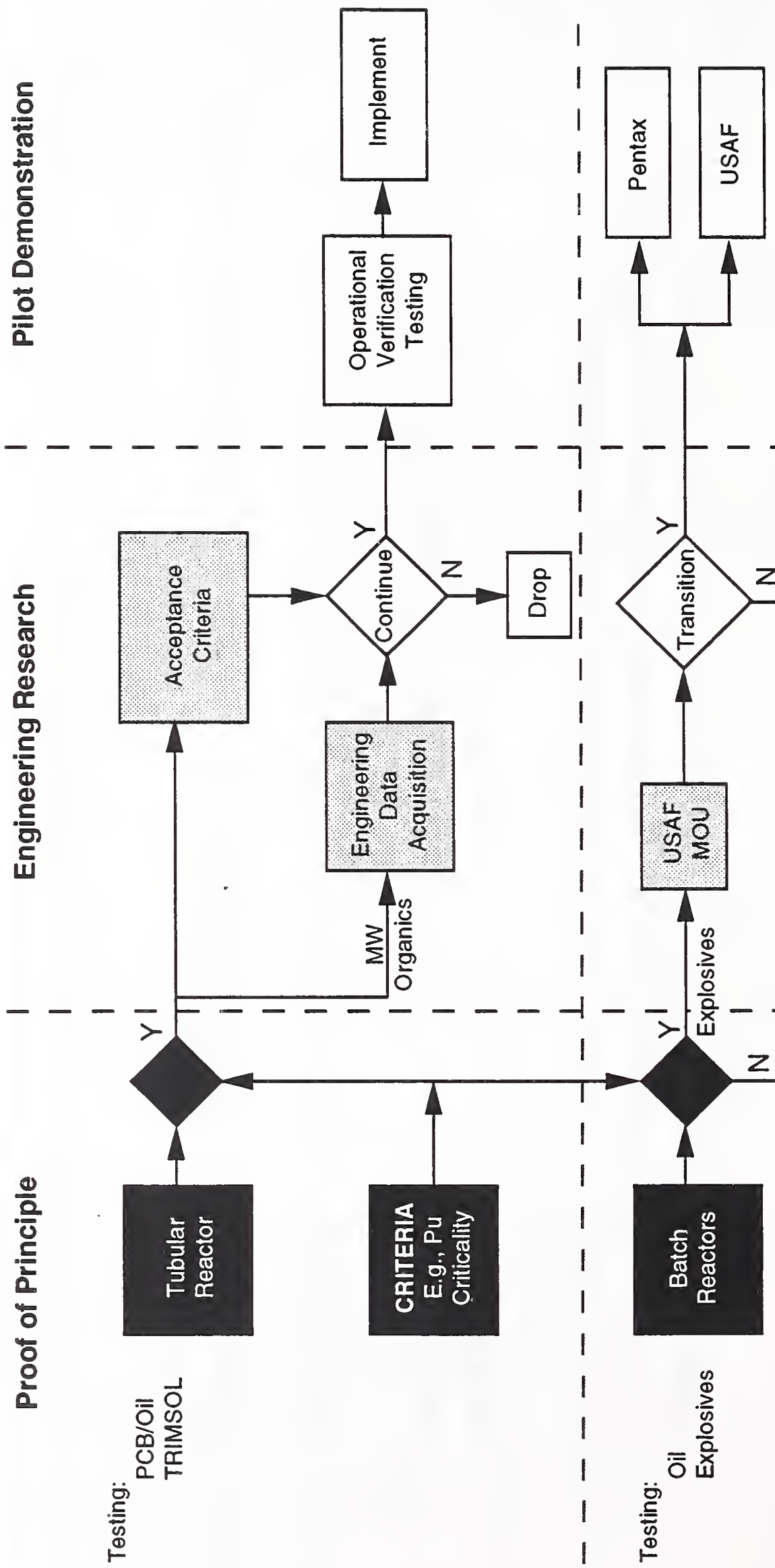

은
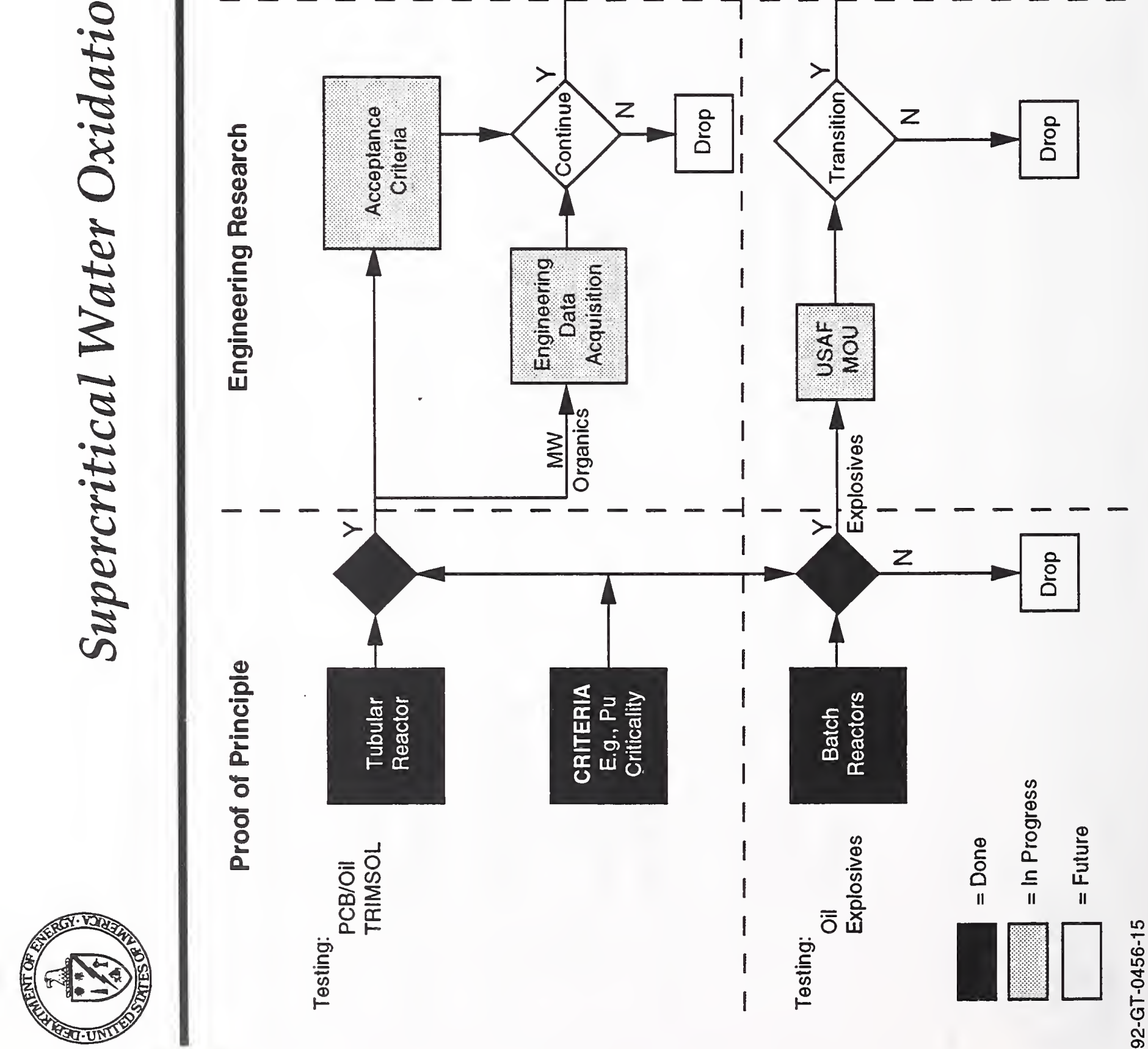


\section{WORKSHOP ON FEDERAL PROGRAMS INVOLVING SUPERCRITICAL WATER OXIDATION}

\section{National Institute of Standards and Technology Gaithersburg, MD 20899 \\ Administration Bldg. July 6-7, 1992}

\section{List of participants}

Dr. Steven J. Buelow

Los Alamos National Laboratory

Mail Stop J567

Los Alamos, NM 87545

Phone 505-667-1178

FAX 505-665-4817

Dr. Ugo Bertocci

National Institute of Standards and Technology

Bldg. 223 Rm. B254

Gaithersburg, MD 20899

Phone 301-975-6017

FAX 301-926-7975

Mr. Bob Chappell

DoE Idaho Operations

785 DoE Place - Mail Stop 1230

Idaho Falls, ID 83402

Phone 208-526-0085

FAX 208-526-5964

Dr. Philippe Chastagner

Westinghouse Savannah River

735AD 106

P.O. Box 616

Aiken, SC 29808

Phone 803-725-3293

FAX 803-725-5377
Dr. Nina Bergan French

Sandia National Laboratories

Livermore, CA 94551-0969

Phone 510-294-3185

FAX 510-294-1015

Dr. Daniel Friend

National Institute of Standards and

Technology

Bldg. 2 Rm. 1305

Boulder, CO

Phone

303-497-5424

FAX 303-497-5224

Mr. T. Mark Hightower

NASA Ames Research Center

M/S 239-11

Moffett Field, CA 94035

Phone 415-604-4443

FAX 415-604-1092

Mr. James A. Hurley

National Institute of Standards and Technology

Bldg. 2 MS 832.01

Boulder, CO 80303

Phone 303-497-3758

FAX 303-497-5044 
Dr. Sheridan Johnston

Sandia National Laboratories

Organization 8103

Livermore, CA 94551-0969

Phone 510-294-2138

FAX 510-294-1004

Dr. Richard F. Kayser

National Institute of Standards and

Technology

Bldg. 221 Rm. A105

Gaithersburg, MD 20899

Phone 301-975-2483

FAX $\quad 301-869-4020$

Mr. Richard E. Kirts

Naval Civil Engineering Laboratory

Code L74B

Port Hueneme, CA 93043

Phone 805-982-1334

FAX 805-982-1409

Dr. Richard Loda

DARPA/DSO/MSD

3701 N. Fairfax Drive

Arlington, VA 22303-1714

Phone 703-696-2283

FAX 703-696-2201

Dr. Andrej Macek

National Institute of Standards and Technology

Bldg. 221 Rm. B306

Gaithersburg, MD 20899

Phone

301-975-2610

FAX

301-926-5002
Dr. Robert Mesmer

Oak Ridge National Laboratory

Bldg. 4500-S MS 6110

P.O. Box 2008

Oak Ridge, TN 37831-6110

Phone

615-574-4958

FAX

615-576-5235

Mr. Dennis Miller

U.S. Dept. of Energy, EM-50

Mail Stop GEO-45

1000 Independence Ave.

Washington, DC 20285

Phone

202-586-3022

FAX

202-586-8872

Ms. Grace Ordaz

U.S. Department of Energy

Mail Stop EM-541

1001 Independence Ave.

Washington, DC 20285

Phone 301-903-7440

FAX

Mr. Chris Otten

Federal Office for Military Technology and Procurement

SG III-3

5400 Koblenz

Germany

c/o Naval Surface Warfare Center,

Carderock Division

Annapolis, MD 21402

Phone 410-267-3732

Fax 410-267-4874 
Mr. Kevin T. Price

U.S. Department of Energy

Idaho Field Office

Idaho Falls, ID 83402

Phone 208-526-5192

FAX 208-526-1184

Lt. Janine Ramirez

WL/MNME

Eglin AFB, FL $32542-5434$

Phone 904-882-4212

FAX $\quad 904-882-3540$
Mr. Anthony T. Rodriguez

David Taylor Research Center

Code 2834

Annapolis, MD 21402-5067

Phone 410-267-2157

FAX 410-267-4874

Dr. Gregory J. Rosasco

National Institute of Standards and Technology

Bldg. 221 Rm. B306

Gaithersburg, MD 20899

Phone $301-975-4813$

FAX 301-926-5002

Dr. Roger Schneider

Rho Sigma Associates 4906 N. Idlewild Ave. Whitefish Bay, WI 53217-5968

Phone

414-332-0138

FAX

Phone 301-975-6023

FAX 301-926-7975

Dr. Michael Rigdon

Institute for Defense Analysis

1801 North Beauregard St.

Alexandria, VA 22311-1772

Phone 703-578-2870

FAX 703-578-2877

Dr. Hratch G. Semerjian

National Institute of Standards and Technology

Bldg. 222 Rm. A317

Gaithersburg, MD 20899

Phone 301-975-3145

FAX 301-975-3845

Mr. Crane Robinson

U.S. Army Armament Research, Development and Engineering Center SMCARAES-P

Picatinny Arsenal, NJ 07806-5000

Phone

201-724-5839

FAX

201-724-3162

Dr. J.M.H. Levelt Sengers

National Institute of Standards and Technology

Bldg. 221 Rm. A105

Gaithersburg, MD 20899

Phone

$301-975-2463$

FAX 
Ms. Carolyn Shapiro

EG\&G Idaho

P.O. Box 1625

Idaha Fall, ID 83415-3416

Phone 208-526-8688

FAX 208-526-9779

Mr. Richard Steeper

Sandia National Laboratories

Div. 8364

P.O. Box 764

Livermore, CA 94551

Phone 510-294-2618

FAX 510-294-1004

Dr. Hank Strauch

BDM International

12850 Middlebrook Rd.

Suite 300

Germantown, MD 20874

Phone 301-601-1113

FAX 301-601-1107

Dr. Wing Tsang

National Institute of Standards and

Technology

Bldg. 222 Rm. A260

Gaithersburg, MD 20899

Phone 301-975-2507

FAX 301-926-4513

Dr. Gideon Varga, CE221

U.S. Department of Energy

1000 Independence Ave. S.W.

Washington, DC 20585

Phone 202-586-0082

FAX 202-586-7114/8134
Dr. James F. Welch

National Institute of Standards and Technology

Bldg. 2 MS 832.01

Boulder, CO 80303-3328

Phone 303-497-3758

FAX 303-497-5044

Dr. Robert E. Zier

BDM International

12850 Middlebrook Rd

Suite 300

Germantown, MD 20874

Phone

301-601-1152

FAX

301-601-1107 


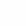




\begin{tabular}{|rr}
\hline NIST-114A & U.S. DEPARTMENT OF COMMERCE \\
(REV. 3-90) & NATIONAL INSTITUTE OF STANDARDS AND TECHNOLOGY \\
& BIBLIOGRAPHIC DATA SHEET
\end{tabular}

1. PUBLICATION OR REPORT NUMBER NISTIR 4920

2. PERFORMING ORGANIZATION REPORT NUMBER

3. PUBLCATION DATE

SEPTEMBER 1992

\section{TITLE AND SUBTITLE}

Proceedings of the Workshop on Federal Programs Involving Supercritical Water Oxidation Technology, July 6-7, 1992.

5. AUTHOR(S)

Edited by Gregory J. Rosasco

6. PERFORMING ORGANIZATION (IF JOINT OR OTHER THAN NIST, SEE INSTRUCTIONS)

U.S. DEPARTMENT OF COMMERCE

NATIONAL INSTITUTE OF STANDARDS AND TECHNOLOGY

GAITHERSBURQ, MD 20899

7. CONTRACT/GRANT NUMBER

$N / A$

8. TYPE OF REPORT AND PERIOD COVERED

9. SPONSORING ORGANIZATION NAME AND COMPLETE ADDRESS (STREET, CITY, STATE, ZIP) N/A

N/A

10. SUPPLEMENTARY NOTES

N/A

11. ABSTRACT (A 200-WORD OR LESS FACTUAL SUMMARY OF MOST SIGNIFICANT INFORMATION. IF DOCUMENT INCLUDES A SIGNIFICANT BIBUOGRAPHY OR UTERATURE SURVEY, MENTION IT HERE.)

The Workshop on Federal Programs Involving Supercritical Water Oxidation, held July 6 \& 7 , 1992 at NIST Gaithersburg, was a follow-on to previous informal meetings held to discuss work in this area. The first was held at the Naval Civil Engineering Laboratory, Port Hueneme, CA in March 1990 and the second at Tyndall Air Force Base, FL in April 1991. As with past meetings, the focus of this Workshop was primarily programmatic not technical. These proceedings include the following:

- An Executive Summary

- List of the attendees, their addresses and phone numbers

- Schedule, including the laboratory visits at NIST, of this two day workshop

- Copies of the speakers' viewgraphs. Some speakers submitted additional material which also is included.

- an SCWO Track Chart which summarizes some of the organizations involved in SCWO technology development and their activities.

12. KEY WORDS (6 TO 12 ENTRIES; ALPHABETICAL ORDER; CAPITALIZE ONLY PROPER NAMES; AND SEPARATE KEY WORDS BY SEMICOLONS)

Chemical agents, chemical kinetics, explosives, hazardous wastes, industrial wastes, materials performance, nuclear mixed wastes, phase behavicr, propellants, supercritical water

\section{AVAILABILITY}

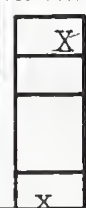

UNLIMITED

FOR OFFICIAL DISTRIBUTION. DO NOT RELEASE TO NATIONAL TECHNICAL INFORMATION SERVICE (NTIS).

ORDER FROM SUPERINTENDENT OF DOCUMENTS, U.S. GOVERNMENT PRINTING OFFICE, WASHINGTON, DC 20402.

ORDER FROM NATIONAL TECHNICAL INFORMATION SERVICE (NTIS), SPRINGFIELD, VA 22161.
14. NUMBER OF PRINTED PAGES

30.7

15. PRICE

A14 
. 
